$$
\text { Teacher }
$$

Internship
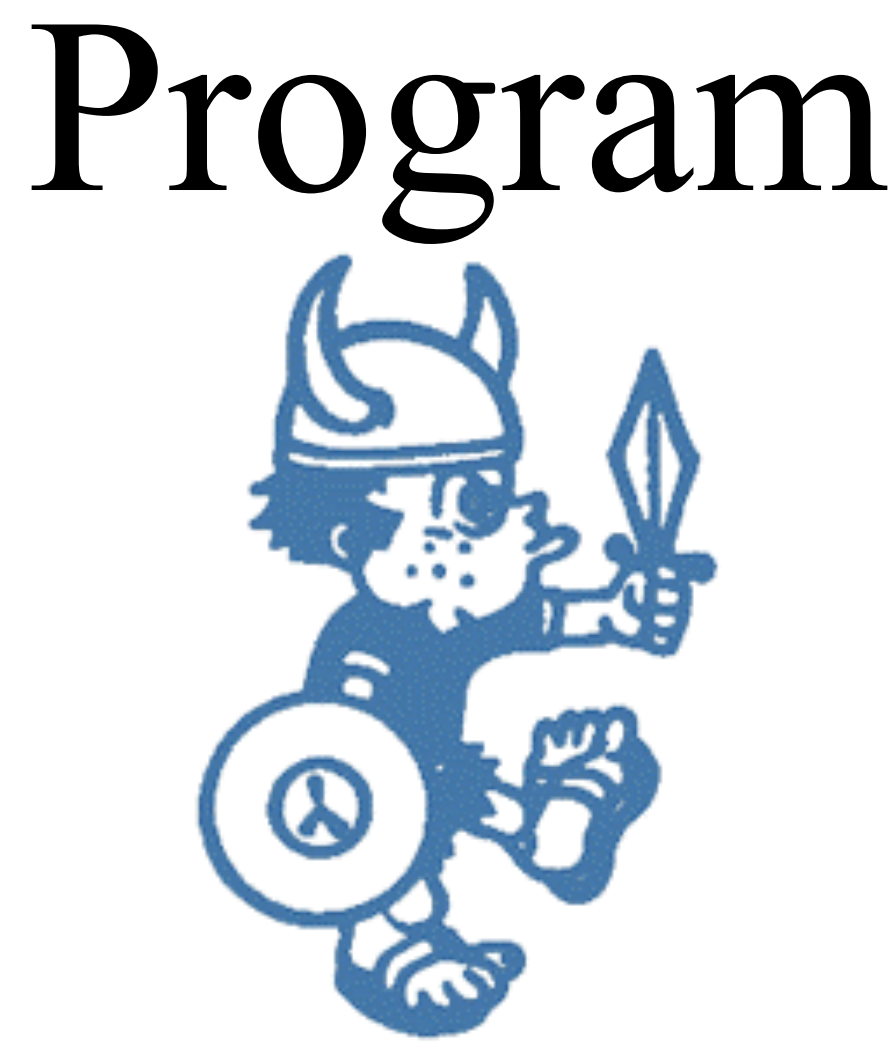

By: Stephanie

Blodgett 


\section{Table Of Contents}

Ag Chemistry Project.............................................................. .

Ag Science Project............................................................

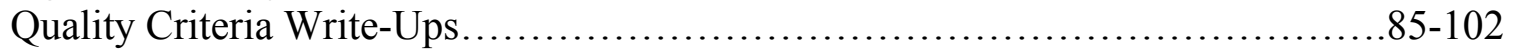

Student Data Sheets...................................................... 103-122

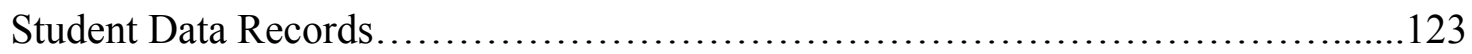

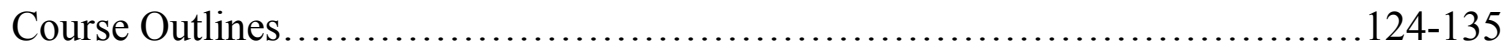

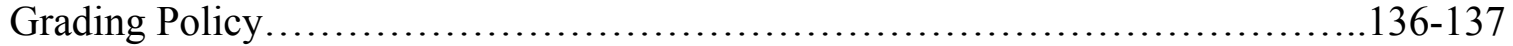

SAE Project Supervision...................................................138-147

SAE Wall Chart................................................................. 148-149

Board Approved Policies.................................................... 150

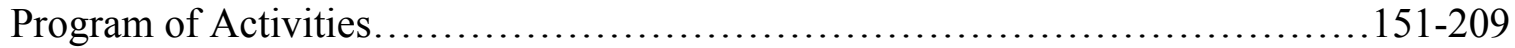

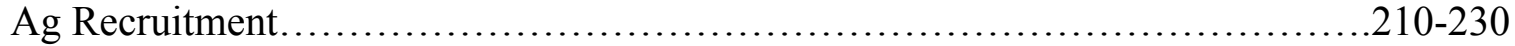

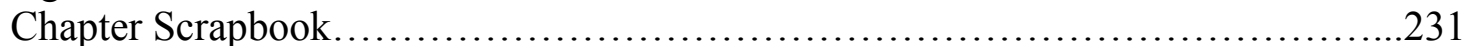

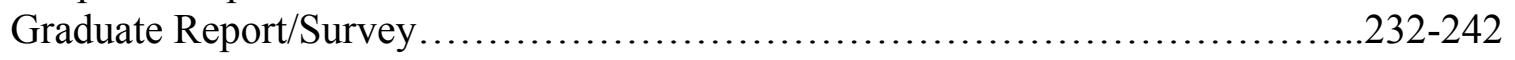

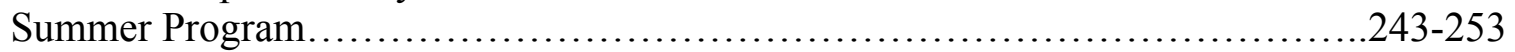

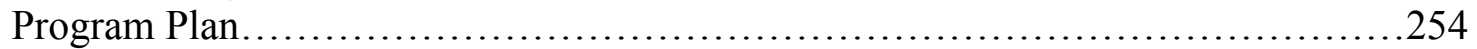

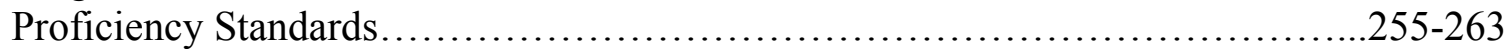

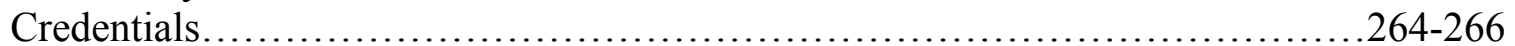

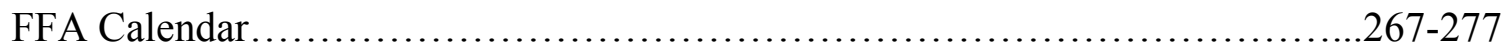

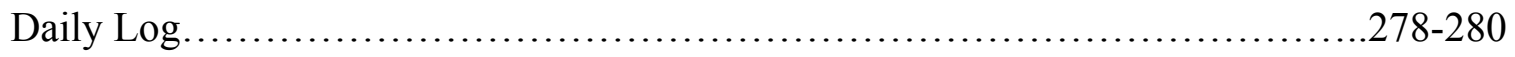

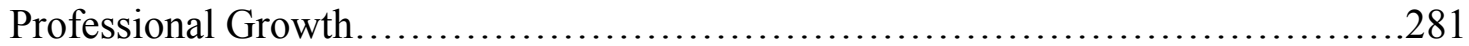

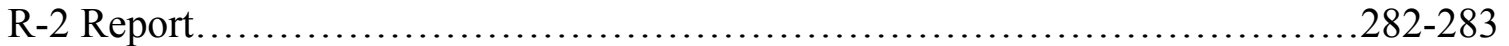

Extra Work Agreement.....................................................284-286

Travel/Conference...........................................................287-290

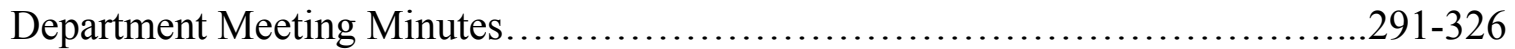

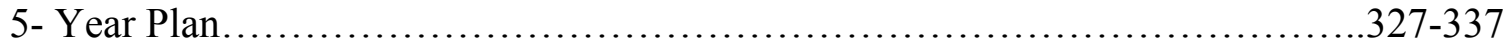

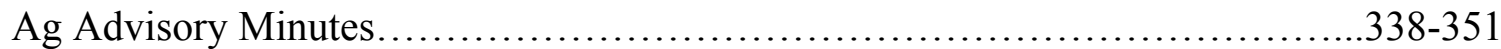

Ag Advisory Program of Activities.............................................

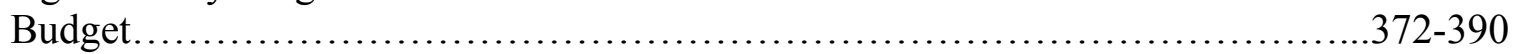

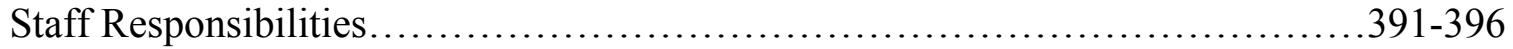

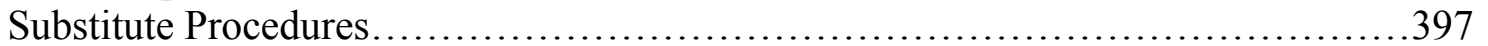

Proficiency for VoAg Students................................................398-402

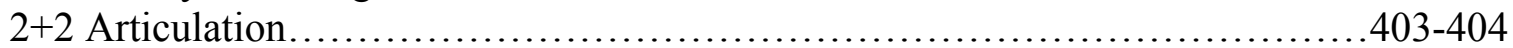

Reimbursements....................................................... 405-410

Student Teacher Ratio......................................................... 411

Lab Activity Awareness....................................................... 412 


\title{
Teacher Internship Program Ag Ed 539
}

\author{
Project: Write the Ag Chemistry Curriculum \\ for the district and get it \\ UC approved.
}

The project that I decided to do for the Teacher Internship Program was to write the ag chemistry curriculum for the district and get it UC approved. In the Salinas Union High School District there has been a push for all classes to meet the A through G requirements and UC approval. In today's time it is tough for an agriculture program in the Salinas area to survive with only electives. Parents, teachers, and administration are pushing for higher API scores and more academic courses. In the last several years our district has added Ag Biology, Ag Chemistry, Ag Government/Economics, Environmental Horticulture, and the Art and History of Floral Design. All of these courses meet a science, history, or art pathway and have been UC approved or are in the process of UC approval. Below are the steps that it took to write the $\mathrm{Ag}$ Chemistry curriculum and get it UC approved so that if someone wanted to they could follow these steps to write curriculum and get it UC approved.

\section{Step 1: Write the Curriculum}

After working at North Salinas High School for one year I began teaching $\mathrm{Ag}$ Chemistry in my second year. I assumed there was curriculum for the course because it was a district approved course, but not one person in the district knew where or what the curriculum looked like. After a science teacher pushed for the course to be UC approved I realized that it was a bigger issue than what I had anticipated and began by first writing the curriculum. To do this I started with the Chemistry curriculum as a base and added the agriculture leadership component. I first had to retype the Chemistry curriculum and put it into our district format. This format included course title, course description, book, prerequisites, major concepts, expected student outcomes, and suggested teacher activities and strategies. Attached is a copy of the Chemistry curriculum that was written.

\section{Step 2: Submit the curriculum to the district science committee}

The next step after writing the curriculum was to write up a curriculum proposal for the district science curriculum committee to approve. This proposal needed to explain the rationale for the modification of the Agriculture Chemistry course, describing the course and providing evidence of supporting requirements that the agriculture departments in the district would provide. Attached is a copy of this course proposal. The only problem was this particular committee only met every couple of months so timing was critical. On February $16^{\text {th }}, 2010$ the Science curriculum committee meeting was held and they approved the new 
Agriculture Chemistry curriculum with the recommendation that the $\mathrm{Ag}$. Chemistry course be taught by a teacher who is credentialed in chemistry and this teacher be limited to the amount of preps.

\section{Step 3: Get it approved for the district.}

Now that the curriculum had been approved by the district science committee, it had to be approved by the district board at their next meeting. I did not actually attend the board meeting due to a conflict, but I know it was brought to the meeting by Janice Souza, an agriculture teacher from Everett Alvarez High School. She informed me that the board approved the curriculum with no problems.

Step 4: Put the curriculum in a format so that it can be UC approved.

The hardest part of the entire process was not getting the curriculum approved for the district, but putting the already written curriculum into a format that could be submitted for UC approval. Every so often the UC approval process is updated and modified to meet their needs. I ran into this exact problem. When submitting anything for UC approval there is a specific format that lab based curriculum or any curriculum for that matter has to follow. Attached is a basic outline of what the format looks like. I had never done this before and no one around me had either. The principal's secretary who is the one at our school to submit this material was unaware until a few months prior that she was suppose to be keeping up on UC approval for courses. She too had never submitted anything for UC approval.

To begin this process, it was suggested by several other agriculture teachers to contact Lloyd McCabe. He not only sent me copies of other UC approved courses, but contacted my regional supervisor to let him know that I needed help. He also worked with a few key people that actually read and approved/not approved courses at the UC level.

I started out just working with him and the curriculum that I had. I put the Ag Chemistry course into a format that I thought the UC system wanted, but I was wrong. It is much simpler than what I had made it. Following the basic UC approval outline really helped me, but in order to actually get it approved Lloyd McCabe put me in touch with someone who walked me through the entire online UC approval format with what needed to be changed. This was a phone conference which allowed me special back access into the UC approval system.

\section{Step 5: Submit for UC approval}

After placing the curriculum into a format following the outline, I attached, I submitted Ag Chemistry for UC approval. There are three ways to do this. There is what is known as full access, limited access, or no access at all which is what I had. I had to write up the material and hand it over to the principal's secretary where she would input the material as well as submit it. Limited access allows you to input the material for UC approval yourself, but does not allow you to submit it. This gives the administration or whoever on your campus is in charge of UC approval a chance to review the material before it is submitted. Full 
access into the UC system gives you the teacher the full power to input the material for UC approval as well as submit it. In order to actually get the course UC approved it took six submissions, before finally receiving a message that my challenge of getting $\mathrm{Ag}$ Chemistry UC approved was finally over. 


\section{UC Approval Format (Basic Outline)}

1. Course Title

2. Subject Area

3. Grade Level

4. Brief Course Description

5. Pre requisites

6. Context for Course (optional)

7. History of Course Development (optional)

8. Textbook information
a) title
b) edition
c) publication date
d) publisher
e) author

9. Supplemental Instructional Materials

10. Course Purpose

11. Course Outline

12. Laboratory Activities

13. Key Assignments

14. Instructional Methods and/or Strategies

15. Assessment Methods and/or Tools 


\title{
High School \\ Ag Chemistry
}

\section{Science Curriculum}

\section{Assessment Instructional Guide}

\author{
Salinas Union High School District
}




\section{Table of Contents}

Course Description: High School Ag Chemistry page 3

SUHSD Student Performance Objectives: Ag Chemistry page 4

Leadership and Team Building Development page 15

$\begin{array}{ll}\text { Investigation and Experimentation } & \text { page } 16\end{array}$

$\begin{array}{ll}\text { Terminology Guide } & \text { page } 18\end{array}$

Instructional Guide: High School Chemistry page 21

Atomic Structure and the Periodic Table page 21

Chemical Bonding and Balancing Equations page 28

Males and Strichinmetry page 36

Gas Laws page 43

Introduction to Solutions page 52

Chemical Bonds and Structure page 58

$\begin{array}{ll}\text { Thermodynamics } & \text { page } 67\end{array}$

$\begin{array}{ll}\text { Solutions } & \text { page } 73\end{array}$

$\begin{array}{ll}\text { Equilibrium } & \text { page } 78\end{array}$

$\begin{array}{ll}\text { Reaction Rates } & \text { page } 84\end{array}$

$\begin{array}{ll}\text { Acids and Bases } & \text { page } 89\end{array}$

$\begin{array}{ll}\text { Safety } & \text { page } 94\end{array}$ 
DEPARTMENT:

COURSE TITLE:

GRADE LEVEL:

LENGTH:

NUMBER OF CREDITS:

PREREQUISITE:

TEXTBOOK:

\section{Agriculture}

\section{Ag Chemistry}

$11-12$

1 year

10 credits

C or better in Algebra

Chemistry Prentice-Hall 2002

SUPPLEMENTARY MATERIALS: Materials provided by the publisher including study guide and lab manual, CD accompaniment, Videodisc Programs, Web Links

COURSE DESCRUPTION:

A lab-based chemistry course which encompasses concepts set forth in the California State Science Content Standards for California Public Schools and the Science Framework for California Public Schools. Laboratory experiences are a major component of the course in addition to traditional instruction. This course meets the California and Salinas Union High School District graduation requirements for physical science. It qualifies as college lab course, meeting the University of California "A-G" requirements.

\section{SOURCES OF CURRICULUM CONTENT:}

The curriculum incorporates all High School Life Science Blueprint content standards adopted by the California State Board of Education 2004, as required. Clarification of topics and unifying themes have been drawn from Benchmarks for Scientific Literacy (American Association for the Advancement of Science) and from National Science Education Standards (National Research Council). Much of the clarification of state standards and development of concept units is a result of meetings with the planning committee composed of high school chemistry teachers in the Salinas Union High School District who met during 2007-08. 


\section{Salinas Union High School District \\ High School Agricultural Chemistry Instructional Guide}

\section{Agriculture Chemistry Course Outline}

\section{Grades: $9-12$}

\section{Major Concepts}

1. Introduction to Chemistry: (Safety and Emergency Response, Laboratory Techniques and Measurement, Significant Figures and Dimensional Analysis)

2. Introduction to Atoms, the Periodic Table, and Nomenclature

3. Conservation of Matter and Stoichiometry

4. Gas Laws

5. Chemical Bonds

6. Thermodynamics

7. Solutions

8. Chemical Equilibrium

9. Rates of Reaction

10. Acids and Bases

11. Organic Chemistry and Biochemistry (after CST)

12. Nuclear Processes (after CST)

\section{MAJOR CONCEPTS/EXPECTED STUDENT OUTCOMES \\ SUGGESTED TEACHER ACTIVTTIES AND STRATEGIES}

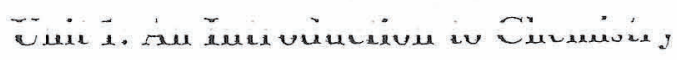

\section{Safety and Emergency Response}

\section{State Standards}

Investigation and Experimentation

1. Scientific progress is made by asking meaningful questions and conducting careful investigations. Students will:

a. Select and use appropriate tools and technology (such as computer-linked probes, spreadsheets, and graphing calculators) to perform tests, collect data, analyze relationships, and display data.

\section{Student Outcomes}

1. Follow all safety rules and precautions as highlighted in the laboratory manual and by the teacher. Students will be expected to pass a safety quiz before the first lab with safety concerns. A grade of $80 \%$ o or better must be obtained before students may participate in laboratory activities.

2. Follow good housekeeping procedures in their laboratory area. This includes good facilities management, equipment and materials storage, chemical handling techniques, and glassware use and storage.

3. Know the location of and how to operate all safety equipment available in the classroom. Protect eyes, face, hands, and body while conducting class and laboratory activities.

4. Dispose of dangerous waste chemicals and materials as prescribed by appropriate standards and laws.

5. Know the appropriate emergency steps to take in the event of an accident or emergency including evacuation procedures. 


\section{Salinas Union High School District \\ High School Agricultural Chemistry Instructional Guide}

\section{Possible Strategies and Activities}

1. Review the appropriate safety procedures before activities and briefly explain specific precautions and hazards associated with the activity.

2. Demonstrate how to store and handle laboratory equipment. Ensure that chemicals are kept in a safe place with limited access to prevent the possibility of hazardous accidents.

3. Check smoke detectors, safety showers, and eyewash facilities on a regular basis. Know how to access all master shutoffs where applicable.

4. Clarify all waste disposal procedures before every activity. Provide separate labeled waste receptacles for waste as needed. Also provide labeled receptacles for broken glass, waste paper, and used matches if applicable.

5. Post your school's accident policy and procedures; make accident reports promptly, accurately, and completely.

\section{Laboratory Techniques and Measurement}

\section{State Standards}

Investigation and Experimentation

1. Scientific progress is made by asking meaningful questions and conducting careful investigations. Students will:

a. Select and use appropriate tools and technology (such as computer-linked probes, spreadsheets, and graphing calculators) to perform tests, collect data, analyze relationships, and display data.

\section{Student Outcomes}

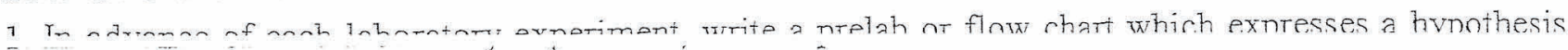
and describes the experimental procedure in the student's own words.

2. Learn the names and functions of the laboratory equipment used in experiments.

3. Understand and use common laboratory terminology.

4. Make all quantitative measurements to the proper level of precision, and state the absolute error for each measurement.

5. Demonstrate competence in performing laboratory procedures. These tasks may include, but are not limited to: transfer of chemicals from stock containers, use of balance (both digital and mechanical, if possible), reading a thermometer, filtration, decantation, operating a Bunsen bumer, reading volumes in graduated cylinders and gas measuring tubes, use of buret, cleaning and rinsing glassware, handling hot equipment and/or solutions, operation of specialized equipment such as colorimeters, $\mathrm{pH}$ meters, or spectrophotometers.

6. Perform appropriate analysis of experimental data, draw logical conclusions, and report findings in a clearly written laboratory report.

\section{Possible Strategies and Activities}

1. Provide guidelines for proper prelab procedures.

2. Identify the equipment used in each laboratory experiment, clarify the function of each item, and insist that students use the proper terms when referring to the equipment during class discussion and in written laboratory reports.

3. Model the use of proper terminology when discussing laboratory procedures, and require students to employ the correct terms when discussing their experiments.

4. Demonstrate how to make quantitative measurements to the highest possible level of precision with each instrument that is used in the laboratory. Follow-up may include putting emphasis on precision 


\section{Salinas Union High School District \\ High School Agricultural Chemistry Instructional Guide}

statements in written laboratory reports as well as consistent modeling when, discussing treatment of quantitative laboratory data during class discussions.

5. Demonstrate the correct procedure for each specialized lab task that is introduced in student experiments, and correct students when necessary while observing their work in, the laboratory. Supporting strategies include requiring students to clearly explain each lab technique in their written flow sheets, and giving a "lab practical" exam in which students' lab skills are formally evaluated.

6. Provide students with a format for writing laboratory reports, evaluate students' reports in a timely manner, and give students suggestions for improving their work as the course progresses.

\section{Significant Figures and Dimensional Analysis (Factor Label Method)}

\section{State Standards}

Investigation and Experimentation

1. Scientific progress is made by asking meaningful questions and conducting careful investigations. Students will:

b. Select and use appropriate tools and technology (such as computer-linked probes, spreadsheets, and graphing calculators) to perform tests, collect data, analyze relationships, and display data.

c. Identify and communicate sources of unavoidable experimental error.

d. Identify possible reasons for inconsistent results, such as sources of error or uncontrolled conditions.

e. Formulate explanations by using logic and evidence.

f. Solve scientific problems by using quadratic equations and simple trigonometric, exponential, and logarithmic functions.

k. Recognize the issues of statistical variability and the need for controlled tests.

$m$. Analyze situations and solve problems that require combining and applying concepts from more than one area of science.

\section{Student Outcomes}

1. Demonstrate a consistent use of units in measurements and calculations.

2. Use the factor label method in the solution of all problems and laboratory calculations assigned throughout the year.

3. Use estimation techniques incorporating dimensional analysis to arrive at approximate values when assigned to do so by the teacher.

4. Demonstrate skills in the use of fractions, scientific notation, exponents, graphing, graph interpretation, and the correct use of significant digits to indicate the appropriate degree of uncertainty.

\section{Possible Strategies and Activities}

1. Provide direct instruction and consistently model the use of appropriate units associated with measurements.

2. Teach factor labeling as a means of solving of problems both from laboratory experiences and from hypothetical situations.

3. Model and provide opportunities for estimation using orders of magnitude; arriving at approximate values; and indicating appropriate degrees of uncertainty.

4. Review basic mathematical skills for use in chemistry.

5. Monitor and evaluate student learning. Make adjustments as shown necessary in the monitoring process, with prompt correction and review.

6. Select appropriate materials and media to enhance learning, including some of the following: measurement lab with significant figures calculations; density lab; graphing lab 


\section{Salinas Union High School District \\ High School Agricultural Chemistry Instructional Guide}

\section{Unit 2: Introduction to Atoms, the Periodic Table, and Chemical Nomenclature}

\section{State Standards}

\section{Atomic and Molecular Structure}

1. The periodic table displays the elements in increasing atomic number and shows how periodicity of the physical and chemical properties of the elements relates to atomic structure. As a basis for understanding this concept:

a. Students know how to relate the position of an element in the periodic table to its atomic number and atomic mass.

b. Students know how to use the periodic table to identify metals, semimetals, non-metals, and halogens.

c. Students know how to use the periodic table to identify alkali metals, alkaline earth metals and transition metals, trends in ionization energy, electronegativity, and the relative sizes of ions and atoms.

d. Students know how to use the periodic table to determine the number of electrons available for bonding.

e. Siudents know the nucleus of the atom is much smaller than the atom yet contains most of its mass.

f. * Siudents know how to use the periodic table to identify the lanthanide, actinide, and transactinide elements and know that the transuranium elements were synthesized and identified in laboratory experiments through the use of nuclear accelerators.

h. *Students know the experimental basis for Thomson's discovery of the electron, Rutherford's nuclear atom, and Millikan's oil drop experiment.

\section{Chemical Bonds}

2. Biological, chemical, and physical properties of matter result from the ability of atoms to form bonds from electrostatic forces between electrons and protons and between atoms and molecules. As a basis for understanding this concept:

a. Students know atoms combine to form molecules by shanng electrons to torm covalent or metallic bonds or by exchanging electrons to form ionic bonds.

\section{Student Outcomes}

1. Students will be able to describe the basic structure of the atom and the experimental evidence that lead to our understanding of atomic structure.

2. Students will know the basic organization of the periodic table.

3. Students will know that compounds are formed when ions are held together by electrostatic forces or when atoms share electrons to form molecules.

4. Students will be able to name and write formulas for ionic and molecular compounds and distinguish between the two by noting whether a metal and a non-metal or two non-metals are combining. In order to do this; students must be familiar with both the locations of metals and non-metals on the periodic table, and with the use and the naming of polyatomic ions.

\section{Possible Strategies and Activities}

1. Provide a historic overview of the history of the discovery of the atom, subatomic particles, and the periodic table. This overview will be accompanied by lab, audiovisual, lecture, and research (including Internet assignments).

2. Explain all the parts of the periodic table including the distinction between groups and families, metals and non-metals, specific families, valence numbers, and how to determine ionic charges.

3. Have students memorize the formula and name for the common polyatomic ions.

4. Have students write IUPAC names for compounds based on their formulas and formulas based on names. 


\section{Salinas Union High School District \\ High School Agricultural Chemistry Instructional Guide}

\section{Unit 3: Conservation of Matter and Stoichiometry}

\section{State Standards}

\section{Conservation of Matter and Stoichiometry}

3. The conservation of atoms in chemical reactions leads to the principle of conservation of matter and the ability to calculate the mass of products and reactants. As a basis for understanding this concept:

a. Sudents know how to describe chemical reactions by writing balanced equations.

b. Siudents know the quantity one mole is set by defining one mole of carbon 12 atoms to have a mass of exactly 12 grams.

c. Siudents hnow one mole equals $6.02 \times 10^{23}$ particles (atoms or molecules).

d. Studenis know how to determine the molar mass of a molecule from its chemical formula and a table of atomic masses and how to convert the mass of a molecular substance to moles, number of particles, or volume of gas at standard temperature and pressure.

e. Students know how to calculate the masses of reactants and products in a chemical reaction from the mass of one of the reactants or products and the relevant atomic masses.

f. * Sizdents know how to calculate percent yield in a chemical reaction.

\section{Student Outcomes}

1. Balance chemical equations using the proper chemical symbols and coefficients. 3 a

2. Utilize Avogadro's hypothesis to solve conversion problems involving atomic weight, molar mass, the mole, and molar volume. $3 \mathrm{~b}, 3 \mathrm{c}, 3 \mathrm{~d}, 3 \mathrm{e}$

3. Students will be able to calculate the percent yield in a chemical reaction.

\section{Possible Strategies and Activities}

1. Provide direct instruction that clarities, detines, and provides a consistem patlen lus suiving pivitin in the laboratory and from hypothetical situations.

2. Give students numerous opportunities to perform molar calculations, using stoichiometric ratios, Avogadro's number, molar mass, and molarity.

3. Provide extensive practice with prompt correction and review of assigned problems.

4. Select appropriate materials in the form of films and software to supplement lecture.

5. Provide laboratory opportunities including calculations of molar mass, mass relationships accompanying chemical changes, and requiring the use of stoichiometric calculations including the calculation of percent yield.

\section{Unit 4: Gas Laws}

\section{State Standards}

\section{Gas Laws}

4. The kinetic molecular theory describes the motion of atoms and molecules and explains the properties of gases. As a basis for understanding this concept:

a. Students know the random motion of molecules and their collisions with a surface create the observable pressure on that surface.

b. Students know the random motion of molecules explains the diffusion of gases.

c. Students know how to apply the gas laws to relations between the pressure, temperature, and volume of any amount of an ideal gas or any mixture of ideal gases.

d. Students know the values and meanings of standard temperature and pressure (STP).

e. Students know how to convert between the Celsius and Kelvin temperature scales.

f. Students know there is no temperature lower than 0 Kelvin. 


\section{Salinas Union High School District}

High School Agricultural Chemistry Instructional Guide

\section{Student Outcomes}

1. Be able to explain the characteristics of gases.

2. Use the following gas law's: Dalton's Law of Partial Pressures, Charles' Law, Boyle's Law, Avogadro's Principle, the Ideal Gas Law, the Combined Gas Law, and Graham's Law. 4c, 4d

3. Be able to graph and exchange the changes of state using heating and cooling curves and label: melting point, boiling point, heat of fusion, heat of vaporization $4 \mathrm{e} .4 \mathrm{f}$

\section{Possible Strategies and Activities}

1. Use models and lecture to illustrate the differences between a solid, liquid and gas on the macro and

2. Define the gas laws and utilize them for problem solving, then provide guided practice and laboratory activities for the students.

3. Select experiments that will facility the students use of laboratory data, and when possible computer graphing software to illustrate heating and cooling curves.

\section{UNIT 5: CHEMICAL BONDS}

\section{State Standards}

2. Biological, chemical, and physical properties of matter result from the ability of atoms to form bonds from electrostatic forces between electrons and protons and between atoms and molecules. As a basis for understanding this concept:

a. Students know atoms combine to form molecules by sharing electrons to form covalent or metallic bonds or by exchanging electrons to form ionic bonds.

b. Students know chemical bonds between atoms in molecules such as $\mathrm{H}_{2}, \mathrm{CH}_{4}, \mathrm{NH}_{3}, \mathrm{H}_{2} \mathrm{CCH}_{2}, \mathrm{~N}_{2}, \mathrm{Cl}_{2}$, and many large biological molecules are covaltul.

c. Students know salt crystals, such as $\mathrm{NaCl}$, are repeating patterns of positive and negative ions held together by electrostatic attraction.

d. Students know the atoms and molecules in liquids move in a random pattern relative to one another because the intermolecular forces are too weak to hold the atoms or molecules in a solid form.

e. Students know how to draw Lewis dot structures.

\section{Students Outcomes}

1. Review the nature and rational of chemical bonding, ionic model, ionic character, oxidation states of families, and covalent bonding.

2. Draw and explain Lewis dot structure, molecular structure, octet rule, valence electrons/pairs.

3. Describe molecular structure, octet rule and exception, resonance.

4. Explain VSEPR model, hybridization, and molecular orbital model.

\section{Possible Strategies and Activities}

1. Students build or observe models of crystal lattices.

3. Build molecular shapes and structures of certain molecules.

4. Use of molecular model sets.

5. Use of Styrofoam balls and colored pipe cleaners to demonstrate valence electrons and molecular shapes. 


\section{Salinas Union High School District}

High School Agricultural Chemistry Instructional Guide

\section{UNIT 6: Thermodymamics}

\section{State Standards}

7. Energy is exchanged or transformed in all chemical reactions and physical changes of matter. As a basis for understanding this concept:

a. Students know how to describe temperature and heat flow in terms of the motion of molecules (or atoms).

b. Students know how chemical processes can either release (exothermic) or absorb (endothermic) thermal energy.

c. Students know energy is released when a material condenses or freezes and is absorbed when a material evaporates or melts.

d. Students know how to solve problems involving heat flow and temperature changes, using known values of specific heat and latent heat of phase change.

\section{Students Outcomes}

1. Explain why energy always flows from warnter to cooler.

2. Describe specific heat is the amount of energy needed to raise the temperature of a substance..

3. Know that specific heat is an inherent property of substances.

4. Chemical and physical changes involve the flow of energy.

5. Be able to solve problems involving specific heat and temperature change.

6. Temperature does not change during a phase change.

\section{Possible Strategies and Activities}

1. Demonstrate calorimeter, Enthalpy, thermo - chemical equations, heats of formation.

2. Solve heat flow problems

3. Calorimeter labs using Styrofoam cups and different metal pieces to identify specific heats of various metals

4. Demonstrate athletic trainer's cold packs (endothermic) and hot packs (exothermic). Liploc oag reactions also work.

5. Show students models of molecular motion of gases, solids and liquids to show energy loss or gain during phase change.

\section{Unit 7: Solutions}

\section{State Standards}

\section{Solutions}

6. Solutions are homogenous mixtures of two or more substances. As a basis for understanding this concept:

a. Students know the definitions of solute and solvent.

b. Students know how to describe the dissolving process at the molecular level by using the concept of random molecular motion.

c. Students know temperature, pressure, and surface area affect the dissolving process.

d. Students know how to calculate the concentration of a solute in terms of grams per liter, molarity, parts per million, and percent composition.

2. Biological, chemical, and physical properties of matter result from the ability of atoms to form bonds from electrostatic forces between electrons and protons and between atoms and molecules. As a basis for understanding this concept:

d. Students know the atoms and molecules in liquids move in a random pattern relative to one another because the intermolecular forces are too weak to hold the atoms or molecules in a solid form. 


\section{Salinas Union High School District \\ High School Agricultural Chemistry Instructional Guide}

\section{Student Outcomes}

1. Be able to state the properties and states of matter verbally and to diagram the states of mater at a macro and micro level. $6 \mathrm{~b}$

2. Compare solids, liquids, and gases and be able to explain the distinguishing characteristics of each. $6 \mathrm{~b}$

3. Be able to explain the structure and properties of liquids, including surface tension, surface area, and how the intermolecular forces in water make it a unique substance. $2 \mathrm{~d}, 6 \mathrm{c}$

4. Be able to explain what a solution is (distinguishing between solute and solvent) including the process of solvation, factors affecting solubility and conductivity, and methods of purification. $6 \mathrm{a}, 6 \mathrm{~b}$

5. Students will be able to calculate the concentration of a solute in terms of grams per liter, molarity, and percent composition. $6 \mathrm{~d}$

\section{Possible Strategies and Activities}

1. Use models and lecture to illustrate the differences between a solid, liquid and gas on the macro and molecular level.

2. Select experiments that will facility the students use of laboratory data, and when possible computer graphing software to illustrate heating and cooling curves, and to calculate heats of phase changes.

3. Supplement lecture with laboratory investigations and demonstrations to illustrate the properties of solutions and the colligative effects of various solutes.

\section{Unit 8: Equilibrium}

\section{Chemical Equilibrium}

\section{State Standards}

9. Chemical equilibrium is a dynamic process at the molecular level. As a basis for understanding this roment.

a. Students know how to use LeChatelier's principle to predict the effect of changes in concentration, temperature, and pressure.

b. Students know equilibrium is established when forward and reverse reaction rates are equal.

\section{Student Outcomes}

1. Describe the equilibrium condition.

2. Describe the microscopic processes that occur in a system when it is at equilibrium. 9b

3. Use Le Chatelier's principle to explain how addition of a catalyst or changes in concentration, temperature, and/or pressure affect a system at equilibrium. 9a

4. Determine equilibrium exp0ressions for a given reaction.

\section{Possible Strategies and Activities}

1. Present examples that clearly illustrate the similarities and differences between steady state and equilibrium conditions.

2. Emphasize the dynamic processes that occur at the microscopic level within a system that is at equilibrium.

4. Give students numerous opportunities to apply Le Chatelier's principle, including written problems, demonstrations, and/or experiments.

5. Illustrate how to derive an equilibrium law expression; then provide practice opportunities with monitoring of student progress.

6. Show several variations of how equilibrium law calculations are used to find unknown concentrations or equilibrium constants. 


\section{Salinas Union High School District \\ High School Agricultural Chemistry Instructional Guide}

\section{Unir 9: Rates of Reaction}

\section{State Standards}

\section{Reaction Rates}

8. Chemical reaction rates depend on factors that influence the frequency of collision of reactant molecules. As a basis for understanding this concept:

a. Students know the rate of reaction is the decrease in concentration of reactants or the increase in concentration of products with time.

b. Students know how reaction rates depend on such factors as concentration, temperature, and pressure.

c. Students know the role a catalyst plays in increasing the reaction rate.

\section{Student Outcomes}

1. Be able to explain how factors such as concentration, temperature, pressure, and the influence of catalysts affect reaction rates. $8 \mathrm{~b}, 8 \mathrm{c}$

2. Be able to graph the change in amount of products versus reactants over time, including the affects of catalyst on the activation energy. 8 a

\section{Possible Strategies and Activities}

1. Give students numerous opportunities to apply Le Chatelier's principle, including written problems, demonstrations, and/or experiments.

2. Graph concentration verses time for iodine clock reaction.

3. Use graphs of activation energy to demonstrate catalyst affects on reactions.

4. Demonstrate catalyst using split tests for oxygen using manganese dioxide in the decomposition of hydrogen peroxide.

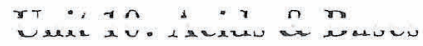

\section{State Standards}

\section{Acids and Bases}

5. Acids, bases, and salts are three classes of compounds that form ions in water solutions. As a basis for understanding this concept:
a. Students know the observable properties of acids, bases, and salt solutions.
b. Students know acids are hydrogen-ion-donating and bases are hydrogen-ion-accepting substances.
c. Students know strong acids and bases fully dissociate and weak acids and bases partially dissociate.
d. Students know how to use the pH scale to characterize acid and base solutions.

\section{Student Outcomes}

1. Demonstrate. how to calculate $\mathrm{pH}$ from a hydrogen-ion concentration in a laboratory exercise utilizing different aqueous concentrations. $5 c$

2. Use a pH meter and hydronium paper to identify solutions that are acidic and basic. $5 \mathrm{~d}$

3. Apply the Arrhenius, Brønsted-Lowery, and Lewis acid-base concepts to lecture problems and homew ork practice exercises. $5 \mathrm{c}$

4. Identify the observable properties of acids, bases, and salt solutions. 5a

5. Compare and contrast the differences between acids that are hydrogen-ion-donating and bases that are hydrogen-ion-accepting substances. $5 \mathrm{~b}$

\section{Possible Strategies and Activities}

1. Clarify how acids are hydrogen-ion-donating and bases are hydrogen-ion-accepting substances.

2. Provide classroom demonstrations that differentiate between acids, bases, and salt solutions. 


\section{Salinas Union High School District \\ High School Agricultural Chemistry Instructional Guide}

3. Monitor student's use of pH meters during laboratory experiments with different strengths of aqueous acidic and aqueous basic solutions.

4. Synthesize the concepts of Arrhenius, Bronsted-Lowery, and Lewis Acid-base solutions in simplifying how each concept is involved in a solution.

5. Evaluate student understanding of how strong bases and bases fully dissociate and weak acids and bases partially dissociate with practice problems, quizzes, and homework.

6. Teachers will facilitate learning with classroom demonstrations, Internet investigations of current topics, and the use of relevant software.

\section{Unit 11: Organic Chemistry}

\section{State Standards}

\section{Organic Chemistry and Biochemistry}

10. The bonding characteristics of carbon allow the formation of many different organic molecules of varied sizes, shapes, and chemical properties and provide the biochemical basis of life. As a basis for understanding this concept:

a. Students know large molecules (polymers), such as proteins, nucleic acids, and starch, are formed by repetitive combinations of simple subunits.

b. Students know the bonding characteristics of carbon that result in the formation of a large variety of structures ranging from simple hydrocarbons to complex polymers and biological molecules.

c. Students know amino acids are the building blocks of proteins.

\section{Student Outcomes}

1. Be able to explain the importance of carbon in the formation of many organic molecules essential to life. $10 \mathrm{a}$

2. Define the role of proteins, nucleic acids, and starch as they relate to biological structure, movement, catalysts, transport, storage, energy transtormation, proteclivil, dill vuleı11. IU

3. Identify the thirty essential elements and know the R-group structure of amino acids and how they combine to form the polypeptide backbone structure of proteins. $10 \mathrm{c}$

\section{Possible Strategies and Activities}

1. Illustrate and provide an opportunity for students to differentiate between simple linear hydrocarbons, hydrocarbons with double and, triple bonds, and molecules that contain benzene rings through demonstration, laboratory experiments, and lecture.

2. Supplement lecture with overheads, film, and models to illustrate the thirty essential elements used by amino acids as the building blocks of protein, and the basic structure of proteins.

3. Explain the role of proteins, nucleic acids, and starch using a systemic approach to the biochemical processes in the human body.

4. Supplement lecture with classroom demonstrations, Internet investigations, laboratory assignments and where appropriate relevant software will be incorporated into content area.

5. Provide opportunities for the students to complete laboratory experiments, analyze data, and write laboratory reports in the above content areas.

\section{Unit 12: Nuclear Chemistry}

\section{State Standards}

\section{Nuclear Processes}

11. Nuclear processes are those in which an atomic nucleus changes, including radioactive decay of naturally occurring and human-made isotopes, nuclear fission, and nuclear fusion. As a basis for understanding this concept: 


\section{Salinas Union High School District \\ High School Agricultural Chemistry Instructional Guide}

a. Students know protons and neutrons in the nucleus are held logether by nuclear forces that overcome the electromagnetic repulsion between the protons.

b. Students know the energy release per gram of material is much larger in nuclear fusion or fission reactions than in chemical reactions. The change in mass (calculated by $\mathrm{E}=\mathrm{mc}^{2}$ ) is small but significant in nuclear reactions.

c. Students know some naturally occurring isotopes of elements are radioactive. as are isotopes formed in nuclear reactions.

d. Students know the three most common forms of radioactive decay (alpha, beta, and gamma) and know' how the nucleus changes in each type of decay.

e. Students know alpha, beta, and gamma radiation produce different amounts and kinds of damage in matter and have different penetrations.

\section{Student Outcomes}

1. Be able to explain how nuclear forces allow protons to overcome their natural repulsion and reside in the nucleus. Ila

2. Be able to state the difference between nuclear fusion and nuclear fission, and be able to relate these differences to the amount of energy generated in each process. $11 \mathrm{~b}$

3. Describe the existence of radioactive isotopes, both natural, and those formed as a byproduct of nuclear reactions, and explain how a radiation counter is used to measure radioactive isotopes. Ilc

4. Compare the different amounts of radiation that alpha, beta, and gamma particles produce, know how the nucleus changes in each type of decay, and be able to describe the penetration ability, and types of damage that each will produce in matter. $11 \mathrm{~d}, 11 \mathrm{e}$

5. Students will be able to explain how a nuclear reactor works, and the importance of chain reactions in this process (including the idea of critical mass).

Donsibln Ctrontamias and Artixities

1. Explain and model nuclear forces as they pertain to atomic structure and illustrate how energy released per gram of material is much larger via nuclear fusion or fission than in previously studied chemical reactions.

2. Use a radiation counter, illustrations, and lecture to demonstrate the radiation emitted from alpha, beta, and gamma radiation and explain the differences in penetration and damage between the three types of radiation.

3. Use guided practice, and examples to show students how to utilize the half-life rule to calculate the amount of material that will decay during a specified amount of time.

4. Use classroom demonstrations, Internet investigations and computer technology where appropriate to compliment lecture and provide the opportunity for students to conduct laboratory experiments, analyze data, and complete laboratory reports to highlight these concepts. 


\section{Salinas Union High School District \\ High School Agricultural Chemistry Instructional Guide}

\section{Leadership \& Team Building Development}

Agriculture and Natural Resources Industry Sector Foundation and Career Pathway Standards

Students will be able to:

- Apply appropriate problem-solving strategies and critical thinking skills to work-related issues and tasks. (FS 5.1)

- Understand the systematic problem-solving models that incorporate input, process, outcome, and feedback components. (FS 5.2)

- Use critical thinking skills to make informed decisions and solve problems. (FS 5.3)

- Understand the characteristics and benefits of teamwork, leadership, and citizenship in the school, community, and workplace settings. (FS 9.1)

- Understand the ways in which pre-professional associations, such as the Future Farmers of America (FFA), and competitive career development activities enhance academic skills, promote career choices, and contribute to employability. (FS 9.2)

- Understand how to organize and structure work individually and in teams for effective performance and the attainment of goals. (FS 9.3)

- Understand leadership, cooperation, collaboration, and effective decision-making skills applied in group or team activities, including the student organization. (FS 9.6)

- Students demonstrate and apply the concepts contained in the foundation and pathway standards. (FS 11.0)

Samnle Artivities and Strateoies.

- Oral and speaking presentations

- Critical Thinking Exercises

- Problem Solving Exercises 


\section{Salinas Union High School District \\ High School Agricultural Chemistry Instructional Guide}

\section{INVESTIGATION AND EXPERIMENTATION}

Scientific progress is made by asking meaningful questions and conducting careful investigations.

Sludents will be able to:

- Plan and conduct a scientific investigation to test a hypothesis.

- Evaluate the accuracy and reproducibility of data.

- Distinguish between variables and controls in a test.

- Design, construct and interpret graphs using data from investigations.

- Apply simple mathematical relationships to determine a missing quantity in a mathematic expression, given the two remaining terms.

The teacher will integrate the investigation and experimentation topics into the content Focus Standards throughout the year, instead of an isolated unit, spiraling content until mastery is achieved by the end of the year. The teacher will devote a minimum of $20 \%$ of the instruction time to hands on experiences in the laboratory.

June, 2005, at the Summer Science Institute at Salinas Union School District, teachers adopted by consensus the following criteria and standards an investigation, activity, or experiment must have to be considered as part of the minimum $20 \%$ required investigation and experimentation Focus Standard.

\section{Definition of lab:}

All labs will molude:

- A clear objective

- A descriptive procedure

- Data collection

- Qualitative and/or quantitative observations

- Analysis of data

- A conclusion-communication of results

An experimental lab will include:

- All of the above and a(n)

1. Hypothesis

2. Control

3. Variable(s)

\section{$\underline{\text { Lab required elements: }}$}

- Title

- Background information (optional)

- Objective with standard(s)

- Questions/problem

- Hypothesis (for experimental labs only)

- Materials listed

- Safety standards (worked into procedure)

- Procedure 


\section{Salinas Union High School District}

High School Agricultural Chemistry Instructional Guide

- Dara

- Analysis of Data

- Conclusion

* Scaffolding of required elements can be changed according to student needs.

\section{Teacher information:}

- Grading rubric (to hand out to students)

- Prelab activities

- Expected results

- Possible difficulties

- Set-up time

- Lab completion time

- Resources/references

- Differentiation

- Vocabulary/skills needed

- Answers to lab questions

Addition demonstrations, activities, manipulatives, and other type of lab investigations are encouraged if they enhance student learning, but are not to be considered as part of the minimum $20 \%$ of investigations and experimentation.

\section{SUGGESTED TEACHING STRATEGIES:}

- Whole group instructions and strategies.

- rometative mrnuns

- Individualized instruction.

- Peer teaching.

- Independent practice.

- Audio-visual aided instruction.

- Individual and or group projects.

- Field trips.

- Interactive media.

- Computer assisted learning.

- Internet activities.

- Laboratory activities.

- Demonstrations.

\section{EVALUATION TECHNIQUES:}

- Teacher developed tests.

- Peer Evaluation.

- Laboratory write-ups.

- Homework.

- Directed study.

- Class participation.

- Independent research and/or experimental projects. 
Salinas Union High School District

High School Agricultural Chemistry Instructional Guide

\section{TERMINOLOGJ' GUIDE}

\section{Standard}

A goal statement that identifies the knowledge and skills to be learned. A content standard specifies what we want students to know and be able to do. The standards identified here are derived from the Science Content Standards for California Public Schools (1998) and serves as the basis of statewide student assessments, the science curriculum framework, and the evaluation of instructional materials. Attention to and familiarity with these standards identified should be the responsibility of the teacher.

\section{Focus Standard}

\section{(Integrated Focus Standards)}

A standard, or parts of a standard, identified by teachers locally as "essential" for students to learn. While teachers may teach more than the identified Focus Standards, they will teach these Focus Standards as targets for student learning and assessment. Cumulatively, the identified Focus Standards form the core of what should be taught, learned and assessed during the school year.

\section{Related California State Standards}

Prior standards identified from the Science Content Standards for California Schools that are vertically aligned to the current Focus Standards for each science content area or grade level. These standards are introduced in prior science classes, but provide support for student leaming of the current content

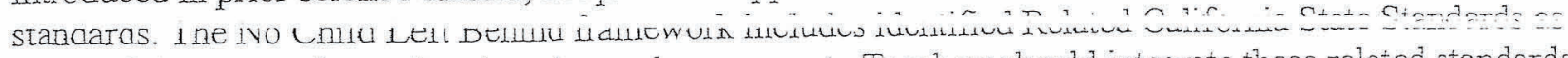
a part of the targets for student learning and assessment. Teachers should integrate these related standards into the current Focus Standards to ensure student understanding.

\section{Enduring Understanding}

The core concepts, principles, theories, and processes that serve as the focal point of curriculum, instruction, and assessment. Enduring Understandings are important, enduring, and transferable beyond the scope of a particular unit. Enduring Understandings identify the "key concepts" or overarching ideas derived from the California Science Content Standards and Framework (2004). Enduring Understandings support the content for that grade level, but may also be applicable at other grade levels. Enduring Understandings are written for student access and understanding at that grade level. Enduring Understandings maybe repeated across the grades to provide "bridges" for students and a sense of connectedness across place and time.

\section{Essential Question}

A provocative question designed to engage student interest and guide inquiry into the important ideas in a field of study. Rather than yielding pat answers, Essential Questions are intended to stimulate discussion and rethinking over time. These may be seen as "doorways" to the Enduring Understandings. 


\section{Salinas Union High School District \\ High School Agricultural Chemistry Instructional Guide}

\section{Integrate}

Integration of curriculum takes on different meanings in different situations. For the purposes of these curriculum guides, integration should be understood as:

- The blending of core concepts in teaching Science lessons/units (i.e., teaching science investigation and experimentation, life science, chemistry, earth science, physical science and/or other science areas simultaneously);

- Teaching more than one subject within a lesson/unit (i.e., Science and Mathematics, Language Arts, History-Social Science, etc.).

- Combining two or more parts of a standard (e.g., Life Science 1.c and 1.f) to teach one lesson/unit.

\section{Benchmark}

Benchmarks are a series of assessments used to determine how students are progressing in their learning throughout the school year. Benchmark assessments may be administered at the end of each quarter, for example, to show "where students are" at that time. Benchmarks provide a snapshot of student achievement and may be indicative if teaching resources and strategies are effective in helping students achieve maximum leaming. Benchmarks are forms of assessment used to inform students and teachers of the student's on-going progress. The results of these assessments are used to continue/extend the learning process and/or modify teaching strategies and resources in order to benefit the student.

\section{Blueprint}

Blueprints are guides developed by the California Department of Education to assist teachers in identifying which standards will be assessed (and to what degree each standard will be weighted in the total assessment) through the California Standards Test and/or No Child Left Behind test. The Science Framerxark for Califnmia Puhlir. Srhnnls is the hluevrint for reform of the science curriculum, instruction, professional preparation and development, and instructional materials in California. The blueprint also identifies the reporting clusters for each test.

\section{Assessments}

Techniques to analyze student accomplishments against specific goals and criteria. Includes paper-pencil tests, exhibits, investigative and experimental labs, exhibits, interviews, surveys and observations. Good assessment requires a balance of techniques because each technique is limited and prone to error. Assessment is sometimes synonymous with evaluation, though a subtle difference exists. A teacher might assess the strengths and weaknesses of a student's performance without placing a value or a grade on that performance.

\section{Assessment Sample}

Sample performance evaluation and district test questions that are keyed to specific objectives and focus standards. Samples include enhanced multiple-choice questions, open-ended questions, and performance tasks. Assessments samples are to serve as a guide for instructors to make meaningful aligned

assessments within the content and context of district, state, and national standards at the appropriate level to ensure student understanding and learning.

\section{Template}

A guide or framework for designers. In its original usage, a template was a thin form, constructed of paper, wood, or sheet metal, whose edge provided a guide for cutting a particular shape. In 


\section{Salinas Union High School District \\ High School Agricultural Chemistry Instructional Guide}

Understanding by Design. the unit-planning template provides a conceptual guide to apply the elements of backward design in developing or refining a unit of study.

\section{Backward Planning}

A process used to design curriculum or units by beginning with the end in mind and designing plans for teaching, resources, learning and assessment toward that end. Why is such a view backward when it seems logical? Many teachers begin their unit design with textbooks, favored lessons, and time-honored activities rather than deriving ideas from targeted goals or standards. In Backward Planning, one starts with the end - the desired results- and then identifies the evidence necessary to determine that the results have been achieved - the assessments. With the results and assessments clearly specified, one can determine the necessary knowledge and skills, and then the teaching needed to equip students to perform.

\section{Understanding by Design}

A theory developed by researchers and educational consultants Jay McTighe and Grant Wiggins with the intent tot teach for understanding using a method of Backward Planning. 


\section{SALINAS UNION HIGH SCHOOL DISTRICT CURRICULUM PROPOSAL}

SCHOOL North Salinas High School, Salinas, Everett Alvarez DEPARTMENT Agriculture TEACHER Stephanie Blodgett, Tom Jones, Stan Uchiyama, Janelle Smith, Felicia Rush, Janice Souza, \& Lindsay Buckner-Almond

Type of Proposal

Pilot Course (Titie)

(for pilot courses both sides of this form must be completed)

X_Major Modification of Existing Course (what course) Agriculture Chemistry

Rationale (Outline the fundamental reason or basis for the proposal. Attach supporting documentation if available)

This course is intended to successfully prepare those students who plan on majoring in agriculture sciences at a four year college and/or university. The curriculum we will be using is the current Salinas Union High School District curriculum and the modification is the addition of the FFA/leadership component. Studies will be made in the following subject areas: nursery soils, fertilizers and special nutrients, pest control and chemical principles.

Description (Briefly describe the proposal only if other than a Pilot Course)

A lab-based chemistry course which encompasses concepts set forth in the California State Science Content Standards for California Public Schools and the Science Framework for California Public Schools. Laboratory experiences are a major component of the course in addition to traditional instruction. This course meets the California and Salinas Union High School District graduation requirements for physical science. It qualifies as college lab course, meeting the University of California "A-G" requirements.

Support Requirements (In-service training, facilities, materials, transportation, etc.) School Agriculture Department to provide materials necessary to teach class. The class should have a working lab facility and access to necessary chemicals. Guest speakers and field trips will be ideal for optimum learning.

Estimated Cost:

School Department Chairperson
Principal
District Subject Area Committee
Display Period From
District Curriculum Development and
Instructional Materials Review Comm.
Curriculum Job Alike
Management Council
Board of Trustees


TO BE COMPLETED FOR PILOT COURSE PROPOSALS

Course Title

Grade Level_ Course Length

Credits_ Prerequisites

Course Outline (list major topics to be covered)

Course Goals and Obiectives (list major aims and objectives to carry them out)

Instructional Resources

Textbook:

Reference Materials:

Other Materials

(Books and instructional materials are subject to adoption in accordance with Board Policy 6161.11) 


\section{SUHSD SCIENCE CURRICULUM COMMITTEE}

MINUTES FOR MEETING: February 16, 2010

Representatives Present: Robin Putney-ESMS, Sandy Hunter-SHS, Paul Quiggle-ASH, Ted Meyenberg NSHS, Serena Telles EAHS, Stephanie Blodgett-NSHS, Janice Souza-EAHS, Claudio Montero-SHS (Chair)

WELCOME: Claudio Montero welcomes all members and calls the meeting to order at 4:15 pm.

MINUTES FROM NOVEMBER MEETING: Minutes were sent electronically to all sites. Minutes were approved. It was brought up by Mr. Montero that minutes should be shared at all sites with all members of each science department.

\section{AGRICULTURAL CHEMISTRY:}

Janice Souza and Stephanie Blodgett presented the proposal to offer an Agricultural Chemistry. This would be the same course as our regular college prep chemistry with the addition of the Agricultural Leadership component. The course will use the same textbook and same curriculum (with the addition of the $5 \%$ ag. leadership). The students in the course will take all the chemistry benchmarks and the chemistry CST as well. The course needs a new course number and therefore SCC and SUHSD Board approval.

SCC recommendation: It was brought up that the Ag. Chemistry course should be taught by a teacher who is credentialed in chemistry and also the teaching assignment should limit the amount of preps for the teacher. The proposal was approved.

\section{SCC BOARD PRESENTATION:}

On Tuesday, March 9, 2010, the SCC will be giving a presentation to the SUHSD board. Mr. Montero shared his draft of the presentation, "The State of Science in SUHSD". The committee went over the draft and offered their input. Claudio asked to have at least one middle school teacher and one high school teacher go to the board meeting with him. Robin Putney and Paul Quiggle are the current volunteers. Thanks so much!

\section{BENCHMARK \#3:}

Benchmark \#3 was given on February $11^{\text {th }}$ at most schools. Robin Putney brought up that there was some confusion with the calendar and the ESMS science benchmark will be given on the $25^{\text {th }}$ of February. The results are in for all of the other schools and we will be going over the results during the next collaboration session. 


\section{REPORT FROM SITES:}

ESMS- Robin Putney brought up that their teachers were wondering when the dates for the 2010-2011 benchmark testing will be scheduled. She mentioned that when the dates are set we need to consider the calendar to avoid tr benchmarks from being scheduled right after a holiday like this upcoming Benchmark \#4.

No other site brought up additional report items

\section{REMINDERS:}

- Please share these minutes with the members of your department.

- Benchmark \#4 for will be given April 9, 2010. ( with 3-day window)

- Next SCC meeting will be Tuesday, March 16th, 2010 at SHS, room 212, 4:15-5:45 pm

- Any corrections to these minutes? Please email me at sahunter@salinas.k12.ca.us

- Any agenda items? Please email them to Sandy Hunter or Claudio Montero, Thank you!

- Mark your calendars! The SCC meeting schedule for the remainder of the year is: March $16^{\text {th }}$, April $_{20}{ }^{\text {th }}$

- A May meeting will take place if needed. 
DEPARTMENT:

COURSE TITLE:

GRADE LEVEL.:

LENGTH:

NUMBER OF CREDITS:

PREREQUISITE:

TEXTBOOK:
Agriculture

Ag Chemistry

$11-12$

1 year

10 credits

C or better in Algebra

Chemistry Prentice-Hall 2002

SUPPLEMENTARY MATERIALS: Materials provided by the publisher including study guide and lab manual, $C D$ accompaniment, Videodisc Programs, Web Links

\section{COURSE DESCRIPTION:}

A lab-based chemistry course which encompasses concepts set forth in the California State Science Content Standards for California Public Schools and the Science Framework for California Public Schools. Laboratory experiences are a major component of the course in addition to traditional instruction. This course meets the California and Salinas Union High School District graduation requirements for physical science.

\section{SOURCES OF CURRICULUM CONTENT:}

The curriculum incorporates all High School Life Science Blueprint content standards adopted by the California State Board of Education 2004, as required. Clarification of topics and unifying themes have been drawn from Benchmarks for Scientific Literacy (American Association for the Advancement of Science) and from National Science Education Standards (National Research Council). Much of the clarification of state standards and development of concept units is a result of meetings with the planning committee composed of high school chemistry teachers in the Salinas Union High School District who met during 2007-08.

\section{Course Purpose}

The purpose of $\mathrm{Ag}$ Chemistry is to provide a chemistry course that covers the curriculum of a regular chemistry course with the agriculture component incorporated. This will enable the student to become familiar with the career technical education when he/she enrolls in college agriculture and science courses. Agriculture Chemistry places a much greater emphasis on career technical education and on leadership ability than does regular chemistry. Agriculture Chemistry involves laboratory investigations of chemistry concepts and requires lab reports. Upon completion of this course, students will:

- Be proficient in all State Standards for chemistry and have a deeper understanding of Agriculture Chemistry and how it relates to Career Technical Education.

- Become proficient in the use of mathematics in chemistry 
- Have experience doing a variety of laboratory activities that involves the use of modern chemistry lab equipment.

- Be familiar with chemistry lab safety procedures and have experience using lab equipment such as crucibles, burets, spectrometers, etc.

- Have gained experience doing independent research into environmental and societal issues that relate to agriculture chemistry.

- In addition to the State Standards for Chemistry, students have learned many of the fundamentals of organic chemistry, electrochemistry, chemical bonding, and how they relate to agriculture.

\section{Course Outlime:}

\section{Major Concepts}

1. Safety and Emergency Response,

2. Laboratory Techniques and Measurement

3. Significant Figures and Dimensional Analysis)

4. Introduction to Atoms, the Periodic Table, and Nomenclature

5. Conservation of Matter and Stoichiometry

6. Gas Laws

7. Chemical Bonds

8. Thermodynamics

9. Solutions

10. Chemical Equilibrium

11. Rates of Reaction

12. Acids and Bases

13. Organic Chemistry and Biochemistry

14. Nuclear Processes

15. Leadership and Team Building

\section{Unit 1: An Introduction to Chemistry}

\section{Safety and Emergency Response}

\section{State Standards}

Investigation and Experimentation

1. Scientific progress is made by asking meaningful questions and conducting careful investigations. Students will:

a. Select and use appropriate tools and technology (such as computer-linked probes, spreadsheets, and graphing calculators) to perform tests, collect data, analyze relationships, and display data.

\section{Student Outcomes}

1. Follow all safety rules and precautions as highlighted in the laboratory manual and by the teacher. Students will be expected to pass a safety quiz before the first lab with safety concerns. A grade of $80 \%$ o or better must be obtained before students may participate in laboratory activities.

2. Follow good housekeeping procedures in their laboratory area. This includes good facilities management, equipment and materials storage, chemical handling techniques, and glassware use and storage.

3. Know the location of and how to operate all safety equipment available in the classroom. Protect eyes, face, hands, and body while conducting class and laboratory activities. 
putting emphasis on precision statements in written laboratory reports as well as consistent modeling when, discussing treatment of quantitative laboratory data during class discussions.

5. Demonstrate the correct procedure for each specialized lab task that is introduced in student experiments, and correct students when necessary while observing their work in, the laboratory. Supporting strategies include requiring students to clearly explain each lab technique in their written flow sheets, and giving a "lab practical" exam in which students' lab skills are formally evaluated.

6. Provide students with a format for writing laboratory reports, evaluate students' reports in a timely manner, and give students suggestions for improving their work as the course progresses.

\section{Significant Figures amd Dimensional Anallysis (Factor Label Method)}

\section{State Standards}

\section{Investigation and Experimentation}

1. Scientific progress is made by asking meaningful questions and conducting careful investigations. Students will:

b. Select and use appropriate tools and technology (such as computer-linked probes, spreadsheets, and graphing calculators) to perform tests, collect data, analyze relationships, and display data.

c. Identify and communicate sources of unavoidable experimental error.

d. Identify possible reasons for inconsistent results, such as sources of error or uncontrolled conditions.

e. Formulate explanations by using logic and evidence.

f. Solve scientific problems by using quadratic equations and simple trigonometric, exponential, and logarithmic functions.

k. Recognize the issues of statistical variability and the need for controlled tests.

m. Analyze situations and solve problems that require combining and applying concepts from more than one area of science.

\section{Student Outcomes}

1. Demonstrate a consistent use of units in measurements and calculations.

2. Use the factor label method in the solution of all problems and laboratory calculations assigned throughout the year.

3. Use estimation techniques incorporating dimensional analysis to arrive at approximate values when assigned to do so by the teacher.

4. Demonstrate skills in the use of fractions, scientific notation, exponents, graphing, graph interpretation, and the correct use of significant digits to indicate the appropriate degree of uncertainty.

\section{Possible Strategies and Activities}

1. Provide direct instruction and consistently model the use of appropriate units associated with measurements.

2. Teach factor labeling as a means of solving of problems both from laboratory experiences and from hypothetical situations.

3. Model and provide opportunities for estimation using orders of magnitude; arriving at approximate values; and indicating appropriate degrees of uncertainty.

4. Review basic mathematical skills for use in chemistry.

5. Monitor and evaluate student learning. Make adjustments as shown necessary in the monitoring process, with prompt correction and review.

6. Select appropriate materials and media to enhance learning, including some of the following: measurement lab with significant figures calculations; density lab; graphing lab 
4. Dispose of dangerous waste chemicals and materials as prescribed by appropriate standards and laws.

5. Know the appropriate emergency steps to take in the event of an accident or emergency including evacuation procedures.

\section{Possible Strategies and Activities}

1. Review the appropriate safety procedures before activities and briefly explain specific precautions and hazards associated with the activity.

2. Demonstrate how to store and handle laboratory equipment. Ensure that chemicals are kept in a safe place with limited access to prevent the possibility of hazardous accidents.

3. Check smoke detectors, safety showers, and eyewash facilities on a regular basis. Know how to access all master shutoffs where applicable.

4. Clarify all waste disposal procedures before every activity. Provide separate labeled waste receptacles for waste as needed. Also provide labeled receptacles for broken glass, waste paper, and used matches if applicable.

5. Post your school's accident policy and procedures; make accident reports promptly, accurately, and completely.

\section{Laboratory Techniques and Measurement}

\section{State Standards}

Investigation and Experimentation

1. Scientific progress is made by asking meaningful questions and conducting careful investigations. Students will:

a. Select and use appropriate tools and technology (such as computer-linked probes, spreadsheets, and graphing calculators) to perform tests, collect data, analyze relationships, and display data.

\section{Student Outcomes}

1. In advance of each laboratory experiment, write a prelab or flow chart which expresses a hypothesis and describes the experimental procedure in the student's own words.

2. Learn the names and functions of the laboratory equipment used in experiments.

3. Understand and use common laboratory terminology.

4. Make all quantitative measurements to the proper level of precision, and state the absolute error for each measurement.

5. Demonstrate competence in performing laboratory procedures. These tasks may include, but are not limited to: transfer of chemicals from stock containers, use of balance (both digital and mechanical, if possible), reading a thermometer, filtration, decantation, operating a Bunsen burner, reading volumes in graduated cylinders and gas measuring tubes, use of buret, cleaning and rinsing glassware, handling hot equipment and/or solutions, operation of specialized equipment such as colorimeters, $\mathrm{pH}$ meters, or spectrophotometers.

6. Perform appropriate analysis of experimental data, draw logical conclusions, and report findings in a clearly written laboratory report.

\section{Possible Strategies and Activities}

1. Provide guidelines for proper prelab procedures.

2. Identify the equipment used in each laboratory experiment, clarify the function of each item, and insist that students use the proper terms when referring to the equipment during class discussion and in written laboratory reports.

3. Model the use of proper terminology when discussing laboratory procedures, and require students to employ the correct terms when discussing their experiments.

4. Demonstrate how to make quantitative measurements to the highest possible level of precision with each instrument that is used in the laboratory. Follow-up may include 


\section{Introduction to Atoms, the Periodic Table, and Chemical Nomenclature}

\section{State Standards}

Atomic and Molecular Structure

1. The periodic table displays the elements in increasing atomic number and shows how periodicity of the physical and chemical properties of the elements relates to atomic structure. As a basis for understanding this concept:

a. Students know how to relate the position of an element in the periodic table to its atomic number and atomic mass.

b. Students know how to use the periodic table to identify metals, semimetals, nonmetals, and halogens.

c. Students know how to use the periodic table to identify alkali metals, alkaline earth metals and transition metals, trends in ionization energy, electronegativity, and the relative sizes of ions and atoms.

d. Students know how to use the periodic table to determine the number of electrons available for bonding.

e. Students know the nucleus of the atom is much smaller than the atom yet contains most of its mass.

f. *Students know how to use the periodic table to identify the lanthanide, actinide, and transactinide elements and know that the transuranium elements were synthesized and identified in laboratory experiments through the use of nuclear accelerators.

h. *Students know the experimental basis for Thomson's discovery of the electron, Rutherford's nuclear atom, and Millikan's oil drop experiment.

\section{Chemical Bonds}

2. Biological, chemical, and physical properties of matter result from the ability of atoms to form bonds from electrostatic forces between electrons and protons and between atoms and molecules. As a basis for understanding this concept:

a. Students know atoms combine to form molecules by sharing electrons to form covalent or metallic bonds or by exchanging electrons to form ionic bonds.

\section{Student Outcomes}

1. Students will be able to describe the basic structure of the atom and the experimental evidence that lead to our understanding of atomic structure.

2. Students will know the basic organization of the periodic table.

3. Students will know that compounds are formed when ions are held together by electrostatic forces or when atoms share electrons to form molecules.

4. Students will be able to name and write formulas for ionic and molecular compounds and distinguish between the two by noting whether a metal and a non-metal or two non-metals are combining. In order to do this; students must be familiar with both the locations of metals and non-metals on the periodic table, and with the use and the naming of polyatomic ions.

\section{Possible Strategies and Activities}

1. Provide a historic overview of the history of the discovery of the atom, subatomic particles, and the periodic table. This overview will be accompanied by lab, audiovisual, lecture, and research (including Internet assignments).

2. Explain all the parts of the periodic table including the distinction between groups and families, metals and non-metals, specific families, valence numbers, and how to determine ionic charges.

3. Have students memorize the formula and name for the common polyatomic ions.

4. Have students write IUPAC names for compounds based on their formulas and formulas based on names. 


\section{Conservation of Matter and Stoichiometry}

\section{State Standards}

\section{Conservation of Matter and Stoichiometry}

3. The conservation of atoms in chemical reactions leads to the principle of conservation of matter and the ability to calculate the mass of products and reactants. As a basis for understanding this concept:

a. Students know how to describe chemical reactions by writing balanced equations.

b. Students know the quantity one mole is set by defining one mole of carbon 12 atoms to have a mass of exactly 12 grams.

c. Students know one mole equals $6.02 \times 10^{23}$ particles (atoms or molecules).

d. Students know how to determine the molar mass of a molecule from its chemical formula and a table of atomic masses and how to convert the mass of a molecular substance to moles, number of particles, or volume of gas at standard temperature and pressure.

e. Students know how to calculate the masses of reactants and products in a chemical reaction from the mass of one of the reactants or products and the relevant atomic masses.

f. *Students know how to calculate percent yield in a chemical reaction.

\section{Student Outcomes}

1. Balance chemical equations using the proper chemical symbols and coefficients. $3 a$

2. Utilize Avogadro's hypothesis to solve conversion problems involving atomic weight, molar mass, the mole, and molar volume. $3 \mathbf{b}, 3 \mathbf{c}, 3 \mathbf{d}, 3 \mathbf{e}$

3. Students will be able to calculate the percent yield in a chemical reaction.

\section{Possible strategies and Activities}

1. Provide direct instruction that clarifies, defines, and provides a consistent pattern for solving problems in the laboratory and from hypothetical situations.

2. Give students numerous opportunities to perform molar calculations, using stoichiometric ratios, Avogadro's number, molar mass, and molarity.

3. Provide extensive practice with prompt correction and review of assigned problems.

4. Select appropriate materials in the form of films and software to supplement lecture.

5. Provide laboratory opportunities including calculations of molar mass, mass relationships accompanying chemical changes, and requiring the use of stoichiometric calculations including the calculation of percent yield.

\section{Gas Laws}

\section{State Standards}

4. The kinetic molecular theory describes the motion of atoms and molecules and explains the properties of gases. As a basis for understanding this concept:

a. Students know the random motion of molecules and their collisions with a surface create the observable pressure on that surface.

b. Students know the random motion of molecules explains the diffusion of gases.

c. Students know how to apply the gas laws to relations between the pressure, temperature, and volume of any amount of an ideal gas or any mixture of ideal gases.

d. Students know the values and meanings of standard temperature and pressure (STP).

e. Students know how to convert between the Celsius and Kelvin temperature scales.

f. Students know there is no temperature lower than 0 Kelvin.

\section{Student Outcomes}

1. Be able to explain the characteristics of gases. 
2. Use the following gas laws: Dalton's Law of Partial Pressures, Charles' Law, Boyle's Law, Avogadro's Principle, the Ideal Gas Law, the Combined Gas Law, and Graham's Law. 4c, 4d

3. Be able to graph and exchange the changes of state using heating and cooling curves and label: melting point, boiling point, heat of fusion, heat of vaporization $4 \mathrm{e} .4 \mathrm{f}$

\section{Possible Strategies and Activities}

1. Use models and lecture to illustrate the differences between a solid, liquid and gas on the macro and molecular level.

2. Define the gas laws and utilize them for problem solving, then provide guided practice and laboratory activities for the students.

3. Select experiments that will facility the students use of laboratory data, and when possible computer graphing software to illustrate heating and cooling curves.

\section{Chemical Bonds}

State Standards

2. Biological, chemical, and physical properties of matter result from the ability of atoms to form bonds from electrostatic forces between electrons and protons and between atoms and molecules. As a basis for understanding this concept:

a. Students know atoms combine to form molecules by sharing electrons to form covalent or metallic bonds or by exchanging electrons to form ionic bonds.

b. Students know chemical bonds between atoms in molecules such as $\mathrm{H}_{2}, \mathrm{CH}_{4}, \mathrm{NH}_{3}, \mathrm{H}_{2}$ $\mathrm{CCH}_{2}, \mathrm{~N}_{2}, \mathrm{Cl}_{2}$, and many large biological molecules are covalent.

c. Students know salt crystals, such as $\mathrm{NaCl}$, are repeating patterns of positive and negative ions held together by electrostatic attraction.

d. Students know the atoms and molecules in liquids move in a random pattern relative to one another because the intermolecular forces are too weak to hold the atoms or molecules in a solid form.

e. Students know how to draw Lewis dot structures.

\section{Students Outcomes}

1. Review the nature and rational of chemical bonding, ionic model, ionic character, oxidation states of families, and covalent bonding.

2. Draw and explain Lewis dot structure, molecular structure, octet rule, valence electrons/pairs.

3. Describe molecular structure, octet rule and exception, resonance.

4. Explain VSEPR model, hybridization, and molecular orbital model.

\section{Possible Strategies and Activities}

1. Students build or observe models of crystal lattices.

3. Build molecular shapes and structures of certain molecules.

4. Use of molecular model sets.

5. Use of Styrofoam balls and colored pipe cleaners to demonstrate valence electrons and molecular shapes.

\section{Thermodynamics}

State Standards

7. Energy is exchanged or transformed in all chemical reactions and physical changes of matter. As a basis for understanding this concept:

a. Students know how to describe temperature and heat flow in terms of the motion of molecules (or atoms).

b. Students know how chemical processes can either release (exothermic) or absorb (endothermic) thermal energy. 
c. Students know energy is released when a material condenses or freezes and is absorbed when a material evaporates or melts.

d. Students know how to solve problems involving heat flow and temperature changes, using known values of specific heat and latent heat of phase change.

\section{Students Outcomes}

1. Explain why energy always flows from warnter to cooler.

2. Describe specific heat is the amount of energy needed to raise the temperature of a substance..

3. Know that specific heat is an inherent property of substances.

4. Chemical and physical changes involve the flow of energy.

5. Be able to solve problems involving specific heat and temperature change.

6. Temperature does not change during a phase change.

\section{Possible Strategies and Activities}

1. Demonstrate calorimeter, Enthalpy, thermo - chemical equations, heats of formation.

2. Solve heat flow problems

3. Calorimeter labs using Styrofoam cups and different metal pieces to identify specific heats of various metals.

4. Demonstrate athletic trainer's cold packs (endothermic) and hot packs (exothermic). Ziploc bag reactions also work.

5. Show students models of molecular motion of gases, solids and liquids to show energy loss or gain during phase change.

\section{Solutions}

\section{State Standards}

Solutions

6. Solutions are homogenous mixtures of two or more substances. As a basis for understanding this concept:

a. Students know the definitions of solute and solvent.

b. Students know how to describe the dissolving process at the molecular level by using the concept of random molecular motion.

c. Students know temperature, pressure, and surface area affect the dissolving process.

d. Students know how to calculate the concentration of a solute in terms of grams per liter, molarity, parts per million, and percent composition.

2. Biological, chemical, and physical properties of matter result from the ability of atoms to form bonds from electrostatic forces between electrons and protons and between atoms and molecules. As a basis for understanding this concept:

d. Students know the atoms and molecules in liquids move in a random pattern relative to one another because the intermolecular forces are too weak to hold the atoms or molecules in a solid form.

\section{Student Outcomes}

1. Be able to state the properties and states of matter verbally and to diagram the states of mater at a macro and micro level. $6 \mathrm{~b}$

2. Compare solids, liquids, and gases and be able to explain the distinguishing characteristics of each. $6 \mathrm{~b}$

3. Be able to explain the structure and properties of liquids, including surface tension, surface area, and how the intermolecular forces in water make it a unique substance. 2d, $6 \mathrm{c}$

4. Be able to explain what a solution is (distinguishing between solute and solvent) including the process of solvation, factors affecting solubility and conductivity, and methods of purification. $6 \mathrm{a}, 6 \mathrm{~b}$

5. Students will be able to calculate the concentration of a solute in terms of grams per liter, molarity, and percent composition. $6 \mathbf{d}$ 


\section{Possible Strategies and Activities}

1. Use models and lecture to illustrate the differences between a solid, liquid and gas on the macro and molecular level.

2. Select experiments that will facility the students use of laboratory data, and when possible computer graphing software to illustrate heating and cooling curves, and to calculate heats of phase changes.

3. Supplement lecture with laboratory investigations and demonstrations to illustrate the properties of solutions and the colligative effects of various solutes.

\section{Equilibrium}

\section{Chemical Equilibrium}

\section{State Standards}

9. Chemical equilibrium is a dynamic process at the molecular level. As a basis for understanding this concept:

a. Students know how to use LeChatelier's principle to predict the effect of changes in concentration, temperature, and pressure.

b. Students know equilibrium is established when forward and reverse reaction rates are equal.

\section{Student Outcomes}

1. Describe the equilibrium condition.

2. Describe the microscopic processes that occur in a system when it is at equilibrium. 9b

3. Use Le Chatelier's principle to explain how addition of a catalyst or changes in concentration, temperature, and/or pressure affect a system at equilibrium. 9a

4. Determine equilibrium exp0ressions for a given reaction.

\section{Possible Strategies and Activities}

1. Present examples that clearly illustrate the similarities and differences between steady state and equilibrium conditions.

2. Emphasize the dynamic processes that occur at the microscopic level within a system that is at equilibrium.

4. Give students numerous opportunities to apply Le Chatelier's principle, including written problems, demonstrations, and/or experiments.

5. Illustrate how to derive an equilibrium law expression; then provide practice opportunities with monitoring of student progress.

6. Show several variations of how equilibrium law calculations are used to find unknown concentrations or equilibrium constants.

\section{Rates of Reaction}

\section{State Standards}

\section{Reaction Rates}

8. Chemical reaction rates depend on factors that influence the frequency of collision of reactant molecules. As a basis for understanding this concept:

a. Students know the rate of reaction is the decrease in concentration of reactants or the increase in concentration of products with time.

b. Students know how reaction rates depend on such factors as concentration, temperature, and pressure.

c. Students know the role a catalyst plays in increasing the reaction rate.

\section{Student Outcomes}


1. Be able to explain how factors such as concentration, temperature, pressure, and the influence of catalysts affect reaction rates. $8 \mathrm{~b}, 8 \mathrm{c}$

2. Be able to graph the change in amount of products versus reactants over time, including the affects of catalyst on the activation energy. 8 a

\section{Possible Strategies and Activities}

1. Give students numerous opportunities to apply Le Chatelier's principle, including written problems, demonstrations, and/or experiments.

2. Graph concentration verses time for iodine clock reaction.

3. Use graphs of activation energy to demonstrate catalyst affects on reactions.

4. Demonstrate catalyst using split tests for oxygen using manganese dioxide in the decomposition of hydrogen peroxide.

\section{Acids \& Bases}

\section{State Standards}

\section{Acids and Bases}

5. Acids, bases, and salts are three classes of compounds that form ions in water solutions.

As a basis for understanding this concept:

a. Students know the observable properties of acids, bases, and salt solutions.

b. Students know acids are hydrogen-ion-donating and bases are hydrogen-ion-accepting substances.

c. Students know strong acids and bases fully dissociate and weak acids and bases partially dissociate.

d. Students know how to use the $\mathrm{pH}$ scale to characterize acid and base solutions.

\section{Student Outcomes}

1. Demonstrate. how to calculate $\mathrm{pH}$ from a hydrogen-ion concentration in a laboratory exercise utilizing different aqueous concentrations. $5 \mathrm{c}$

2. Use a $\mathrm{pH}$ meter and hydronium paper to identify solutions that are acidic and basic. $5 d$

3. Apply the Arrhenius, Bronsted-Lowery, and Lewis acid-base concepts to lecture problems and homework practice exercises. 5e

4. Identify the observable properties of acids, bases, and salt solutions. 5a

5. Compare and contrast the differences between acids that are hydrogen-ion-donating and bases that are hydrogen-ion-accepting substances. $\mathbf{5 b}$

\section{Possible Strategies and Activities}

1. Clarify how acids are hydrogen-ion-donating and bases are hydrogen-ion-accepting substances.

2. Provide classroom demonstrations that differentiate between acids, bases, and salt solutions.

3. Monitor student's use of $\mathrm{pH}$ meters during laboratory experiments with different strengths of aqueous acidic and aqueous basic solutions.

4. Synthesize the concepts of Arrhenius, Brønsted-Lowery, and Lewis Acid-base solutions in simplifying how each concept is involved in a solution.

5. Evaluate student understanding of how strong bases and bases fully dissociate and weak acids and bases partially dissociate with practice problems, quizzes, and homework.

6. Teachers will facilitate learning with classroom demonstrations, Internet investigations of current topics, and the use of relevant software.

\section{Organic Chemistry}

\section{State Standards}


10. The bonding characteristics of carbon allow the formation of many different organic molecules of varied sizes, shapes, and chemical properties and provide the biochemical basis of life. As a basis for understanding this concept:

a. Students know large molecules (polymers), such as proteins, nucleic acids, and starch, are formed by repetitive combinations of simple subunits.

b. Students know the bonding characteristics of carbon that result in the formation of a large variety of structures ranging from simple hydrocarbons to complex polymers and biological molecules.

c. Students know amino acids are the building blocks of proteins.

\section{Student Outcomes}

1. Be able to explain the importance of carbon in the formation of many organic molecules essential to life. 10a

2. Define the role of proteins, nucleic acids, and starch as they relate to biological structure, movement, catalysts, transport, storage, energy transformation, protection, and buffering, $10 \mathrm{~b}$

3. Identify the thirty essential elements and know the R-group structure of amino acids and how they combine to form the polypeptide backbone structure of proteins. 10c

\section{Possible Strategies and Activities}

1. Mlustrate and provide an opportunity for students to differentiate between simple linear hydrocarbons, hydrocarbons with double and, triple bonds, and molecules that contain benzene rings through demonstration, laboratory experiments, and lecture.

2. Supplement lecture with overheads, film, and models to illustrate the thirty essential elements used by amino acids as the building blocks of protein, and the basic structure of proteins.

3. Explain the role of proteins, nucleic acids, and starch using a systemic approach to the biochemical processes in the human body.

4. Supplement lecture with classroom demonstrations, Internet investigations, laboratory assignments and where appropriate relevant software will be incorporated into content area.

5. Provide opportunities for the students to complete laboratory experiments, analyze data, and write laboratory reports in the above content areas.

\section{Nuclear Chemistry}

\section{State Standards}

Nuclear Processes

11. Nuclear processes are those in which an atomic nucleus changes, including radioactive decay of naturally occurring and human-made isotopes, nuclear fission, and nuclear fusion. As a basis for understanding this concept:

a. Students know protons and neutrons in the nucleus are held together by nuclear forces that overcome the electromagnetic repulsion between the protons.

b. Students know the energy release per gram of material is much larger in nuclear fusion or fission reactions than in chemical reactions. The change in mass (calculated by $\mathrm{E}=\mathrm{mc}^{2}$ ) is small but significant in nuclear reactions.

c. Students know some naturally occurring isotopes of elements are radioactive, as are isotopes formed in nuclear reactions.

d. Students know the three most common forms of radioactive decay (alpha, beta, and ganma) and know how the nucleus changes in each type of decay.

e. Students know alpha, beta, and gamma radiation produce different amounts and kinds of damage in matter and have different penetrations.

\section{Student Outcomes}

1. Be able to explain how nuclear forces allow protons to overcome their natural repulsion and reside in the nucleus. Illa 
2. Be able to state the difference between nuclear fusion and nuclear fission, and be able to relate these differences to the amount of energy generated in each process. $11 \mathrm{~b}$

3. Describe the existence of radioactive isotopes, both natural, and those formed as a byproduct of nuclear reactions, and explain how a radiation counter is used to measure radioactive isotopes. Ille

4. Compare the different amounts of radiation that alpha, beta, and gamma particles produce, know how the nucleus changes in each type of decay, and be able to describe the penetration ability, and types of damage that each will produce in matter. $11 \mathrm{~d}, 11 \mathrm{e}$

5. Students will be able to explain how a nuclear reactor works, and the importance of chain reactions in this process (including the idea of critical mass).

\section{Possible Strategies and Activities}

1. Explain and model nuclear forces as they pertain to atomic structure and illustrate how energy released per gram of material is much larger via nuclear fusion or fission than in previously studied chemical reactions.

2. Use a radiation counter, illustrations, and lecture to demonstrate the radiation emitted from alpha, beta, and gamma radiation and explain the differences in penetration and damage between the three types of radiation.

3. Use guided practice, and examples to show students how to utilize the half-life rule to calculate the amount of material that will decay during a specified amount of time.

4. Use classroom demonstrations, Internet investigations and computer technology where appropriate to compliment lecture and provide the opportunity for students to conduct laboratory experiments, analyze data, and complete laboratory reports to highlight these concepts.

\section{Leadership \& Team Building Development}

Agriculture and Natural Resources Industry Sector Foundation and Career Pathway Standards

\section{Students will be able to:}

- Apply appropriate problem-solving strategies and critical thinking skills to workrelated issues and tasks. (FS 5.1)

- Understand the systematic problem-solving models that incorporate input, process, outcome, and feedback components. (FS 5.2)

- Use critical thinking skills to make informed decisions and solve problems. (FS 5.3)

- Understand the characteristics and benefits of teamwork, leadership, and citizenship in the school, community, and workplace settings. (FS 9.1)

- Understand the ways in which pre-professional associations, such as the Future Farmers of America (FFA), and competitive career development activities enhance academic skills, promote career choices, and contribute to employability. (FS 9.2)

- Understand how to organize and structure work individually and in teams for effective performance and the attainment of goals. (FS 9.3)

- Understand leadership, cooperation, collaboration, and effective decision-making skills applied in group or team activities, including the student organization. (FS 9.6)

- Students demonstrate and apply the concepts contained in the foundation and pathway standards. (FS 11.0) 
Sample Activities and Strategies:

- Oral and speaking presentations

- Critical Thinking Exercises

- Problem Solving Exercises

\section{Laboratory Instruction}

1. In advance of each laboratory experiment, write a prelab or flow chart which expresses a hypothesis and describes the experimental procedure in the student's own words.

2. Learn the names and functions of the laboratory equipment used in experiments.

3. Understand and use common laboratory terminology.

4. Make all quantitative measurements to the proper level of precision, and state the absolute error for each measurement.

5. Demonstrate competence in performing laboratory procedures. These tasks may include, but are not limited to: transfer of chemicals from stock containers, use of balance (both digital and mechanical, if possible), reading a thermometer, filtration, decantation, operating a Bunsen burner, reading volumes in graduated cylinders and gas measuring tubes, use of buret, cleaning and rinsing glassware, handling hot equipment and/or solutions, operation of specialized equipment such as colorimeters, $\mathrm{pH}$ meters, or spectrophotometers.

6. Perform appropriate analysis of experimental data, draw logical conclusions, and report findings in a clearly written laboratory report.

For all Labs students will be able to:

- Plan and conduct a scientific investigation to test a hypothesis.

- Evaluate the accuracy and reproducibility of data.

- Distinguish between variables and controls in a test.

- Design, construct and interpret graphs using data from investigations.

- Apply simple mathematical relationships to determine a missing quantity in a mathematic expression, given the two remaining terms.

The teacher will integrate the investigation and experimentation topies into the content Focus Standlards throughout the year, instead of an isolated unit, spiraling content untill mastery is achieved by the end of the year. The teacher will devote a mimimum of $20 \%$ of the instruction time to hands on experiences in the laboratory.

All labs will also include:

- A clear objective

- A descriptive procedure

- Data collection

- Qualitative and/or quantitative observations

- Analysis of data

- A conclusion - communication of results

An experimental lab will include:

- All of the above and a(n)

1. Hypothesis

2. Control

3. Variable(s)

Lab required elements: 
- Title

- Background information (optional)

- Objective with standard(s)

- Questions/problem

- Hypothesis (for experimental labs only)

- Materials listed

- Safety standards (worked into procedure)

- Procedure

- Data

- Analysis of Data

- Conclusion

*Scaffolding of required elements can be changed according to student needs.

\section{Labs:}

\section{Laboratory Techniques and Measurement:}

Emulsification of Fat (1a): Students use Petri dishes to demonstrate how water, vegetable oil, and whole milk emulsify. When a toothpick touches the milk, the detergent temporarily destroys the surface tension. The detergent emulsifies any fat in the milk, causing the colors to move from the outside to the center.

\section{Significant Figures and Dimensional Analysis}

Layers of Liquids (1e): Students will use various liquids to demonstrate the property of density. Students to use glycerol, water, corn oil, and alcohol to show differences of densities of the liquids.

Matter (1e, 1f, 1m): Students will use a match and an electronic balance to determine the mass of the match. Students will then light the match, let it burn for 5 seconds, blow it out, measure the mass of the remaining match. Students to determine how you account for the change in mass and where the matter is that appears to have been lost.

Beverage Density Determination (1e, 1f, $1 \mathrm{~m}$ ): Students use pipettes and electronic balances to calculate the density of several common liquids, and then graph this data to determine the relative sugar content

Surface area and percent yield (1b, 1e, 1f, 1m): Students to use a Bunsen burner and steel wool to design an experiment to determine what gauge steel wool will produce the higher yield of iron oxide.

\section{Introduction to Atoms, the Periodic Tablle, and Nomenclature}

Small Particles (1h): Students to use vanilla extract and a balloon to analyze how small atoms actually are and how molecules are in motion.

Descriptive Chemistry of the elements $(1 \mathrm{~b}, 1 \mathrm{c})$ : Students will use a small hammer, hydrochloric acid, a conductivity apparatus and small samples of elements to determine the pattern of the properties of the represented elements. Each element will be tested using a hammer, hydrochloric acid, and a conductivity apparatus.

Flame Test (1a, 1b, 1c, 1d): Students will observe the characteristic colors by common cations using a flame test. 
Observing a Chemical Reaction ( $3 a$ ): Using a .01M Potassium permanganate solution and $.01 \mathrm{M}$ of Sodium hydrogen sulfite solution students to perform and observe a chemical reaction in which one of the reactants is completely used up and the other is in excess.

Sand Bag $(3 b, 3 c)$ : Students to determine how many grains of sand are in a bag of sand that they are given. Each grain of sand weighs approximately .05 grams. Using what they know about moles, students are to solve the problem.

Percent Composition of Gum (3a, 3b, 3c, 3d, 3e, 3f): Students to calculate the percent composition of solid sweeteners in gum to infer whether the sugar is a coating or is mixed throughout the gum.

Baking Soda ( $3 a, 3 b, 3 d, 3 e)$ : Using baking soda, a crucible, and a Bunsen burner students to predict how much sodium bicarbonate is produced when baking soda decomposes. Students will use stoichiometry to predict the mass of a product and compare it with the experimental mass.

Hydrogen Rockets (3a 3e): Students to generate hydrogen gas and oxygen gas and prepare small micropipette rockets using various concentrations. Lab report explores the relationship between the correct stoichiometric ratio of reactants and the distance the rocket traveled.

\section{Gas Laws}

Balloons ( $4 a, 4 e)$ : Students to use a balloon, string, a ruler and a bucket of ice to determine how temperature change affects the air in a balloon.

Carbon Dioxide (4c): Students will use Avogadro's principle and the combined gas law to calculate and compare the density of baking soda, vinegar, and oxygen.

Hot Air Balloon ( $4 a, 4 b, 4 c, 4 d, 4 f)$ ): Students creatively design and build a hot air balloon using any possible materials available (trash bags, dry cleaning bags, candles, pipe cleaners, glue, tooth picks, Styrofoam, paper, string, clay, matches, wood pieces, etc) Students are to use what they know about the ideal gas law and the affects of outside factors to construct a hot air balloon that will fly.

Boyle's Law ( $4 a, 4 b, 4 c, 4 d, 4 e)$ : Students use gas syringes to obtain data to use in graphing the relationship between the pressure and the volume of the gas.

\section{Chemical Bonds}

Versatile Metals (2a): Students to use samples of copper wire, a light socket with bulbs, and a battery to see how a variety of processes can be used to shape metals into different forms.

Making Ionic Compounds (2a), : Using magnesium ribbon, a crucible, ring stand and ring, a clay triangle, Bunsen burner, distilled water, a stirring rod, and a conductivity tester students will demonstrate the formation of ionic bonds. Students to heat the 
magnesium ribbon until a dry solid product forms, add distilled water and check the conductivity of the solution.

Treatment of Steel (2c): Students to observe how the properties of a metal change as rate of cooling changes and will hypothesize how the size and type of crystal formed affect properties.

Plant Chromatography (2b): Students to use various leaf samples, ethanol and chromatography paper to separate and analyze the various pigments in leaves.

Single Replacement Reaction $(2 a, 2 b)$ : Students to analyze the chemical reaction between copper and silver nitrate using a ring stand, small iron ring, funnel, Erlenmeyer flask and filter paper.

Hard Water (2a): Using distilled water, hard water, filter paper, washing soda, and dish detergent students will compare how hard and soft water differs in their ability to clean. Students will also determine how hard water can be softened.

Observing a Precipitate(2a, 2d): Students to combine Sodium Hydroxide and Epsom salts along with distilled water to observe what happens when two colorless solutions are mixed causing a double replacement reaction.

Hydrogen Pop (Chemical Change) (2a): Students to use hydrochloric acid, zinc, and lighted splint to observe how hydrogen gas reacts with oxygen in the air to produce gaseous water. The reaction produces a pop.

\section{Thermodymamics}

Temperature of a Reaction (7a, 7b): Students will observe an exothermic reaction by mixing solutions of hydrochloric acid and sodium hydroxide. Students to determine if there is a temperature change when the chemical reaction takes place.

Enthalpy of Fusion for Ice (7c, 7d): Using a foam cup, thermometer, stiming rod, ice, water and a balance, students will use experimental data to calculate the enthalpy of fusion of ice.

Calorimetry $(7 \mathrm{c}, 7 \mathrm{~d})$ : Students to investigate the amount of energy contained in a potato chip. The burning of a potato chip releases heat stored in the substances contained in the chip. The heat will be absorbed by a mass of water. Students to use a large potato chip, a beaker, evaporating dish, thermometer, ring stand with ring, wire gauze, matches, a stirring rod, and a balance.

\section{Sollutions}

Solution Formation $(6 a, 6 b)$ : Students to combine ammonium chloride and water and calcium chloride and water to investigate whether the dissolution of two different solids in water is an exothermic or endothermic process.

\section{Chemical Equilibrium}

Equilibrium $(9 \mathrm{a}, 9 \mathrm{~b})$ : Using a beaker, graduated cylinder, water, and two straws students to fill both the graduated cylinder and beaker with $20 \mathrm{~mL}$ of water. Students are to then transfer water simultaneously from the graduated cylinder to the beaker 
and from the beaker to the graduated cylinder using the straws. Students will discover that equilibrium does not mean equal amounts of reactant and product.

Le Chatelier's Principle (9a): Students predict and then confirm the effect of a change in temperature, and then a change in concentration, would have on a chemical equilibrium.

\section{Rates of Reaction}

Speeding Reactions $(8 \mathrm{a}, 8 \mathrm{~b}, 8 \mathrm{c})$ : Students to combine dry active yeast and hydrogen peroxide to observe how an enzyme increases the rate of a chemical reaction.

Effervescent Tablet $(8 \mathrm{a}, 8 \mathrm{~b})$ : Students to determine the effect of temperature on the rate of a chemical reaction. Students will combine a piece of the effervescent tablet and water at room temperature, and a piece of the effervescent tablet and water using a hot plate.

Popcorn ( $8 \mathrm{a}, 8 \mathrm{~b})$ : Students to use oil, com kernels, and a hot plate to determine how many corn kernels, how much oil, and how various factors affect the potential to make the perfect popcorn.

Concentration and Reaction Rate (8a, 8b): Using four test tubes, a test tube rack, and proper safety equipment students to observe how different amounts of hydrochloric acid dissolve a piece of magnesium ribbon at different rates depending upon the concentration of hydrochloric acid.

\section{Acids and Bases}

Household Products (5a): Using red litmus paper, blue litmus paper, a microplate, 6-8 household products and phenolphthalein students to use two indicators to distinguish whether each household product is acidic or basic.

Soils ( $5 a, 5 b, 5 c, 5 d)$ : Students to use neutral litmus paper, phenolphthalein, $\mathrm{pH}$ paper, mortar and pestle, and three types of soil samples to determine first if each of the soils are acidic or basic, second to find what type of plants can grow in these soils, and third if they would find these soil types in the area in which they live.

Buffer Investigation ( $5 \mathrm{~b}, 5 \mathrm{c}, 5 \mathrm{~d})$ : Students to make a buffer solution using acetic acid and sodium acetate, observing the behavior of the buffer by adding small amounts of acid and base. Data is collected using $\mathrm{pH}$ probes and graphed for the lab report.

Orgamic Chemistry and Biochemistry

Motor Oil (10a, 10c): Knowing that the molecules of motor oil have long chains of carbon atoms, students will compare the viscosities of motor oil and water as well as the viscosities of two weights of motor oils.

Making an Ester (10a, 10b, 10c): Using salicylic acid, methanol, distilled water, beral pipette, and concentrated sulfuric acid students will prepare an ester by reacting alcohol with an organic acid. 
Chain Reactions (11a): Students will model a nuclear chain reaction using dominoes. They will manipulate the arrangement of the dominoes in order to maximize the rate at which the chain reaction occurs.

Modeling Radioactive Decay $(11 \mathrm{a}, 11 \mathrm{c}, 11 \mathrm{~d}, 11 \mathrm{e})$ : Students will use a model to examine the concept of half life of radioactive isotope using pennies, a large plastic cup, and graph paper.

\section{Kev Assigmments}

- Weekly homework assigned primarily from the textbook

- Lab reports

- Research assignments on topics that relate to societal and environmental issues that involve agricultural chemistry.

\section{Instructional Methods and/or Strategies}

Students in Agriculture Chemistry are given lectures which often include demonstrations, weekly homework assignments primarily from the textbook or handouts, weekly labs, and one research project. Students are encouraged to ask questions during the lectures and are encouraged to come in after school for help with assignments. Students usually work with a lab partner for every lab. Tests are given every few weeks, the graded tests are promptly returned, gone over, and students are encouraged to save them to use to study for future tests/finals.

\section{Assessment Methods/Tools}

Assessment of student performance is based on tests, lab participation and lab reports, completion of homework, class work, and one research paper/research project. Ten percent of their grade is based upon Supervised Agricultural Experience Program projects and another ten percent of their grade is based upon FFA. 


\section{Add New / Reinstate Courses}

View New Course

This is a resubmission for the course Agriculture Chemistry

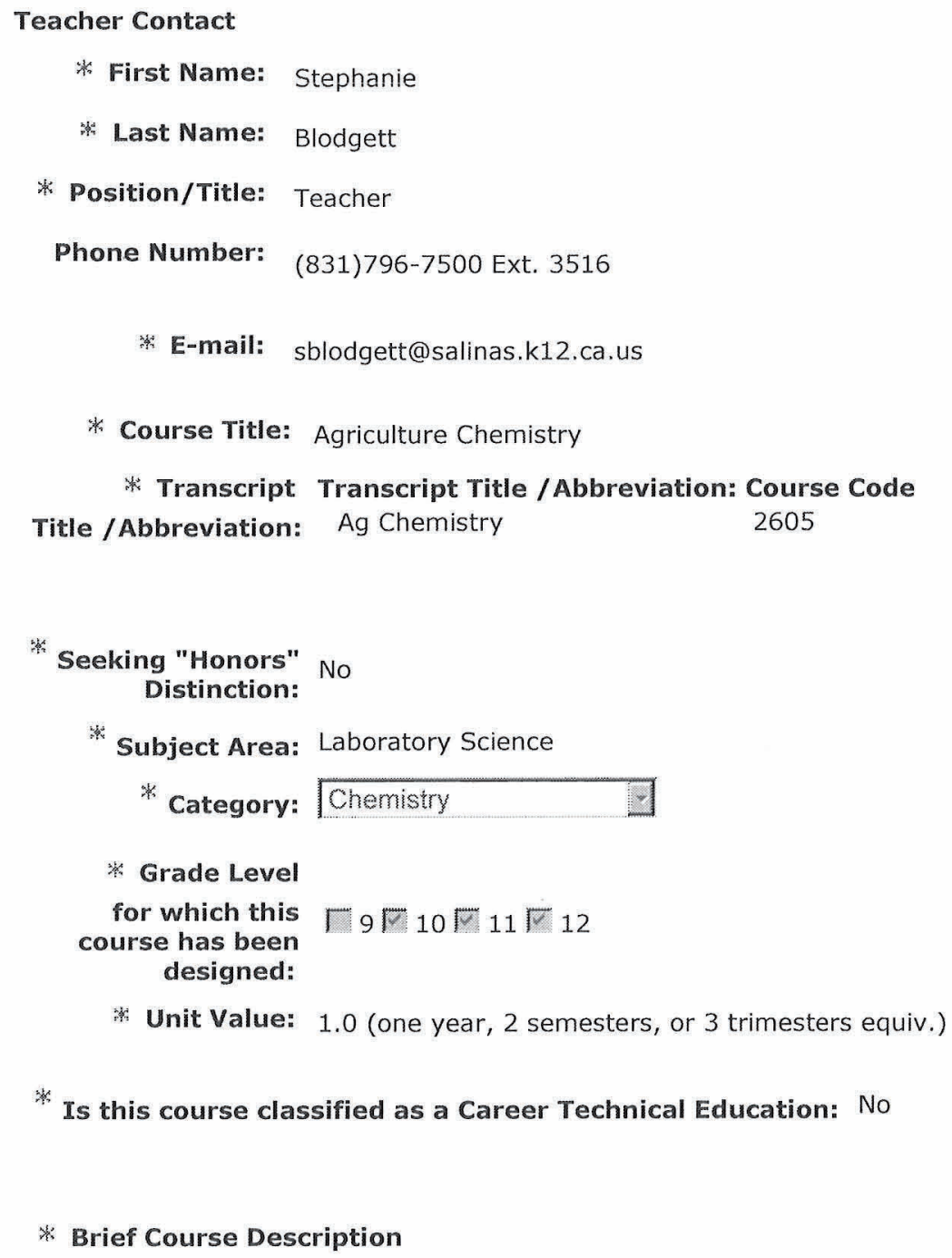


Framework for California Public Schools. Laboratory experiences are a major component of the course in addition to traditional instruction. This course meets the California and Salinas Union High School District graduation requirements for physical science. It qualifies as college lab course, meeting the University of California "A-G" requirements.

\section{Pre-Requisites}

C or better in Algebra or instructors permission. - Required

\section{Co-Requisites}

\section{Context for Course (optional)}

Students will learn a lab based chemistry course with a focus on agriculture and technology. This course will encompass concepts set forth in the California State Science Content Standards for California Public Schools as well as the Agriculture and Natural Resources Industry Sector Standards for California Public Schools. During this course students will learn about the composition and behavior of matter, atomic and molecular structure, conservation of matter and stoichiometry, chemicals and their properties and nuclear processes. Additionally, students will study practical skills development, academic skills development, and developing values including personal aspirations. The course addresses laboratory experiments in order to connect chemical science concepts with agricultural applications, including written and reporting skills. This courses also has a focus on students participation in the FFA program and the values and knowledge contained within.

\section{History of Course Development (optional)}

The curriculum incorporates all High School Life Science Blueprint content standards adopted by the California State Board of Education 2004, as required. Clarification of topics and unifying themes have been drawn from Benchmarks for Scientific Literacy (American Association for the Advancement of Science) and from National Science Education Standards (National Research Council). Much of the clarification of state standards and development of concept units is a result of meetings with the planning committee composed of high school chemistry teachers in the Salinas Union High School District who met during 2007-08.

\section{Textbooks}

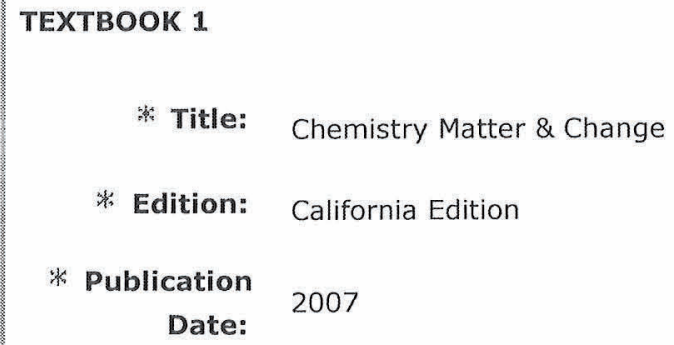


* Publisher: Glencoe

* Author(s): Laurel Dingrando

URL

Resource:

Usage: Primary Text

Read in entirety or near entirety

\section{Supplemental Instructional Materials}

FFA Course Manuel

SUPPLEMENTARY MATERIALS: Materials provided by the publisher including study guide and lab manual, CD accompaniment, Videodisc Programs, Web Links

\section{is Course Purpose}

Students will learn a lab based chemistry course with a focus on agriculture and technology. This course will encompass concepts set forth in the California State Science Content Standards for California Public Schools as well as the Agriculture and Natural Resources Industry Sector Standards for California Public Schools. During this course students will learn about the composition and behavior of matter, atomic and molecular structure, conservation of matter and stoichiometry, chemicals and their properties and nuclear processes. Additionally, students will study practical skills development, academic skills development, and developing values including personal aspirations. The course addresses laboratory experiments in order to connect chemical science concepts with agricultural applications, including written and reporting skills. This courses also has a focus on students participation in the FFA program and the values and knowledge contained within.

\section{凉 Course Outline}

Unit One Atomic Structure and the Periodic Table Main Concepts The atom is composed of protons, neutrons and electrons. Bohr's model of the atom Periodic table Focus Understandings The atom is the basic unit of matter Elements are placed on the periodic table in a specific Arrangement Readings-Chemistry, Glencoe MCGraw Hill -Pages 86-197 The structure of the Atom, Electrons in Atoms, Periodic Table and Periodic Law Reading-AGChemistry, FFA rules and regulation handbook Activity-Agriculture and Natural Resources-AG chemistry lab Finding Density Students apply algebraic techniques to solve rate problems, work problems and percent mixture problems. Activity-Agriculture and Natural Resources-AG chemistry how to make presentations and reports Unit Two Chemical bonding and Balancing Equations Main Concepts Naming and writing of chemical formulas is essential for writing balanced equations AG Chemicals used in agriculture. Farming practices and chemical controls Chemical bonding is a result of electron sharing or transfer The number of atoms for each element must be 
equal on both sides in a balanced equation Focus Understandings A molecule is two or more atoms chemically combined In a chemical reaction the amount of reactants have to equal the amount of products Chemical bonds are all about electrons Readings-Chemistry, Glencoe MCGraw Hill-pages 210-292 Ionic compounds, covalent bonding and Chemical Reactions Agriculture and Natural Resources-students organize and describe distributions of data by using a number of different methods, including frequency tables, histograms, standard line and bar graphs, stem and leaf displays, scatter plots, and box and whisker plots Unit Three-Moles and Stoichiometry Main Concepts The mole is the central unit in stoichiometry Conservation of atoms in reactions leads to the conservation of matter and ability to calculate the mass of products and reactants Focus Understandings Matter cannot be created or destroyed Moles count atoms Readings-Chemistry, Glencoe MCGraw Hill - Pages 308-370 Measuring matter, mass and the mole, moles of compounds, empirical and molecular formulas and the formula for hydrate. Included are readings related to Stoichiometeric calculations and limiting reactants and percent yield. Activity-Agriculture and Natural Resources-students research and pick their on FFA project. Unit Four-Gas Laws Main Concepts Gas laws explain the properties of gases Kinetic molecular theory describes the motion of atoms and molecules Focus Understandings All gases follow a group of predictable properties Temperature is a measurement of kinetic energy of particles Readings-Chemistry, Glencoe MCGraw Hill -Pages 418-440 Students will understand Boyle's law, Charle's Law and how it relates to Avogadro's principle. The students will also understand Ideal Gas Law and apply stoichiometry to this law. Agriculture and Natural Resources-students will understand how the gas laws apply to agricultural industry. Unit Five-Intro to Solutions Main Concepts Solutions are homogenous mixtures of two or more substances Random molecular motion can be described by the dissolving process at the molecular level Focus Understandings Solutions are homogenous mixtures Substances that dissolve become solutions Readings-Chemistry, Glencoe MCGraw Hill - Pages 452-476 Students will understand solution, solvents and solutes. They will understand heterogeneous and homogeneous mixtures and properties of solutions. Agriculture and Natural Resources-students will understand how to mix chemical together to create solutions animal health and in the farming industry. Researching and making community connects for real life examples for using mixtures in agriculture. Unit Six-Chemical Bonds and Structure (Biochemistry) Main Concepts Biological, chemical, and physical properties of matter result from the ability of atoms to form bonds There are electrostatic forces between electrons and protons as well as between atoms and molecules. Focus Understandings Electrons are responsible for the formation of chemical bonds ReadingsChemistry, Glencoe MCGraw Hill -Pages 662-761 Students will understand Voltaic cells, types of batteries and electrolysis. Students will understand Alkanes, Isomers and hydrocarbons and Petroleum. Agriculture and Natural Resourcesstudents will understand how chemical and physical properties effect matter and how this relates to the balance of soil in farming. Unit Seven--Thermodynamics Main Concepts Energy is exchanged or transformed in all chemical reactions and physical changes of matter Focus Understandings Energy flows from warmer to cooler Specific heat is the amount of energy needed to raise the of a substance Specific heat is an inherent property of substances Chemical and physical changes involve the flow of energy Energy flows during chemical physical changes Temperature does not change during a phase change Readings-Chemistry, Glencoe MCGraw Hill -Pages 488-513 Students will understand energy and chemical change using thermochemical equations. Students will be able to calculate enthalpy change. Agriculture and Natural Resources-students will understand how energy flows in our natural environment and how chemical 
properties affect the flow of energy. Unit Nine--Equilibrium Main Concepts Chemical equilibrium is a dynamic process at the molecular level Focus Understandings During chemical equilibrium forward and reverse reactions are occurring at the same rate Readings-Chemistry, Glencoe MCGraw Hill -Pages 558-575 Students will understand Equilibrium and the state of dynamic balance Students will demonstrate factors affecting chemical equilibrium through experimentation Agriculture and Natural Resources-students will build a business plan and develop outcomes for an agricultural market of their design. Unit TenReaction Rates Main Concepts Chemical reaction rates depend on factors that influence the frequency of collision of reactant molecules Focus Understandings Concentration, temperature and pressure can affect reaction rates ReadingsChemistry, Glencoe MCGraw Hill -Pages 528-546 Students will understand the model of reaction rates and the factors affect them. Students will demonstrate different reactions and record rates and effects. Agriculture and Natural ResourcesStudents will be able to distinguish between hypothesis and theory as scientific terms. They will also formulate explanations by using logic and evidence. Unit Eleven-Acids and Bases Main Concepts Acids, bases, and slats are three classes of compounds that form ions in water solutions Focus Understandings Acids donate hydrogen ions and bases accept hydrogen ions Readings-Chemistry, Glencoe MCGraw Hill -Pages 594-617 Students will understand acids and bases Students will demonstrate different reactions and record rates and effects. Agriculture and Natural Resources-Students will use soil samples and research to apply acidic and basic nature of soil to farming practices.

\title{
*aboratory Activities
}

\section{Laboratory Techniques and Measurement}

\author{
State Standards \\ Investigation and Experimentation
}

1. Scientific progress is made by asking meaningful questions and conducting careful investigations. Students will:

a. Select and use appropriate tools and technology (such as computer-linked probes, spreadsheets, and graphing calculators) to perform tests, collect data, analyze relationships, and display data.

\section{Student Outcomes}

1. In advance of each laboratory experiment, write a prelab or flow chart which expresses a hypothesis and describes the experimental procedure in the student's own words.

2. Learn the names and functions of the laboratory equipment used in experiments. 
3. Understand and use common laboratory terminology.

4. Make all quantitative measurements to the proper level of precision, and state the absolute error for each measurement.

5. Demonstrate competence in performing laboratory procedures. These tasks may include, but are not limited to: transfer of chemicals from stock containers, use of balance (both digital and mechanical, if possible), reading a thermometer, filtration, decantation, operating a Bunsen burner, reading volumes in graduated cylinders and gas measuring tubes, use of buret, cleaning and rinsing glassware, handling hot equipment and/or solutions, operation of specialized equipment such as colorimeters, $\mathrm{pH}$ meters, or spectrophotometers.

6. Perform appropriate analysis of experimental data, draw logical conclusions, and report findings in a clearly written laboratory report.

\section{Possible Strategies and Activities}

1. Provide guidelines for proper prelab procedures.

2. Identify the equipment used in each laboratory experiment, clarify the function of each item, and insist that students use the proper terms when referring to the equipment during class discussion and in written laboratory reports.

3. Model the use of proper terminology when discussing laboratory procedures, and require students to employ the correct terms when discussing their experiments.

Demonstrate how to make quantitative measurements to the highest possible level of precision with each instrument that is used in the laboratory. Follow-up may include putting emphasis on precision

1. statements in written laboratory reports as well as consistent modeling when, discussing treatment of quantitative laboratory data during class discussions.

2. Demonstrate the correct procedure for each specialized lab task that is introduced in student experiments, and correct students when necessary while observing their work in, the laboratory. Supporting strategies include requiring students to clearly explain each lab technique in their written flow 
sheets, and giving a "lab practical" exam in which students' lab skills are formally evaluated.

3. Provide students with a format for writing laboratory reports, evaluate students' reports in a timely manner, and give students suggestions for improving their work as the course progresses.

\section{Significant Figures and Dimensional Analysis (Factor Label Method)}

\section{State Standards}

Investigation and Experimentation

1. Scientific progress is made by asking meaningful questions and conducting careful investigations. Students will:

b. Select and use appropriate tools and technology (such as computer-linked probes, spreadsheets, and graphing calculators) to perform tests, collect data, analyze relationships, and display data.

c. Identify and communicate sources of unavoidable experimental error.

d. Identify possible reasons for inconsistent results, such as sources of error or uncontrolled conditions.

e. Formulate explanations by using logic and evidence.

f. Solve scientific problems by using quadratic equations and simple trigonometric, exponential, and logarithmic functions.

k. Recognize the issues of statistical variability and the need for controlled tests.

m. Analyze situations and solve problems that require combining and applying concepts from more than one area of science. 


\section{Student Outcomes}

1. Demonstrate a consistent use of units in measurements and calculations.

2. Use the factor label method in the solution of all problems and laboratory calculations assigned throughout the year.

3. Use estimation techniques incorporating dimensional analysis to arrive at approximate values when assigned to do so by the teacher.

4. Demonstrate skills in the use of fractions, scientific notation, exponents, graphing, graph interpretation, and the correct use of significant digits to indicate the appropriate degree of uncertainty.

\section{Possible Strategies and Activities}

1. Provide direct instruction and consistently model the use of appropriate units associated with measurements.

2. Teach factor labeling as a means of solving of problems both from laboratory experiences and from hypothetical situations.

3. Model and provide opportunities for estimation using orders of magnitude; arriving at approximate values; and indicating appropriate degrees of uncertainty.

4. Review basic mathematical skills for use in chemistry.

5. Monitor and evaluate student learning. Make adjustments as shown necessary in the monitoring process, with prompt correction and review.

6. Select appropriate materials and media to enhance learning, including some of the following: measurement lab with significant figures calculations; density lab; graphing lab

\section{Unit 2: Introduction to Atoms, the Periodic Table, and Chemical}




\section{Nomenclature}

\section{State Standards}

\section{Atomic and Molecular Structure}

1. The periodic table displays the elements in increasing atomic number and shows how periodicity of the physical and chemical properties of the elements relates to atomic structure. As a basis for understanding this concept:

a. Students know how to relate the position of an element in the periodic table to its atomic number and atomic mass.

b. Students know how to use the periodic table to identify metals, semimetals, non-metals, and halogens.

c. Students know how to use the periodic table to identify alkali metals, alkaline earth metals and transition metals, trends in ionization energy, electronegativity, and the relative sizes of ions and atoms.

d. Students know how to use the periodic table to determine the number of electrons available for bonding.

e. Students know the nucleus of the atom is much smaller than the atom yet contains most of its mass.

f. *Students know how to use the periodic table to identify the lanthanide, actinide, and transactinide elements and know that the transuranium elements were synthesized and identified in laboratory experiments through the use of nuclear accelerators.

h. *Students know the experimental basis for Thomson's discovery of the electron, Rutherford's nuclear atom, and Millikan's oil drop experiment.

\section{Chemicall Bonds}

2. Biological, chemical, and physical properties of matter result from the ability of atoms to form bonds from electrostatic 
forces between electrons and protons and between atoms and molecules. As a basis for understanding this concept:

a. Students know atoms combine to form molecules by sharing electrons to form covalent or metallic bonds or by exchanging electrons to form ionic bonds.

\section{Student Outcomes}

1. Students will be able to describe the basic structure of the atom and the experimental evidence that lead to our understanding of atomic structure.

2. Students will know the basic organization of the periodic table.

3. Students will know that compounds are formed when ions are held together by electrostatic forces or when atoms share electrons to form molecules.

4. Students will be able to name and write formulas for ionic and molecular compounds and distinguish between the two by noting whether a metal and a non-metal or two nonmetals are combining. In order to do this; students must be familiar with both the locations of metals and non-metals on the periodic table, and with the use and the naming of polyatomic ions.

\section{Possible Strategies and Activities}

1. Provide a historic overview of the history of the discovery of the atom, subatomic particles, and the periodic table. This overview will be accompanied by lab, audiovisual, lecture, and research (including Internet assignments).

2. Explain all the parts of the periodic table including the distinction between groups and families, metals and nonmetals, specific families, valence numbers, and how to determine ionic charges.

3. Have students memorize the formula and name for the common polyatomic ions. 
4. Have students write IUPAC names for compounds based on their formulas and formulas based on names.

Unit 3: Conservation of Matter and Stoichiometry

State Standards

\section{Conservation of Matter and Stoichiometry}

1. The conservation of atoms in chemical reactions leads to the principle of conservation of matter and the ability to calculate the mass of products and reactants. As a basis for understanding this concept:

a. Students know how to describe chemical reactions by writing balanced equations.

b. Students know the quantity one mole is set by defining one mole of carbon 12 atoms to have a mass of exactly 12 grams.

c. Students know one mole equals $6.02 \times 10^{23}$ particles (atoms or molecules).

d. Students know how to determine the molar mass of a molecule from its chemical formula and a table of atomic masses and how to convert the mass of a molecular substance to moles, number of particles, or volume of gas at standard temperature and pressure.

e. Students know how to calculate the masses of reactants and products in a chemical reaction from the mass of one of the reactants or products and the relevant atomic masses.

f. *Students know how to calculate percent yield in a chemical reaction.

\section{Student Outcomes}

1. Balance chemical equations using the proper chemical symbols and coefficients. 3 a 
2. Utilize Avogadro's hypothesis to solve conversion problems involving atomic weight, molar mass, the mole, and molar volume. $3 \mathrm{~b}, 3 \mathrm{c}, 3 \mathrm{~d}, 3 \mathrm{e}$

3. Students will be able to calculate the percent yield in a chemical reaction.

\section{Possible Strategies and Activities}

1. Provide direct instruction that clarifies, defines, and provides a consistent pattern for solving problems in the laboratory and from hypothetical situations.

2. Give students numerous opportunities to perform molar calculations, using stoichiometric ratios, Avogadro's number, molar mass, and molarity.

3. Provide extensive practice with prompt correction and review of assigned problems.

4. Select appropriate materials in the form of films and software to supplement lecture.

5. Provide laboratory opportunities including calculations of molar mass, mass relationships accompanying chemical changes, and requiring the use of stoichiometric calculations including the calculation of percent yield.

\section{Unit 4: Gas Laws}

State Standards

\section{Gas Laws}

4. The kinetic molecular theory describes the motion of atoms and molecules and explains the properties of gases. As a basis for understanding this concept:

a. Students know the random motion of molecules and their collisions with a surface create the observable pressure on that surface. 
b. Students know the random motion of molecules explains the diffusion of gases.

c. Students know how to apply the gas laws to relations between the pressure, temperature, and volume of any amount of an ideal gas or any mixture of ideal gases.

d. Students know the values and meanings of standard temperature and pressure (STP).

e. Students know how to convert between the Celsius and Kelvin temperature scales.

f. Students know there is no temperature lower than 0 Kelvin.

\section{Student Outcomes}

1. Be able to explain the characteristics of gases.

2. Use the following gas laws: Dalton's Law of Partial Pressures, Charles' Law, Boyle's Law, Avogadro's Principle, the Ideal Gas Law, the Combined Gas Law, and Graham's Law. $\mathbf{4}$ c, $\mathbf{4 d}$

3. Be able to graph and exchange the changes of state using heating and cooling curves and label: melting point, boiling point, heat of fusion, heat of vaporization $\mathbf{4 e .} \mathbf{4 f}$

\section{Possible Strategies and Activities}

1. Use models and lecture to illustrate the differences between a solid, liquid and gas on the macro and molecular level.

2. Define the gas laws and utilize them for problem solving, then provide guided practice and laboratory activities for the students.

3. Select experiments that will facility the students use of laboratory data, and when possible computer graphing software to illustrate heating and cooling curves. 


\section{UNIT 5: CHEMICAL BONDS}

\section{State Standards}

2. Biological, chemical, and physical properties of matter result from the ability of atoms to form bonds from electrostatic forces between electrons and protons and between atoms and molecules. As a basis for understanding this concept:

a. Students know atoms combine to form molecules by sharing electrons to form covalent or metallic bonds or by exchanging electrons to form ionic bonds.

b. Students know chemical bonds between atoms in molecules such as $\mathrm{H}_{2}, \mathrm{CH}_{4}, \mathrm{NH}_{3}, \mathrm{H}_{2} \mathrm{CCH}_{2}, \mathrm{~N}_{2}, \mathrm{Cl}_{2}$, and many large biological molecules are covalent.

c. Students know salt crystals, such as $\mathrm{NaCl}$, are repeating patterns of positive and negative ions held together by electrostatic attraction.

d. Students know the atoms and molecules in liquids move in a random pattern relative to one another because the intermolecular forces are too weak to hold the atoms or molecules in a solid form.

e. Students know how to draw Lewis dot structures.

\section{Students Outcomes}

1. Review the nature and rational of chemical bonding, ionic model, ionic character, oxidation states of families, and covalent bonding.

2. Draw and explain Lewis dot structure, molecular structure, octet rule, valence electrons/pairs.

3. Describe molecular structure, octet rule and exception, resonance.

4. Explain VSEPR model, hybridization, and molecular orbital 
model.

\section{Possible Strategies and Activities}

1. Students build or observe models of crystal lattices.

3. Build molecular shapes and structures of certain molecules.

4. Use of molecular model sets.

5. Use of Styrofoam balls and colored pipe cleaners to demonstrate valence electrons and molecular shapes.

\section{UNIT 6: Thermodynamics}

\section{State Standards}

7. Energy is exchanged or transformed in all chemical reactions and physical changes of matter. As a basis for understanding this concept:

a. Students know how to describe temperature and heat flow in terms of the motion of molecules (or atoms).

b. Students know how chemical processes can either release (exothermic) or absorb (endothermic) thermal energy.

c. Students know energy is released when a material condenses or freezes and is absorbed when a material evaporates or melts.

d. Students know how to solve problems involving heat flow and temperature changes, using known values of specific heat and latent heat of phase change. 


\section{Students Outcomes}

1. Explain why energy always flows from warnter to cooler.

2. Describe specific heat is the amount of energy needed to raise the temperature of a substance..

3. Know that specific heat is an inherent property of substances.

4. Chemical and physical changes involve the flow of energy.

5. Be able to solve problems involving specific heat and temperature change.

6. Temperature does not change during a phase change.

\section{Possible Strategies and Activities}

1. Demonstrate calorimeter, Enthalpy, thermo - chemical equations, heats of formation.

2. Solve heat flow problems

3. Calorimeter labs using Styrofoam cups and different metal pieces to identify specific heats of various metals.

4. Demonstrate athletic trainer's cold packs (endothermic) and hot packs (exothermic). Ziploc bag reactions also work.

5. Show students models of molecular motion of gases, solids and liquids to show energy loss or gain during phase change.

Unit 7: Solutions

State Standards

\section{Solutions}

6. Solutions are homogenous mixtures of two or more substances. As a basis for understanding this concept:

a. Students know the definitions of solute and solvent. 
b. Students know how to describe the dissolving process at the molecular level by using the concept of random molecular motion.

c. Students know temperature, pressure, and surface area affect the dissolving process.

d. Students know how to calculate the concentration of a solute in terms of grams per liter, molarity, parts per million, and percent composition.

2. Biological, chemical, and physical properties of matter result from the ability of atoms to form bonds from electrostatic forces between electrons and protons and between atoms and molecules. As a basis for understanding this concept:

d. Students know the atoms and molecules in liquids move in a random pattern relative to one another because the intermolecular forces are too weak to hold the atoms or molecules in a solid form.

\section{Student Outcomes}

1. Be able to state the properties and states of matter verbally and to diagram the states of mater at a macro and micro level. 6b

2. Compare solids, liquids, and gases and be able to explain the distinguishing characteristics of each. $\mathbf{6 b}$

3. Be able to explain the structure and properties of liquids, including surface tension, surface area, and how the intermolecular forces in water make it a unique substance.

$2 \mathrm{~d}, 6 \mathrm{c}$

1. Be able to explain what a solution is (distinguishing between solute and solvent) including the process of solvation, factors affecting solubility and conductivity, and methods of purification. 6a, 6b

2. Students will be able to calculate the concentration of a 
solute in terms of grams per liter, molarity, and percent composition. 6d

\section{Possible Strategies and Activities}

1. Use models and lecture to illustrate the differences between a solid, liquid and gas on the macro and molecular level.

2. Select experiments that will facility the students use of laboratory data, and when possible computer graphing software to illustrate heating and cooling curves, and to calculate heats of phase changes.

3. Supplement lecture with laboratory investigations and demonstrations to illustrate the properties of solutions and the colligative effects of various solutes.

\section{Unit 8: Equilibrium}

\section{Chemical Equilibrium}

\section{State Standards}

9. Chemical equilibrium is a dynamic process at the molecular level. As a basis for understanding this concept:

a. Students know how to use LeChatelier's principle to predict the effect of changes in concentration, temperature, and pressure.

b. Students know equilibrium is established when forward and reverse reaction rates are equal.

\section{Student Outcomes}

1. Describe the equilibrium condition.

2. Describe the microscopic processes that occur in a system when it is at equilibrium. $\mathbf{9 b}$ 
3. Use Le Chatelier's principle to explain how addition of a catalyst or changes in concentration, temperature, and/or pressure affect a system at equilibrium. 9a

4. Determine equilibrium exp0ressions for a given reaction.

\section{Possible Strategies and Activities}

1. Present examples that clearly illustrate the similarities and differences between steady state and equilibrium conditions.

2. Emphasize the dynamic processes that occur at the microscopic level within a system that is at equilibrium.

4. Give students numerous opportunities to apply Le Chatelier's principle, including written problems, demonstrations, and/or experiments.

5. Illustrate how to derive an equilibrium law expression; then provide practice opportunities with monitoring of student progress.

6. Show several variations of how equilibrium law calculations are used to find unknown concentrations or equilibrium constants.

\section{Unit 9: Rates of Reaction}

State Standards

\section{Reaction Rates}

8. Chemical reaction rates depend on factors that influence the frequency of collision of reactant molecules. As a basis for understanding this concept:

a. Students know the rate of reaction is the decrease in concentration of reactants or the increase in concentration 
of products with time.

b. Students know how reaction rates depend on such factors as concentration, temperature, and pressure.

c. Students know the role a catalyst plays in increasing the reaction rate.

\section{Student Outcomes}

1. Be able to explain how factors such as concentration, temperature, pressure, and the influence of catalysts affect reaction rates. $\mathbf{8 b}, \mathbf{8 c}$

2. Be able to graph the change in amount of products versus reactants over time, including the affects of catalyst on the activation energy. $\mathbf{8 a}$

\section{Possible Strategies and Activities}

1. Give students numerous opportunities to apply Le Chatelier's principle, including written problems, demonstrations, and/or experiments.

2. Graph concentration verses time for iodine clock reaction.

3. Use graphs of activation energy to demonstrate catalyst affects on reactions.

4. Demonstrate catalyst using split tests for oxygen using manganese dioxide in the decomposition of hydrogen peroxide.

\section{Unit 10: Acids \& Bases}

\section{State Standards}

\section{Acids and Bases}

5. Acids, bases, and salts are three classes of compounds that form ions in water solutions. As a basis for understanding 
this concept:

a. Students know the observable properties of acids, bases, and salt solutions.

b. Students know acids are hydrogen-ion-donating and bases are hydrogen-ion-accepting substances.

c. Students know strong acids and bases fully dissociate and weak acids and bases partially dissociate.

d. Students know how to use the pH scale to characterize acid and base solutions.

\section{Student Outcomes}

1. Demonstrate. how to calculate $\mathrm{pH}$ from a hydrogen-ion concentration in a laboratory exercise utilizing different aqueous concentrations. $5 \mathrm{c}$

2. Use a pH meter and hydronium paper to identify solutions that are acidic and basic. $\mathbf{5 d}$

3. Apply the Arrhenius, Brønsted-Lowery, and Lewis acid-base concepts to lecture problems and homework practice exercises. $\mathbf{5 c}$

4. Identify the observable properties of acids, bases, and salt solutions. 5 a

5. Compare and contrast the differences between acids that are hydrogen-ion-donating and bases that are hydrogen-ionaccepting substances. $\mathbf{5 b}$

\section{Possible Strategies and Activities}

1. Clarify how acids are hydrogen-ion-donating and bases are hydrogen-ion-accepting substances.

2. Provide classroom demonstrations that differentiate between acids, bases, and salt solutions. 
3. Monitor student's use of $\mathrm{pH}$ meters during laboratory experiments with different strengths of aqueous acidic and aqueous basic solutions.

4. Synthesize the concepts of Arrhenius, Brønsted-Lowery, and Lewis Acid-base solutions in simplifying how each concept is involved in a solution.

5. Evaluate student understanding of how strong bases and bases fully dissociate and weak acids and bases partially dissociate with practice problems, quizzes, and homework.

6. Teachers will facilitate learning with classroom demonstrations, Internet investigations of current topics, and the use of relevant software.

\section{Unit 11: Organic Chemistry}

\section{State Standards}

\section{Organic Chemistry and Biochemistry}

10. The bonding characteristics of carbon allow the formation of many different organic molecules of varied sizes, shapes, and chemical properties and provide the biochemical basis of life. As a basis for understanding this concept:

a. Students know large molecules (polymers), such as proteins, nucleic acids, and starch, are formed by repetitive combinations of simple subunits.

b. Students know the bonding characteristics of carbon that result in the formation of a large variety of structures ranging from simple hydrocarbons to complex polymers and biological molecules.

c. Students know amino acids are the building blocks of proteins.

\section{Student Outcomes}

1. Be able to explain the importance of carbon in the formation of many organic molecules essential to life. 10a 
2. Define the role of proteins, nucleic acids, and starch as they relate to biological structure, movement, catalysts, transport, storage, energy transformation, protection, and buffering. $10 \mathrm{~b}$

3. Identify the thirty essential elements and know the R-group structure of amino acids and how they combine to form the polypeptide backbone structure of proteins. 10c

\section{Possible Strategies and Activities}

1. Illustrate and provide an opportunity for students to differentiate between simple linear hydrocarbons, hydrocarbons with double and, triple bonds, and molecules that contain benzene rings through demonstration, laboratory experiments, and lecture.

2. Supplement lecture with overheads, film, and models to illustrate the thirty essential elements used by amino acids as the building blocks of protein, and the basic structure of proteins.

3. Explain the role of proteins, nucleic acids, and starch using a systemic approach to the biochemical processes in the human body.

4. Supplement lecture with classroom demonstrations, Internet investigations, laboratory assignments and where appropriate relevant software will be incorporated into content area.

5. Provide opportunities for the students to complete laboratory experiments, analyze data, and write laboratory reports in the above content areas.

\section{Unit 12: Nuclear Chemistry}

State Standards

\section{Nuclear Processes}

11. Nuclear processes are those in which an atomic nucleus changes, including radioactive decay of naturally occurring 
and human-made isotopes, nuclear fission, and nuclear fusion.

As a basis for understanding this concept:

a. Students know protons and neutrons in the nucleus are held together by nuclear forces that overcome the electromagnetic repulsion between the protons.

b. Students know the energy release per gram of material is much larger in nuclear fusion or fission reactions than in chemical reactions. The change in mass (calculated by $\mathrm{E}=\mathrm{mc}^{2}$ ) is small but significant in nuclear reactions.

c. Students know some naturally occurring isotopes of elements are radioactive, as are isotopes formed in nuclear reactions.

d. Students know the three most common forms of radioactive decay (alpha, beta, and gamma) and know how the nucleus changes in each type of decay.

e. Students know alpha, beta, and gamma radiation produce different amounts and kinds of damage in matter and have different penetrations.

\section{Student Outcomes}

1. Be able to explain how nuclear forces allow protons to overcome their natural repulsion and reside in the nucleus. Ila

2. Be able to state the difference between nuclear fusion and nuclear fission, and be able to relate these differences to the amount of energy generated in each process. IIb

3. Describe the existence of radioactive isotopes, both natural, and those formed as a byproduct of nuclear reactions, and explain how a radiation counter is used to measure radioactive isotopes. IIc

4. Compare the different amounts of radiation that alpha, beta, and gamma particles produce, know how the nucleus changes in each type of decay, and be able to describe the penetration ability, and types of damage that each will produce in matter. $\mathbf{1 1} \mathbf{d}, \mathbf{1 1} \mathbf{e}$ 
5. Students will be able to explain how a nuclear reactor works, and the importance of chain reactions in this process (including the idea of critical mass).

\section{Possible Strategies and Activities}

1. Explain and model nuclear forces as they pertain to atomic structure and illustrate how energy released per gram of material is much larger via nuclear fusion or fission than in previously studied chemical reactions.

2. Use a radiation counter, illustrations, and lecture to demonstrate the radiation emitted from alpha, beta, and gamma radiation and explain the differences in penetration and damage between the three types of radiation.

3. Use guided practice, and examples to show students how to utilize the half-life rule to calculate the amount of material that will decay during a specified amount of time.

4. Use classroom demonstrations, Internet investigations and computer technology where appropriate to compliment lecture and provide the opportunity for students to conduct laboratory experiments, analyze data, and complete laboratory reports to highlight these concepts.

Key Assignments

Unit One Atomic Structure and the Periodic Table Periodic Table GamePuns Atomic mass of candium ( $p 126$ ) Unit Two Chemical bonding and Balancing Equations Dry Lab-writing chemical formulas Graphic organizer-flow chart for naming compounds Conductivity lab-testing conductivity of salt water Making ionic compounds-pg $163 \mathrm{Lab}-\mathrm{pH}-$ names and formulas of ionic compounds Precipitation reactions-formation of solids Unit Three-Moles and Stoichiometry Lab-measuring mass as means of counting Lab-conservation of mass-analysis of baking soda Stoichiometry lab-predict mass of object Unit Four-Gas Laws Lab--Boyles 
laws-vacuum pump Lab--BB's and pressure Lab--Carbon dioxide from antacids Demo-perfume/ammonia from side to side in room-Graham's Law Lab--Reaction of Acids with Carbonates Unit Five-Intro to Solutions Cartoon video creation to illustrate concept Lab--electrolytes Unit SixChemical Bonds and Structure Lab-food labels, cosmetics-writing ionic compounds Lab-build models of crystal lattices Lab-surface tension and mercury-hydrogen bonding Unit Seven--Thermodynamics Phase change lab-observe and graph temperature change or lack thereof as ice melts and water evaporates Lab-calorimeter lab using metal and Styrofoam cups to identify specific heats of various metals Lab-athletic trainer's cold packs and hot packs for endo and exothermic reactions Model molecular motion of gases, solids and liquids to show energy loss or gains during phase change Unit Eight--Solutions Demo-sugar cube vs. powdered sugar dissolving, gas pressure in soda can Lab-crystal growing-supersaturated solutions Lab-solutions showing molarity by color Home lab-molarity of drink from home Unit Nine-Equilibrium Water cycle as model of equilibrium Graphing-identify when equilibrium reached Equilibrium of iron thiocynate Unit Ten-Reaction Rates Lab-students graph concentration vs. time for iodine clack reaction or sulfur clock reaction Lab-splint test for oxygen Graph-activation energy Lab demodecomposition of hydrogen peroxide using catalysts Inquiry lab-baking soda and vinegar-filling up balloons Unit Eleven-Acids and Bases pH paper lab-use at home Lab-experiment with wide variety of indicators Lab-substances on the $\mathrm{pH}$ scale Lab-titration Mapping the $\mathrm{H}+$ ions Predict products of reactions Lab-Conductivity meter to understand full dissociation vs. partial dissociation in common household substances. Key Assignments-Detailed descriptions of the Key Assignments including tests and quizzes. How do assignments incorporate topics? Include all major assignments that students will be required to complete. Key assignments in Agricultural Chemistry comprise two parts, the chemistry focus and the agriculture foundation focus. Key assignments in chemistry include the labs listed and described in Laboratory Assignments as well as the all quizzes, tests, and final exams. Students also participate in the school wide science fair at the end of the year. The agricultural component of the agriculture chemistry course includes but is not limited to the requirements of the FFA program as described in the FFA manual used in the course as a supplemental text.

\section{* Instructional Methods and/or Strategies}

Students will use a variety of strategies to learn the material covered in agricultural chemistry including but not limited to Vocabulary Review of prior information Hands-on wet labs Dry paper labs Direct instruction Textbook readings DVD Computer websites Coursework Homework Use of state standards Graphic organizers Mini assessments District wide benchmark review

\section{絭 Assessment Methods and/or Tools}

Students will utilize a wide range of tools for assessment including quizzes, tests, coursework, homework, district generated benchmarks, state testing, and final 
exams for this course for all of the units involved. Additionally unit specific assessment will include but is not limited to the following for each unit: Unit One Atomic Structure and the Periodic Table Quiz of elements and symbols-desired outcome $80 \%$ correct Identify form periodic chart specific elements and their info. Quick write on atomic structure Unit Two Chemical bonding and Balancing Equations Mini-assessment of writing formulas and naming compounds Graphic organizer comparing and contrasting ionic and covalent bonds Unit Three-Moles and Stoichiometry Practice problems - predict the mass of product Factor labeling dimensional analysis on stoichiometry problems Calculate molar mass from chemical formula Unit Four-Gas Laws Solve problems given initial conditions of temperature, pressure and volume Use the ideal gas equatiton to find the fourth variable Use Dalton's law to solve for pressure equations Unit Five-Intro to Solutions T-chart of compare and contrast solute and solvent Unit Six-Chemical Bonds and Structure Draw and label crystal lattice structure Quick writeelectrostatic forces Draw and label molecule diagrams during phase change Quick write-intermolecular forces Lewis dot structures of elements and covalent compounds Quick write-valence electrons and Lewis dot structures Octet rule Draw arrows on periodic table -trends in ionization energy, electro negativity, and size of atoms Unit Seven--Thermodynamics Quick write-differences between temperature and heat flow Identify-exothermic and endothermic reactions Graph reaction including reactants, transition stages, products and levels of potential energy Problem solving - temperature, specific heat Unit Eight--Solutions Quick write- how temp, pressure and surface area affect dissolving of solutes Draw and label particle size and solution dissolution Calculate concentration of solutes in terms of gram per liter, molarity, parte per millions and percent Unit NineEquilibrium Write equilibrium equations Draw and label and diagram of a solution in equilibrium Draw and label diagrams of solutions in equilibrium and with changes in pressure and temperature Unit Ten-Reaction Rates Quick write changes in temperature of pressure can affect reaction rate Quick write-how a catalyst can affect reactions Interpret reaction curves by lowering activation energy by a catalyst Unit Eleven-Acids and Bases Graphic organizer-properties of acids, basses and salts Quick write- $\mathrm{pH}$ scale and values for acids and bases Quick write-relationship of dissociation of acids and bases and their properties such as electrical conductivity

\section{Print Close}

Send e-mail to: hsupdate@ucop.edu 


\section{Teacher Internship Program Ag Ed 539}

\section{Project: Re-Write the Ag Science Curriculum for the district and put into a format that can be UC approved.}

The second project that I decided to do for the Teacher Internship Program was to re-write the ag science curriculum for the district and put it into a format that could be UC approved. Like I said before, in the Salinas Union High School District there has been a push for all classes to meet the A through $G$ requirements and UC approval. In today's time it is tough for an agriculture program in the Salinas area to survive with only electives. The traditional agriculture class does not exist anymore and most programs are getting rid of those courses. At North Salinas however, we would like to keep as many of the traditional ag courses as possible. Rewriting the curriculum and putting it in a format that can be UC approved will help to keep the Ag Science course in the district.

\section{Step 1: Gather Previous Curriculum}

The Agriculture Science Curriculum for the Salinas Union High School District has not been updated in years. At North High Stan Uchiyama showed me at least three different curriculums that had been adopted throughout his years at North Salinas High School. In order to begin I took those curriculums and combined them to see what I liked or did not like about each one. I also looked at the State Standards for Ag Science as well as the other pathways that tie into ag science.

\section{Step 2: Start with District Course Outline}

I took my Ag Chemistry Curriculum and a few other curriculums in the district to use as a base for the Ag Science curriculum. This curriculum included a course title, grade level, length, number of credits, prerequisites, textbook, course description, sources of curriculum content, course outline, major concepts, and expected student outcomes. From there I started to input the Ag Science material that I had gathered into the appropriate format.

Step 3: Combine the Ag Science curriculums and incorporate State Standards to form new curriculum

Looking at the three Ag Science curriculums I was shown as well as the State Standards I took bits and pieces from all three to form the new curriculum. I mainly used the most recently approved curriculum, modified, and added to in order to produce a better representation of today's agriculture. The course outline included major concepts such as California Agriculture, Animal Science, Plant Science, Agricultural Business Management and Leadership. The next 
part was to input major concepts and expected student outcomes. I am currently still in the process of modifying the curriculum. As of now I am by no means satisfied with the work that I have done. I did not realize that this process would take as long as it has, but by the beginning of next school year (Fall 2011) a curriculum will be finished. I could very well submit this curriculum to the district as well as UC approval as an elective, but I am not sure that this is the path I want to take with this curriculum. I would like to push for a Life Science district approved course and a lab based UC approved course as well. I have provided a copy of the curriculum I have written at this time. 


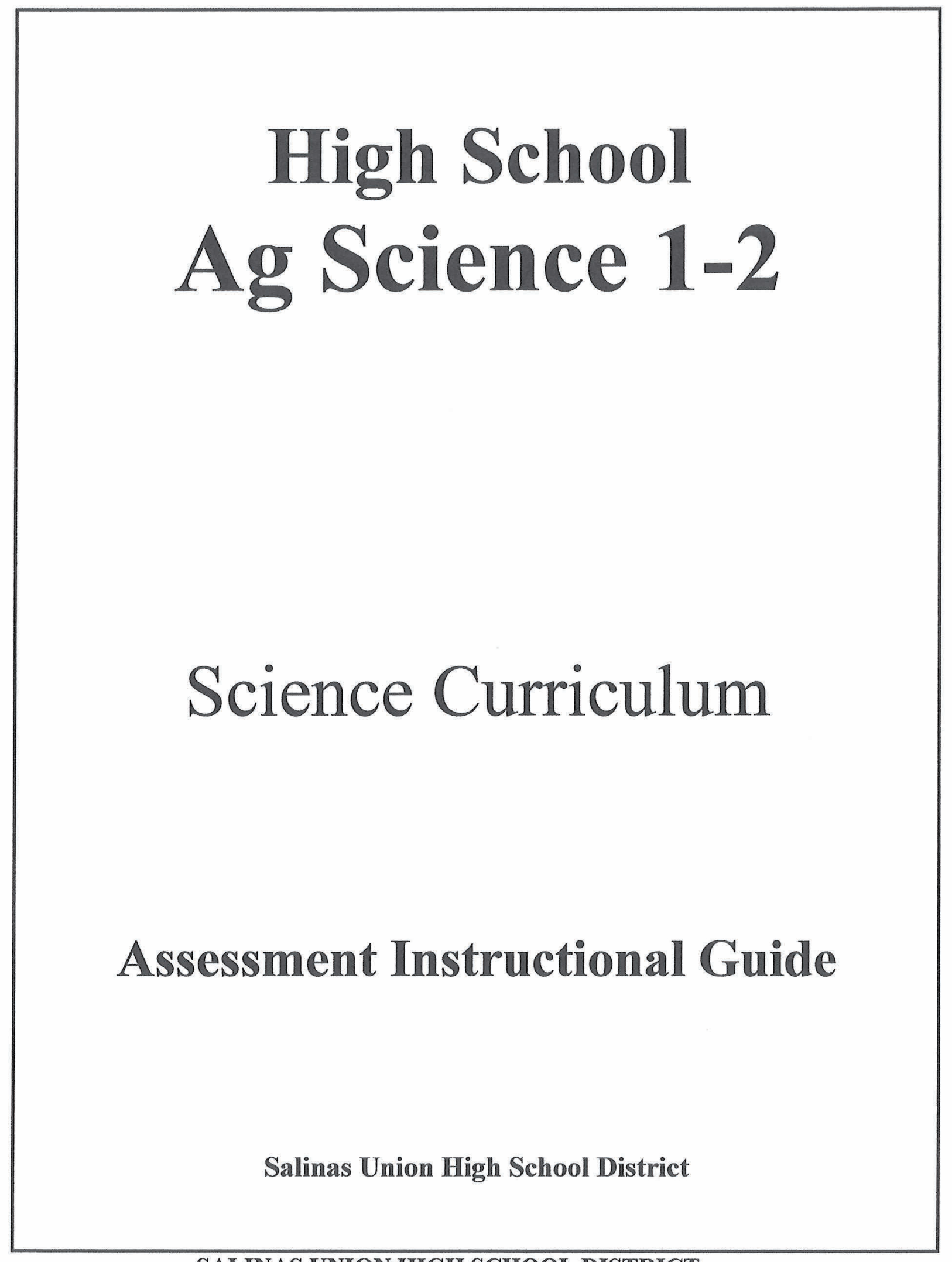

SALINAS UNION HIGH SCHOOL DISTRICT 


\section{DEPARTMENT:}

COURSE TITLE:

GRADE LEVEL:

LENGTH:

NUMBER OF CREDITS:

PREREQUISITE:
Agriculture

Ag Science 1-2

9-12

1 year

10 credits

None

TEXTBOOK:

SUPPLEMENTARY MATERIALS: Materials provided by the publisher including study guide and lab manual, CD accompaniment, Videodisc Programs, Web Links (SciLinks).

\section{COURSE DESCRIIPTION:}

This is a year course open to all students. It is strongly recommended for all beginning AgriScience and horticulture students. Students are given an insight into the importance of the agriculture industry in our lives and to society. Introductory study units cover agriculture supply and services, ornamental horticulture, agricultural mechanics, plant and animal science, agribusiness, and natural resources, and agriculture products and processing. Student four year program plan and involvement in the Department's supervised project program will complement areas of instruction. This course meets the California and Salinas Union High School District graduation requirements for Biological science or Vocational Education graduation requirements.

\section{SOURCES OF CURRICULUM CONTENT:}

The curriculum is formed from the California Agriculture and Natural Resources Industry sector. 


\section{Agriculture Science Course Outline}

\section{Grades 9-12}

\section{Major Concepts}

1. California Agriculture

2. Animal Science

3. Plant Science

4. Agricultural Business Management

5. Leadership

\section{MAJOR CONCEPTS/EXPECTED STUDENT OUTCOMES}

\section{MAJOR CONCEPT:}

California Agriculture

A. Students will understand the ecomomic importance of the agricultural sector in California and be able to identify the leading state's production areas and commodities.

1. Identify the major agricultural production areas of California and list three commodities produced in each.

2. List the dollar value of the five leading agricultural commodities produced in the local community

3. List the dollar value of the five leading agricultural commodities produced in the state.

B. Students will understand the interrelationship of agriculture and society in California, including the factors that influence agricultural activities.

1. List three problems faced by California farmers that are caused by population shifts and social and technological trends.

2. List five government agencies that influence and affect agricultural production in California

C. Students will understand the impact of agricultural production on the environment and the natural resources of Califormia

1. Describe the major watersheds of California

2. Identify the major forest regions of California

3. List three environment effects of production agriculture in California 


\section{MAJOR CONCEPT:}

Animal Science

A. Students will understand the importance of domestic animalls and their roles in moderm society.

1. Describe the importance of animal domestication.

2. Identify within each domestic species four livestock enterprises that are part of production agriculture in the United States.

3. Identify the major animal sources of protein in the world.

B. Students will develop a basic understanding of animal behavior, morphology, taxonomy, general reproductive traits, and natural sellection.

1. Visually identify the external anatomical parts of the pig, cow, horse, chicken, goat, and sheep.

2. Describe generally how conception takes place and make a chart listing the gestation period of the cow, horse, pig, chicken, goat, and sheep.

3. Describe the difference between a species and a subspecies (variety) and provide examples from among domestic animals.

4. Observe how the offspring and dam of a domestic species interact and identify each other (e.g., sight and smell). Discuss the observations. (This activity can be done with either live or videotaped animals).

C. Students will develop a basic understanding of the structure, function, and maintenance of the major body systems (e.g., the dligestive system) and their components.

1. Use appropriate terminology when describing direction and position on the vertebrate body (dorsal, ventral, etc).

D. Students will develop a basic understanding of the theory of inheritance and the genetic basis for animal selection

1. Describe the process of fertilization in mammals.

E. Students will dlevelop an understanding of the factors involved in animal mutrition, animal feeding, and the basic feedstuffs available for that purpose.

1. Describe the six classes of nutrients and identify examples of feeds containing each.

2. Visually identify six common feed additives.

3. Describe a simple feeding regime for market swine, lambs, and cattle.

4. Either individually or through a cooperative effort, feed an animal through a full production cycle. 
5. Develop an appreciation for the importance of feeding regimes in animal management and compute a balanced ration, using the Pearson Square or nutrient requirement and feed composition tables.

F. Students will understand the concept of animal health. They will become familiar with methods of identification of unhealthy animals, preventive measures, treatment, and the casual agents of common health problems in animals of economic importance.

1. List five predisposing conditions that cause health problems in animals.

2. Demonstrate a method of control for an internal and external parasite.

3. Read and interpret the information found on medication labels. (For example: dosage, storage, antidotes).

4. Identify specific ways that infectious agents may gain entrance and do harm to an animal.

5. Properly determine the body temperature of an animal.

G. Students will develop an appreciation for the factors involved in and the ability to evaluate and select livestock for specific uses.

1. List four uses of livestock evaluation for the producer.

2. Differentiate between ideal modern and old fashioned types of meat animals, using two market species. Live animals or photos may be used as examples.

3. Evaluate two species of livestock on a comparative basis, in groups of our, using live animals or photos.

H. Students will understand the basis for meat grading and develop an appreciation of the variety of products available from meat animals.

1. Visually identify six wholesale and retail cuts each of beef, pork, and lamb.

2. List the USDA grades for beef, pork, and lamb.

\section{MAJOR CONCEPT:}

Plant Science

A. Students will understand the growth and development of plants, including the functions of plant parts, reproductive systems, and auxins

1. Identify plant parts and their functions.

2. List the necessities of human's lives that are furnished by plants.

3. Classify plant samples as annuals, biennials, or perennials.

4. Explain the difference between sexual and vegetative propagation and demonstrate each method.

5. List six basic plant growth requirements and indicate the importance of each. 
6. List factors that cause poor seed germination.

7. Raise a plant from a seed or cutting to a marketable or harvestable size.

B. Students will understand the role of soill in plant production, including factors that affect soill productivity.

1. Discuss the function of soil as related to plant growth, development, and maintenance.

2. List the basic types of soil texture and structure.

3. Describe soil texture and understand its importance to soil tilth, water use, and fertility needs.

4. Collect and test a soil sample.

C. Students will understand the role of fertilizers in agricultural production.

1. List the primary, secondary, and macro and micronutrient materials.

2. List a source for each of the primary elements (i.e. N-Nitrogen; P-Phosphorus, and K-Potassium).

3. Select from a list, the information that must be stamped on fertilizer bags according to law.

4. Calculate the content of N-P-K in a fertilizer container.

D. Students will understand the role of irrigation in plant production.

1. Name four tapes of irrigation systems.

2. List three ways to determine when to irrigate.

E. Students will understand the importance of pest control is agricultural production and appreciate the need for web pesticide application procedures.

1. List the four major types of plant pests.

2. Visually identify, collect, and dry mount 15 common weed samples.

3. Discuss weed competition and how it affects plant populations.

4. List four results of plant disease.

5. List ways the insects cause losses in plants.

6. Visually identify the three regions of an insect's body.

7. Label a drawing depicting the life cycle of an insect.

F. Students will develop an appreciation for safety in the workplace and the proper use of tools.

1. Store tools, equipment, and materials properly.

2. Identify the major classifications of fires and describe how to extinguish each.

3. Demonstrate the proper use of a fire extinguisher 
G. Students will understand the basic applications of measurement in calculating volume and distance and will develop an appreciation for the difference between the U.S. Customary and metric systems.

1. Accurately measure objects with a ruler or tape for use in the calculation of area or volume.

2. Measure items in fractions of inches.

3. Differentiate between U.S. Customary and metric units for linear and liquid measurement and convert units from U.S. Customary to metric and metric to U.S. Customary.

\section{MAJOR CONCEPT:}

Agricultural Business Management

A. Students will appreciate the importance of keeping accurate records of business transactions in agriculture.

1. List reasons for keeping records.

2. List the purpose of an inventory.

B. Students will understand the basic role of financial credit in agriculture, including sources and costs of farm credit.

1. Calculate simple interest, using the straight-line (alternate) method.

\section{MAJOR CONCEPT:}

Leadership \& Team Building Development

Agriculture and Natural Resources Industry Sector Foundation and Career Pathway Standards

\section{A. Students will be able to:}

1. Apply appropriate problem-solving strategies and critical thinking skills to workrelated issues and tasks. (FS 5.1)

2. Understand the systematic problem-solving models that incorporate input, process, outcome, and feedback components. (FS 5.2)

3. Use critical thinking skills to make informed decisions and solve problems. (FS 5.3)

4. Understand the characteristics and benefits of teamwork, leadership, and citizenship in the school, community, and workplace settings. (FS 9.1)

5. Understand the ways in which pre-professional associations, such as the Future Farmers of America (FFA), and competitive career development activities 
enhance academic skills, promote career choices, and contribute to employability. (FS 9.2)

6. Understand how to organize and structure work individually and in teams for effective performance and the attainment of goals. (FS 9.3)

7. Understand leadership, cooperation, collaboration, and effective decision-making skills applied in group or team activities, including the student organization. (FS 9.6)

8. Students demonstrate and apply the concepts contained in the foundation and pathway standards. (FS 11.0)

Sample Activities and Strategies:

- Oral and speaking presentations

- Critical Thinking Exercises

- Problem Solving Exercises Supervised Occupational Experience 


\section{SALINAS UNION HIGH SCHOOL DISTRICT AGIRISCIENCE AND TECHNOLOGY CURRICULUM \\ SAMPLE ACADEMIC YEAR PLANNING CALENDAR \\ YEAR \#1}

\begin{tabular}{|c|c|c|}
\hline MONTH & TOPIC & CURRICULUM \\
\hline \multirow[t]{2}{*}{ September } & California Ag & $\mathrm{I}-\mathrm{A}, \mathrm{B}$ \\
\hline & Leadership & \\
\hline \multirow[t]{2}{*}{ October } & Supervised Agricultural Experiences & $\mathrm{VI}-\mathrm{A}$ \\
\hline & Leadership & $\mathrm{V}-\mathrm{B}, \mathrm{C}$ \\
\hline \multirow[t]{2}{*}{ November } & Agribusiness Leadership & IV - A \\
\hline & & $\mathrm{V}-\mathrm{D}$ \\
\hline \multirow[t]{4}{*}{ December } & Agribusiness & IV - A \\
\hline & Animal Science & II - A \\
\hline & Animal Science & II $-\mathrm{B}$ \\
\hline & Leadership (year 2) & $\mathrm{V}-\mathrm{C}$ \\
\hline \multirow[t]{4}{*}{ January } & Animal Science & II $-\mathrm{B}$ \\
\hline & Leadership (year 2) & $\mathrm{V}-\mathrm{C}$ \\
\hline & Animal Science & $\mathrm{II}-\mathrm{C}$ \\
\hline & Animal Science & $\mathrm{II}-\mathrm{D}$ \\
\hline \multirow[t]{3}{*}{ February } & California Ag & $\mathrm{I}-\mathrm{C}$ \\
\hline & Plant Science & III - A \\
\hline & Animal Science & $\mathrm{II}-\mathrm{H}$ \\
\hline \multirow[t]{4}{*}{ March } & Plant Science & III - B \\
\hline & Plant Science & III - C \\
\hline & Supervised Agricultural Experience & $\mathrm{VI}-\mathrm{A}$ \\
\hline & Employability & $\mathrm{VII}-\mathrm{A}$ \\
\hline \multirow[t]{3}{*}{ April } & Plant Science & $\mathrm{III}-\mathrm{D}$ \\
\hline & Careers & VIII - A \\
\hline & Employability & $\mathrm{VII}-\mathrm{B}$ \\
\hline \multirow[t]{2}{*}{ May } & Career & VIII - B \\
\hline & Plant Science & III - E \\
\hline June & Catch up & \\
\hline
\end{tabular}




\section{Quality Criteria \\ One \\ Curriculum and Instruction}

The curriculum at North Salinas High School has been organized and sequenced around the career pathways for the district. The career pathways include Agriscience, Horticulture science, Agricultural welding, and Agricultural mechanics. Each class has been assessed and placed in an organized path based on the needs of all of the Agriculture programs in the district. The curriculum is written in a form that includes the course description, goals, objectives, and outlines. The curriculum identifies the major instructional strategies, texts, supplemental materials, etc. In the past, every year students were given a district course manual which showed a flow chart of these career pathways, but because of the budget and the cost of printing all of these handbooks, the district stopped. The only time students might see this flow chart is during class registration. However, those already in the agriculture program fill out a data sheet that utilizes this chart every year.

All classes in the agriculture department at North Salinas High School meet the vocational education requirements, electives, or physical/life science requirements needed for graduation. The Agriculture Earth science, Ag Biology, and Ag Chemistry courses meet the University of California A-G requirements. There are several other courses that are in the process of being UC approved as well. In all of the Agricultural classes offered, FFA as well as SAE is taught. Every student has a record book which is maintained throughout the year, and every year from there on for those that continue with the program. Record books are kept in department files until one year following their graduation. 
Each course incorporates higher order of thinking utilizing group and individual decision making. We have access to, and use computer hardware and software as instructional tools as well as guest speakers, etc. Every teacher, however, is different in the way he/she goes about it depending on the course taught. An agriculture careers unit is a part of every course and each course has been designed to meet the needs of all students. We work with all student populations to the best of our ability.

When students are registered into classes, counselors are not following the recommended sequencing of courses according to our flow chart. Counselors are educated on how this works, but have been trained to get classes scheduled for students which will push them more academically. They look at and treat all agricultural courses as an elective. This is one area in which the agriculture department at North Salinas needs to work on. We need to better communicate, build up relationships with counselors, and be more active with the registration process. 


\section{Quality Criteria \\ Two \\ Leadership and Citizenship \\ Development}

At North Salinas High School students enrolled in an agriculture class develop leadership, citizenship, interpersonal and employment skills by being involved in community service projects and individual or competitive activities. North Salinas High School Future Farmers of America (FFA) received its charter in 1960 and the program is supported by the school, district, community, as well as the school board.

The Agriculture Department at North Salinas has four agriculture teachers. Two are full time agriculture teachers, the third is a part time agriculture teacher, and the fourth teaches one agriculture class. Each teacher in the program uses the three integral circles in order to grade our students. Every student enrolled in an agriculture class is automatically a member of the FFA. FFA activities, as well as the Supervised Agricultural Experience (SAE) program, are just as important as the technical skills learned in the classroom. Ten percent of a student's grade each semester is based on the students FFA activities. Students are required to complete 4 FFA activities per semester to build leadership skills. Two of the four activities must be meetings while, the other two activities can be chosen from a variety of options including conferences, public speaking events, community service, fundraisers, farm work days, etc. Of course, students can go above and beyond the four activities and earn extra points towards their grade.

Students in the program are encouraged to move beyond the chapter level activities to promote the growth of individual leadership skills for future use. All activities are previously approved by an advisor as well as the school activities 
director. Each one is run by either our Chapter FFA leadership officers or committee members and is overseen by at least one FFA advisor. 


\section{Quality Criteria \\ Three \\ Practical Application of Occupational Skills}

At North Salinas High School practical application of occupational skills is accomplished through classroom instruction, activities, or entrepreneurship. In all of the Agriculture classes at North High including Ag Chemistry, Ag Earth Science, Ag Mechanics, Ag Welding, Ag Science, and Ag Government/Econ students are given the opportunity to do hands on activities that explore that sector of the agriculture industry.

Not only do students get the opportunity to develop occupational skills through classroom instruction and activities, but all students are required to conduct an out of class SAE project. As mentioned earlier 10 percent of a students grade per semester is based on FFA activities, but another ten percent of a student's grade per semester is based upon their SAE project. Each student is to keep up to date records on the hours worked, jobs completed, money spent or earned, calendar of events, FFA activities, community service, etc. This is documented in the students' record books which the students continue to work on throughout the school year. There are three ways in which we verify that the projects are being conducted: one being a signed document by parents and students stating that parents know about this project, the second being pictures of their project from beginning to end, and last is actual project visits conducted by the ag teachers. Every project conducted by a student will have a business agreement, budget, financial statement as well as any documented property.

An ag truck is available for any one of the agriculture teachers to utilize for SAE projects or any other integral activities to the program. 


\section{Quality Criteria \\ Four \\ Qualified and Competent \\ Personnel}

All of the agriculture vocational education teachers are competent and qualified to teach at North Salinas High School. There are two full time teachers including myself and Stanley Uchiyama. One part time teacher, Tom Jones and Margaret Nororian who is teaching one ag class.

All of the ag teachers at North High hold a valid credential that allows us to teach an agricultural course. Stan has a Standard secondary for life credential and has been teaching for over 40 years; Margaret has a Standard secondary for life credential and has been teaching for 35 years; Tom has an Agriculture Specialist credential and has been teaching for 5 years and this is my third year teaching agriculture with an agriculture specialist credential. By holding these credentials it shows that each of us has a minimum of 3000 hours of occupational experience in agriculture allowing each of us to teach in the field of ag.

Each instructor at North Salinas uses a variety of instructional strategies and materials to enhance student learning in order to meet the needs of all of the students. We diversify instruction using demonstrations, labs, activities, and technology. Professional development is a continuous process in order to enhance or expand our knowledge or skills in vocational education. Each year we attend curriculum planning days, and workshops in the various aspects of agriculture. Every year, Stan, Tom, and I attend the California Agriculture Teachers Conference in order to improve or better develop our vocational 
instruction skills. This year we have all participated in the fall regional CATA meeting, the spring regional CATA meeting, and over four sectional meetings. 


\section{Quality Criteria \\ Five \\ Facilities, Equipment and \\ Materials}

The facilities, equipment, instructional materials and supplies that North Salinas High School Agriculture Department has to offer is a combination of both new and old. We are slowly increasing the use as well as the improvement in technology. Everything that is used helps to meet the needs of our instructional objectives as well as the individual needs of all of our students.

In the last several years the woodshop room has added two drill presses, a plainer as well as a jointer. There are already two table saws and a miter saw in the shop. We have slowly been improving the facilities in this room to make it usable for the ag mechanics courses offered at North Salinas.

The welding program has been well maintained over the last 40 years due to the management efforts of Stanley Uchiyama. Over the last several years we have added two MIG welders along with a recently added metal bender.

The science courses offered in the agriculture department including the $\mathrm{Ag}$ science, Ag Earth science as well as the Ag Chemistry has slowly been building up its materials. I teach both of the $\mathrm{Ag}$ Chemistry Courses and three $\mathrm{Ag}$ Earth Science courses. When I started working at this school, the ag department did not have much to offer in the way of lab equipment. We had a class set of dissecting microscopes and compound microscopes, but that was the extent of what I have had. Over the years we have added glassware, smocks, safety glasses, and even chemicals. The science department has also been gracious enough to share the equipment that we do not have. Along with that we do not have lab facilities for our students within our program. We have managed using 
our own rooms or have swapped classes for a period or two with a science teacher in order to access a lab facility and necessary equipment. We try as much as possible to utilize our facilities and equipment to accomplish our labs, but our science department is very willing to help with any additional support/equipment needed.

In addition, three years ago, North Salinas received a specialized grant to improve our small animal facilities as well as the greenhouses on campus. The two greenhouses received new fiberglass, but the fiberglass that was put up was not of quality or grade so we have been trying to replace the fiberglass by working with a company in Salinas. We did however purchase enough equipment for the small animal facility, to enable us to raise rabbits on campus. We are currently trying to work on access and security to the facility. Without proper access or security the facility is not yet usable. 


\section{Quality Criteria Six \\ Community, Business and Industry Involvement}

At North Salinas we have both an Agriculture advisory committee as well as an Ag Booster's club. Our Ag advisory committee is made up of community, business, industry, parents, students, and other individuals who possess the skill and knowledge of the occupations in our area.

The advisory committee provides guidance to our program in terms of developing and implementing a Program Improvement Plan. They provide advice, support, counsel, written recommendations to instructional classes, budget, program recruitment, facilities, equipment and materials, program planning, job placement, class size, new technology, etc. They communicate not only with the community, but to the administration about the needs and direction of the program.

Over the last few years our advisory committee has taken a much more important role in standing up for the departments needs. We currently only have one ag stipend and 3 main ag teachers. Our small animal facility as well as our greenhouses are not very functional due to the fiberglass grade and lack of security for students to maintain projects using these facilities. We have also been struggling in working with our counselors to place students in our agriculture classes so the ag advisory has been taking more of a fighting stance on the issues.

Our advisory committee meets almost monthly throughout the year in order to provide input on program decisions including students of all needs. The 
advisory committee minutes for each meeting are given to the principal to look over. 


\section{Quality Criteria Seven \\ Career Guidance}

All of the students involved in the ag program at North Salinas complete a student data sheet showing individual course goals and pathways. These data sheets are kept on file and updated on a yearly basis for students that remain in the agriculture program. The students receive instruction, guidance and counseling by each of the agriculture instructors on developing their career plans and career paths. Students are made aware of the $2+2$ articulation agreements with Hartnell Community college in Ag Science 1-2 as well as Ag Construction. In the last few years the agriculture teachers in the area have also tried to articulate other classes with Hartnell, but nothing has come of the few meetings we have had. For those students that do not remain in the ag program from year to year their data sheets are kept on file just in case they come back into the program one, two, or three years down the road.

Our counselors and other resource personnel do provide career guidance, but are not very supportive of the agricultural program. Information as well as recruitment materials have been provided to the counselors on numerous occasions, but the counselors do not seem to utilize the resources. In the past few years it has been a struggle working with the counselors to help them see the opportunities that the agriculture program has to offer. In the near future it is our goal to better establish a working relationship with the counselors and get them more involved with our program to show them why the ag program is important to our school as well as our community. Individual career plans are not followed when a student registers for their classes. 


\section{Quality Criteria \\ Eight \\ Program Promotion}

North Salinas has a recruitment plan implemented every year to inform students, parents, and administration of the opportunities that the agricultural program has to offer. We do not apply the actual plan until half way through the second semester. At North Salinas High school we put in about eight bulletins that go into detail about the ag program and encourage students to take an agricultural class. We also give the administrators and counselors a packet of information about our program. This year the ag instructors talked to all of our ag classes to see who would be interested in taking another agriculture class next year. On a sheet of paper students wrote down their name, identification number, the class they were interested in, and why they wanted to take that particular course. We worked with the counselors to see if all of those students were enrolled or could be enrolled in our classes.

At the middle school level, we usually take students in the agriculture program to give presentations at all of the feeder schools about a week before they register. We also give the middle school's administration, counselors, and teachers a packet of program information. This year however we were not informed of the recruitment dates in time so we only presented to one middle school.

Other than the usual recruitment plan, throughout the year the ag program holds two barbeques, every other year we go on a Salinas Ag Industry tour, every other year we go to the Ag Farm Show, we take students/animals to Monterey County Ag day, we participate in a number of community events, we put on our Chapter's FFA week, we hold our annual awards banquet, and 
complete supervised agricultural experience project visits. This year we also created a promotional brochure of our program, but have yet to implement it. All of these activities are used to promote the agriculture program at North High reaching students, parents, teachers, counselors, administrators, community members etc. 


\section{Quality Criteria \\ Nine \\ Program Accountability and Planning}

The only annual program assessment that I know of uses the input from the agriculture teachers, the ag advisory committee, and the ag incentive grant review. This assessment usually takes place during the meetings of the advisory committee throughout the year. We look at the classes, student achievement, retention rate, program goals, program completer input, as well as the ag incentive grant recommendations/commendations made by the regional supervisor.

Students that complete the core curriculum or a career pathway are not given a standard test, but after they graduate they are given a survey to fill out assessing the program. North Salinas High School does not give a career pathway test due to students in the program switching career pathways so often. The agriculture department uses this information to modify or adjust program goals or direction. This information is shared with the advisory committee members and administration.

Within the program there is an annual assessment including a program improvement plan that is updated every year. The plan is decided by the ag instructors, administrators, and the agriculture advisory committee members. A copy is even filed with Mr. Greg Beard.

Along with a program improvement plan is a comprehensive five year program plan that has been updated and filed with the regional supervisor. Every year we review, we update, and we add to our five year program plan. 
The program plan for North Salinas High school is extremely detailed and organized. It includes all of the necessary information required by the state and more. A copy can be found in Mr. Beard's office. 


\section{Quality Criteria \\ Ten \\ Student Teacher \\ Ratio}

Over the last several years North Salinas High School, like every other high school, has been struggling to maintain our student teacher ratio. The agriculture department especially is dependent upon sustaining a student teacher ratio that ensures effective instructional and safe working conditions. In the last few years however, we have been able to meet the student teacher ratio. Every year all of our classes definitely exceed the student teacher ratio, but by the end of October our class sizes have dropped tremendously. This year our student teacher class period average ratio on October $26^{\text {th }}$ was 1 to 23 in the $\mathrm{Ag}$.

Sciences and 1 to 23 in the Shop classes. 


\section{Quality Criteria \\ Eleven \\ Full Year Employment}

Being an agriculture teacher means that effective instruction extends far beyond the regular school day, year, or classroom. Currently only one agriculture teacher at North Salinas High school receives an agriculture stipend for a full year in order to perform these basic duties. The ag teachers as well as our ag advisory committee members have been fighting for at least one more stipend for the last two years, but so far nothing has come of it.

Not only during the year do we only have one stipend, but during the summer only one teacher receives an extra work agreement. This last year Mr. Uchiyama was kind enough to split his hours with me so that two agriculture teachers were provided a summer contract equally making about $\$ 2000$ each.

Lastly a supervision period has not been granted to any of the agriculture teachers in the district in years and I do not think we will ever receive that period back. As far as receiving another agriculture stipend, we are hoping with a new principal and Mr. U's communication efforts with the superintendent that we can get one more ag stipend. 


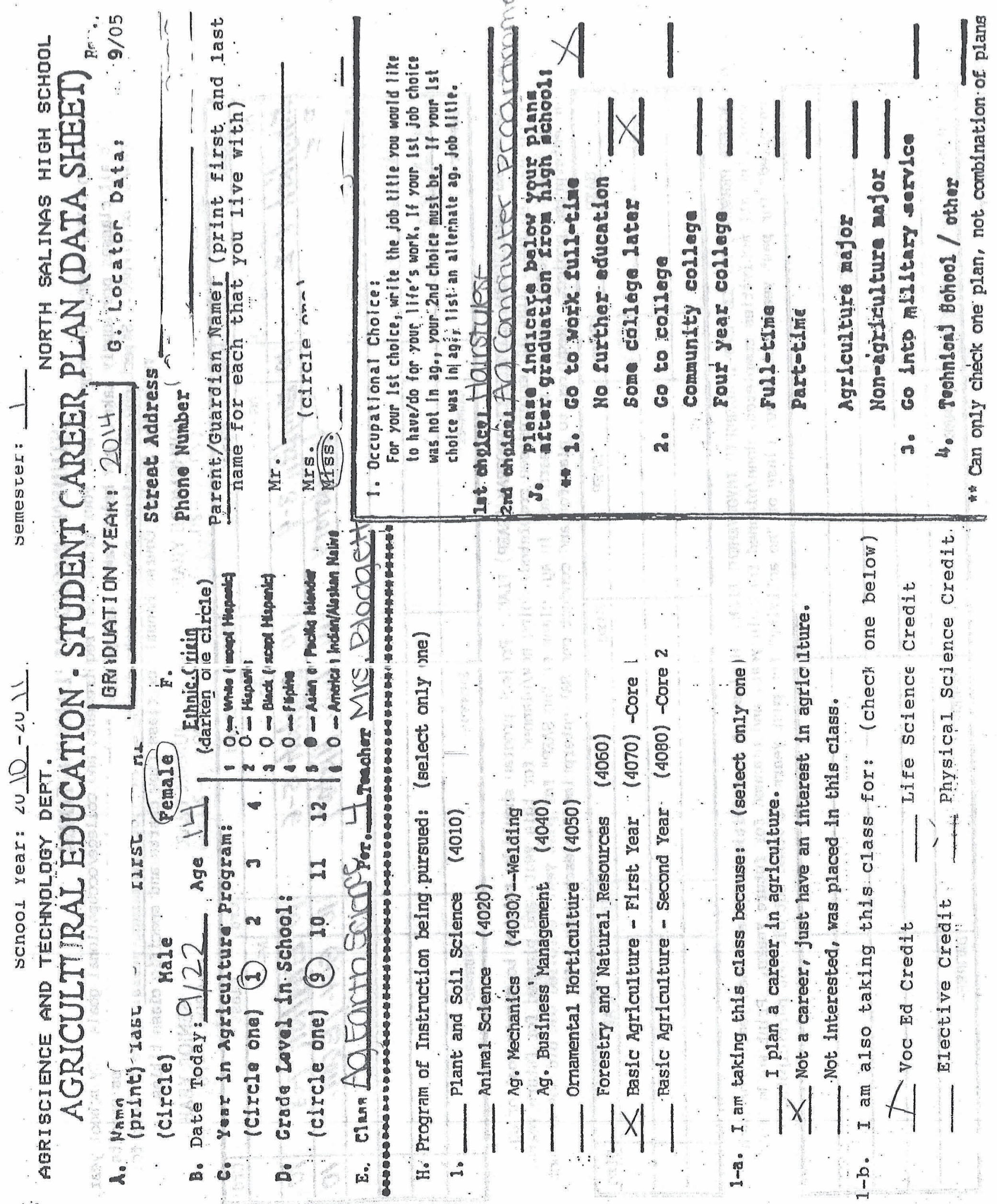




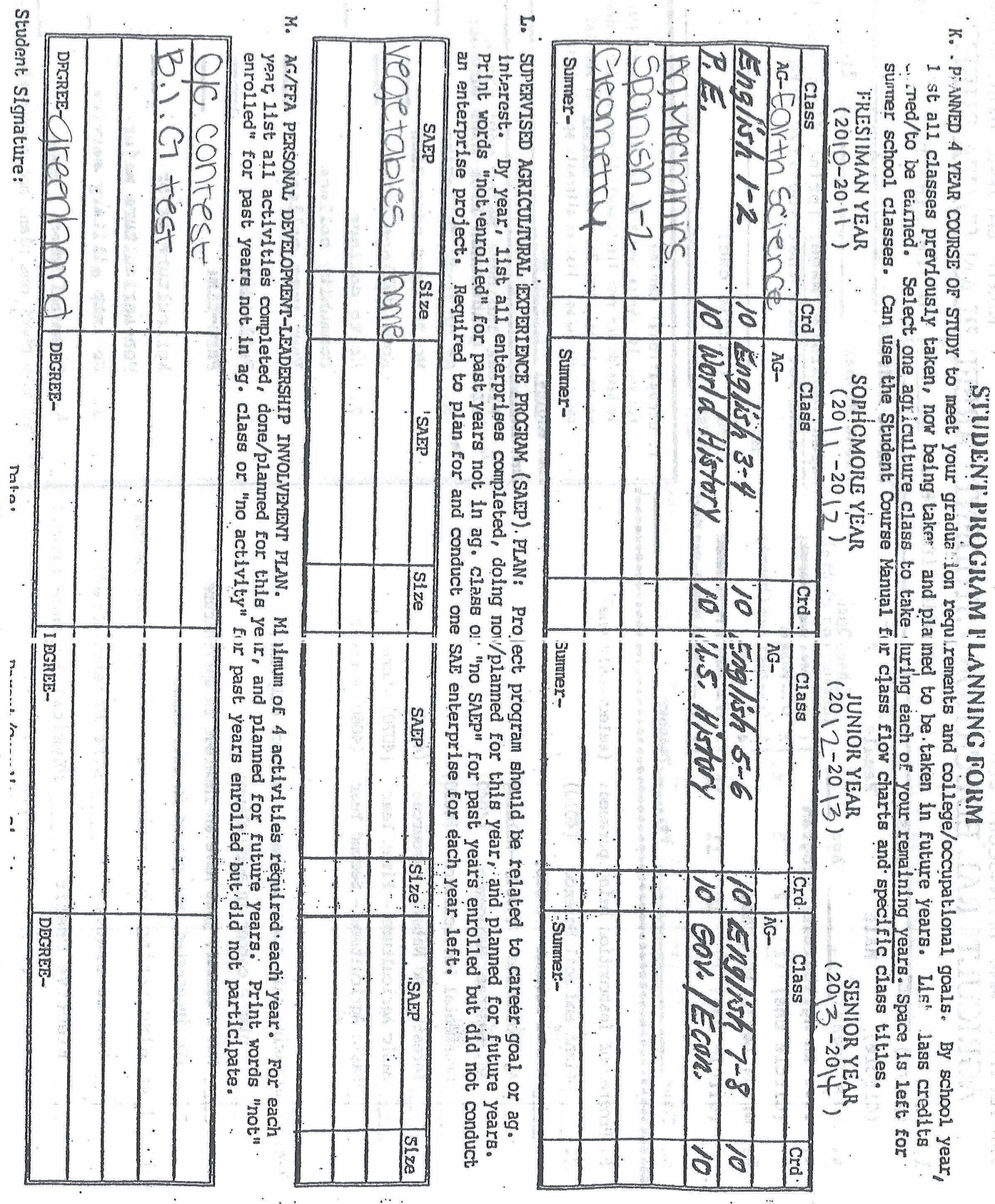




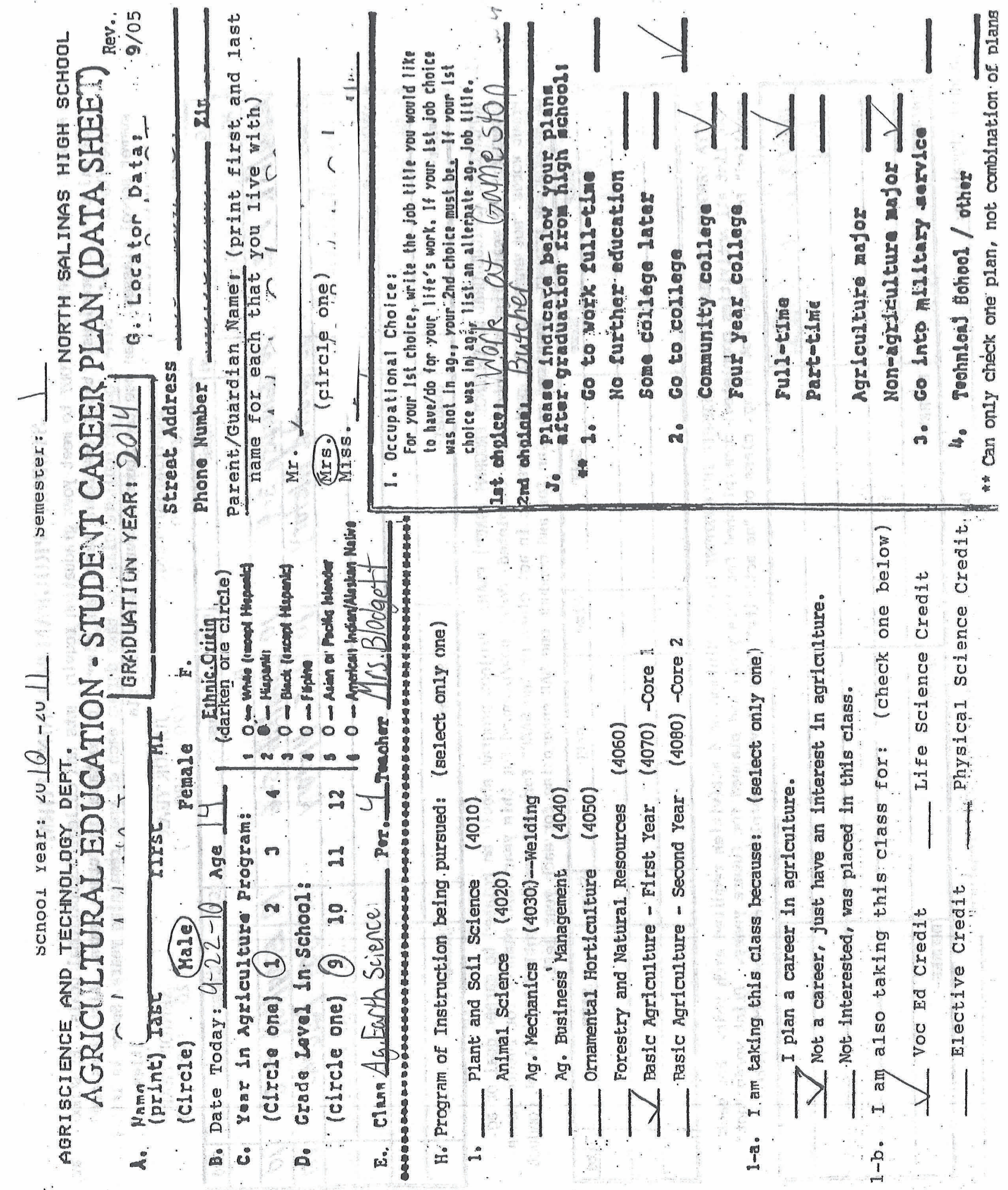




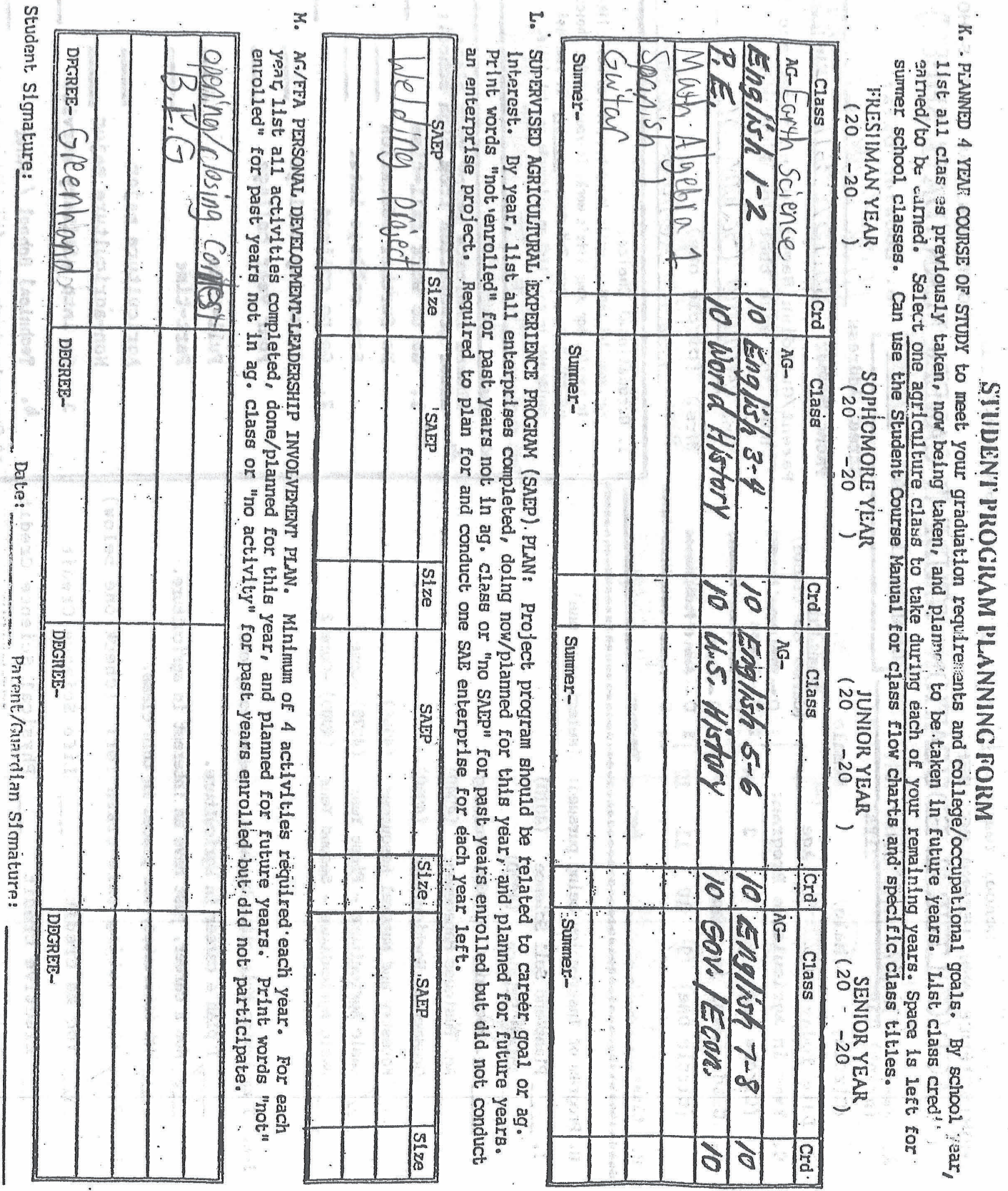




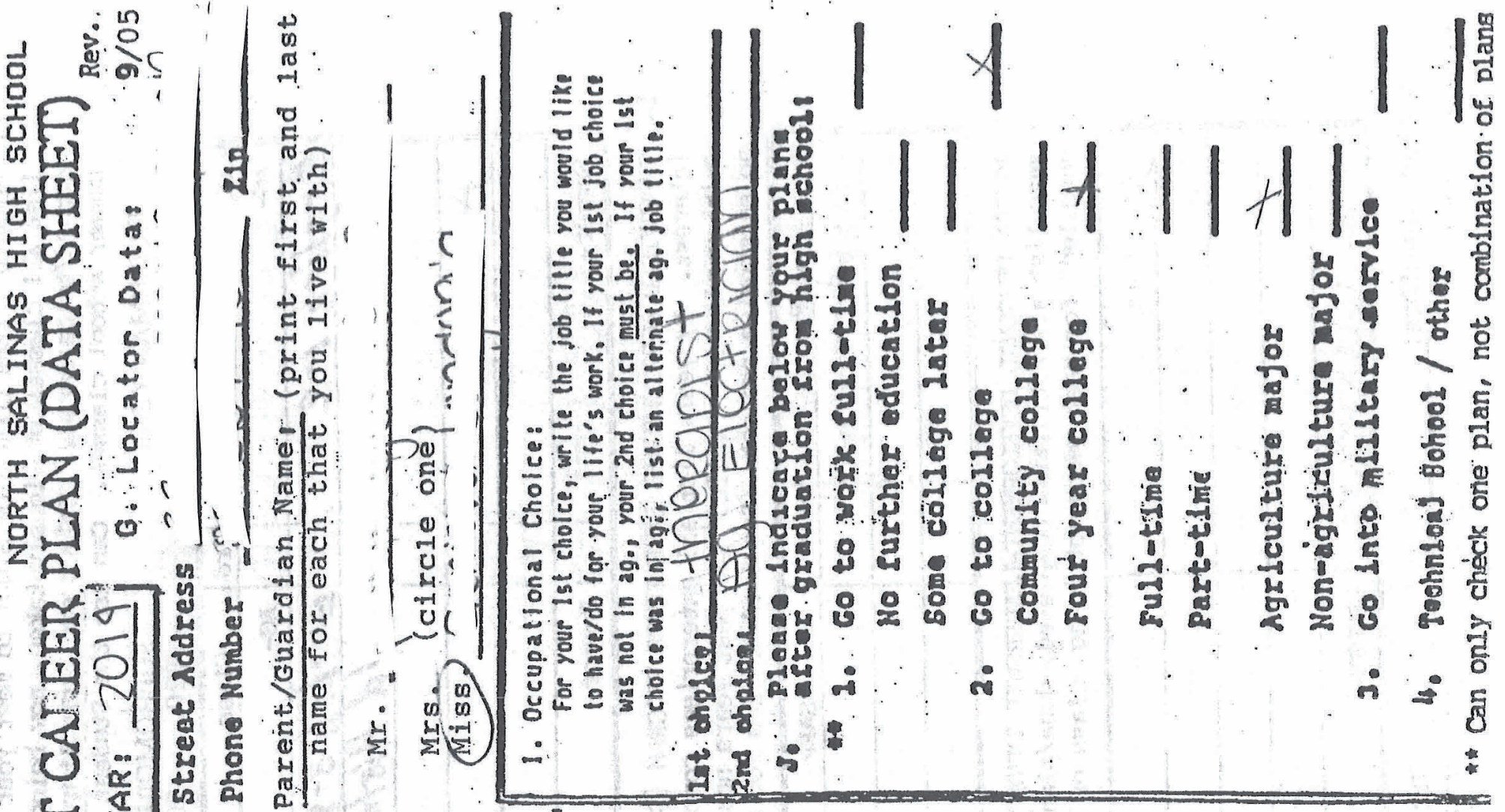

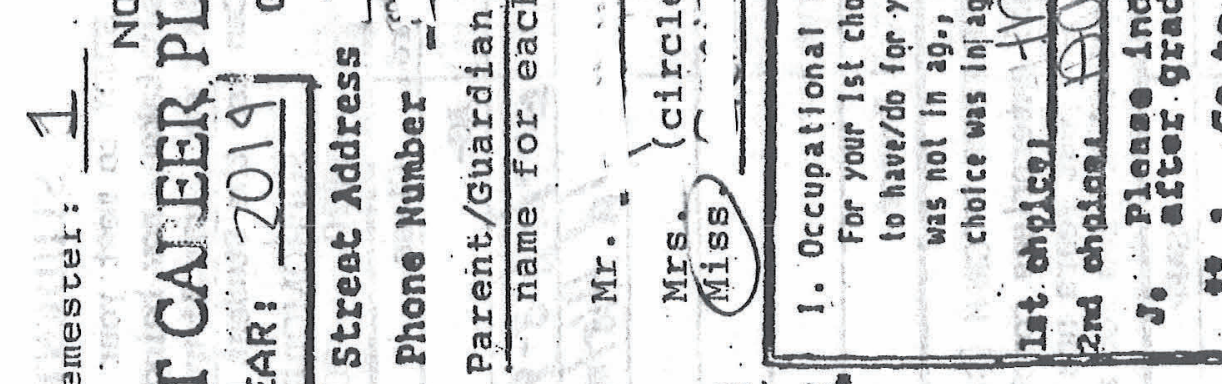

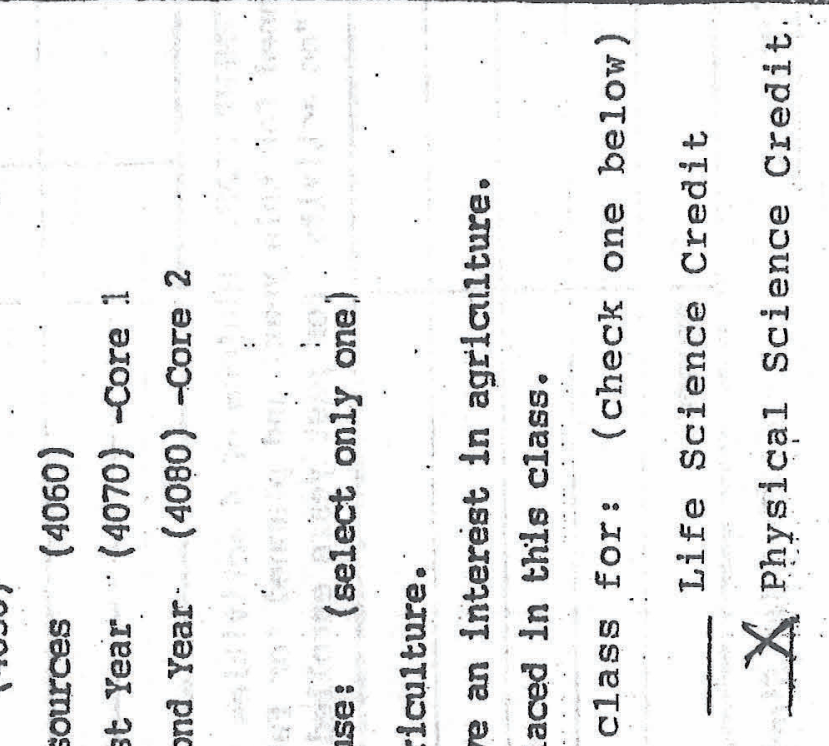

年变

O

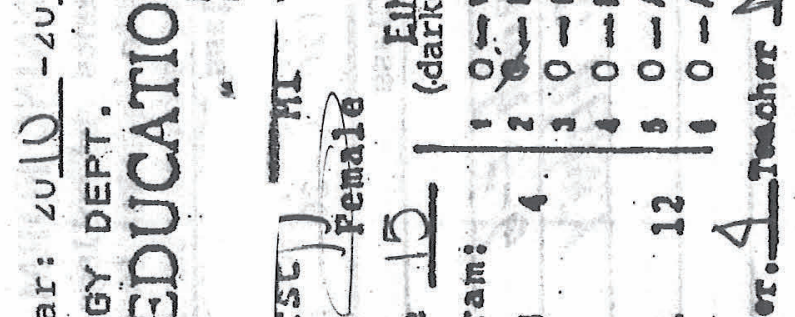

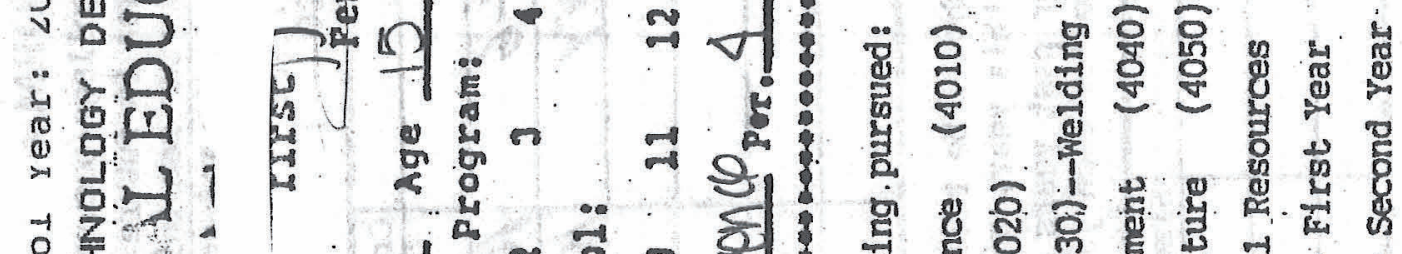

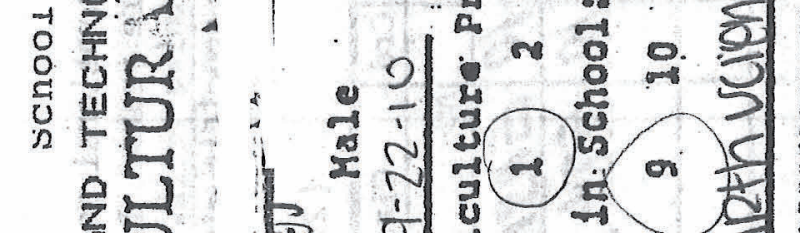

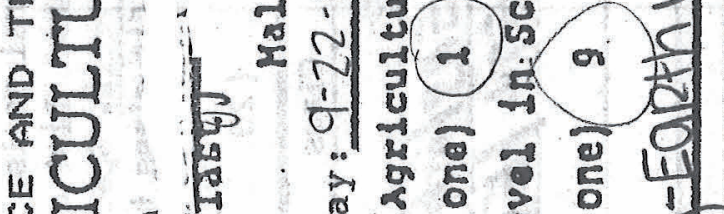

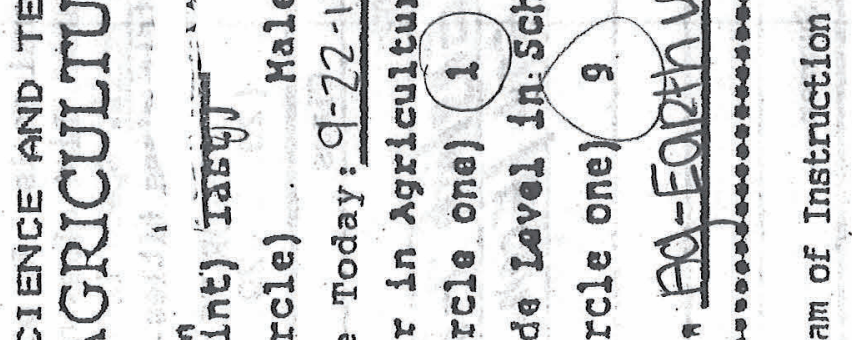

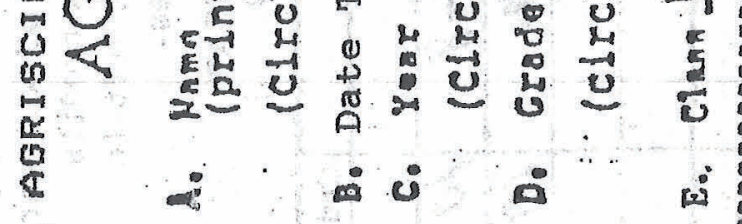

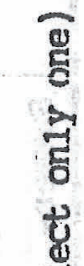

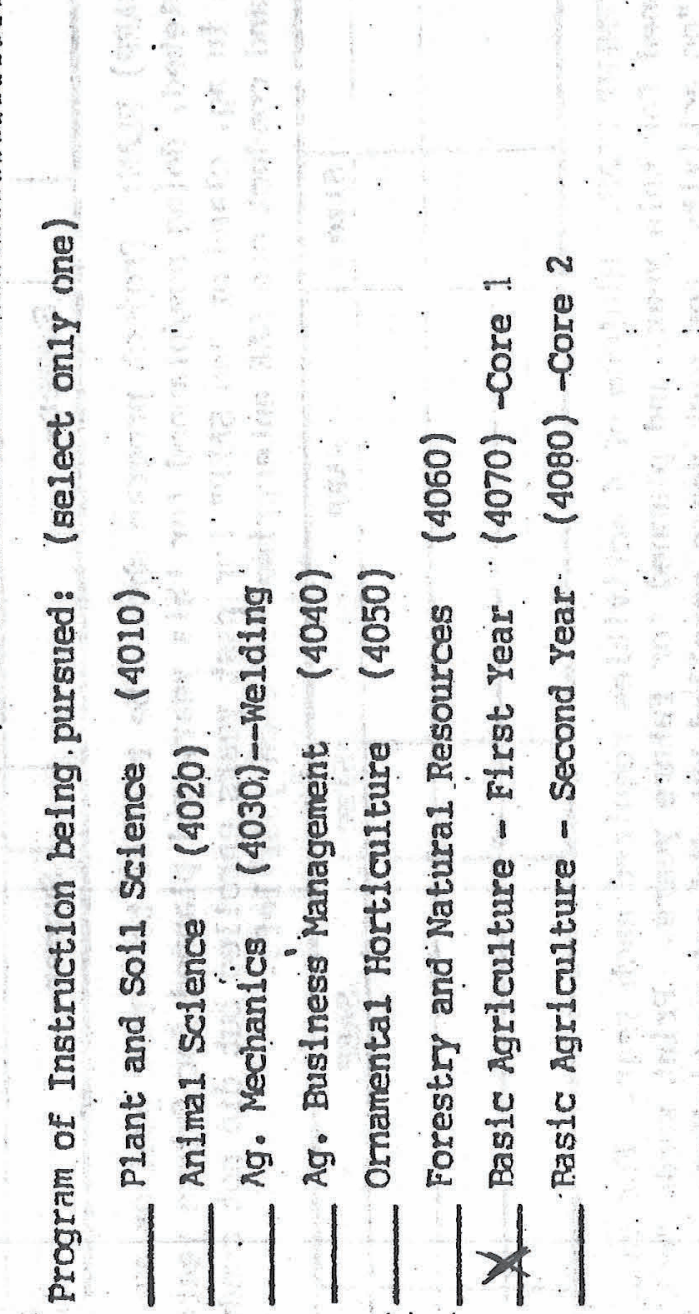

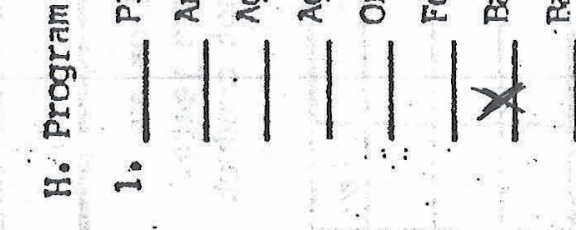

पू

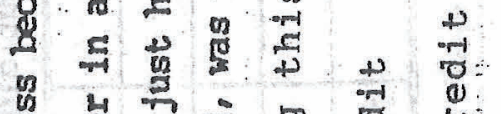

荡

- $\frac{4}{8}$ है $\frac{\pi}{0}$ है

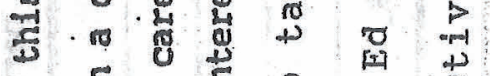

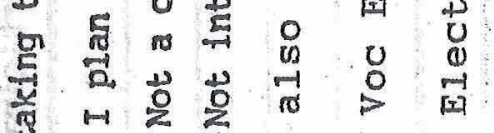

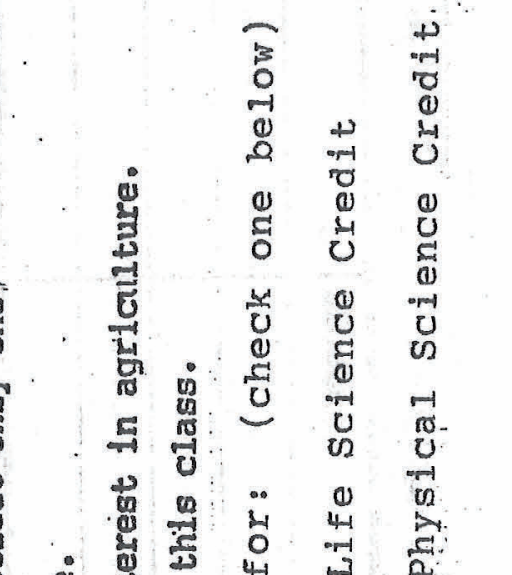

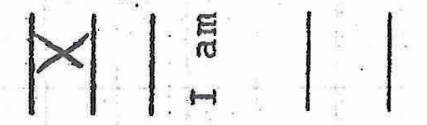

$\stackrel{i}{1}$

$\frac{0}{1}$ 


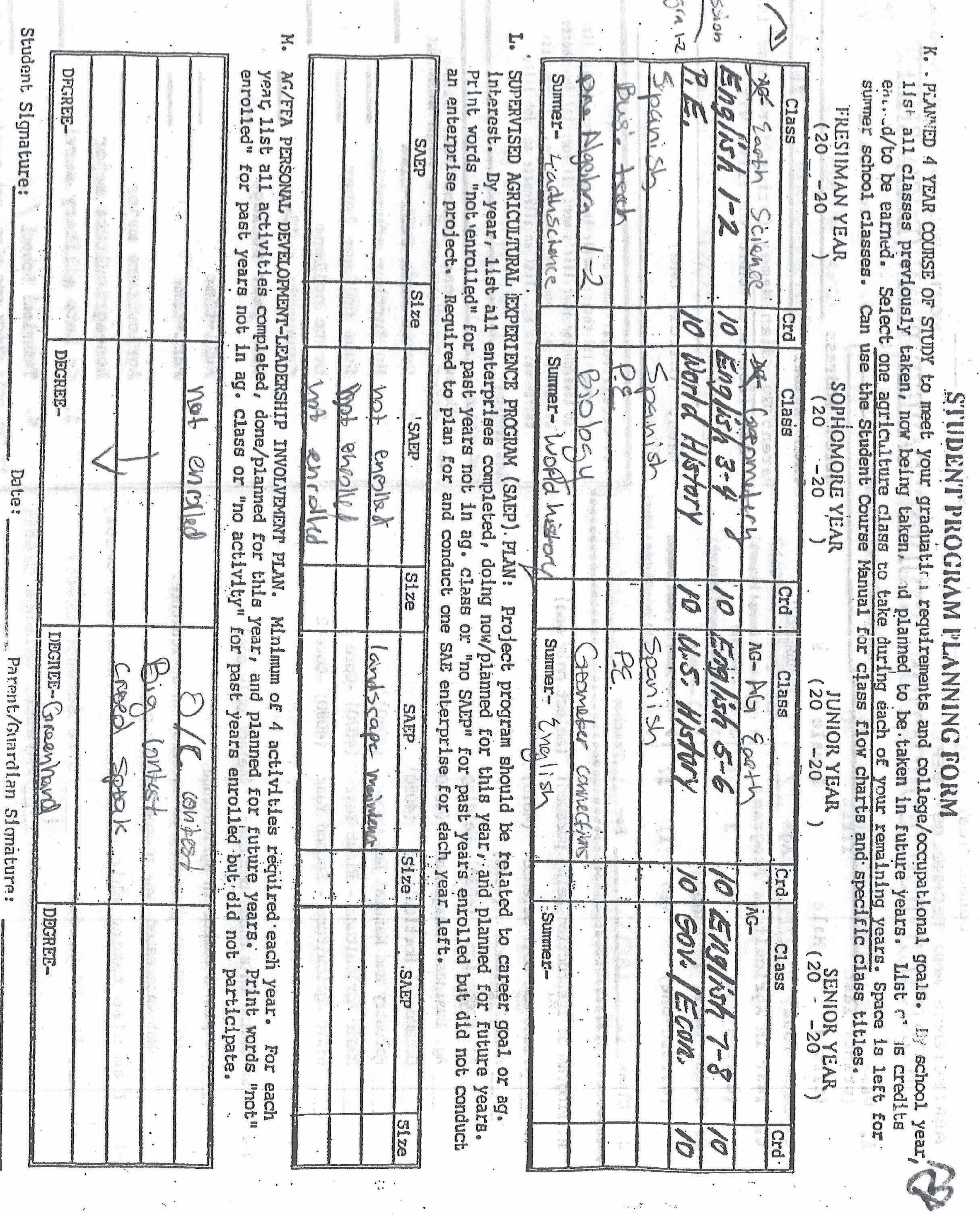




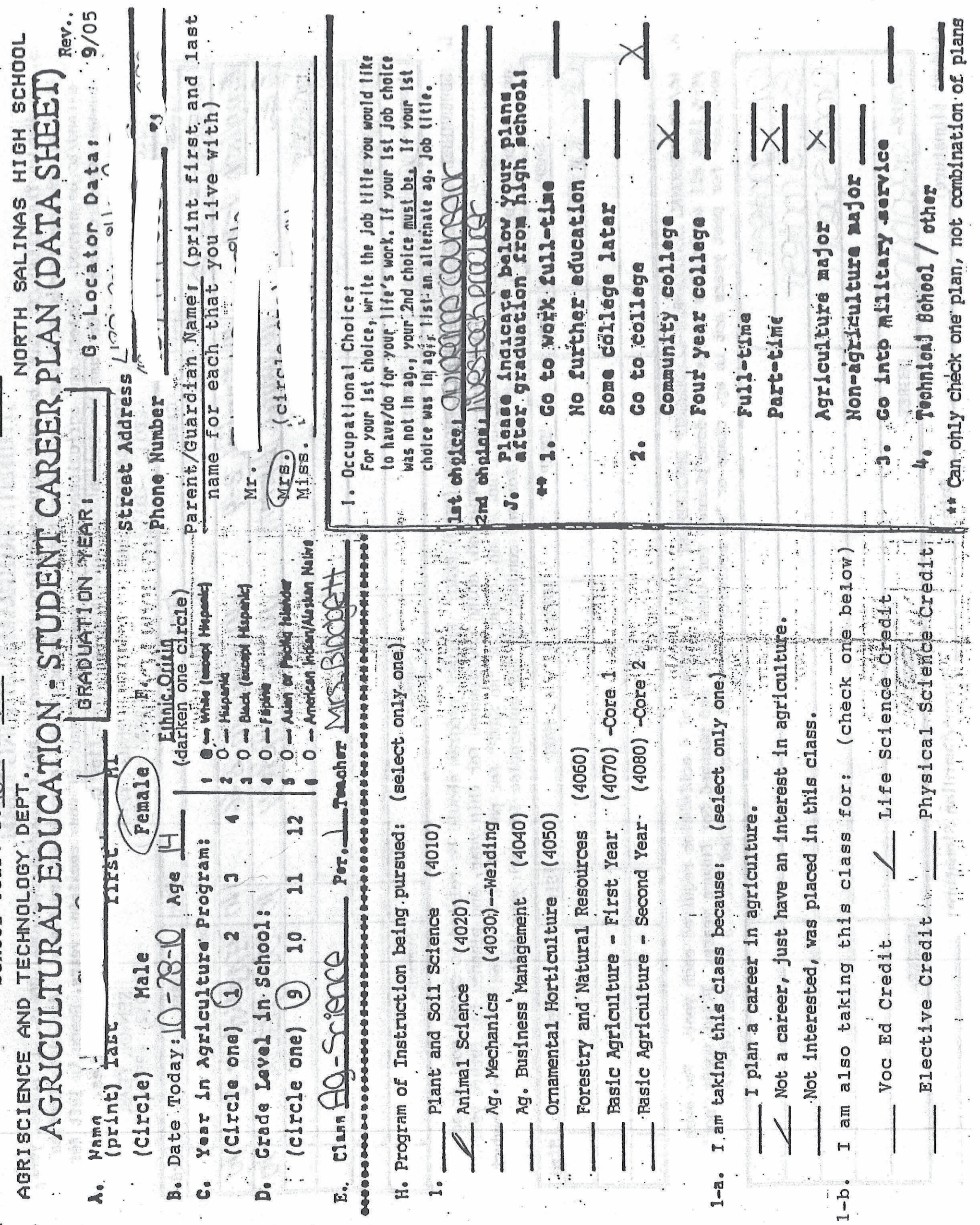




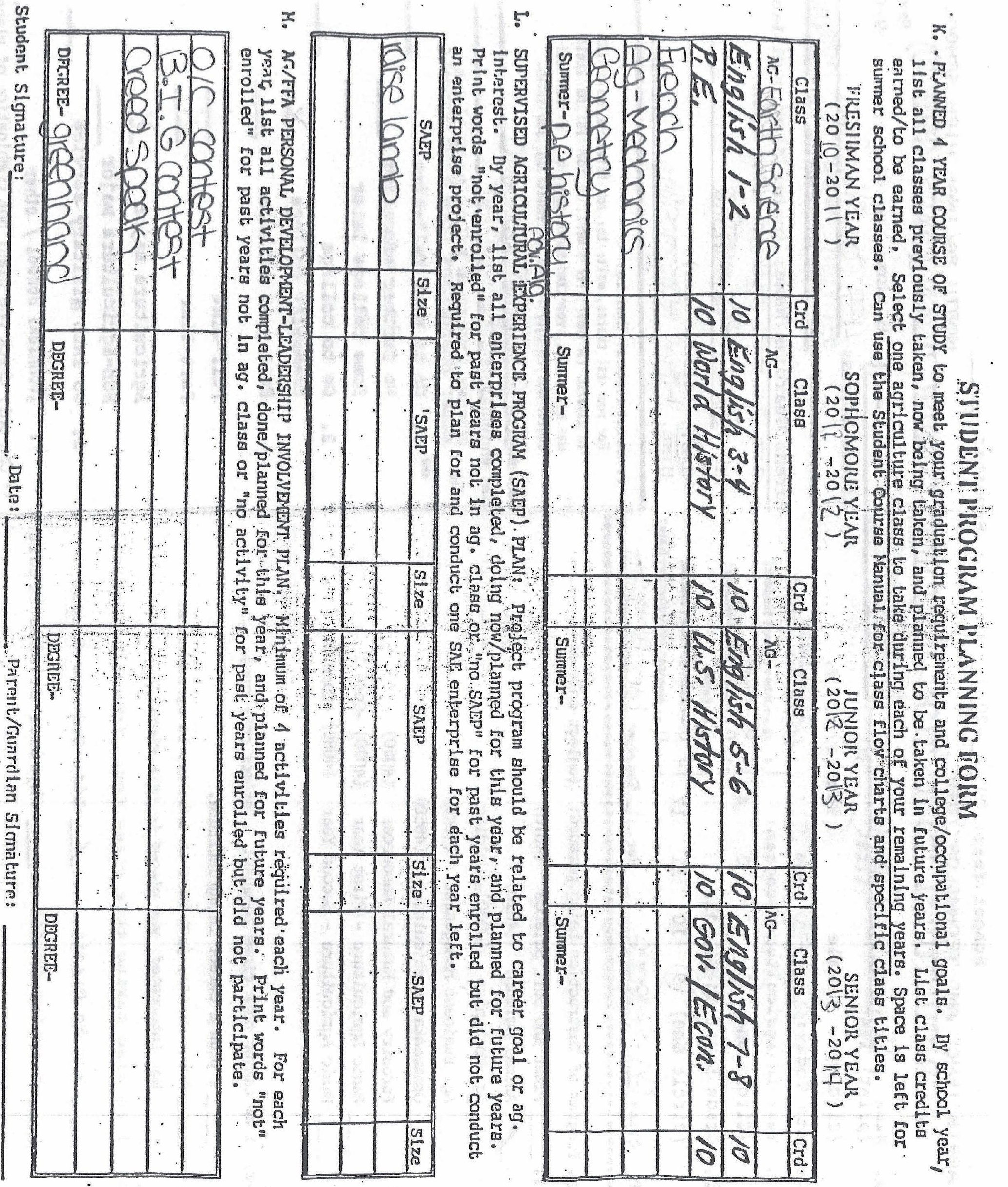




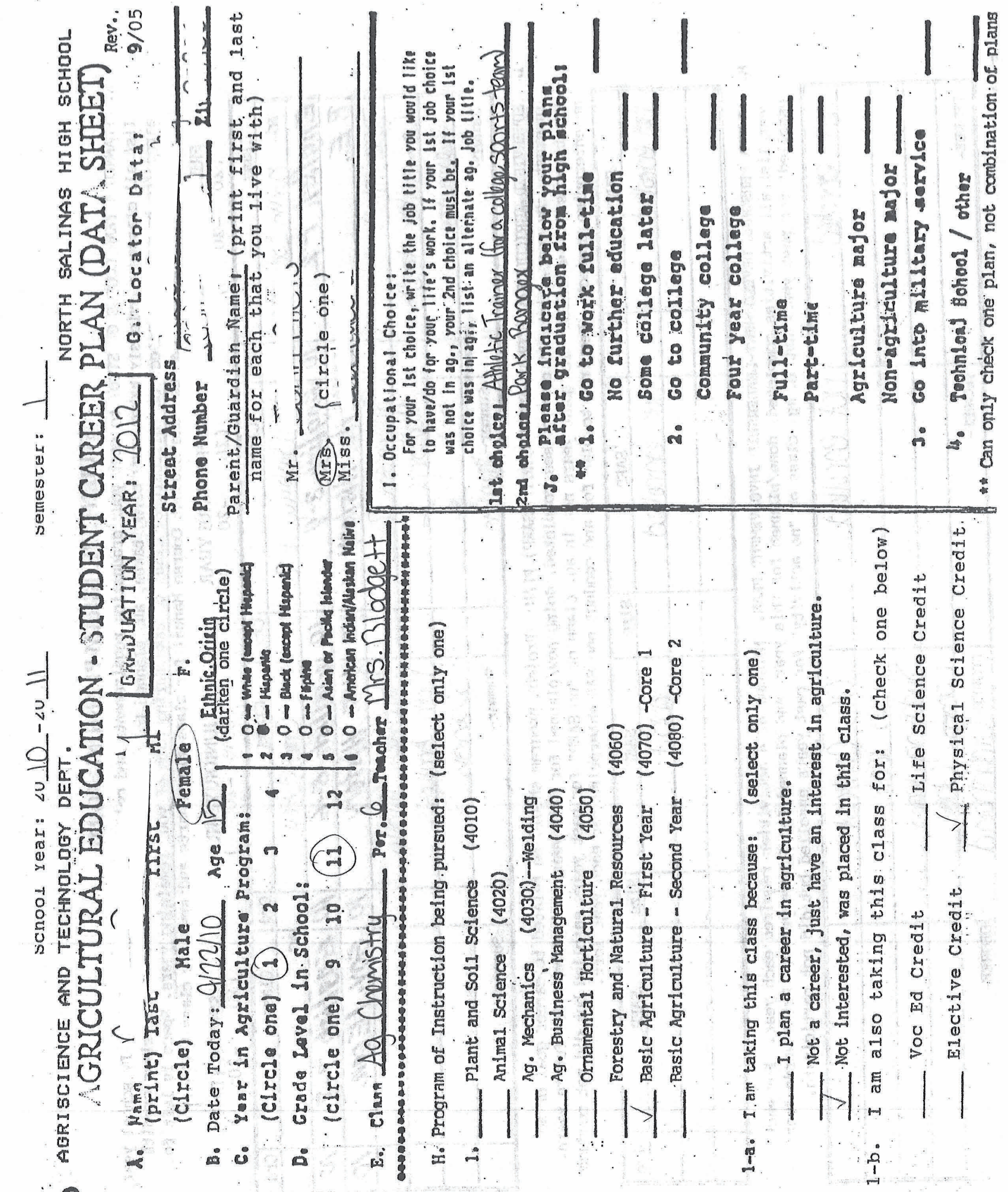




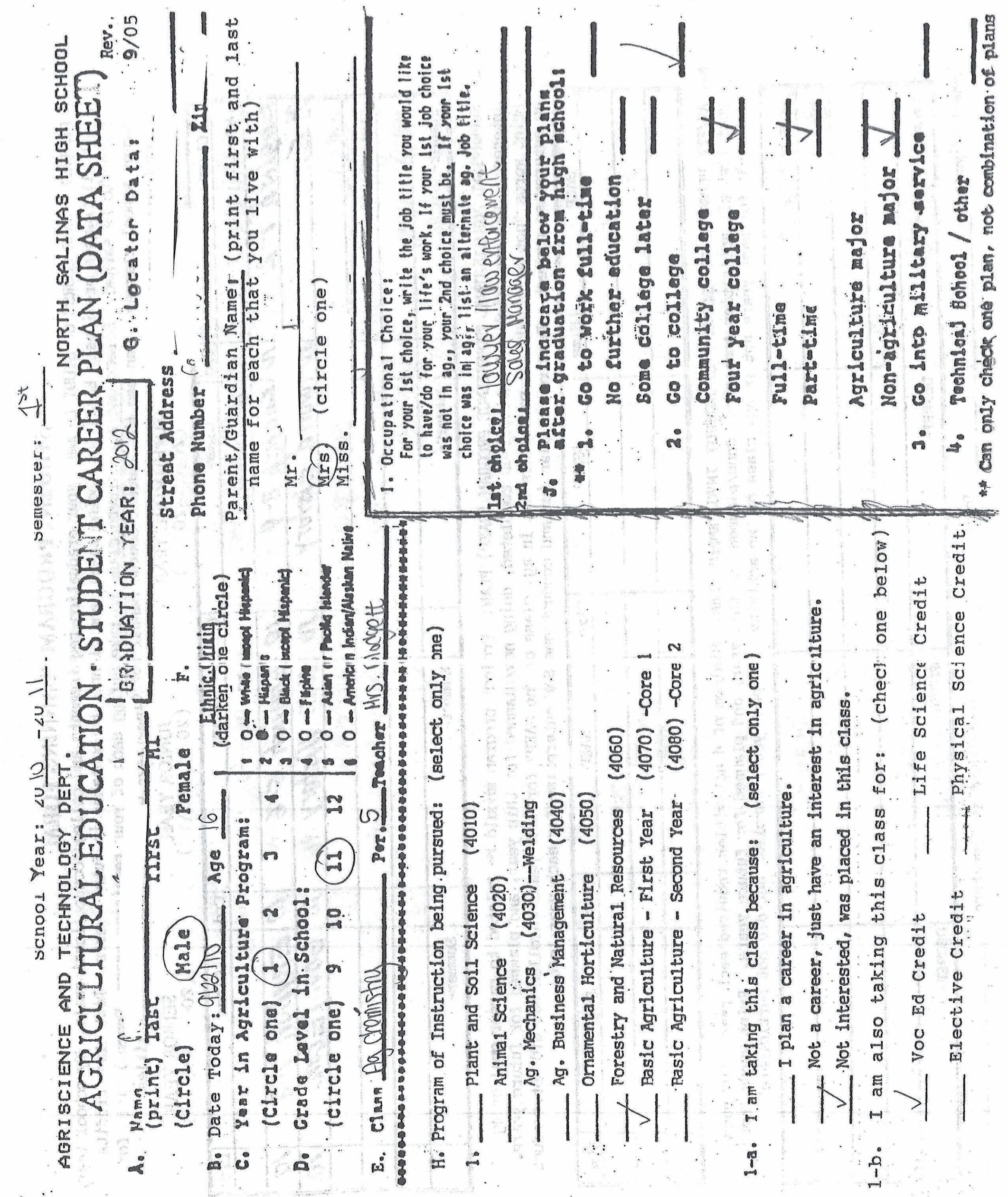




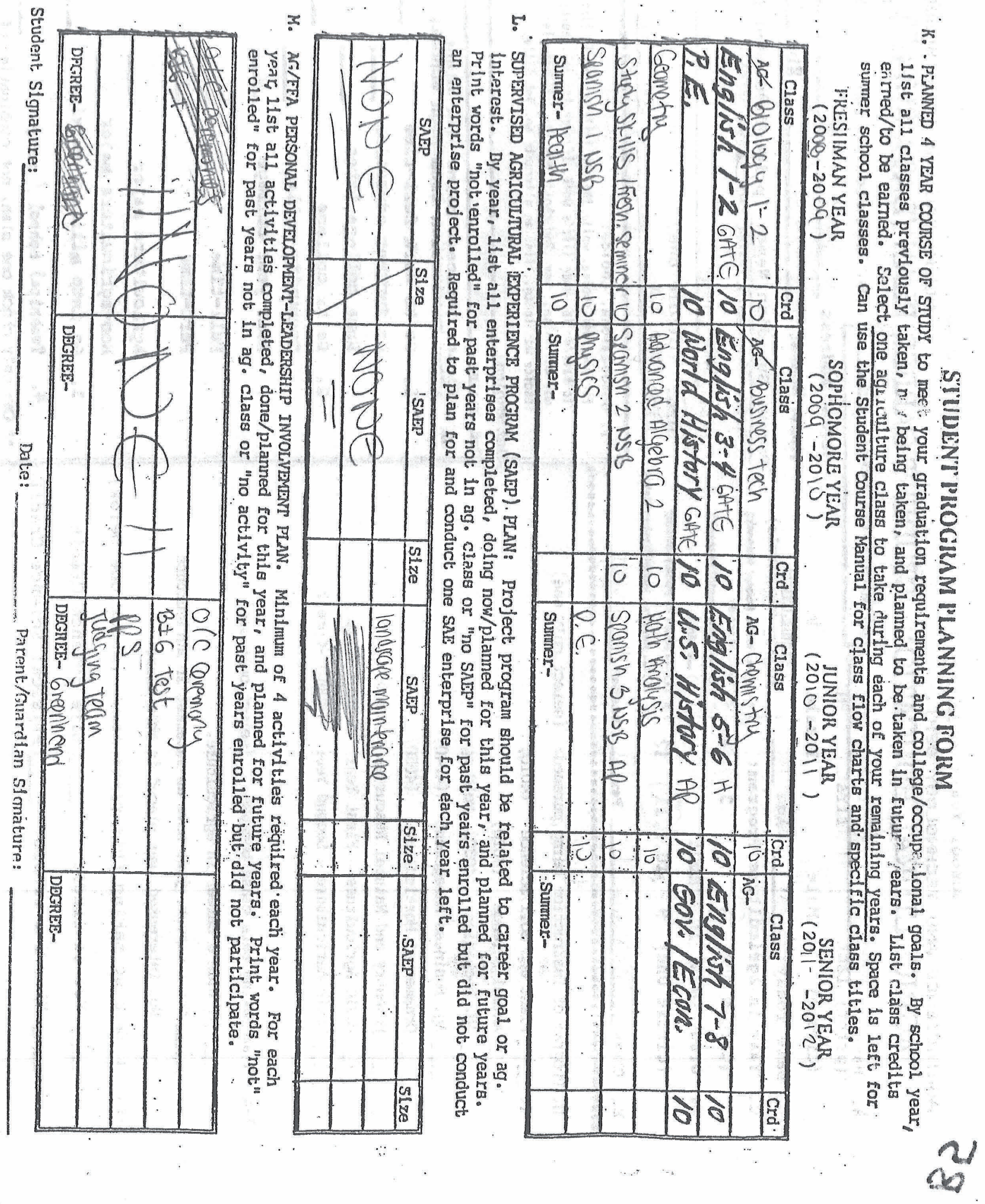




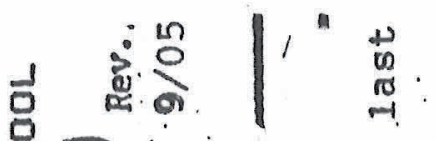

오요

品

㒘

政

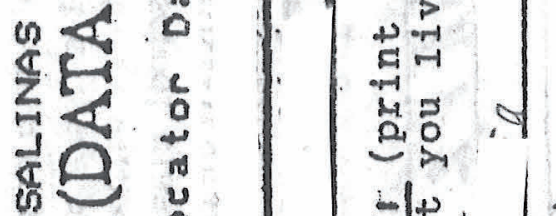

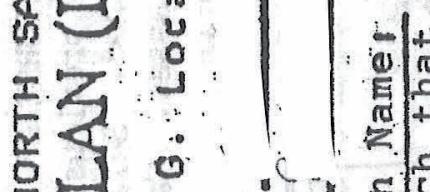

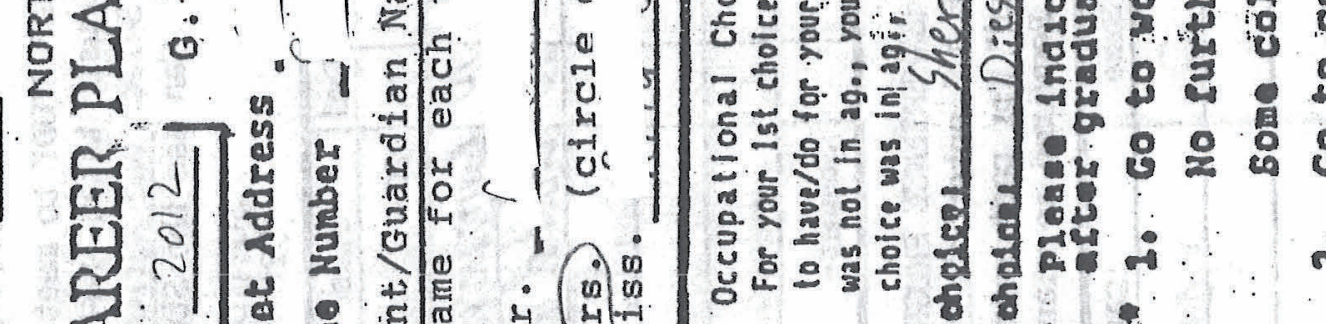

$-1$.

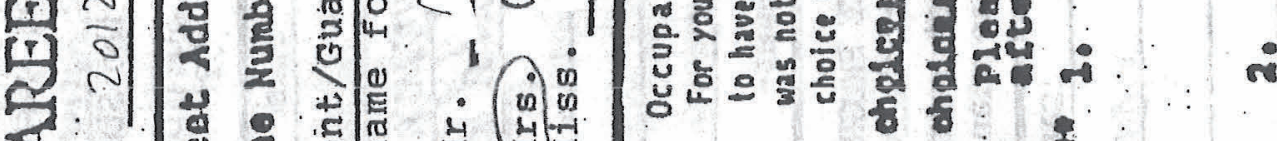

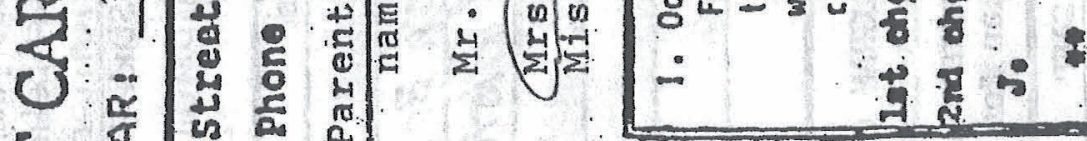

$\rightarrow \frac{\pi}{\text { I }}$ के है

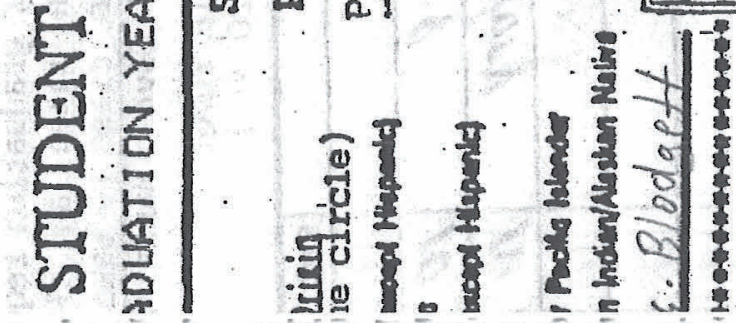

$\Rightarrow \quad-\frac{a}{a}$.

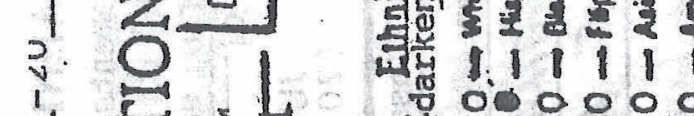

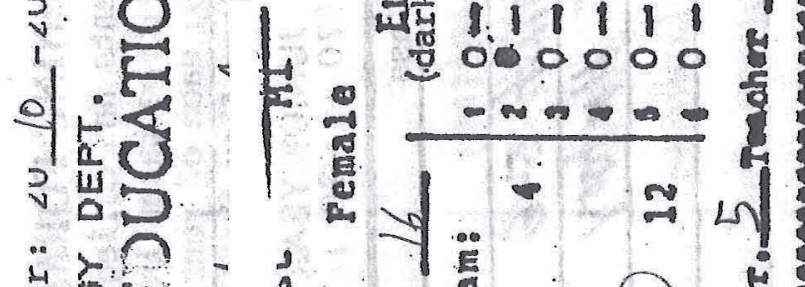

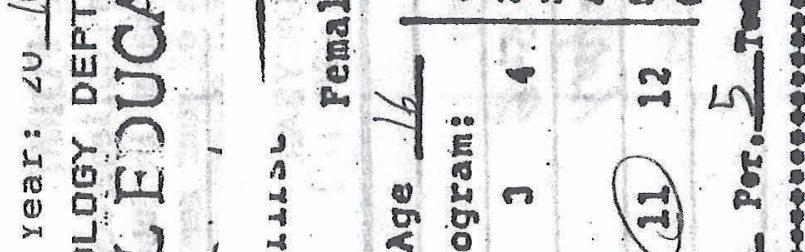

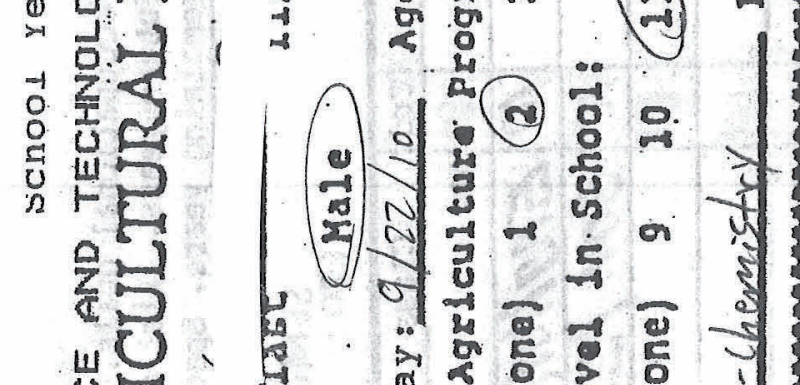
面然

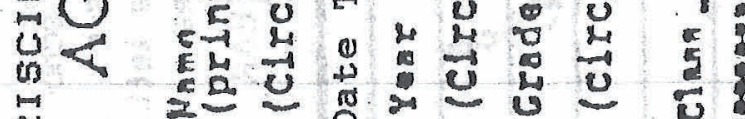

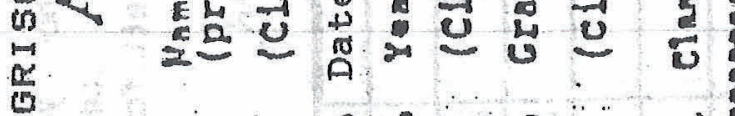

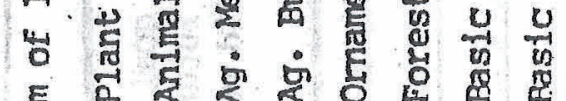

(1)

$$
\therefore 0^{\circ} \circ \text { a }
$$

$\sqrt{\frac{m_{0}}{2}}$

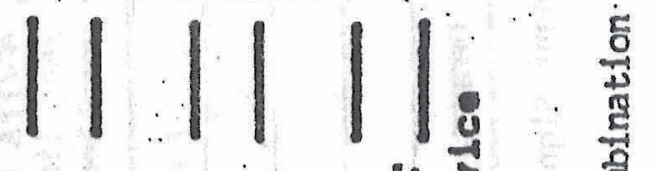

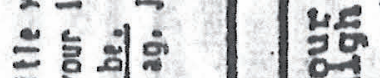

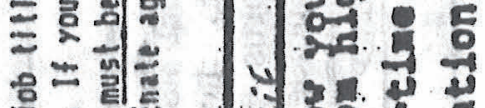

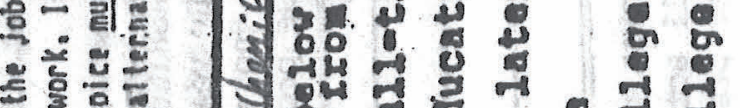

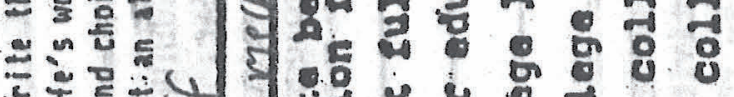

훔늘

픈요

1

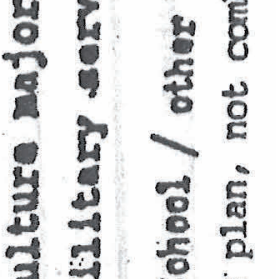

प्य है ए

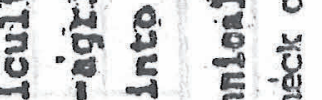

ते

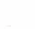




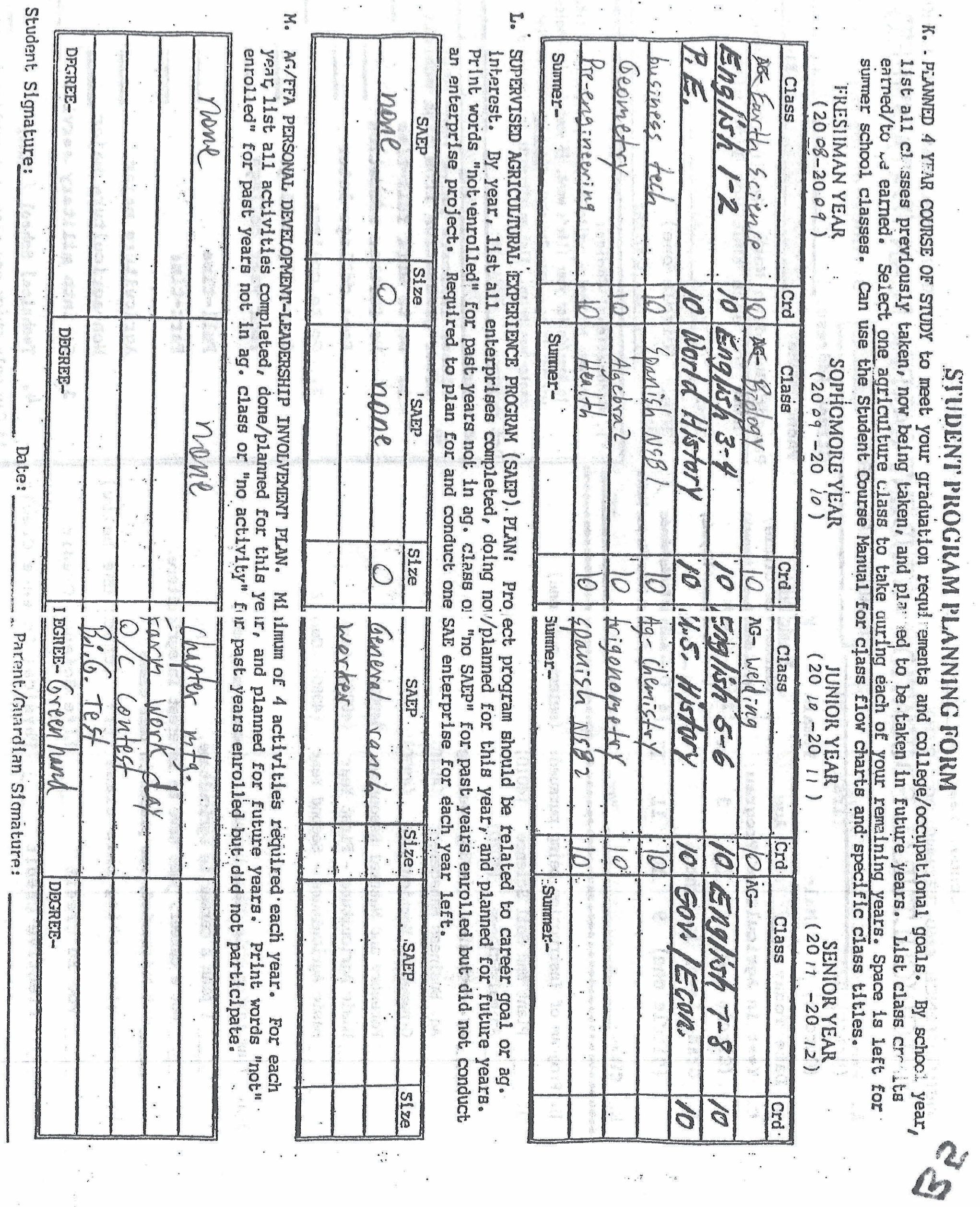




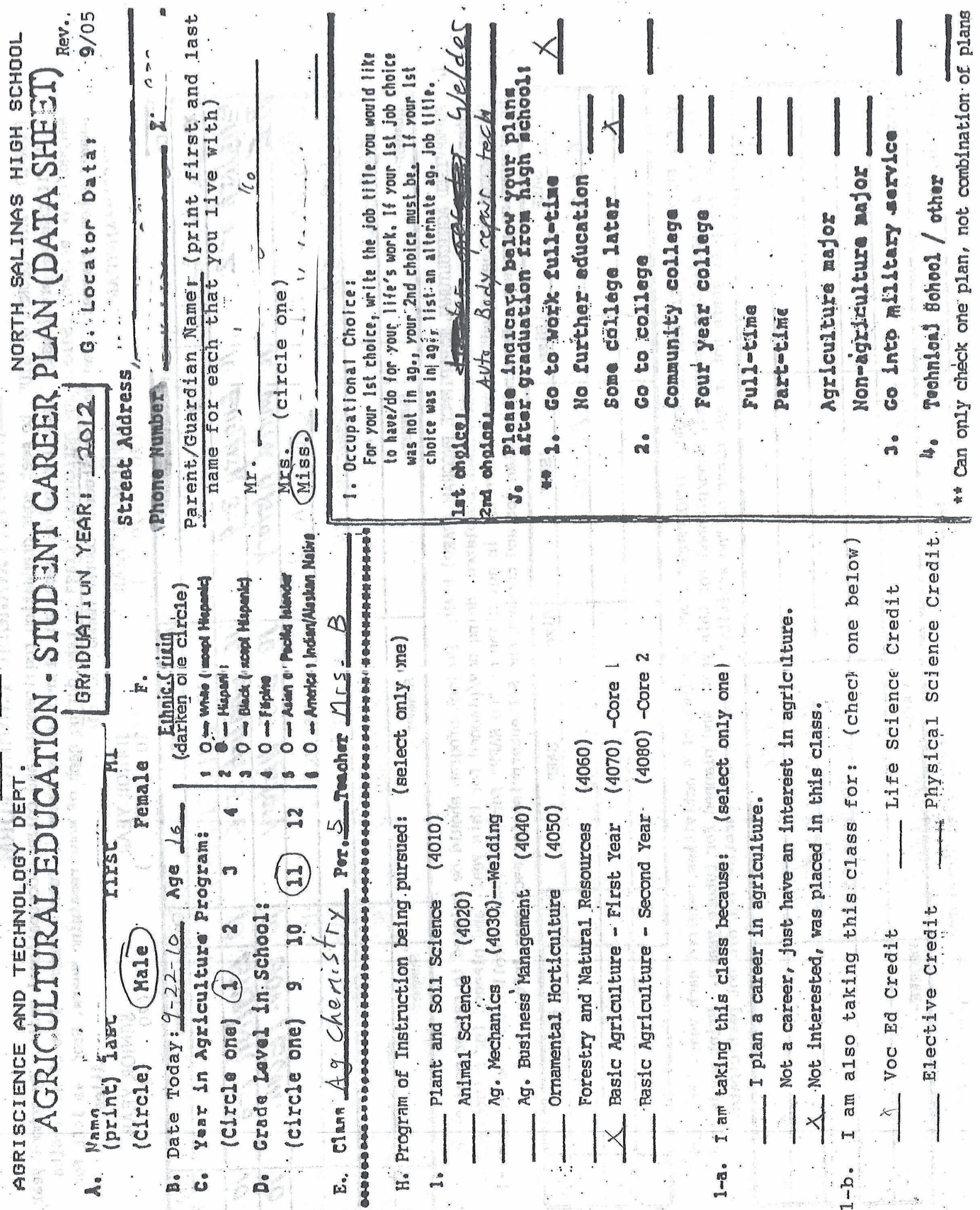




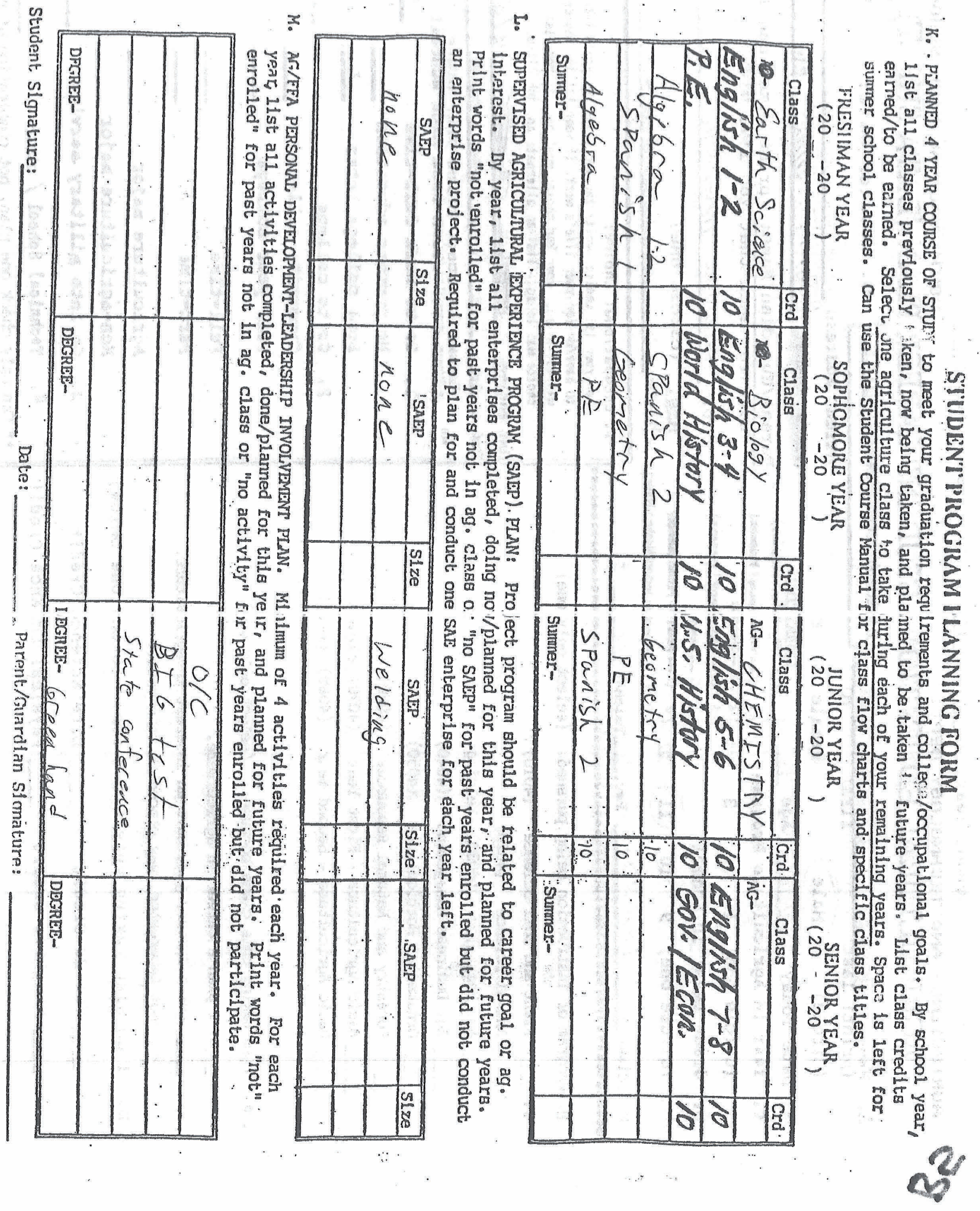




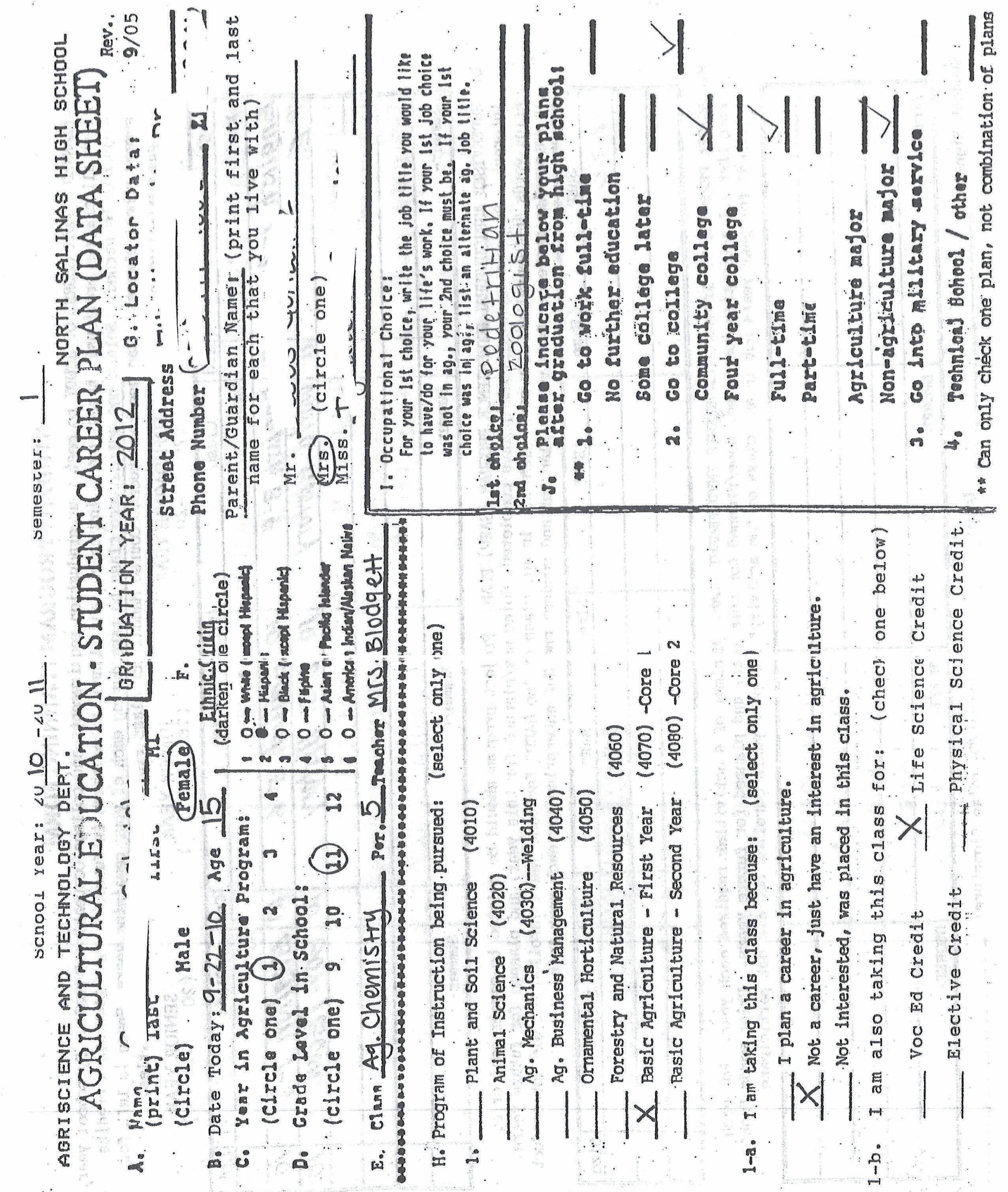




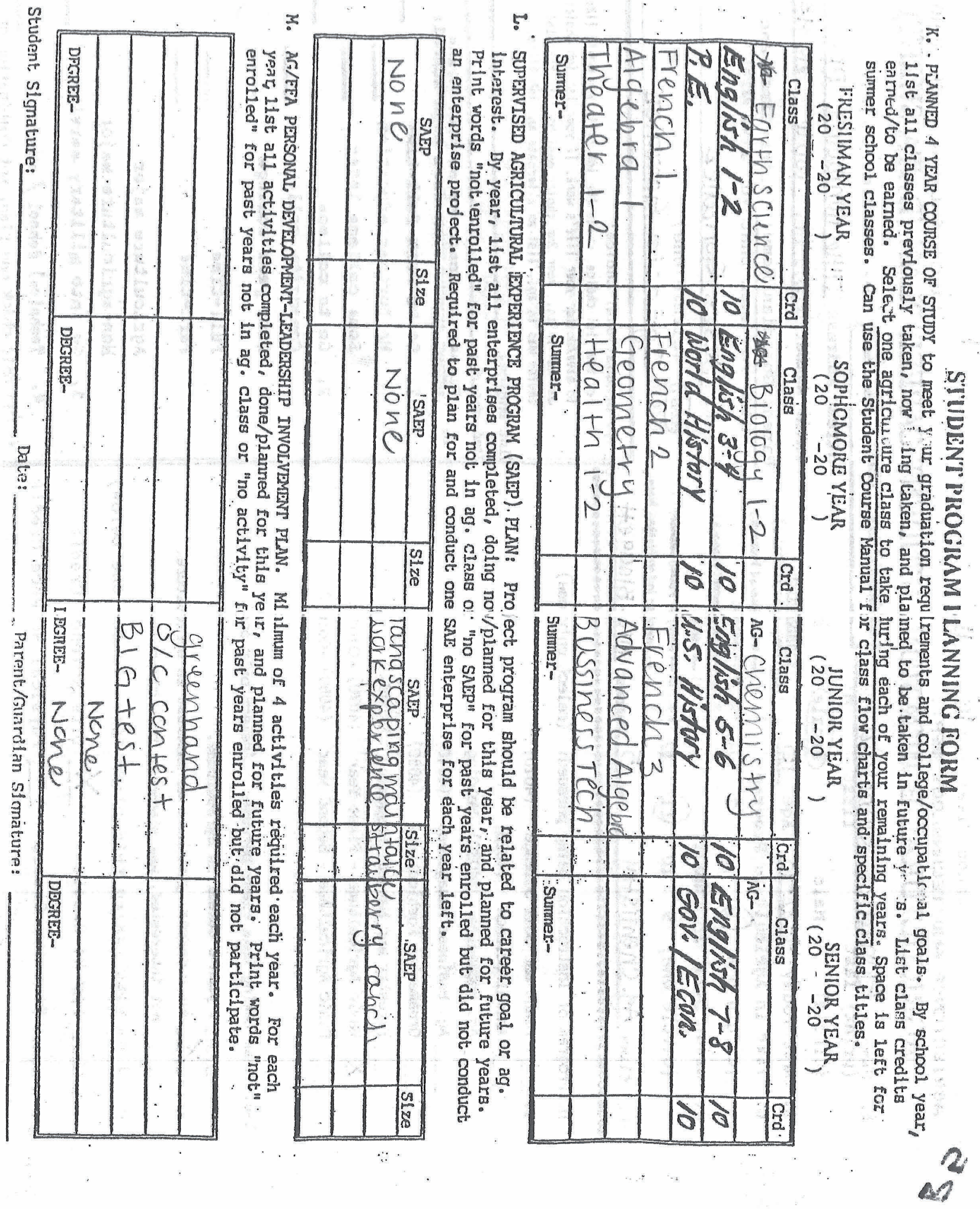




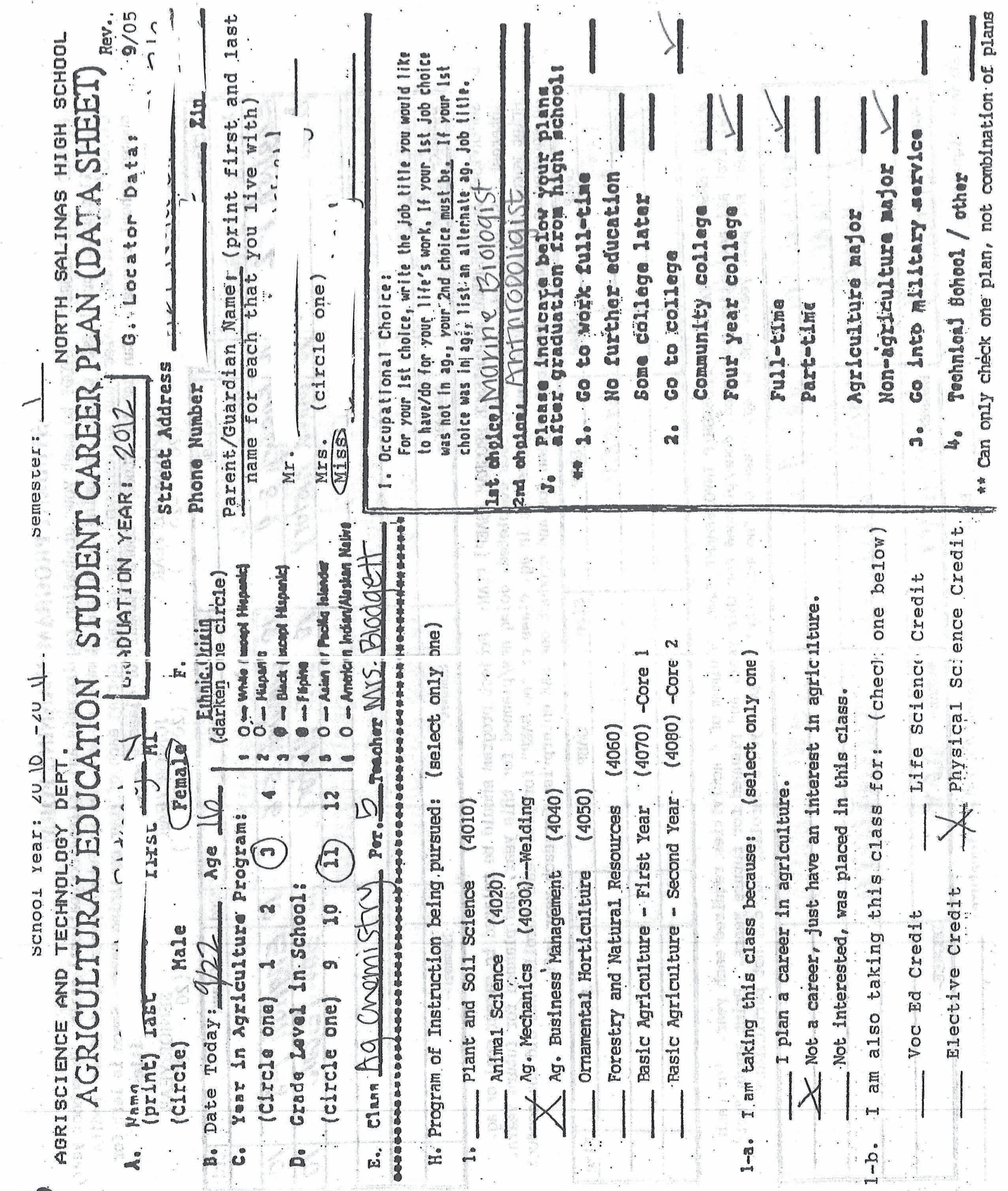




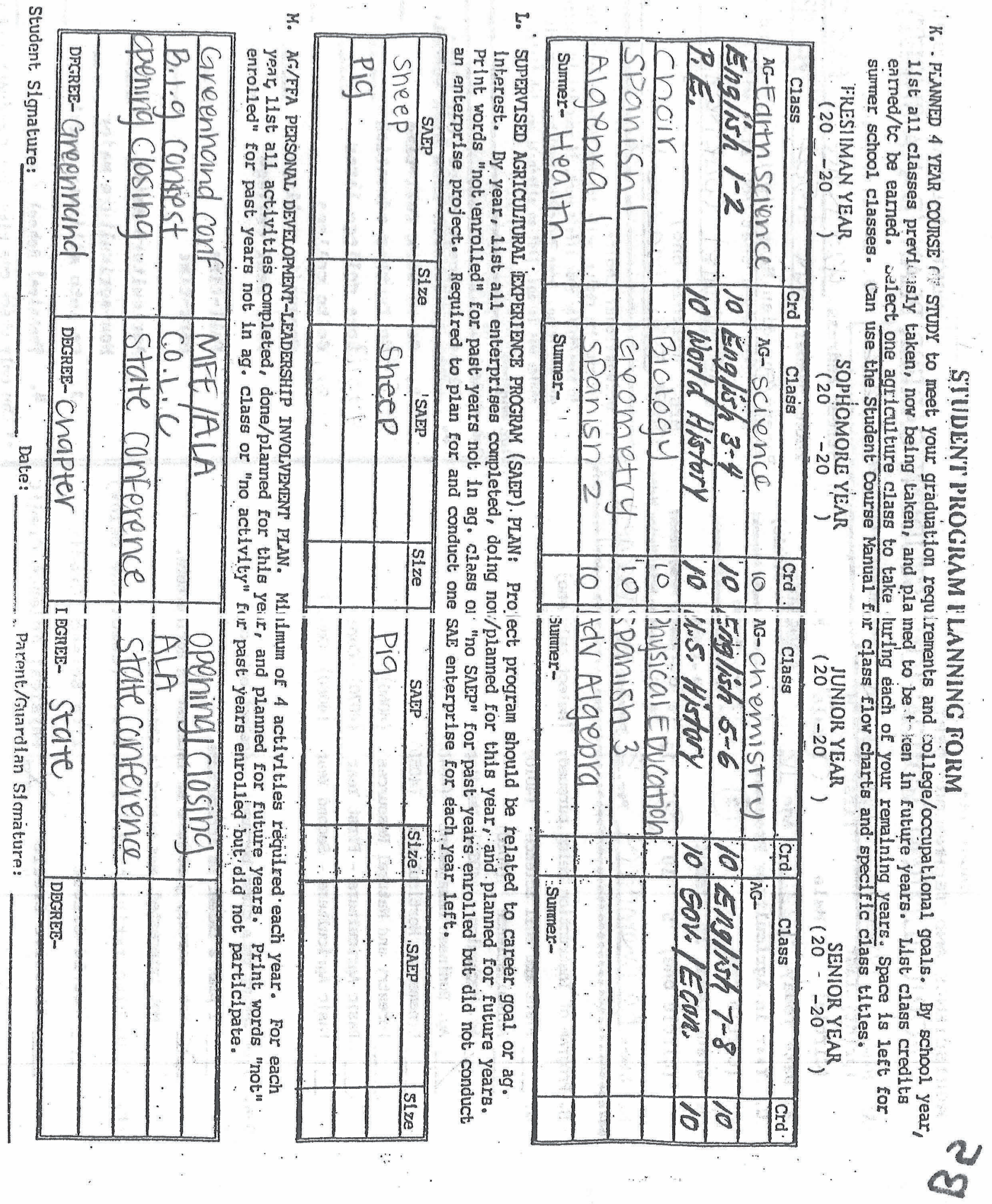




\section{Permanent Vocational Agriculture Student Records}

Below are pictures of our agriculture student records kept on file. Data sheets are identified by current students, organized by teacher/period, or by graduation year.
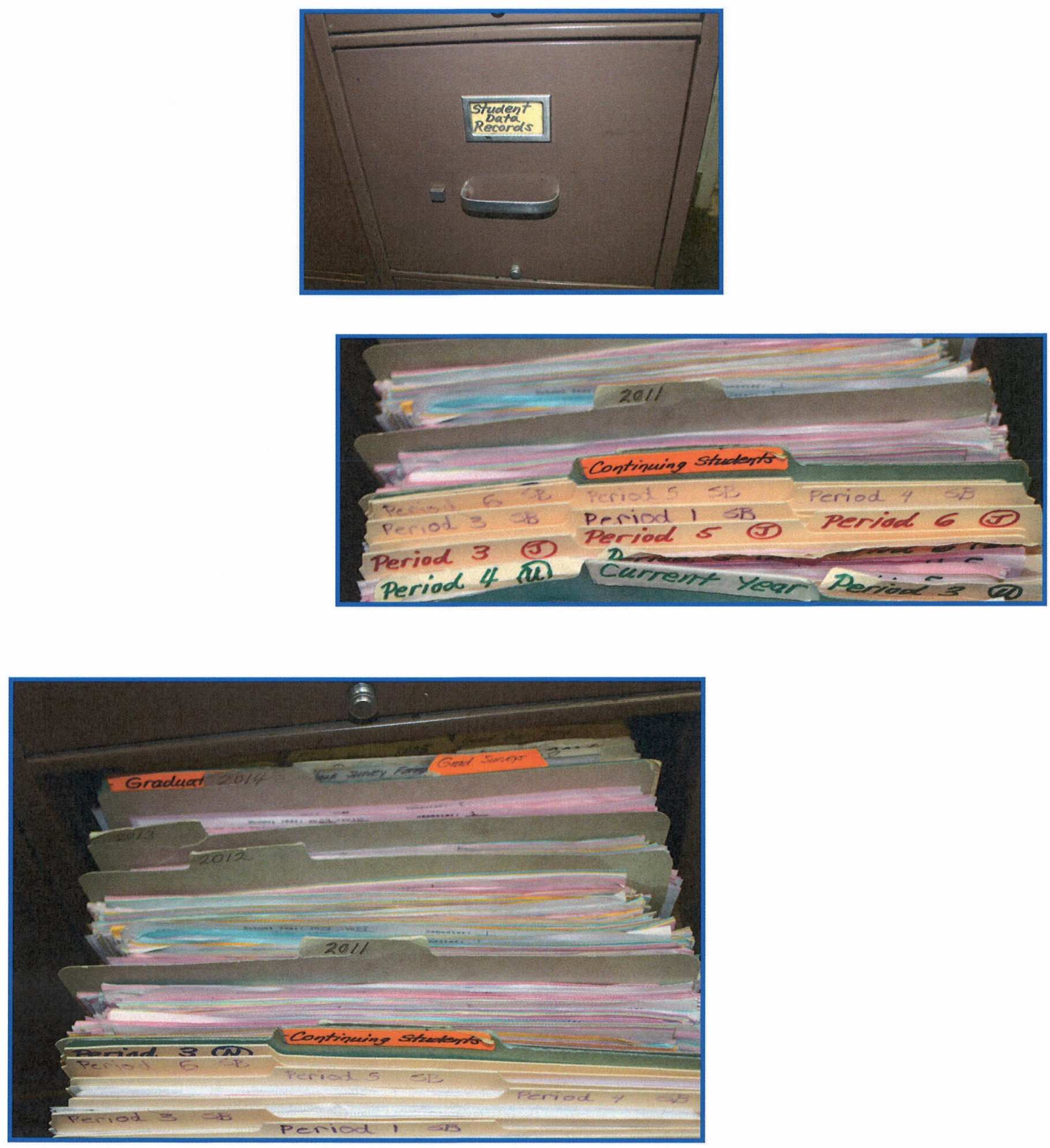


\section{Agriculture and Technology Department \\ Agriculture Chemistry \\ Grades 11-12 \\ Instructor: Mrs. Blodgett}

Course Description: Agricultural Chemistry is a lab-based course aligned to the California Content Standards for Chemistry and will include an agricultural component. This course studies the composition and behavior of matter. Atomic and molecular structure; conservation of matter and stoichiometry; chemicals and their properties; and nuclear processes are studied. Classroom demonstration and laboratory activities are an integral part of this course. (Meets the UC laboratory admission requirement in science) 10 credits.

Textbook: Text: Barr, Caputo, Davisson, Harris, and Hirschfeld; Chemistry: Matter and Change. McGraw Hill Companies.

* Prerequisites: A grade of " $\mathrm{C}$ " or better in Algebra 1-2 or approval of the instructor

Expectations: Students are expected to arrive on time and come prepared for class each day. It is important that students exercise consideration for the educational rights of others to study and learn, including their personal property rights, conducting themselves responsibly and respectfully at all times including the avoidance of fights, use of profanity, any form of harassment or other vulgarities. Students are also expected to follow school as well as classroom rules and safety procedures. In individual assignments, students are expected to do their own work.

Required Materials: Students need to bring to class each day: a blue/black ink pen and pencil, a three ring binder, a notebook and assigned textbook. Colored pencils, pens, etc. may be required to complete certain assignments.

\section{Attendance}

I. Absences: When you have been absent or missed class notes/handouts, YOU are responsible for obtaining your work in a timely manner. Makeup work will be done as homework and due within the number of days absent before work is considered late. If requested long term absences (more than 5 days) will have a reasonable work make up schedule. If tests need to be made up, they will be given during lunch time or as arranged with your teacher in a timely manner.

Invalid absences: Truancy, cutting class, and absences without a valid excuse WILL NOT be able to make up work missed.

III. Tardly: When you are NOT SEATED in your assigned seat in the classroom when the bell rings. Students who are tardy the first time will be given a warning, the second time will be a 10 minute detention; the third time will be 20 minutes, etc. Additional tardies will follow school policy. During lunch detention some kind of work must be done or additional time will be issued.

* To avoid receiving a "tardy slip" for being late, a school note or pass is required when you first walk into class after the bell has rung. 


\section{Class Grading Policy:}

$90 \%-100 \%=A$
$80 \%-89 \%=B$
$70 \%-79 \%=C$
$60 \%-69 \%=D$

I. Quarter/Semester Grade: Your grade is based upon your participation in the three components of all California Ag. Education programs:

A) Classroom Performance and Productivity

B) Ag/FFA Personal Development (Leadership activities)

C) Verified conduct of a long term Ag. Project (SAEP)

* Ag/FFA Personal Development Activities and Supervised Agriculture Experience Program (SAEP) Grades- Your participation in Ag/FFA leadership activities, personal development events, and the Ag. Project program are recognized as INTEGRAL and long-term homework assignments for all student in ag. classes. There importance to your learning and self-development allow both program components to be taught and used in class grading. At a minimum, you will be required to participate in FOUR AG/FFA activities (two of these four must be FFA chapter meetings each semester) and conduct one SAE project (validated by project record books) during the year. Grading is based upon a percentage ( $20 \%$ total) of each students earned semester classroom points. A student cannot pass this class when AG/FFA activity and SAEP points become the only basis for receiving a passing grade.

II. Productivity Grade: A daily point system evaluating work ethic and attendance will be used in determining your quarter and semester grades. Except for validated ag program and excused absences, a point deduction system for unexcused absences and tardiness will be part of student "Productivity" grading.

III. Citizenship Grade: Given each quarter this grade is determined by your behavior, attendance, and student responsibilities:

$\mathrm{O}=$ Outstanding, $\mathrm{S}=$ Satisfactory, and $\mathrm{U}=$ Unsatisfactory

- Automatic $U$ in citizenship will be given if you receive 5 invalid tardies, 5 invalid absences, or a combination of the two in a grading period.

- Automatic $U$ in citizenship will also be given if you receive less than $50 \%$ during a grading period.

Assignments: When an assignment is not identified with where to place your name you must identify the assignment yourself using the top right/left hand comer with:

1) your full name (first and last name- no initials)

2) the class title and period

3) the date

- Use only blue/black ink when using pen to complete assignments. Other colors used on the assignments/tests will be deducted an automatic 5 points.

Late Assignments: If the assignment is within one week late it will be deducted 20\%; the second week and thereafter it will be deducted 30\%. The last week of any grading period no late work will be accepted, except for that week's work. 


\section{Other:}

- Follow your teacher's directions especially during an emergency situation.

- Students will show courtesy and respect towards substitute teachers. "Zero productivity" points, an unsatisfactory citizenship grade, or an automatic referral will be given to students who are listed on the Substitute Teacher"s "Problem Student" list.

- Phones, Ipods, other electronic devices, skateboards etc. are not to be used during class time. Please, let's avoid these items being taken away from you. Thanks!

- Food and drinks are not permitted in the classroom during class time. Please make sure to do this before coming to class. 


\title{
Agriculture and Technology Department
}

\author{
Agriculture Earth Science \\ Grades 9-12. \\ Instructor: Mrs. Blodgett
}

Course Description: Agriculture Physical Science is a one year science course that is designed to successfully prepare those students with career interests in agriculture. This course will provide the student with theories and principles related to agricultural sciences and physical science. There is emphasis on developing values, aspirations, and attitudes that promote the student's personal involvement with the scientific explorations that promote the discoveries of the future. The course addresses laboratory experiments in order to connect physical science concepts with agricultural applications, including written and reporting skills. (Meets U.C. elective science requirements/and high school Physical Science graduation requirements) 10 credits

\author{
Textbook: Prentice Hall Earth Science Tarbuck ISBN\# 0-13-125897-4 \\ * Prerequisites: None
}

Expectations: Students are expected to arrive on time and come prepared for class each day. It is important that students exercise consideration for the educational rights of others to study and learn, including their personal property rights, conducting themselves responsibly and respectfully at all times including the avoidance of fights, use of profanity, any form of harassment or other vulgarities. Students are also expected to follow school as well as classroom rules and safety procedures. In individual assignments, students are expected to do their own work.

Required Materials: Students need to bring to class each day: a blue/black ink pen and pencil, a three ring binder, a notebook and assigned textbook. Colored pencils, pens, etc. may be required to complete certain assignments.

\section{Attendance}

1. Absences: When you have been absent or missed class notes/handouts, YOU are responsible for obtaining your work in a timely manner. Makeup work will be done as homework and due within the number of days absent before work is considered late. If requested long term absences (more than 5 days) will have a reasonable work make up schedule. If tests need to be made up, they will be given during lunch time or as arranged with your teacher in a timely manner.

Invalid absences: Truancy, cutting class, and absences without a valid excuse WILL NOT be able to make up work missed.

11. Tardly: When you are NOT SEATED in your assigned seat in the classroom when the bell rings. Students who are tardy the first time will be given a warning, the second time will result in 10 minutes of lunch detention; the third time will be 20 minutes, etc. Additional tardies will follow school policy. During lunch detention some kind of work must be done or additional time will be issued.

* To avoid receiving a "tardy ticket" for being late, a school note or pass is required when you first walk into class after the bell has rung. 


\section{Class Grading Policy:}

$90 \%-100 \%=\mathrm{A}$
$80 \%-89 \%=\mathrm{B}$
$70 \%-79 \%=\mathrm{C}$
$60 \%-69 \%=\mathrm{D}$

1. Quarter/Semester Grade: Your grade is based upon your participation in the three components of all California Ag. Education programs:

A) Classroom Performance and Productivity

B) Ag/FFA Personal Development (Leadership activities)

C) Verified conduct of a long term Ag. Project (SAEP)

* Ag/FTA Personal Development Activities and Supervised Agriculture Experience Program (SAEP) Grades- Your participation in Ag/FFA leadership activities, personal development events, and the Ag. Project program are recognized as INTEGRAL and long-term homework assignments for all student in ag. classes. There importance to your learning and self-development allow both program components to be taught and used in class grading. At a minimum, you will be required to participate in FOUR AG/FFA activities (two of these four must be FFA chapter meetings each semester) and conduct one SAE project (validated by project record books) during the year. Grading is based upon a percentage (20\% total) of each students earned semester classroom points. A student cannot pass this class when AG/FFA activity and SAEP points become the only basis for receiving a passing grade.

II. Productivity Grade: A daily point system evaluating work ethic and attendance will be used in determining your quarter and semester grades. Except for validated ag program and excused absences, a point deduction system for unexcused absences and tardiness will be part of student "Productivity" grading.

III. Citizenship Grade: Given each quarter this grade is determined by your behavior, attendance, and student responsibilities:

$\mathrm{O}=$ Outstanding, $\mathrm{S}=$ Satisfactory, and $\mathrm{U}=$ Unsatisfactory

- Automatic $U$ in citizenship will be given if you receive 5 invalid tardies, 5 invalid absences, or a combination of the two in a grading period.

- Automatic U in citizenship will also be given if you receive less than $50 \%$ during a grading period.

Assignments: When an assignment is not identified with where to place your name you must identify the assignment yourself using the top right/left hand corner with:

1) your full name (first and last name- no initials)

2) the class title and period

3) the date

- Use only blue/black ink when using a pen to complete assignments. Other colors used on the assignments/tests will be deducted an automatic 5 points.

Late Assignments: If the assignment is within one week late it will be deducted 20\%; the second week and thereafter it will be deducted 30\%. The last week of any grading period no late work will be accepted, except for that week's work. 


\section{Other:}

- Follow your teacher's directions especially during an emergency situation.

- Students will show courtesy and respect towards substitute teachers. "Zero productivity" points, an unsatisfactory citizenship grade, or an automatic referral will be given to students who are listed on the Substitute Teacher's "Problem Student" list.

- Phones, Ipods, other electronic devices, skateboards etc. are not to be used during class time. Please, let's avoid these items being taken away from you. Thanks!

- Food and drinks are not permitted in the classroom during class time. Please make sure to do this before coming to class. 


\section{A G-MECHANICS/WOODSHOP COURSE \\ Syllabus 09/11}

\section{WHAT IS AG-MECHANICS/WOODSHOP?}

Ag Mechanics/Woodshop is a ten credit grade 9-12 course that introduces students to a variety of ag-mechanics and woodshop skills. Students will learn advanced skills in the agricultural mechanics field. Topics may include equipment operation, safety, agricultural structures, plumbing, electricity, concrete, rope work, safety and maintenance, and project design and construction. Students will also be provided an understanding of agriculture and society, agriculture business management, and employability and leadership development. The course is designed to enhance a student's problem solving abilities and make practical connections to classes across the curriculum as well as real world applications. Students will work alone and with partners and participate in hands-on activities.

Ag-Mechanics/Woodshop can consist of the following areas of study:

\begin{tabular}{|c|c|}
\hline (): Construction Technology & (-) Basic construction techniques \\
\hline () Basic Electricity & (). Gluing and fastening \\
\hline () Basic Plumbing & (-) Wood cutting tools and techniques \\
\hline (-) Measurement & (-) Board feet calculations \\
\hline (-) Basic Wood Tools & (-) Project estimates \\
\hline (-) Basic Electrical Tools & (-) Calculation of project costs \\
\hline () Basic Plumbing Tools & (): Weather Monitoring \\
\hline () Concrete Basics & (-) Personal and Business Communication \\
\hline (-) Rope Knots and uses & (-) Time Management \\
\hline -) Career Exploration & (-) Employment Skills \\
\hline
\end{tabular}

Each student can expect to work on a variety of assignments and tasks in each area of study. Approximately every three weeks of instruction, students will be evaluated on their summary and assignment notebook.

Students will be working with extremely expensive and technical equipment. The instructor will insist that each student act responsible and respect the shop equipment. Students failing to follow the rules will lose their privileges in the shop.

Ag-Mechanics/Woodshop meets the Vocational Education requirement for high school graduation. The course may be used to fulfill elective requirements. 


\begin{tabular}{|c|c|c|c|}
\hline \multicolumn{4}{|c|}{ Name of Course: Agric } \\
\hline \multicolumn{4}{|c|}{ Name of Teacher: Mr. Jones } \\
\hline \multicolumn{4}{|c|}{ E-maill Address: tjones@monterey.k12.ca.us } \\
\hline \multicolumn{2}{|c|}{ Voicemail \#:796t. 274} & Room Number: 730 & Preparation Period: 2 \\
\hline I. & \multicolumn{3}{|c|}{$\begin{array}{l}\text { Course Description / Outline (reflects meeded skills): } \\
\text { Students will learn advanced skills in the agricultural mechanics field. Topics may } \\
\text { include equipment operation, safety, agricultural structures, plumbing, electricity, } \\
\text { concrete, rope work, safety and maintenance, and project design and construction. } \\
\text { Students will also be provided an understanding of agriculture and society, } \\
\text { agriculture business management, and employability and leadership development. }\end{array}$} \\
\hline III. & \multicolumn{3}{|c|}{$\begin{array}{l}\text { Materials Provided (mame of textbook, videos, software, other resources): } \\
\text { Introduction to Agricultural Mechanics }\end{array}$} \\
\hline III. & \multicolumn{3}{|c|}{$\begin{array}{l}\text { Materialls Required: } \\
10-12 \text { ' Tape Measure, Safety Glasses, Shop apron or coat, Combination Lock, } \$ 20 \\
\text { Materials use Fee, } 70 \text { page notebook, }\end{array}$} \\
\hline
\end{tabular}


IV. Goals (knowledge to be acquired, technical skills, etc., specific references to ESLRs and state standards):

A) 1. Calculate Area, Volume, Board Feet

2. Use given materials in efficient methods to least waste/cost saving principles.

B)1.Identify parts of Engine

2.Conduct Maintenance Requirements for engines

3.Perform Troubleshooting, Fixing, Repairing

Conduct Proper Lubrication procedures

C)1.Assemble projects in both wood and metal

2.Refit common farm tools

3.Use taps, dyes, drills, and common fasteners

4. Construct projects

5 Draft a 3-D drawing for project design

D) 1.Understand Physical properties of Concrete

2.Perform estimation of quantities, figuring costs, and job quotes

E) 1. Conduct operation and care of plumbing tools

2. Identify and use properly types of fittings

3. Carry out layout and measuring skills

F)1. Complete splicing and connection of circuit boards

2. Install lighting circuit and receptacle circuits

3. Understand and comprehend safety principles with electricity

G)1. Perform Basic Welding Techniques

2. Use and understand the differences in the processes with respect to ARC, MIG, TIG

3. Complete projects in metalworking use Bending, Drilling, Marking, and Sawing metal.

H) 1. Perform Oxy-Fuel basic techniques

2. Properly change fuel bottles

3. Perform cutting of metal using the Oxy-Fuel process

I)1. Tie a single knot ties

2. Tie double knot ties

J)1. Identify Pole Barn structures

2. Identify and understand Steel Structure Building codes and properties

3. Conduct typical Farm Fencing operations

4. Identify Farrowing Facilities strains and stresses. 


\begin{tabular}{|c|c|}
\hline $\mathrm{V}$. & 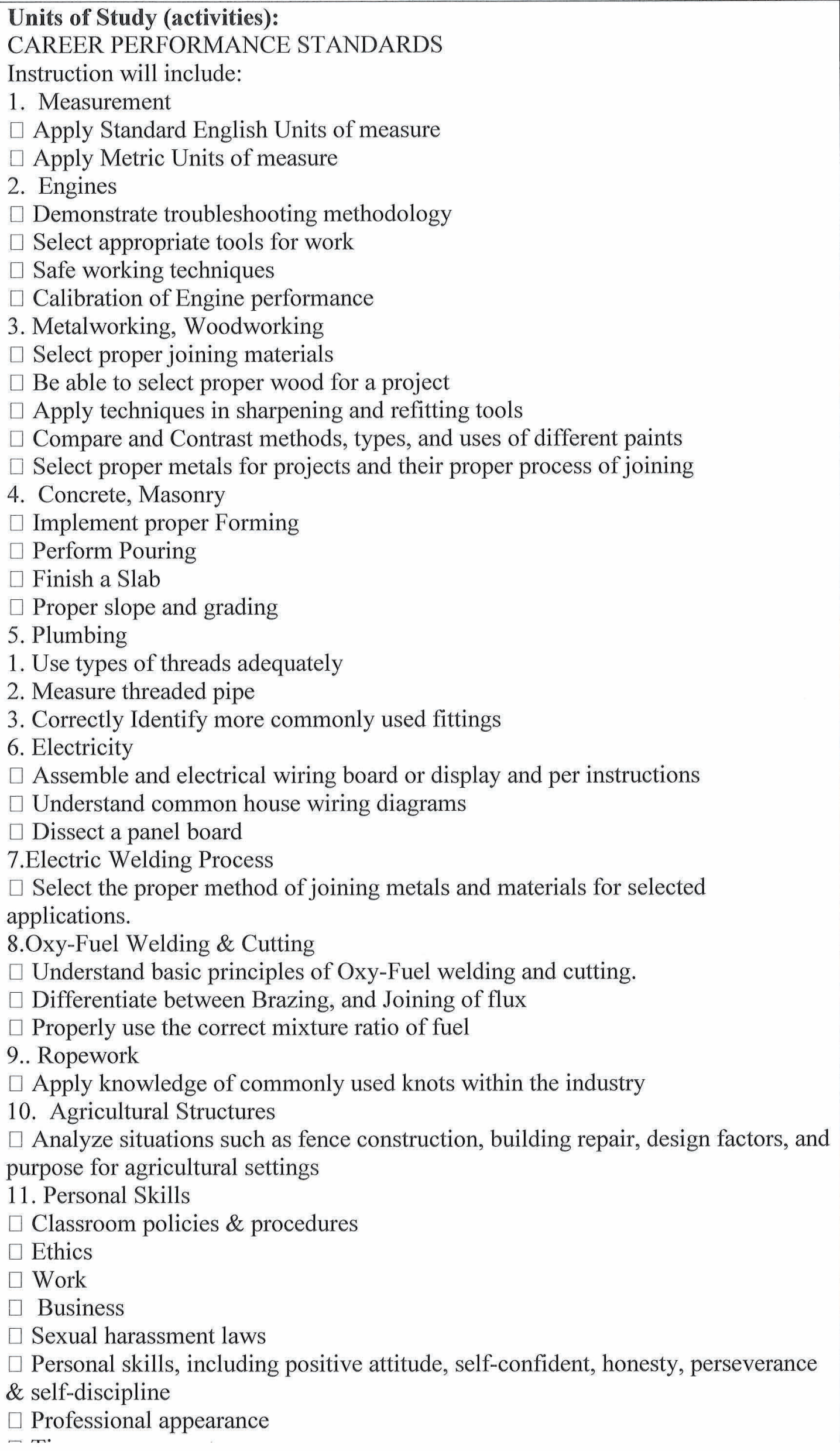 \\
\hline
\end{tabular}




\begin{tabular}{|l}
\hline VI. \\
Methods of Assessment (tests, portfolios, projects, essays, etc.): \\
Tests, Weekly Class Participation Evaluations, Semester Project, Homework, \\
Project write-ups, etc \\
\hline VIII. \\
Methods to Accommodate Language Learners and Other Target Populations: \\
The classroom should be predictable and accepting of all students. \\
Instructional activities should maximize opportunities for language use. \\
Instructional tasks should involve students as active participants. \\
Instructional interactions should provide support for student understanding. \\
Instructional content should utilize student diversity \\
VIIII. Units or Activities that Address Language and Math Standardls Needled for \\
High School Exit Exams: \\
Measurement, Concrete, Welding, Career Awarness \\
Class Procedures: \\
Students must be in their seats when the tardy bell rings. \\
Students must bring all materials to class. \\
Homework that is assigned for the next class meeting is due the day the student \\
returns to school. It will not be accepted late \\
A project grade will be dropped one letter grade for each day it is late. When \\
students are excused for an abscence, it is their responsibility to check with the \\
teacher to arrange a make-up time for tests, quizzes, etc. \\
No food or drinks are allowed in the classroom or shop areas. \\
Personal grooming is not allowed in the classroom or shop areas. \\
Daily attendance is required for optimal progress. Students are responsible for all \\
work missed during their absence \\
Tardiness will be handled according to school policy. Please try to arrive in class on \\
time. \\
When the tardy bell sounds at the beginning of class, students are expected to : \\
(a) be seated in assigned seat \\
(b) have materials ready \\
(c) be quiet and attentive waiting for the teacher to start class . \\
DISRUPTIVE BEHAVIOR WILL NOT BE TOLERATED IN CLASS. \\
If a student causes disruption, the teacher may: \\
(a) call the parents \\
(b) refer student to dean and/or academic director \\
(c) assign student appropriate detentions \\
Mutual respect for all is expected. \\
Follow directions the FIRST TIME they are given. \\
Raise your hand and wait to be recognized BEFORE talking. . \\
ABSOLUTELY, NO ONE IN THE SHOP WITHOUT PERMISSION FROM MR. \\
JONES \\
IX
\end{tabular}




\section{Behavioral Expectations (and consequences):}

Be Prepared for Classes. This includes being on time (to teacher's expectation), having all the necessary materials, and having done any assigned class work.

Be Fully Involved in the Educational Process. Full involvement means being responsible for the daily work, keeping track of assignments, using one's notebook, participating in classroom activities and other school projects, and helping the learning process whenever possible.

Be Respectful. Being respectful of oneself, others, property, and the environment is important to us at Gonzales High School. This includes the avoidance of activities which could lead to the injury of anyone in our school, abusive language, and littering.

Obey School District Policy. Major offenses listed below are those actions which, because of their severity, warrant an immediate referral to an administrator:

Alcohol Drugs Use of tobacco Fighting and assault Harassment Disobedience Gambling Truancy Theft Weapons and dangerous objects

Basic Classroom Progressive Discipline Policy

Step \#1 Verbal warning (one-on-one talk with teacher).

Step \#2 Detention time (assigned and monitored by teacher).

Step \#3 Double detention time and a telephone call to parents by teacher - notify assistant principal - miss next activity.

Step \#4 Parents, student, teacher and administrator conference.

Step \#5 Notification of administration .

XI. Gradling Pollicy:

Standard Based Grading Scale is in effect.

$\begin{array}{lll}\text { FFA } & \text { - } & \mathrm{A}=92-100 \% \\ \text { SAE-Project req }- & 10 \% & \mathrm{~B}=82-91 \% \\ \text { Test / Quizes - } & 15 \% & \mathrm{C}=72-81 \% \\ \text { Workpoints - } & 30 \% & \mathrm{D}=63-71 \% \\ \text { Project Assessment }-30 \% & \mathrm{~F}=0-62 \%\end{array}$

Total - $100 \%$

Eight (8-minimum) FFA Activity Points are required per semester required to pass 
AGRICULTURE PROJECT (SAEP) and PERSONAL DEVELOPMENT PROGRAM COMPONENTS (adopted December, 1992, revised 11/08)

The program of agricultural education instruction at North Salinas High School is composed of three components, which are integral to each other. These instructional components are (1) Classroom Instruction; (2) Supervised Agriculture Experience Program (SAEP) or "ag. project program;" and (3) the Ag/FFA personal development program.

The application of learned skills and attitudes through the ag. projects and FFA programs are recognized as INTEGRAL (intra-curricular) to classroom instruction. To be considered successful in meeting the Agriculture Department's program objectives, student involvement in all three program components is necessary. To emphasize the importance of SAEP and Ag/FFA program involvement, the following grading policy shall apply to students enrolled in agriculture classes at North Salinas High School.

As part of the student's classroom instruction grade, twenty percent (10\% SAEP and $10 \%$ FFA) of each student's final semester grade shall be based on the student (1) conducting a long-term out of class time, agricultural enterprise project that can be validated by personal records; and (2) participating in four Ag/FFA personal development activities (two must be Chapter meetings) during each semester of class enrollment. These requirements will be considered as long-term "homework" (non-class time) assignments. Satisfactory SAEP documentation and FFA involvement will earn full grade percent values. Involvement beyond the minimum requirements will result in extra credit grades earned towards the student's final semester classroom grade.

Each student must conduct, at least, ONE ownership (investment required) or non-ownership (work experience) agricultural enterprise project that requires $75 \%$ out-of-class time to complete. First year students must have an ag. enterprise project planned or in operation by the start of their second semester of class enrollment. All first year students are required to have their project in operation by the end of the second month of the second semester of enrollment. "Continuing" students must conduct an ag. enterprise project during each semester of class enrollment. Continuing student projects should show "growth," improvement, and/or diversity of experiences for each year of involvement.
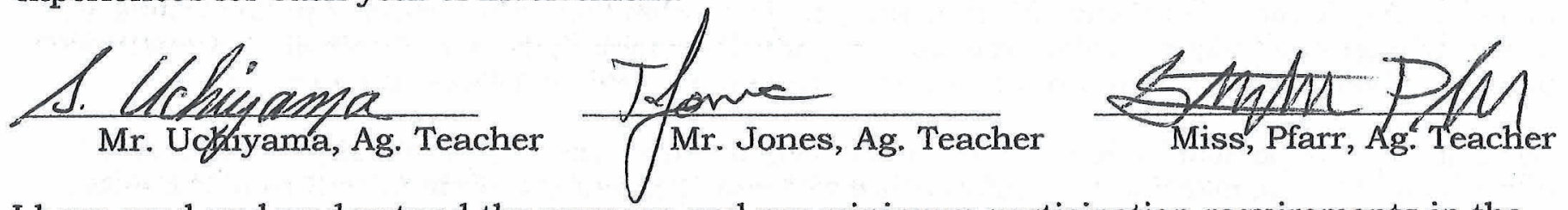

I have read and understand the purpose and my minimum participation requirements in the Ag/FFA personal development and Ag. Project (SAEP) components of North Salinas High School's agriculture education program.

Student's Signature: Date:

I support the Agriculture Department's efforts to provide the fullest of learning experiences and opportunities for my son/daughter, I shall encourage and support him/her to take an active part in the Ag/FFA personal development and Ag. Enterprise Project (SAEP) programs.

Parent/Guardian Signature: Date:

References: SB187, Vo-Ag Program Standards, CATA Policy Statement on FFA and SAEP, State AgEd Plan, State Ag. Advisory Committee, and Public Law 81-740, $81^{\text {st }}$ Congress of the United States. 
The development of leaders and competent people in all facets of agriculture is a goal of agricultural education. The primary aim of the FFA Student Organization is the development of agricultural leadership, cooperation, and citizenship among high school students enrolled in agriculture classes. More specifically, imparting knowledge, broadening abilities, and instilling attitudes that contribute to the development of leaders are activities recognized as appropriate and essential in California's agricultural education programs. In fact, such competencies and attitudes which relate to the ability to interact with people, make decisions, follow through on responsibilities, follow directions, direct the activities of others, take initiative, etc. are often over-looked job competencies. We cannot send young people into the labor market if they are ill-equipped as people.

\section{FFA PERSONAL DEVELOPMENT PROGRAM}

The FFA is a teaching strategy that provides practical, action oriented opportunities for students to engage in leadership, practical skills application, and other personal development activities. The FFA's Program of Activities are designed to develop attitudes conducive to good character development, good citizenship, building of self confidence, skills acquisition, responsibility, initiative, and working in harmony with others. FFA is the heart of agriculture education in the sense that it's activities are what it is all about---young people, how they develop to achieve immediate success and future goals.

In the classroom, FFA helps to bridge the gap between school and work. FFA is NOT an extracurricular club. FFA members may participate in a variety of local, regional, state and national sponsored activities. Some activities take place during the school day or in the classroom. Because FFA opportunities go beyond the class boundaries, many activities are also conducted after school, evenings, weekends, holidays, and during the summer. Also, they may be conducted at locations far removed from the school campus and the Salinas area.

In many instances, the student selects which activities to participate in and when they are able to participate in them. School eligibility standards do apply to participation in off-campus FFA activities when class time is missed. A student may waive their participation requirement in-lieu of completing a special project related to the intent of the FFA participation requirement.

\section{SUPERVIED AGRICULTURE EXPERIENCE PROGRAM (SAER) OI "AG. PROJECT"}

The "Ag. Project" program is a learn by doing experience. Through it, students reinforce learning by applying skills and knowledge acquired in the class. A student's SAEP is one way of extending instruction beyond the walls of the classroom. Through this teaching strategy, hands-on learning experiences and business skills are available to the student that would not otherwise exist in the school. Given the minimum requirements, students select their ag. project to conduct. The variety of projects range from raising market livestock, gardening, yardwork, ag. work experience jobs, small animals, ag. construction projects, raising rabbits, natural resources, rural recreation, welding, conservation projects, etc.

Some of the more popular projects require a financial investment and parental support for successful completion. In turn, many of these projects are sold and often bring a profitable return to the student. Financial investment in an ag. project is not required nor is it a criteria for successful project completion. Certain projects may be successfully conducted on the school campus, at home, or others may require an off-campus location to conduct the project. Although it is a very visible part of our project program, attendance at County Fairs IS NOT required in determining the successful completion of a student's ag. project. Like the FFA component, school eligibility standards apply to off-campus SAEP activities where class time is missed.

Special programs and services are available to assist students in financing, selecting, obtaining, housing, and selling of their ag. project. More details are available from the student's ag. teacher.

Participation in the FFA program of activities is not required in order for a student to successfully conduct and complete an agricultural project. However, lack of FFA participation DOES LIMIT the student's opportunity to participate in higher level SAEP recognition activities. 
North Salinas High School Agriculture Department SAE Project Supervision

Student Name. Danielle Ni to liana Ramos, Student Name: Danielle Niter Iliana Date: $2 / 26 / 11$ Projects): Breeding Raw bits

Record Book: Yes No

Parent Contact:

Yes

No

Project condition: Went to a Rabbit breeders show in Turlock, Each student picked/bought a breeding rabbit.

Recommendations: Students were told to boy a cage, feed, waterier, bowl, and bedding.

commendations: All of the students were able to get decent quality rabbits for a good price.

Student Signature:

Parent Signature:

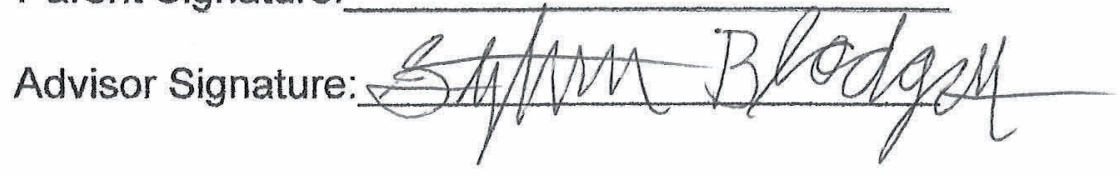




\section{North Salinas High School Agriculture Department SAE Project Supervision}
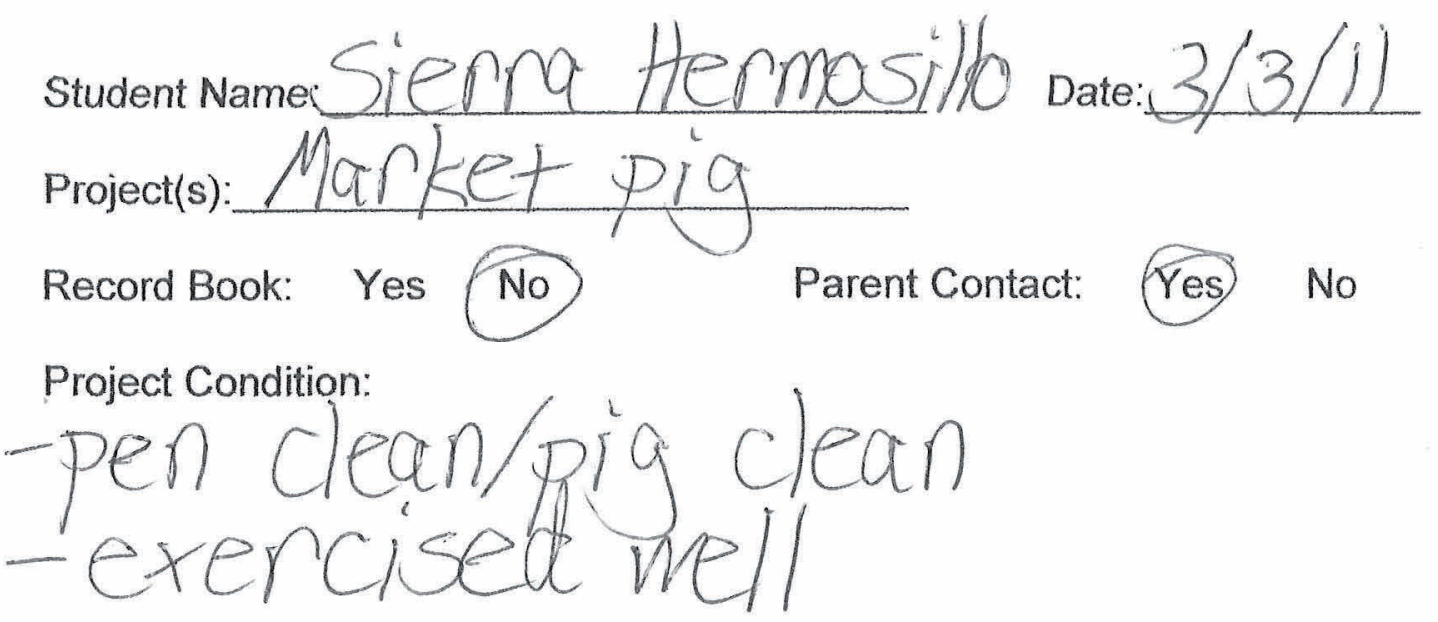

- Decommendations:

Commendations:

Student Signature:

Parent Signature:

Advisor Signature: \& 3 beteft 
North Salinas High School Agriculture Department SAE Project Supervision

student Name: liana Ramos Project(s):Breeting Rabbit

Record Book:

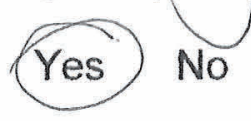

Parent Contact:
Date: $3 / 10 / 11$

Project Condition:

- cage clean
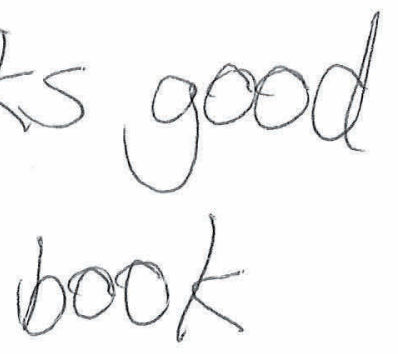

Recommendations:
- weight?

- practice showing monk with

- record book is up to date.

Student Signature:

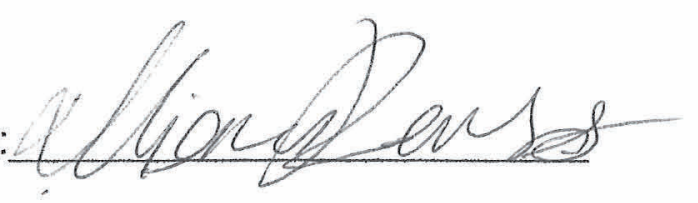

Parent Signature:

Advisor Signature:

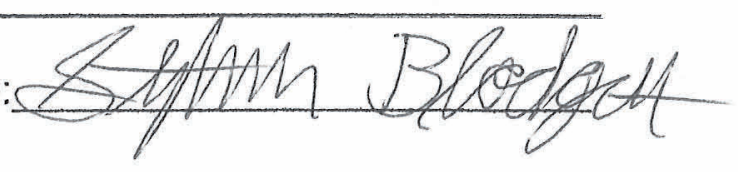


North Salinas High School Agriculture Department SAE Project Supervision

student Name: Sydney Coakley

Date: $3 / 15 / 11$ Project(s):Manket pig

Record Book: Yes No

Parent Contact:

Yes

No

Project Condition:

- nice looking pig

- just switched feed to show tee to try

- continue doing what is already being done.

Recommendations:

Commendations:

- pig seems to like the feed

Student signature:Sydnoy Cake

Parent Signature:

Advisor Signature: \&. B beat got 
North Salinas High School Agriculture Department SAE Project Supervision

Student Name: Diana Figueroa Date: $4 / 7 / 11$ Projects: Breeding rabbit

Record Book: Yes No Parent Contact: Yes No

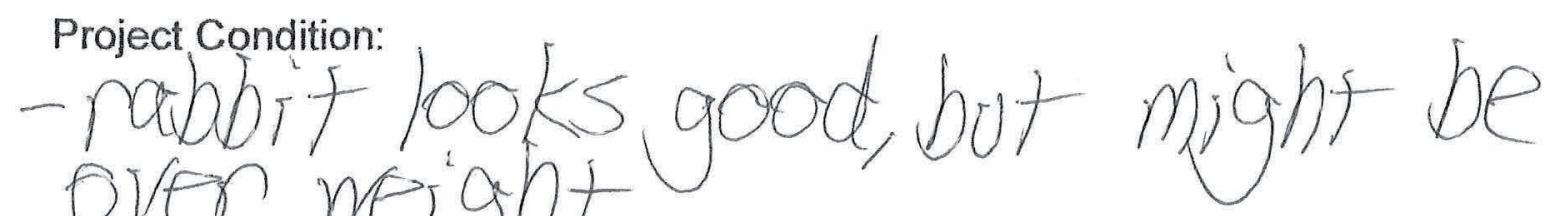

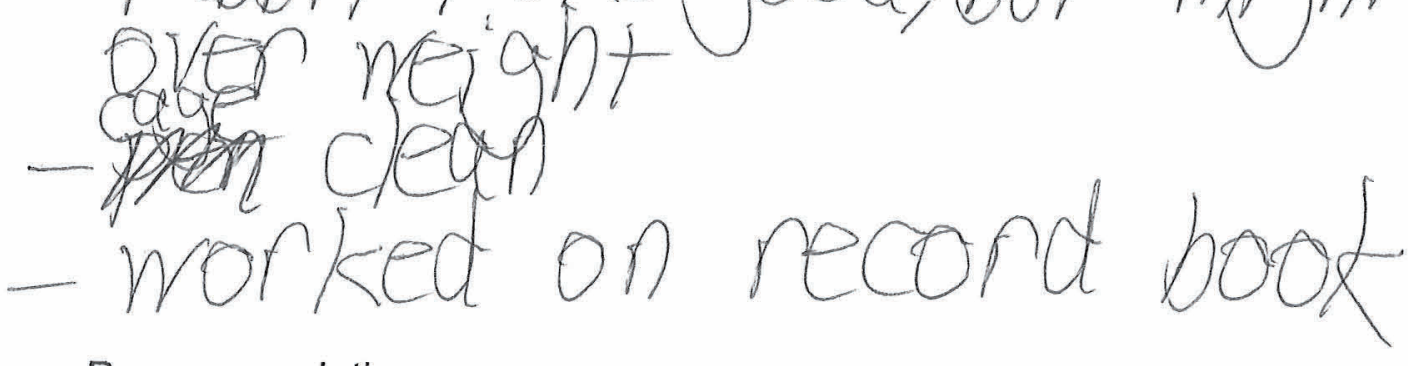

- recommendations:

- weigh more regularly

- work on record book update

Commendations:

Student Signature:Dirana Figueroa

Parent Signature:

Advisor signature: Blot er B oft 
North Salinas High School Agriculture Department SAE Project Supervision

Student Name: Rebecca Collins Date: $4 / 4 / 11$ Projects: Market lamb

Record Book: Yes No Parent Contact: Yes No Project Condition:

- Pen dean, lamb fooks.good.

- Health check for ring worm/ fungus

- show practice.

Recommendations:
- contivive to practice showing

Commendations:

- has been working with lamb( great)

student signature:Peleccas Collins

Parent Signature:

Advisor Signature:

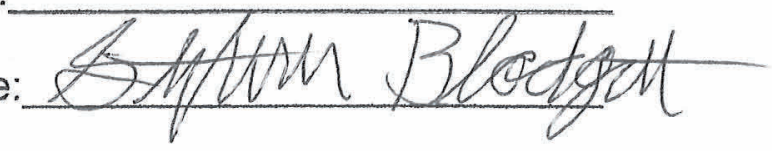


North Salinas High School Agriculture Department SAE Project Supervision

student Name: Cheyenne Silvers Date: $4 / 12 / 11$ Projects): Market lamb

Record Book: Yes No Parent Contact: Yes No

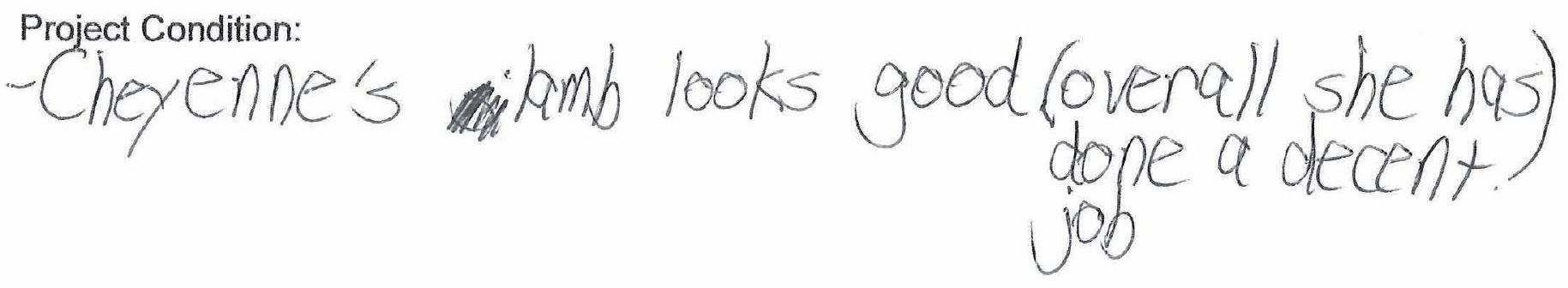

- Recommendations: fair and stop focusing so much on steen

- Braces(lamb) perfect

Student signature: Oluyuo Sienese

Parent Signature:

Advisor Signature:

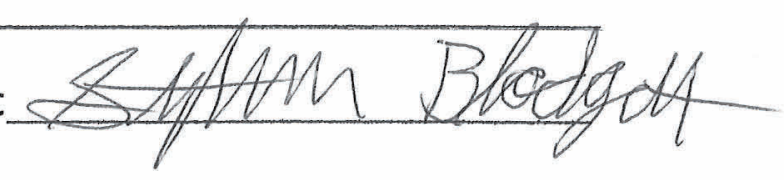


North Salinas High School Agriculture Department SAE Project Supervision

Student Name: Cheyenne Stevens Data: 4/21/11 Projects: Market steen

Record Book: Yes No Parent Contact: Yes No

- Steen looks extremely good, but worried about weight

- maybe neigh in for Santa Cruz County
Fair just in case.

- Recommendations: step n mope often

- completely hasten ho kph

Student Signature:

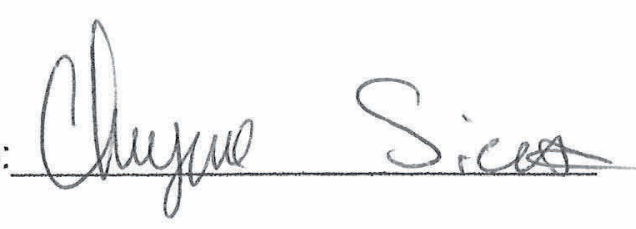

Parent Signature:

Advisor Signature: 
North Salinas High School Agriculture Department SAE Project Supervision

Student Name:Cheyeme Silvers, Victoria Silva Projects): Market Steers (weighed steers at fol

Record Book

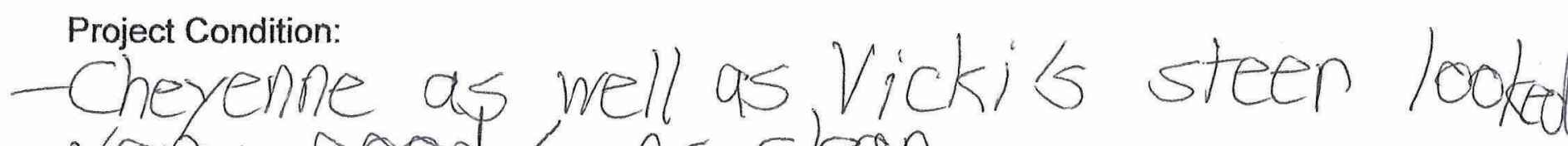

Parent Contact:

No

Yes No very good/pens clean.

- Cheyenne's steen weighed 1,030 ks

- Vickie steer weighed 920 los Recommendations: Recommendations: needs to watch the weight
- Cheyenne needs

Commendations:

- both steers are gaining weight well.

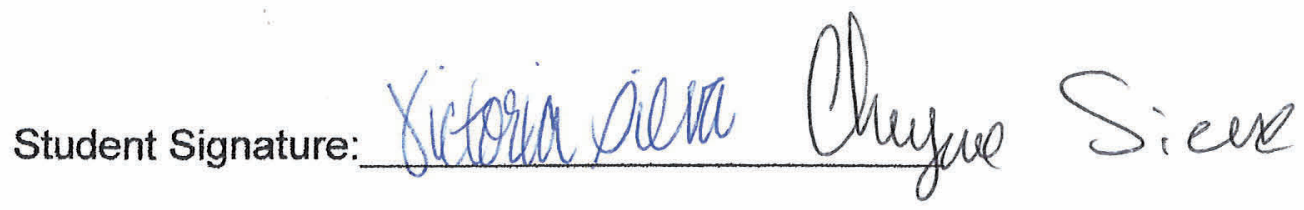
Parent Signature:

Advisor Signature

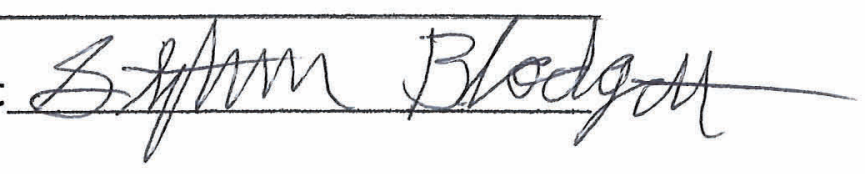


North Salinas High School Agriculture Department SAE Project Supervision

Student Name: Victor ria Silva Date: 4/25/11 Projects): Market steer

Record Book: Yes

No)

Parent Contact:

Yes

No

- project Condition: clean/animal

Recommendations:
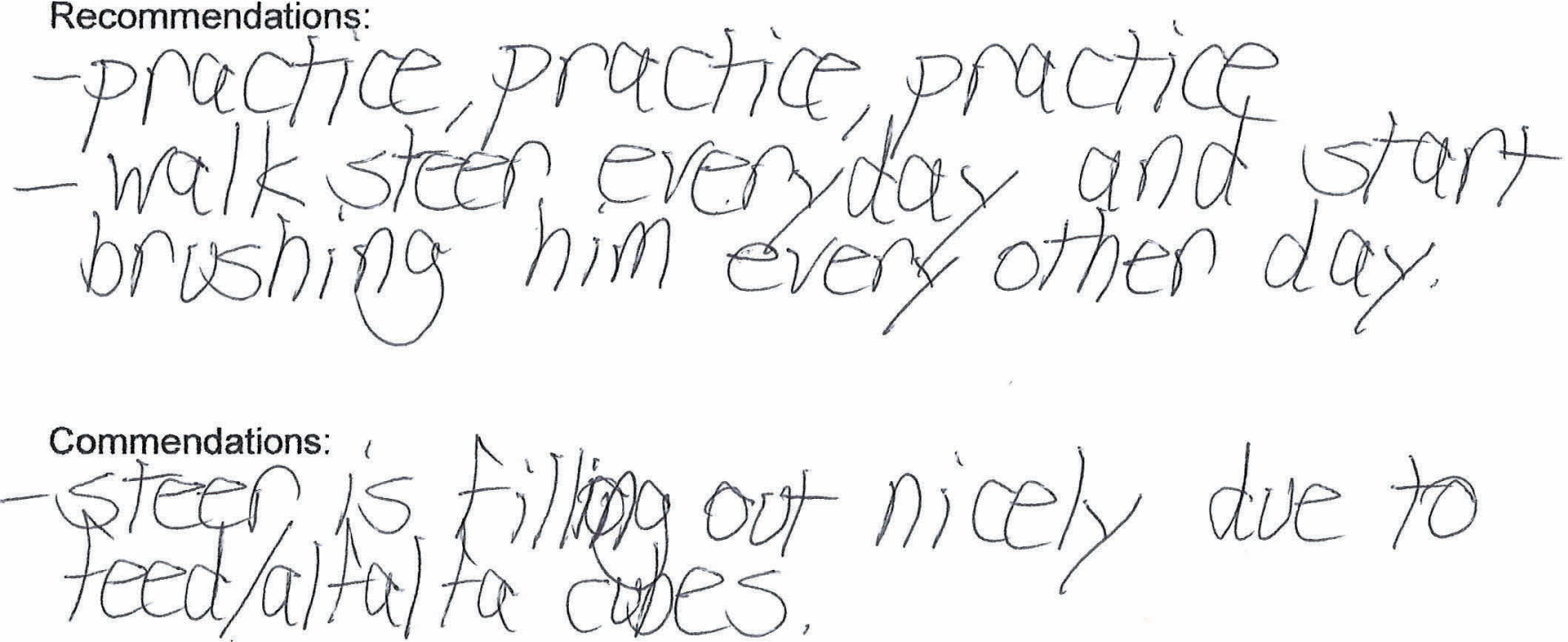

Student Signature: (Noforle Dicta

Parent Signature:

Advisor Signature 


\section{Wall Chart of SAE Visits}

A wall chart of the SAE visits no longer exists in the agriculture department at North Salinas High School. Now, with how big the department has grown it is not possible to visit every SAE project. We as a department do not visit every project. 


\section{SAE Summary}

North Salinas Agriculture department stopped filling out and maintaining an SAE summary by individual student when the folders for each student were no longer available. 


\section{Board Approved SAEP/FFA Policy Statement}

To our knowledge there is no board approved policy statement pertaining to department SAEP procedures, and operations statement or FFA as being an integral part of the Ag program. Our policy statement has been used for so long that it is the policy for the department. 


\section{0-2011 Program Of Activities}

North Salinas FFA (c)ivis? Scholarship

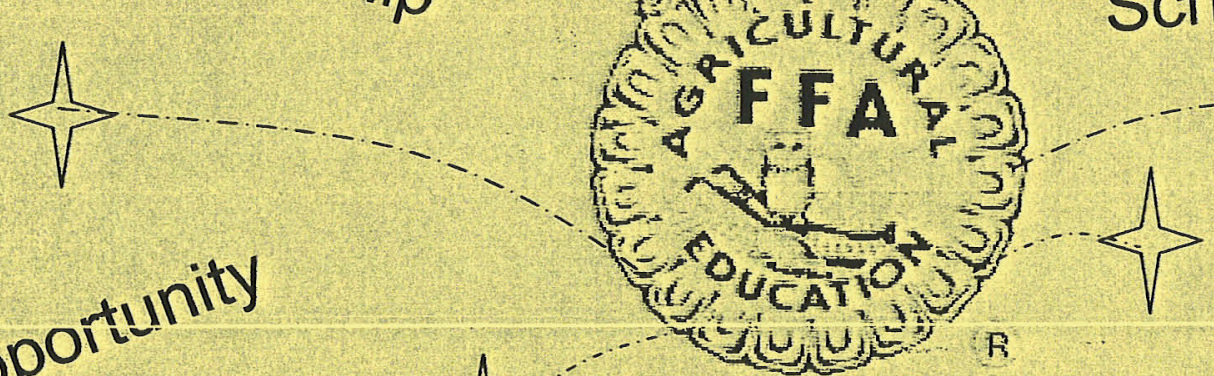

opportunity

Agriscience And

Technology Dept

North Salinas

High School 


\section{Officer's Challenge To FFA Members}

The FFA Program of Activities (POA) events calendar and operations guide was developed so that YOU, the members, could plan your involvement in FFA leadership, career skills, the agriculture enterprise project program, and other activities of our Agriscience and Technology Department

Your officer team has spent a lot of time and thought in developing this year's Program of Activities. We believe that the many different kinds of activities will provide something of interest for everyone. The FFA program offers many opportunities for success and it's up to YOU to take advantage of the opportunities to better yourself and become more successful. We know about opportunities and success because we gave FFA a try and liked what we accomplished.

We want your active participation and we want YOU to experience the great feeling of accomplishment and pride in being an FFA member. "Viking Aggies" ARE part of the "Blue and Gold" traditions of the FFA. Join us!

\section{Your Officer Team,}

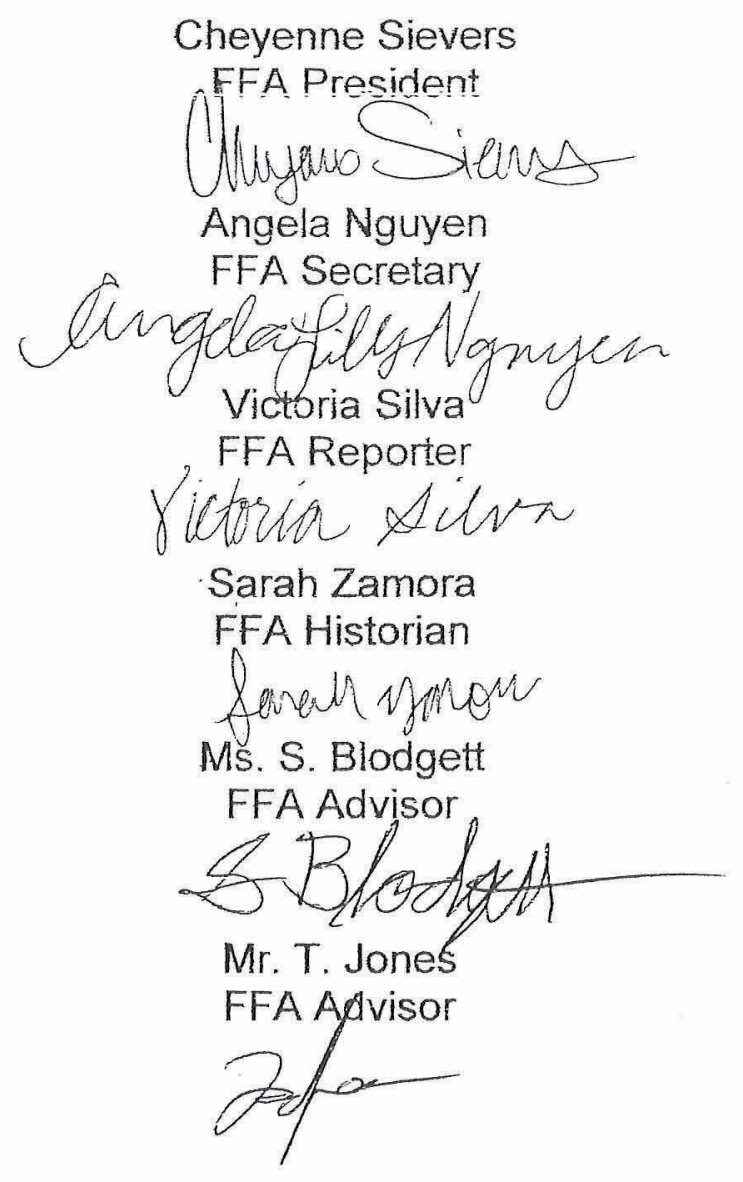

Sydney Coakley

FFA Vice President

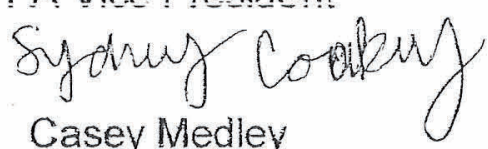

FFA Treasurer

Casey Medlu.

Adrian Lustre

FFA Sentinel

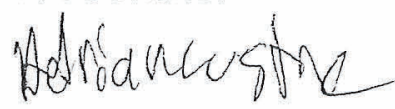

Mr. S. Uchiyama

FFA Advisor

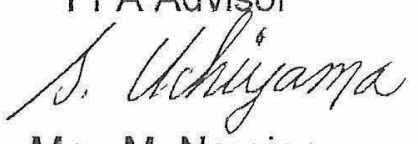

Mirs. M. Noroian

FFA Advisor

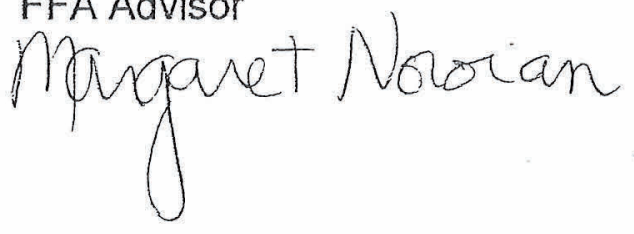




\section{The FFA Organization}

FFA is a dynamic youth organization within public high school agricultural programs in America. FFA changes lives and prepares students for premier leadership, personal growth and career success. FFA is a national student organization with 495,046 students in 7,242 chapters located in our nation's public schools, Puerto Rico, and the Virgin Islands. The Mission of the FFA is to make a positive difference in the lives of students by developing their potential for PREMIER LEADERSHIP, PERSONAL GROWTH, and CAREER SUCCESS through agricultural education.

Organized in November, 1928, the FFA is an integral/intra-curricular part of all agriscience and technology education programs. Members are engaged in a wide variety of agricultural education and career development activities, leading to over 300 career opportunities in the food, fiber, and natural resources industry. No national youth organization enjoys greater freedom of self government under adult counsel than the FFA.

FFA activities encourage members to learn through active participation how to conduct and take part in public meetings; to speak in public; to buy and sell coo9peratively; to solve their own problems; to finance themselves and to assume civic responsibility. The FFA has no outside affiliation and there is no secrecy in any of its ceremonies.

The FFA exists today because of a cooperative spini and a desire on the part of students 14 to 21 years of age to have a national, state and chapter organization system in which they can secure practical business experience, develop leadership and career skills, and enjoy good fellowship with others who have a common interest in agriscience and agribusiness. Members strive for improved agriculture, to better their communities, achieve a more satisfying home life and to become responsible citizens.

The National Center of the FFA is located in Indianapolis, Indiana. The FFA New Horizons Magazine, the National FFA supply Service, leadership and educational programs, and other supportive services are coordinated there.

The North Salinas FFA Chapter supports the activities of the California FFA Association and the National FFA organization and encourages personal skills development and achievements in agriculture education. Our 187 Chapter members are part of the 64,201 FFA members which make up the 317 Chapters in the California Association FFA. 


\section{Din}

FFA makes a positive difference in the lives of students by developing their potential for premier leadership, personal growth, and career success through agricultural education.

To accomplish this mission, FFA:

- Develops competent and assertive agricultural leadership.

- Increases awareness of the global and technological importance of agriculture and its contribution to our well-being.

- Strengthens the confidence of agriculture students in themselves and their work.

- Promotes the intelligent choice and establishment of an agricultural career.

- Encourages achievement in supervised agricultural experience programs.

- Encourages wise management of economic, environmental and human resources of the community.

- Develups inlejpersoral skills in teamivork, communications, human relations and social interaction.

- Builds character and promotes citizenship, volunteerism and patriotism.

- Promotes cooperation and cooperative attitudes among all people.

- Promotes healthy lifestyles.

- Encourages excellence in scholarship.

\section{The Agricultural Education Mission}

The mission of the Agricultural Education is to prepare and support individuals for careers, build awareness and develop leadership for the food, fiber and natural resources systems. 


\section{0-2011 Chapter Officers}

President Cheyenne Sievers

Vice President Sydney Coakley

Secretary Angela Nguyen

Treasurer Casey Medley

Reporter Victoria Silva

Sentinel Adrian Lustre

Historian Sarah Zamora

Chapter Advisors North Salinas Chapter

Mr. Stan Uchiyama

Mr. Tom Jones

Mrs. Stephanie Blodgett

Mrs. Margaret Noroian
State Advisors

California Association FFA

Mr. Bob Heuval, Head State

Supervisor Agriculture

Education

State Dept. of Education

Sacramento

Mr. Josiah Mayfield

Assistant State FFA Advisor

Calif. Association FFA

Agriculture Education

State Dept. of Education
Regional Advisors
South Coast Region

Mr. Greg Beard

Reg. Supervisor

Ag. Education

Cal Poly, San Luis Obispo

\section{Administration}

Mr. Jim Earhart

District Superintendent

Mr. Tim Vanoli

Associate Supt.-Instruction

Mr. Jim Earhart. Assoc. Supt-Business Service

Mr. Alejandro Hogan. Assoc. Supt-Human Resources

Mr. Randy Bangs Director ROP/Career Education Mrs. Gina Muller. Mr. Augie Caresani. Asst. Director ROP/Career Education

Mr. Jill Camron. Principal

Mr. Bernie Nuno Assistant Principal, Facilities

Mrs. Jean Ashen. Assistant Principal, Business

Mr. Margaret Noroian Assistant Principal, Instruction .Director of Student Activities.

\section{Board of Trustees}

Salinas Union High School District

Mr. Phillip Tabera, President

Cherl Larison, Vice President

Patty Sa'enz, Board Clerk

Mr. James Reavis Mrs. Lila Cann

Mrs. Kathryn Ramirez Mrs. Evamarie Martinez 


\section{0-2011 Parent Agriculture Boosters Club}

President Mrs. Terry Mendez

Vice President Mr. Richie Mendez

Secretary. Mrs. Syble Beardslee

Treasurer Mrs. Julie Medley

Public Relations Mrs. Elaine Benadom

\section{Agriculture Advisory Committee}

Mr. Josh Ruiz, Chairman Tanimura \& Antle

Mr. Gary Peterson, Vice Chairman P \& P Sales

Mr. Paul Kohatsu Church Bros.

Mr. Vince Ferrante Dole Fresh Veg.

Mr. Dale Wallace Don Chapin Co.

Mr. Rick Winburn Smurtit-stone Containers

Mr. Tom Collier Collier Feed \& Pet Supply

Mr. Andy Madolora Tamagni Farms

Mr. Mike Johnson 101 Livestock Market

Ms. Rebecca Collins Student Representative

Ms. Terry Mendez Ag Boosters Rep.

Mr. Augie Caresani. School Principal

Mr. Stan Uchiyama, Secretary Ag Teacher/Dept. Chair

Mr. Tom Jones Ag Teacher

Mrs, Stephanie Blodgett Ag Teacher

Mirs. Margaret Noroian Ag Teacher

\section{FFA Chapter Divisions and Program Activities}

1. Executive Committee Cheyenne Sievers, Chapter President Officers and Committee Chairman

2. Member Activity Development Division.

- Officer Advisors- Cheyenne Sievers, President and Sydney Coakley, Vice President

- Program Areas - Leadership, Scholarship, Member Development, Alumni, SAEP, and Agriculture Career Skills.

3. Chapter Activity Development Division

- Officer Advisors-Angela Nguyen, Secretary and Casey Medley, Treasurer

- Program Areas-State and National Activities, Cooperation, Conduct Of Meeting, Recreation, Chapter Safety, Earnings and Savings, Ag. Boosters, and Recruitment.

4. Community and School Activity Development Division.

- Officer Advisors-Victoria Silva, Reporter, Adrian Lustre, Sentinel.

- Program Areas-Community Service, Public Relations, School and Community Improvement, and Ag. Advisory Committee.

The purpose of FFA committees is to give members an opportunity to build leadership and cooperation skills through planning and conduct of Chapter activities. Activity assignments to the three divisions may not fit the division titles they are assigned to, giving all committees the opportunity to conduct a variety of activities. 
Chapter officers serve a vital leadership function in the FFA. Officers organize, conduct, and supervise Chapter activities for the benefit of the FFA program and its membership. Officers manage the chapter and perform duties not commonly known to members. The following are general duties expected of ALL CHAPTER OFFICERS:

1. Be able to lead by example. Follow the FFA Code of Ethics and CATA Curricular code of Conduct.

2. Meet school scholarship and citizenship eligibility requirement standards. Maintain a satisfactory scholarship record.

3. Have a basic working knowledge of parliamentary procedure.

4. Be willing to accept responsibility and understand the duties of fellow officers and chairpersons.

5. Memorize parts in FFA ceremonies involved in.

6. Attend $90 \%$ of chapter and executive committee meetings, and as many of the chapter activities as possible.

7. Help plan events and programs for the Program of Activities guide.

8. Help plan chapter meeting agenda and conduct special programs.

9. Help with end of year Parent-Member awards banquet.

10. Work with activity chairpersons in planning committee meetings and in the conduct of their activities.

11. Wear FFA jacket at chapter meetings. Wear full dress uniform at special ceremonies and programs, banquets, and other designated activities.

12. Be familiar with chapter constitution/by-laws.

13. Have a genuine interest in being a part of the officer team. Be proud to be a member of the FFA and stand ready to defend its aims and purposes Encourage other members to get involved.

14. Attend designated chapter officer leadership training conferences.

15. Serve as judges for chapter level contests.

16. Help with the nominating, screening, and election process for chapter officers and other leadership positions.

Each officer has specific responsibilities that are a natural part of their office and are assigned other duties which have been agreed to by the officer team. They are as follows:

\section{The President Shall:}

1. Preside over meetings according to accepted rules of Parli-Pro.

2. Appoint special committees and supervise their progress.

3. Serve as the official representative of the chapter.

4. Work with the Vice President to Coordinate chapter activities and their progress to completion; and to develop the annual chapter Program of Activity guide.

5. Prepare chapter and officer meeting agendas and conduct the meetings.

6. Conduct chapter meeting and activity evaluations.

7. Regularly meet with FFA Advisors to discuss chapter activity progress, Program of Activity needs, special problems, etc.

8. Serve as student representative on the Ag. Advisory Committee.

9. Conduct officer nominations, screening, and election process.

10. Conduct old and new officer review meeting at end of year.

11. Award officer and chairmen pins.

12. Conduct honorary member degree ceremonies.

13. Organize end of year Parent-Member Awards banquet. 


\section{The Vice President Shall:}

1. Assume the duties of the President when necessary.

2. See that the Program of Activities is being followed-work with other officers to coordinate committee work progress.

3. Work with the President to develop the Chapter's annual Program of Activity guide. Assemble and distribute the POA in a timely manner.

4. Construct and maintain active member involvement wall chart.

5. Serve as student representative in the Ag Booster Club.

6. Organize FFA workers for Ag Booster's summer activities if needed,

7. Insure that chapter "Thank You" letters are written and sent.

8. Keep record of officer meeting and activity attendance.

9. Complete and submit Superior Chapter award application to State FFA Association.

\section{The Secretary Shall:}

1. Prepare, present, and keep records of chapter and execurtive committee meeting minutes, including financial decisions made.

2. Keep a neat and accurate Official FFA Secretary book, including treasurer and committee reports. Must turn in at end of year.

3. Work with the President to develop Chapter meeting agenda.

4. Be responsible for chapter correspondence.

5. Keep chapter resource files and records drawer organized.

6. Keep member meeting attendance records.

7. Complete chapter activity summary report to state association.

8. Have on hand for each meeting:

a. Official Secretary's Book with meeting minutes.

b. Appointed committee member lists.

c. Program of Activity copy.

d. The Official Manual.

-..--- Chapter constitution and bylaws.

9. Issue membership/l.D. cards.

10. Update and turn in copy of Chapter Constitution to ASB office.

11. Complete and submit Star Advisor award application to State FFA Association.

\section{The Treasurer Shall:}

1. Develop Chapter wide activity budget for Program of Activity guide.

2. Receive and deposit chapter funds and issue necessary receipts.

3. Send out bills to members to collect debts to the Chapter.

4. Complete purchase order requests for chapter supply and equipment needs, pay chapter bills and reimbursement claims.

5. Prepare the membership roster and FFA affiliation fees to State FFA Association by October $15^{\text {th }}$ of each year.

6. Maintain a neat and accurate official FFA Treasurer's Book, Turn in at end of year for audit.

7. Assist with all chapter fundraising activities.

8. Prepare monthly treasurer's reports for chapter meetings.

9. Be in charge of special year long ag. jacket, hat sales, etc.

The Reporter Shall: (work with Historian)

1. Prepare and distribute a semester chapter newsletter.

2. Write and submit chapter and member achievement items for printing in school's daily student bulletin.

3. Write news articles to local newspaper and other community media.

4. Write articles for school newspaper, Viking Voice, etc.

5. Send news articles and pictures to sectional, regional, state and national FFA reporters in a timely manner. 
6. Help coordinate FFA Week activities and publicity programs.

7. Work with local newspapers, radio, and television media to promote FFA activities.

8. Submit chapter and member achievement recognition reports to School Board in a timely manner.

The Sentinel Shall:

1. Fill out school calendar reservation forms for meetings and chapter activities.

2. Submit monthly meeting notice reminders for Student Bulletin and other activity announcements.

3. Set-up room for meetings and cleanup room after the meeting.

4. Keep chapter equipment and paraphernalia in proper condition.

5. Welcome visitors and introduce guests at meetings and events.

6. Keep the meeting room comfortable and assist in keeping order.

7. Assist in taking charge of candidates for degree ceremonies.

8. Conduct chapter recreation and special meeting activity programs.

9. Do monthly activity wall calendar.

10. Organize special meeting refreshments.

11. Turn in chapter officer name list to ASB office.

The Historian Shall: If one is not elected, duties shall be added to the Chapter Reporter.

1. Order Chapter scrapbook, filler pages, etc.

2. Maintain an attractive and up to date scrapbook of memorabilia and current year's history; display it at meetings and appropriate functions.

3. Serve as chapter photographer, order film, develop pictures, and be responsible for the chapter's FFA camera.

4. Help the Reporter with writing news articles and school's student bulletin notices.

5. Enter scrapbook in county fairs and other competitions.

\section{Committee Chairperson Responsibilities}

Committee chairpersons are the important "key" to the successful planning and completion of selected chapter activities.

1. To attend executive committee and chapter meetings.

2. To select committee members and hold regular committee meetings.

3. To keep records of committee meeting decisions and member attendance.

4. To work with FFA Officer-Advisor in planning, conducting, and completing committee activities.

5. To determine activity participation credit guidelines and complete necessary ASB/School or District activity approval forms.

6. To develop activity budgets, complete expense purchase orders, and bill members for money due.

7. To coordinate activity publicity and the writing of thank you letters.

8. To perform officer ceremony parts in the absence of other officers.

9. To serve as a leadership example to other FFA members and be willing to accept. responsibility.

10. To be familiar with the FFA Code of Ethics and CATA Curricular Code of Conduct.

11. Should own an FFA jacket and know the proper use of the jacket.

12. To be familiar with the Chapter Constitution and have a basic knowledge of parliamentary procedure.

13. Have a genuine interest in being part of a leadership team. Be proud to be a member of the FFA and stand ready to defend its aims and purposes. 


\section{Activity Planning Guide}

1.5 Line SpaciffEA Program of Activities North Salinas Chapter

Committee Name:

.5 pt after paragraph spacing

Meeting Date:

Title of Acivity:

Activity Date:

Budget: \$

Est. Profit: \$

Activity Goal:

Chairman:

Officer Advisor:

Committee 1 .

Members: 2.

3.

4.

5.

6.

Members Absent:

\section{Guide For Activity Accomplishment}

1. Hold committee meeting to plan the activity: decide what needs to be done-select date, time and place of activity, facilities and equipment needed, supplies and materials needed, decorations, entertainment, transportation, decide on rules and procedures, make a budget, food or refreshment needs, awards or recognition, extra help needed, setup and cleanup of activity, participation credit sheets, decide how purchases or income will be handled, etc.

2. Select chairman and people for jobs to be done-write down job assignments and main activity decisions made.

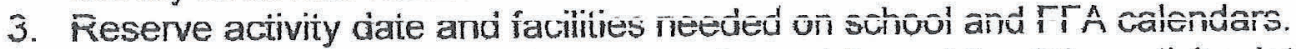

4. Decide how to inform members, parents, and the public of the activity details. Do you need information fliers, sign-up sheets, announcements in student bulletin, posters made, newspaper articles written, etc.?

5. Plan at least one progress meeting to make sure assigned jobs are being done. Chairman needs to regularly check on work progress being made.

6. Conduct the Activity.

7. After the activity - hold a committee meeting to evaluate and "wrap up" activity needs, determine member credits, write thank you notes, etc.

Activity Assignments and Decision Making Job Assignements/Decisions Made

Person Responsibility 


\section{Executive Committee}

Made up of Chapter Officers, Division, and Committee Chairmen. Its purpose is to coordinate chapter activities and make decisions necessary to carry out the Chapter's Program of Activities and operations between monthly meetings. The following functions have been identified:

1. Program of Activity neview and planning

2. Meeting agenda planning and special program development.

3. Activity budget approvals, purchasing and bill payment processing.

4. Review/approve news media relations activities.

5. Review/approve FFA reports, award and degree applications, etc.

6. Review officer applications; conduct screening and election process.

7. Review County Fair and other participation eligibility requirements.

8. Review Chapter representative/delegate selection process for local, section, region and state activities.

9. Act as a special problem-solving team when necessary.

10. Writing of "Thank You" letters not done by others.

\section{Member Activity Development Division}

- Program Areas-Leadership, Scholarship, Member Development, Alumni, SAEP, and Agricultural Career Skills.

- Officer-Advisors-President and Vice President

The purpose of this division is to encourage Chapter members to build their individual leadership skills and to provide life skills development activities which will improve the whole student. To achieve this purpose the following activity descriptions have been identified:

1. Leadership activities that help the individual develop technical, human relations and decision making leadership skills to enhance personal success.

2. Healthy lifestyle activities that promote the well being and self-esteem of the student either mentally or physically.

3. Supervised agriculture experience activities that promote student involvement and growth through agriculturally related experience and/or entrepreneurship.

4. Scholarship activities that develop an attitude for life long learning experience.

5. Agricultural career skills activities that develop agricultural occupation and career skills through a progressive learning environment.

Member Division Activities to Conduct

1. Leadership Development contests-Creed speaking, Parli-pro, public speaking, BIG, Open/Closing ceremonies, marketing job interview and specialized skills development contests.

2. Organize Chapter Project Competition judging and awards program.

3. Member involvement in section, region, state and national conventions/meetings/leadership conferences, including finances.

4. Coordinate Leadership and Scholarship grade level awards.

5. SAEP proficiency awards-application and selection process.

6. Hold and judge SAEP record book contest for members.

7. Distribute Chapter FFA Degree applications and conduct ceremony.

8. Do Ag Calendar Sales fundraiser and ordering.

9. Scholarship application distribution and Ag. Booster Scholarship.

10. Get Alumni member involvement as judges, class speakers, etc.

11. Construct SAEP ID/visitation wall chart.

12. Parent-Member awards banquet program organizing and awards order. 


\section{Chapter Activity Development Division}

- Program Areas-State and National Activities, Cooperation, Conduct of Meetings, Recreation, Chapter Safety, Earnings and Savings, Ag.Boosters, and Recruitment.

- Officer/Advisors-Secretary and Treasurer

The purpose of this division is to encourage the chapter to provide group activities and services to its members. The activities/services provided may involve promoting increase member participation, financial responsibility, a positive image, interactions with support groups and cooperation through organized activities. To achieve this purpose the following activity descriptions have been identified:

1. Chapter recruitment activities conducted to increase the agricultural education enrollment and/or FFA membership and encourage greater participation.

2. Chapter financial activities conducted to encourage and improve thrift among its members and financial standing through earnings, savings, and investments.

3. Chapter public relations activities conducted to promote a positive image and inform students, parents, school officials, and the community of chapter and member accomplishments.

4. Chapter leadership activities conducted to develop teamwork and cooperative skills in chapter officers, committees and members.

5. Chapter support group activities conducted to develop and maintain positive relations between the FFA and parents.

\section{Chapter Division Activities to Conduct}

1. Special activities for Chapter meetings.

2. Fair equipment preparation, purchases, repairs, etc.

3. Maintain FFA dinner/banquet supply inventory.

4. Plan and conduct new member orientations and Barbeque.

5. Conduct Beef Jerky fundraising activity.

6. Distribute Greenhand Degree applications and conduct ceremony.

7. Construct member activity participation chart/point awards chart.

8. Safety promotion-organize school farm/Dept. clean-up workdays.

9. Middle school recruitment brochures and parent's night display.

10. Plan for and conduct FFA Week activities, including publicity.

11. Ag. Boosters - belp with activities as needed.

12. Organize Chapter recreation activities and barbeques.

13. Parent-Member awards banquet facilities RSVP, set-up and cleanup. 


\section{Community \& School Activity Development Division}

- Program Aneas - Community Service, Public Relations, School and Community Improvement, and Ag. Advisory Committee.

- Officer/Advisors-Reporter and Sentinel

The purpose of this division is to develop FFA partnerships with other groups and organizations to make the school and community a better place to live and work. School and community involvement can serve to improve the community's economic, environmental, and human resources. To achieve this purpose the following activity descriptions have been identified:

1. Chapter ag awareness and economic activities conducted to improve the economic welfare of the community; and to help create an awareness in order to help the public become better informed of the food system and related agricultural issues.

2. Chapter support group activities conducted to develop and maintain positive relations between the FFA, community leaders, and industry.

3. Chapter environment activities conducted to preserve natural resources and develop more environmentally responsible indrviduals.

4. Chapter public relations activities conducted to promote a positive image and inform students, parents, school officials and the community of chapter and member accomplishments.

5. Chapter citizenship activities and human resources activities conducted to promote and encourage members to become active, involved citizens of their school and community; and to improve the welfare and well-being of members and citizens of the community.

\section{Community and School Division Activities to Conduct}

1. School Farm construction and repair projects/workdays.

2. County Fair ag exhibit feature booths and educational displays.

3. Organize petting zoos for elementary schools, etc.

4. Salvation Army "bell ringing" Kettle drive.

5. ASB holiday canned food and "toys" collection drive participation.

6. Organize ag facility and school improvement projects.

7. Conduct Donkey Basketball fundraising game.

8. Maintain display area/showcase for activity pictures, etc.

9. Work with Ag. Advisory Committee on annual Spring Agribusiness tour or Calif. Farm Equipment Show trip.

10. Organize Community Service Club program presentations.

11. Conduct Firewood sales fundraiser.

12. Parent/Member awards banquet invitations and food arrangements.

\section{Use of the President's Gavel During Meetings}

Two taps of the gavel calls the meeting to order

Three taps of the gavel signals all of the members to stand.

One tap of the gavel seats members, restores order, or indicates final action on a voted item.

Series of sharp taps is also used to restore meeting order. 


\section{FFA Member's Responsibilities}

FFA is an organization of, by and for its members. For a chapter to operate effectively and to be successful, each member must take an active part. Successful member responsibilities are:

1. To attend meeting regularly and on time.

2. To participate in chapter activities, accept responsibility, and serve on committees.

3. To be able to recite the opening ceremony response when the President asks "FFA members, why are we here?"

4. To maintain school eligibility and a satisfactory scholarship record.

5. To be familiar with the Chapter Program of Activities, Constitution and have a basic knowledge of parliamentary procedure.

6. To participate in leadership skills development activities.

7. To conduct an appropriate SAEP during each year of enrollment; improving their agricultural skills, leading to advancement in FFA degrees.

8. To support the efforts of the Chapter Officers and activity Chairmen.

9. To be familiar with the aims and purposes of the FFA, emblem, symbols, colors, motto, creed, and FFA salute.

10. To follow the FFA Code of Ethics and CATA Curricular Code of Conduct; conducting themselves respectfully and practicing good sportsmanship.

11. To own an FFA jacket and know the proper use of the FFA jacket.

12. To dress appropriately for FFA activities and be familiar with the Official Uniform Dress requirements for members.

13. To take pride in the FFA and promote the industry of agriculture,

\section{Member's Preamble to FFA Meetings}

The following statement should be memorized by all FFA members and spoken, in unison, during the opening ceremonies at all FFA meetings. As an FFA member you must learn your member's part for opening ceremonies. After the President states, "Thank you. FFA members why are we here?" ( 3 taps of the gavel signals members to stand) All members stand and repeat the following preamble:

"To practice brotherhood, honor agricultural opportunities and responsibilities and develop those qualities of leadership which an FFA member should possess."

All members are seated after the President makes one tap of the gavel. 
Chapter History - Leadership and Award Recipients

North Salinas FFA Chapter No, 284

Founded January, 1960

Outstanding Agriculture Students

(Highest Honor-Perpetual School Plaque)

Freshman

1965 Harold Terry

1966 Ken Beadelston

1967 Maddy Green

1968 David Mathews

1969 Cheryl Tharp

1970 Mikel Ann Smith

1971 David Chester

1972 Vicki Taylor

1973 Janet Stubblefield

1974 Mike Davis

1975 Marie Gourley

1976 Suzanne Ammons

1977 Kristi Krites

1978 Joann Curry

1979 Georgia Carpenter

1980 Randy Dreadin

1981 Lucy Garcia

1982 Esteban Morales

1983 Bart Walker

1984 Bart Haycraft

1985 _-_._.

1986 Jimmy Kessinger

1987 Elizabeth Howell

1988 Kristine Johnson

1989 Ann Beck

1990
Sophomore

John Schoch

Harold Terry

Walter Morisoli

Leroy Mallonee

Jim Dezelle

Teryl Tharp

Mark Gourley

David Chester

Dobbie Romines

Mark Tideman

Linda Death

Mike Davis

Marie Gourley

Mike Raine

Kim Rodriguez

Sam Trotter

Lucio Chavez

Warren Kahn

Lucy Garcia

Esteban Morales

Patty Morales

Vince Ferrante

John Corea

Jimmy Kessinger

Joey Serafin

Kristie Johnson
Junior

Bill Schoch

John Schoch

Ronnie Hall

Walter Morisoli

Bob Monares

Cathy Deen

Linda Massolo

Paul Forney

David Chester

Debbie Romines

Mark Tideman

Donna Blose

Marie Gourley

Denmis Murfin

Kim Rodriguez

Sam Trotter

James Crouse

Cioriano Morales

Cara Hande1

Andrea Williams

Patty Morales

Julie Skillett

Bart Spurling

Jimmy Kessinger

Joey Serafin
Senior

Jerry Miracle

Bill Schoch

Carl Harviston

Harold Terry

Charlene Kearney

Bob Monares

Cathy Dean

Louie Grossi

Panl Forney

Bill Svetz

Debbie Romines

Mark Tideman

Kari Mocattini

Randy Witham

Nancy Stubblefield

Kristi Crites

Sam Trotter

James Crouse

Cipriano Morales

Cara Handel

Esteban Morales

Patty Morales

Julie Skillett

Bart Spurling

Johnny Gray Jr. 


\begin{tabular}{|c|c|c|c|}
\hline 1991 Kristi Abercrombie & Mike Johnson & Tracy Beck & Kristine Johnson \\
\hline 1992 Mike Harris & & Mike Johnson & \\
\hline 1993 Andy Kalstrom & Tori Walls & Shawn Zachary & Mike Johnson \\
\hline 1994 Robyn Thornton & Mike Harris & $\begin{array}{l}\text { Thea Rhodes } \\
\text { Tim Lewis }\end{array}$ & Tori Walls \\
\hline 1995 Diane Boyer & Casey Roland & - & Thea Rhodes \\
\hline 1996 Josh Ruiz & Diane Boyer & Casey Roland & Irene Hintze \\
\hline 1997 Rebecca Hunzie & Josh Ruiz & Diane Boyer & Casey Roland \\
\hline 1998 & Randa Hilton & Josh Ruiz & Diane Boyer \\
\hline 1999 Nicholas Fieguth & Katherine Gnat & Randa Hilton & Cindy Parry \\
\hline 2000 Jennifer Innocenti & Noel Hall & Katherine Gnat & Amanda Wallace \\
\hline 2001 Melisa Warren & Jennifer Innocenti & Noel Hall & Katie Gnat \\
\hline 2002 Jeremy Gattis & Sarah Schoch & Jennifer Innocenti & Jackie Page \\
\hline 2003 Matt Collins & Jeremy Gattis & Jordan Rogers & Jennifer Innocenti \\
\hline 2004 Ivan Sarabia & Matt Collins & Christina Jones & Jordan Rogers \\
\hline 2005 Leslie Wayman & & Amanda Huerta & Emieline Elias \\
\hline 2006 Javier Cortez & Stefani Faro & Kasey \& Lyn Lingbeck & Amanda Huerta \\
\hline 2007 Katrina Albano & Elizabeth Corona & Joseph Hernandez & Kasey Lingbeck \\
\hline 2008 Tyler Gibson & Katrina Albano & Elizabeth Corona & 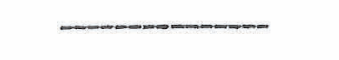 \\
\hline 2009 Cheyenne Sievers & Tyler Gibson & Katrina Albano & Elizabeth Corona \\
\hline 2010 Victoria Silva & Sydney Coakley & . & Angela Gilliam \\
\hline
\end{tabular}

\section{PAST NORTH SALINAS FFA CHAPTER PRESIDENTS \\ 1984 Lucy Garcia}

1970 Bob Monares

1971 John Cunha, Jr.

1972 David Casey

1973 Paul Forney

1974 Jim Elliat

1975 Debbie Romnes

1976 Kim Hafen

1977 Tony Chuha, Jr.

1978 Wanda Hix

1979 Mike Raine, Jr.

1980 Kim Rodriguez

1981 ??

1982 Bill Lane

1983 Lucy Garcia

$\begin{array}{ll}1985 & \text { Elizabeth Morales } \\ 1986 & \text { Patty Morales } \\ 1987 & \text { Vince Ferrante } \\ 1988 & \text { Bart Spurling } \\ 1989 & \text { Johnny Gray, Jr. } \\ 1990 & \text { William Cummings } \\ 1991 & \text { Kristine Johnson } \\ 1992 & \text { Karen Skillett } \\ 1993 & \text { Mike Johnson } \\ 1994 & \text { Mike Harris } \\ 1995 & \text { Tim Lewis } \\ 1996 & \text { Casey Roland } \\ 1997 & \text { Diane Boyer } \\ 1998 & \text { Josh Ruiz } \\ 1999 & \text { Cindy Parry }\end{array}$


2000 Morgen Roland

2000 Rebecca Hunzie

2001 Katic Gnat

2002 Jennifer Innocentie

2003 Jennifer Innocentie

2004 Christina Jones

2005 Amanda Huerta
2006 Leslie Wayman

2007 Javier Cortez

2008 Elizabeth Corona

2009 Elizabeth Corona

2010 Angela Gilliam

AMARICAN FFA DEGREE HOLDERS

(Highest Degree from National Organization)

$\begin{array}{llll}1970 & \text { Harrold Terry } & 2000 & \text { Diane Boyer } \\ 1990 & \text { Vince Ferrante } & 2001 & \text { Cindy Parry } \\ 1994 & \text { Karen Skillett } & 2002 & \text { Amanda Wallace } \\ 1997 & \text { Thea Rhodes } & 2005 & \text { Jennifer Innocentie } \\ 1999 & \text { Thea Roland } & 2007 & \text { Christina Jones } \\ 2000 & \text { Nicholas Josh Ruiz } & & \end{array}$

GOLDEN STATE FFA DEGREE HOLDERS

(Highest Degree from State Association)

$\begin{array}{ll}1969 & \text { Haruju Terry } \\ 1971 & \text { Catherine Deen } \\ 1973 & \text { John Cunha Jr., David Casey } \\ 1974 & \text { Linda Reisig } \\ 1976 & \text { Mark Tideman } \\ 1977 & \text { Carey Witham } \\ 1978 & \text { Marie Gourley, Randy Witham, } \\ & \text { Wanda Hix, Arleen Chester } \\ 1979 & \text { Kim Rodriguez } \\ 1980 & \text { Kristi Crites } \\ 1983 & \text { Lucia Garcia } \\ 1984 & \text { Linda Lane } \\ 1987 & \text { Julie Skillett, Vince Ferrante } \\ 1988 & \text { Bart Spurling } \\ 1989 & \text { Joey Serafin } \\ 1991 & \text { Tiwn Serafiß, Tracy Beck, } \\ & \text { Kristine, Johnson } \\ 1992 & \text { Karen Skillett }\end{array}$

13994 Thea Rhodes

1995 Mike Harris, Tim Lewis

1996 Casey Roland, Diane Boyer

1999 Josh Ruiz

2000 Randa Hilton, Rebecca Hunzie, Cindy Parry, Erica Schwoidt, Amanda Wallace

2001 Katie Gnat, Noel Hall

2002 Jennifer Innocenti, Jackie Page

2005 Christine Jones, Daniel Marynak

2006 Atmanda Huerta

2007 Kasey Lingbeck, Lynn Lingbeck

2008 Elizabeth Corona, Javier Cortez

2009 Celeste Celedonio, Katrina Albano

2010 Joshua Shatraw 


\section{STAR CHAPTER FFA DEGREE RECIPIENTS}

(Most Oustanding of Degee Holders)

STAR GREENHAND

1975 Lori Tharp

1976 Lori Glenn

1977 Jack Gulick

1978 Kenny Michels

1979 Georgia Carpenter

1980 Randy Dreadin

1981

1982 Esteban Morales

1983 Kurt Calender

1984 Bart Haycraft

1985 Julie Skillett

1986 Kristin Bever

1987 Joey Serafin

1988 Kristine Johnson, Timmy Serafin

1989

1990

1991 Mike Johnson

1992 Mike Harris

1993

1994 Tracy Fahey

1995 Jason Hunzie

1996 Josh Ruiz

1997 Sean Schmidt

1998 Brian Gertsch

1999 Katherine Gnat

2000 Jennifer Innocenti

2001 Sarah Schoch

2002 Jordan Rogers

2003 Kaitlin Schoch

2004 Amanda Huerta

2005 Leslie Wayman

2006 Morgana Sevans

2007 Katrina Albano

2008 Tyler Gibson

2009 Cesar Lopez

2010 Casey Medley
STAR CHAPTER

Mark Tideman

Tony Cunha, Jr.

Randy Witham

Nancy Stubblefield

Kim Rodriguez

Kim Rodriguez

Lucy Garcia

Brad Gay

Esteban Morales

Patty Morales

Julie Skillett

Jimmy Kessinger

Joey Serafin

Karen Skillett

Mike Johnson

Tori Walls

Casey Rolland

Diane Boyer

Josh Ruiz

Amanda Wallace

Rebecca Hunzie

Jennifer Innocenti

Erin Moore

Brooke Winburn

Christina Jones

Amanda Huerta

Leslie Wayman

Amanda Alvarez, Elizabeth Corona

Katrina Albano

Joshua Shatraw

Angela Gilliam
Cheri Wiggins

Nancy Villalobos
Casey Roland

Cindy Parry

Randa Hillton 


\section{MOST ACTIVE MEMBER - GOLD PIN LEVEL}

1996 Darrell Leckbee (Inspiration for award)

1997 Diane Boyer, Josh Ruiz

1998 Diane Boyer, Josh Ruiz, B. Gertsch

1999 Randa Hilton, Brian Gertsch, Cindy Perry

2000 Amanda Wallace, Noel Hall, Randa Hitton

2001 Katie Gnat, Jennifer Innocenti

2002 Jennifer Innocenti

2003 Jennifer Innocenti

2004 Ann Marie Martin

2005 Amanda Huerta

2006 Lauren Coakley

2007 Elizabeth Corona

2008 Elizabeth Corona

2009 Katrina Albano

2010 Angie Gilliam

SENIOR BANK OF AMERICA AWARD

AGRICULTURE

1972 John Cunha

1975 Kim Rogers

1976 Mark Tideman

1977 Kari Mocettini **

Andrea Knappen

1978 Arleen Chester

1979 Nancy Stubblefield

1980 Kim Rodriguez **

Kristi Crites

1982 James Crouse

1983 Bill Lane

1984 Lucia Garcia **

Cara Handel

1985 Danny Bledsaw

1986 Brenda Wiggs

1987 Julie Skillett

1988 Bart Spurling

1991 Kristine Johnson

1992 Karen Skillett

1994 Kevin Abercrombie

1995 Thea Rhodes
SENIOR AGRICULTURE DEPARTMENT

$\underline{\mathrm{KEY}}$

1972 John Cunla

1975 Debbie Romines

1976 Marc Fanning

1977 Linda Gnesa

1978 Ellen Barne

1979 Dennis Murfin

1980 Joyce Bollinger

1982 Kathy Brown

1983 Cipriano Morales

1984 Margie Ingvalson

1985 Esteban Morales

1986 Patty Morales

1987 Vince Ferrante

1988 Mark MeHaney

1991 Jennifer Silva

1992 Tracy Beck

1994 Cory Walls

1995 Tim Lewis 


$\begin{array}{llll}1996 & \begin{array}{l}\text { Irene Hintze ** } \\ \text { Randy Gillenwater }\end{array} & 1996 & \text { Irene Hintze } \\ 1997 \text { Casey Roland } & 1997 & \text { Robyn Thornton } \\ 1998 & \begin{array}{l}\text { Diane Boyer } \\ \text { Josh Ruiz }\end{array} & 1998 & \text { Amanda Peterson } \\ 1999 \text { Cindy Perry } & 1999 & \text { Darrell Leckbee } \\ 2000 & \text { Rebecca Hunzie } & 2000 & \text { Chris Fieguth } \\ & & \text { Amanda Wallace } \\ 2001 & \text { Katie Gnat } & 2001 & \text { Adam Jacop } \\ 2002 & \text { Jackie Page } & 2002 & \text { Noel Hall } \\ 2003 & \text { Jennifer Innocenti ** } & 2003 & \text { Jeff Eastin } \\ & \text { Brooke Winburn } & & \\ 2004 & \text { Jordan Rogers } & 2004 & \text { Cory Peebles } \\ 2005 & \text { Christina Jones } & 2005 & \text { Christina Jones } \\ 2006 & \text { Amanda Huerta } & 2006 & \text { Amanda Huerta } \\ 2007 & \text { Amanda Alvarez } & 2007 & \text { Kasey and Lynn Lingbeck } \\ 2008 \text { Joseph Hernandez } & 2008 & \text { Joseph Hernandez } \\ 2009 & \text { Elizabeth Corona } & 2009 & \text { Elizabeth Corona } \\ 2010 & \text { Melissa Sanchez } & 2010 & \text { Angela Gilliam } \\ \text { (*** VocEd Plaque Winner) } & & \\ & & & \end{array}$

DEKALB AGRICULTURAL ACCOMPLISHMENT AWARDS

DEKALB Genetics Corporation, Monsanto Seeds

(Perpetual School Plaque--Jr./Sr. Student)

$\begin{array}{llll}1976 & \text { James Wyrick } & 1994 & \text { Mike Harris } \\ 1977 & \text { Donna Blose } & 1995 & \text { Tim Lewis } \\ 1978 & \text { Marie Gourley } & 1996 & \text { Casey Roland } \\ 1979 & \text { Mike Raine } & 1997 & \text { Diane Boyer } \\ 1980 & \text { John Gnesa } & 1998 & \text { Josh Ruiz } \\ 1981 & \text { Kenneth Neff } & 1999 & \text { Brian Gertsch } \\ 1982 & \text { Mike Trotter } & 2000 & \text { Randa Hilton } \\ 1983 & \text { Brad Gay } & 2001 & \text { Adam Jacop } \\ 1984 & \text { Steve Garcia } & 2002 & \text { Noel Hall } \\ 1985 & \text { Danny Bledsaw } & 2003 & \text { Jennifer Innocenti } \\ 1986 & \text { Jimmie Turner } & 2005 & \text { Christina Jones } \\ 1987 & \text { Vincent Ferrante } & 2006 & \text { Amanda Huerta } \\ 1988 & \text { John Corea } & 2007 & \text { Kasey Lingbeck } \\ 1989 & \text { Joey Serafin } & 2008 & \text { Lynn Lingbeck } \\ 1990 & & 2009 & \\ 1991 & \text { Timmy Serafin } & 2010 & \text { Cheyenne Sievers } \\ 1992 & \text { Tracy Beck } & & \\ 1993 & \text { Shawn Zachary } & & \end{array}$




\section{HONORARY ADULT CHAPTER FFA MEMBERS}

Mr. Stanely Milford, Mr. Orville Helm, Mr. Albino Cotta, Mr. Robert C. Wills, Mr. William Kearney, Mrs. Sandy Terry

1970 Mr. David Weakley

1971 Mr.James Shinn and Mrs. Eve Matuszek

1972 Mr. Wayne McDaniel and Mr. G. Deleissegues

1973 NSHS Custodial Staff

1974 Mrs. Pat Parker and Mrs. Joyce Tharp

1975 Mr. Richard Everly and Mrs. E. Tomblinson

1976 Mr. Malcolm Young and Mr. David Meyers

1977 Mr. Don Barnes and Mr. Richard Gourley

1978 Mr. John Clemenger and Mr. Frank Hix

1979 Mr. Gary Sampson and Mrs. Margaret Noroian

1980 Mrs. Lorriane Rodriguez and Mrs. S. Crites

1981 Mr. Sam Trotter and Mr. Doug Forbord

1982 Mr. G.H. Viales and Mr. Stanley Uchiyama

1983 Mr. Roy Miller and Mrs. Carolyn Barnes

1984 Mr. Lionell Handel (MB FFA Section-1996)

1985 Mr. Ken Westall

1986 Mr. Tony Parker and Mr. Jerry Skillett (MB FFA Section-1991)

1987 Mr. Bob Boteilho

1988 Mr. Tony Singh and Mrs. Millie Kesinger

1989 Mrs. Diane Johnson and Mrs. Lavina Cummings

1991 Mrs. Syble Kinzer

1992 Mr. Dick Johnson and Mr. Dan Beck

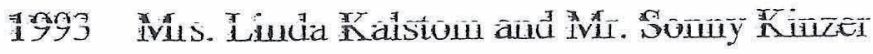

1994 Mr. tom Collier

1996 Mr. Paul Kohatsu

1997 Mrs. Kathy Boyer and Mrs. Vikki Krebbs

1998 Mrs. Michelle Roland and Mr. Steven Luis

1999 Mrs. Jill Cahoon and Mr. Curtis Parry

2000 Mr. Jerry Skillett (FFA Section level), Mr. John Favaro and Mr. Gary Peterson

2001 Mrs. Jami Wallace and Mrs. Mrs. Laurie Rogers

2002 Mr. Rick Winburn, Mrs. Claudia Innocenti, and Mr. Gary Peterson

2003 Mrs. Mary Winburn and Mr. Dale Wallace

2004 Mrs. Mary McNeese

2005 Mr. Albert Uribe and Mrs. Rhonda Wallace

2006 Miss Janelle Smith and Miss Cecilia Ritterbush

2007 Miss Markie Severtson, Mr. Alfredo Corona, and Mrs Margaret Corona

2008 Mr. Tom Collier (MB Section Blue and Gold Award), Mr. Ken Faro, Mrs. Lisa Faro, Mrs. April Lingbeck, and Mr. August Caresani

2009 Mr. Tom Jones and Mrs. Stephanie Blodgett

2010 Mr. Josh Ruiz 


\section{ADULT OUTSTANDING FFA SERVICE PLAQUE}

( 5 or More Years of Volunteer Service)

1978 Mr. Frank Hix

1979 Mr. Laxxy Hirahara, Mr. Fred Hooker III

1980 Mr. Donald Barnes

1981 Mrs. Leona Mathieson

1983 Mrs. Louise Sitva

1986 Mrs. Carolyn Barnes

1988 Mr. G. H. Viales

1991 Mrs. Lavina Cummings

1992 Mr. Jerry Skillett
1995 Mr. Lionell Handel

1996 Mr. Roy Miller

2002 Mr. Paul Kohatsu

2003 Mrs. Laurie Rogers

2005 Mr. Dale Wallace, Mrs. Claudia Imocenti Mrs. Rhonda Wallace

2007 Mr. Tom Collier

2010 Mr. Ken Faro and Mrs. Lisa Faro

IMM PHLLIPS MEMORIAL SCHOLARSHIP WINNERS

(Founder and First Ag. Booster President, 1970)

$\begin{array}{ll}1973 & \text { John Cunha Jr. } \\ 1974 & \text { Linda Reisig } \\ 1975 & \text { Kim Rodgers } \\ 1976 & \text { Carey Witham } \\ 1977 & \text { Kari Mocettini } \\ 1978 & \text { Wanda Hix } \\ 1980 & \text { John Gnesa } \\ 1981 & \text { Regina Bronson } \\ 1982 & \text { Kathy Brown } \\ 1983 & \text { Cipriano Morales } \\ 1984 & \text { Lucia Garcia } \\ 1985 & \text { Esteban Morales and Etta Phillips } \\ 1986 & \text { Jimmie Turner } \\ 1987 & \text { Julie Skillett and Vincent Ferrante } \\ 1988 & \text { Mark McHaney } \\ 1990 & \text { Elizabeth Howell } \\ 1991 & \text { Kristine Johnson } \\ 1992 & \text { Tracy Beck and Karen Skillett } \\ 1993 & \text { Mike Johnson } \\ 1994 & \text { Tori Walls and Kevin Abercrombie } \\ 1995 & \text { Thea Rhodes and Tim Lewis } \\ 1998 & \text { Diane Boyer } \\ 1999 & \text { Cindy Parry } \\ 2010 & \text { Angela Gilliam }\end{array}$


FFA ADVISORS/DEPARTMENT STAFF

$$
\text { ( }+ \text { Program Managers) }
$$

\begin{tabular}{|c|c|}
\hline $1960-1962$ & Mr. Murl Craft ++ \\
\hline $1960-1965$ & Mr. Don Mullen ++ \\
\hline $1965-1968$ & Mr. Orville Helm ++ \\
\hline $1966-1967$ & Mr. Ralph Loya \\
\hline $9968-1971$ & Mr. H. Wayne McDaniel \\
\hline $1968-1975$ & Mr. Stan Tharp ++ \\
\hline $1970-1973$. & Mr. Hank Eaton \\
\hline $1971-1976$ & Mrolown Cemenger- ROP \\
\hline 1975-1981 & Mr. Joe Kiely \\
\hline $1976-1977$ & Ms. Penny Griffin \\
\hline $1976-1991$ & Ms. Margaret Piffero/Noroian- ROP \\
\hline $1981-1989$ & Mr. Tony Parker \\
\hline $1989-1990$ & M. Ir.Ted Meyenberg \\
\hline \multirow[t]{2}{*}{$2001-2003$} & Mrs. Annemarie Sullivan \\
\hline & Current \\
\hline 1970-Present & Mr. Stanley Uchiyama ++ \\
\hline 2007-Present & Mr. Tom Jones \\
\hline 2008-Present & Mrs. Stephanie Blodgett \\
\hline 2010 Present & Mús. Margaret Noroian \\
\hline
\end{tabular}




\section{0-2011 FFA Chapter Activity Budget}

Area and Activity Description

Previous Year's Balance Forward (7/1/10)

Expense

Income

\# 190-FFA Gen, \#195-FFA Foundation, \#180 School Farm, \#185- FFA EnterpriseProjects

Chapter Activities- General

$\$ 7,617.11$

Special Meeting Refreshments - 4 Mtgs x $\$ 40$

Officer and Chapter Operations Materials and Supplies

Chapter Leadership Awards - Creed, Speaking, BIG

CDE Judging Skills Team Pins

New Member Barbecue - Food and Supplies

Greenhand and Chapter Degree Ceremony - Food and Supplies

Officer, Greenhand, and Chapter FFA Degree Pins

Scrapbook - Supplies, Film Purchase and Development

Student Recruitment and Display Materials

$\mathrm{Ag}$ Calendar and Cookbook Sales

FFA Week Activities - Promotion Materials, Pizza Lunch

FFA Casual Ag Jackets - 20 × 65

FFA Banquet Awards - Pins, Certificates, Plaques, Ribbons

FFA Banquet - Food, Supplies, and Decorations

Officer Training - POA, Planning Trips, Meetings

ervised Agricultural Experiences Program

Equipment Purchase, Repairs, Blade Sharpening, Etc

Livestock Medical and Show Supply Purchase, Sales

Record Book Contest Awards

School Farm Repairs/Construction/Fencing Supply

Farm Livestock Rent, Work Fines, Pen Cleaning Fines, etc

Chapter Project Competition-Awards, Food, Supplies

Fairs and Shows

Livestock Exhibit Decorations, Signs, Plants, Etc

Cleaning Equipment - Brooms, Shovels, Rakes, Forks, Etc

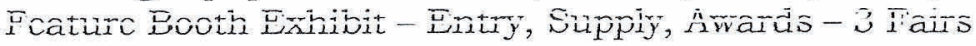

Livestock Education Displays - Supply and Awards-MCF, SCCF,

Scrapbook - Entry, Supply, Awards - SVF and MCF

Clean Pen Premium Awards - MCF, SCCF

Livestock Judging Contest - MCF and SBCF

160

100

D

50

80

60

80

150

150

400

75

600

200

1300

600

600

250

500

400

50

600

100

400

250

250

75

60

B

D

S

S

S

B

$B$

S

D-B

$S$

B

D-B

B

D

D

$\mathrm{D}$

D

60

75

Section and Region FFA Activities

Delegate Expenses to Section Meetings - 2 Del x $2 \mathrm{Mtg}$

Section FFA Trophy Fund Assessment

Fall Region Officer Leadership Conf-7 Members@, \$50

Region Spring Meeting - Officers and Members $8 @ \$ 10$

Greenhand Conference-12 Members@\$30

FFA Excellence and Adv LDR Conf - 11 Members@ @100

State and National FFA Activities

State Leadership Conf - 2 Delegates - Reg and Meals

State FFA Judging Finals Entry Fees - 3 Teams x $\$ 20$

FFA Membership Packet for Graduates - 6 Members@ @8.50

National Convention Trip - Student Sponsorship

Total

Balance Forward to 2011-2012

$\$ 6,136.11$

Key: Matching/Income Sources; S (Students), B (Ag Boosters), D ( Ag Department)

Activity expense/income not labeled or exceeds income are Chapter funds 


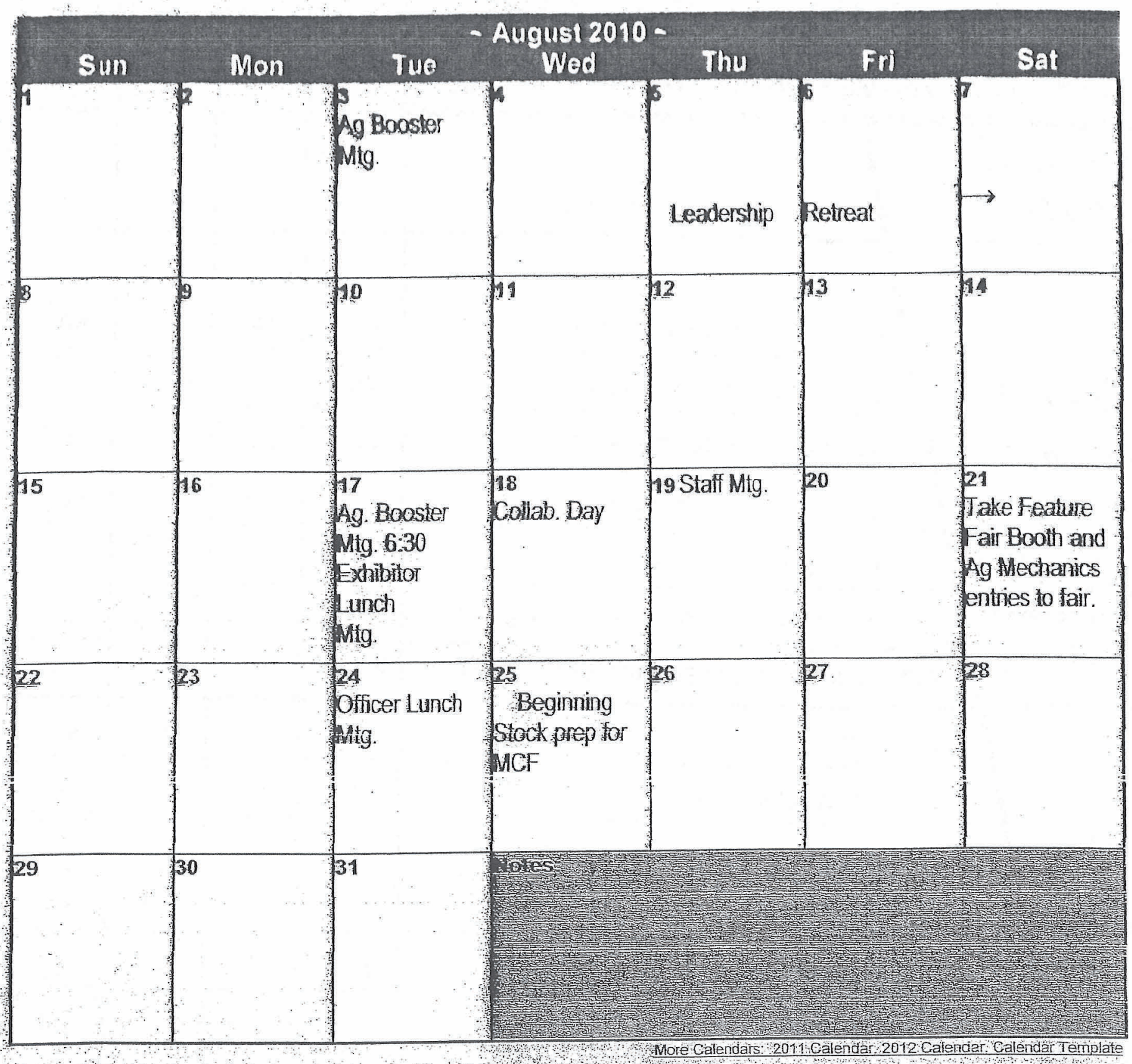


Precedence of Miotions For Parli-Pro A motion. lower on the chart is out of order, if a motion above it is being considered. main motions have the lowest precedence.

\section{Privileged motions}

1. QUESTICN OF PRIVILACE

2. POINT Of INECIMATION

Incidental

3. PAPLIAYENTAPY INQUIRY motions

Have No Iank of precedence anong thenselves. but tare. precedence over subsiliaty xotions.

4. DIVISION GF THE HOUSE

5. APFEAI DECISION CF THE CHAIP

6. POINT CF OPDWR

7. HITHDPA (withcut objection)

OBJECTICN TO AN ACCEPTAYCE OI WITHIRAYAL

7. IAY CN THE TABID

8. POEVICUS QUESICN

Sutsidiary Motions:

9. FCSTPOAE DEFINITELY

10. PES TO A COMUITTEE

11. ANDDMENT TO AN AMENDMEIT

12. AMENDMEMT

13. POSTFCNE INDEEINITELY

14. MAIN MOTIONS (a.11)

main

a. TAKE FPOM THE TABIE

b. TC RECCNSIDES (roored by provaling side) YTS

$\begin{array}{ll}2 & 0 \\ 8 & 0 \\ 2 & 8 \\ a & 0\end{array}$

$\frac{5}{4}$

YES

NO

\begin{tabular}{|c|c|c|c|c|}
\hline YES & NO & No & No & NCNE \\
\hline YES & NO & No & NO & NCNE \\
\hline YES & No & Xo & No & NCNE \\
\hline YES & NO & NO & NO & NCNE \\
\hline$Y \cong S$ & $Y \equiv \bar{S}$ & Y:S & NO & MAJ. \\
\hline YES & No & NO & No & MONE \\
\hline$Y \equiv S$ & NO & NO & NO & NON \\
\hline NO & No & No & NO & MAJ \\
\hline NO & YES & NO & NO & $\mathrm{MAJ}$ \\
\hline No & YES & No & $\mathrm{NO}$ & $2 / 3$ \\
\hline NO & $Y \mp S$ & $Y \geq=$ & $Y Z S$ & MAI \\
\hline
\end{tabular}

\begin{tabular}{|c|c|c|c|c|}
\hline YES & NO & No & No & NCNE \\
\hline YES & NO & No & NO & NCNE \\
\hline YES & No & Xo & No & NCNE \\
\hline YES & NO & NO & NO & NCNE \\
\hline$Y \cong S$ & $Y \equiv \bar{S}$ & Y:S & NO & MAJ. \\
\hline YES & No & NO & No & MONE \\
\hline$Y \equiv S$ & NO & NO & NO & NON \\
\hline NO & No & No & NO & MAJ \\
\hline NO & YES & NO & NO & $\mathrm{MAJ}$ \\
\hline No & YES & No & $\mathrm{NO}$ & $2 / 3$ \\
\hline NO & $Y \mp S$ & $Y \geq=$ & $Y Z S$ & MAI \\
\hline
\end{tabular}

\begin{tabular}{|c|c|c|c|c|}
\hline YES & NO & No & No & NCNE \\
\hline YES & NO & No & NO & NCNE \\
\hline YES & No & Xo & No & NCNE \\
\hline YES & NO & NO & NO & NCNE \\
\hline$Y \cong S$ & $Y \equiv \bar{S}$ & Y:S & NO & MAJ. \\
\hline YES & No & NO & No & MONE \\
\hline$Y \equiv S$ & NO & NO & NO & NON \\
\hline NO & No & No & NO & MAJ \\
\hline NO & YES & NO & NO & $\mathrm{MAJ}$ \\
\hline No & YES & No & $\mathrm{NO}$ & $2 / 3$ \\
\hline NO & $Y \mp S$ & $Y \geq=$ & $Y Z S$ & MAI \\
\hline
\end{tabular}

YES

\begin{tabular}{|c|c|c|c|c|}
\hline YES & NO & No & No & NCNE \\
\hline YES & NO & No & NO & NCNE \\
\hline YES & No & Xo & No & NCNE \\
\hline YES & NO & NO & NO & NCNE \\
\hline$Y \cong S$ & $Y \equiv \bar{S}$ & Y:S & NO & MAJ. \\
\hline YES & No & NO & No & MONE \\
\hline$Y \equiv S$ & NO & NO & NO & NON \\
\hline NO & No & No & NO & MAJ \\
\hline NO & YES & NO & NO & $\mathrm{MAJ}$ \\
\hline No & YES & No & $\mathrm{NO}$ & $2 / 3$ \\
\hline NO & $Y \mp S$ & $Y \geq=$ & $Y Z S$ & MAI \\
\hline
\end{tabular}

\begin{tabular}{|c|c|c|c|c|}
\hline YES & NO & No & No & NCNE \\
\hline YES & NO & No & NO & NCNE \\
\hline YES & No & Xo & No & NCNE \\
\hline YES & NO & NO & NO & NCNE \\
\hline$Y \cong S$ & $Y \equiv \bar{S}$ & Y:S & NO & MAJ. \\
\hline YES & No & NO & No & MONE \\
\hline$Y \equiv S$ & NO & NO & NO & NON \\
\hline NO & No & No & NO & MAJ \\
\hline NO & YES & NO & NO & $\mathrm{MAJ}$ \\
\hline No & YES & No & $\mathrm{NO}$ & $2 / 3$ \\
\hline NO & $Y \mp S$ & $Y \geq=$ & $Y Z S$ & MAI \\
\hline
\end{tabular}

\begin{tabular}{|c|c|c|c|c|}
\hline YES & NO & No & No & NCNE \\
\hline YES & NO & No & NO & NCNE \\
\hline YES & No & Xo & No & NCNE \\
\hline YES & NO & NO & NO & NCNE \\
\hline$Y \cong S$ & $Y \equiv \bar{S}$ & Y:S & NO & MAJ. \\
\hline YES & No & NO & No & MONE \\
\hline$Y \equiv S$ & NO & NO & NO & NON \\
\hline NO & No & No & NO & MAJ \\
\hline NO & YES & NO & NO & $\mathrm{MAJ}$ \\
\hline No & YES & No & $\mathrm{NO}$ & $2 / 3$ \\
\hline NO & $Y \mp S$ & $Y \geq=$ & $Y Z S$ & MAI \\
\hline
\end{tabular}

\begin{tabular}{|c|c|c|c|c|}
\hline YES & NO & No & No & NCNE \\
\hline YES & NO & No & NO & NCNE \\
\hline YES & No & Xo & No & NCNE \\
\hline YES & NO & NO & NO & NCNE \\
\hline$Y \cong S$ & $Y \equiv \bar{S}$ & Y:S & NO & MAJ. \\
\hline YES & No & NO & No & MONE \\
\hline$Y \equiv S$ & NO & NO & NO & NON \\
\hline NO & No & No & NO & MAJ \\
\hline NO & YES & NO & NO & $\mathrm{MAJ}$ \\
\hline No & YES & No & $\mathrm{NO}$ & $2 / 3$ \\
\hline NO & $Y \mp S$ & $Y \geq=$ & $Y Z S$ & MAI \\
\hline
\end{tabular}

1

\begin{tabular}{|c|c|c|c|c|}
\hline YES & NO & No & No & NCNE \\
\hline YES & NO & No & NO & NCNE \\
\hline YES & No & Xo & No & NCNE \\
\hline YES & NO & NO & NO & NCNE \\
\hline$Y \cong S$ & $Y \equiv \bar{S}$ & Y:S & NO & MAJ. \\
\hline YES & No & NO & No & MONE \\
\hline$Y \equiv S$ & NO & NO & NO & NON \\
\hline NO & No & No & NO & MAJ \\
\hline NO & YES & NO & NO & $\mathrm{MAJ}$ \\
\hline No & YES & No & $\mathrm{NO}$ & $2 / 3$ \\
\hline NO & $Y \mp S$ & $Y \geq=$ & $Y Z S$ & MAI \\
\hline
\end{tabular}

\begin{tabular}{|c|c|c|c|c|}
\hline YES & NO & No & No & NCNE \\
\hline YES & NO & No & NO & NCNE \\
\hline YES & No & Xo & No & NCNE \\
\hline YES & NO & NO & NO & NCNE \\
\hline$Y \cong S$ & $Y \equiv \bar{S}$ & Y:S & NO & MAJ. \\
\hline YES & No & NO & No & MONE \\
\hline$Y \equiv S$ & NO & NO & NO & NON \\
\hline NO & No & No & NO & MAJ \\
\hline NO & YES & NO & NO & $\mathrm{MAJ}$ \\
\hline No & YES & No & $\mathrm{NO}$ & $2 / 3$ \\
\hline NO & $Y \mp S$ & $Y \geq=$ & $Y Z S$ & MAI \\
\hline
\end{tabular}

\begin{tabular}{|c|c|c|c|c|}
\hline YES & NO & No & No & NCNE \\
\hline YES & NO & No & NO & NCNE \\
\hline YES & No & Xo & No & NCNE \\
\hline YES & NO & NO & NO & NCNE \\
\hline$Y \cong S$ & $Y \equiv \bar{S}$ & Y:S & NO & MAJ. \\
\hline YES & No & NO & No & MONE \\
\hline$Y \equiv S$ & NO & NO & NO & NON \\
\hline NO & No & No & NO & MAJ \\
\hline NO & YES & NO & NO & $\mathrm{MAJ}$ \\
\hline No & YES & No & $\mathrm{NO}$ & $2 / 3$ \\
\hline NO & $Y \mp S$ & $Y \geq=$ & $Y Z S$ & MAI \\
\hline
\end{tabular}

\begin{tabular}{|c|c|c|c|c|}
\hline YES & NO & No & No & NCNE \\
\hline YES & NO & No & NO & NCNE \\
\hline YES & No & Xo & No & NCNE \\
\hline YES & NO & NO & NO & NCNE \\
\hline$Y \cong S$ & $Y \equiv \bar{S}$ & Y:S & NO & MAJ. \\
\hline YES & No & NO & No & MONE \\
\hline$Y \equiv S$ & NO & NO & NO & NON \\
\hline NO & No & No & NO & MAJ \\
\hline NO & YES & NO & NO & $\mathrm{MAJ}$ \\
\hline No & YES & No & $\mathrm{NO}$ & $2 / 3$ \\
\hline NO & $Y \mp S$ & $Y \geq=$ & $Y Z S$ & MAI \\
\hline
\end{tabular}

\begin{tabular}{|c|c|c|c|c|}
\hline YES & NO & No & No & NCNE \\
\hline YES & NO & NO & NO & NCNE \\
\hline YES & No & NO & No & NCNE \\
\hline YES & NO & No & NO & NCNE \\
\hline$Y \nexists S$ & $Y \geq S$ & Y.S & NO & MAJ. \\
\hline YES & No & NO & No & HON: \\
\hline YES & NO & MO & MO & MON \\
\hline NO & No. & No & No & MAJ. \\
\hline NO & YES & NO & NO & NAS \\
\hline No & YES & No & $\mathrm{NO}$ & $2 / 3$ \\
\hline NO & $Y \Xi S$ & $Y=?$ & $y=3$ & MAI \\
\hline
\end{tabular}

\begin{tabular}{|c|c|c|c|c}
\hline NO & $Y E S$ & $Y \Xi$ & $Y \Xi$ & MAJ \\
\hline NO & $Y E S$ & $Y \equiv S$ & NO & MAJ \\
\hline NO & $Y E S$ & $Y \Xi S$ & $Y \Xi S$ & MAI \\
\hline NO & $Y E S$ & NO & MAU \\
\hline NO & $Y E S$ & NO & NO & MAJ \\
\hline YES & YES & YES & NO & MAJ \\
\hline
\end{tabular}




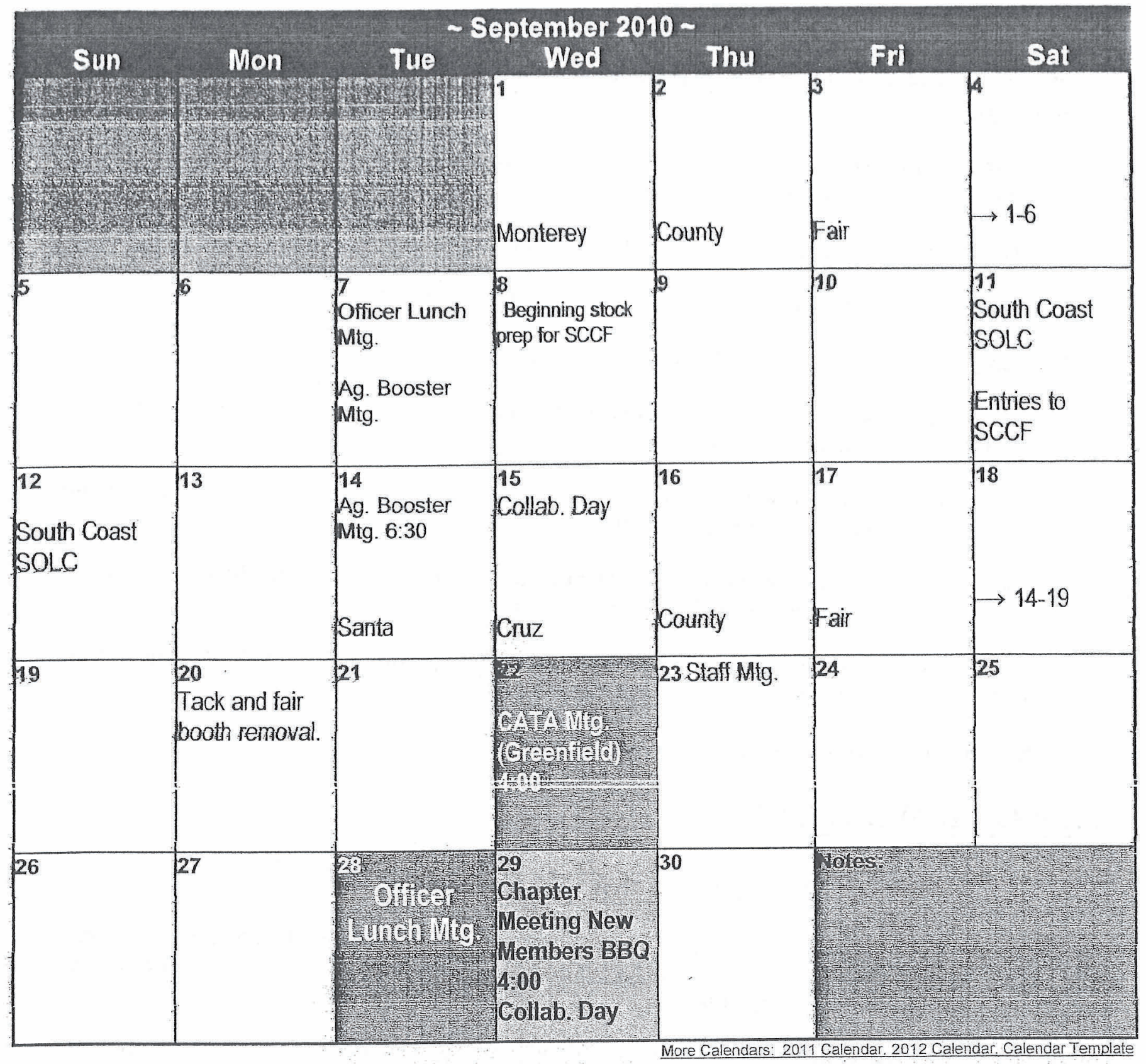




\section{When You Look Good...}

\section{The FFA Code of Ethics}

We will conduct ourselves at all times in order to be a credit to our organization, chapter, school and community by:

1. Developing my potential for premier leadership, personal growth, and career success. Make a positive difference in the lives of others.

2. Dressing neatly and appropriately for the occasion.

3. Showing respect for the rights of others and their property.

4. Being courteous, honest and fair with others at all time.

5. Communicate in an appropriate, purposeful and positive manner. Refraining from loud, boisterous talk, swearing and horseplay.

6. Demonstrating good sportsmanship in the show ring, judging contests, and meetings. Being modest in winning and generous in defeat.

7. Take pride in our organization by attending meetings promptly, respecting the opinion of others in discussion, and actively participate in activities.

8. Conduct and value a supervised agricultural expenience program; in our exhibits, and in the occupations of agriscience, farming and ranching.

9. Appreciate and promote diversity in our organization.

\section{$\ldots$ FFA Looks Great}

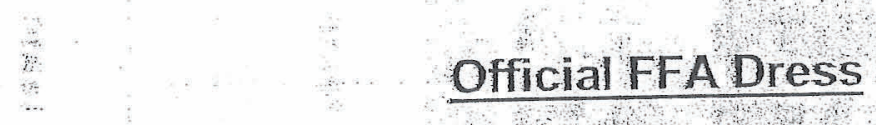

The official dress for female members is to be black knee length skirt, white blouse, official FFA scarf, black shoes, neutral color hose, and official jacket zipped to the top. Bläck slacks may be worn for outdoor activities, such as judging contests.

The official dress for male members is to be black slacks, white shit, official FFA tie, black shoes, black socks and the official jacket zipped to the top.

The official FFA show uniform shall consist of white trousers, white dress shirt or blouse (short or long sleeved) with the FFA emblem attached to the left pocket and the official FFA necktie or scarf is to be worn. The official FFA jacket is optional; if worn, the shirt emblem is not required. Hats or headgear of any kind shall not be worn with the official show uniform while showing at fairs and shows. Wearing of tennis shoes is discouraged.

The official dress uniform is to be worn at all contests and events that require official dress for participation, FFA ceremonies, public relations, events, etc. Consult with your FFA Advisor for dress modifications for certain contest and events. The WELL DRESSED FFA members ALWAYS leave others with a good impression of our organization. Wear your uniform with dignity, pride, and belief in yourself. 


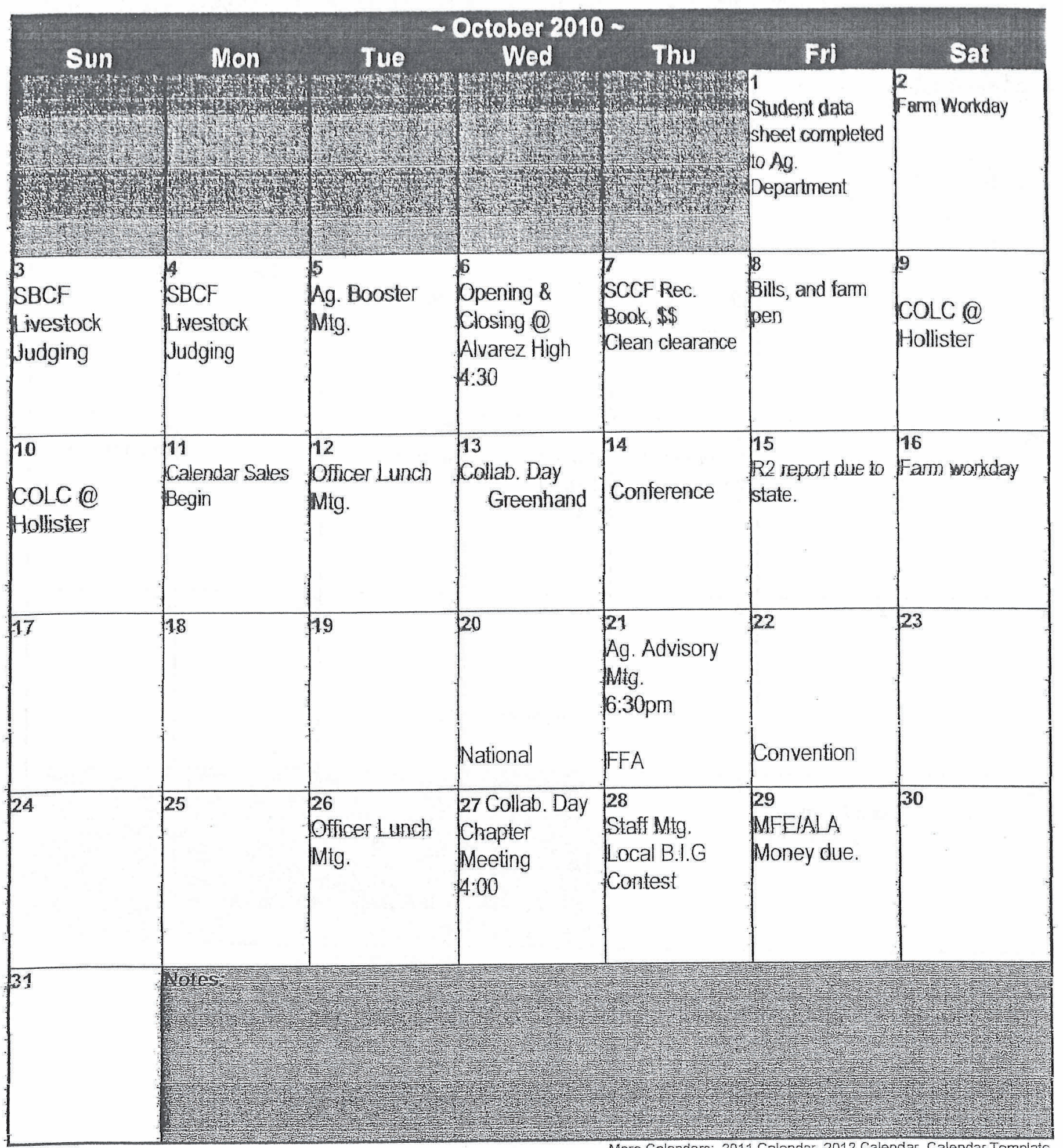

More Calendars: 2011 Calender. 2012 Calendar. Calendar Template 


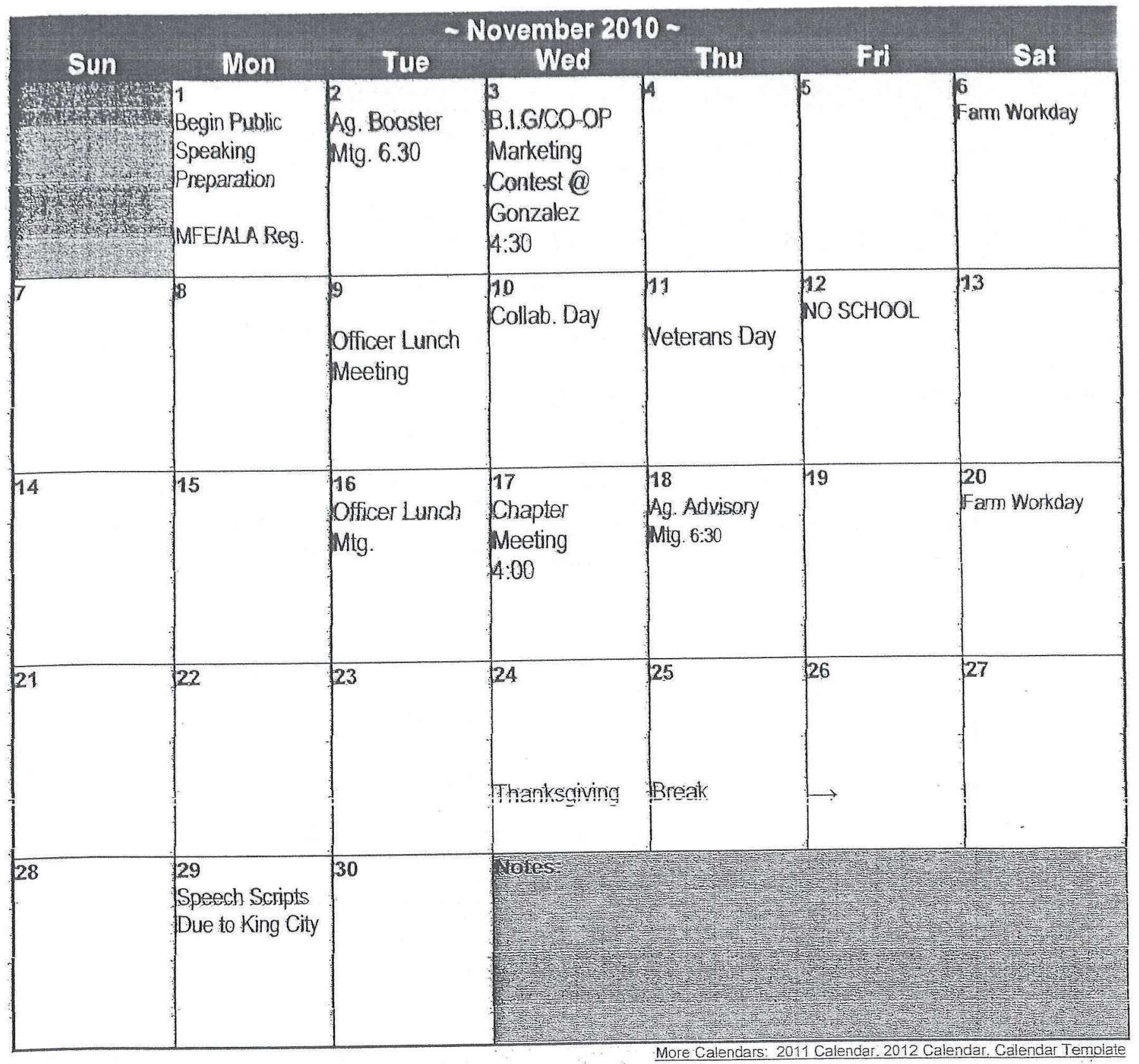




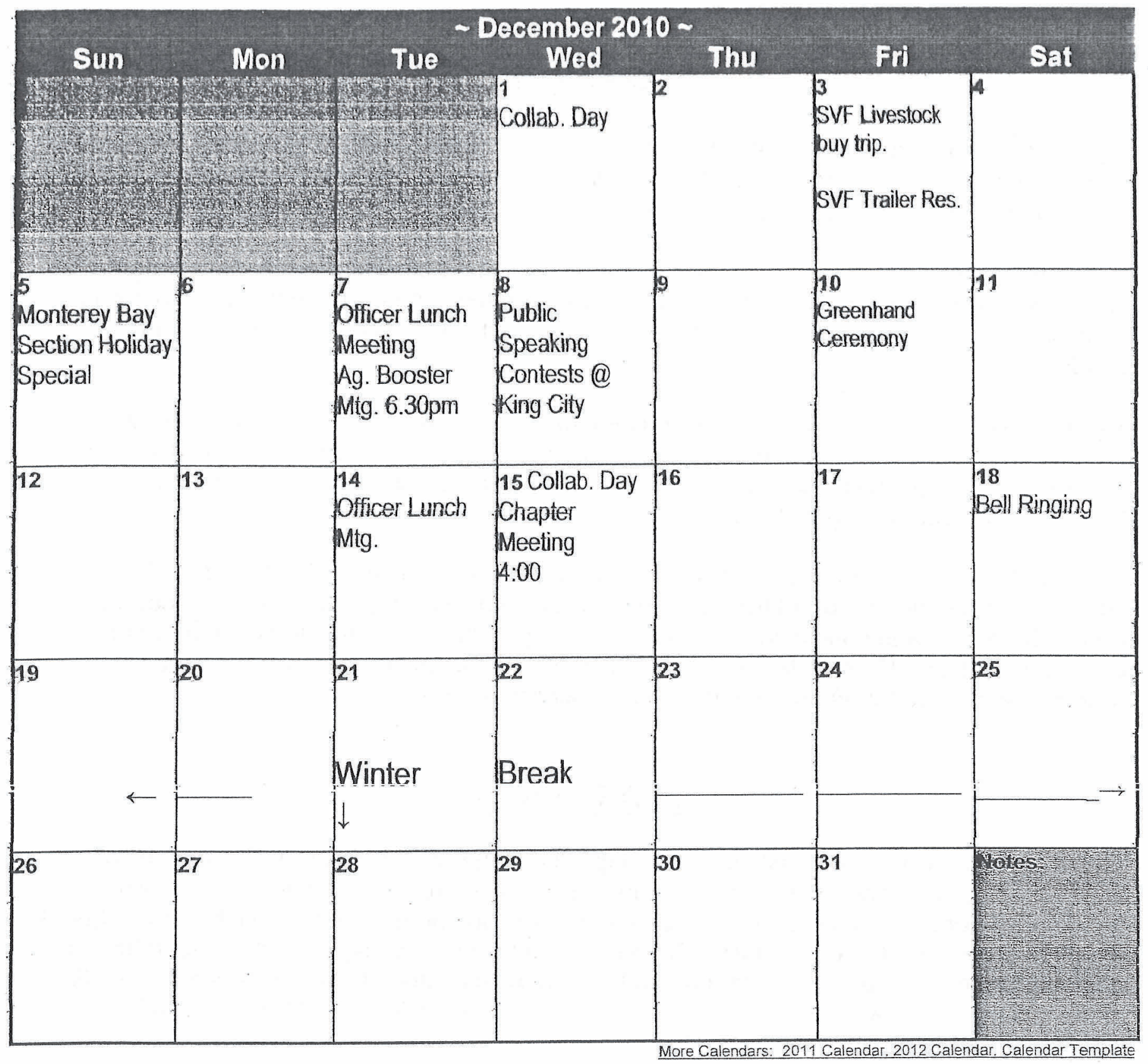




\title{
The FFA Motto
}

\author{
"Learning to do, \\ Doing to learn; \\ Earning to live, \\ Living to serve."
}

Learning to Do.... As agriculture education students in high school, FFA members study the economic and scientific principles of agriculture and learn skills needed in agribusiness, ag. production and other agricultural occupations.

Doing to Learn..... Each member is required to conduct an agricultural experience program at home or on a ranch/farm, or an agricultural work experience program, under the supervision of their agriculture teacher. The student thus gets practical experience to supplement his study.

Earning to Live.... The Supervised agricultural experience program is designed to provide income so that the FFA member may expand their agricultural enterprises as they learn, or save toward further education, to the end that they will be successfully established and become a self-supporting, substantial citizen.

Living to Serve.....Development of competent, aggressive agricultural leadership and citizenship is a primary aim of the FFA. Practical experiences in parliamentary procedure, committee work, and conducting business of an organization are provided through regular chapter meetings. Cooperation with other groups, and the assumption of community responsibilities are taught through community service activities.

\section{FFA Degrees}

There are four degrees of active membership — "Greenhand", "Chapter FFA", "State FFA", and "American FFA" degrees. These degrees of membership are contingent on definite accomplishments in connection with agriculture education programs in the high school. Local chapters determine the individual's advancement in the first two degrees of membership, the state determines the third, and the national organization confers the fourth degree. Specific levels of attainment with respect to agriculture earnings, investments, leadership, and scholarship are set up for each degree.

"The bronze pin of the Greenhand, the silver Chapter FFA pin, the golden charm of the State FFA degree, and the gold key of the American FFA degree await those whom earn them." 


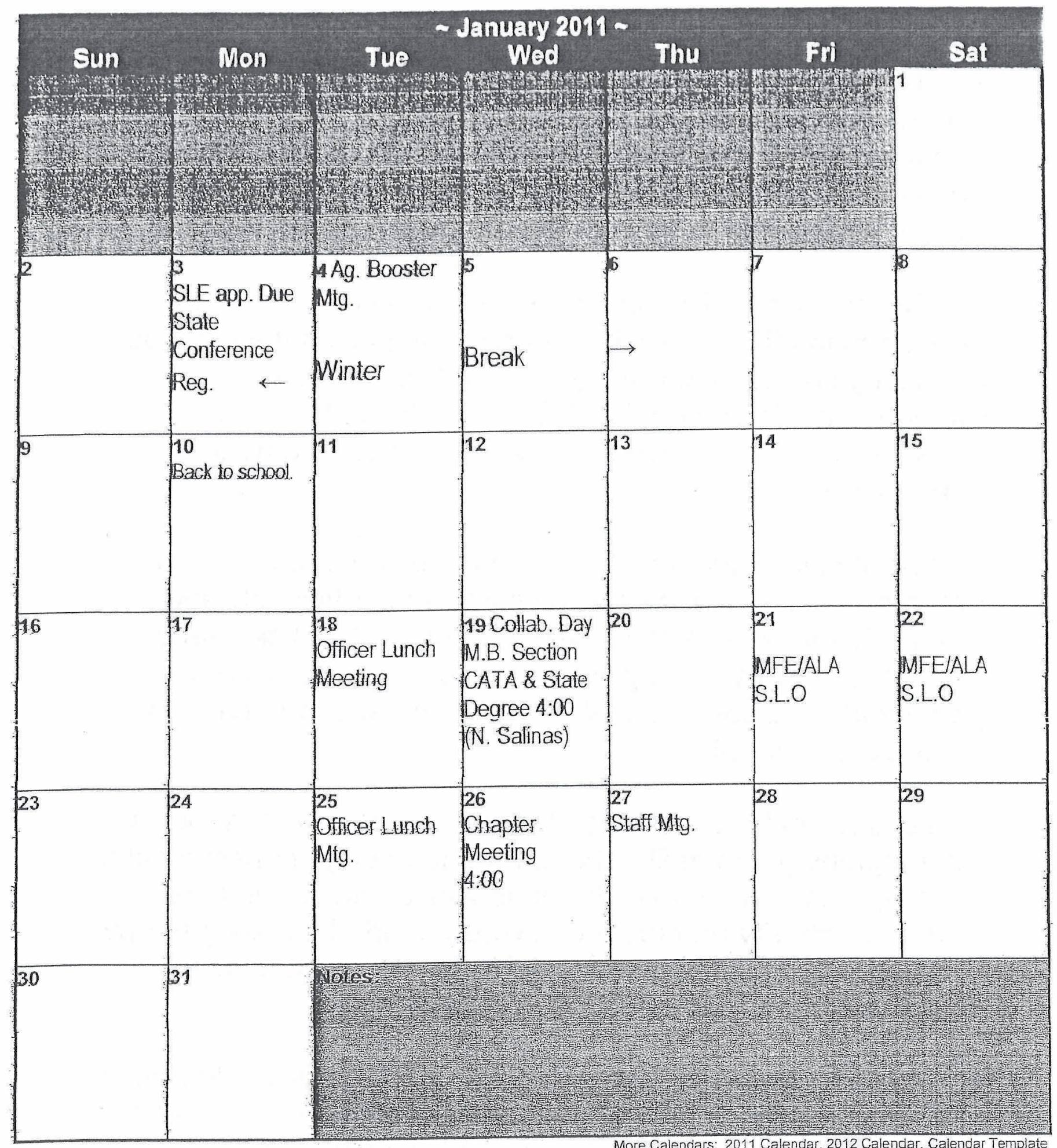

More Calendars: 2011 Calendar. 2012 Calendar. Calendar Template 


\section{The FFA Creed}

1. believe in the future of agriculture, with a faith born not of words but of deeds-achievements won by the present and past generations of agriculturalists; in the promise of better days through better ways, even as the better things we now enjoy have come to us from the struggles of former years.

I believe that to live and work on a good farm, or to be engaged in other agricultural pursuits is pleasant as well as challenging; for I know the joys and discomforts of agricultural life and hold an inborn fondness for those associations which, even in hours of discouragement, I cannot deny.

I believe in leadership from ourselves and respect from others. I believe in my own ability to work efficiently and think clearly, with such knowledge and skill as I can secure, and in the ability of progressive agriculturists to serve our own and the public interest in producing and marketing the product of our toil.

I believe in less dependence on begging and more power in bargaining; in the life abundant and enough honest wealth to help make it so-for others as well as myself; in less need for charity and more of it when needed; in being happy myself and playing square with those whose happiness depends upon me.

I believe that American agriculture can and will hold true to the best traditions of our national life and that I can exert an influence in my home and community which will stand solid for my part in that inspiring task.

The creed was written by E. M. Tiffany and adopted at the $3^{\text {rd }}$ National Convention of the FFA. It was revised at the $38^{\text {th }}$ Convention of the FFA. It was revised at the $38^{\text {th }}$ Convention and the $63^{\text {rd }}$ Convention. 


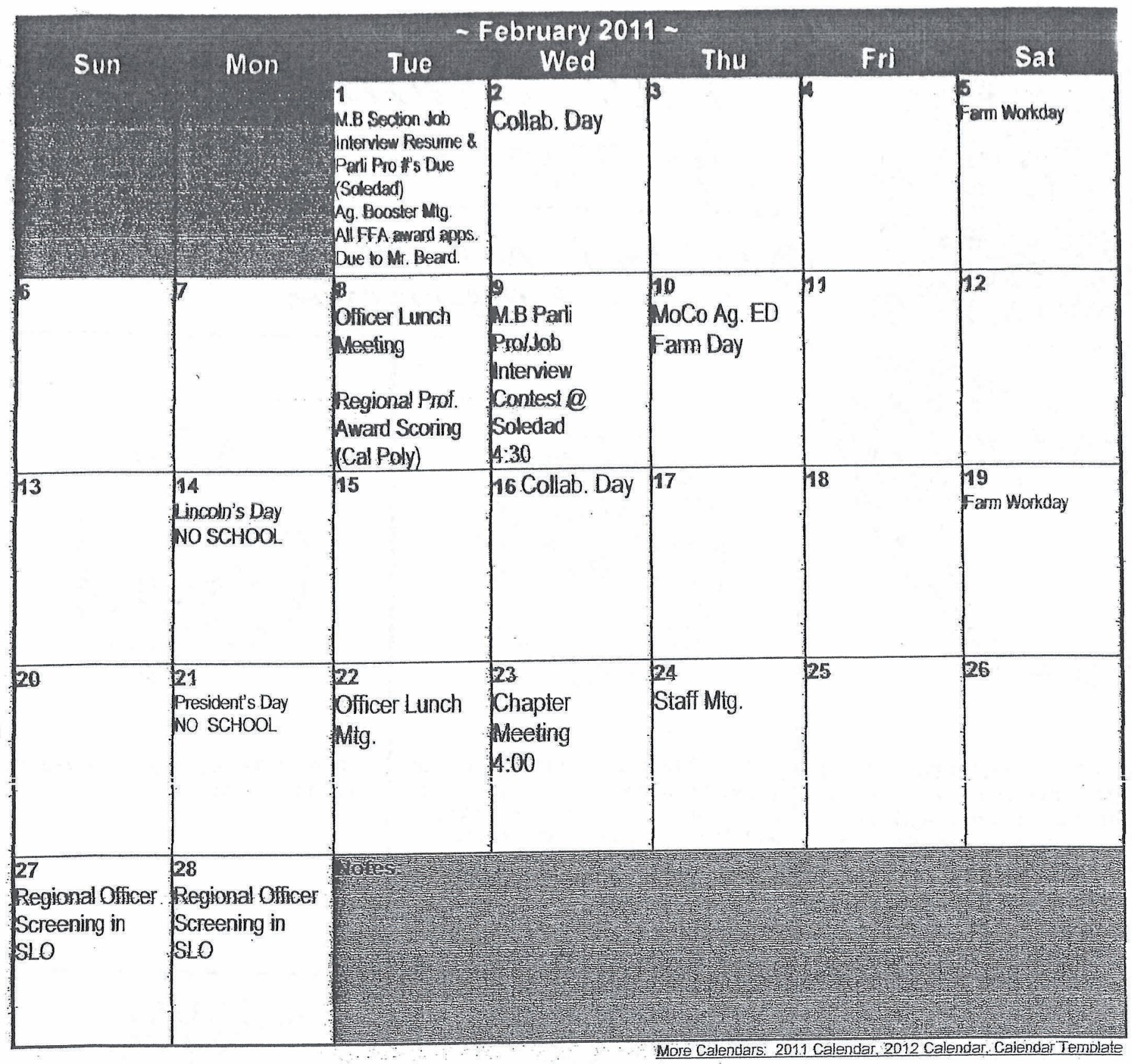




\section{FFA EMBLEM ELEMENTS}

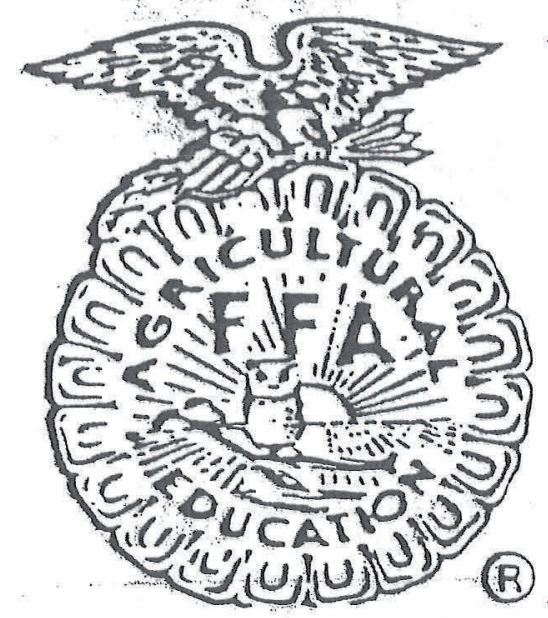

\section{Five Symbols Make up The FFA Emblem \\ (B) Emblem is a registered frademant of the FFA Organization}

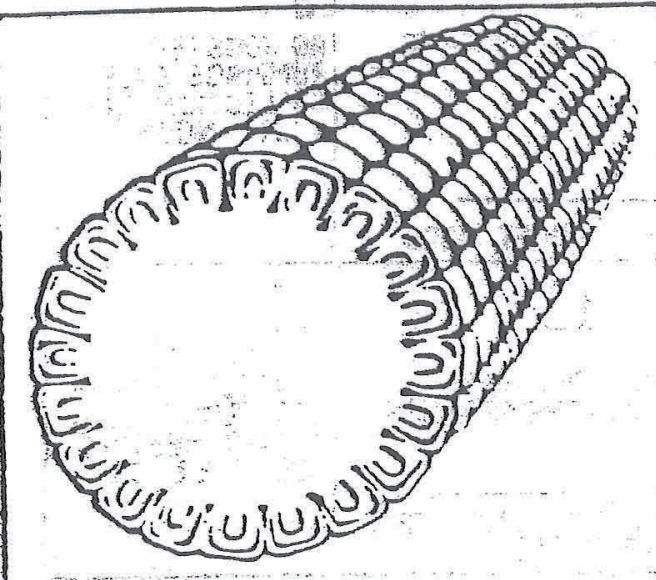

A CROSS SECTION OF AN EAR I OF COnN is the baskground. Corn rez-esents our common agricultura! inlerests, is native to Ame ica and is g-ovin in every siate.

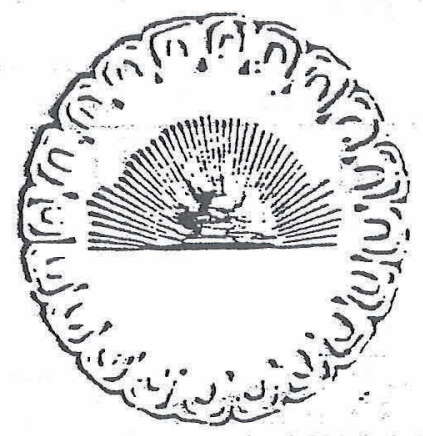

The RISING SUN, in the center of the embiem, symbolizes progress in acricullure and the conkidence that all FFA members have in the future.

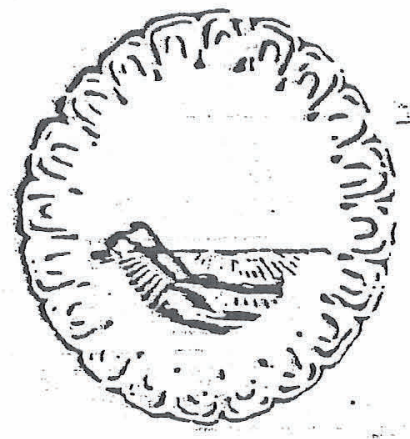

The PLOW is the symbol of labor and ziliage of the soil.

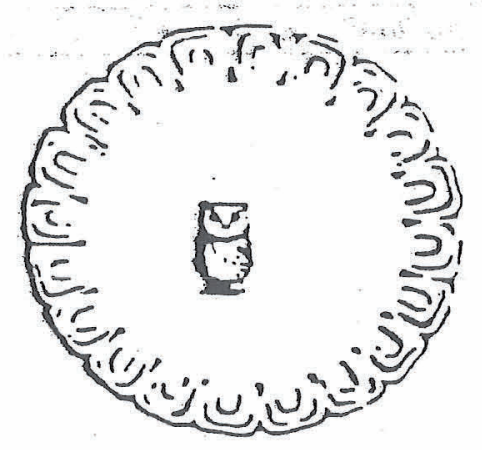

The OWL is the symbol of wistom and knowiledge.

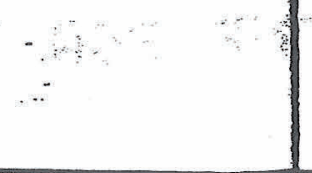

The EAGLE is symbolic of the national scope of the FFA.

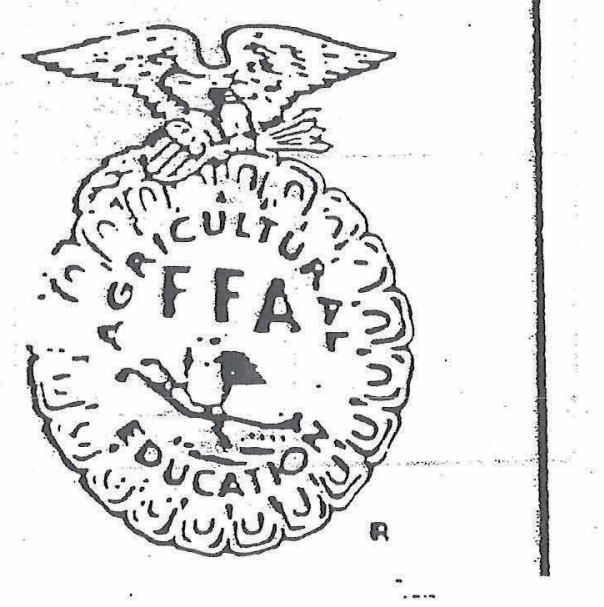

The words -AGRICULTURAL EDUCATION ${ }^{-}$surTounding the lerters "FFA" Iell us that FFA is an imporzant part of the agricutturs? education program. 


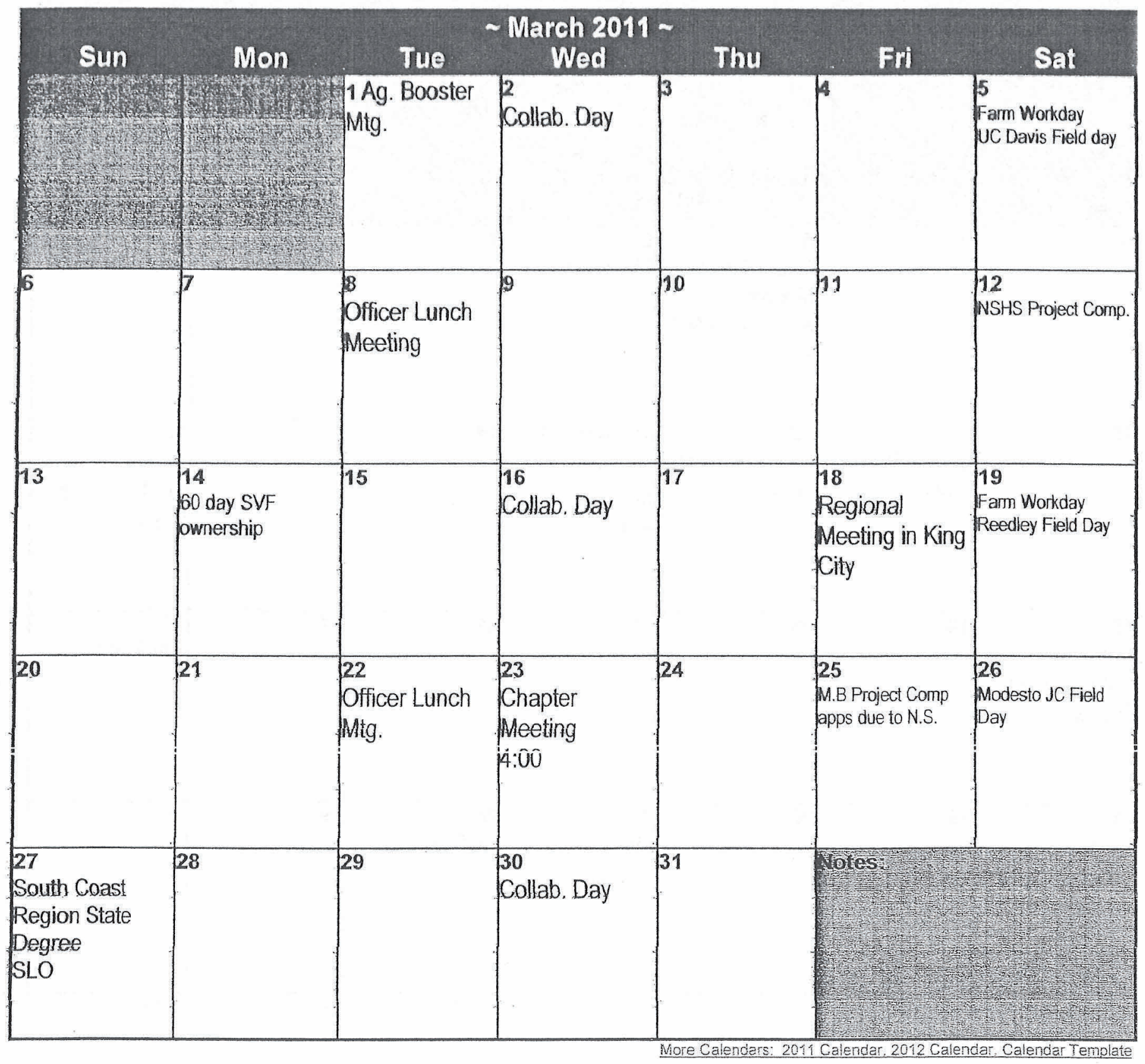




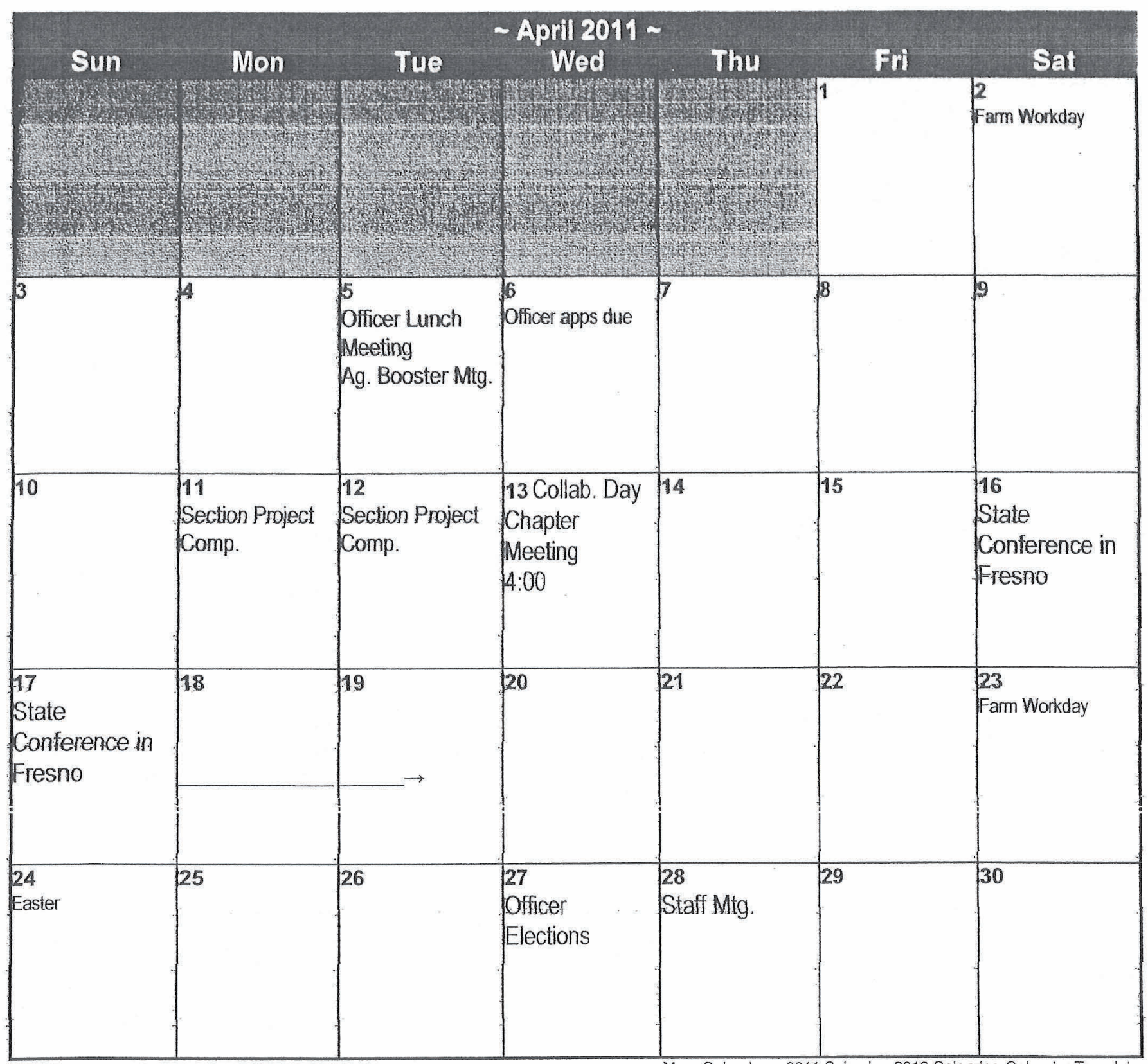

Mare Calendars: 2011 Calendar. 2012 Calendar. Calendar Template 


\begin{tabular}{|c|c|c|c|c|c|c|}
\hline Sun & Mon & Tue & $\begin{array}{c}\text { - May } 2011 . \\
\text { Wed }\end{array}$ & Thu & Fni & Sat \\
\hline SVFF Fair Prep & 2 & $\begin{array}{l}3 \\
\text { Officer Lunch } \\
\text { Meeting } \\
\text { Ag. Booster } \\
\text { Mtg. }\end{array}$ & $\begin{array}{l}4 \text { Collab. Day } \\
\text { Lunch Chapter } \\
\text { Meeting. } \\
\text { Project Comp. } \\
\text { Banquet @ } \\
\text { Marnell } \\
6: 30\end{array}$ & 5 & $\begin{array}{l}6 \\
\text { Chapter } \\
\text { Banquet } \\
6: 00 \mathrm{pm}\end{array}$ & $\begin{array}{l}7 . \\
\text { Farm Workday } \\
\text { SVF Fair Booth and } \\
\text { Ag. Mech. Setup }\end{array}$ \\
\hline 8 Trailer Setup SVF & 9 & 10 & Salinas & 12 & $\begin{array}{l}13 \\
\text { Fair }\end{array}$ & $\begin{array}{l}14 \\
\text { SVF }\end{array}$ \\
\hline $\begin{array}{l}15 \\
\text { MCF Fair buying trip }\end{array}$ & 16 & 17 & 18 Collab Day. & 19 & 20 & $\int_{\text {Farn Workday }}^{21}$ \\
\hline 22 & $\begin{array}{l}23 \\
\text { CATA MB Section } \\
\text { Planning Mig. @ } \\
\text { Salinas High. }\end{array}$ & 24 & $\begin{array}{l}25 \\
\text { Chapter Mtg. } \\
\text { lunch. }\end{array}$ & 26 & 27 & 28 \\
\hline 29 & 30 & 31 & Noles & & & \\
\hline
\end{tabular}




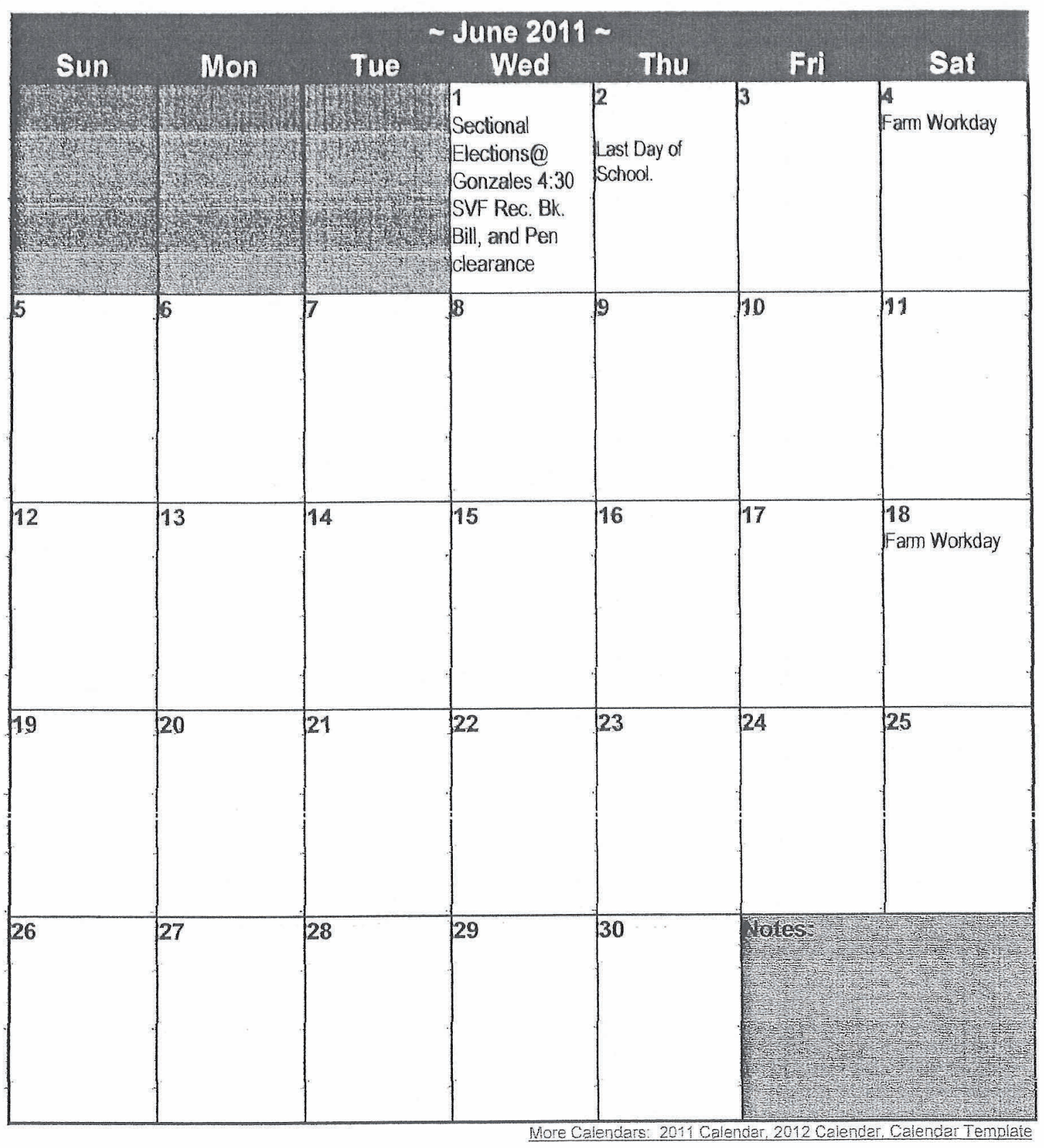




\section{CALIFORNIA ASSOCIATION FFA \\ 2010-11 \\ SOUTH COAST REGION DUE DATES}

All State Due Dates are Postmark Dates

October 1

Call in number of attendees at COLC (Hollister)/CATA Conference (805-756-2803)

October 15

Program Enrollment Data Reports (R2) Due (Department to Place On-Line)

Ag Incentive Grant Report of Expenditures Due (Department to Mail to Greg Beard)

FFA Membership Roster Due (Department to Place On-Line)

Ag Incentive Grant Graduate Follow-Up (Department to Place On-Line)

November 15

Agriculture Incentive Grant Checklist (Department to Submit to Greg Beard)

Program Plan Updates Due (Department to Submit to Greg Beard)

This includes: Five Year Equipment Acquisition Schedule, Chart of Staff Responsibilities,

FFA Program of Work, Advisory Committee Roster, and Advisory Committee minutes for the past year.

ALA Registration Due (Chapter to Mail to FFA Financial Office)

MFE Registration Due (Chapter to Mail to FFA Financial Office)

December 5

Road Show Registration Due (805-756-2803)

December 15

Agriculture Incentive Grant Checklist (Department to Submit to Greg Beard)

January (Check your Regional Calendar)

Star in Ag Placement Applications Due (Chapter to Submit at Sectional Scoring)

Star in Agribusiness Applications Due (Chapter to Submit at Sectional Scoring)

Star Farmer Applications Due (Chapter to Submit at Sectional Scoring)

․ State Degree Applications Due (Chapter to Submit at Sectional Scoring)

January 3

$\square \quad$ SLE Applications Due (Chapter to Mail to Asst. State Advisor)

January 3

State Conference Committee Chair Applications Due (Chapter to Mail to Asst. State Advisor)

February 1

Agri-Entrepreneurship Applications Due (Chapter to Mail to Greg Beard)

Agriscience Student Applicationś Due (Chapter to Mail to Greg Beard)

Agriscience Teacher Applications Due (Chapter to Mail to Greg Beard)

Hall of Chapter Applications Due (Chapter to Mail to Asst. State Advisor) 
H.O. Sargent Applications Due (Chapter to Mail to Asst. State Advisor)

National Chapter Application Due (Chapter to Mail to Asst. State Advisor)

National Scholarship Applications Due (Chapter to Mail to National FFA)

Star Administrator Applications Due (Chapter to Mail to Greg Beard)

Star Advisor Applications Due (Chapter to Mail to Greg Beard)

Star Counselor Applications Due (Chapter to Mail to Greg Beard)

Star Reporter Applications Due (Chapter to Mail to Greg Beard)

State Band Applications Due (Chapter to Mail to Asst. State Advisor)

State Choir Applications Due (Chapter to Mail to Asst. State Advisor)

State Scholarship Applications Due (Chapter to Mail to Asst. State Advisor)

State Superior Chapter Applications Due (Chapter to Mail to Asst. State Advisor)

State Talent Applications Due (Chapter to Mail to Asst. State Advisor)

State Conference Scholarship Applications Due (Chapter to Mail to Asst. State Advisor)

February 8

Proficiency Award Applications Due 9:00 a.m. to Greg Beard

February 15

State Nominating Committee Applications Due (Chapter to Mail to Greg Beard)

State Officer Applications Due (Student to Mail to Asst. State Advisor)

March 1

State Conference Registration Due (Chapter to Mail to FFA Financial Office)

April 1

State Officer Banquet Requests Due (Chapter to Mail to Asst. State Advisor)

May 17

American Degree Applications Due (Chapter to Mail to Greg Beard)

May 3

WLC Scholarship Applications Due (Chapter to Mail to Asst. State Advisor)

June 1

National Band Applications Due (Student to Mail to Asst. State Advisor)

National CDE Certification Forms Due (Chapter to Mail to Asst. State Advisor)

National Choir Applications Due (Student to Mail to Asst. State Advisor)

National Convention Delegate Applications Due (Student to Mail to Asst. State Advisor)

National Talent Applications Due (Student to Mail to Asst. State Advisor)

National Proficiency Applications Due (Chapter to Mail to Asst. State Advisor)

June 30

Ag Incentive Grant Applications Due (Department to Mail to Greg Beard)

July 1

National Convention Scholarship Applications Due (Chapter to Mail to Asst. State FFA Advisor) 


\title{
North Salinas FFA Chapter, NO. 284 \\ NORTH SALINAS HIGH SCHOOL
}

\section{CONSTITUTION AND BY-LAWS}

\author{
REVISED \\ OCTOBER, 2010 \\ SALINAS, CA
}


Faurided - Ianuary P: 1960 NOPTH GALINAE HIBH ECHODL SALINAS, EALIFUEMIA

\section{APTICLE I. NAME, AMMS, AND PURPOSE OF THE ORGANIZATIBN}

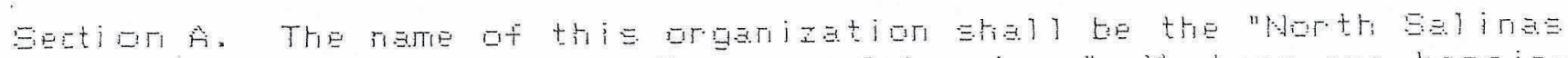
Chapter. Future Farmere of Amerieg." Member. are hereinaftr referred to as "Future Farmere df america" or the letters

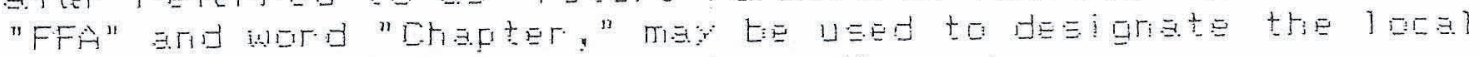
group it: ectiuitieg, or member. thereof.

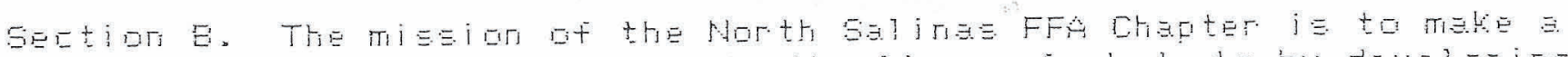

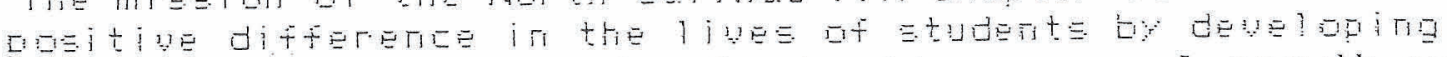
their potentiel for premier leadership personal growth and

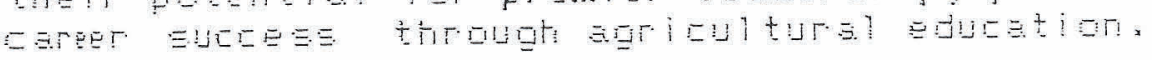

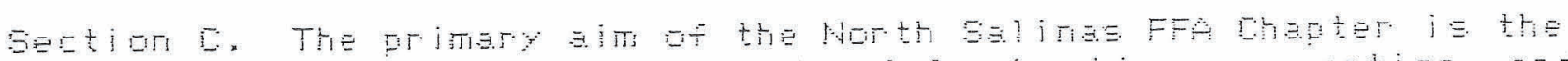

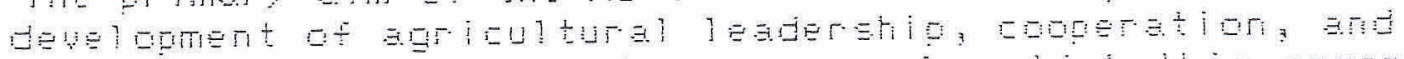

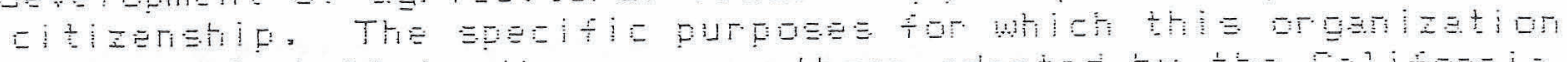

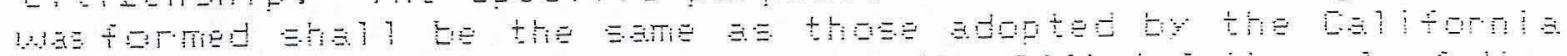

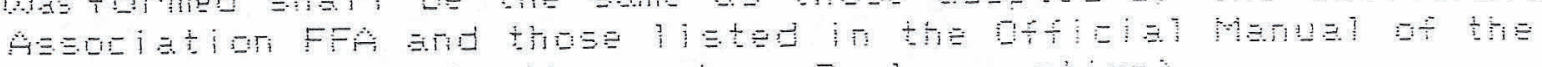

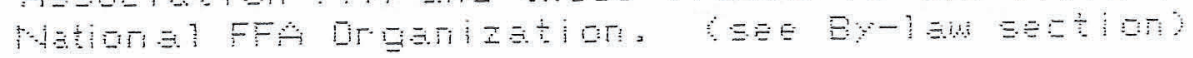

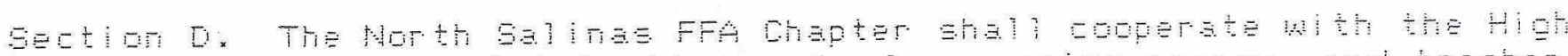

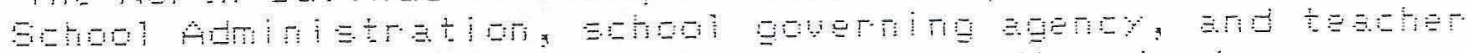

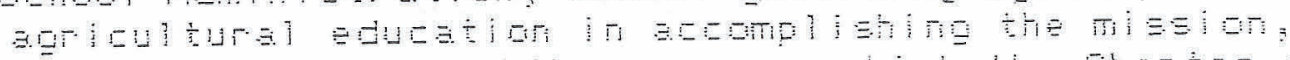

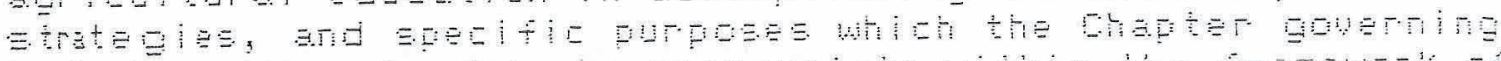

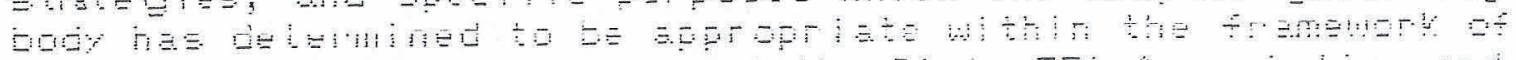

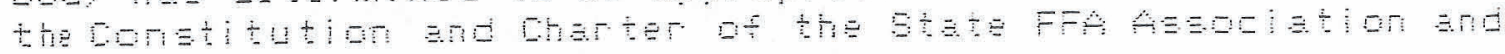
Nationa FFA Organization.

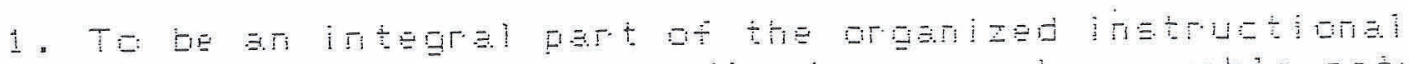

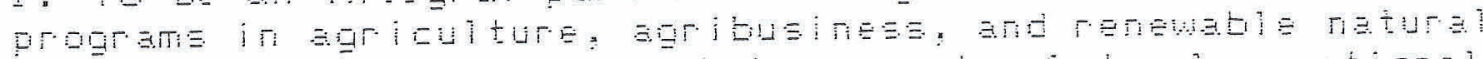

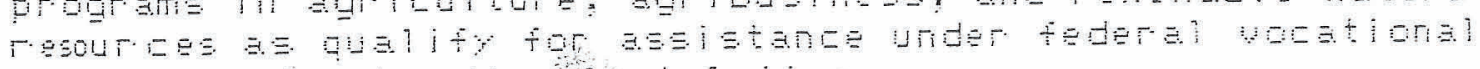

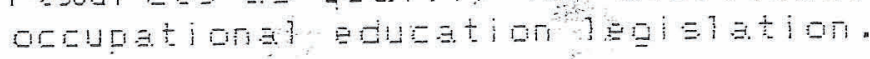

2. To build the corfidence of etudente in themeelue and their. work by developirig desirable wark hatite end the eftective use

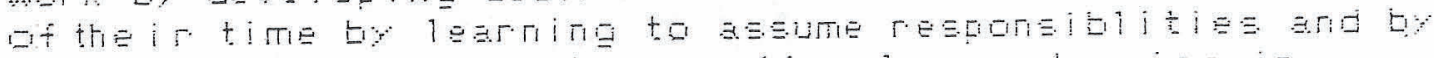
deuelopirg öreonal and oreupational competencies ir

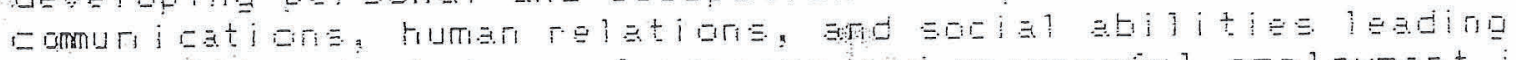
to intelligert choices of rareers and gucceseful emigloyment in Egriculture.

: To foster programe and artiuities whith will develop leadership, character, gcholarehip and occupat orial pride,

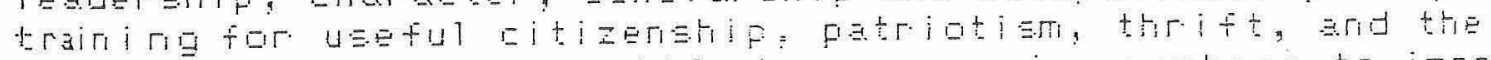
improvement in community life by encouraging miembere to improue the home and it. Eur. Qunding..

4. To recogrize indiujdual achievement in acholarehip ard agricul tural experience progreme and other indiudual achieus mente by prouiding auardj to deseruing members. 
5. To encourage members in the development of indiuidual supervised agricultural experience programs and establistiment in agriculture.

6. To develop anowledge and appreciaticn of cur agricultural heritage and to encourage member's participation ir wortry undertakinge for the improvement of agriculture.

7. To advance the cause of agricultural education in the public schools of California.

\section{ARTICLE II. DEFINITION OF AGRICULTURAL EDUCATION and SUPERUI SED AGRI CULTURE EXPERIENCE PROGRAM}

Section A. For the purposes of this Constitution, agricultural educatior stiall include agriculture programs/clasces qualifying for. reimbursement under the provisions outlined in the california State Plars for Vocational Education. The term agricultural education is syronymous with the words vocational agriculture.

Section B. For the purpose of this Constitution, Supervised Agricultural Experience Frograme shall include programs that are of a productive riature and/or practical field, laboretory, er agribusires work experience programs directed or superuised by qusi ified agriculture eduration instructor in a school

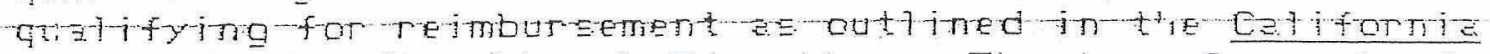
State Plar fur yacationel Educetion. The tern Superul sed Agriculturs] Experience Frogram $i s$ synonymous with the letters

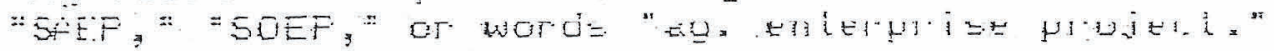

\section{ARTICLE III. ORGAHIZATION}

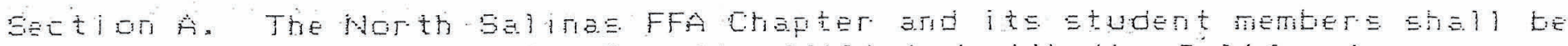
a chartered local unit affiliated with the california Aseociation FFA, which iE Effiliated with the lational FFA organization. It is orgarized on ly iria public sctuad providing programe which meet Etardarde for agriculturel education as outlined in the califorria state Plan for vecationsl Education: FFA actiuities, SAEF, and related programs shall be integral to classroom instruction.

Sectior E. The Nor th Sal inas FFA chafter ghall te iri the South CoEst FEgion FFA ard MortereY Eay SEction FFA; both geographical sutidisiens of the state and regional FFA organizations respectively.

Section C. The annual Frogram of Agtivities and cperating budget of this cliapter stial l be planned ty the elected student officers and the FFA Adwi sor. Thie Frogram of factiuities and regular work ot this chapter stall be conducted by the studerit officers, division committee chairmen, and the active chapter members.

Section D. The advisor of the local chapter must be a qualified agriculture educetion teacher currently teaching reimbursable agriculture education classes at North Salinas High School. 
Section E. FFA Booster/support group formation stall be supforted by the FFA Chapter and its members. Their involuement is encouraged and actiuities shall be in hamony with the purposes and Frooram of fictiuities of the FFA Chapter: and coriducted fer an benetit of FFA members.

Eection F. Local Young Farmeryanchere Chapter of the Leliforria Yaug FarmerfRancher's Association aridor FFA Alumri Grganization activities are Supported by the North Salinas FFA Chapter.

Section 5. Chapter records, member records. program information: and equipment inventories chall be maintained and kept within the facilities provided for by the worth Salinas Hi gt: Scticil

Haricul ture Department.

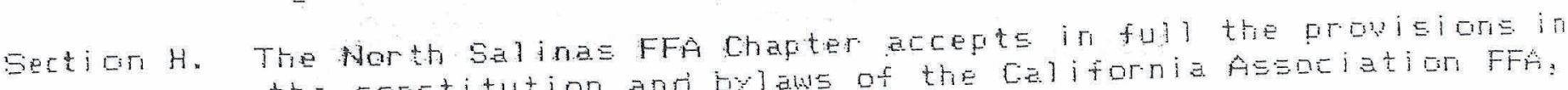
the constitution and by aws of the [a]ifornia Assdeiation FFA E. wel] a those of the National FFA Drgenizatigr.

\section{ARTICLE IU. MEMBERSHIF AND ELIGIBILITY}

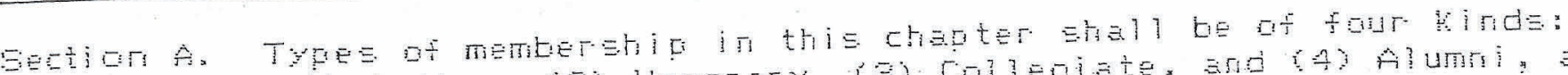

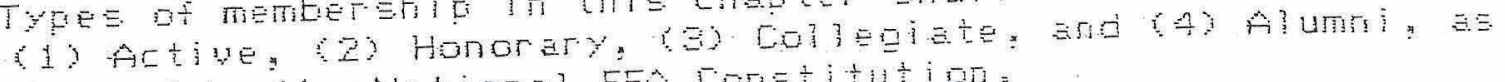
A

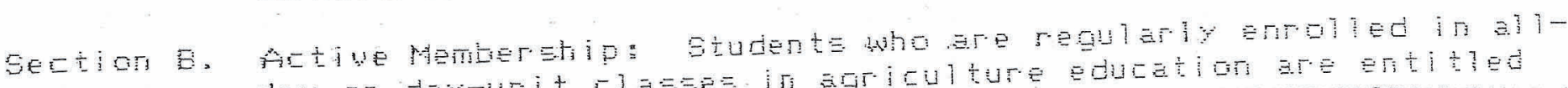

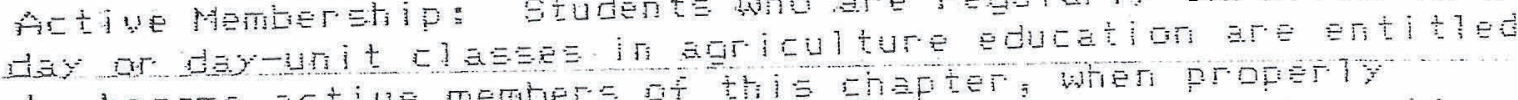

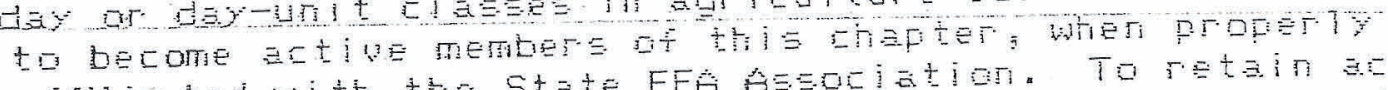

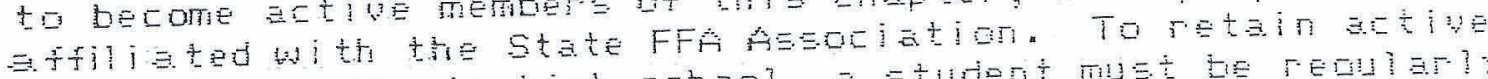

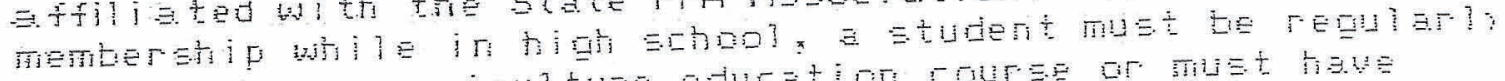

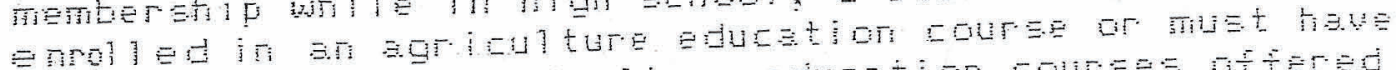
[ETIE

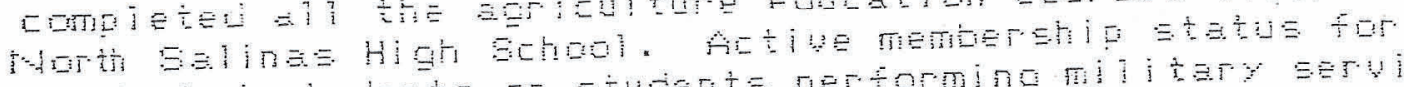

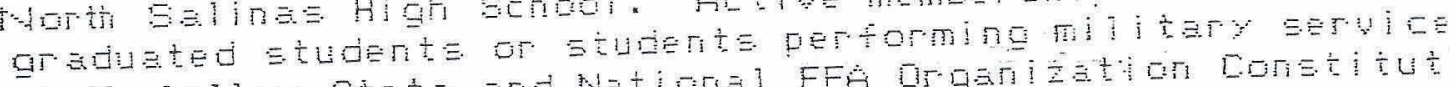

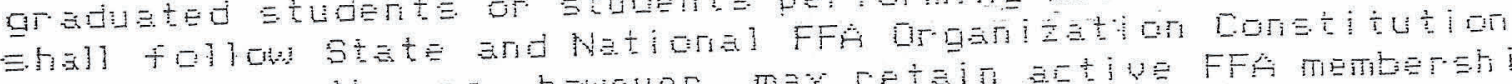

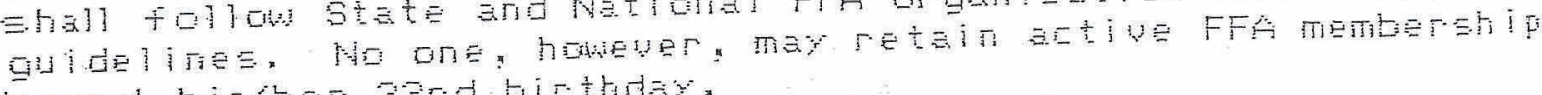

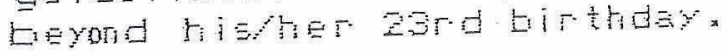

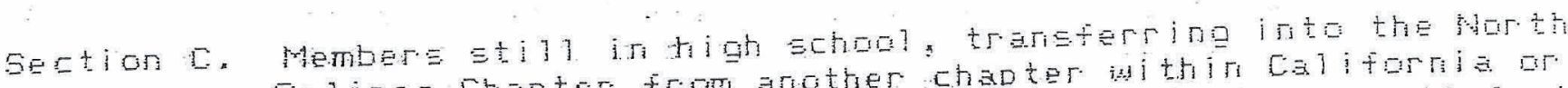
Saliras Chapter from another chapter iathir cal ifarria ar angther state of the United States hiof present Eatistastury Euderize of the ir godd etending in that chapter or states shall

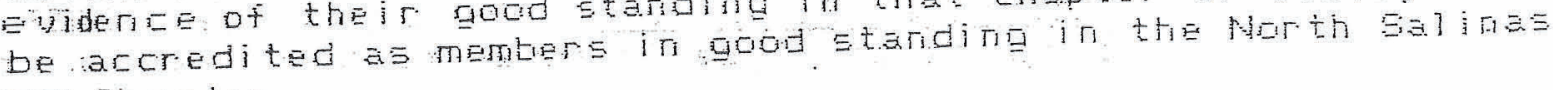
FFA CHEFter.

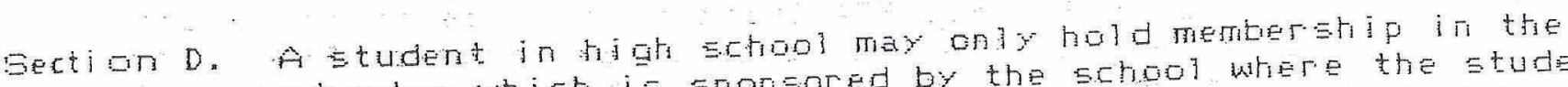
chapter which $i \equiv$ spongored by the school where the studert is Errolled in agriculture education. Should a Etudent be errolled ir agriculture education classes at more thar are E. Ihool, the Etuderit must be a memiter at the srhool within whose legal distriet boundaries the etudent l ives or primary satioul Ef enro] lmert. A Etudent who is out of high school and wishes to maintair. FFA affiliation may on $y$ do $\equiv 0$ a FFAchapter to uhich helshe belonged at the tine of leaxirg high school.

Eectigr E. The regular work of this chapter shall te carrigd or by the actiug membership. 
Section F. Active members in good ctanding may vote on all business brought before the chapter and Ear participate in all FFA chapter activities. An active member chall be considered in good standing when:

1. They attend local chapter meetings wi th reasonable requiarity.

2. They show an interest in and take part in the affaire af the chapter.

3. Are certified ty the FFA aduigor ac Btate FFA AEEgriatiors. affialiated member and meeting minimum school eligibility requirements.

Section G. Special active member criteria for shapter membere ghall be determined in the fall of each year, specifically to determine GAEP livestock exhibitor eligibility at courity districts or state faire.

section H, Upon termiration ot active FFA memberehip, fog. Eedeteryalumi menterohip ghal be operi to former. FFA members.

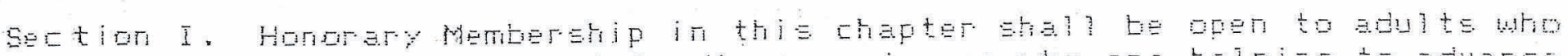

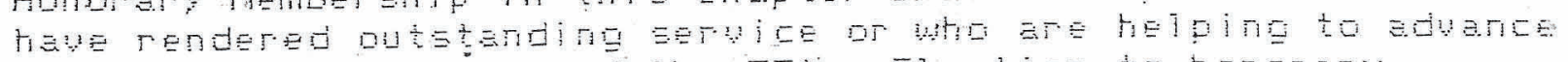
agricuture education and the FFA Eleztion to hongery

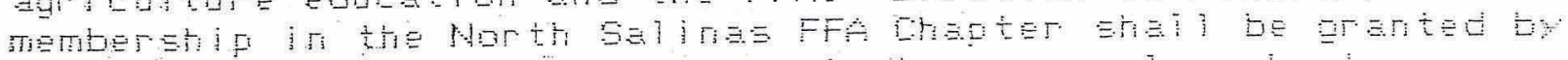

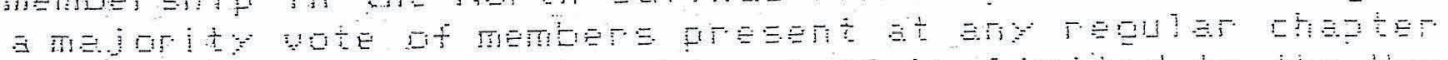
neeting, Honorery memberetip eriall be i mited to the Honorery DisT TEF FFA DEQTEE.

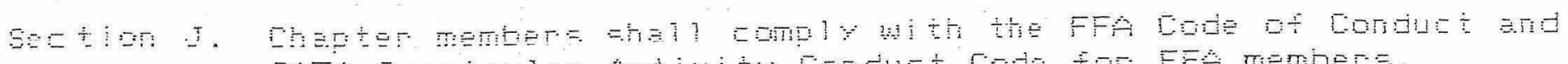

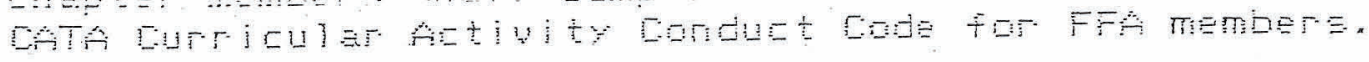

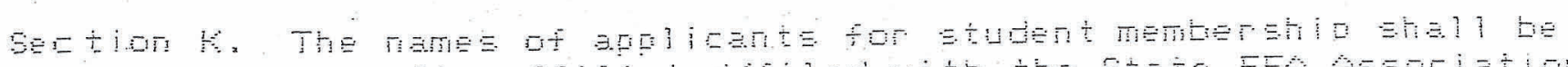
aniug?

\section{AFTICLE U, ACTIUE MEMBEESHIF DEBREES AND PRIUILEGES}

Sectior A. There erel i be four degrees of artive memberefip besed upon

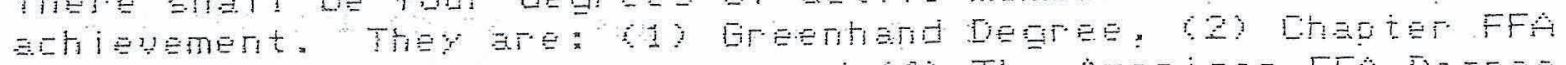

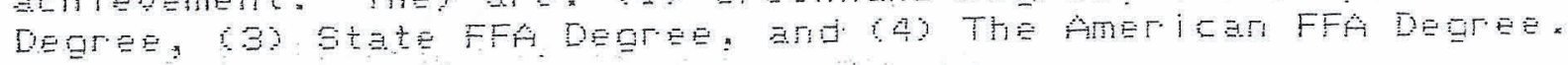

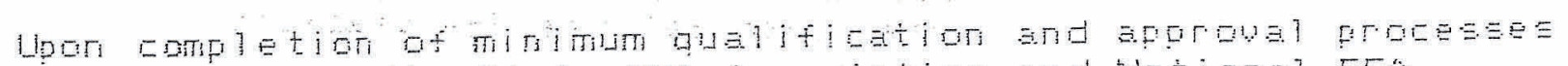

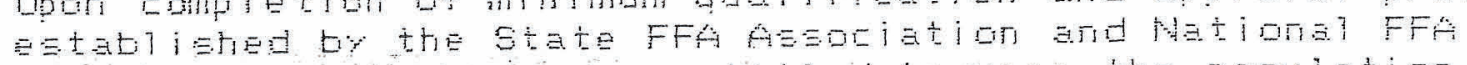
brgarizatior, member are ertitied to bear the regulatior emblem fin af their respezive degrea.

Sertign B. Greentarid Degree or meeting the folloming mirimum qualification=: the Greentand Degrea may be coriferred by the Ehapter:

1. Ee regularly enrolizd in a agriculture eduation cour Ee for an agricultura] oreupation and tiave $\equiv$ atisfactory and aca ertable plare far a program of Euperui sed agriaul tura occupational experiente.

2. Learr and exphati the FFA Creed, Motto, Selute, and the FFA MiEEion Statement. 
3. DeEErite the FFA emblem, colore, arid symtile.

4. Explain the proper use of the FFA jactet.

5. Hewe Satisfactary knawledge of the histary of the organization

6. Krow the duties and responsitilities af FFA members.

7. Personally own or have accese to the Dfficial FFa Manual.

B. Submit written application for the degree for atapter record

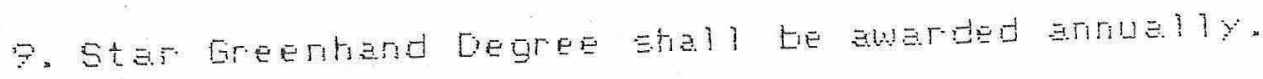

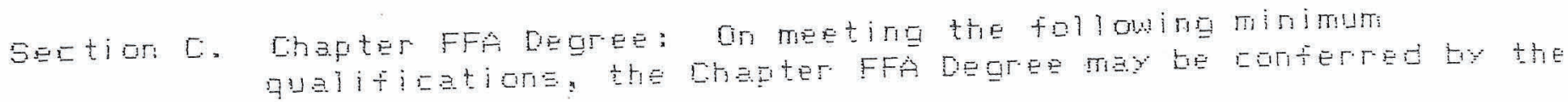
chater:

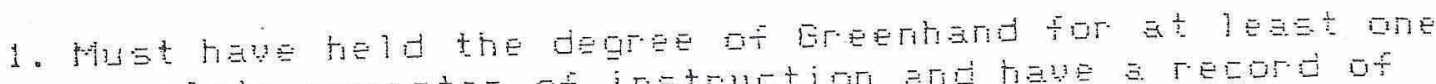

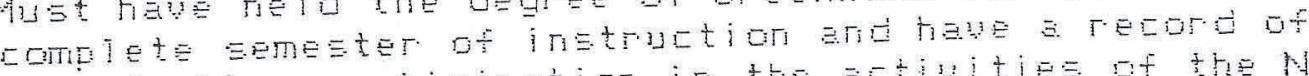
gati

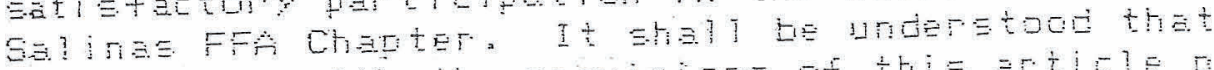

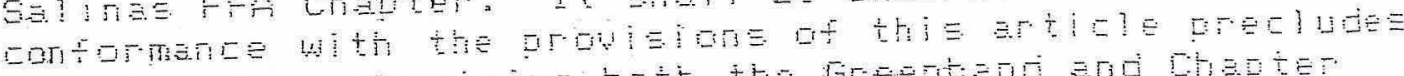

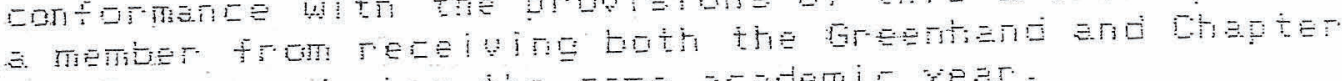

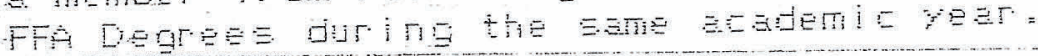

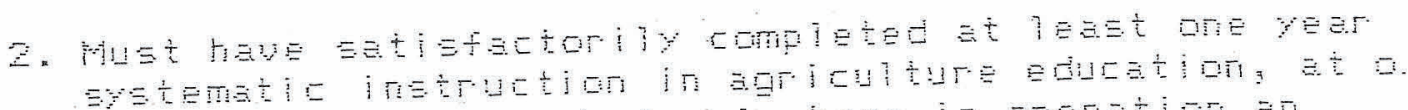

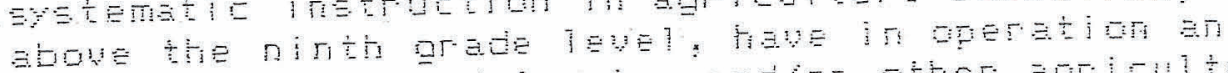

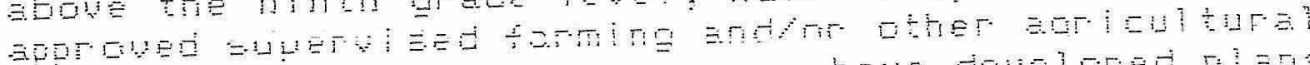

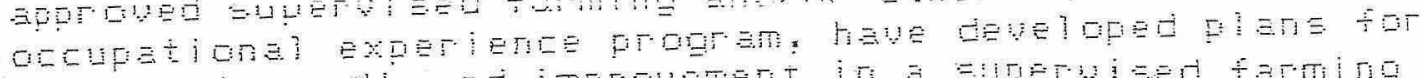

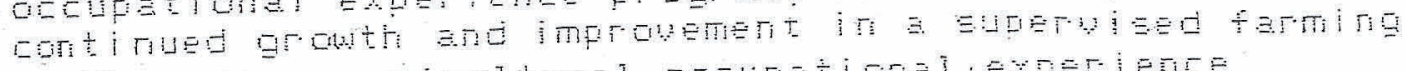
and

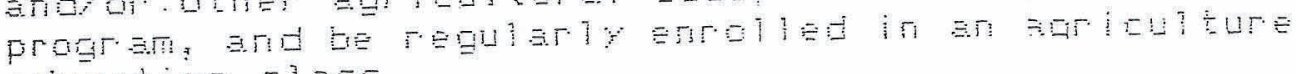

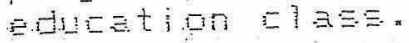

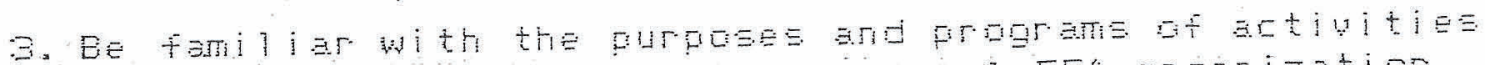

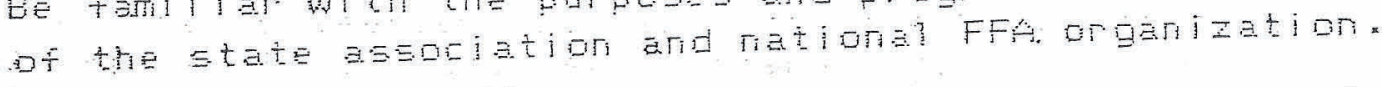

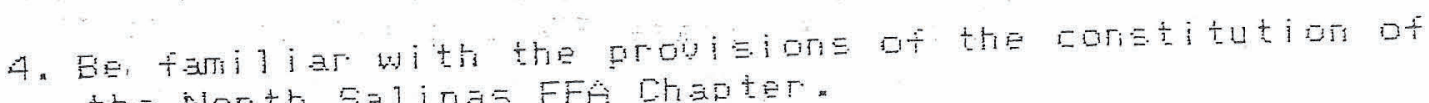
the north Salinas FFA Dhapter.

5. $E=\operatorname{tami} 1$ ar w i th Par l amentary Pricedure.

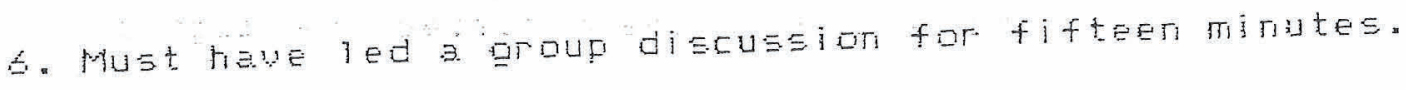

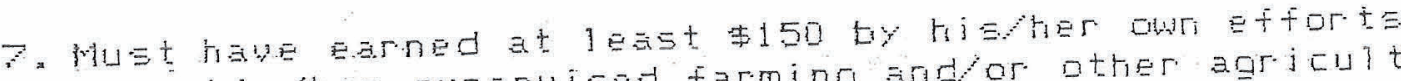

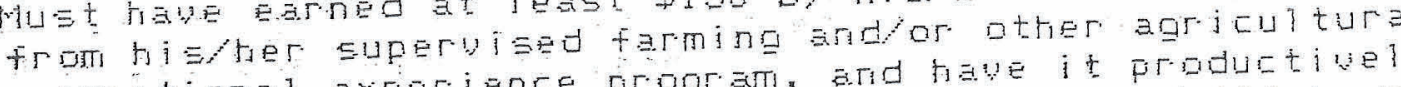

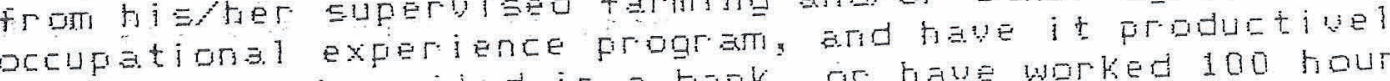
inuested or defogited ir a tank as have warked 100 hour

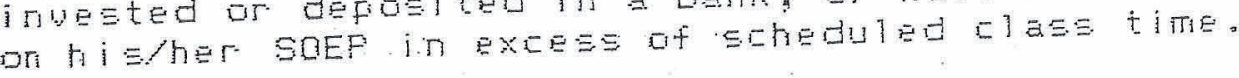

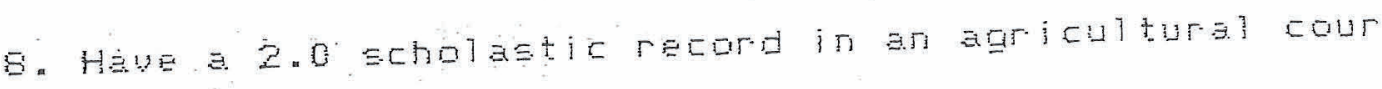

F. Star Ehapter FFA Degree andar Etapter Agritusines

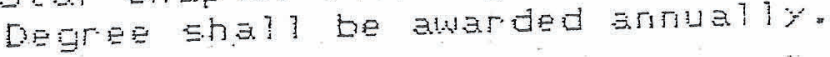


SECTION D. State FFA Degree: The State FFA Degree is conferred by the California Association FFA. To be eligible to be elected to. the state FFA Degree a member must meet all of the followir minimum qualifications:

1. Must have held the chapter FFA Degree for at least one year immediately preceding application to the state FFA Degree.

2. Heve been an active member of the FFA for at least two years preceding application for the State FFA Degree.

3. At the time of application for the state FFA Degree,

a. must have completed at least two years of instruction in agriculture education, at or above the ninth grade lewel, which included an agricultural Superwised Decupational Experiente Frogram; and

b. must be regularly enrolled in an agriculture educatior clas at the Eecoridary education level, an agriculturs course at the post-secondary education levely or te a graduate ot a secondary agriculture eduration frogram who is engaged irian agricultural cecupation.

G. Haue worked for a minimum of 500 hour scheduled clase time; on hishther Superyjsed Agriculturel

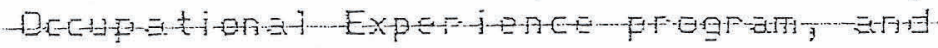

a. must have earneg by his her dur efforts from an agr icuiturai enterprise or other agriculturaijyrelated work ard deposited in a tank or otherwise productively inuested at least \$iono; or

b. Stroh an investment rogt of at 3 east $\neq 2,000$ in depreciable property inventary; or

c. have earned by his/ter own efforts fram agricultural enterprise or other agriculturally-related wrok and deposited in a bank or otherwise productively jriveste: a combination of dollars and unpaid labor hours whi ch when sumed, equal at least $1,000,00$. When qualifying tased on a combination of dollars and hours, the hours counted for quel ification must be unpaid labor hours of agriculturally-related work which are hours iriexess of class time and which are hours over and above the minimum of 500 hours require: in (4) above. Under no circumatarices shall a member. be qualified for the degree when qualifying based or combiration of dollars and hours without posting a minimum of $\$ 750.00$ of earned and productively investel income.

For the purposes of this subsection, productively invested is defined as any, all, or any combinationof the following: amounts held in secured liquid financiaj inwestments, expenses paid for educational purposes, financial support of the immediate family? and/or that proportion of the purchase price of a motor vehicle which is used for in the conduct of 
5. Demonstrate leadership ability by:

A. performing ter profedures of parliamentary law of demanetrate proficiency in parliamentary law as evidenced by paseing a written examination frepared and administered by the local agriculture instructor:

b. giving a Eix-minute specti OR lead a group discuseior for forty-minutes on a topic relating to agriculture or the FFA:

E. Eerving as an officer, committeg cheirperebr, ar participating member of a committee

d. participating in at least five distirctly different FFA actiuities at the chapter level:

e. participetirg in at least two distinctly different non-FFA Ectrod actiujties wich are conducted outeide of normel clasetime:

f. partisipatirg an artivities for community improvenent eE evidenced by participeting in at ieset two dietiritiy different actiuities to the extent of epending et least 20 houre of perecrea this: which

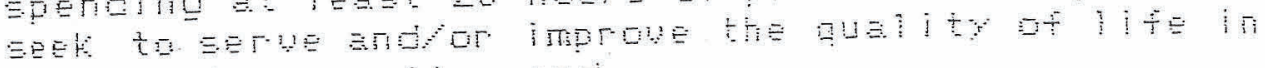
the lonel comminity and

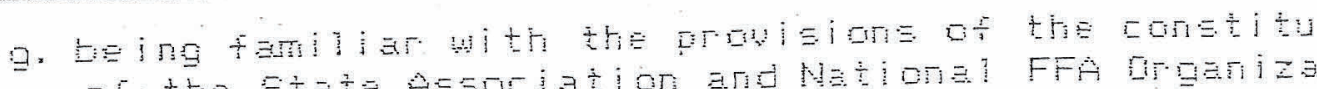
of the Stete Association and Netionel FFA Drgeniza

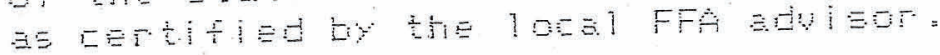

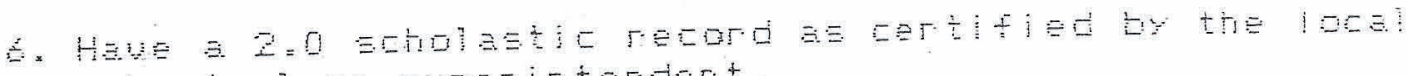
yrinizipal or enperintendent.

7. Heve perticipated ir the plenring and completion uf the chapter Frogran of Activities.

E. Have participated in at least fue fFA betiulties aboue the Ehapter level.

P. Wr itten applifytion and thorough, complete, and accurate

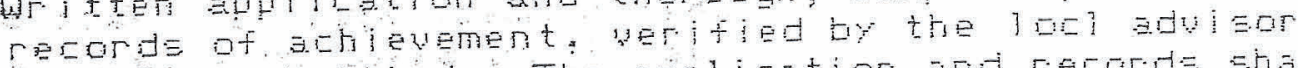
shal 1 be sutmitted. The appliration and recorda sha\} be based on the member o our entries ir the las iforria Uocatoral Agr fulture. Fecord Eook which i E cur.rently approued by tre Cal ifornia ctate staff for Agricultural Education as the official recordtook for Califorria vocationel Agriculture Etudents. Such a record book $i=$ the only Eutstantiation a member may use for the purpose of epplying for adwanced degrees and awards in this association. A comittee afpuinted by the state adyjeor will then review the records and submit its findinge to the Etate aduisor: who will wake hisher recommendatc to the state Exerutive Comimitteex Jhe Exscutiug committeg will nominate and elect the cardidates whic a. beer, found qualified to receive the degree.

10. HuEt recejue severty percent rays of the possible paint or hisher Record Book Scare. 
11. Ey appliatioli. Califarnia Star. Farmel and star in Agritusiries atwarde chall tue awarded arnually.

Section $E$. American FFA Degree. The gualifications for the American FFA Degree shall be those set forth in the National FFA Constitution.

Sectian F. A record of all FFá Deqree applications shall be kept in the office of the jocal chapter.

ARTICLE UI. OFFICERS, DUTIES, NOMINATIONS AND ELECTIONS

Section $A$. The ejected officers of the chapter shall be fresident, Vice Fresident, Secretary, Treasurer, Reporter, Sertirel, and Historian. The local FFA Aduisor stiall be the teacher of agricultural education ir the school where the thater is located.

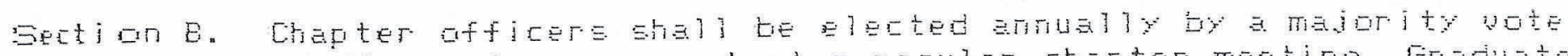
of the membere present at a regular chepter meetiro. Graduated

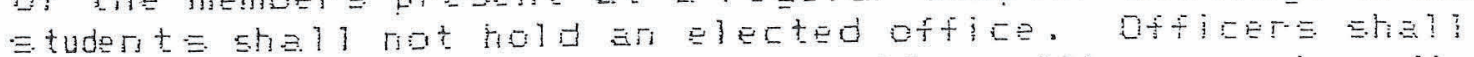
performi the duties of the rr respective offices as described in

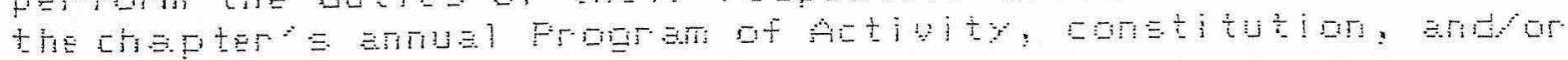
[ificia] FFA Mantial =

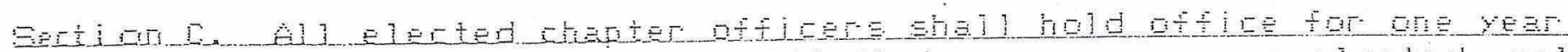

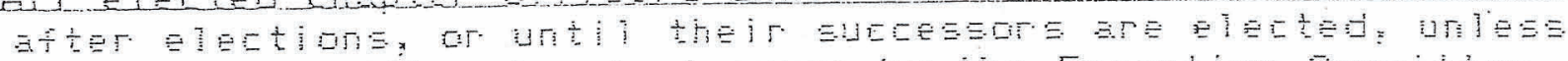

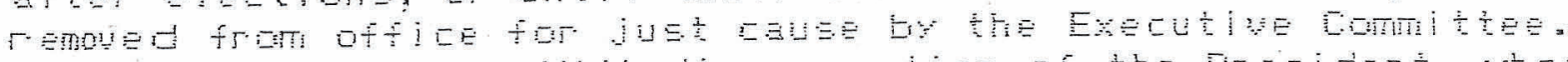
ondor FFa Adui

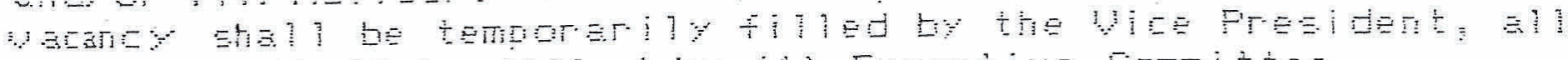

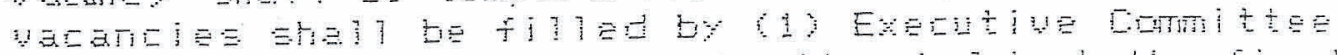

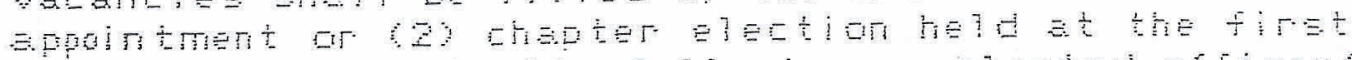

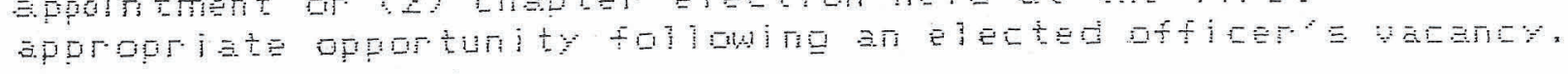

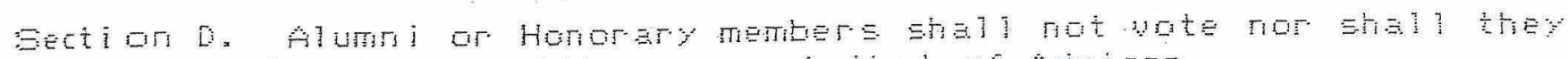
hold any office except that or hivisor.

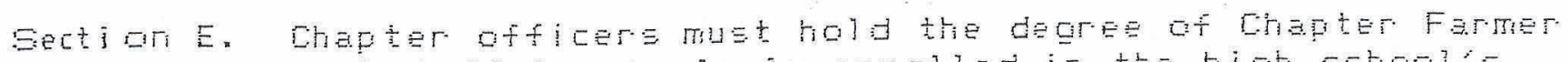
and sha? l be regulariy enrolled iri the high actidol' agricj] ture education progr sm.

Section F. Chapter afficerj shajl be elected by gecret ballot and

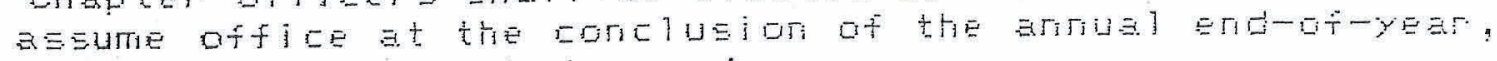
parent-member awards tanguet.

Section G. An officer may be impeached with due reasor and impeachement by a twa-thirds vote of the chapter memeerstip is required.

Sectidr H. Chaired by the Fresident, the Executive comimitte shall Eerve a the Chapter's rew officer rominating and screering

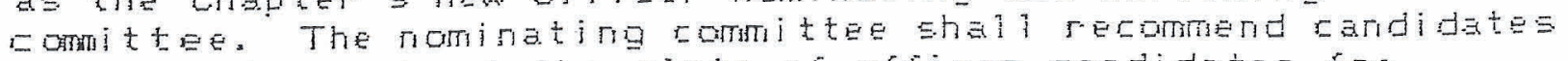
for ejection and set the 3 ate of officer candidates ror 上曰]lotirig. 


\section{ARTICLE UII. EXECUTIVE COMMITTEE, DIUISION CHAIRMEN, AND DUTIES.}

Section A. The officers and FFA Aduisor of the chapter together with th committee chairmen in charge of the major diuisions of the program of activities shall contitute the chapter Executive committee. The Executive committee shall have full polier to act on tehalf of the chapter and canduct busiress in-tietween meeting dates, and as necescary in accordance with actions taken at Ehepter meetirige.

Sectidr E. The FFA Adui Eor shall be a voting member.

section c. The duties of the executive camittee ghall be as follows:

1. MeEt regularly to plen meeting programe. make decisons. arid canduct chapter business.

2. Enforce the constitution and Eylawe. FFA Code of Ethics. and policies establi jhed by the chater.

3. Revi

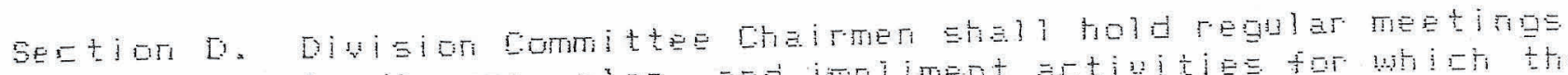

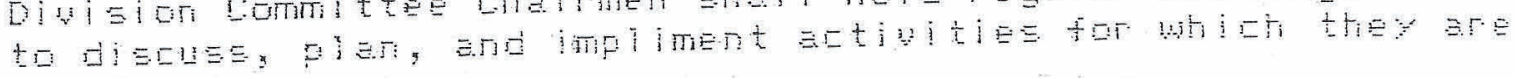
responstitis:

ARTITLE UII, CHAPTEF, SETTION, REGIMA, AUD STATE HEETINGS

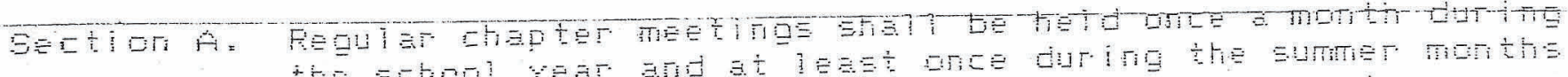

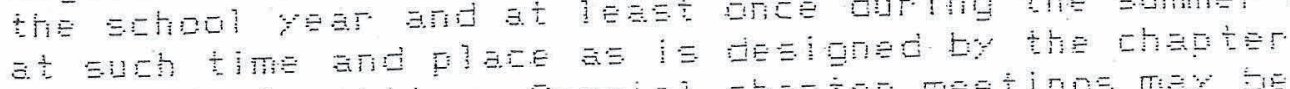

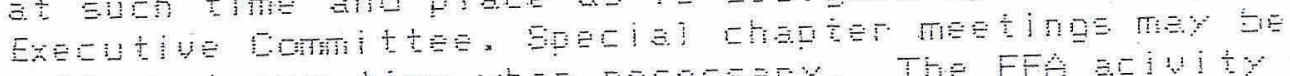

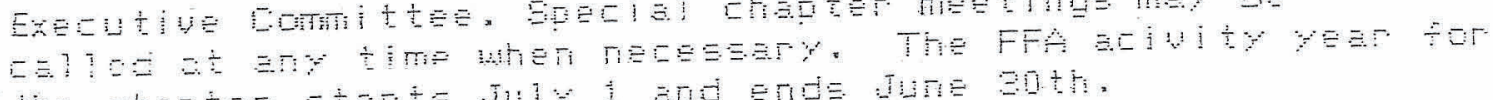
the barter Etarta JuY i and ende June eoth.

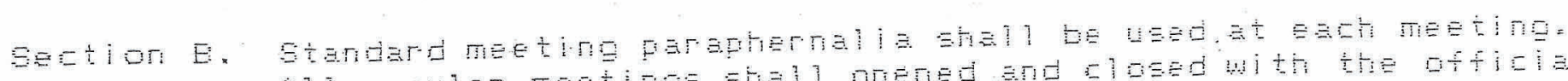

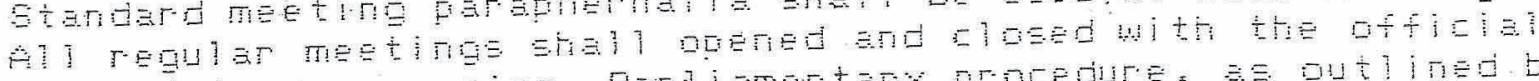

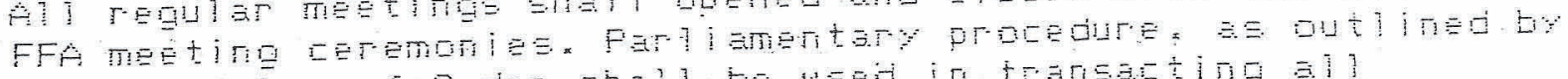

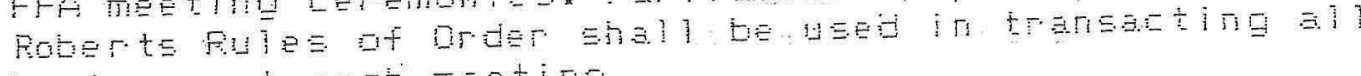

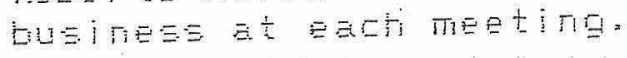

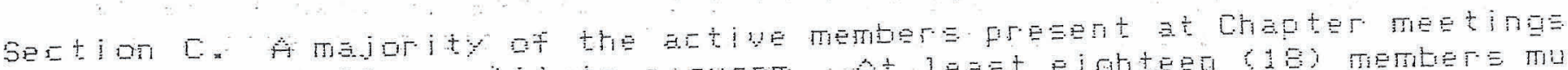

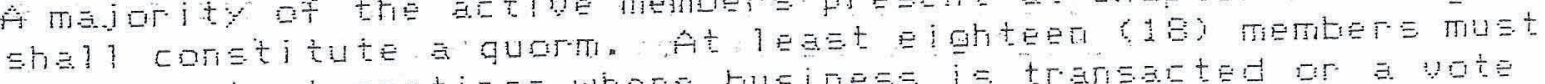

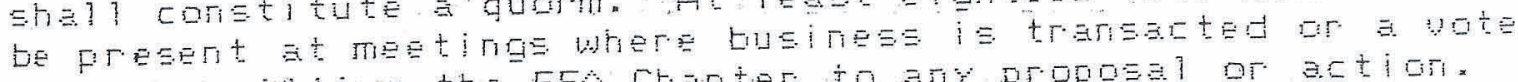
teken commlting the FFA Chapter to any propoeal or action.

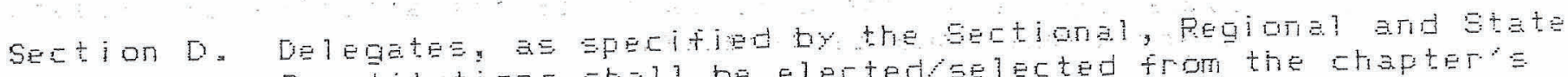
constitutions shall be elected/selected from the chapter actiue member ship to represent the shapter at the respective meetirige, conferences, or gather irge reduiring chapter representation and woting, dther delegates may be riamed as

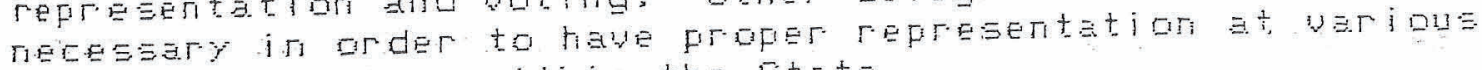
other. FFA meEtinge withir the state. 


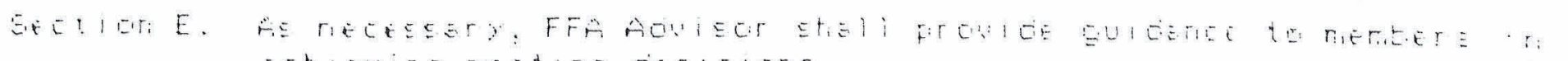

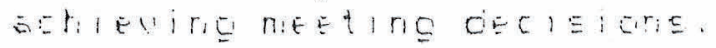

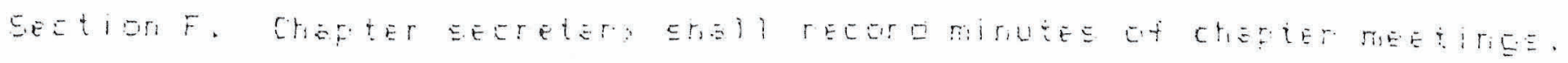

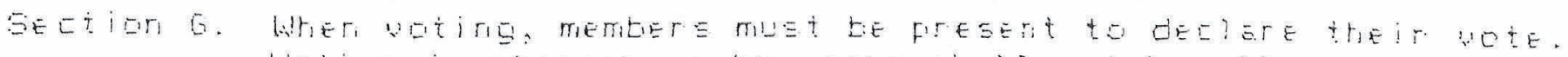

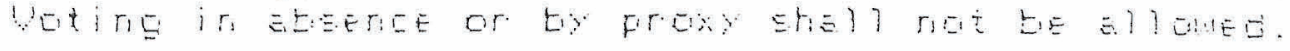

\section{ARTICLE IX. MEMEER AFFILIATION AND FINANEES}

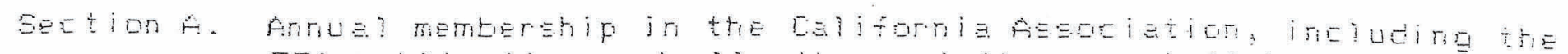

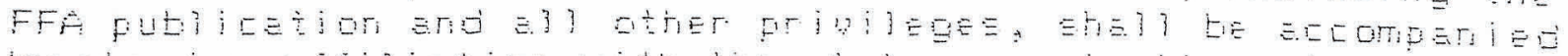

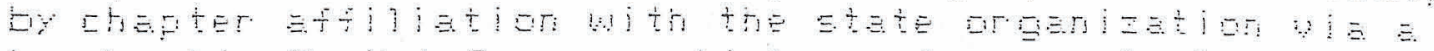
Leaderehif Packet Frogran which requires each chepter to

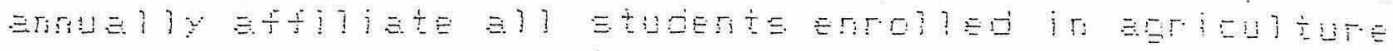

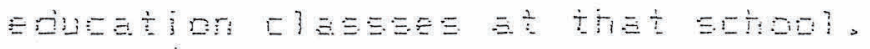

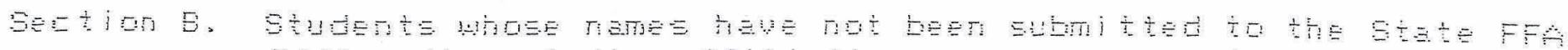

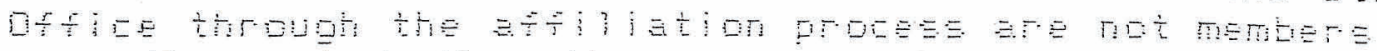

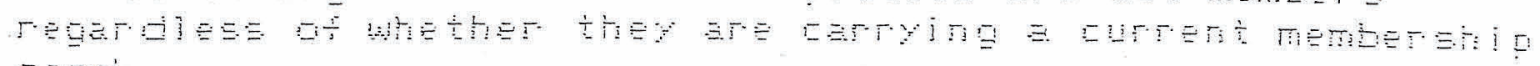
cers.

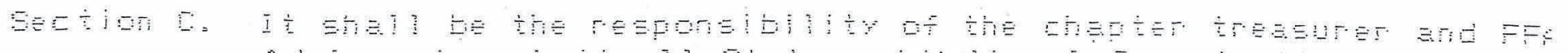

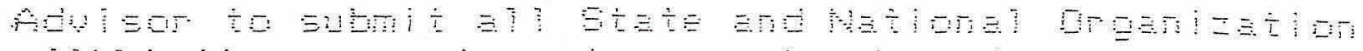

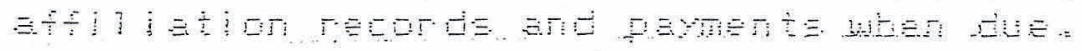

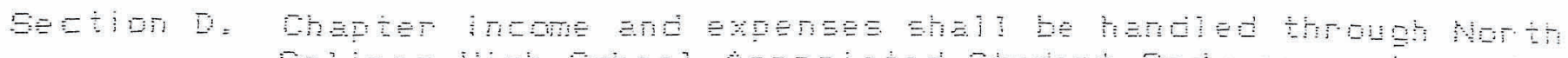
SE?

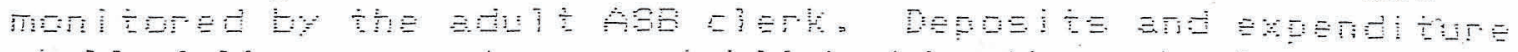

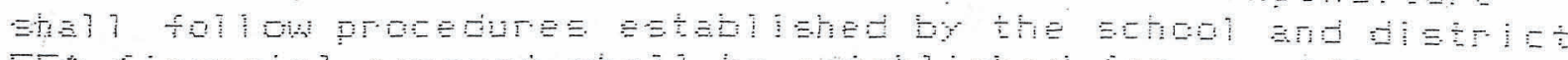

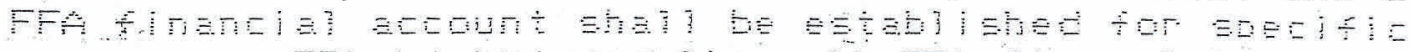

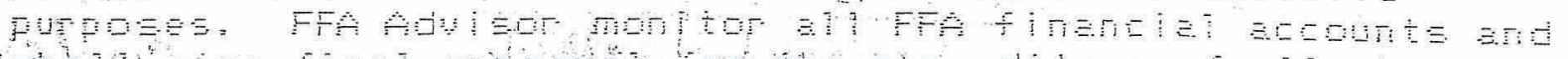

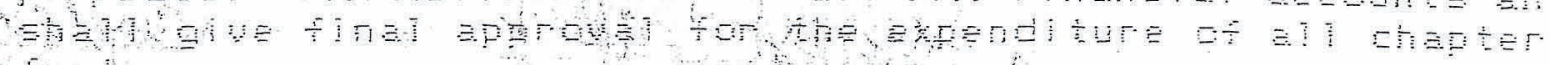
$\div$ ind

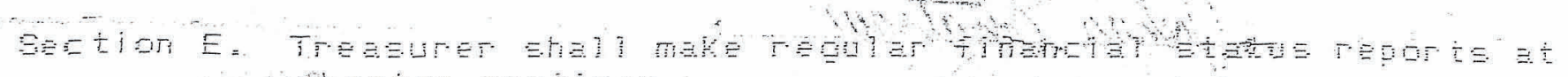

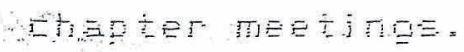

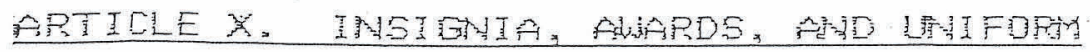

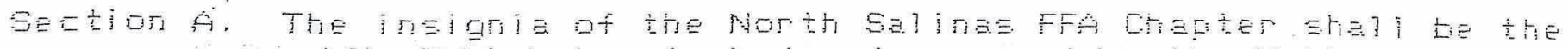

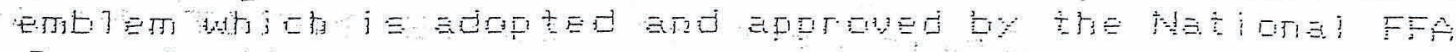
Or.g巳ization.

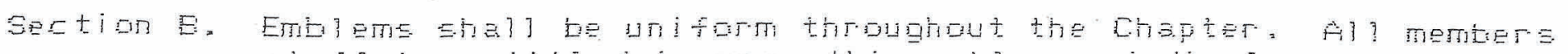

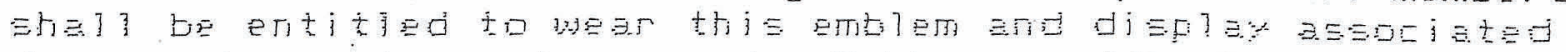

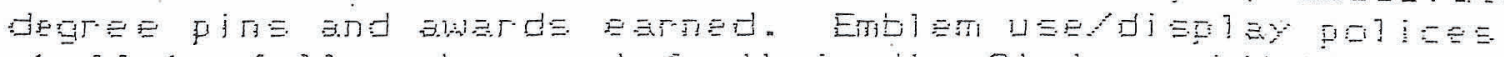

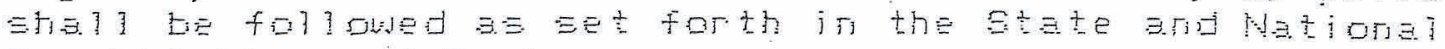

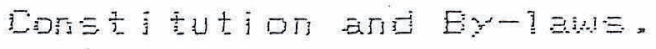

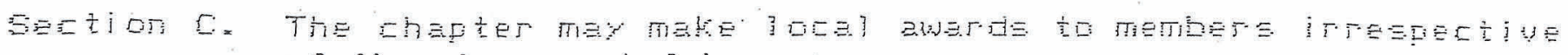

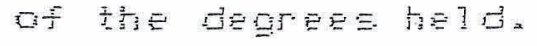

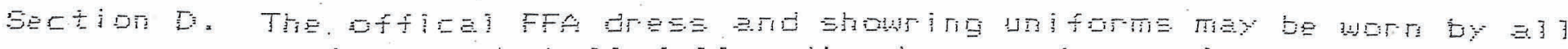

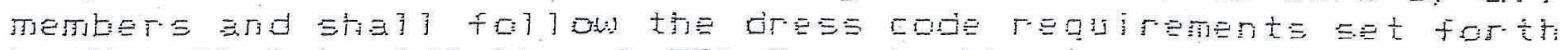

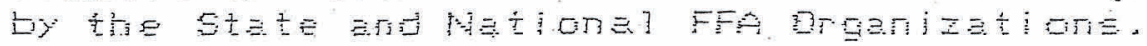


AFTICLE XI. CHAFTEF CONSITITUTION, GMENDMENTS, AND ETLALIS

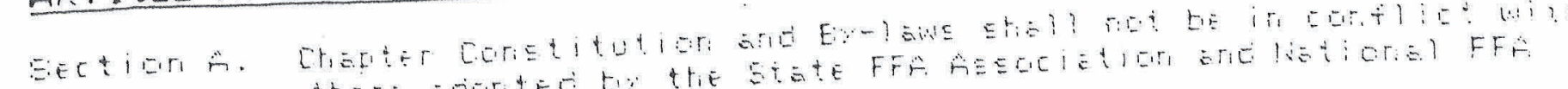

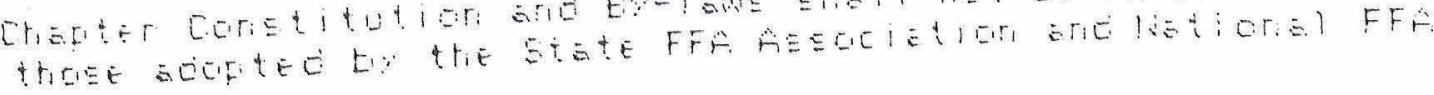
Grogn i zaticr.

Eectigr E. The Chapter Frogram of fictivity guide sno conterta Ehai

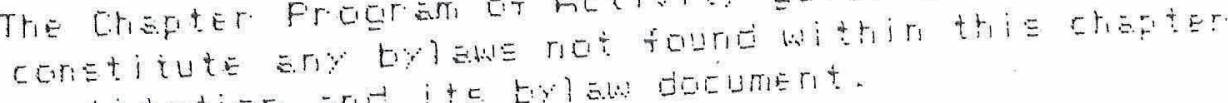
Eonstitutior snd itc bylab document.

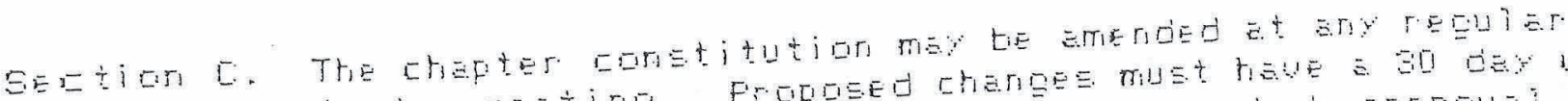

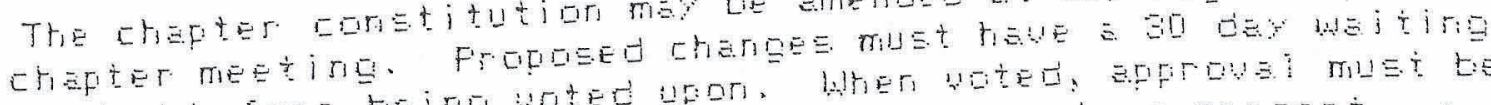
perigd before beirg woted upon. When woted, epprous must be

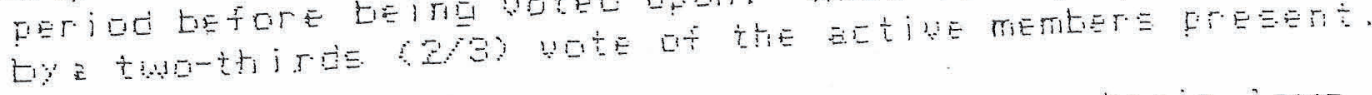

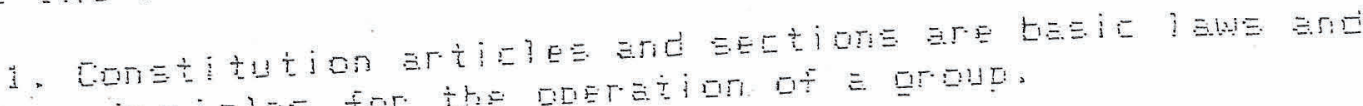

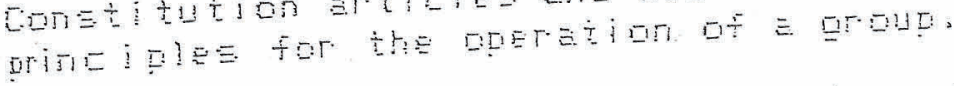

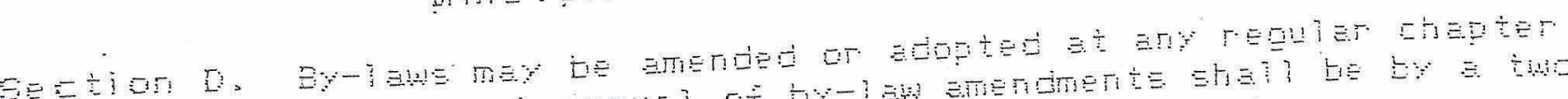

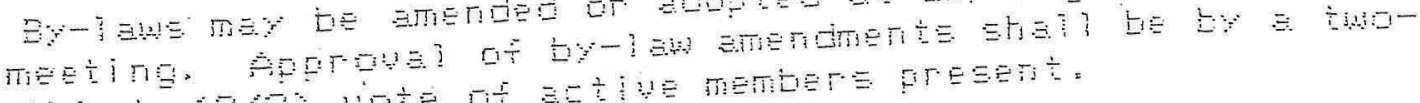
thit

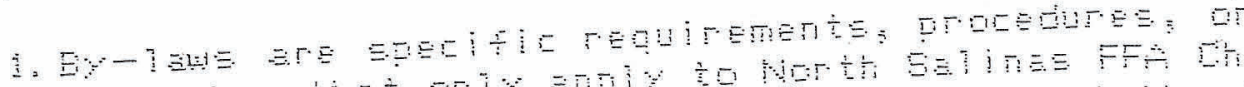
[0] [ I

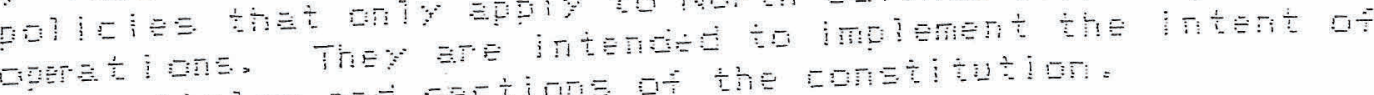
tre artites and getione ot the tonetitution

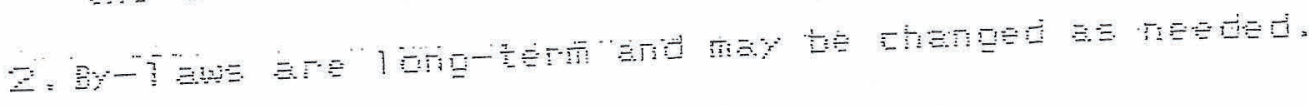

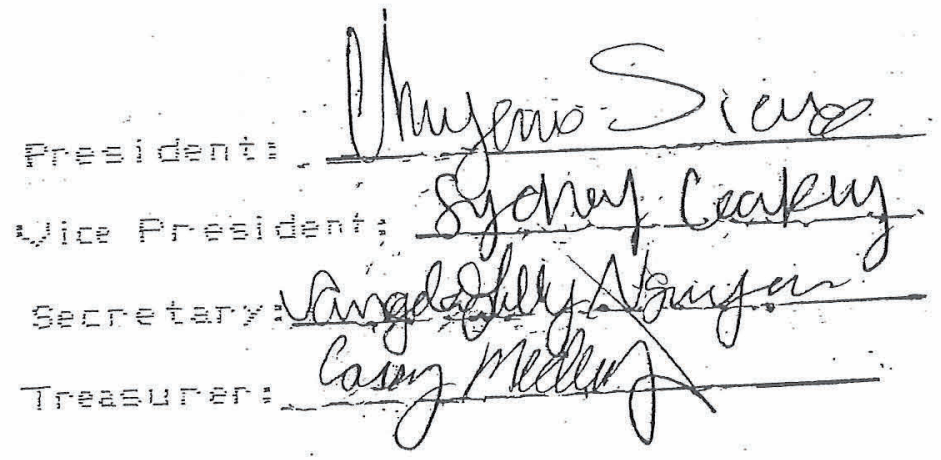
FEFT: Syopres ceakens.

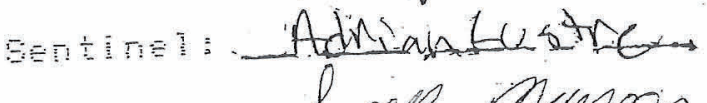

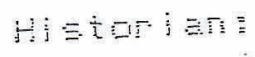
FFA औUH

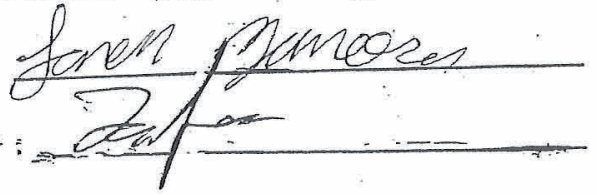

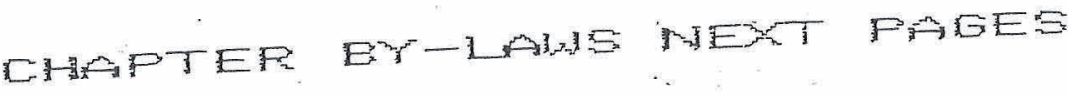


NORTH SALINAS CHAPTER NO. 284. FUTURE FARMERS OF AMERICA Fourided--January ? 1960 NOFTH SALINAS HIGH SCHOOL SALINAS, CALIFORNIA

\author{
$E r-1 \rightarrow \mu, S$ \\ Adopted January: 1960 - Fevised detober: 2007
}

THE FULLOUIMG EY-LAWE MATEH TO EPEEIFIC AFTIELES AMD EECTIDHE

OF THE NOFTH EALINAE FFA CONGTITUTION.

\title{
AFTICLE I. NAME AND PURFOSE OF THE ORGANIZATIDN
}

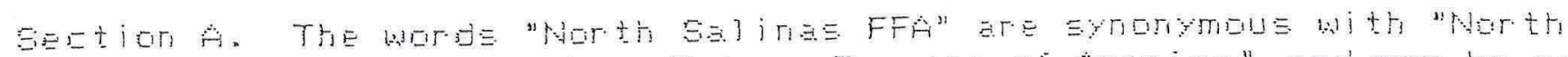

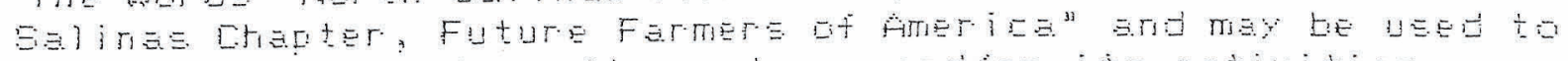
jontify the Ehapter: jte membere, ander ite artiojtises.

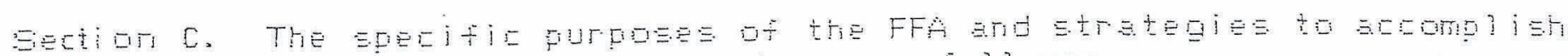
FFA' mi

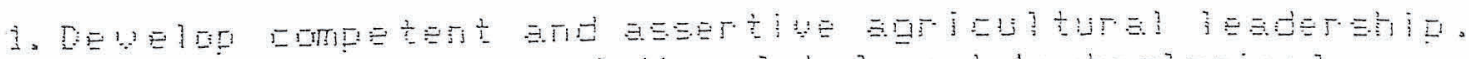

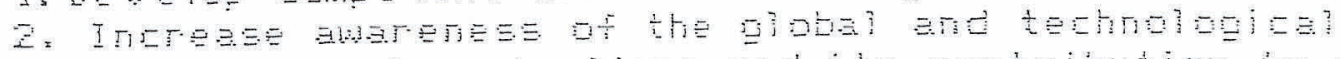

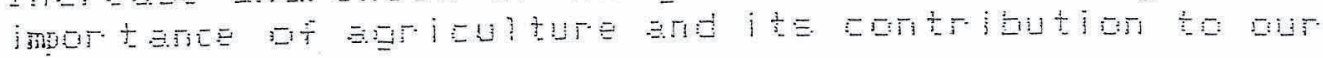
ine a l t we ing.

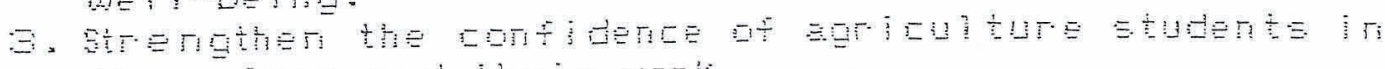
thengelues and thelr worlt.

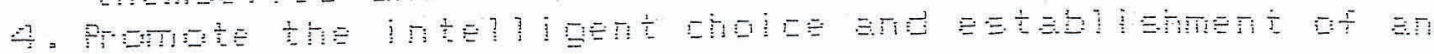
arriculturei zereer =

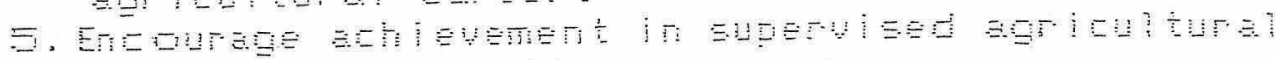

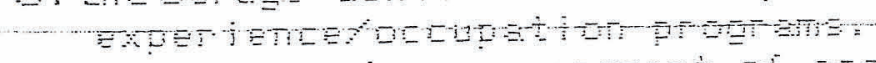

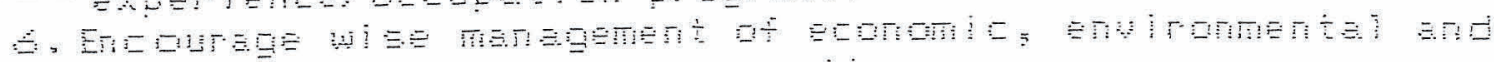

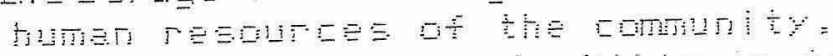

7 Deve a d It

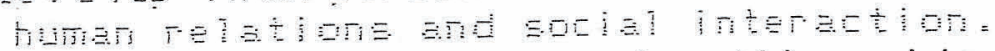

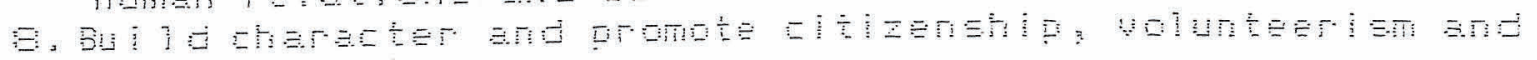
petriction.

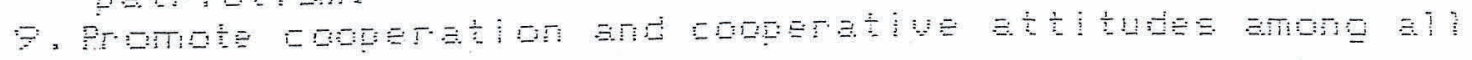
people

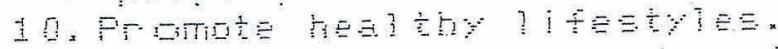

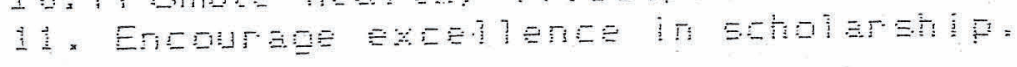

\section{ARTICLE III, DFEAMIZATIDUH}

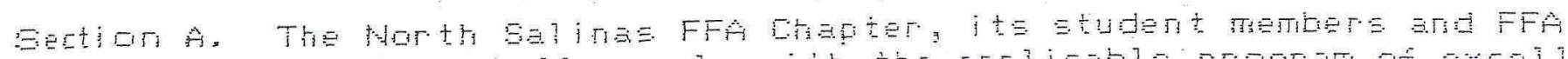

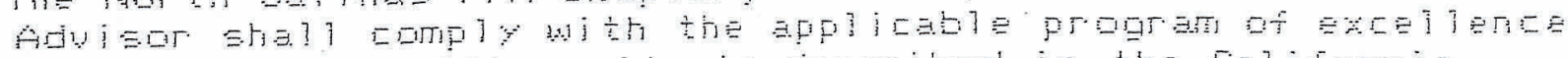

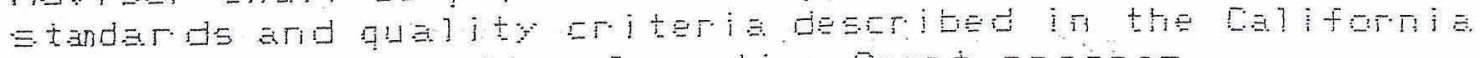

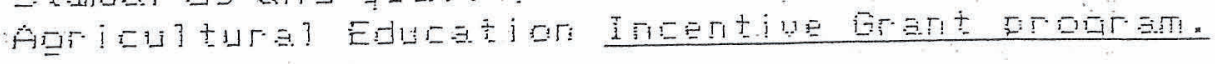

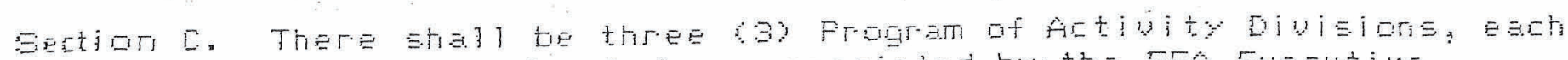
with Eeperate chairmeri appririted by the FFA Executive

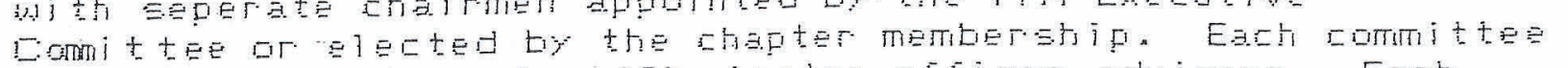

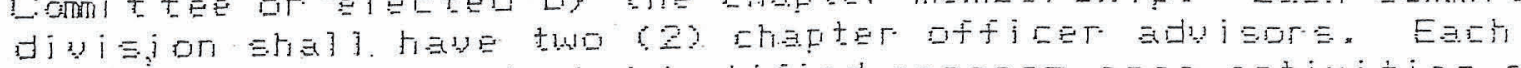
amitioe be dE

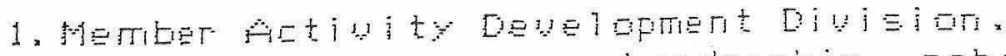

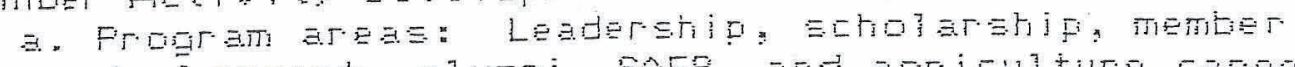

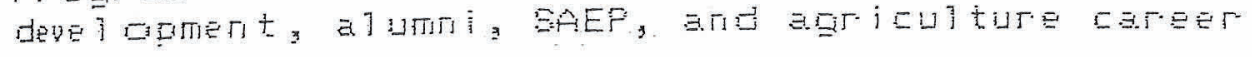
$\equiv$ Li i $11 \equiv x$

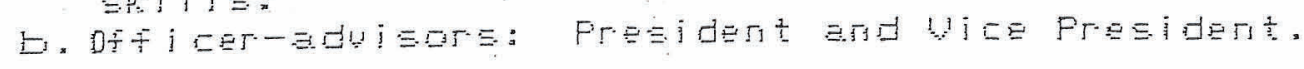


2. Chepter Aetiuty develapment Divisian.
a. Frogram areas: State and rationsl activities. cocperation, conduct of meetirge, recreation. chapter Eafety. earririgs and savige. Ag. Eogeter and recruitment.
b. Dfficer-aduisare: Becretary and Treasurer.

3. Community and School activity development division.

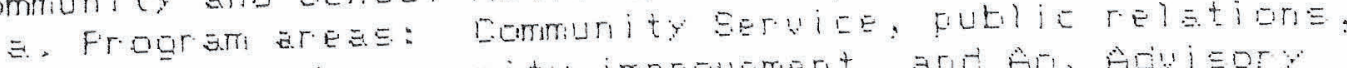
echool and commuity improvement, and AD. Adui ary commi t tee.

b. Dfficer-aduiedre: Feparter and Sentinel

Eection D. Administretion and superyision of FFA actiulties je the reEponsitility of the Ecrool" EAgriculture pepartment and FFA

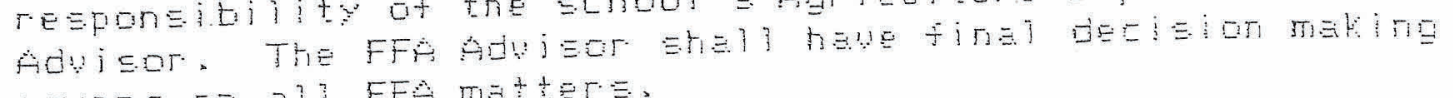
pouners on al] FFA mat?

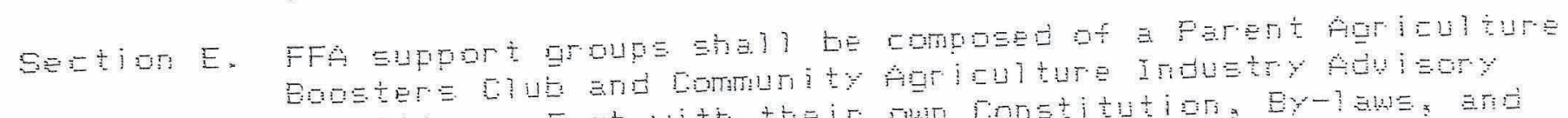

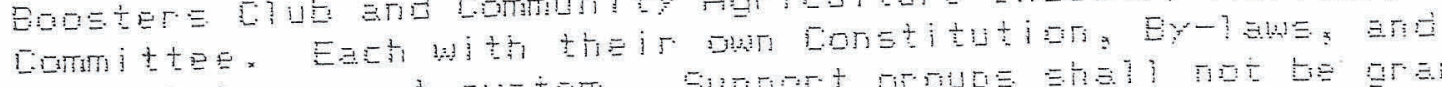

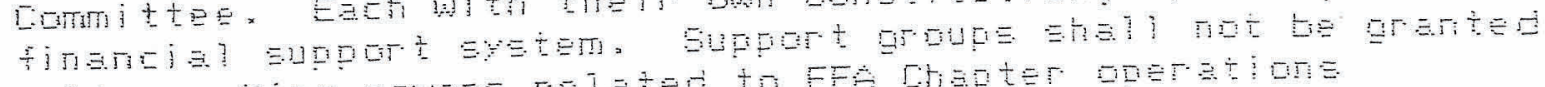

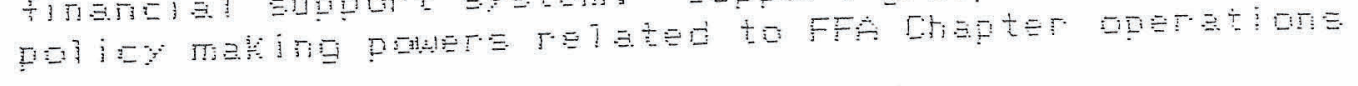

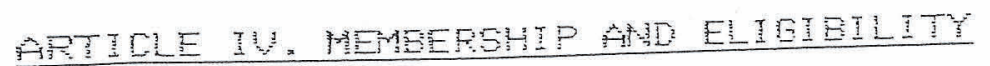

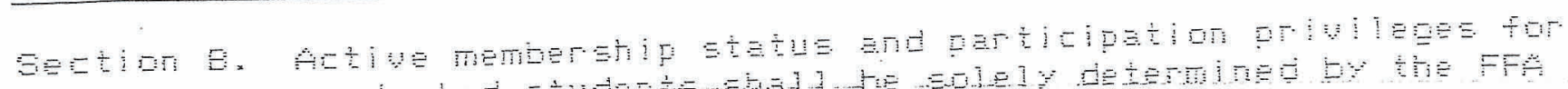
gr ady

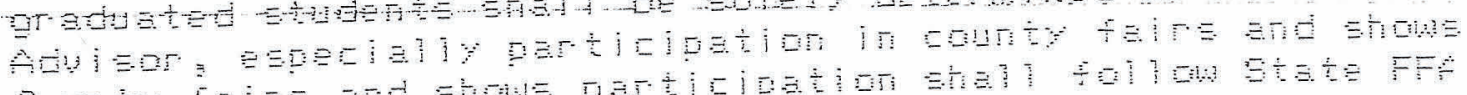

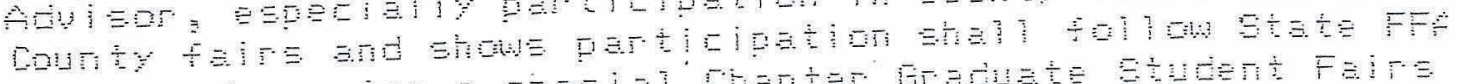

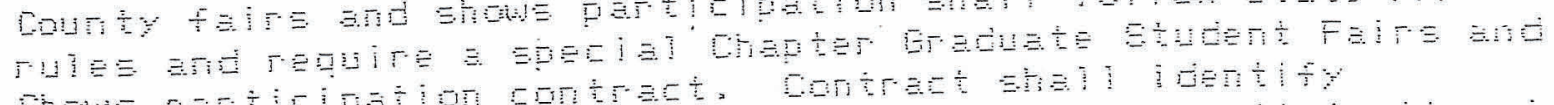

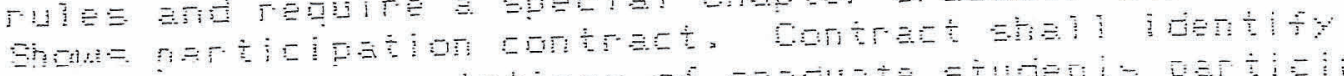

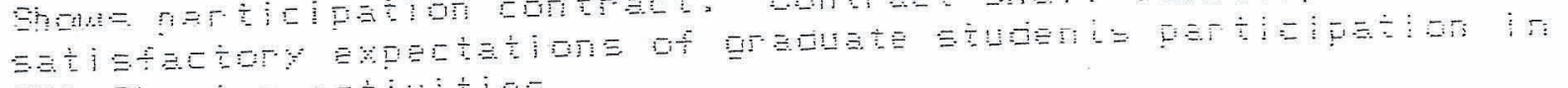

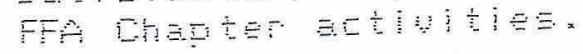

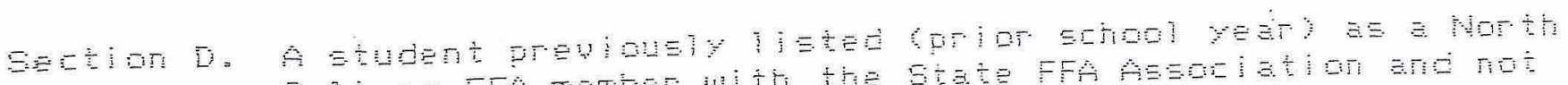

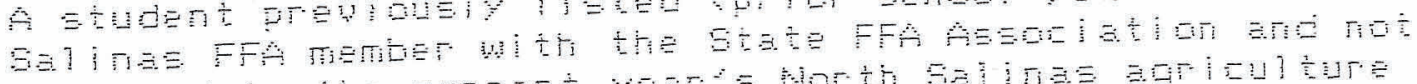

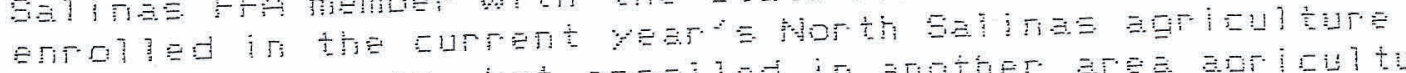

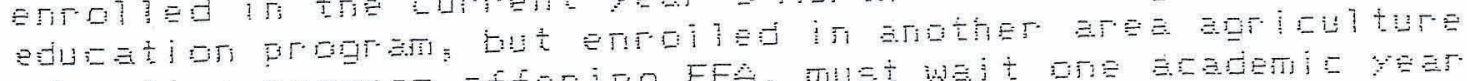

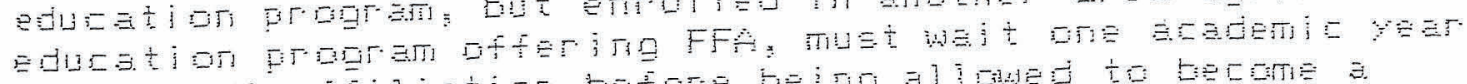

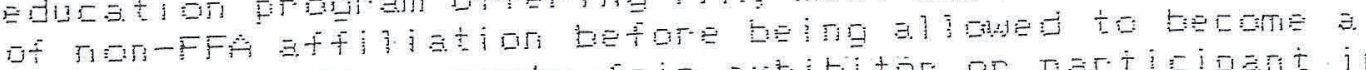

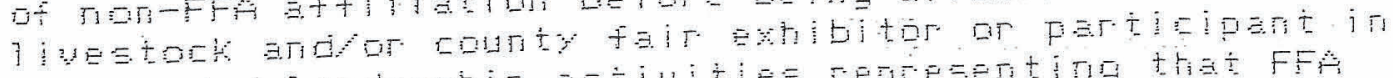

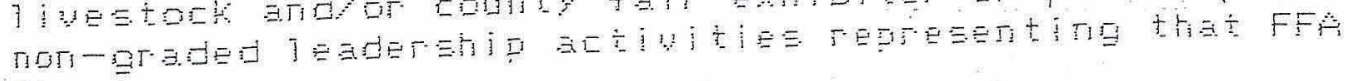
Etrepter.

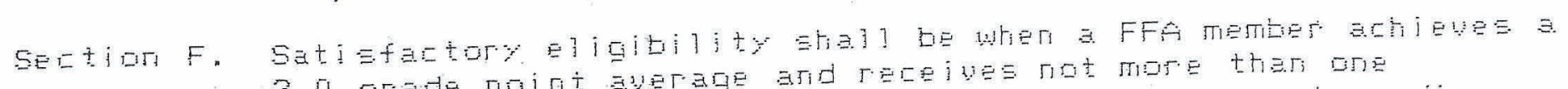
2.0 grade poiri guerage ard receives not mare thari gne

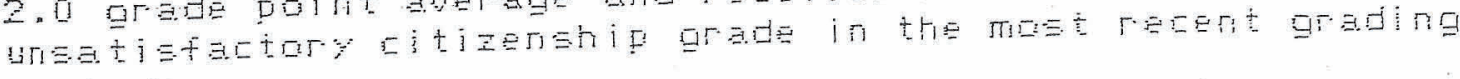
period.

1. School idertifieg ineligitie suderte may not participat in courty fajo ard struls. FFA competition Euente. epecial field tripj, leaderetrip confererces, tigla electe ] aderehip positions, or participate ir ary other

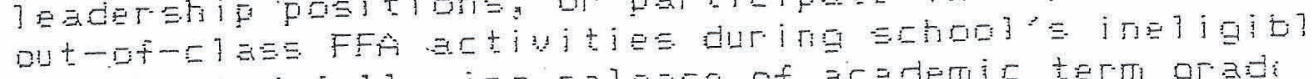

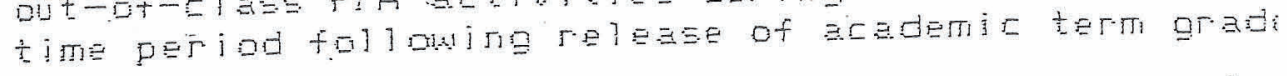

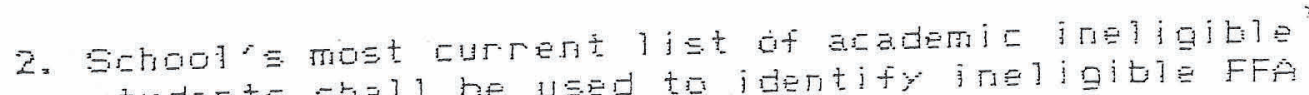

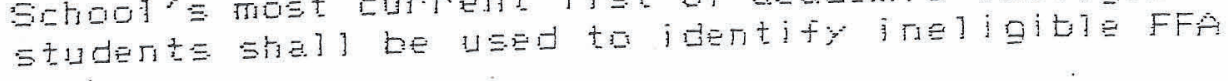
ment:DE: . 
Section G. FFA members conducting SAEF livestock Enterprises intended for show and or Eale at courity faire ar ather fFA livestack exhititions Ehall be required to complete the FFA Chapter' Livestock Exhibitor Eligitility participation contract.

1. Eligibility contrect shall cover, but not be limited to the following areas:

a. Understandirig arid purfose.

b. Chapter and ar SAEP meeting attendance-eo\% at tendarice.

E. FFA actiuity farticifation-z differert actipity per semester.

1. Academic achievement in agriculture cless-an F gradz in agrieulture i conjidered eutomatic ineligitility.

e. Eligitility clearence-authorized by Ectod animini Etration arly.

f. Livegtock rare and manggement.

9. Livestock dispoga\}-erterprise termination.

h. SAEF record keEping and seles check re] ease-rid checks relesegj until alj enterprise obligatione heve tean conpleted.

i. County fair pertormance grading:

j. Trangprtation, perticipetion rules and dedte.

H. Houging and cheperones.

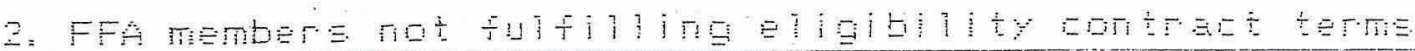

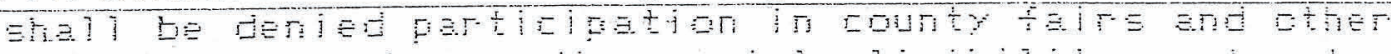

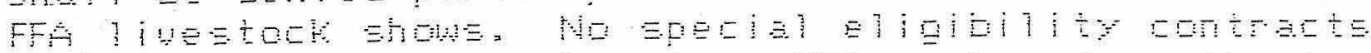

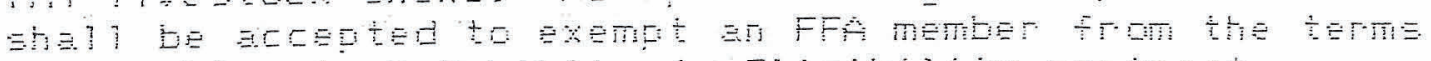

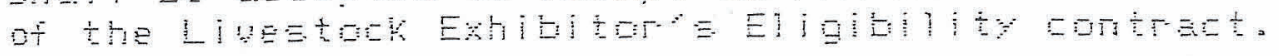

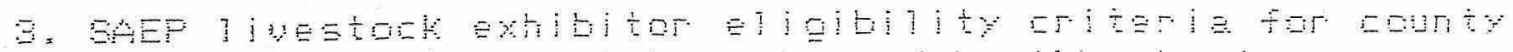

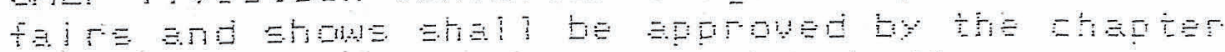

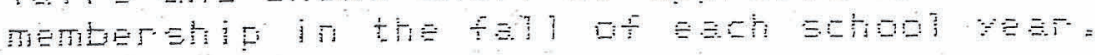

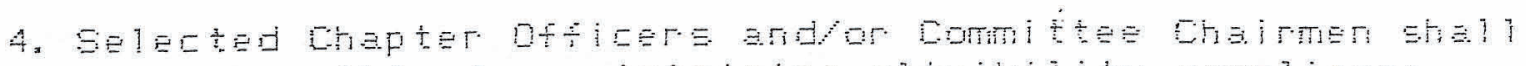

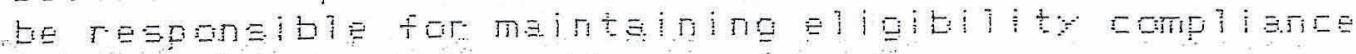

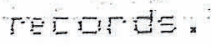

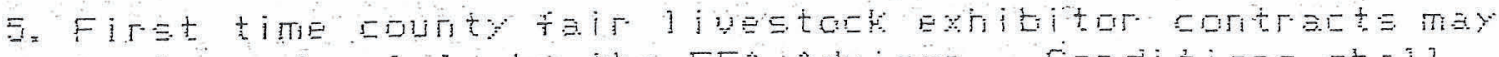

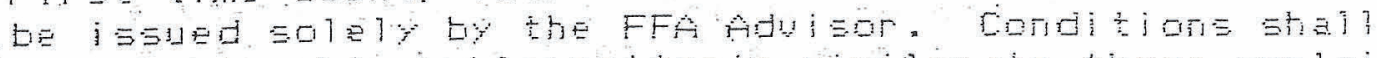

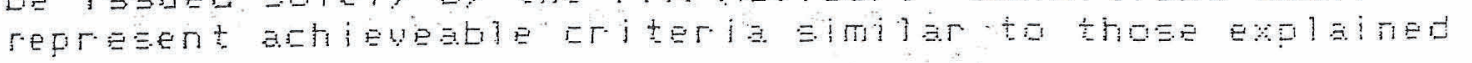
stove.

Bection I. Hon ary Memberahif in this chepter shel1 be 1 imited to awarding two az) Hororary Chapter FFA Degreas fer year =

\section{ARTICLE $U$. ACTIUE MEMBERSHIP DEGREES and PRIUILEGES}

Section C. Ehapter FFA Degreen or mesting the foll owing mirimim qualificatione, the Chapter FFA Degree may be conferred ty the chapter:

6. Must present a formal d-e minute uninterrupted speech ui th mianuecript or haue jed a group diseusejon for fiften miritites. 
Eection A. Greenhand officere may be elected if the need is decided by majority vote of the chapter membership. Greenhand officers shall be subordinate to the regular chapter officer team. Greenhand officer term of service and duties shall be determi ned by the Chapter. Executive committee.

Section E. If reeded, Chapter historian, parliamentarian, chaplain: Jristudent Aduicor may be elected by majority vote of the chapter membershif. Each shall be a member of the Chapter Executi ye Committee and eerve a one year term.

Section C. Chapter FFa officers must attend 90 percent Df monthly executive committee and chapter meetinge, chapter. Fresident shall kep attendance recorda. Auarding of officer seruice fins etall be determined by the FFA Executive comm ttee end prejented at the erid-of-year Parent-Member Auarda banauet.

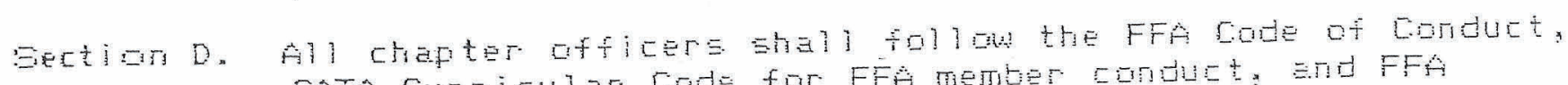
CATÁ Curricular CodE for FFA member conduEt: End FFA uniforma jakket uEe criteria.

Seation E. Chapter Grficers must te enrolled ir on-cample agricultue

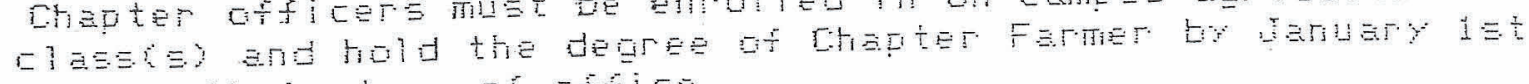
during their termi of G+Fice.

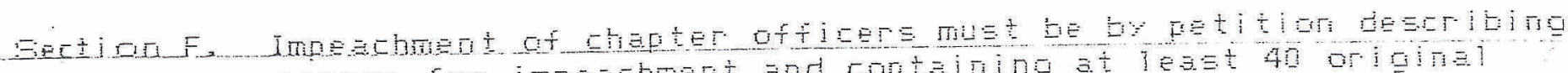
reason for impachnert and conteining at leset 40 or ignel

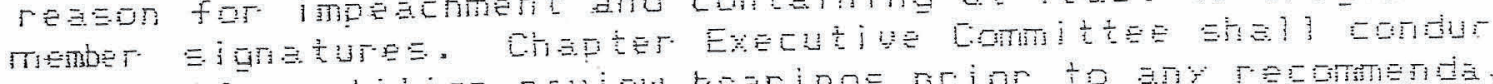

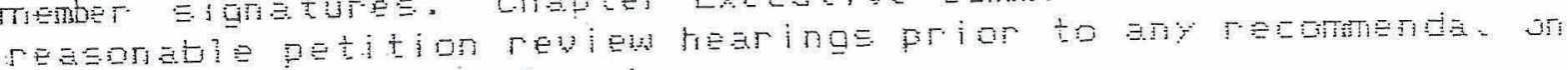
tor or ageitiet impesctimerit.

Seztion G. The FFA Executive Committee mey add additional chepter members

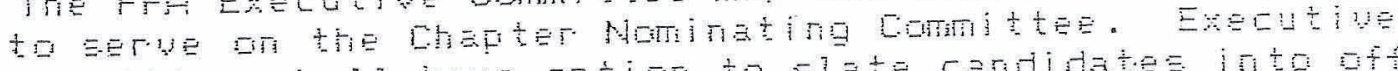
committee shall haye option to slate candidates into office best sulted to candidaté gualificatione and experiences.

\section{ARTICLE UYI. CHAPTER. SECTION: REGION, AND STATE MEETIMGS}

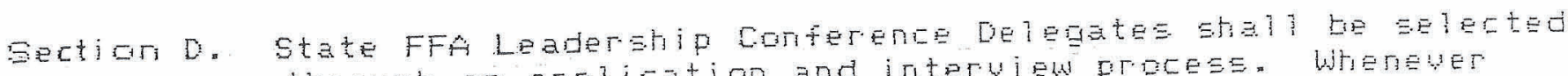
through an application and interview process. Whereuer possible, all cotiference expenses ghall de pait for by the fFA Chapter.

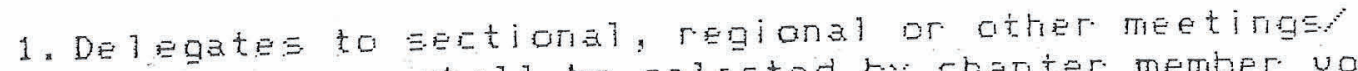
conferences shall be selected by chapter member vote. Executi we committee selection, and/or presidental appointment.

Z.Highest raking chapter officer at any-aboye-shapter FFA meetings actiuity, conference, or competition ghall act ar beh 31 f of the chapter and its members.

Sextion F. Chapter secretary Etall use the official FFA Eecretary Eno' hep records of chapter meting minutes and other related documentsyiformation common to the office of secretary. Eomlete Secretary book shall be filed with the ASE Club Activity Clerk in June of each year. 
BETICLE IX. MEIEEF: AFFILIATIOW AND FINANEE

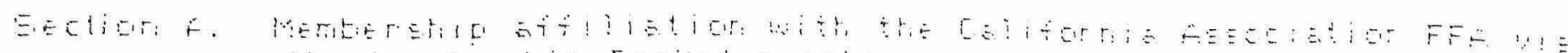

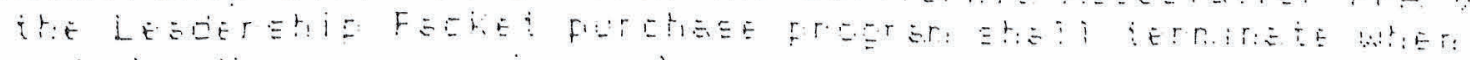

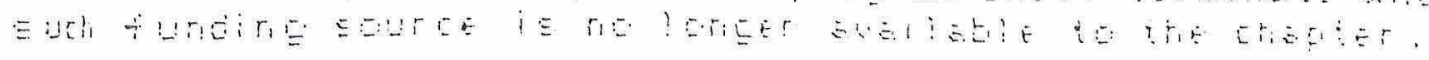

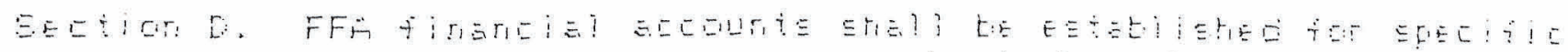

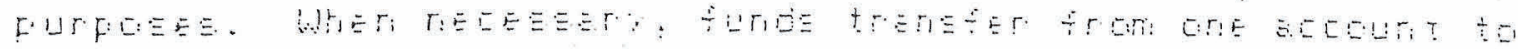

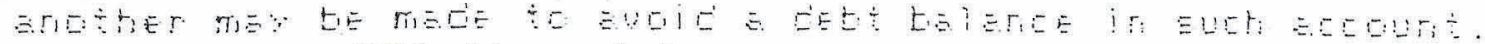

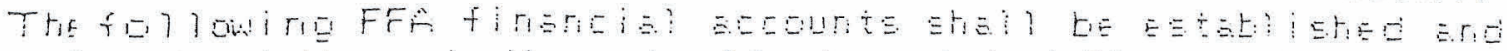

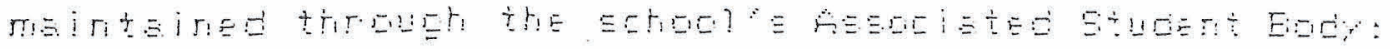

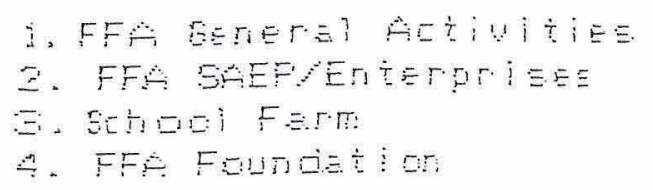

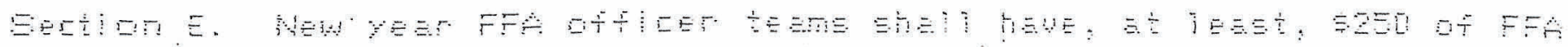

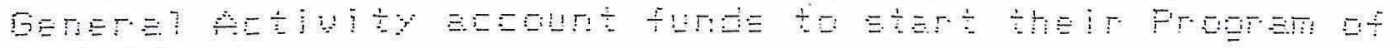

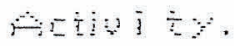

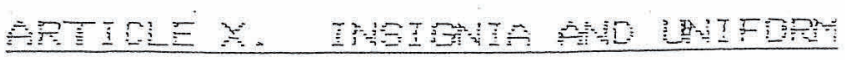

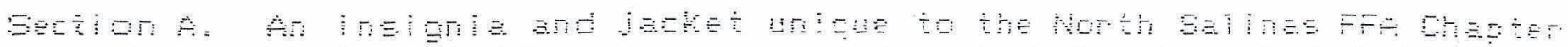

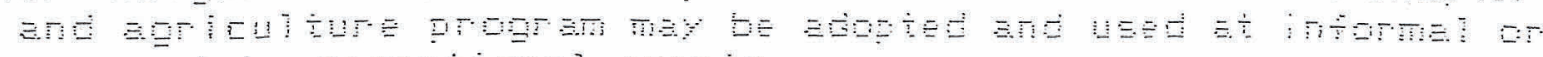

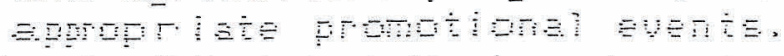
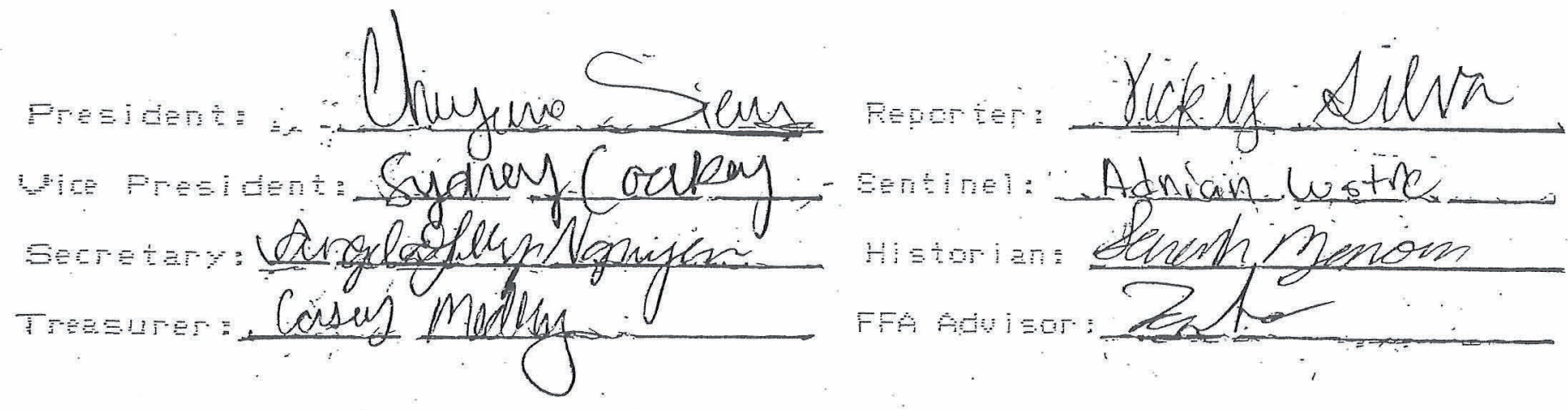


\section{PRE-REG. AG. PROGRAM RECRUITMENT PRESENTATION (GVMS, HMS, WMS) $3 / 09$}

SET-UP/NEEDS: 3-4 Student Team. 5-8 Minute Presentation. Program part assignments. Personal written scripts done and reviewed. Presentation practice. FFA Uniform Dress. FFA banners, easel stand, FFA flags, Program Brochure handouts, duct tape, 3 Program Circles (class, SAE, FFA) poster.

\section{PROGRAM PRESENTATION/INFORMATION SEQUENCE}

(Ag. Program and Course Description section of Student Manual)

(Class flow chart, FFA and SAEP achievement flow charts)

** All help with Program Brochure distribution when students first enter.

1. INTRODUCTION TO DAY'S PROGRAM PRESENTATION.

a. Why are we here? Tell purpose of presentation and what hope to achieve?

1. Here to tell you about North High's Agriculture Program that will give you leadership training, a chance to travel up and down our State, earn money, learn new skills, and will help you to meet your credits for graduation.

b. Tell about general Ag. and FFA program information. Only give you an idea of how much opportunity for involvement there is in our agriculture program.

Other students will give you more details in their presentation.

1. Class Offerings-have 10 different agriculture classes in plant and animal sciences, welding, horticulture, business, and construction mechanics.

2. Graduation Credits-Ag. classes meet graduation requirements in 4 ways. Agriculture classes will give you biological science, physical science, vocational ecucaition, and elective graduation creciils.

3. We also have a greenhouse, small animal lab. building, and a school farm.

4. Our FFA Chapter is part of the largest student organization in California.

5. The FFA project program can teach you new skills and earn you money c. Introduce self and team members (member name only).

1. Who you are? Years in Ag.? Background or connection with Ag.? Why in Ag. Program? Benefits of Ag. and FFA to you? Awards or achievements earned? SAE Projects conducted? Other interests? Future education 86 career goals?

2. Emphasize: Do not need to live on farm or ranch. Get opportunity to learn something different, get positive and life-long experiences.

2. Introduce self. TALK ABOUT SAE PROJECT PROGRAM, KINDS, OPPORTUNITIES, BENEFITS.

a. Chance to learn new skills and experiences by raising animals, plants, or build projects for county fair exhibits and sale (describe examples). Participate in 3 County Fairs. Trips taken? Awards? Can get started this summer. Describe your projects.

3. Introduce self. TALK ABOUT FFA PROGRAM. OPPORTUNITIES, LEADERSHIP EVENTS, BENEFITS.

a. FFA Activities-Give individual and team leadership building and public speaking experiences (give examples). Participate in regional and state FFA activities all yearlong (describe some). Make new and lasting friendships (examples). Trips taken? Degrees and awards? Describe your involvement. 
Page 2 - MS Recruit Program

4. Introduce self. TALK ABOUT AG. SCIENCE 1-2, AG. BIOLOGY, ORNAMENTAL HORTICULTURE CLASSES. (see Student Manual class descriptions) a. Short description of what is studied, learned, or done in the class. Greenhouse use? b. Graduation credits received for taking class?

c. Trips taken, class competitions, special projects,etc.?

5. TALK ABOUT AG. WELDING and AG. MECHANICS CLASSES. (see Student Manual class descriptions)

a. Short description of what is studied, learned, or done in the class.

b. Graduation credits received for taking class?

c. Trips taken, class competitions, special projects, etc.?

6.

TALK ABOUT AG. EARTH SCIENCE CLASS, and USE OF SCHOOL FARM, GREENHOUSE, SMALI ANIMAL LAB. (see Student Manual class descriptions)

a. Short description of what is studied, learned, or done in the class.

b. Graduation credits received for taking class.?

c. Trips taken, class competitions, special projects, etc.?

d. Describe what school farm, greenhouse, and small animal lab is and how they are used?

7. and EVERYONE COME TO FRONT AND STAND IN GROUP.

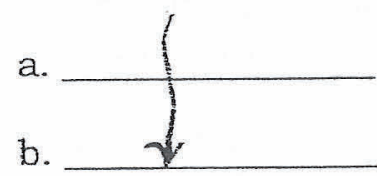

c.

d. Repeat/summary of SAEP program. One sentence format. Repeat/summary of FFA program. One sentence format. Repeat/summary of Ag. Classes to take---Ag. Science 1-2, Welding, Ag. Mechanics, Ornamental Horticulture. One sentence format.

Repeat/summary of Graduation Credits earned-Biology, Physical, Vocational, or Elective credits earned.

One sentence format.

8. ASK FOR ANY QUESTIONS? ANY PERSON CAN ANSWER. Important to give measureable/specific answers.

a. Thank students for attention and turn program over to Mr. Caresani. 
(1) Good Moming everyone. My name is Tylen Bibson. I I un here with Melissa, Angief. Ensily to talk to you about the most turn auk actine cinbs on oun coppus, The Nonth sulinas FAt, in the hopes of vecruiting you to be future viting Assiess. The FFA is a national organication othat gives you Lerbership training, a chance

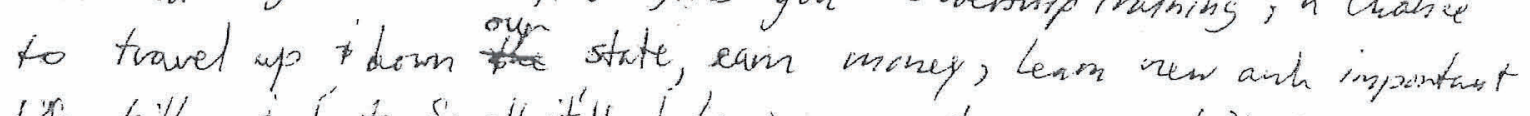

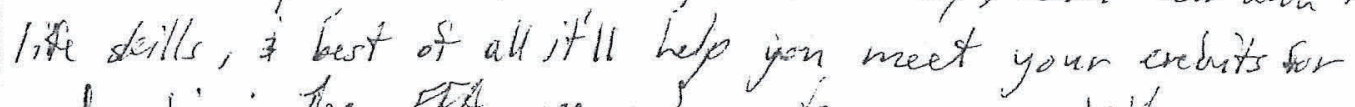
grabuation: The PAA opeens hoors to many Increhible oppourtumitios

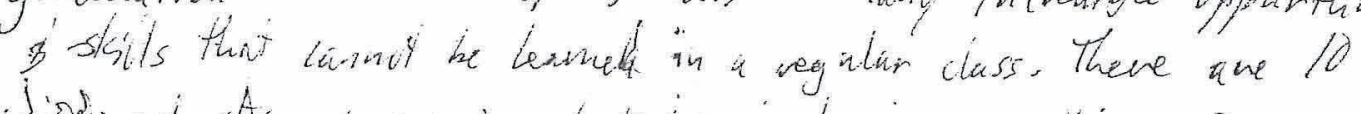

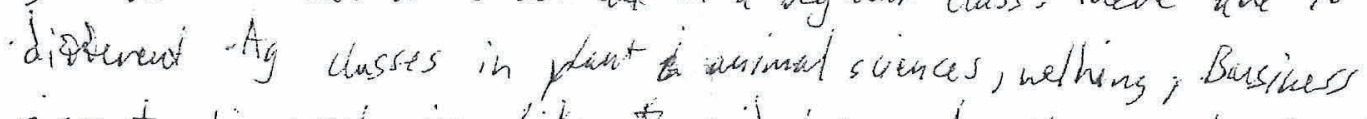

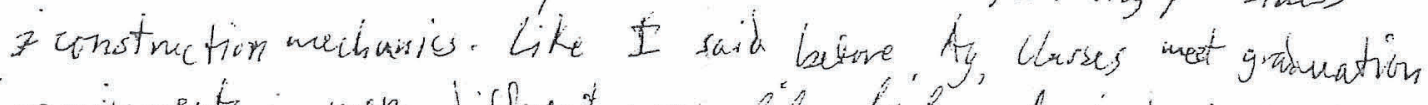

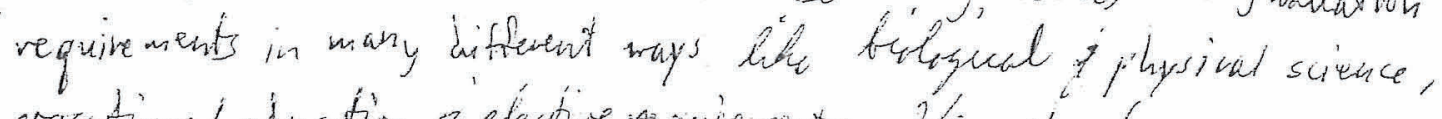

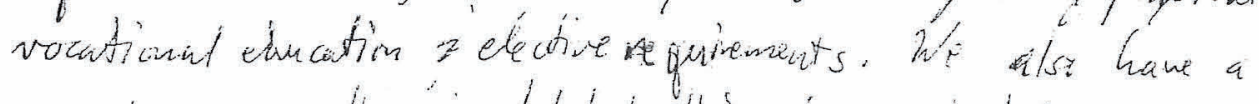
greenhouse, smath animat lab builling i a school tarm in which in which to key your PAR projects:

Self Intiv

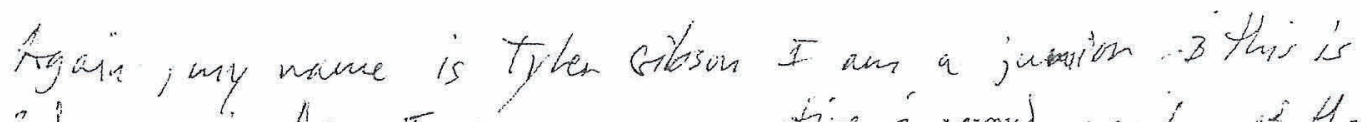

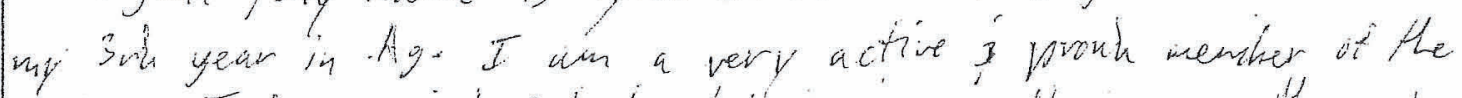
PEt

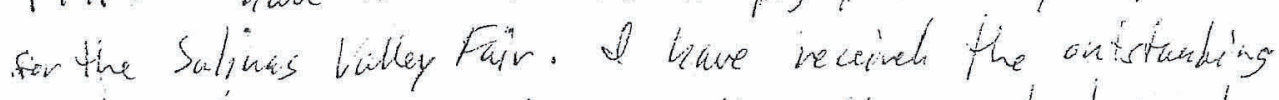

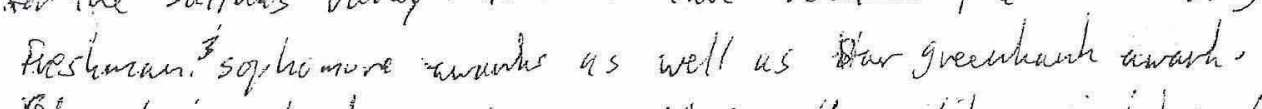

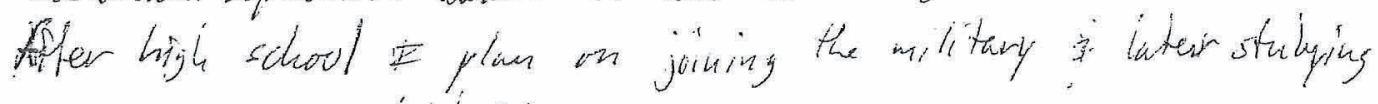
Fo berome a matrine bittujist. 
(1)

(2)

$$
\text { EMILY Karo }
$$
Hey every on My name is emily faro. I am freshman. tr eve been indued in ag for byers. I currently as m raising my lot lamb. His hame is Ralphie. I raise him at the school farm and go twice a day to feed and care fo Ralphie.

(2)

Imam here to talk to you about a SAE. SAE stands for supervised Agricultural experience. SAE is pretty much an FFA project that you do out of class. your FFA project can be lots of things,

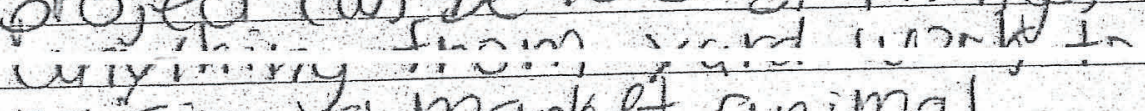
raising a market animal.

(3)

If you do a market animal as your SAE, you can raise pigs, sheep, gats, steers, chickens and Rabbits. There are 3 different fairs you can show at, salinas valley in may, Monterey county in september, and santa cruz county in september. 
(4)

some of the best parts about raising a mar bet animal is you get to have a lot of fun Another thing is, you get to keep the money after yous ell your animal.

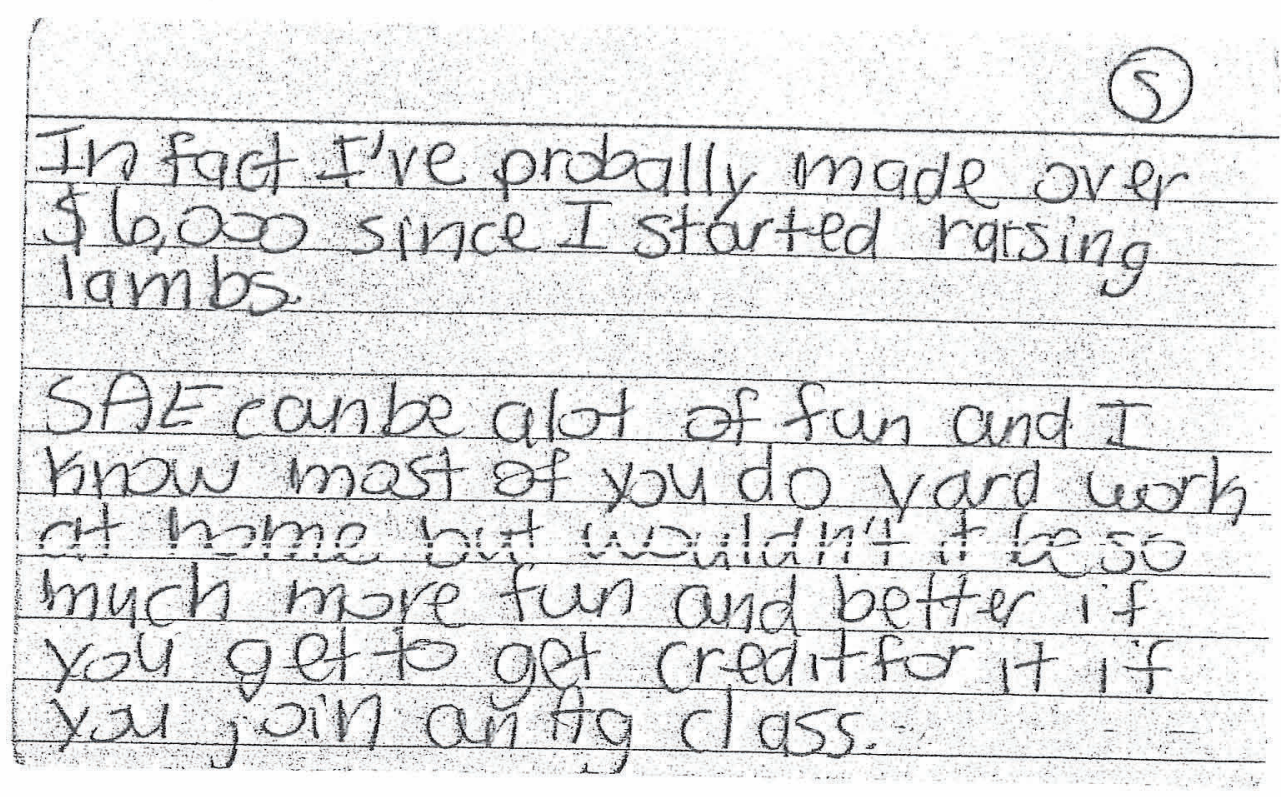

ENDING

(6)

body we talked about the different types of I FA projects xu can do such as pigs and sheep and all the fun you can have going to frA activities and you get to learn a bt from your frA broject. 
morning everyone! How are we all doing morin? * * Alright well as we intoned earlier, my name is Angie Gilliam am a senior in high school and this is hay

(3) worth year being invaded in the Ag programs. I have been around agriculture for over E years. And 1 also have raised ten sheep and 7 pigs. In here to talk to you about FFH.

How many of you want to have a job when you get older?

In FFA, there's countless events that will strength your leadership skills and prepare you for life journeys. Many of the leadership events that we get to go to provide us with public speaking skills and the confidence to speak in front of large crowds. Honestly, if it wast for FFA, and all the activities l would not have the courage and confidence to be standing right here in front of a large crowd. We also get to travel in and down California for different conferences and events. While were out ai these innings, we meet people who become our friends forever. Advanced Leadership Academy, Made for excellence, Greensand Conference, and my favorite, State. Convention! are just a few of the many fun events that nave the chance to attend. $\rightarrow$

Angle Gilliam 


$$
\begin{aligned}
& \text { Melissa n } \\
& \text { Sanchez }
\end{aligned}
$$

(4)
Ag- Science 1-2, Ag. BiO, OH

3 of the many Ag. classes North High offers Are. Ag. Science 1-2, Ag-Biolvay and ornamental haticuture. Aq. Science -intro to ag.

- Ag Basics Such as natural resources Ag.Bio-Designed for clear bound students with

(2)

interest in Ag.

- this class heaps connect big ideas of life science to Agriculture.

ornamental Horticulture -

- teaches about plants and crops. and how to grow

them

- complete your Biological science vocational ed -or elective credits.

fa. earth science

(3)

- meets physical science credits.

- teaches about soil science

- hands on learning

school farm - is a place for Ag Students to keep their fair animals 
greenhouse -

(1)

Un campus and lets students grow their plant projects on campus:

small anima $a b$ - is currently under construction and will be used for small animals like rabbits and chickens.

(5)

The aq classes that we offer are tons of fun and I encourage you to sign up for an ag class. 


\section{MEMIORMTUUM}

Ta: Middle School Fre-registration Coordinators and Teachers of 8th Grade Students Atteriding North Sal inas High School

FROM: Mr. S. Lehiyama, Agricultural Teacher

SUBuECT: Agricultural Classes-

credit Status and Enroliment Pre-registration

DATE:

As you assist students during the class pre-registration process, please fani North Salinas High School information below. Agriculture las students to meet their high school asses cffer many alternatiues for

(1) AGRICULTURE WELDING $1-2$. AG. MECHANICS $1-2$ ALL AG. SCIENCE TYPE CLASSES are Classified a and and meet the Distriet' $\equiv$ vocationial - lass requiremente for graduation.

- AD. Welding 1-2, Ac. Mechanics $1-2$. High. shop-type classes beirg offered at North

(3) AGRICULTURE SCIENCE $1-2$ meets the 10 eredit Intraduction to Bialagy selore - busils.

(3) AGRICULTURE BIOLOGY class gives 10 eredits of Introduction to biolagy

(4) AGRICULTURE PHYSICAL SCI TNCE Searthi SEience fCCEpted as a UC and CSil entrance of physical

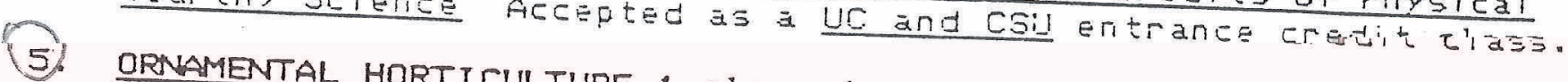

ORNAMENTAL HORTICULTURE 1 elass is a news class that will study indoor and outdoor decorative plants and thein rel will study and man agement.

(5)

Student involvement in FFA and Âg. Enterprise Project activities are academic grades.

If you are familiar with our Ag/FFA program and have seen or heard kow the good things that enrollment has done for students, you at North High. studients will benefit by taking agricultural classes detailed pragram and those interested, the this notice and consideration of encouraping your fou your review of a part of North High's agricultural prograng your students to become 


\section{CALIFORNIA AGRICULTURAL EDUCATION}

\section{Components of an Agricultural Education Program}

They all fit together in harmony. One can not function properly without the others.
A complete Agricultural Education Program includes each.

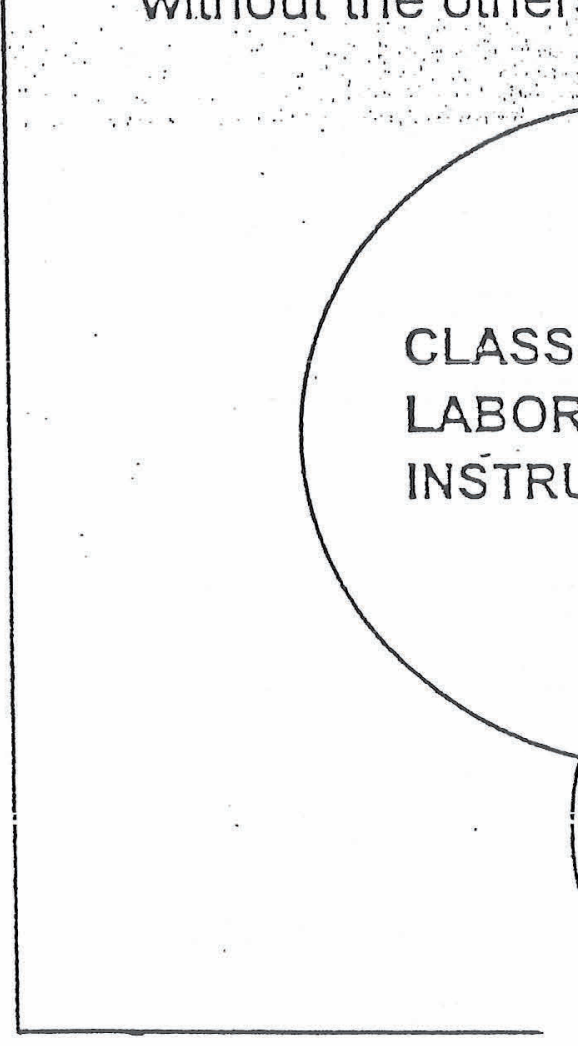

CLASSROOM-LABORATORY INSTRUCTION

AGRICULTURE SCIENCE 1-2

ANIMAL-PLANT SCIENCE

LIUESTOCK-CROPS PRODUCTION

$A G$. BUSINESS MANAGEMENT

$A G$. BIOLOGY

AG. PHYSICAL SCIENCE

AGRI CULTURE WELDING

$A G$. MECHANICS

ROP AG. CLASSES

$A G$. WORK EXPERIENCE

\section{FFA ACTIUITIES}

Chapter, Section, Region, State, and National Levels

FFA LEADERSHIP CONFERENCES CONUENTIONS \& SPECIAL EVENTS FFA OFFICER POSITIONS COMMITTEE CHAIRMEN ACTIUITY ORGANIZING PUBLIC SPEAKING EVENTS CAREER SKILLS COMPETITIONS FFA ACHIEVEMENT DEGREES PARLIAMENTARY PROCEDURE TRAVEL OPPORTUNITIES COMAUNITY SERUICE PROJECTS RECREATIONAL ACTIUITIES ALUARDS AND RECOGNITION MEETING NEW PEOPLE
SUPERUISED AGRI CULTURA: EXPERIENCE Ag. Enterprise Projects

LIVESTOCK PRODUCTION SMALL ANIMAL PRODUCTION PQULTRY \& EXOTIC ANIMALS FOOD CROPS PRODUCTION BUSINESS MANAGEMENT HOME GARDEN GROWING FLOWERING \& HOUSE PLANTS SHRUB AND TREE PRODUCTION BUYING, ADVERTISTIPG, SALE BERRY AND FRUIT PRODUCTION AG. WORK EXPERIENCE JOBS LANDSCAPE INISTALLATION LANDSCAPE MAINTENANCE MONEY MANAGEMENT \& PROFITE HOME IMPROVEMENT CONSTRUCTION PROJECTS WILDLIFE \& CONSERVATION 
AGRI CULTURAL EDUCATION PROGRAM COMPONENTS EXPLAINED

EACH OF THE THREE CIRCLES SCLASSROOM-LABDRATORY INSTRUCTION, FFA ACTIUITIES, AND SUPEFUISED AGRICULTLIRAL EXPERIENCE PROJECTS) ARE EQUALLY IMPORTANT PARTS OF ALL AGRICULTURAL EDUCATION PROGRAMS. THEY ARE INTEGRAL TO THE SUCCESS OF AGRICULTURE STUDENTS. THE WORD INTEGRAL MEANS ESSENTIAL QR NECESSARY FUR COMPLETENESS.

THE CLASSROOM-LABORATORY INSTRUCTION PART PROVIDES ORGANIZED TEACHING AND STUDY, TECHNICAL KNOWLEDGE, ANO LABORATDRY EXPERIENCES IN SPECIFIC AGRICULTURAL SIJEJECTS. IT MAKES STUDENTS ALARE OF THE IMPORTANCE OF AGFICULTURE IN EVERYONE'S LIUES AND HOW COMPLEX THE AGRICULTURE INDISTRY IS. CLASSROOM INGTRUCTION GIVES STUDENTS OPFORTLNITIES FOF CAREER SELELTIDN AND TRAINING.

THE SUPERUISED AGRI CULTLIFAL EXFERIENCE PROIECT (SAEP) FART FROUIDES STUDENTS WITH "LEARN BY DOING" EXFERIENCES EY CONDUCTING ANA AG. ENTERFFISE OF INTEREST. THE AG. FROJEET TEACHES STLIDENTS RESPONSIBILITY, BUSINESS PRACTICES, DECISION MAKING, AND MDNEY MANAGEMENT. TO BE ABLE TO LISE KNDLLEDGE AND SKILLS LEAFNED IN THE CLASSRDOM TO REAL-LIFE SITUATIONS IS THE GOAL OF AN SAEP.

THE FFA ACTIUITIES FART BUILDS LEADERSHIF AND COMMUNICATION SKILLS, AND HELFS TO IMPROVE A STUDENT'S CHANCE'S OF BECOMING MORE SUCCESSFUL IN THE WORLD OF WORK. FFA ACTIUITIES TEACH STLIDENTS HOW TO WORK WITH OTHERS, PLAN AND CONDUCT GRQUP ACTIUITIES, AND PROUIDES AWARDS TO STLIDENTS FOR, THEIR ACHIEVEMENTS. FFA ACTIUITIES ALSO DFFER VALUABLE CAREER TRAINING SKILLS BECAUSE of THE MANY CONTESTS AVAILABLE.

IT HAS EEEN PRONEN THAT INULLUEMENT IN CLASSROOM INSTRLICTION, FFA ACTIUITIES, AND THE SUPERUISED AGEICULTURAL EXPERIENCE FROJECT WILL

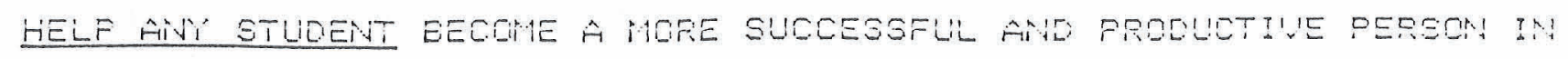
LIFE. 


\section{CAREER PREPARATION PATHS FOR AGRICULTURAL EDUCATION}

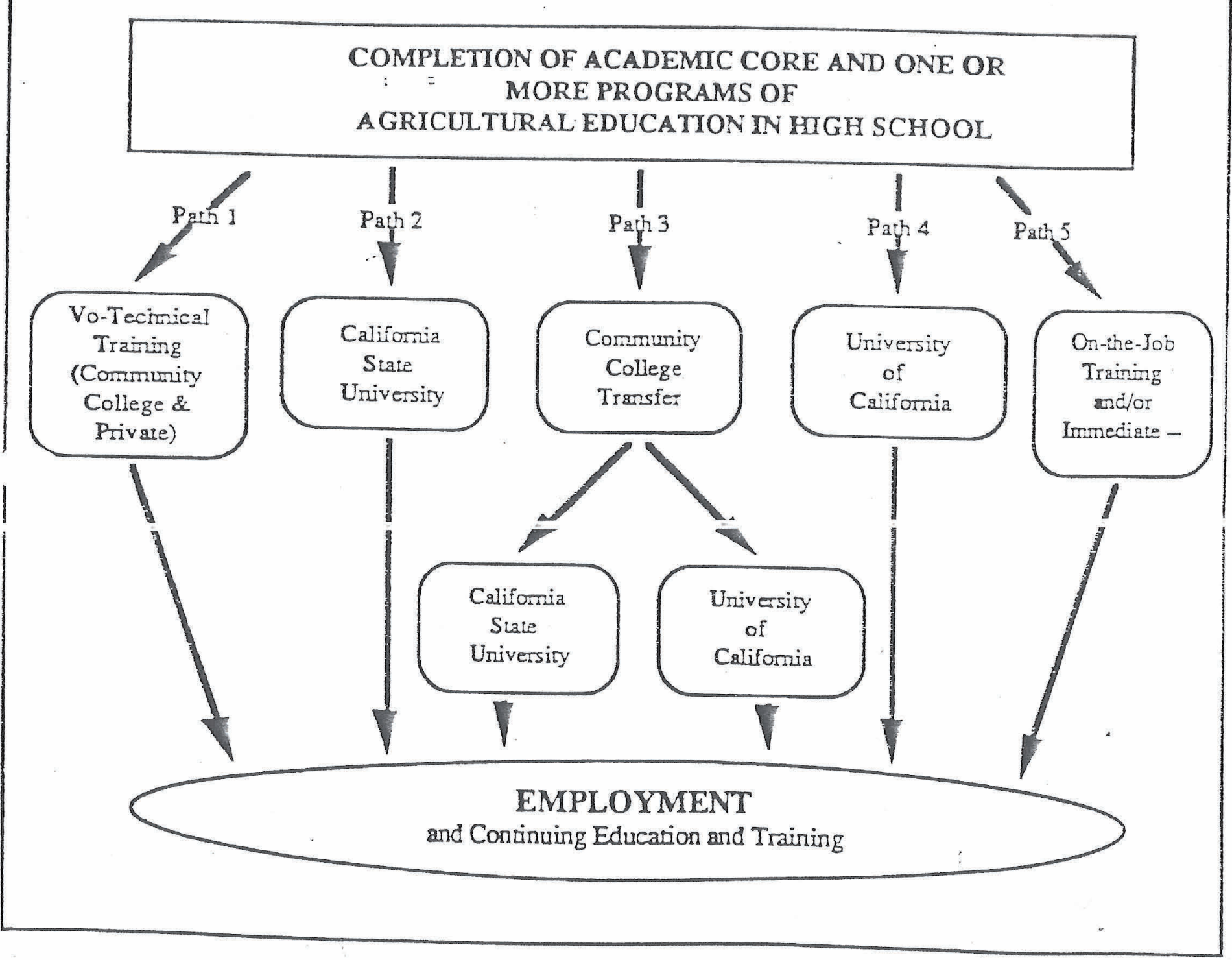

The career paths displayed above are an example of opporturities available to agricultural education students. The paths may remain constant over time; however, retraining loops for adults should be expected as they retum for additional training to learn about new technologies or prepare to enter new or emerging occupations. 


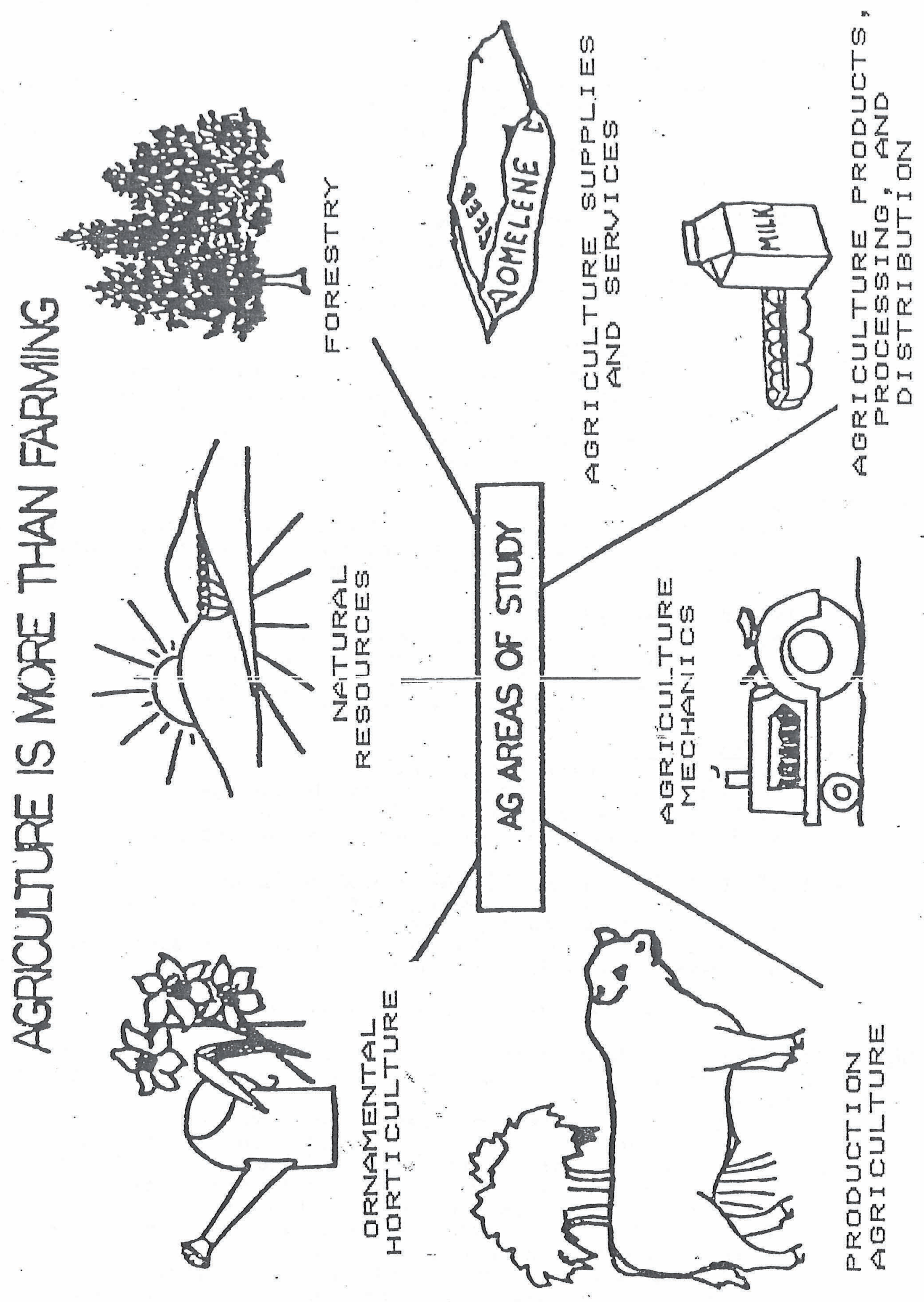


THE SEUEN CAREER INDUSTRIES (PATHWAYS) OF AGRICULTURE ( $8 / 03$ )

1. PRODUCTION AGRICULTURE--FOOd Crops and A A imals.

a. Livestock - beef, sheep, swiriè, dairy, poultry, horse, goats, rabtits, fish farms and exotic animals.

b. Crops - vegetables, grains, farage, fruits, nuts, citrus, vineyards, special crops.

c. Crop and animal research, farm and ranch management, etc.

2. AGRICULTURE SUPPLIES AND SERUICES

a. Specialization in seeds, fertilizers, pesticides, livestock feeds, equipment, banking, repairs.

b. Advertising, sales, marketing, transportation.

c Veterinary services, management and consulting, etc.

3. ORNAMENTAL HORTICULTURE (decorative plants)

a. Landscapirg - design, installation, maintenaree.

b. Turf and golf couress - production, greenskeeper.

c. Flower fol iage, shrubs, and tree production.

d. Flori Etry - cut flowers and arrangements.

e. Orrimental pest and disease control, etc.

4. FORESTRY

a. Paper and lumber products, lumber mills, logging, sales. -

b. Tree production, forest management, wood technology.

c. Fecreation (hunting, fishing, camping) services, etc.

5. AGRI CULTURE MECHANICS

a. Equifment construction, inspectian, machinist.

b. Fepairs and services to equipment, seles.

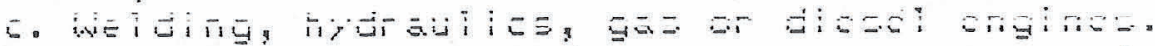

d. Engineering and design, surveyar, researeh, etc.

6. NATURAL RESOURCES

a. Forest and Fark Management

b. Wetlands. Water Management and hydrology.

c. Soil Maragement, Rangeland Management.

d. Fish and Wildife Management. Marine Biology.

7. AGRICULTURE PRODUCTS, PROCESSING, and DISTRIBUTION

a. Canririg, freezing, meat processing, inspection.

b. Packaging, product grader, equipment aperator.

c. Product transpartation, distribution, product buyer

d. Research, new food product development, etc.

$++t+t+t+t+t+t+t+t+t+t+t+t+t+t+t+t+t+t+$

AGRISCIENCE PROFESSIONS Jobs that can $f i t$ in any of the Ag. Career

Pathways. Requires aduariced knowledge, special training, and usually a college degree.

2. Ag teacher, extension Epecialist, journalist.

七. Ag Lawyer, accountant, economist, lator relations.

c. Animal or plant geneticist, siliviculturist, bicengineer.

d. Physiologist, botanist, biochemist, zoologist

e. Bacteriologist, entamalagist, pathologist.

f. Marine biglagist, food chemist, ueterinary medicine.

9. Nutritionist, engineers, resesrch.

h. Managers, marketing, consultants, advertising.

i. Computer specialists, international marketing, etc. 


\section{All students in ag. clavires 2. Section - Monterey Bay section. FFA 3. Region - South Coast Region FFA 4. State - California Asoociation Fi=P 5. National- National FFA organization nembers}

\section{What is FFA?}

FFA is a dynamic youth organization within agricultural educarion that changes lives and prepares students for premier leadership, persona! growit and cirest sucus. FFA was created in 1928 as Future Farmers of America; the name was changed in 1988 to the National FFA Organization to represent the growing diversity of agriculture. Today, almost half a million student members are engaged in a wide range of agriculoural education activities, leading to over 300 career opportunities int the food, fiber and natural resources industry. Studehr success remains the primary mission of FFA.

What do the letters F-F-A stand for?

The letters FFA seand for Future Farmers of America; however, the official name of the organization was changed in 1988 to the Nacional FFA Organization. Over the past 75 years, FFA and agriculural education have grown to encompass all aspects of agriculture, from produc:cion farming, agribusiness and forestry to biotechnology, marketing and food processing.

\section{The Mission and Strategies}

FFA makes a positive difference in the lives of students by developing their potential for premier leadership, personal growth and career success through agricuitural education.

To accomplish this mission, FFA:

- Develops competent and assertive agricultural leadership.

- Increases awareness of the global and technological importance of agriculture and its contribution to our well-being.

- Strengthens the confidence of agriculture students in themselves and their work.

- Promotes the intelligent choice and establishment of an agricultural career.

- Encourages achievement in supervised agricultural experience programs.

- Encourages wise management of economic, environmental and human resources of the community.

- Develops interpersonal skills in teamwork, communications, human relations and social interaction.

- Builds character and promotes citizenship, volunteerism and patriotism.

- Promotes cooperation and cooperative attitudes among all people.

- Promotes healthy lifestyles.

- Encourages excellence in scholarship. 


\section{Emblem}

The national FFA emblem, consisting of five symbols, is representative of the history, goals and future of the organization. As a whole, the emblem covers the broad spectrum of FFA and agriculture. Each element within the emblem has unique significance.

The cross section of the ear of corn provides the foundation of the emblem, just as com has historically served as the foundation crop of American agriculture. It is also a symbol of unity, as corn is grown in every state of the nation.

The rising sun signifies progress and holds a promise that tomorrow will bring a new day glowing with opporturity.

The plow signifies labor and tillage of the soil, the backbone of agriculture and the historic foundation of our country's strength.

The eagle is a narional symbol which

serves as a reminder of our treedom and ability to explore new horizons for the future of agriculture.

The owh, long recognized for its wisdom, symbolizes the knowledge required to be successful in the industry of agriculture.

The words "Agricultural Education" and "FFA" are emblazoned in the center to signify the combination of learning and leadership necessary for progressive agriculture.

The emblem and the letters "FFA" are protected by uademark registration in the U.S. Patent Office and by Public Law 225, 105 th Congress.
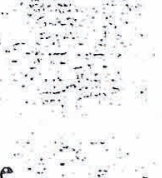

$\therefore$
$\therefore$
$\therefore$
$\therefore \quad$
$\therefore$




\section{IDEA LIST-- AG/FFA ACTIUITY PARTICIPATION and AWARDS \\ STUDENT LEADERSHIP AND PERSONAL SKILLS DEVELOPMENT PROGRAM
(reVised $11 / 9 \mathrm{3}$ )}

REQUIREMENT. The setting of personal goals and achievements are just as important to a person's success as their planning for college entrance or job placement. Every student in agriculture is required to participate in a minimum of two different $A Q / F F A$ activities per semester. To get the most from the $A g / F F A$

leadership program, one of the four yearly activities should include the achieument of an FFA Degree, participation in a skills development/leadership event, or attend a leadership conference. Some Aq/FFA events/achievements require a step-by-step advancement process or are limited to certain arade levels. Awards are not considered an actiuity, but are the result of your level and quality of activity participation. Ten Dercent of your class arade is based on your minimum Ag/FFA activity inuoluement.

\section{EXAMPLES OF}

GENERAL AG/FFA ACTIUITIES---EVERYONE CAN PARTICIPATE IN

(chapter actiuities will yary from year-ta-year)

- Attend monthly FFA meetings.

- Help with school AgFFA displays.

- Participate in FFA Week activities.

- Help uith Ag.FFA Recruitment actiuities.

- Help with County Fair Booth exhibits.

- Get parents involved in Ag. Booster Club.

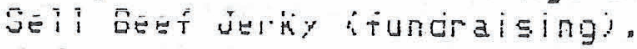

- Sell Ag. Calendare (fundraising).

- Help with Echool canned food drives.

- Help with Firewood sales fundraiser.

- Sell Donkey Basketball tickets.

- Participate in Schoor Farm wark days.

- Be on a Chapter activity committee.

- Be on Ag. Marketing (Co-Dp Quiz) Team.

- Participate in Job Intervieu contest.
- Attend New Student. Artiuity.

- Attend binter fiuards Dinner.

- Attend Parent-Member Awards

Eanquet at end-of-year.

- Help on Petting zoo exhibits.

- Help with fair equipment preparations.

- Enter spring Chapter SAE Project competition.

- Saluation Army Christmas Kettle fundraising.

- Participate in Chapter SAEP Record Keeping Contest.

- Exhibit prajects at County Fairs.

\section{Career Development} Events ${ }^{\oplus}$ (CDEs)

Also called "Judging Teans" and are made up of three members. Requires team practices (January - May) and three to five week-end travel trips to contests. Individual and team awards given. Some contests have grade level participation restrictions: are one person competitions and held only once during the year. State and National championship titles auarded.

(See attached State and National Career Development contest 1 ist.) 
STUDENT GUIDE FOR FFA DEGREE ACHIEUEMENTS, LEADERSHIP. DEUELOPMENT EUENTS, LEADERSHIP ROLES, AND ANARDS BY GRADE LEUEL

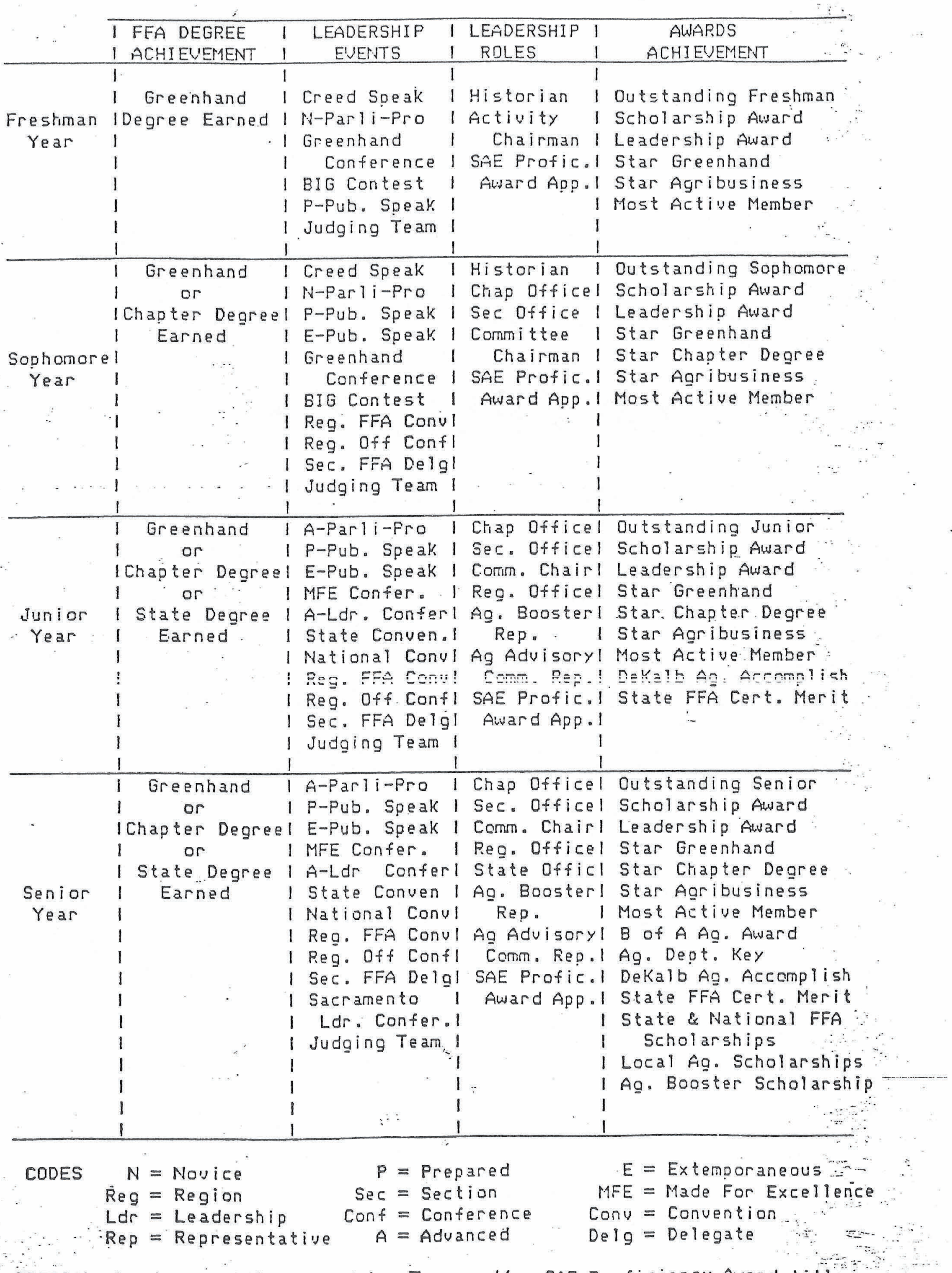

SPECIAL: Must name kind of Judging Team and'or SAE Proficiency Award title. 
AGRI SLIENCE AND TECHNIILOGY DEPT.

\section{SAE programs}

\section{IDEA LIST-D-ENTERPRISE PROJECTS and AWARDS \\ SUPERUISED AGRICULTURE EXPERI ENCE PROGRAM \\ (revised $11 / 78$ )}

REQUIREMENT. Every student in agriculture is required to conduct a minimum of one agriculture experience project (SAEP) for each year they are enrolled in an agriculture class. The minimum SAEP standards are: (a)"must be agricultural; (b) must involue out-of-class labor by the student: (c) no financial investment is required; and (d) must be of sufficient size and challenge to allow for regular (4 activity per month minimum). long-term record keping. Enterprise projects do not haue to be related to a specific agriculture class (example: You can raise plants or animals and be enrolled in a welding class). First year students need to have plans to conduct an SAEP and show reasonable effort to accomplish their selected enterprise. Continuing students should conduct an on-going SAEP each year that shows "growth" or diversity. Ten percent of class grade is based on your SAEP involvement.

Enterprise projects are classified as OuNERSHIP and NON-ChNERSHIP. Qiunershio enterprises are those that you pay expenses for, hope to make an income and profit from, and where-you make the decisions (manage) what happens to the enterprise. Plon-ounershid enterprises are those that you do not have any control ouer what is done and you may or may not get paid for your labor.

\section{EXAMPLES OF}

ANIMAL ENTERPRISE PROJECTS-CLINERSHIP

Raised for meat food sales, eggs sales, milk production, pet sales, wool production, pleasure, training, or competitive shouing. Raise for treeding purpases. \{sa?\} offapring as feeder or repiacement stack).

\begin{tabular}{|c|c|}
\hline IUESTOCK & $=1$ \\
\hline SUA I RE & Fateits \\
\hline Sheep & Ehigrens \\
\hline BeEf Catt & Turkey= \\
\hline Dairy cattle & Freasante \\
\hline Drop C.31, & D」にks \\
\hline Hor $\Xi E \Xi$ & Gee \\
\hline Goat: & Euзі\} \\
\hline
\end{tabular}

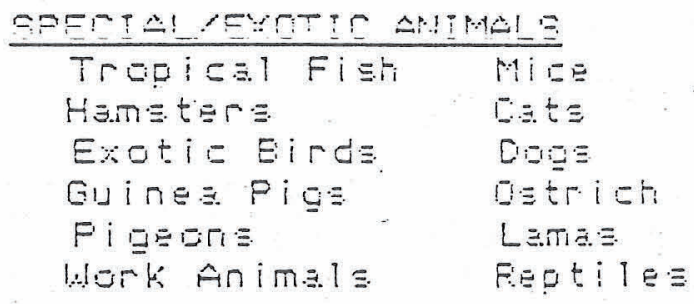

CROPS AND GARDEN FOOD ENTERPRISE PROJECTS--OWNERSHIP

Grow as a home garden for fanily food consumption. Sell vegetables to neighbors or at Farmer's Markets. Erow transplants for sale or oroly livestock feed, care for tree crope.

UEGETABLES. FRUITS. AND RERPIES.

Vege tables--fall/winter types

Vegetables--spring/summer types

Fruit Tree Crops. Grape Vineyard.

Strauberries, Raspberries, etc.
SPECIALL CRDF PFO.TECTS

Vegetable Sesdling Transplants

Qat or Alfalfa Hay Prouduction

Feed Grain Production

Fasture or Silage crops

Christmas Tree Production

\section{OPNAMENTAL HORTI CULTURE ENTERPRISE PROJECTS--CWNERSHIP}

Graw house and garden plants for home use, landscaping and beautification. Sall plants to neighbors and nurseries. Sell cut flowers for floral arrangements or orow specialty plants. Conduct landseaping jobs for profit.

Plant Production

Flower Garden

Foliage House Plants

Tree Seedlings.

Shrub seedlings

Flower Trananlante
Feid liark Exgerience

Eet bun equipment and sufplies to conduet landacape maintenance - jabs for neighters or st hims.
Soecialty Proiecte Sell Flouser Arrangements Make Terrariums Tropital Fish Fiants Cut Flouer Sales rartile a Eurrulant Plant 
Frouide own equipment, materials, and supplies to construct-projects for own needs or 5 ale to others. Repair projects for profit. Perform job services for profit.

Constructed Pro.jects

Livestork Tack Euxes

Ear-becue Pits \& Farks

Sheep Triming Tables

Beef Trimming Chutes

Fence Parrels and Gates

Utility Trailers

Tool Boxes. Signe

Sulire Transport Crate

Truek Racks

Electrical, Flumting Syetems

Rabbit cages.

Landaraping Strue tumers

Special Equipment/ToGl Making
Paid Wark Exoerierice

Get cun equipment arid supplies to conduct constructior/refa ir. jobs for neightors ar at home.

Fenairira Frojects

Emall Engine Fepair

Sharpen Hand Togl E

Garden Tool Fiepair

Pepair construeted Equipment

Pedesign Equipment

other Fiefair Needs

AGRICULTURE BUSINESS MANAGEMENT ENTERPIRSE PROJECTS-- NON-OWNERSHIP Regular and on-going agricultural work performed for someone else. You may geb paid, receive no pay, or get paid sometimes.

Landscape Mainterinee-rarduork

Feed Stare Clerkhlarker

General Ranch or Farm horker.

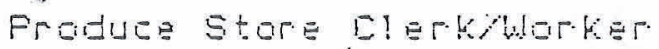

lidelding shop worker

Dairy Farm Worker

Arg. Serviceisuply Co. Werker.

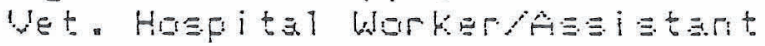
Auctian Yard barker.

Aq. Har dware Etore Clerklubrer

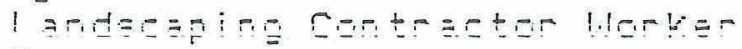

produre Salig Co. linkker
Ir igetar for Farmifanch

Fogd Proceseing Flant horker

Euteher Stare Clerkyorker

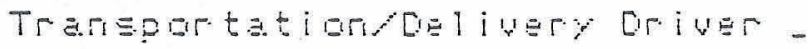

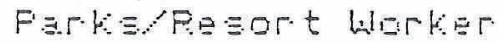

Firelugid cuttiriggales hurker

Equipmert Bal EEREFair Ca. hurker Nurearygarden Center blorker

Pet Stare Cler.k. SPLA harker.

Tractor Dri tuer.

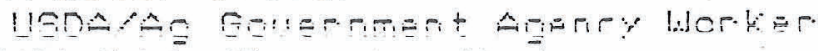

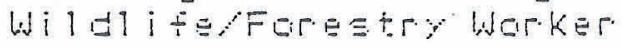

\section{SPECIAL INTEREST ENTERPRISE PROJECT AREAS}

In addition to the more cominon project ideas listed above, enterprise projects car also be conducted in Natural Resources. Forestry, Rural Recreation, Wildi ife Management, Soil Conservation, and Ag. Research

Growing Trees for Refarestation

River/Wildife Habitat Cleanup

Wildife Surueysprotection

Wildife/Fish Habitat Construction
Wi dife,Fish Habitat Festoration

Soil Conservation Pl antings

Nature Trail. Construction

Parks and Récreation Area Cleanup

\section{SAEP PROFICIENCY AWARDS--EUERYONE CAN APPLY FOR ONE}

Awards given in ounership and non-ounership SAE areas at Chapter, Section, Reqion, State, and National levels. No restriction on grade level qualification.

(See attached Proficiency Awards listing.) 


\section{CALIFORNLA DEPARTMENT OF EDUCATION AGRICULTURAL EDUCATION}

\section{SUPERVISED AGRICULTURAL EXPERIENCE PROJECTS}

A project is acceptable as an SAE until that product leaves the students control. For example: If a student owns an apple orchard and sells at a road side stand, the student could also make apple pies and sell them in the family restaurant. However, if the student did not own the apple trees, then making apple pies would not be considered an acceptable project.

Acceptable Entrepreneurship SAE's:

Agriscieace Fair (outside of class)

Agricultural Mechanics

Aquaculture

Bees

Breeding and Market Beef

Breeding and Market Goats

Breeding and Riding Horses

Breeding and Marketing Lizards, Snakes,

Mice, etc.

Breeding and Market Rabbits

Breeding and Market Sheep

Diccdies äd iviânitet Swiut

Breeding Dogs

Chickens - Meat and Eggs

Christmas Trees

Dairy Cattle

Dairy Replacement Heifers

Emu Production

Field Crop Production

Firewood Business

Floral Business

Fruit Tree Production

Game Bird Production

Garden Production

Greenhouse Production

Horseshoeing Business

Hydroponics Production

Home Animal Care Service

Llama Production

Nut Tree Production

Ûstrich Production

Raising Worms

Ratite Production

Turkey Production

Veal Calves

Vegetable Crop Production

Vine Production

Wildlife Assistant or Field Worker

Acceptable Placement SAE's:

Ag Chemical Salesperson

Feed Store

Ag Equipment Operator

Ag Publicity Director or Writer

Fish Harvester

Floral Worker

Ag Pest Worker

Food Safety Assistant

Garden Lawn or Landscape Worker

Ag Research Lab or Field Assistant

Hatchery Worker

Ag Water Sample Collector

Ag Welder

Horse Stable Worker

Ag Worker at Fair

House Plant Care Worker

Conservation Resources Worker.

Landscape Maintenance Worker

Dog Kennel Worker

Meat Cutter/Processor

Farm Worker (Crops or Livestock)

Parts Store Worker in Agriculture Business 


\section{Chapter Scrapbook}

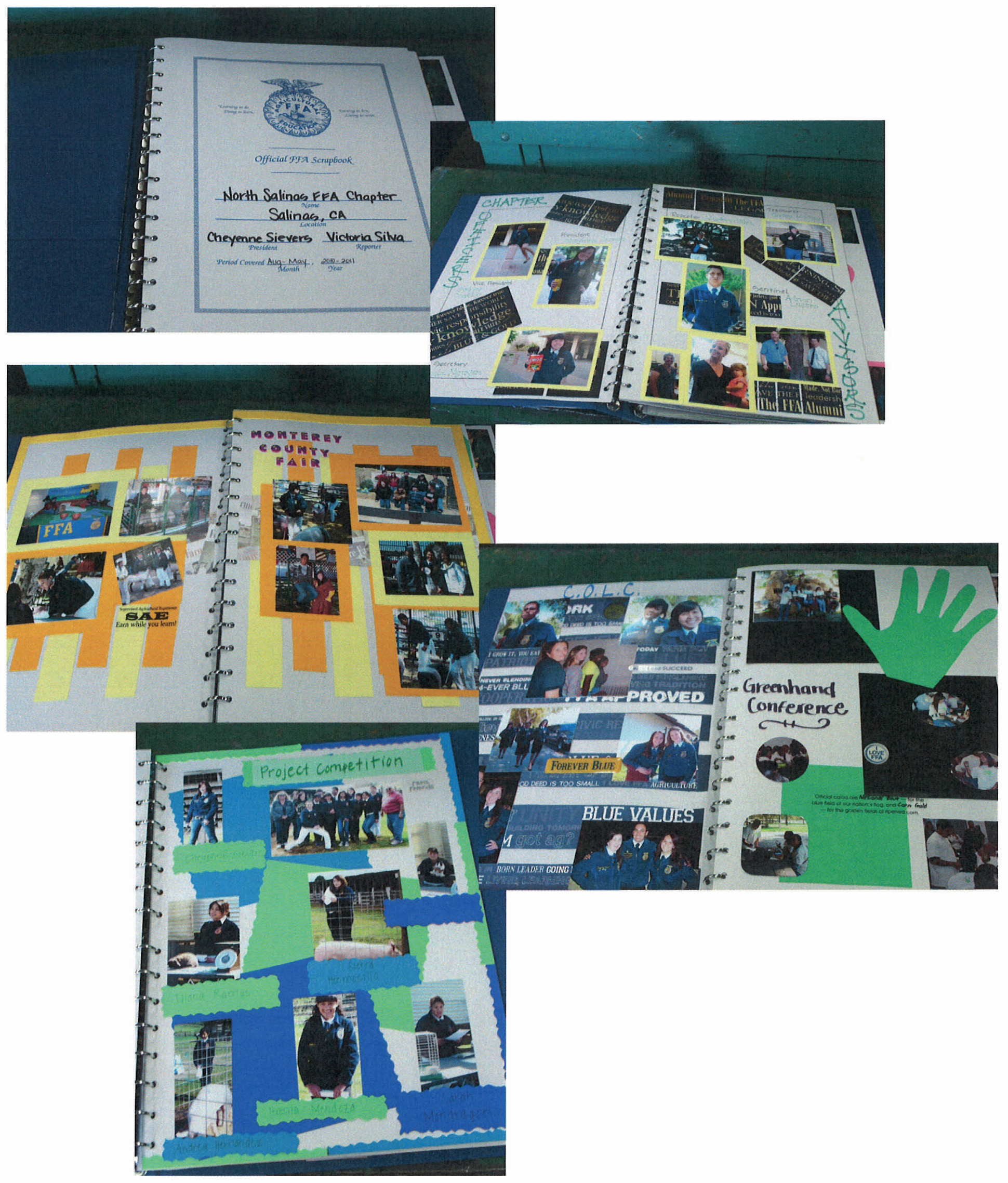




\section{Graduate Follow-up Report \\ Year $=2010$}

\# CA0200 Salinas - North Salinas

I i Salinas HS

53 Kip Dr.

Salinas, CA 93906

Printed: 10/4/2010 11:52:46 PM

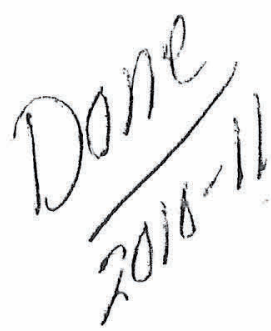

\begin{tabular}{|c|c|}
\hline Total Seniors (Year=2009): & 37 \\
\hline $\begin{array}{l}\text { Total Seniors having completed } \\
3 \text { or more years of Ag Instruction: }\end{array}$ & 1 \\
\hline \multicolumn{2}{|l|}{ Program Completer Status } \\
\hline Two Year College Non-Ag Major & 1 \\
\hline
\end{tabular}

CalAaEd Home I R-2 Home I R-2 Online Home I Roster | Teacher I FAQ I Help | Log Out Site developed and maintained by ATI Net, Califormia State University, Fresno with funds from the California Department of Education,
Agricultural Education Unit.

\section{Graduate Follow-up}

\# CA0200 Salinas - North Salinas

North Salinas HS

55 Kip Dr.

Salinas, CA 93906

Year: 2010 Go

Last Name First Name Graduate Status

Sanchez Melissa Two Year College-Non-Ag Major

Printed: 10/4/2010 11:50:56 PM

Count: 1 
GRADUATE SURVEY SUMMARY

FOLIOH-UP OF VO-AG PROGRAM GRADUATE PIACGMENT

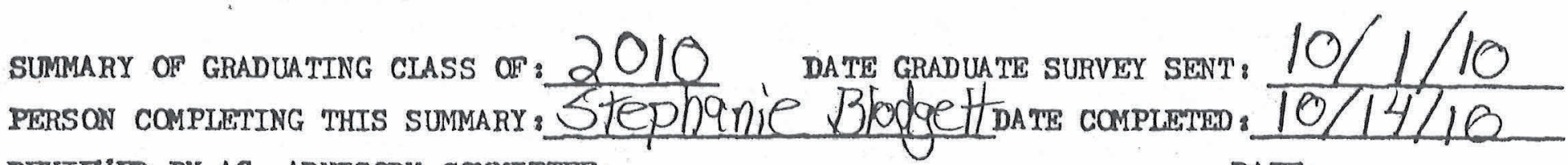
REVIEW BY AY. ADVISORY COMMITTEE:

(chalrpers on verification)

DATE 8

I. GENERAL SURVEY DATA

1. Total number of program graduates andor completors in $\frac{20 / 0}{(\text { year })}=$

2. Total number of program graduates and/or completors surveyed...... =

a. Nunber male: $Q=0$ \% of group surveyed

b. Number female: $2=100 \%$ of group surveyed

3. Total number of program graduates and/or completors responding.... = to survey.
a. Number male:
0
0
go of group surveyed
b. Number female: $=$ $0(2$ $\%$ of group surveyed

4. Return rate of graduates and/or completors surveyed (male \& female) $=100 \%$

\section{ADVANCED EDUCATION DATA}

1. Number that went on to advanced edueation...............

(a) Number pursuing education in agriculture studies $=50 \%$

b. Number pursuing education in non-agric. studies. $1=50 \%$

c. Number in full-time advanced education............. $=50 \%$

d. Number in part-time advanced education, no work. . 1 $=50 \%$ included.

\section{MORK FORCE ENTRANCE DATA}

1. Number that went to work directly after graduation....... $\quad=0$

a. Number that went to work in agriculture occupation for which they had prepared for

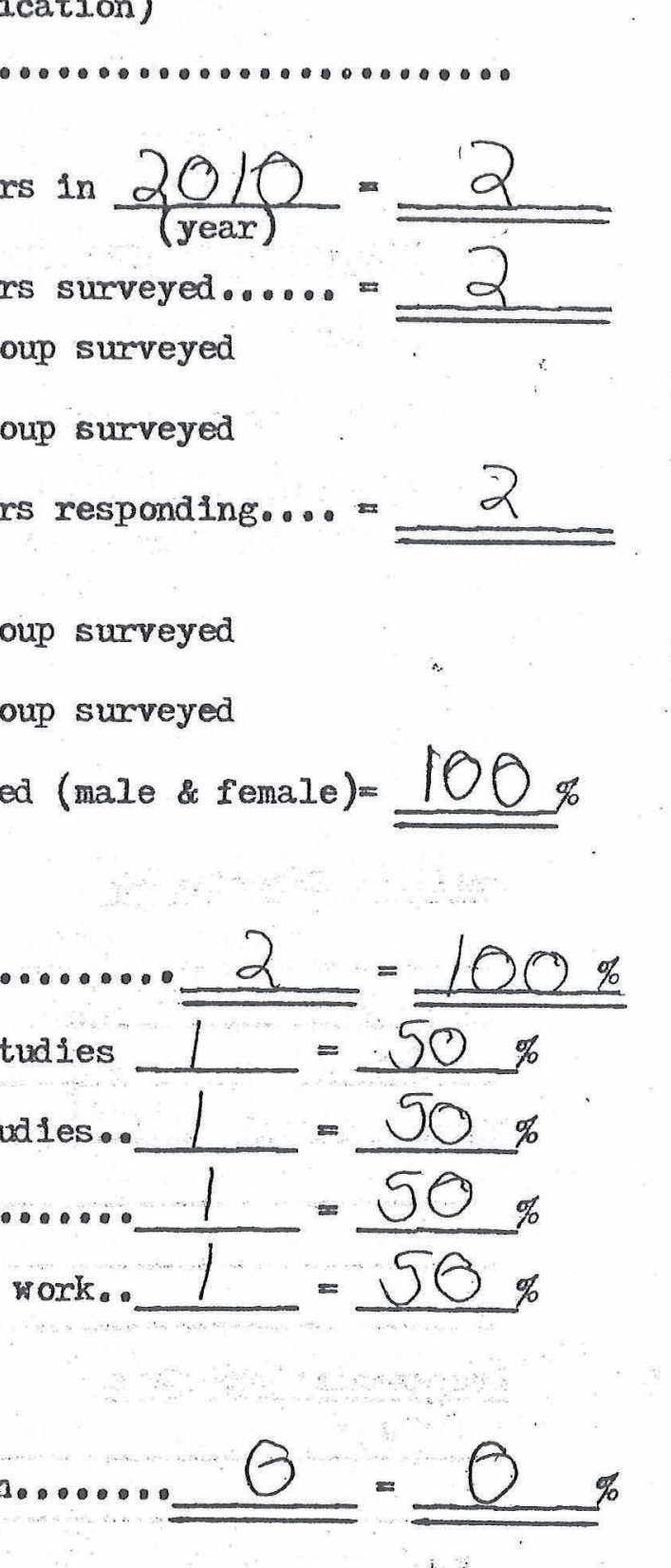

(b.) Number that went to work in agriculture occupations different than that for which they prepared for..

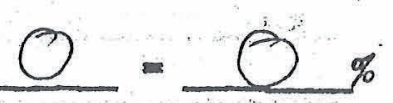

Number that went to work in non-agriculture

occupations.

d. Number working full-time in their occupation.
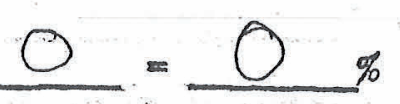

a. Wumber working puli-tine in their occupation.....-

e. Number working part-time in their occupation..... $\bigcirc=$ (2) (no advanced education included) 
IV. COMBINATION ADVANCED EDUCATION AND WORK DATA

1. Number of graduates and/or program completors that are both

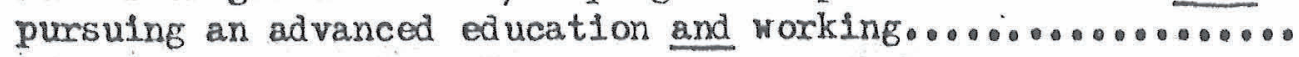

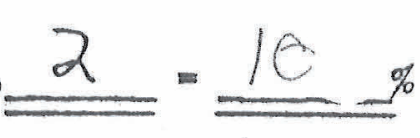

(a.) Number pursuing education In agriculture and working part-time in agriculture........

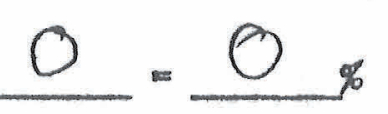

6. Number pursuing education in agrlculture and working paxt-time in non-agriculture area...

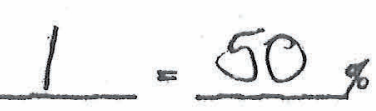

- (c) Number working part-time in agriculture and pursuing education in non-agriculture studies

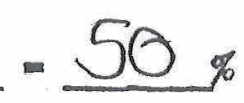

V. PIACEIENT IN AGRICULTURE DATA (Refex to parts II, III, and IV)

1. Number pursuing advanced education in agriculture studies

2. Number that went to work directly in an agric. occupation

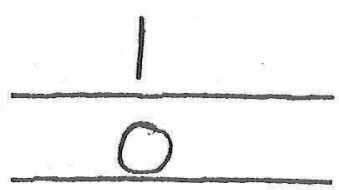

3. Number pursuing part-time education in agriculture or working part-time in an agriculture occupation

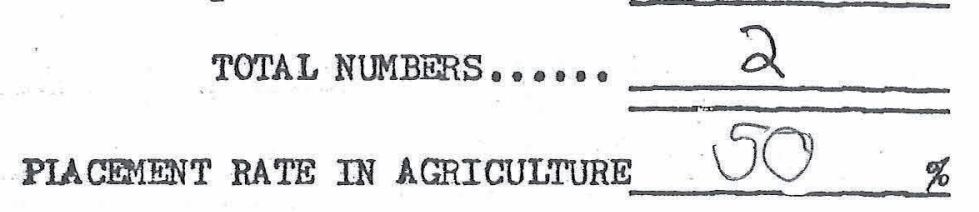

SURVEY RESPONSE COMMERTS

Positive Experiences

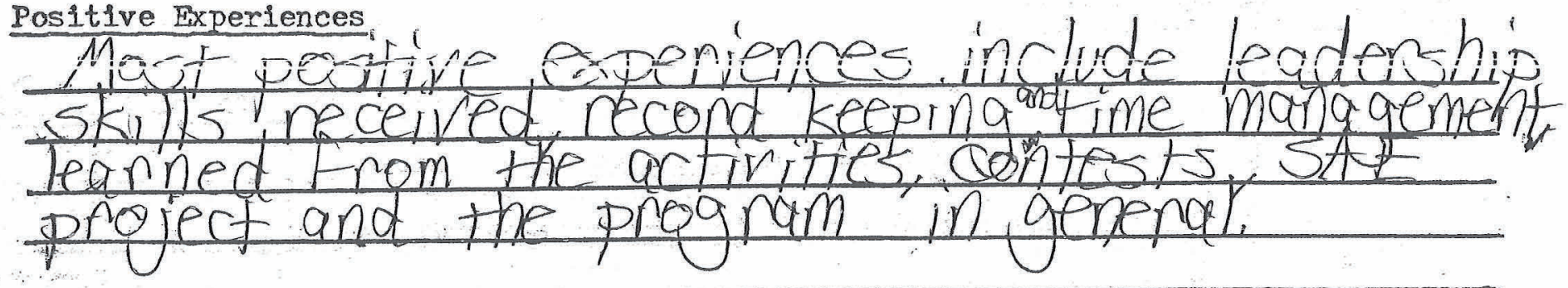

Recommendations Made

none 


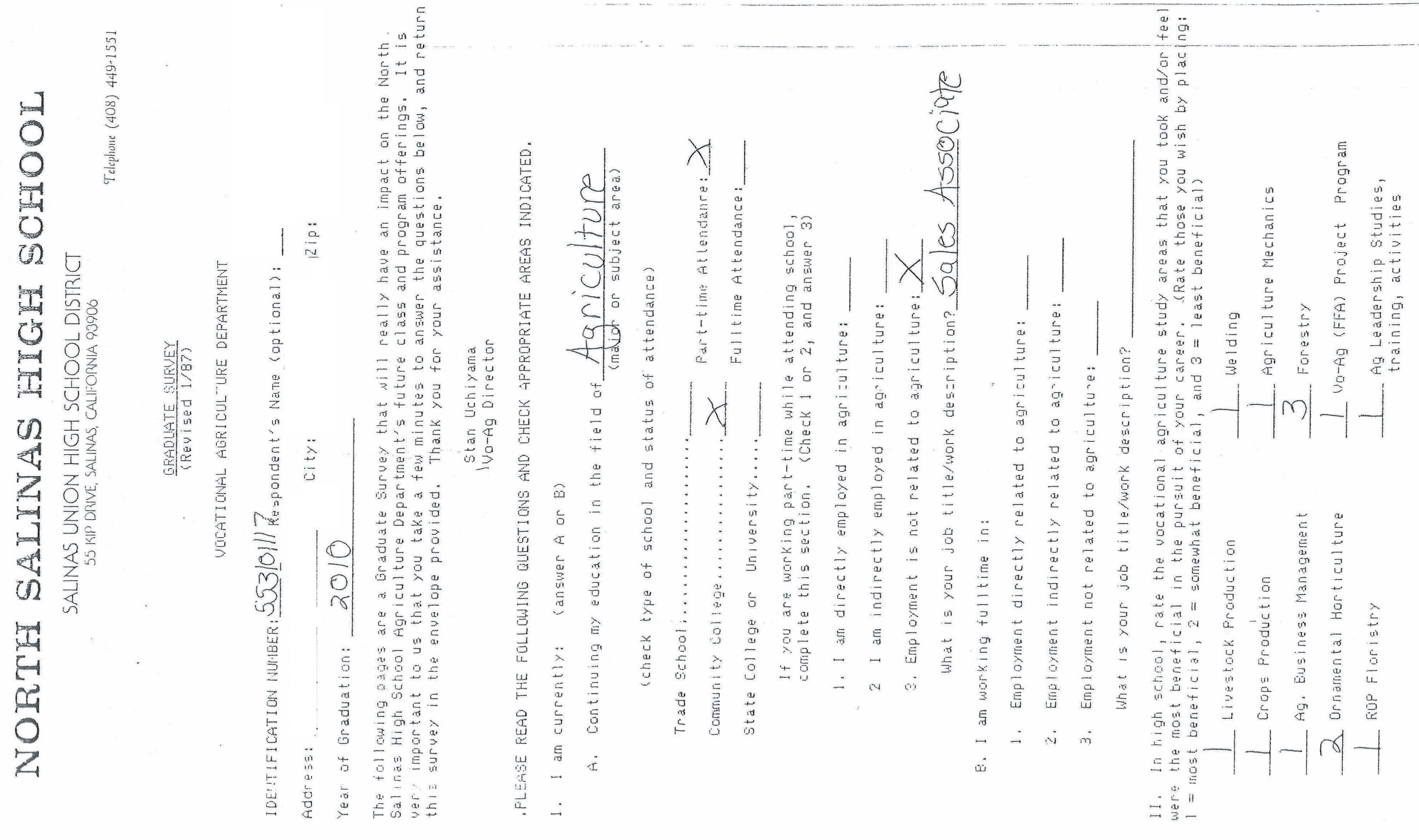



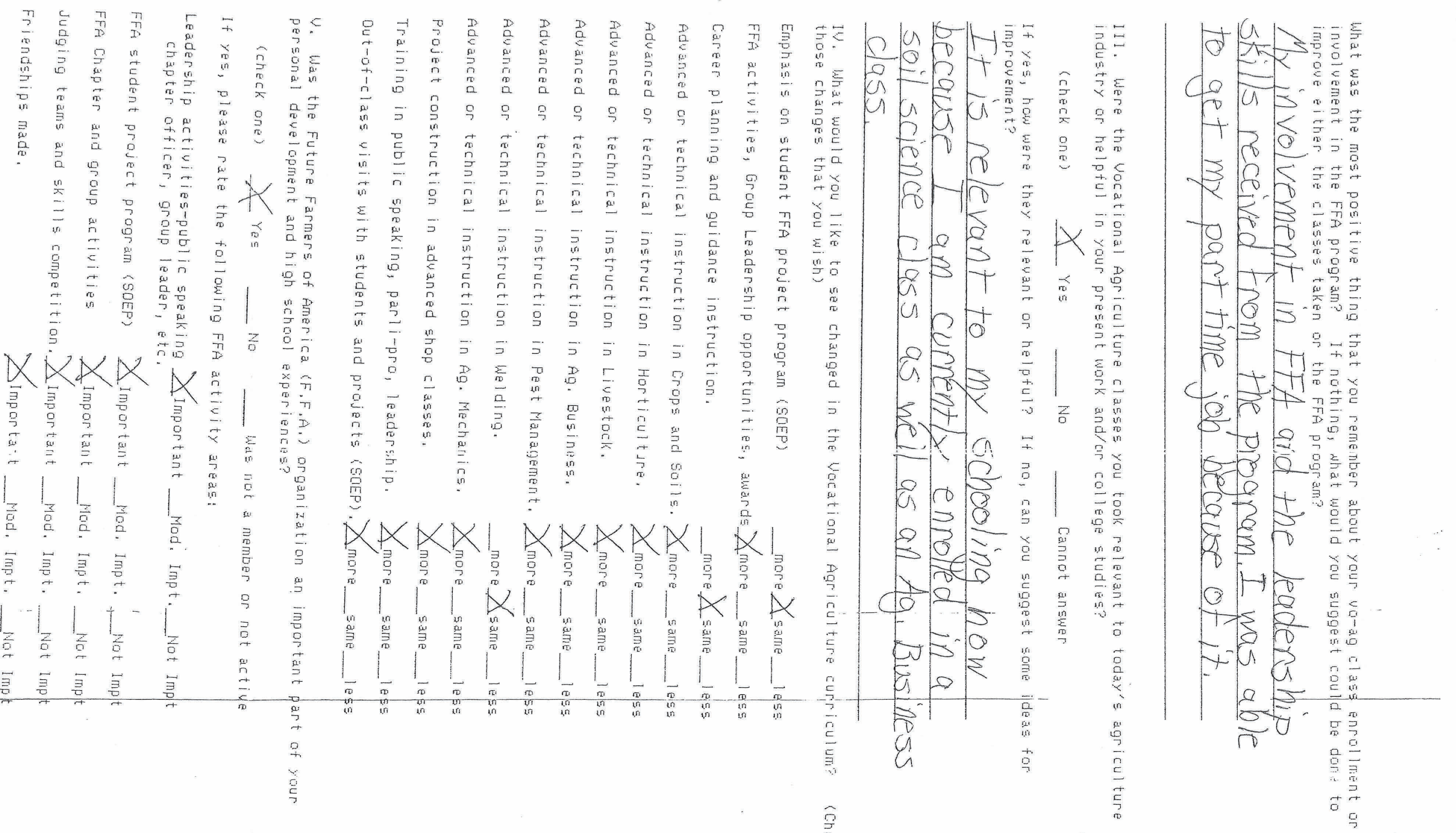

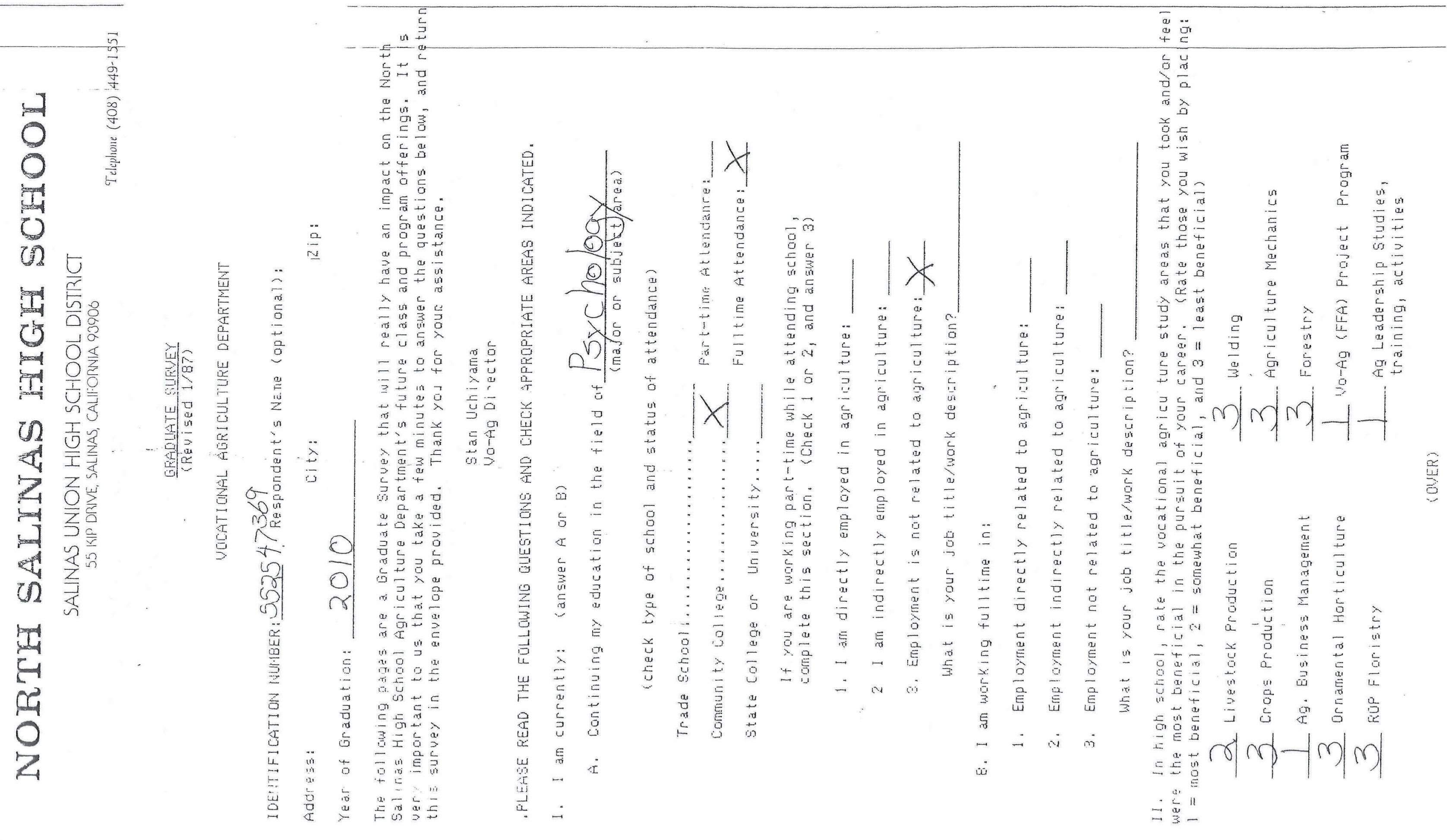

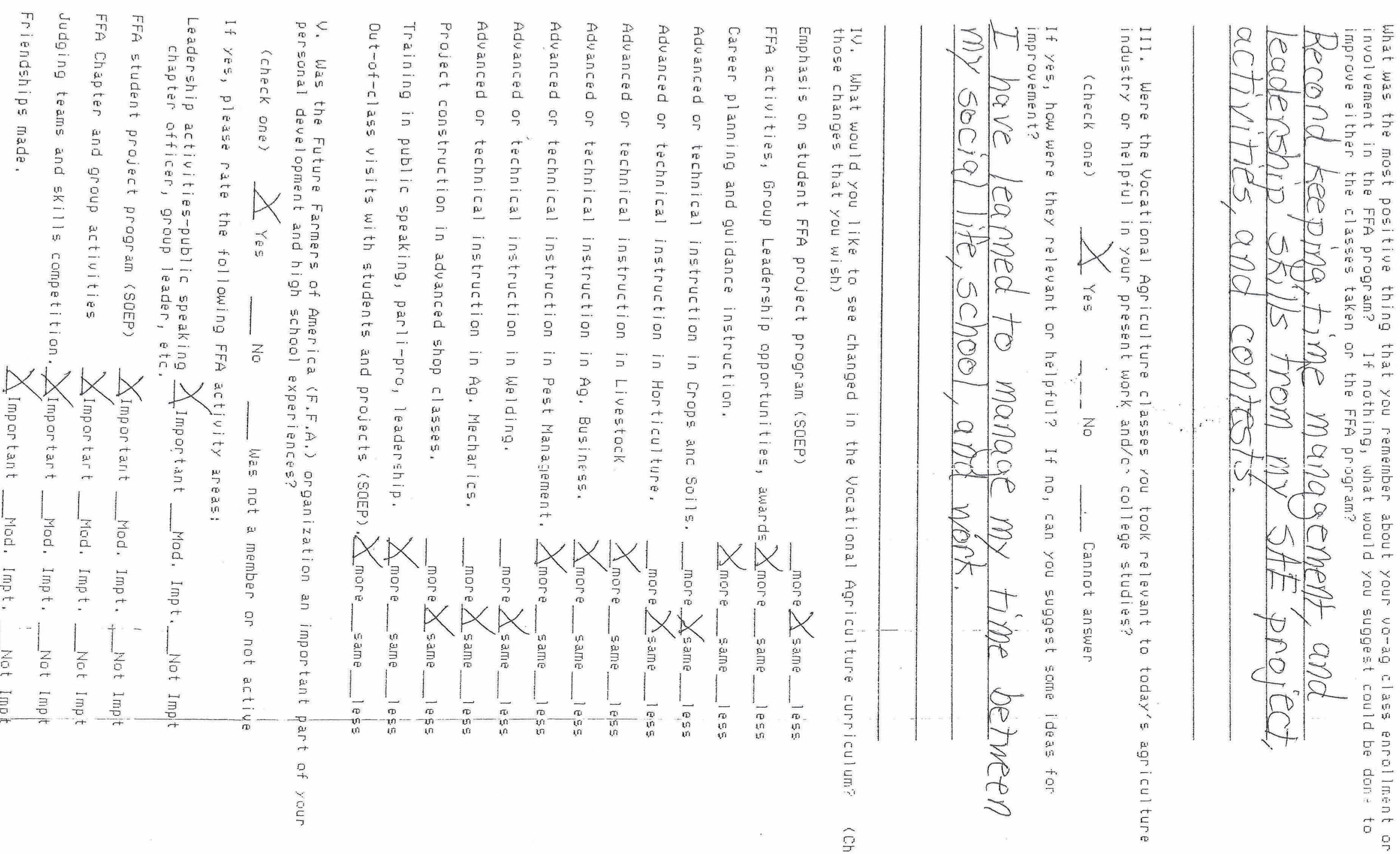
SUMMARY OF GRADUATING CLASS OP: 2009 DATE GRADUATE SURVEY SENT: PERSON completing this sumary Stephanie Blodge to to completed: REVIEWED BY AG. ADVISORY COMMITTEE:

I. GENERAL SURVEY DATA

1. Total number of program graduates and/or completors in $\frac{2009}{\text { (year) }}=$

2. Total number of program graduates and or completors surveyed...... =
a. Number male:
$0=$
() $\%$ of group surveyed
b. Number female: $=100 \%$ of group surveyed

3. Total number of program graduates and/or completors responding.... = to survey.
a. Number male: \% of group surveyed
b. Number female: $=$ 100 \% of group surveyed

4. Return rate of graduates and/or completors surveyed (male \& female) $=100 \%$

\section{ADVANCED EDUCATTON DATA}

1. Number that went on to advanced education.............. $1=100 \%$

(a) Number pursuing education in agriculture studies $\bar{\theta}=$

b. Number pursuing education in non-agric. studies. $0 \%$

c. Number in full-time advanced education.......... $100 \%$

d. Number in part-time advanced education, no work. . 0 $=100 \%$ included.

III. WORK FORCP ENTRANCE DATA

1. Number that went to work directly after graduation........ ( ) () $\%$
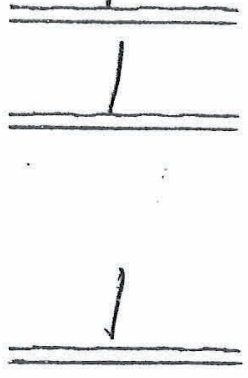
1. Number of graduates and/or program completors that are both pursulng an advanced education and working...

$\cdots 1=10 \rho \%$

(a.) Number pursuing education in agriculture and working part-time in agrlculture.
$\ldots$

$\theta=O \%$

b. Number pursulng education in agriculture and working part-time in non-agriculture area...

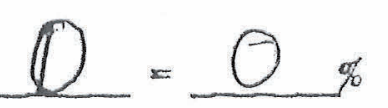
(C) Number working part-time in agriculture and
pursuing education in non-agriculture studes

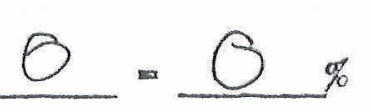

V. PIACFATENT IN AGRTCULTURE DATA (Refer to parts II, III, and IV)

1. Number pursulng advanced education in agriculture studies

2. Number that went to work directly in an agric. occupation

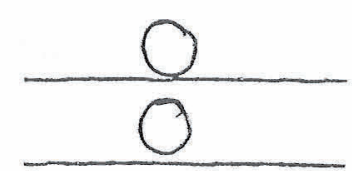

3. Number pursuing part-time education in agriculture or working part-time in an agriculture occupation

TOTAL NUMBERS .

PLACEMENT RATE IN AGPICULTURE

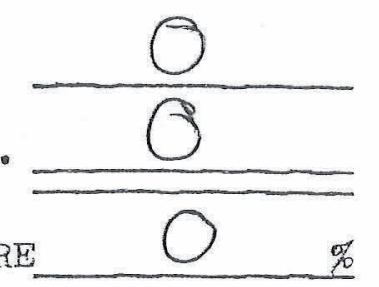
SURVEY RESPOHSE COMNESTIS

Positive Experiences

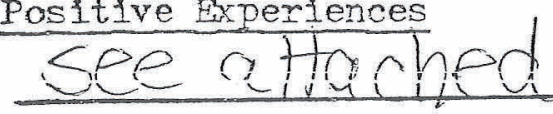




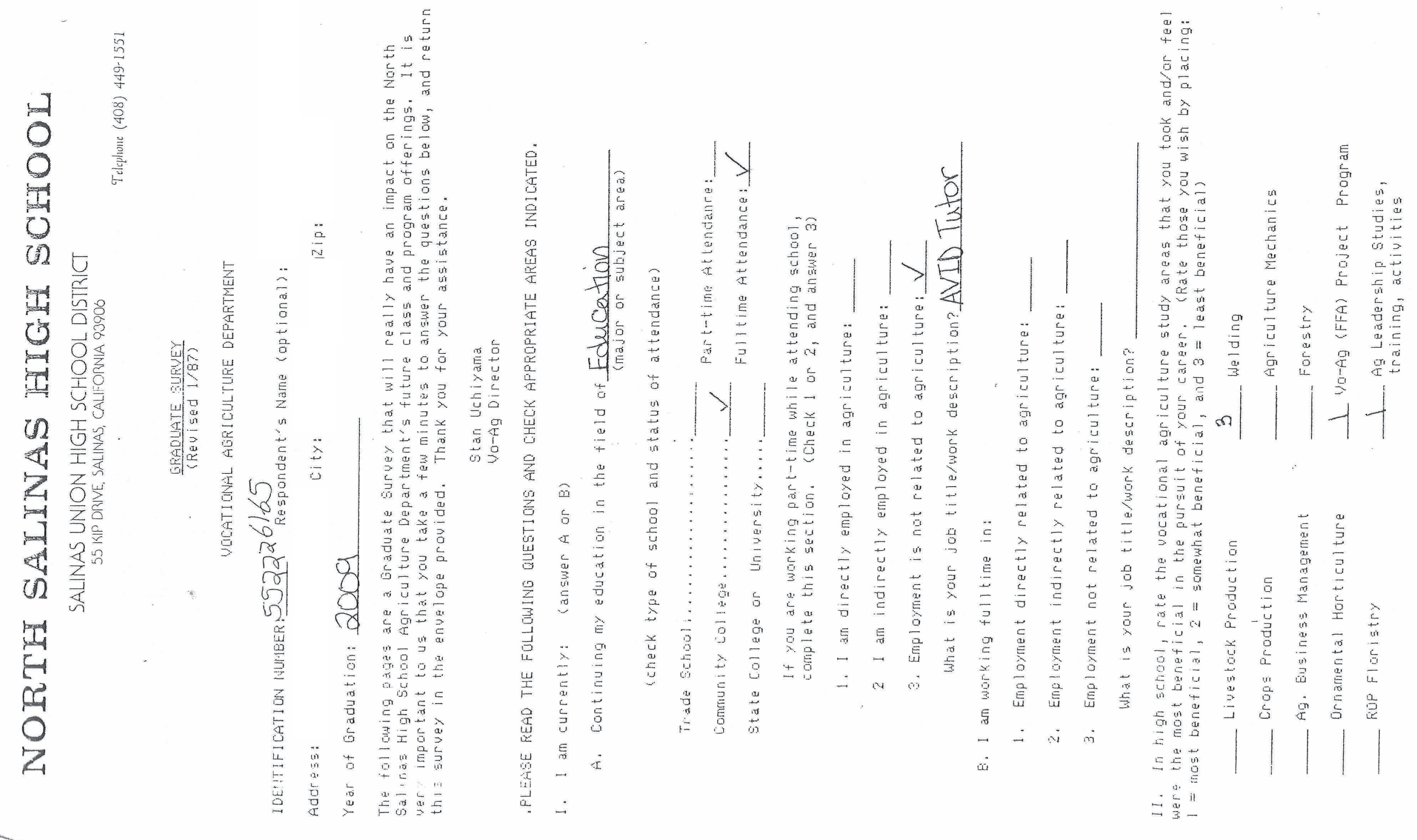






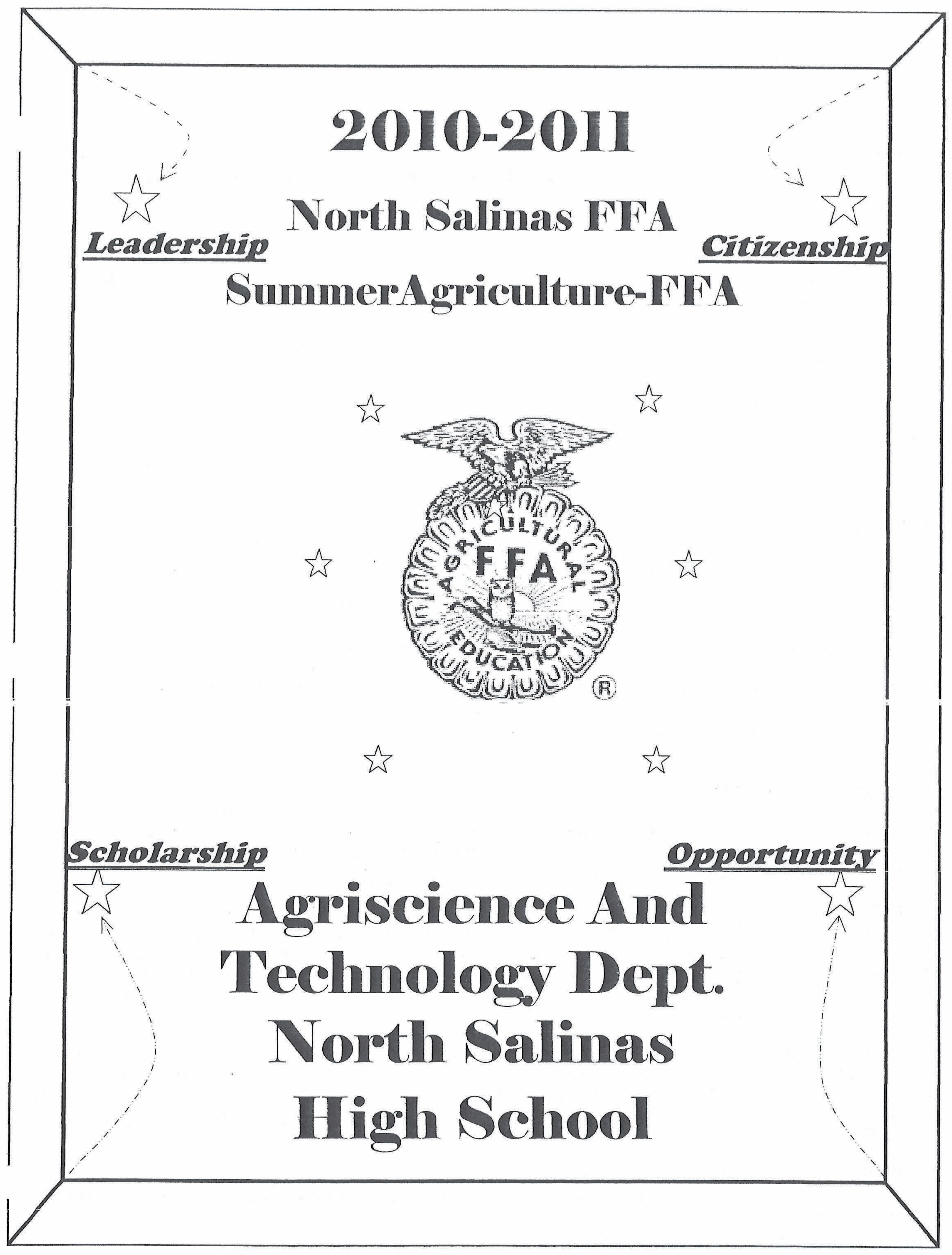




$$
\text { (TEN. } 7 / 10 \text { ? }
$$

The attached calendar of actiuities was set-uo to help you plan your participation in our summer agr iculture/FFA program. READ THE INFORMATION BELOW AND KEEP IT IN A HANDY PLACE FOR FUTURE REFERENCE: YOU ARE RESPONSIELE TO KEEP YOURSELF INFORMED AND TO REASONABLY PARTICIPATE IN OUR SUMMER PROGRAM.

WORK EXPERIENCE MEMEERS: If you are workirig part or full-time at an agriculture related job this summer, Keep your work hours and pay in your project record book. You Do Nor have to atterd any work experience clasees to be invalved.

ORTGMENTAL HORTICULTURE: Students with home landscape maintenance or wanting to start plant production projecte need to cantact Mr. Uchiyame. Monterey County fair hás a wide variety of plant categories that can be entered. Potted plast orowing space is ayaj]able at the school's greerihouse.

UEGETABLE GaRDENS: Gardens should be planted by the end of Jume for Monterey Fair produce entries. Vegetatiles sold or eaten at your own dinner table can be recorded as a project income ir your record book.

LIUESTOCK PROJECTS: Feed them properly, regularly, and exercise them to keep thejr. muscle torie in good shape and their appetites high. Don't ouer-feed or under-feed, have plerty of fresh water auailable, and use salt blacks. Aucid exercising your animale during the hotter part of the day. If you go on any trips, be eure to have sameane take good care of your anjmi. Linestock weighing should be dane at least three times a month and mare in

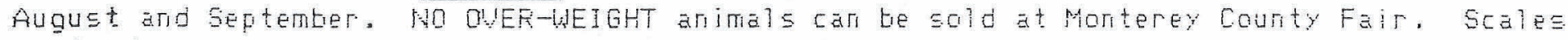
ean be torrowed if you warut to weigh your animal more ofter. Figs should tre mormed ance each month. Sheep should be warmed ir June and July, and keep their hooves trimmed. Perform reqular "heal th checke" or ycur animale. Practice animal "shoumanghip" shill

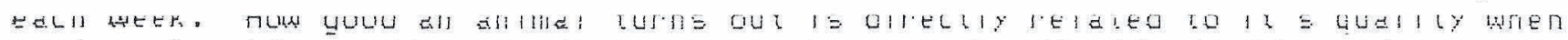
purchaeed and hou well it is being cared for-cogo for the blue! If you have a protlem call Mr. Uehiyame or a vet. Dor't wait until the probiemi gets worse.

SHOW LNIFORMS, EQUIPMENT, ETC.: Get your show uniform reEds now---nat in August. Purchase or borraw any needed livestock equipment arid groming supplies. Euild, repair. or repaint your tack boxes befare the fair. Plane are auailable for building a tack trox. If needed, lamb grooming tables can be borroned fram school. Dorit thait until the last minute to da these neceseary thingen

WELDING AND AGRICULTURE MECHANICS: Work an thase new project ideas and finish the old ones. Monterey and Santa Cruz Fairs would like to have large exhibits this year. See the calendar for the dates that the shop will be open. If we do not get a reasonable number of people to use the shop facility within the first half-hour of each scheduled time, the shop will be closed. Contact Mr. Uchiyama if you would like to use the shop for project work during other times.

COUNTY FAIR ENTRY DEADLINES: Entry forms received by the fairs after the deadi ine date are not accepted--complete your entry forms and mail them early. See the calendar or a Fair. Premium Book for fair entry form deadline dates and when completed projects have to be taken to the fair and checked in for judging. IT IS YOUR RESPONSIBILITY TO MEET ENTRY DEADLINE DATES.

MEETINGS: Eueryone planning to show their projects at the fair must atterd Chapter. metings to receive project and actiuity information, complete fair entry forms, and receive other important information. A phone comithe will be set-up to contact all interested members about meetings and activities. If you do not get a call about a meeting, etc. call Mr. Uchirama or orie of the FFA Officers to canfirm the activity and date. FFA afficers don't farget your executive cammittee meetings. 


\section{EUERYONE IS EXPECTED TO HELP DO THEIR SHARE}

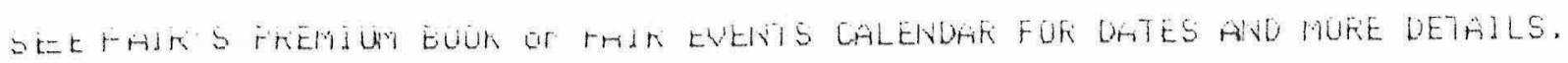

ENTRY PAYMENT AND DUE DATES TO FAIR OFFICE. All livestock, Ag Science, Ag. Mechanics, eg., O.H., etc. entry forms are due June 30 th. Rabbit and Poultry forms are due July gth. Late entries WILL NOT be accepted.

LIUESTOCK AUCTION BUYERS. Now is the time to write your buyer letters for selling your market animal. Better yet, make personal contact with your potential buyers--.-this always works out the best. Bid cards and details can be obtained from Mr. Lchiyama. Early contacts give the best results. Sending out buyer letters a week or two before the fair usually will get you poor or no results. For help, contact Mr. Uchiyama.

FEATURE BOOTH EXHIBIT. Help will te needed to make some extibit changes to insure that the booth will be ready, for fair exhitition. Bortt will be put up on Saturday, August 4 th, 11 am -5 pm only. People will be needed for booth set-up. A firet place will bring our Chapter needed prize money to finance our FFA activities.

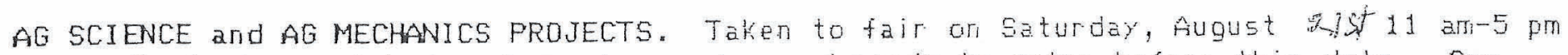
only. Frojects need to be cleaned-up, etc. and ready to enter tefore this date. See ca]endar for "Open-Shop" days/nights so you can make plans to work on your projects.

UEGEATBLE CROPS and FRESH FLONERS. Entries checked-in an Sunday, Auguet Zzad 10 a

OTHER FFA ENTRIES THAT YOU MAY EE INTERESTED IN -SEe OpEn Junior Honie Arts Depar tment and FFA Feature Garden Eections of Entry Guide Book.

FFA SCRAPBOOK. Dne-tun people are needed to up-date the O9-10 FFa Scraptook for Moco fir entry, we have the book, materials, pictures, ete. This could mean more prize money for FFA activity use:

LIUESTOCK EDUCATIONAL DISPLAY, WI1] neEd to help conetruct a $4 \times 4$ fod I lvestock information display. We heve or can get necescary materials. Check-in i a Hondar, August 30 th, $5 \mathrm{fm}$.

MCF JR. BUILDING HOSTING. One of our. Fair jobs is to hast the FFA and $4-H$ building, Thursday, Sept 2,20\%, 6-8 pri. We are required to heve 4-5 people and 2 adults to help work our shift. Top half of FFA uniform required. You will be aseigned a time to work.

LIUESTOCK JUDGING SCHEDULE. See attached fair event calendar.

\section{SANTA CRUZ COUNTY FAIR \\ (September $14-19$ th?}

\section{SEE FAIR PREMIUM BOOK ON EVENTS CALENDAR FOR MORE DETAILS}

LIUESTOCK 60 DAY OWNERSHIP DEADLINE. for pigs, sheef, and market goats is JULY 13 th, 30 day owrership for rabbits is August 12 th.

ENTRY COMPLETION, FEES, AND DUE DATE TO FAIR OFFICE. All Entries are due on Satur.day, August 4, 2007. Premium books and entry forms are auailable for completion. Contact Mr. Uchiyama.

LIVESTOCK JUDGING SCHEDULE. See attached fais calendar and details. 
RECORD BOQKS: New books or additional record book paqes are auailable upon request. If you need help in the record book contact Mr. Uchiyama. Livestock project owners will not get their fair sales check until all bills are paid, borrawed equipment returried, record hook up-dated, buyer letter sent, school farm pens clearied, etc. Be sure to keep track sur animal's weight and weight gains, medical treatments, feed purchases, special jobs done, weekly feeding and care labor, farm work days, and other project related work. Record other ag. Jabar performed igardening, yardwork, helping others with their praiect, etc.). Keep your records up-to-date.

FAIR PREPARATION OF EQUIPMENT AND CHAPTER EXHIBITS: Everyone is required to help out with the Chapter"s efforts in getting equipment ready for the fair, feature booth work, and with other fair displays. When called, be ready to offer some tielp in these areas. EXTRA FAIR DUTY WILL GO TO THOSE WHO DO NOT HELP.

PARENT AG. BOOSTER ACTIUITIES: It is of benefit to each member that all of us help and participate in the Ag. Eogster activities. It is not an unreasonable requirement for parents to give some of the ir time for these meetings and activities. Remember. Eooster. activities benefit everyone!

PROJECT BILLS: Ee sure to pay your project bill a for medical treatmente, School farm rentrines, or other past debts prior to going to the fair.

LIUESTOCK OWNERSHIF: Remember the chnership deadline dates sefe calendar) for Manterey county and Santa Cruz County Fairs. This means that the animal is in your possession and reqular care. Male sure you taue a bill of sale for your animal, Cortact Mr. Urtiyama if you need help in purchastrg your livestock projects.

FAIR RULES, PARTICIPATION CONTRACTS, CHAPERONE AGREEMENTS, ENO LIUESTOCK TRANSFORT WAIUERS: New livestock project omers need to get copies of these and get then eigred ar eturned to Mr. Uehiyama. This is a must? older-experienced exhititore revienthe contracts and agreements, ete. weed at the last fair. Copies ayailable upon request.

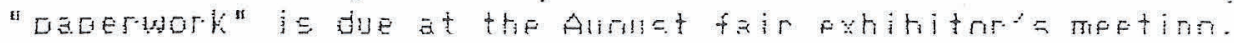

LIUESTOCK INSURANCE: FFA has a program for insuring market animale agairet accidentäl death or lose. The cost is very reaconabie and all project ownere should give seridus thought to protecting their market animal investments. Livestock fept at the school Farm are required to purchase insurance. To purchase this insurance. Contact lí. Hohiyana.

SCHOOL FARM LIUESTOCK OHNER: Always think safety and work Eafely while at the Farm. Do not go to the farm alone, take a friend. Wo one is to tie there after darkrese or before surise. Check the chalkboard iri the barri for messages, ete. keep the Farm and your animal pens clean. Stratu works best to keep your pigs warm at night and needs to be changed when dirty or does not "fluff up." Straw and wocd shavings in the barn are not to be used for farm pens--buy your oun bedding needs. Durers of pigs will have to replace the dirt in the pens where pigs made holes, etc. Pen damages-wili be repaired or paid for by the animal's owner. As it is developed, Farm-use agreements, 1 iability waivers, and cleaning deposit fees may be implemented at the Farm. Adjust your schedules to he]p on farm workdays. There is always cleanup, refair, or construction work to be dane--everyone is expected to helP. WO WORK OR LON WORK HOUR CREDITS--YOU WILL BE CHARGED A PROFATED DA] LY RENTAL FEE (\$,50/day), OR MAY HAUE TO REMOUE YOUR ANIMAL FROM THE FAFM!

OFFICER PLANIING WORKSHOPS: All FFA Officere are expected to attend the actioity planing Titetirigs that are agreed to.

QUESTIONS AND PROJECT NEEDS: I am sure that some things may not have been mentioned within this 7 ist of reminders. If you have ary questions, contact Mr. Uchiyama i449-717w. at $796-7536)$. When leaving phone messages, please give your rame, néd, and a return phore number. Plan ahead. Days where no activity is scheduled are days when Mr. Il h iyama may not be auailable to help you. 


\section{LIVESTOCK PROJECTS: \\ AN FFA MEMBER'S RESPONSIBILITIES}

1. TAKE CARE OF YOUR ANIMAL!

- Feed, house, and handle it properly.

- Treat it humanely. Be conscious of it's comfort and needs.

- Observe it carefully and te able to tell when it is ill or in distress. When it is sick, take corrective actions quickly.

- Keep your animal parasite free (Worm It Unce A Month!)

- Exercise, handle, brush, wash, trim hogfe, etc. regularly.

- Keep water supply fresh and don't feed old or moldy feeds.

- Use salt blocks arid store feed properly.

2. CARRY DUT YOUR PROJECT RESPONSIBILITIES!

- Feed and care for your animal yourself, reqularly. Coon't expect Mom, Dad, or anyone e]se to do $j t$ ?

- Plan to pay all your project expenses. If you borrow money to buy your animal, arrange to pay it back (ewen-to your parents)

- Takie care of borrowed equipment and respect property.

- Keep grod SÄEp records and keep them up-to-date. Records are valuable management tools.

3. BE A GOOD NEIGHBOR!

- Keep manuare arid wet straw zleaned up. Practice go fly cantrol.

- Keep corrals and penje clean, neat, and free of trash.

- Keep the dust and riai ge dowri.

- If you have rejghbors who might object to your keeping an animal, wi it them to introduce yourself and tell them about your fFA project.

LEARN SOMETHING FROM YOUR PROJECT!

- Don't just pour feed and water into it ard hope that it will make weight. Weint rour arimal rin a ramilar bas

- Read ard Etudy sbout treeds ard mienagement.

- Attend FFA project information meetings, show s. Eeminare, ete.

- Teach ard shou others what you've learned.

5. HAUE FUN WITH YOUR ANIMAL!

- Get to know your animal, halter break it, care for it.

- Talk to other FFA members, breeders, leaders "judge and share experierices; learn from one another.

- Take pictures, this is a major FFA project you should be proud of.

- Exhibit your animal for local schools. field days, or fairs.

6. YOU HAVE RESPONSIBILITIES WHEN EXHIBITING YOUR ANIMAL:

- You arid your animal should be clean arid well-groúmed. A lot of people are seeing animals for the first time through you and your FFA project. Leave a good impression!

- Keep pens, al leys and equipmert clean.

- Be courtecus to everyone, especially the visiting public. Help your fell ow FFA members.

- Da you awn work, you're supposed to be learring.

- Invite people to come and watch the Junior Livestock fuction.

7. IF YOU HAUE A MARKET ANIMAL PROJECT AND LIKE IT, plán tó raise additional animals riext year, OR get started in a breeding project.

- GET INWOLUED IN OTHER FFA PROJECTS THAT WILL COMPLIMENT YOUR LIUESTOCK project such as: veterinary studies, ag. mechanics, marketing, welding, crops, nutrition studies, judging teams, or whatewer else comes to mind. 
1. Thou shall not feed, train, and care for the animal for thou are tryi to teach the FFA member responsibility.

2. Thou stiall forgive the FFA member for making mistakes in the show rirg for thou hast made mistakes too.

3. Thou shall not get mad when thy FFA member forgets items in the show box, for one day thau may forget the showbox.

4. Thou shalt help the show maragement for they are doing a job that thou. would not want to do.

5. Thou shalt see that thy FFA member is on time for all show activities for thou would not want to wait on another.

6. Thou shalt make Eure thy FFA member has animal E entered and registration papers in order by the designated time.

7. Thou shalt teach the fFA member that winning a blue ribbon is a desirable goal but making friends along the way is a more worthy goal.

8. Thou shalt not complain about the judge for it is hiefter opinion that has teen sought.

9. Thol shalt remember that 1 ivestack projects are teachirg projecte, rot riecessarily money-making projects.

10. Thou shalt remember that the livestock project is a family project that shall te enjoyed and supported by the entire family. 


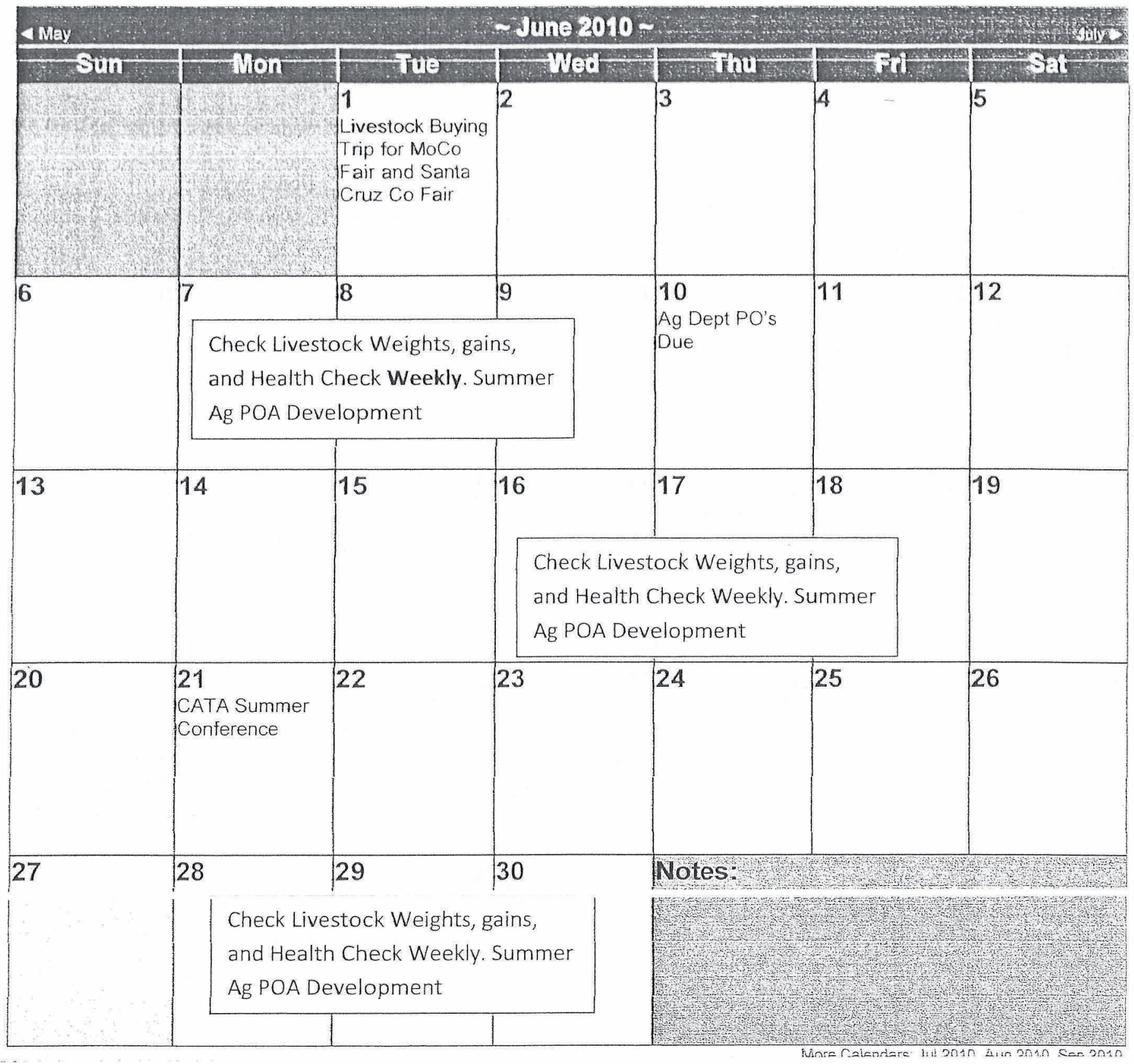




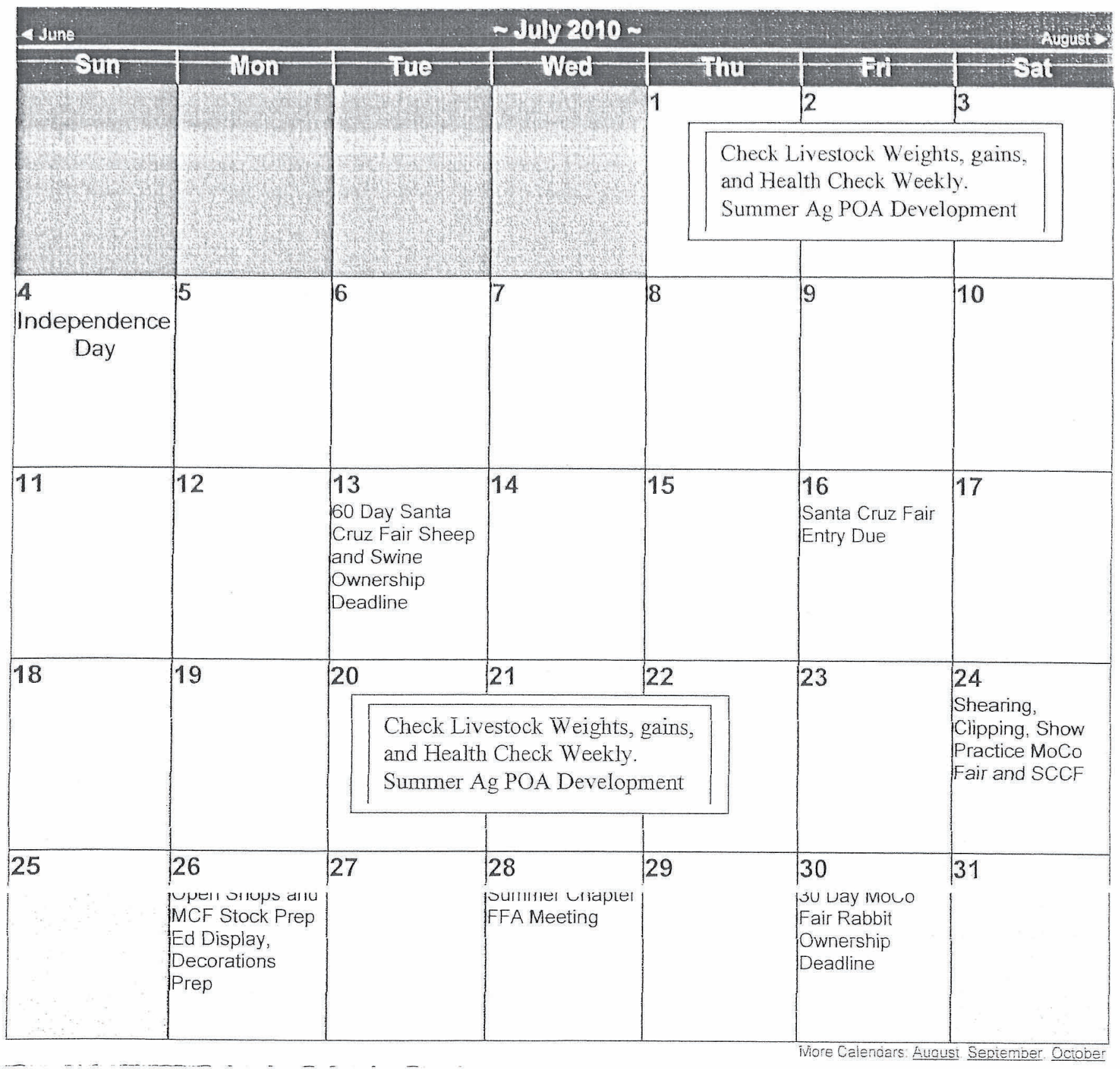




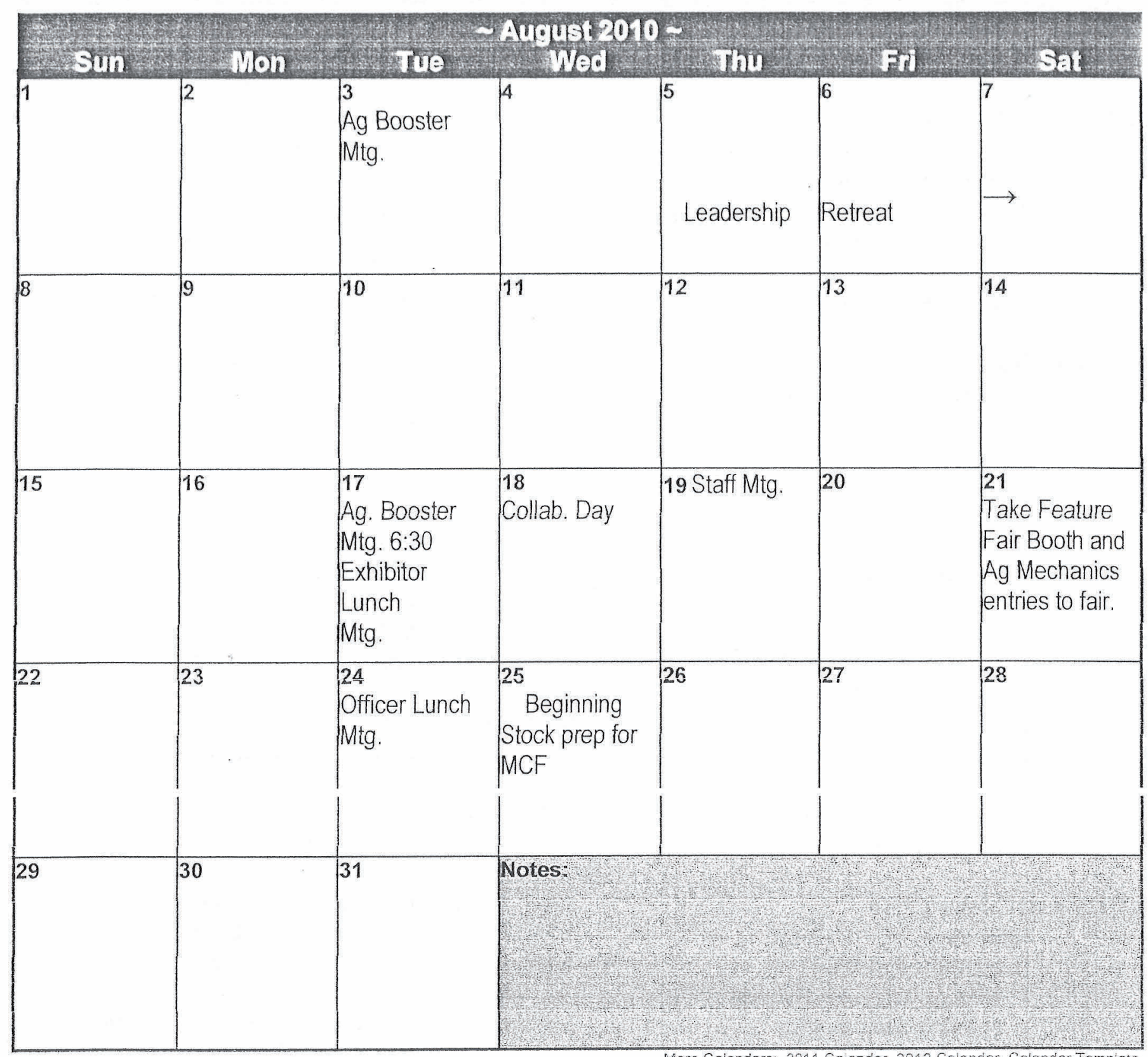

More Calendars: 2011 Calender 2012 Calendai. Calencar Temolate 


\section{Janion Ltvestorh Schedule}

SUNDAT, APRIL 18, 2010

.0 a.m. 4.H \& FFA lunior Horse Show

TUESDAX, AUGUST 31, 2010

2:00-6:00 p.m. Sheep \& Swine Official Weigh In

4:00 - 8:00 p.m. Sr. \& Jr. Poultry Coop-In

5:00 p.m. Market Goat Official Weigh in at Sheep Scale

6:00 - 7:00 p.m. Beef Official Weigh In

7:00 p.m. Livestock in Place

7:00-8:00 p.m. Market Broiler Weigh in

WEDNESDAY, SEPTEMBER 1,2010

9:00 a.m. Dairy Goat \& Market Goat Show -

Little Livestock Lawn (Following is show order)

- Showmanship - 4-H Followed by FFA

- Breeding Classes - 4-H Followed by FFA

- Club/Chapter Groups - 4-H Followed by FFA

- Market Classes - 4-H Followed by FFA

- Pee Wee Showmanship

9:00 a.m. Swine Show - Auction Ring

(Following is show order)

- Showmanship - 4-H Followed by FFA

- Master Showmanship

- Breeding Classes - 4-H Followed by FFA

- Club/Chapter Groups - 4-H Followed by FFA

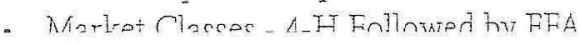

- Feeder Classes - 4-H Followed by FFA

7:00 p.m. Best Dressed Goat - Little Livestock Lawn

THURSDAY, SEPTEMBER 2, 2010

9:00 a.m.

Sheep Show - Auction Ring

(Following is show order)

- Showmanship - 4-H Followed by FFA

- Master Showmanship

- Breeding Classes - 4-H and FFA

- Club/Chapter Groups - 4-H Followed by FFA

- Market Classes - 4-H Followed by FFA

- Feeder Classes - 4-H Followed by FFA

5:00 p.m.

Lamb Blocking Contest \& Junior Lamb

Lead Entries Due - Jr. Fairboard Office

\section{FRIDAY, SEPTEMBER 3, 2010}

9:00 a.m.

Rabbit Pee Wee Showmanship Entry Forms

Due - Little Livestock Office

9:00 a.m. Rabbit Show - Little Livestock Lawn

- Jr. Rabbit Show

ı:00 a.m. Rabbit Showmanship - Little Livestock Lawn

- Pee Wee Showmanship

- Rabbit Showmanship

9:00 a.m.

Beef Show - Auction Ring
(Following is show order)

- Showmanship - 4-H Followed by FFA

- Master Showmanship

- Club/Chapter Group - 4-H followed by FFA

- Market Beef - 4-H followed by FFA

- Beef Breeders - 4-H followed by FFA

- BeefFeeders - 4-H followed by FFA

- Bred \& Fed - 4-H followed by FFA

12:00 p.m. Lamb Blocking Contest - Show Ring 2

1:00 p.m.

3:00 p.m.

Jr. Market Broiler Judging -Little Livestock Lawn

Poultry Show - Little Livestock Lawn

- Jr. Poulury Showmanship - A-H Followed by FEA

- Pee Wee Showmanship

- Avian Bow?

- Jr. \& Sr. Breed Show

3:00 p.m. Dairy Show - Auction Ring

(Following is show order 4-H \& FEA show together)

- Showmanship

- Club/Chapter Groups - 4-H Followed by FFA

- Dairy Breeding

5:00 p.m. Junior Lamb Lead - Community Stage

6:00 p.m. Livestock Judging - Location TBA

SATURDAY, SEPTEMBER 4, 2010

10:00 a.m. Junior Livestock Auction

SUNDAY, SEPTEMBER 5, 2010

9:00 a.m. Sr. Rabbit Show - Litile Livestock Lawn

10:00 a.m. 4-H \& FFA Round Robin-Auction Ring

Noon - 1:00 p.m. JLA Picture Distribution - Auction Ring

1:00 p.m.

4:00 p.m.

4-H \& FFA Vegetable Judging Contest - Location TBA

Pee Wee Showmanship Entries Due

(Except Rabbits) - Junior Fairboard Office

5:00 p.m. Pee Wee Showmanship Sheep \& Swine

Followed by Adult Showmanship - Auction Ring

MONDAY, SEPTEMBER 6, 2010

9:00 a.m. Awards Breakfast Ceremony

12:30 p.m. Wool Auction

3:00 - 5:00 p.m. Buyer \& Trophy Thank You Letters Due-

Auction Ring

4:00 - 8:00 p.m. Sr. \& Jr. Poultry Released

5:00 p.m. Walkout of Tack and Begin Removing

Decorations

7:00 p.m. Driveout of Tack and Animals Not Sold at

Auction

EVERYTHING MUST BE REMOVED FROM THE

FAIRGROUNDS BY

7:00 PM. TUESDAY, SEPTEMBER 7, 2010. 


\begin{tabular}{|c|c|c|c|c|c|c|c|c|}
\hline \multicolumn{2}{|c|}{ C010 Livestock Schedule } & $\begin{array}{l}\text { MON } \\
\text { Sept } 13\end{array}$ & $\begin{array}{l}\text { TUES } \\
\text { Sept } 14\end{array}$ & $\begin{array}{l}\text { WED. } \\
\text { Sept } 15\end{array}$ & $\begin{array}{l}\text { THURS. } \\
\text { Sept } 16 \\
\end{array}$ & $\begin{array}{l}\text { FRI. } \\
\text { Sept } 17 \\
\end{array}$ & $\begin{array}{l}\text { SAT. } \\
\text { Sept } 18\end{array}$ & $\begin{array}{l}\text { SUN } \\
\text { Sept } 19\end{array}$ \\
\hline Pre-Fair & Market Beef Ear Tagging & \multicolumn{7}{|c|}{ Wednesday, May 19, 2010, 4:00 - 7:00 PM, 101 Livestock Sales Yard-MANDATORY } \\
\hline Check-In & $\begin{array}{l}\text { All Hogs/Beef Arrive } \\
\text { All Lambs/Goats Arrive for Vet } \\
\text { Check* }^{*}\end{array}$ & $\begin{array}{l}\text { 8:00am-7:00pm } \\
3: 30-7: 00 \mathrm{pm}\end{array}$ & & & & & & \\
\hline \multirow{2}{*}{ Weigh-In } & $\begin{array}{l}\text { Market and Carcass Hogs, Lambs, } \\
\text { Goats }\end{array}$ & $3: 30-7: 00 \mathrm{pm}$ & & & & & & \\
\hline & Market and Carcass Beef & 5:30-6:30pm & & & & & & \\
\hline \multicolumn{2}{|c|}{ All Junior Livestock in place } & 7:00pm & & & & & & \\
\hline \multicolumn{2}{|c|}{ Ice Cream Social/Exhibitor's Meeting } & 7:30pm & & & & & & \\
\hline SHEEP & $\begin{array}{l}\text { 4-H Groups, Market \& Feeders } \\
\text { 4-H Showmanship } \\
\text { FFA Groups, Market \& Feeders } \\
\text { FFA Showmanship } \\
\text { Supreme Showman } \\
\text { 4-H/FFA \& Breeding }\end{array}$ & & $\begin{array}{l}9: 00 \mathrm{am} \\
* * \\
\star \star \\
* * \\
\star \star \\
\star * \\
\end{array}$ & & & & & \\
\hline \multirow[t]{4}{*}{$\begin{array}{l}\text { GOAT } \\
\text { DIVISIONS }\end{array}$} & $\begin{array}{l}\text { MARKET GOATS } \\
\text { 4-H Market, Feeders \& Groups } \\
\text { 4-H Showmanship } \\
\text { FFA Market, Feeders \& Groups } \\
\text { FFA Showmanship } \\
\text { Supreme Showman } \\
\text { Commercial/Registered Meat Does } \\
\end{array}$ & & $\begin{array}{l}1: 00 \mathrm{pm} \\
\star \star \\
* * \\
* * \\
* * \\
* * \\
* *\end{array}$ & & & & & \\
\hline & $\begin{array}{l}\text { JUNIOR DAIRY GOATS } \\
\text { 4-HIFFA Showmanship } \\
\text { 4-H/FFA }\end{array}$ & & & & & $\begin{array}{l}9: 00 a m \\
* *\end{array}$ & & \\
\hline & JUNIOR BREEDING BOER GOATS & \multicolumn{7}{|c|}{ There will be no Junior Breeding Boer Goat Show this year. } \\
\hline & BEST-DRESSED GOAT CONTEST & & & & & & & $1: 00 \mathrm{pm}$ \\
\hline SVINE & $\begin{array}{l}\text { 4-H Showmanship } \\
\text { 4-H Market \& Feeders } \\
\text { FFA Showmariship } \\
\text { FFA Market \& Feeders } \\
\text { Sunreme Shnwmsn } \\
\text { 4-H/FFA Breeding } \\
\end{array}$ & & & $\begin{array}{l}9: 30 \mathrm{am} \\
* * \\
* * \\
* * \\
* * \\
* * \\
\end{array}$ & & & & . \\
\hline BEEF & $\begin{array}{l}\text { 4-H Showmanship } \\
\text { 4-H Market \& Feeders } \\
\text { FFA Showmanship } \\
\text { FFA Market \& Feeders } \\
\text { Supreme Showman } \\
\text { Junior Beef Carcass Judging } \\
\text { 4-H/FFA Breeding } \\
\end{array}$ & & & & $\begin{array}{l}9: 00 \mathrm{am} \\
* * \\
* * \\
* * \\
* * \\
* * \\
* \\
*\end{array}$ & & & \\
\hline $\begin{array}{l}\text { DAIRY } \\
\text { CATTLE }\end{array}$ & $\begin{array}{l}\text { 4-H/FFA Showmanship } \\
\text { 4-H/FFA Dairy Cattle } \\
\end{array}$ & & & & $* *$ & & & \\
\hline RABBITS & $\begin{array}{l}\text { Check in } \\
\text { Judging } \\
\text { Showmanship }\end{array}$ & & & & & $\begin{array}{l}\text { 8:30am } \\
9: 30 \mathrm{am} \\
9: 30 \mathrm{am} \\
\end{array}$ & & \\
\hline \multicolumn{2}{|c|}{ OPEN CARCASS Division } & & & & $6: 00 \mathrm{pm}$ & & & $10: 30 \mathrm{am}$ \\
\hline Pee Wee Sho & wmanship & & & 5:00pm & & & & \\
\hline Adult Showma & anship & & & $7: 00 \mathrm{pm}$ & & & & \\
\hline \multicolumn{2}{|c|}{$\begin{array}{l}\text { Livestock Judging - Registration } \\
\text { Livestock Judging - Contest }\end{array}$} & & & & & $\begin{array}{l}\text { 5-5:30pm } \\
6: 00 \mathrm{pm} \\
\end{array}$ & & . \\
\hline \multicolumn{2}{|c|}{ Auction Buyers Registration Begins } & & & & & & 9:00am & \\
\hline \multicolumn{2}{|c|}{ Jr. Livestock Auction } & & & & & & 10:00am & " \\
\hline \multicolumn{2}{|c|}{ Parade of Champions - Check Fair Guide } & & & & & & 6:30pm & \\
\hline Round Robin & $\begin{array}{l}\text { 4-H/FFA Senior Round Robin } \\
\text { 4-H Jr./FFA Novice Round Robin }\end{array}$ & & & & & & & $9: 00 \mathrm{am}$ \\
\hline \multicolumn{2}{|c|}{ Awards Ceremony } & \multicolumn{7}{|c|}{$\begin{array}{l}\text { Sunday, September } 19,2010,3: 00 \mathrm{PM} \\
\text { Thank You Letters due in Livestock Office within } 24 \text { hours of show where award was won. }\end{array}$} \\
\hline \multicolumn{2}{|c|}{$\begin{array}{l}\text { Tack \& Livestock Release } \\
\text { Auction Checks Released }\end{array}$} & \multicolumn{7}{|c|}{ Sunday, September $19,2010,5: 00 \mathrm{pm}$} \\
\hline \multirow{2}{*}{\multicolumn{2}{|c|}{$\begin{array}{l}\text { Auction Checks Released } \\
\text { Junior Super Steer Awards }\end{array}$}} & \multicolumn{7}{|c|}{ Wednesday, September 29,2010,3:00-7:00 pm - Fairgrounds Main Office } \\
\hline \multirow{2}{*}{\multicolumn{2}{|c|}{$\begin{array}{l}\text { Junior Super Steer Awards } \\
\text { zss Contest Results and Awards BBQ }\end{array}$}} & \multicolumn{7}{|c|}{ Date to be determined - Grange Hall } \\
\hline & & Date to be determin & d-Grange & Hall & & & & \\
\hline
\end{tabular}




\section{Comprehensive Program Plan}

A copy of our comprehensive program plan is on file at Cal Poly in Mr. Greg Beard's office. 


\section{MGRICULTURE SICIEMICE $1-2$

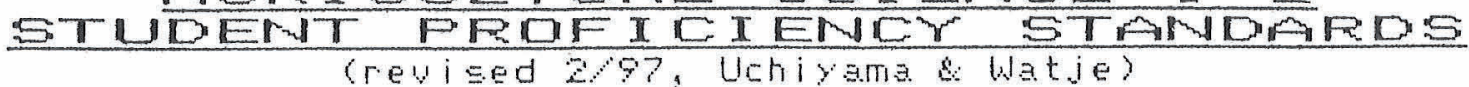

Upon completion of this course, all students will, at a minimum, have 2 satisfactory understanding of; be able to perform; or will have accomplished the following criteria identified as important proficiencies for Salinas. Union High School District agriculture education students.

\section{UNITED STATES AND INTERNATIONAL AGRICULTURE}

1. Ee able to identify geographical locations con map of U.S.) of production areas--corn, cotton, dairy, wheat, range and forage, citrus, vegetatile, etc.

2. Understand the economic importance of the top 5 U.S. agricultural export and import commodities.

3. Have a knowledge of National Free Trade Agreement (NFTA) as it applies to agricuture.

4. Know what the USDA is and have a knowledge of what their function $i 5$

\section{CALIFORNIA AGRI CULTURE}

1. Have a knowledge of the 7 cal ifornia agricultural production regions and their main commodities produced.

2. Understand the economic importance of the californi A Agriculture to the State's economy and econamy of the United States.

3. Have a knowledge of the top 5 agricultural commodities produced in Cal i for nia.

4. Know 5 agricultural commodities that are $90-100 \%$ produced in [:a] ifornia.

5. Know what the CDFA is and have a knowlege of their seruices. MONTEREY COUNTY AGRICULTURE

1. Be able to deecribe the difference betueen North county and South county agriculture-commodities grown, growirg season (climate) canditions, and water availability.

2. Krow the top 10 agricultural commodities produced in Monterey county.

3. Have a knowledge of governmental agencies found in Monterey County and their function. Examples: Ag. Commissioner. Ag. Extension Service, Soil Conservatian Society, etc. 
4. Have a knowledge of epecial agriculture interest groups and boards serving Monterey County. Example: Farm Bureau, Grower-Shipper Vegetable Association, Young Farmers, Cattlemen's Associatin, Calif. Agricultural Production Association, Calif. Women in Agriculture, etc.

5. Understand the economic importance of Monterey County agricul ture to 1 ocal and state economies.

\section{ANIMAL SCIENCES}

1. Understand the importance of domestic livestock to food production and services to mankind.

2. Know the major external body parts of cattle, swine, sheep, horses, poultry, rabbits, dairy goats, and dairy cattle.

3. Know the correct sex terms for cattle, swine, sheep, hor ses, poultry, rabbits, and goats.

4. Know the estrus and gestation cycle days for cattle, swine, sheep, horses, poultry, rabbits. and goats.

5. Be able to calculate the dressing percent for all meat animals.

6. Knowledge of and be able to calculate average daily gains, feed conversion ratios, and daily feeding percentages of all meat animals.

7. Identify three breeds of cattle, swire, sheep, horses poultry, rabbits, goats, and dairy cattle.

8. Identify the wholesale cuts of cattle, swine, sheep, and poultry.

9. Know the difference between monggastric and ruminant digestive systems.

10. Understand the function of and be able to identify major body organs. Example: Heart, liver, lunge, small and large intestines, etc.

11. Understand the function of the respiratory and circulatory şstemミ.

12. Identify the major parts of the male and female reproductive systemi.

13. Use a compcund mierascope to identity and label the parts a sperm ce 11.

14. Make written or aral report an a Eelected internal or external livestock parasite.

15. Know proper storage of, disposal of, and demonstrate safe handling of 1 ivestack medicines. Vaccines, dusts, oral tolus and pomders, feed addi tives.

16. Know acceptable methods of restraining livestock and demonstrate proper techniques for intramuscular and subeutaneous injections, including proper injection locations.

7. Able to read and understand medicine label information and orections. 
18. Make written or oral report on a selected livestock disease.

19. Know proper methods of handling livestock for transport and safety in daily handling practices, including correct completion af CDFA cattle transportation $\leqq$ ips.

20. Identify alfalfa hay, oat hay, grase hay, corn, oats, barley, wheat, milo, cottonseed, linseed, molasses, and fish meal feed

ingredients.

21. Able to read and understand a feed labe?.

22. Know the difference between concentrate and roughage feeds, and purpose of levels of protein in feeds.

23. Understand acceptable feeding practices and daily nutritional requirements (feed and water needs) of livestock.

24. Define a balanced ration.

25. Know the acceptatile market weights and grades for cattle, sheep, and swine.

26. List 10 acceptable management practices for the daily care of livestork.

27. Identify 10 commonly used pieces of equipment associated with veterinarian science, cattle, sheep, swine, and horse prduction, including describing their. use.

28. Design a satisfactory housing and fencing facility for student selected livestock species.

29 Know normal body temperature range for selected livestock species, demonstrate how to take body temperature, and read a livestock thermame ter.

30. Understand the major factors in selecting cattle, sheep, and swire for market and breeding purposes.

31. Deseribe 1 commonly used method of marketing Salinas Valley 1 ivestock.

PLANT SCIENCE

1. Understand the importance of plants to food, shelter, and clothing production.

2. Identify the major plant parts and describe their function. Example: roots, stems, leaves, bud, flower, and seed parts.

3. List 3 reasons why plants are important to mankind.

4. Understand the scientific classification system of plants.

5. Know the use claseification of agricultural plants (vegetables, grains, fruits, nuts, citrus, uines, agronomic, forage, etc.) and $i$ ist 3 examples of each.

6. Understand seed germination process and be able to calculate seed germination percent. 
7. List 3 differences between $p$ lant and animal cells:

8. Understand plant process of diffusion, osmosis, xylem and phloem tissue functions.

9. Identify 10 common edible portions of vegetable.

10. Make a collection of 10 vegetable crop eeeds common to salinas Valley.

11. Identify 5 weeds common to Sal inas Valley.

12. Propagate plants from sexual and asexual methods.

13. Complete a report on selected vegetable, ornamental, or other crop diseases.

14. Identify 5 harmful and beneficial insects common to agricultural plants.

15. Know the 4 layers of a soil profile and describe their characteristics.

16. Know the 3 primary elements $(N-P-K)$ of a fertilizer mixture and the purpose of each element.

17. Know how to read a fertilizer label and calculate the actual

fertilizer amount in the bag.

18. List 3 types of irrigation syetema used in the Salinas Yalley and describe how they are used.

19. Understand the importance of water quality and conservation to Salinas Valley erop production.

20. Underetand the importance of Eafe handling, application, and disposal of agricultural chemicals.

21. Identify 10 large implements commonly used in Salinas Valley crop production.

22. Understand acceptable techniques used in harvesting, proceseing, storage, and transporting of major Salinas Valley crops.

23. Deseribe 1 commonly used method of marketing Salinas Valley crop progducts.

\section{LEADERSHIP}

1. Understand the FFA organization structure, achievement opportunities, and FFA leaderehip programe by Ecoring 80\% on local Best Informed Greenhand written test.

2. Complete minimum requirements for and peceive the FFA Greenhand Degree.

3. Understand the basic princifles of public speaking and give a 3 minute speech on an aricultural topic.

4. Demonstrate the ability to cooperatively work with other studente by participating in 2 PrA Chapter activities or as a team member, in opening and closing Ceremony and Farliamentary frocedure contest. events. 
5. Understand 5 parliamentary procedure skills--main motion, amendment, pefer to committee, previous question, postpone/table motion, and voting processes.

6. Attend and participate in 2 Chapter. FFA meetings.

7. Conetruct a four year plan for pereonal leadership development.

\section{SUPERVISED OCCUPATI ONAL EXPERIENCE}

1. Able to explain the difference between ownership and non-ownership enterprise programs.

2. Construct a four year plan for soe involvement, including plans for growth.

3. Complete application for Chapter or section enterprise proficiency auard.

\section{AGRI CULTURE BUSINESS MANAGEMENT}

1. List 3 reasons why record keeping is an important management practice.

2. Know the purpose of each section of the Calif. Wo-Ag Record Book and commonly used record keeping terms.

3. Keep an up-to-date Calif. Yo-Ag Recard Bock on student selected enterprise by completing the following book sections:
a. Front cover and planning calendar.
b. Calendar of events and operations.
c. Enterprise business agreement.
d. Enterpri se budget.
e. Activity and financial journals.
f. Enterprise and personal financial statement.
g. FFA, Echool, and community involvement listing.

\section{EMPLOYABILITY}

1. Interpretate newspaper job listing descriptions.

2. Understand techniques for job search and types of jobs associated with levels of preparation and training.

3. Devel op a personal resume using a computer.

\section{CAREERS}

1. Name the $7 \mathrm{ag}$. accupational areas and list 3 jots related to each area.

2. Complete a job application in 20 minutes.

3. Complete student Program Planning and Career choice data sheet. 
PART 1--GENERAL PROGRAM COMPLETION PROFICIENCY STANDARDS STUDENT PROGRAM COMPLETERS IN AGRISCIENCES, AG WELDING? MECHANICS, AND HORTICLILTLIRE PRGGRAM PATHWAYS (TEW. $11 / 98$ )

Name : Date Started: Graduation Year:

Agriculture Program Pathways: Check/write-in Classes Completed

Agriculture Scierice Welding/Ag Mech Ag Science $1-2$ $A n / P l a n t ~ S c i$. Lystk/Crofs Ag Bus Mgmt Weldirig $1-$ ? Welding $3-4$ Hor ticulture Ag Sri $1-2$ Hort. $1-2$ Grow bir n
Farestr.y/NR

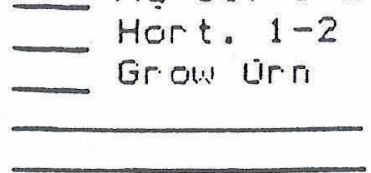

Aụ Siri $1-2$

Forestry $1-3$ Ag BuS Mgmt

Combined with pathway class proficiency standards, "PROGRAM COMPLETER" students will have learried, demonstrated, or provided satisfactory evidence of completing $70 \%$ of the competericies listed below. Each competency be verified when you have demonstrated or ualidated that proficiency. Euidence of competency completion can be achieved through classroom instruction, participation in Ag/FFA leadershipsskills events, SAE involvement, or other valid high school experiences. A "Program Completer" is any student who has completed two years of pathway class sequence above the $1-2$ level.

\section{A. COMMUNICATIONS}

molyr ver.

1. Oral speech presentation - 6-8 minutes prepared, 4-6 minutes extemporaneous, or creed contest participation. Title and where?

2. Oral group discussion/demonstration - 20 minutes. Title and where?

3. Prepare written research project on agrirultural or envirenmental subject, ineluding bitliography.

Title and for?

4. Write effective busiriess letter. Purpose?

5. Write thank you letter with supportive evidence iridicating your sincerity.

Reasenn written?

ó. Telephone procedure - used properly arid rourterusil. Adult verified:

7. Prepare commodity infermation sales facket. Re asori?

\section{B. COMPUTER COMPETENCY}

1. Properly operate computer, including start-up, shut-down pirocedure, arid printer use. Computer and printer trand?

2. Reliably load, save, and copy files or flopoy \& hard drives. Program used? 
macip wer.

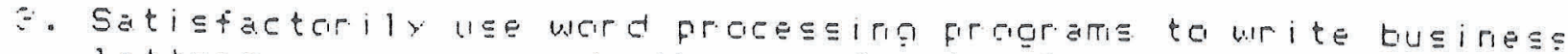
letters, resumies. arid ather orofessirnal doeuments.

Prcigr ami used?

4. Satisfactorily use desktop putishirg frogram ta make piaster, advertisemerte. etc. that irtegrate grafiles with text.

Frogram used \&. What rreated?

5. Satistactorily caristruct a spreadshet for tata callectirig. Created for?

t. Satisfactorily use saEp enterprise computer records pregram for orie entergi ise or for dne vear of SAEF regords. Year computer records kept:

\section{CAREER PLANINING AND DEUELOPMENT}

1. Prepare a plari fer caregr euccesea ingluding a feur-year plan for graduation, SAFP, and AglfFA leadership artivitr invalvemient.

3. Prefare a career researet refict, iricluding iet descriptich, education preparation, rareep advantagss and disaduantages. and advaricement cipporturites.

Report Title?

3. Ciomplete an emplarment applicaticin.

For?

4. Prepare a personal resume.

5. Write a "letter of application" for a ibb aduertisement. Fer $\because$

e. Prepare a portfolio af wark samples photgoraphe and raptione of activities, achievemente, arit other informaticn resded to find and apply for jets. (teacher ris. oa your oortfolia)

7 . Be a member of a non-leadership agl skills (iudging) competitive team whose activities relate to your career ocel and or major SAEP interest. Team is oroanized. conducts team prartices, anr competes ini 2 or more events as a team.

Team title?

\section{LEADERSHIP DEUELOPMENT}

1. Complete self-evaluation on work habits and attitudes. PURFOSE?

2. Demenetrate atility to farticipate injconduct a meeting utilizing Parliamentary Law: make motions, amendments, refer. to committer, tatile, arid votirion. How validated?

3. Act as chaiperson for a meeting or discussion. Title?

4. Write a wark plan and set priorities for task accomflishment. Wrark plan for: 
moisp ver.

5. Instruct and sufervise employefs on a jot task. Task title?

6. Fualuate worker performance.

Task done?

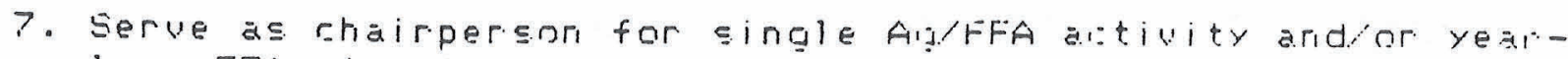
lorig FFA standirig committee.

Title?

Q. Farn FFA Greentiand Degree or leadership award.

9. Perform and document 20 hours of volunteer work. Adult verified?

10. Completed leaderehip trainirig cenference/program. Titles?

11. Completed full term as FFA ijficer, any level. Titles?

\section{E. SUPERUISED AGRICULTURE EXPERIENCE PROGRAM (SAEP)}

1. Prepare a plan for starting a emall business--identify 8-10 major operations to be completed.

Enterprise title?

2. Prepare a budget for a proposed small business. Enterprise title?

3. Prepare agreements with all farties participating in propesed small business.

Enterprise title?

4. Keep satisfartory on-going records (3 mo minimum) of SAE activities using approved record keefing system.

SAE title \& system used?

5. Maintain monthly 3 mo min) journal talarice etatements of SAE finarieial and labor actiutes showing profit and lose status. Enterprise title? Year:

6. Keep record of 10-15 specifir and different skil is/knowledige learried resulting from SAaEF.

SAEF title?

7. Demonstrate ability to maintain arid romplete depreriatle property inventory records for one full year.

How validated?

8. Complete income summary statement to measure SAEF succese.

9. Achieve minimum FFA degree respertive to SAEP inuolvement. Chapter Deoree _ State Degree American Degree

10. Complete profiriency award appliration in your safp area. Title? 


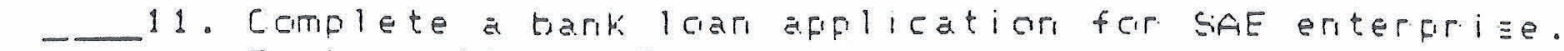
Eank applied to?

12. Receive grade of eo\% gr more or arie tull year of sife recard Fecord book year:

\section{F. COMMUNITY CIUICS AND SCHOOL PRIDE}

Aetivities must be different from thase validated atowe.

1. Ee a member af a eshool club ar group. Nome:

2. Farticipated in a scheol campus improuement project ar schoel invalued community eervice activity. Deseritie: Hour : :

3. Ee a member of a community organizatien or group. Name:

4. Participated in a mon-school related community service event. Describe: Hours: 


\section{STEPHANIE LYN PFARR}

DOCUMENT NUMBER: 080091598

VALCO: $04 / 03 / 2008$ to $05 / 01 / 2013$

DOCUMENT TrTuE: Preliminary Single Subject Teaching Credential

\section{SUBJECT(S) AND AUTHORIZATION(S):}

Agriculture

(R1S) This document authorizes the holder to teach the subject area(s) listed in grades twelve and below, including preschool, and in classes organized primarily for adults.

(R142) This document authorizes the holder to provide the following services to English learners: (1) instruction for English language development in grades twelve and below, including preschool, and in classes organized primarily for adults; and (2) specially designed content instruction delivered in English in single-subject-matter (departmentalized) courses as authorized on this document. This authorization also covers classes authorized by other valid, non-emergency credentials held, as specified in Education Code Section 44253.3.

\section{RENEWAL CODE(S):}

(R14I) This credential may not be renewed. To qualify for the clear credential, the holder of this document must complete a Commission-approved Induction program including Verification of Completion by the program sponsor.

Specific information pertaining to credential renewal requirements may be obtained under

Credential Renewal Statements and Requirements at www.ctc.ca.gov

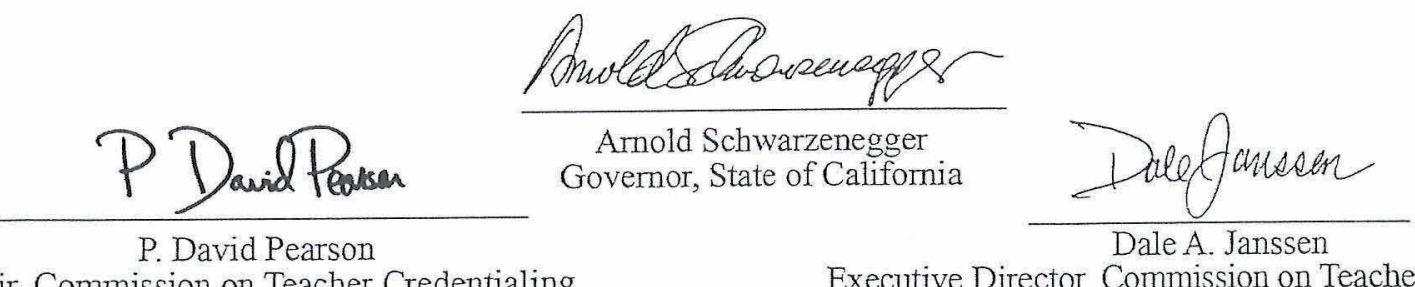

Chair, Commission on Teacher Credentialing Executive Director, Commission on Teacher Credentialing 


\section{STEPHANIE LYN PFARR}

DOCUMENT NUMBER: 080091599

VACXD: $04 / 03 / 2008$ to $05 / 01 / 2013$

DOCUMENT TITLE: Clear Specialist Instruction Credential (Agriculture)

\section{SUBJECT(S) AND AUTHORIZATION(S):}

Agriculture

(R3A1) This credential authorizes the holder to teach agriculture in grades twelve and below, including preschool, and in classes organized primarily for adults. It also authorizes the holder to develop and coordinate curriculum, develop programs, and deliver staff development for agriculture education programs coordinated by school districts or county offices of education.

\section{RENEWAL CODE(S):}

(R15P) The term of this credential is limited by the term of the prerequisite credential. To renew this credential, the holder must also renew the prerequisite credential.

Specific information pertaining to credential renewal requirements may be obtained under

Credential Renewal Statements and Requirements at www.ctc.ca.gov

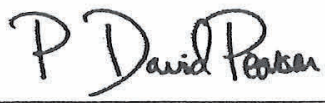

P. David Pearson

Chair, Commission on Teacher Credentialing

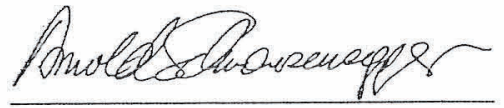

Arnold Schwarzenegger Governor, State of California

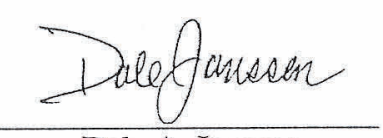

Dale A. Janssen

Executive Director, Commission on Teacher Credentialing 


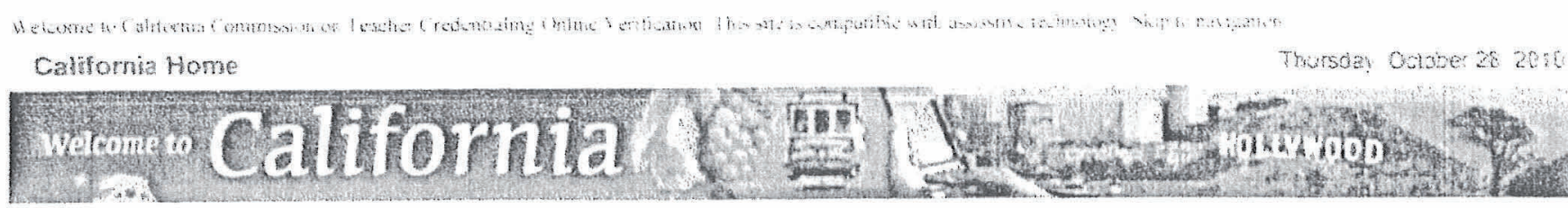

\section{California Teacher \\ Credential Look-up and Renewal}

Search for Credential for a
Public School Teacher
Search for a Teacher's
Application Status and
Gredentials Held
Renew Credentials
Direct Application
(Non-Recommendation Only)
Track Payment
(Renewal Only)
Track Payment
(Recommendation Onlv)
Start the IHE Login Page
Start the Student Applicant
Login
Frequently Asked Questions
Glossary of Credential
Terms

California Commission on Teacher Credentialing Web Site

Governor's Home Page

Contact the Commission on Teacher Credentialing

\section{California Commission on Teacher Credentialing}

$$
\text { (6) My CA }
$$

\section{Selected Credential Helo}

The application status and credential information was last updated on 10/28/2010. Local employing agencies have the flexibility to assign individuals to serve in subject areas other than those authorized on credentials. The Commission, at one time, issued documents without assigning any document number. Assigning a document number to these records was necessary to be able to display them online. The document numbering assigned to display those records will appear as "NONE1, NONE2, NONE3, etc."

\section{Details of Selected Credential}

Name: BLODGETT, STEPHANIE LYN

Document Tifle Certificate of Clearance (valid five years if issued on or after 7/1/2007)

Document Number 060078262

Renewal Code(s) NA

Issuance Date $03 / 28 / 2006$

$<$ Return to Summary

New Search 


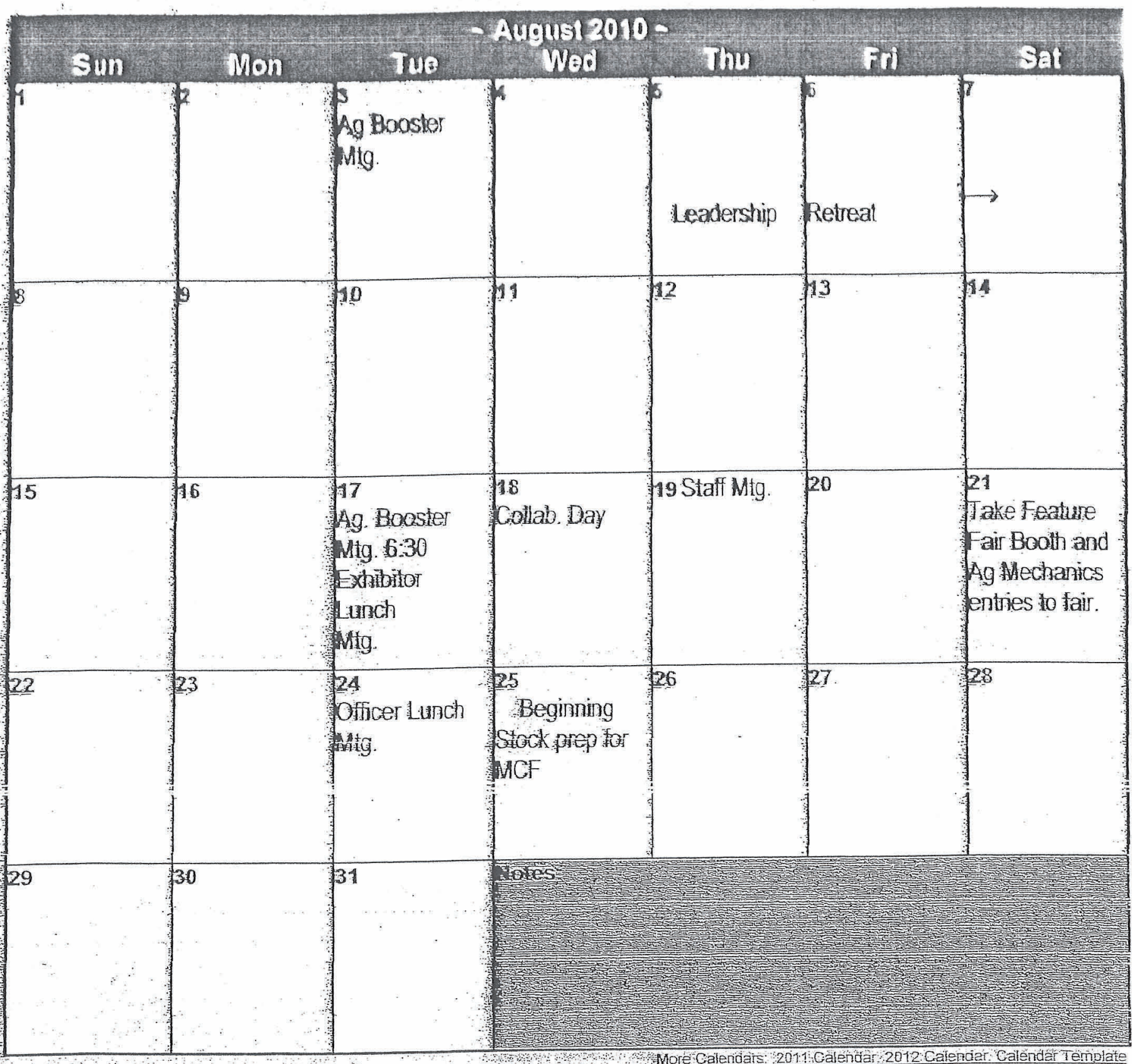




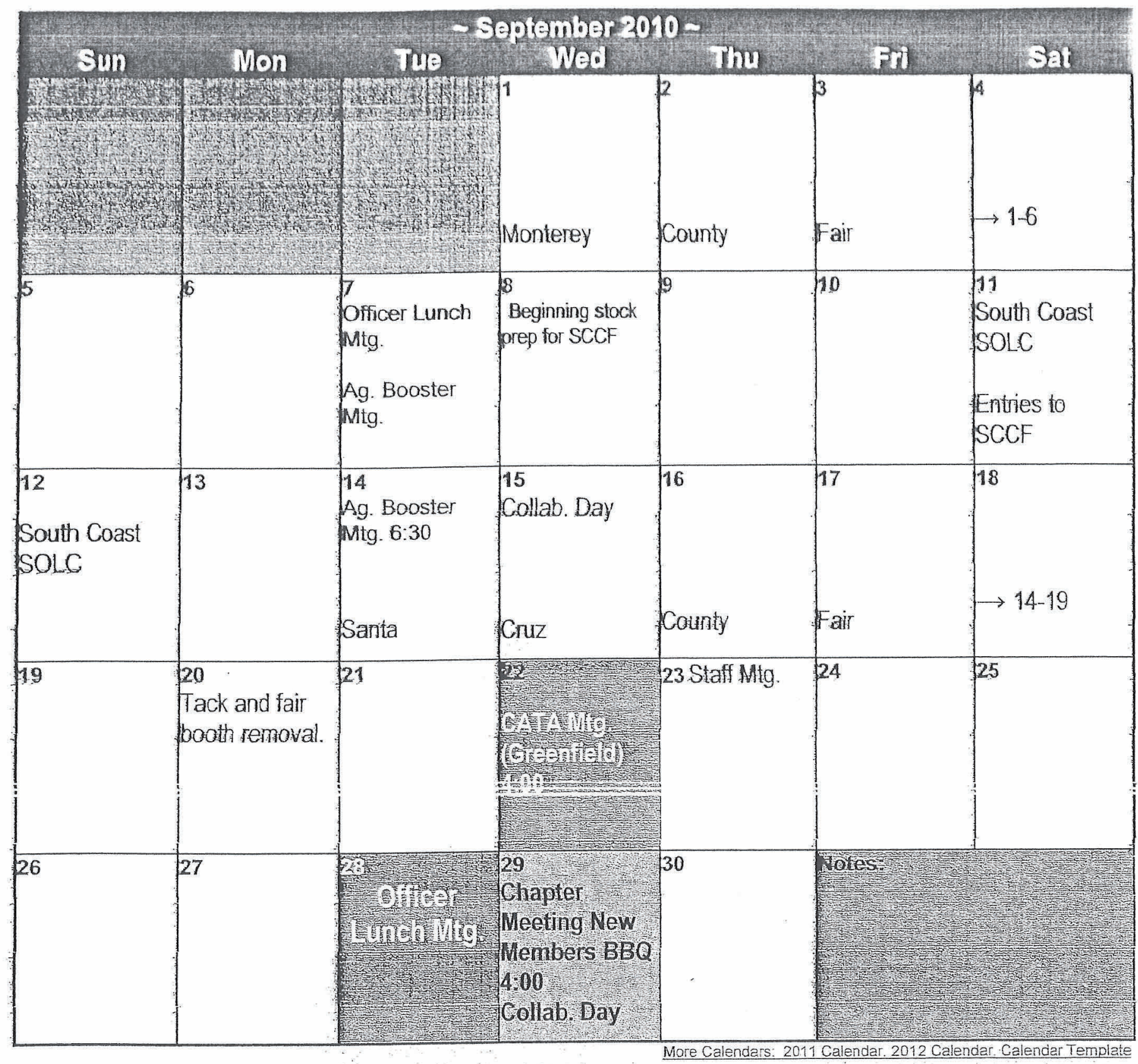




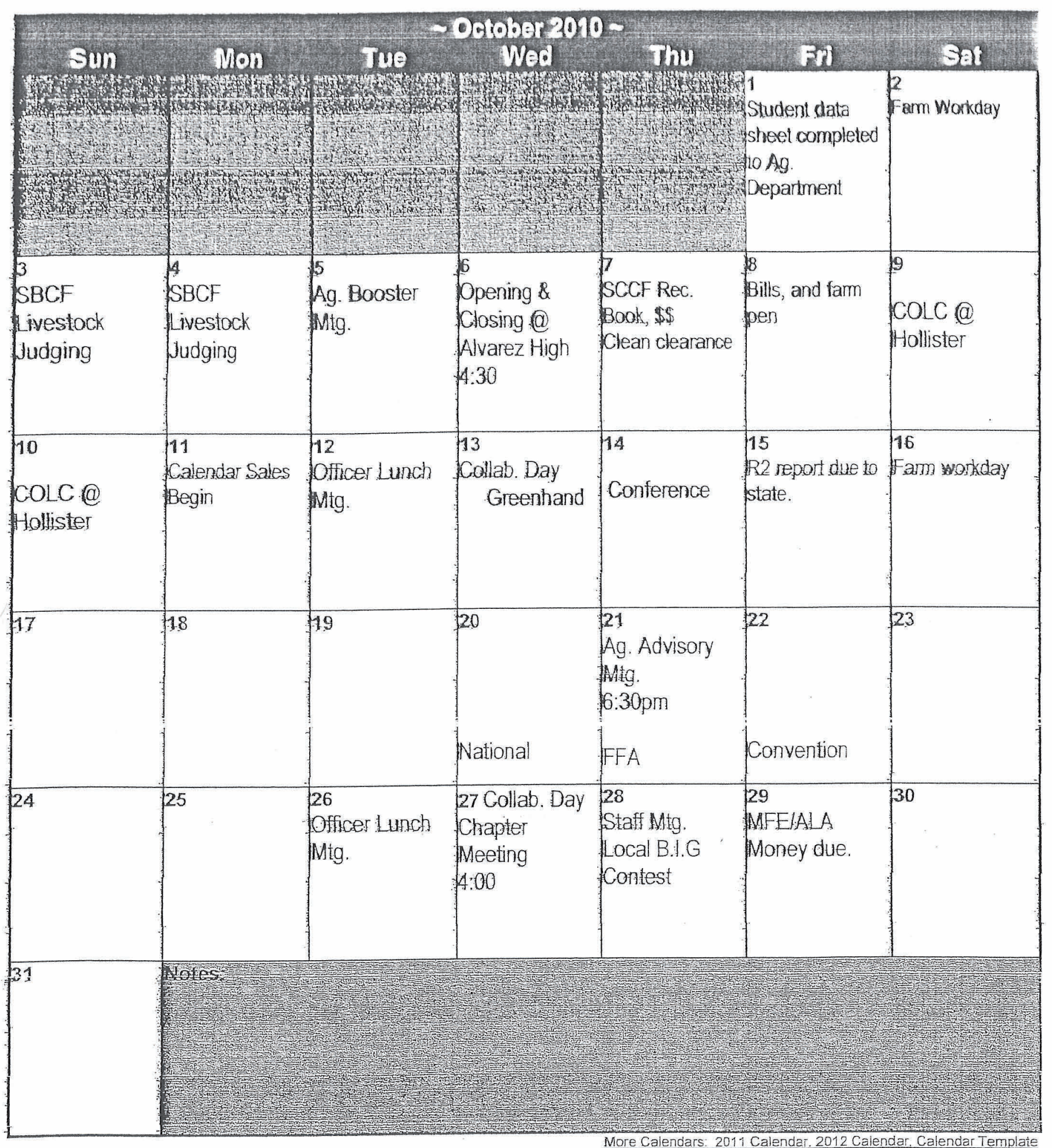




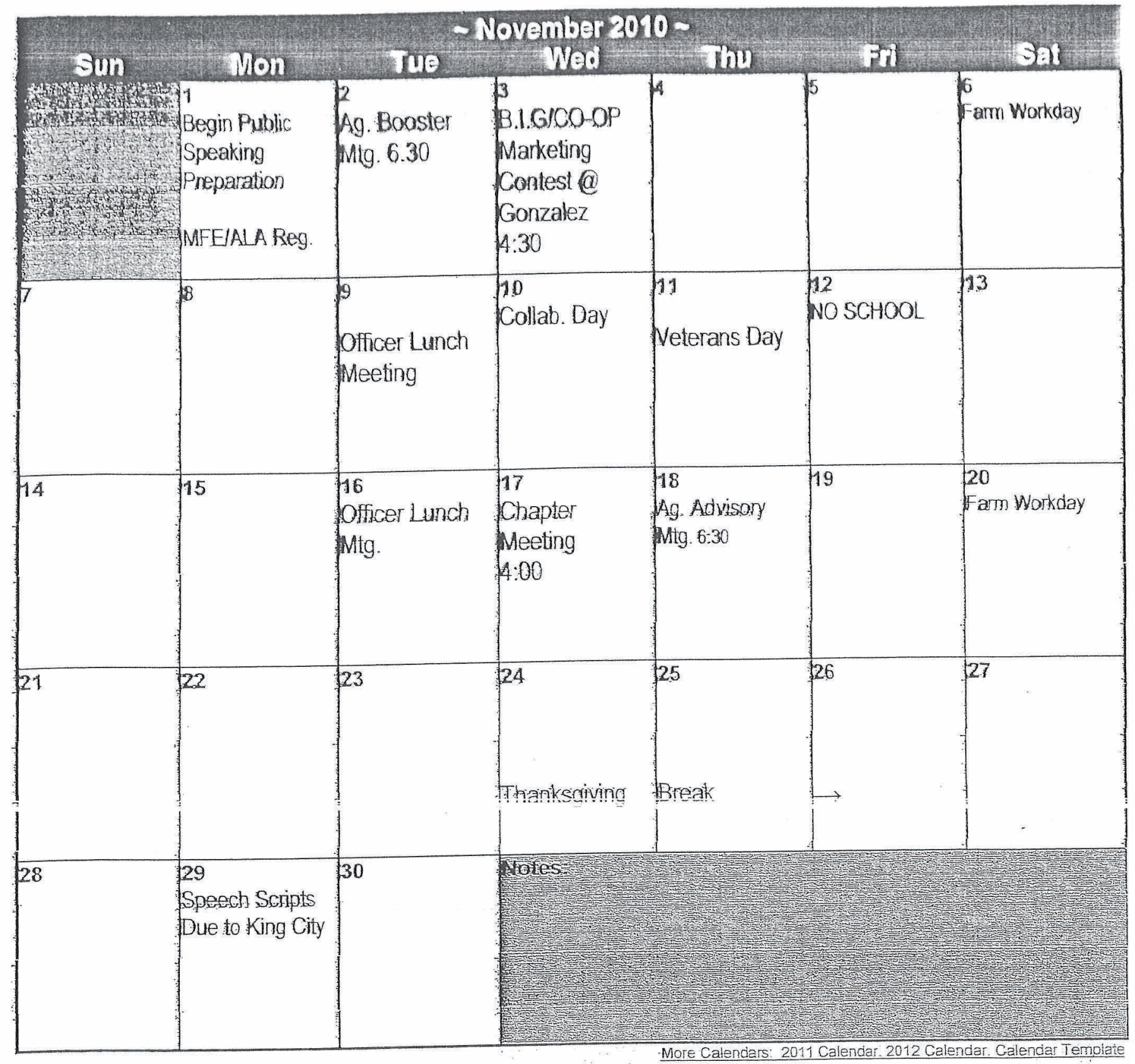




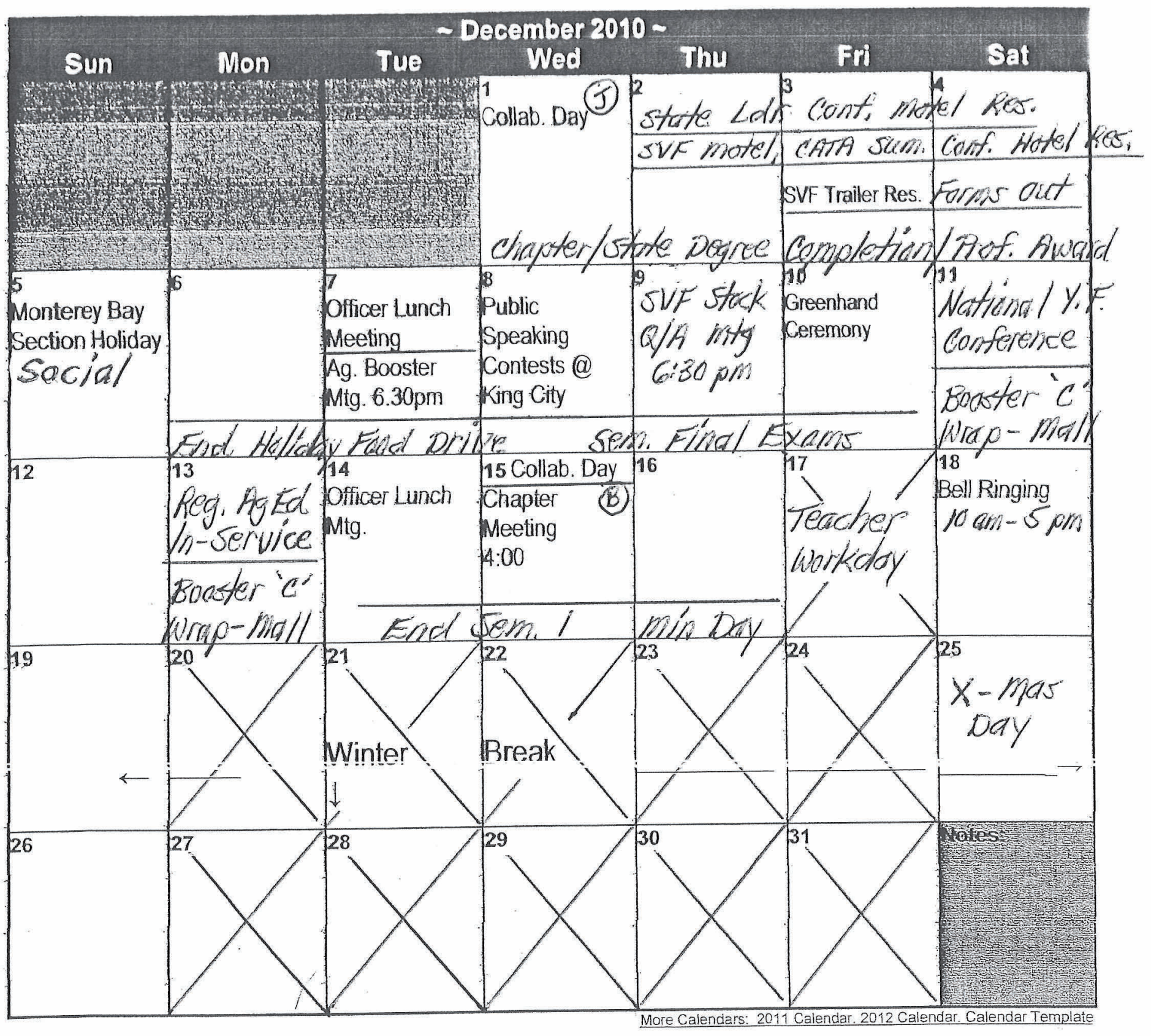




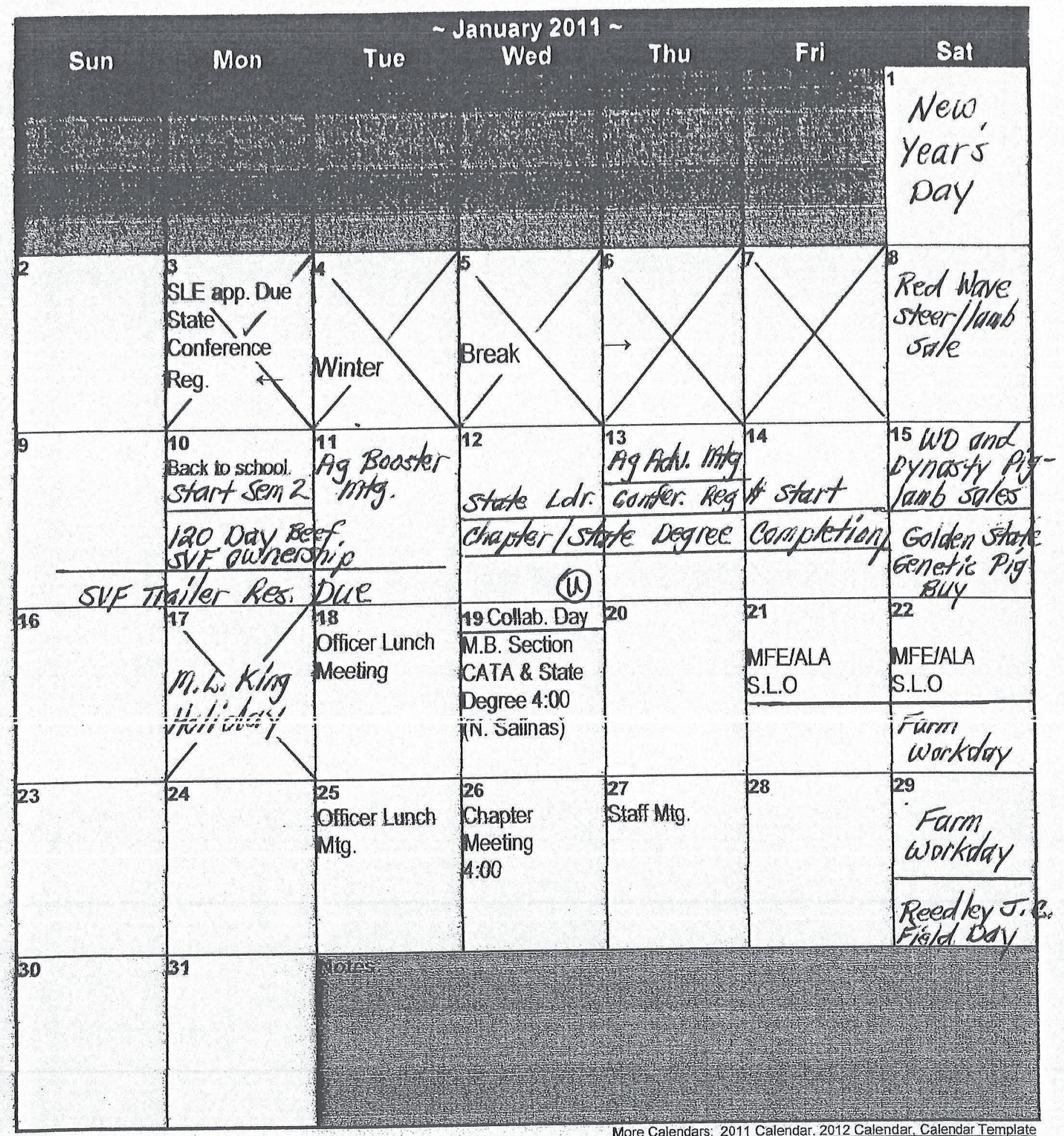

More Calendars: 2011 Calendar. 2012 Calendar, Calendar Template 


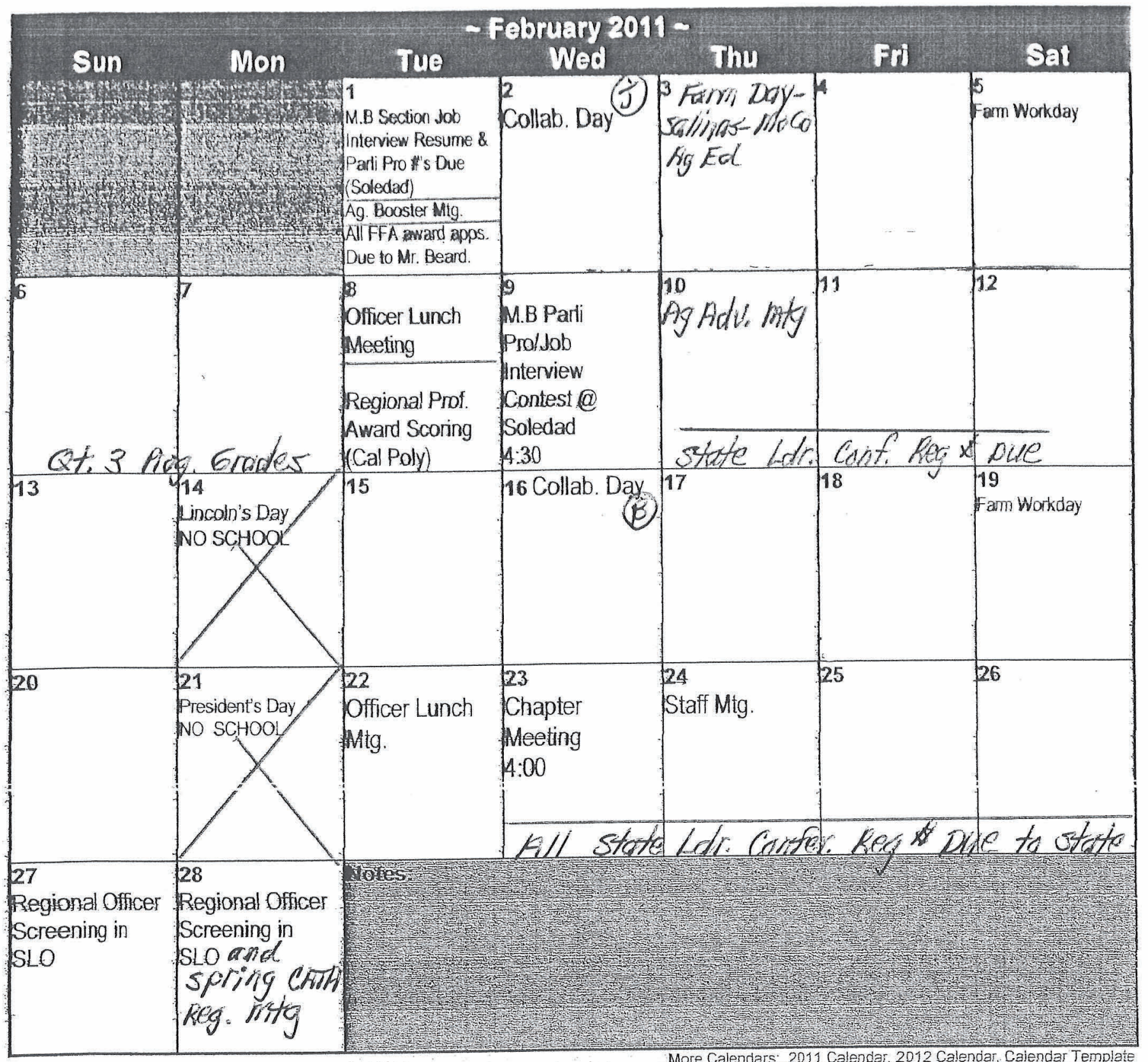




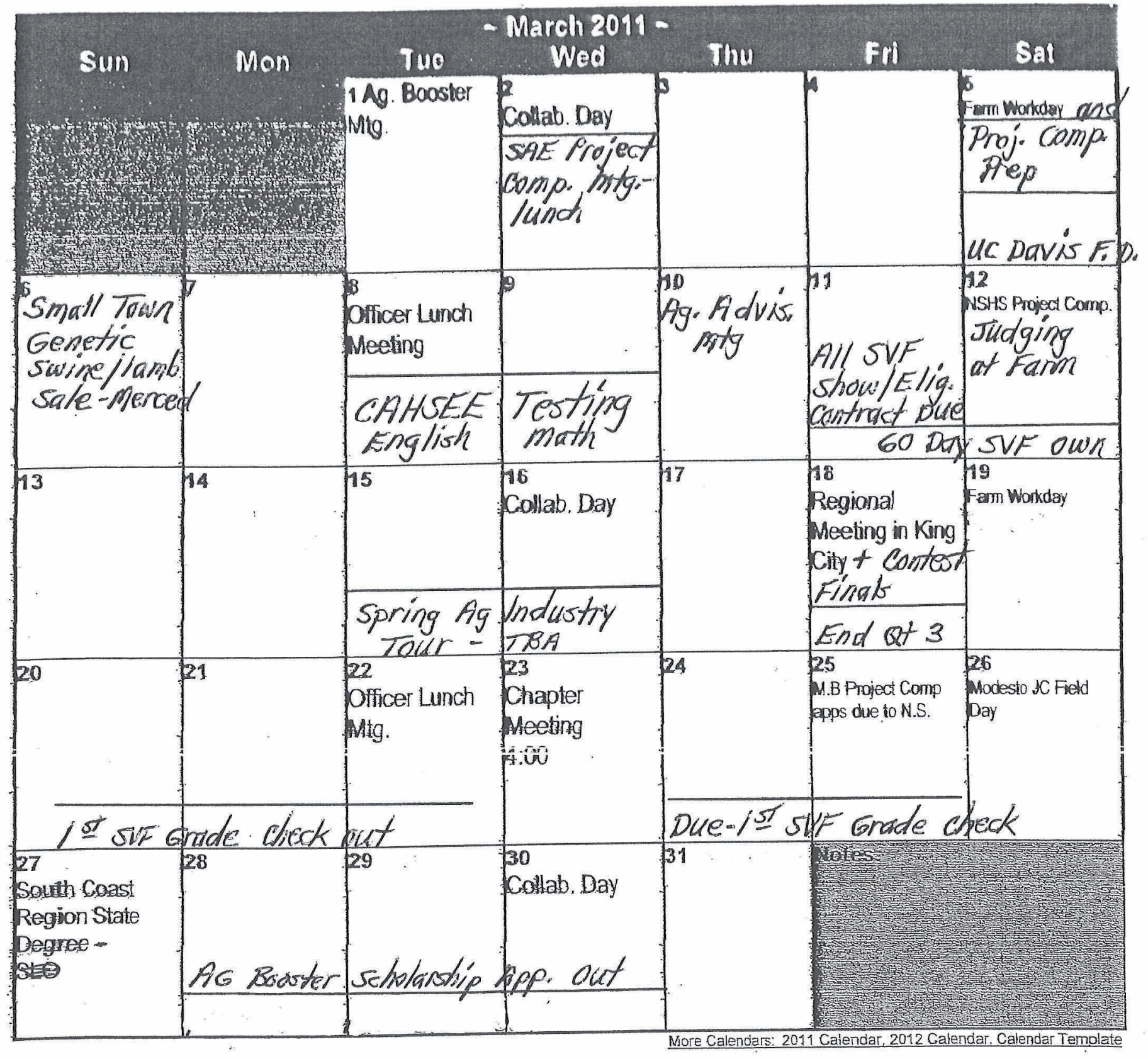




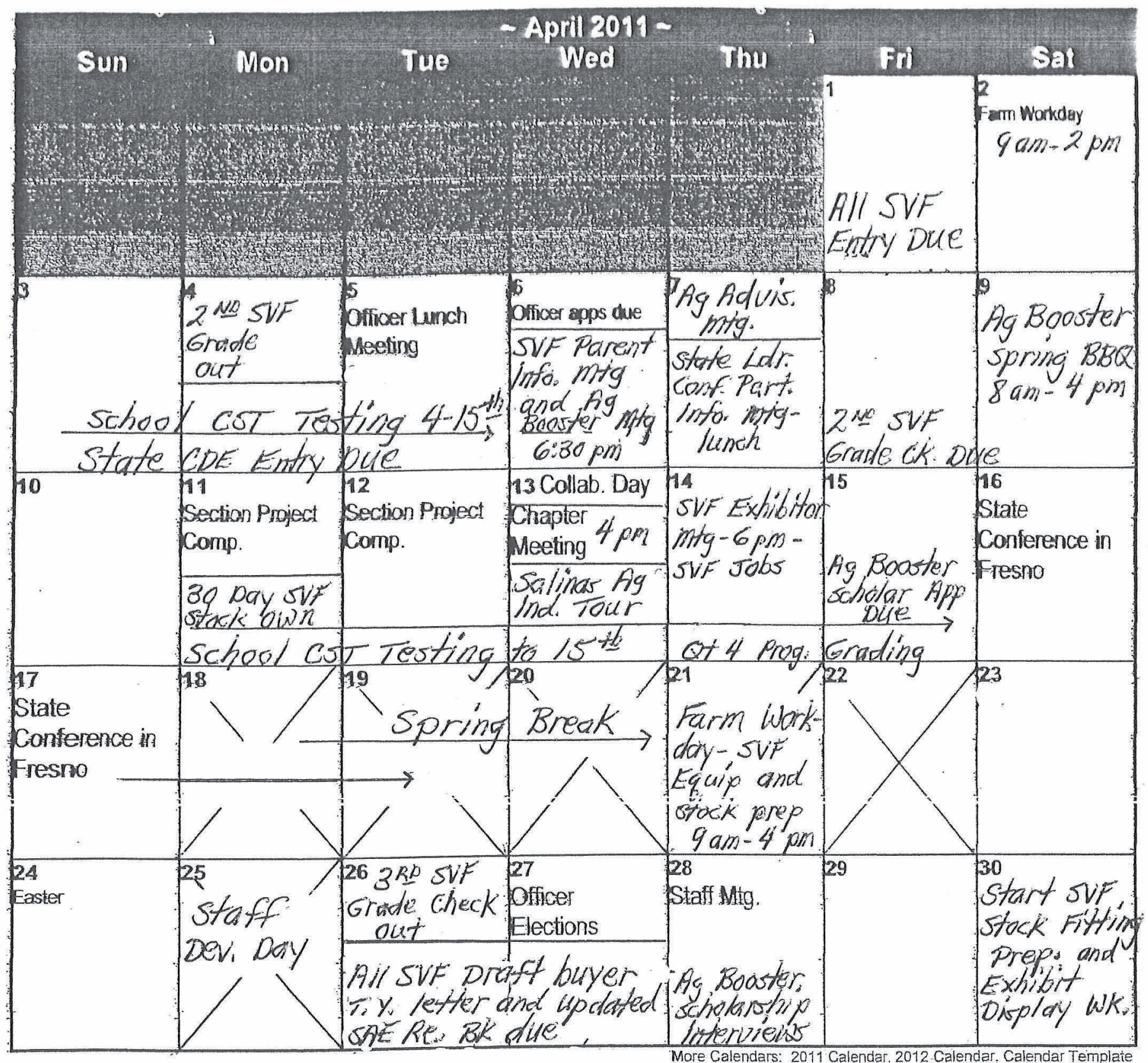




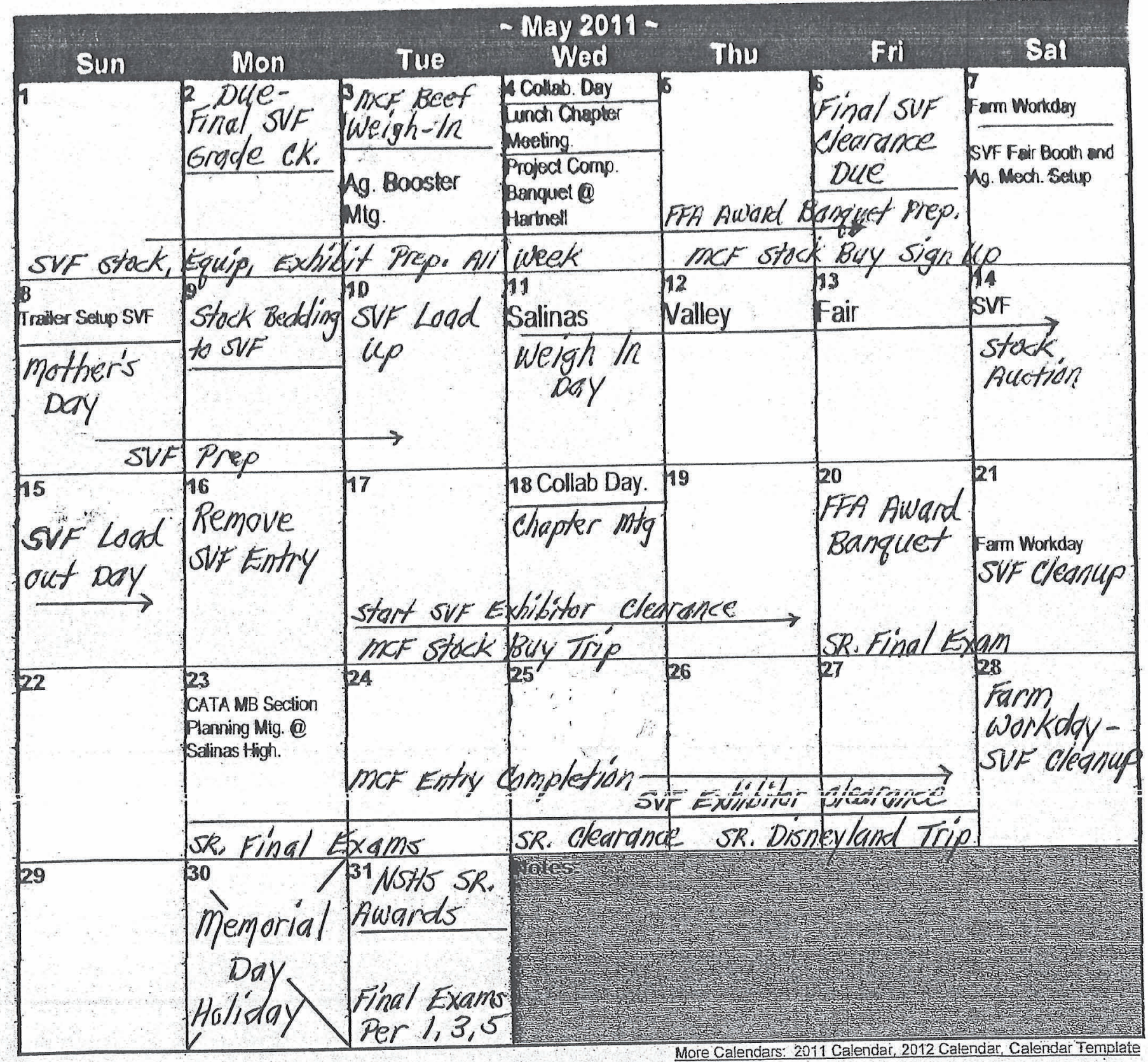




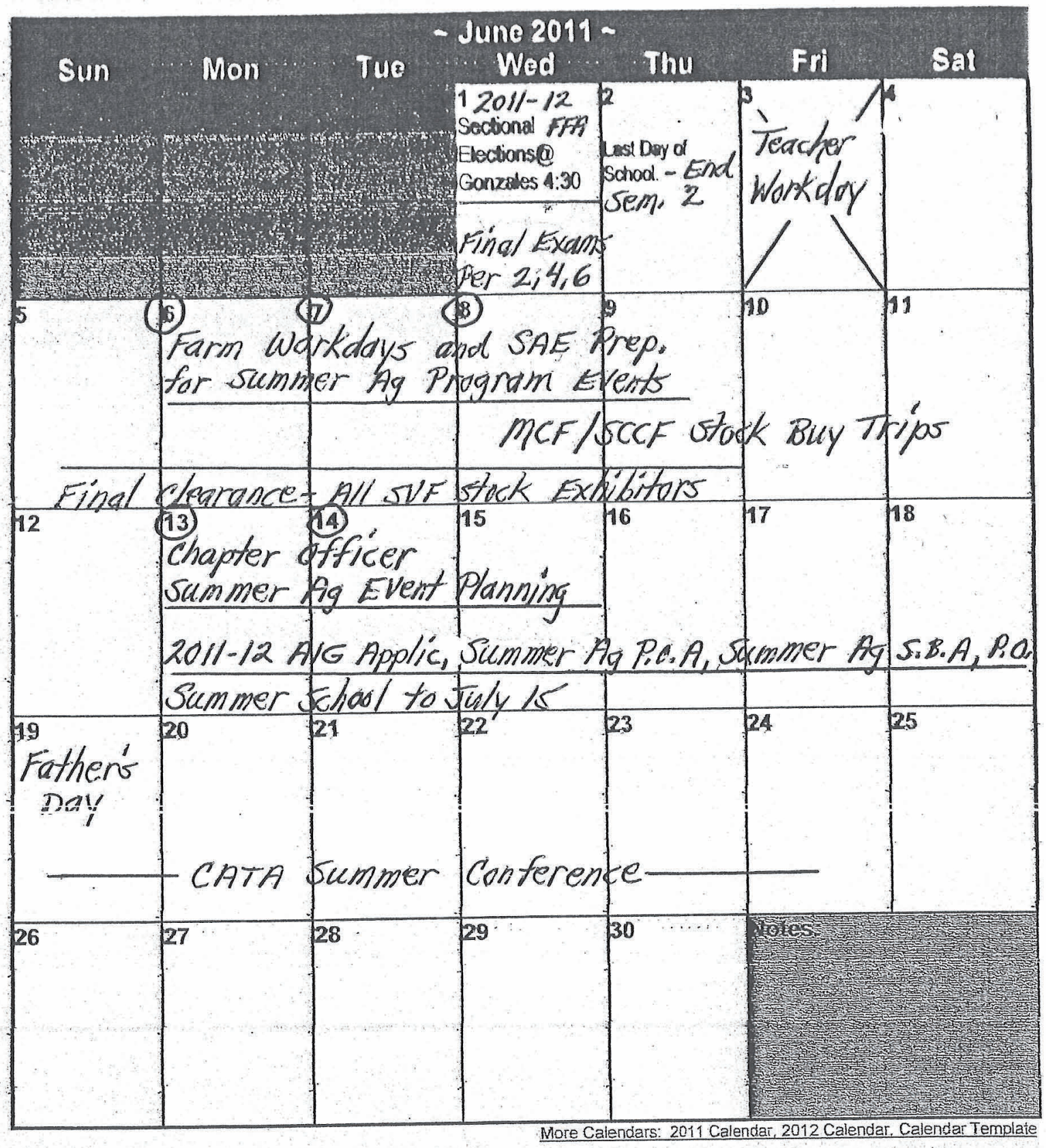




\section{Daily Log}

I do not keep a daily log for the current year, but I plan on starting a daily log for this next year because I would like to be able to justify any of my hours spent on the job or justify what I was doing on a certain day if anyone was to ever question me. A copy of our departments daily log form is provided. 


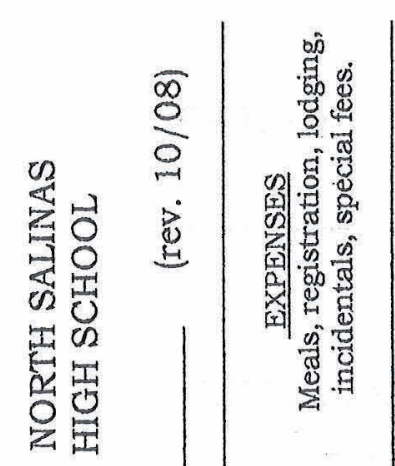

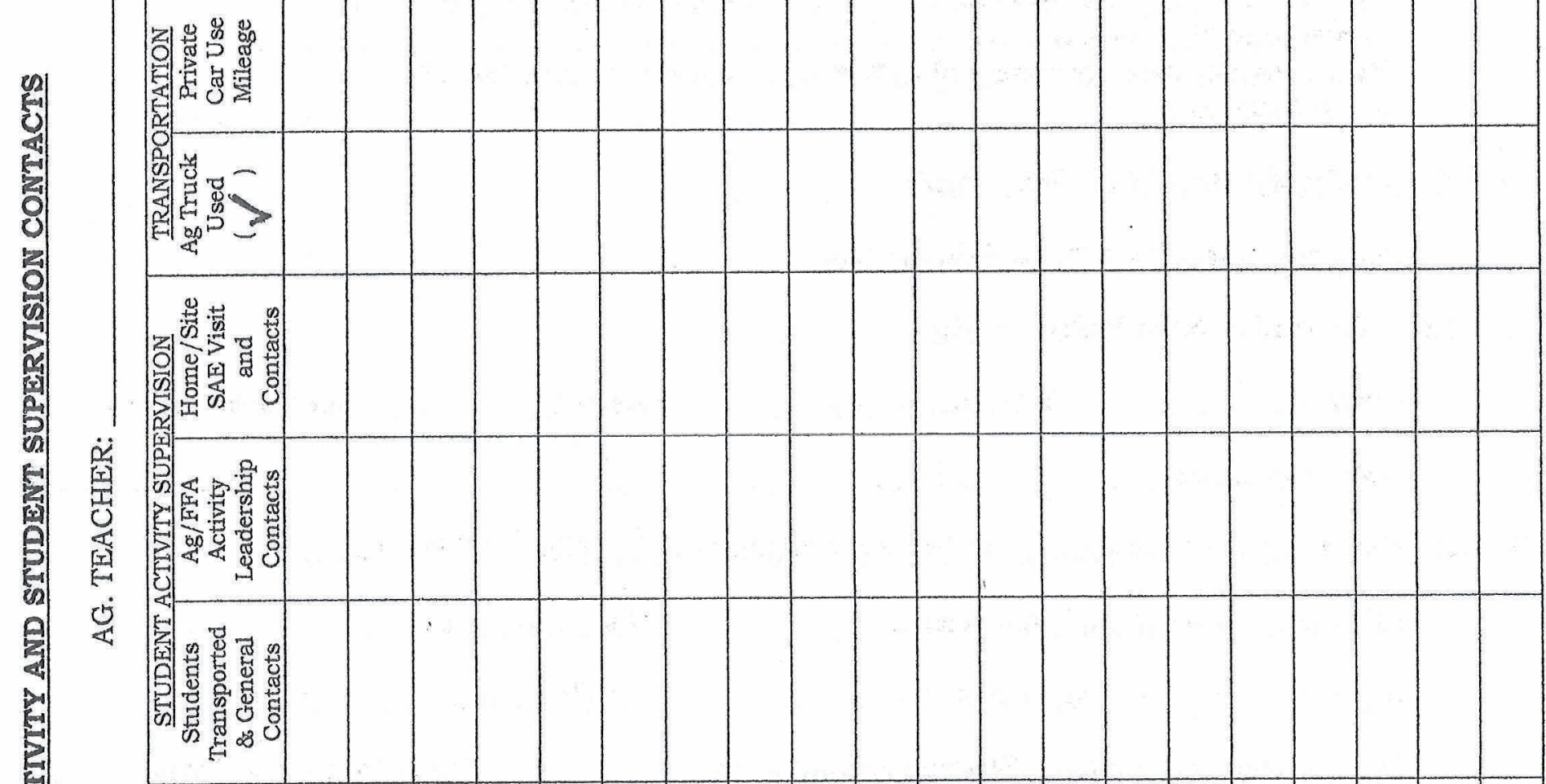

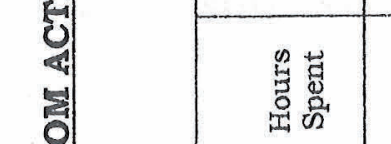

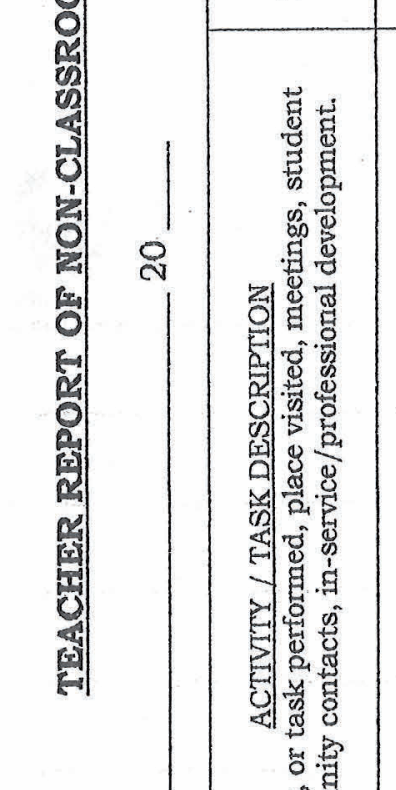

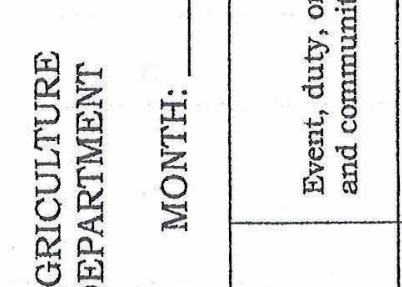


MONTH: , 20

TEACHER:

1. TOTAL NON-CLASSROOM JOB HOURS SPENT:

2. TOTAL STUDENT ACTIVITY AND ENTERPRISE PROJECT SUPERVISON CONTACTS MADE

a. Transportation of, general/follow-up, recruitment, misc. contacts.......

b. Ag/FFA activity supervision, CDE skills team training, leadership development contacts.

c. Home/work site enterprise/placement project visits, and SAEP related contacts

3. TOTAL PRIVAE CAR MILE TRAVELED:

a. Ag Truck use - see truck mileage log.

4. JOB REQUIRED EXPENSES MADE:

Meals: \$__ Registration: $\$ \_$L____ Special Fees: $\$$

Other Expenses:

5. MEETINGS AND PROFESSIONAL DEVELOPMENT ACTIVITIES ATTENDED:

FFA Chapter and/or Committees:

Ag. Department:

CATA:

Ag. Advisory:

Ag. Booster:

School/District, Admin,, Student Confer. Mtg:

Other Prof. Dev. Mtg:

6. AGRICULTURE, COMMUNITY, BUSINESS RELATIONS CONTACTS:

Agribusiness and/or Farmer:

Misc. Business:

Community Group and/or Service Club contacts:

7. SPECIAL ACTIVITIES CONDUCTED OR JOB TASK ACCOMPLISHMENTS.

a.

b.

c.

d.

e.

f.

g.

h:

i. 


\section{Professional Growth Activities}

Every year I attend four sectional meetings, two regional meetings (fall and spring) and the California Agriculture Teachers Association conference. I also attend the regional winter agriculture education in-service (road show) and attended my last New Professionals conference. Not only that, but I was a BTSA support provider this past year. In the future, I plan to continue attending these professional growth activities and perhaps a few more as North Salinas High Schools budget allows. 


\title{
CALIFORNIA ASSOCIATION FFA
}

\section{AFFILIATION MATERIAL ORDER FORM}

The Salinas - North Sallinas Chapter of FFA is requesting 343 Leadership Packets in order to facilitate instruction at the start of school. Ship materials to:

\author{
\# CA0200 \\ North Salinas HS \\ $55 \mathrm{Kip}$ Dr. \\ Salinas, CA 93906
}

Please sign below indicating you understand that your chapter must affiliate at least as many students as you have ordered packets for, and at least as many students as will be

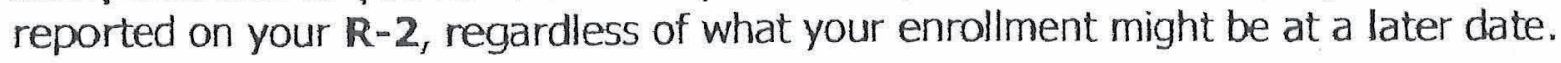

Advisor signature:

Please allow for two weeks delivery from the date your order is received in our office.

\begin{tabular}{|l|c|c|r|}
\hline Description & Qty & Rate & Amount \\
\hline Student & 343 & 8.50 & $2,915.50$ \\
\hline Others & & 7.00 & 0.00 \\
\hline Shipping & 343 & & 55.00 \\
\hline & \multicolumn{2}{|c|}{ TOTAL } & $\$ 2,970.50$ \\
\hline Less "Early Order" & \\
Amount & \\
\hline Net Payment & \\
\hline
\end{tabular}

A Purchase Order addressed to California Association, Future Farmers of America - Vo Ag Student Leadership Program must accompany thing form and malled to:

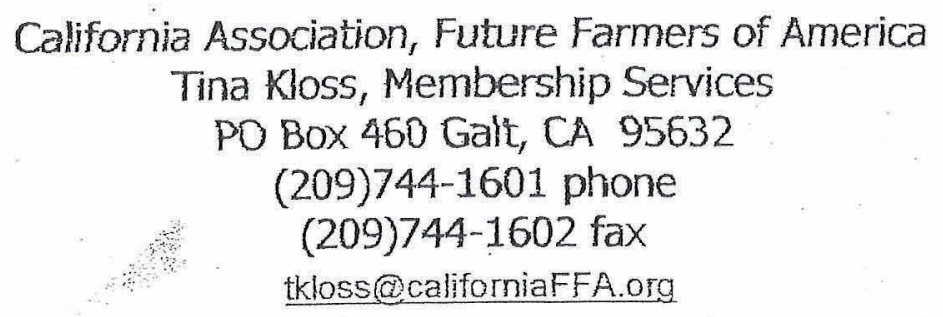

Printed: 07/20/2010

Print Student List 


\section{North Salinas HS \\ R2 Student Report \\ Year:2010}

\begin{tabular}{|l|l|l|}
\hline ProgName & Male & Female \\
\hline Ag Bus Mgt & 11 & 7 \\
\hline Ag Mech. & 90 & 28 \\
\hline Agriscience & 113 & 89 \\
\hline An. Science & 0 & 4 \\
\hline O.H. & 0 & 1 \\
\hline
\end{tabular}

\begin{tabular}{|l|l|l|l|l|l|l|l|}
\hline ProgName & White & Black & Hispanic & Filipino & $\begin{array}{l}\text { American } \\
\text { Indian }\end{array}$ & $\begin{array}{l}\text { Asian/Pacific } \\
\text { Islander }\end{array}$ & Others \\
\hline Ag Bus Mgt & 2 & 0 & 0 & 0 & 0 & 0 & 10 \\
\hline Ag Mech. & 13 & 5 & 0 & 0 & 1 & 2 & 89 \\
\hline Agriscience & 19 & 9 & 0 & 0 & 1 & 1 & 159 \\
\hline $\begin{array}{l}\text { An. } \\
\text { Science }\end{array}$ & 2 & 0 & 0 & 0 & 0 & 0 & 2 \\
\hline O.H. & 1 & 0 & 0 & 0 & 0 & 0 & 0 \\
\hline
\end{tabular}

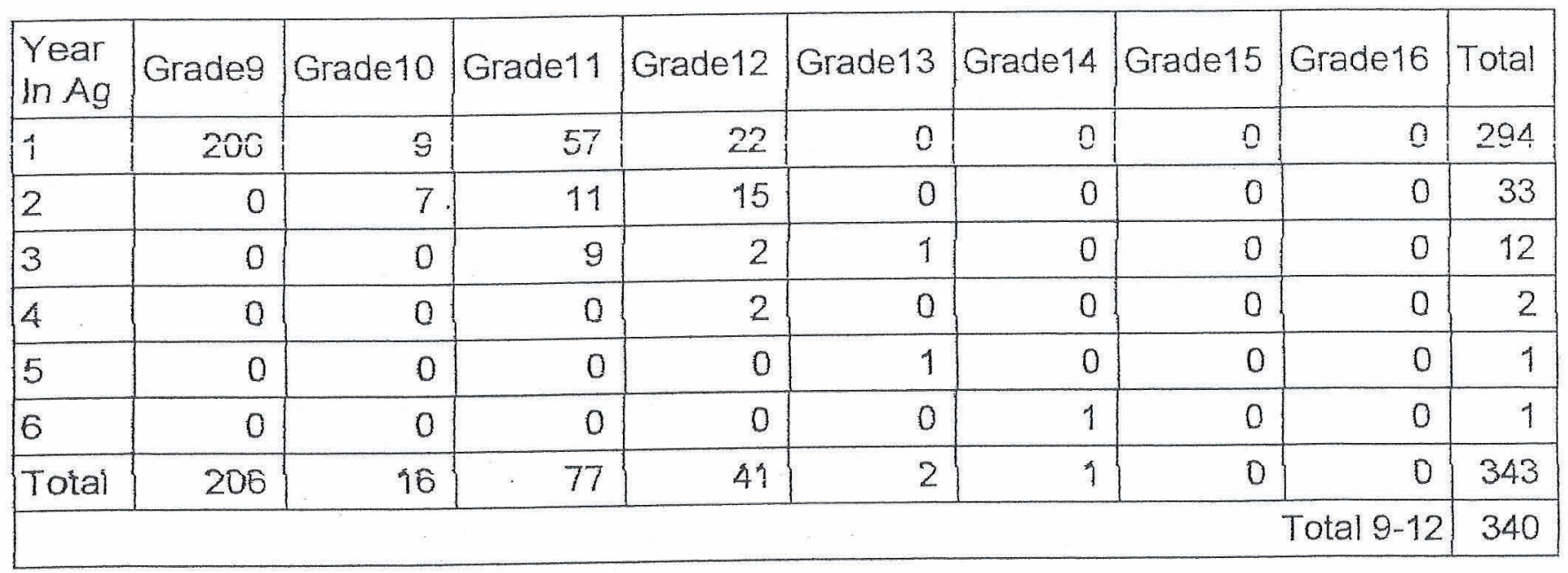

Printed: 10/13/2010 9:52:40 PM 
DATE: June 18, 2009

TO: Mr. A. Caresani, Principal, North Salinas High School

FROM: Stan Uchiyama, Vo-Ag Teacher

\section{SUBJECT: EXTRA WORK AGREEMENT PROPOSAL: 2009-2010 SUMMER AGRICULTURE PROGRAM VO-AG TEACHER EMPLOYMENT}

NEED, IMPORTANCE, AND RATIONAL FOR SUMMER VO-AG PROGRAMS. (Refer to SB-187 State Vo-Ag Advisory Committee Report adopted by State Board of Education; 2005-2006 Vo-Ag Incentive Grant Compliance Review Quality Standard Number 11, Full Year Employment and Standard Number 3, Practical Application of Occupational Skills.):

Effective instruction in Vocational Education in Agriculture extends far beyond the regular school day, school year, and school environment. The basic component of instruction occurs as group instruction in the classroom, shop, or field during regularly scheduled classes September through June. The unique character of California Vo-Ag education programs encourages continued instruction during the summer months, July through August.

The vocational agriculture teacher's supervision of student agricultural/occupational experiences (SAE) is to (1) provide individual instruction as needed; (2) assist the student in selecting and conducting occupational experiences which are relevant to his or her occupational objectives and which include opportunities to reinforce learning and apply skills and knowledge in class or shop; (3) counsel the student in employer-employee relationships; (4) assist in locating suitable employment for program students and graduates; (5) provide opportunities for student

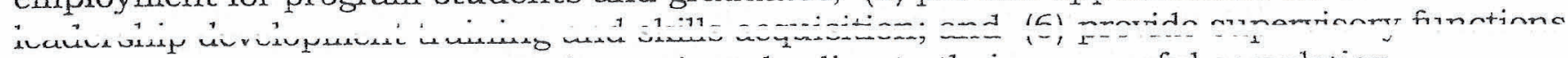
and activities for student enterprise projects leading to their successful completion.

Since the instructional program is considered to be best accomplished on a year-round basis for reasons stated previousiy, and since FFA is an integral part of the agriculture education instructional program, it follows that the FFA advisors(s) should be available to supervise and conduct those FFA leadership and enterprise project activities which are scheduled for the summer months and other times outside the regular class schedule. To meet the Summer Vo-Ag program objectives, needs of participating students and to maintain Ag. Program continuity, teacher compensation is necessary.

BACKGROUND: The 2007-08 Summer Vo-Ag program provided $\$ 5314$ (130 hours at $\$ 37.53$ per hour, including teacher benefits) for 1 FTE agriculture teacher at North Salinas High School. Per annual Ag. Incentive Grant specifications and compliance evaluations, \$4000 in additional state funds were received and used for non-salaried program expenditures during the 2008-09 school year.

For the 2009-10 year, our School will continue to have a summer ag. program that will involve 1 FTE ag. teacher. Our 2009-10 enrollment will, again, qualify our school to receive additional State Ag. Grant funding for non-salary Ag. Program use. Without 2009-10 District Summer Ag. Program salary support our school will not qualify to receive the full Ag. Grant funds available to us. Receiving the maximum amount of Ag. Grant funds is critical to the effective year-long operation of North High's active agriculture program. 
PROPOSAL: Two FTE North High ag. teachers be retained to be responsible for the conduct of student enterprise projects, school farm management supervision, FFA student leadership activities, applicable contact with incoming students and parents, Ag. Booster Club activities, summer program management needs, Ag. facilities and equipment maintenance, and supervision of student participation in the Monterey and Santa Cruz County Fairs. Documented teacher activity logs for summer employment have far exceeded the hourly allocations for any one past summer employment period. Minimum teacher duties are proposed and teachers will submit detailed activity description logs and time sheets to validate their performance of described duties. Although it is not equitable to surrounding School Districts providing summer $\mathrm{Vo}-\mathrm{Ag}$ employment for their teachers, the following 2009-10 Summer Vo-Ag Program employment plan is proposed:

I. Summer Ag. Program Support Expenses:

* Ag truck operational costs: Paid by our site budget allocations.

* Teacher expenses and private car mileage: Paid from our school's 2009-10 Ag. Grant or other available North High funding sources.

II. Ag. Teacher Summer Ag. Program Employment:

* Two FTE ag. teachers. 260 employment hours, $\$ 37.53$ hourly rate $=\$ 9,758$ (130 hours/FTE)

-- Teacher hour allocations are for July-August, 2009 and June, 2010. We will save an optional 8 hours for June, 2010 use.

-- 70 students involved with 21 County Fair (MoCo and S.C.C.F) animal, 108 ag. mechanics, 47 ag. science, Chapter Feature Booth, and livestock educational exhibits.

\section{IMPLEMENTATION, COMPLIANCE CRITERIA, AND MINIMUM TEACHER DUTIES FOR SUMMER VO-AG PROGRAM.}

1. Construct a calendar of activities for FFA leadership development and SAEP activities for July and August.

2. Descriptive activity logs will be kept by teachers to verify their performance of duties and shall be used to validate monthly salary allotments.

3. Teachers shall be responsible for the up-keep and maintenance of the agriculture truck, school farm facility, school site facilities, and equipment, as required by our school's ag. program needs.

4. Teachers will make reasonable project supervision contacts with involved students. Number of supervision visits/contacts made shall be according to student project type and/or needs.

5. Hourly salary time may include time spent for summer program/ag. department administration work, community relations, and other related activities of benefit to the supervised project, Vo-Ag and FFA summer program. Time spent shall be balanced with student supervision time. 
6. Teachers will conduct FFA and Ag. Program activities and supervise those events which are necessary to the proper functioning of the FFA Chapter and Vo-Ag programs during the summer months, including work with support groups, new activities, and other necessary duties related to successful summer program operations.

7. Out-of District travel (School Business Agreements) and reimbursement requests shall be approved by and/or filed with school administration.

8. Teachers make plans to contact or involve incoming freshman students and potential new students. Such plans shall be the design of each teacher, pending availability or reasonable implementation time.

RESPONSE: To allow for agriculture staff coordination of summer program duties, calendar schedule development and miscellaneous payroll filing deadlines, I await your timely response to my proposal. Thank you for your consideration.

Cc: Mr. T. Jones

Ms. S. Pfarr 


\section{MEMORANDUM}

TO: Mr. Randy Bangs, Director VEA/ROP

FROM: Stan Uchiyama, Ag. Dept. Chairman, NSHS

SUBJECT: 2009-2010 Request for TEACHER SUBSTITUTE TIME, CONFERENCE REGISTRATION FEES, AND LODGING EXPENSES from allocated VEA/Perkins funding source.

DATE: December 9, 2009

Coples TO: Mr. T. Jones, Miss. S. Blodgett, and Mr. A. Caresani

In planning this year's agriculture teacher professional in-service and FFA student activities program, I REQUEST YOUR APPROVAL for substitute teacher time and/or conference registration and lodging fees to support the following activities and dates. Depending on duration and supervision/chaperone requirements, two or three teachers are required to satisfactorily conduct the designated activity. Request based on May 28, 2009 memo allocating VEA funds available for use by the three teacher NSHS Ag. Staff, which is:

$\Leftrightarrow$ Six days substitute teacher expenses for professional development.

- Six days substitute teacher expenses for FFA Leadership travel.

$\Leftrightarrow \$ 2000$ registration ( $\$ 1000$ per teacher), lodging, meals, and other travel expenses.

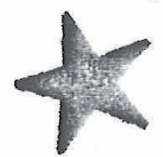

Special request: With a third ag. teacher added to our staff, I request an additional $\$ 650$ in VEA travel and conference support funds? Please respond.

Ag Incentive Grant has limited non-salary teacher funds to cover meals, some registration or in-service fees, and lodging expenses not requested below. In addition, NSHS site funds have been requested to complete funding needs not requested below. Whenever possible, travel will be by school's agriculture truck and/or school van, including approved VEA or other travel forms not listed below. If you see any administrative support problems, I would like to meet with you to discuss some kind of workable solution to your concerns. I appreciate your consideration of my request and I await your timely reply. Thank you.

TRAVEL REQUESTS (SCHOOL BUSINESS AGREEMENTS) HAVE BEEN OR WILL BE SUBMITTED.

October 10-11, 2009: REGISTRATION. South Coast Region CATA Fall Meeting and Chapter FFA Officer Leadership Conference. Attendance, per Ag Incentive Grant evaluation criteria.

** Three teacher registration fee. $\$ 150$

December 14, 2009:

THREE DAY SUTSTITUTE AND REGISTRATION. Region AgEd

In-Service Day. Attendance, per Ag Incentive Grant evaluation criteria.

** Three teacher and Department registration fee.....\$125

** Three day teacher substitute expense.................\$375

February 22, 2010:

THREE DAY SUBSTITUTE AND REGISTRATION. Spring Region CATA In-Service meeting and FFA Region Officer screening. Attendance, per Ag Incentive Grant evaluation criteria.

** Three teacher registration fee........................\$ 90

** Three day teacher substitute expense.................\$375

March 19, 2010:

TWO DAY SUBSTITUTE. Region FFA Leadership Conference and FFA Contest finals. Student Delegate attendance required by State FFA Association and Ag Grant evaluation criteria.

** Two day teacher substitute expense....................\$250 


\section{Mission Trails Regional Occupational Programs}

QT: Agriculture and Natural Resources

FROM: $\quad$ Eric Deleissegues

DATE: $\quad$ May 28, 2009

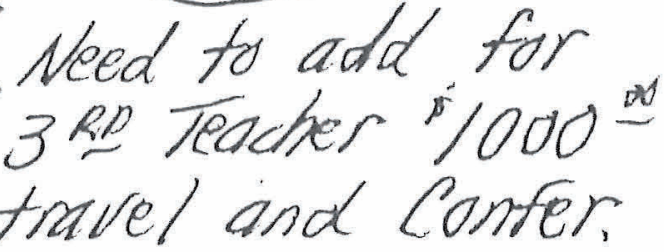

SUB JECT: Agriculture and Natural Resources VEA Allocations for 09-10

As part of the district 5-year plan the following gives you the district allocation for the Agriculture and Natural Resources Department this school year. Please note that there are three district categories for this allocation.

\section{A. CURRICULUM DEVIELOPMENT}

To align the Agriculture and Natural Resources Department with the state CTE model curriculum and framework. In addition CTE instructors will align their new curriculum with the embedded academic standards from Language Arts, Math, Science \& Social Studies.

Allocation: Each teacher $=3$ teacher $\times 2=6$ day $=1 / 5 / 5$ Two release days for the department to meet at ROP.

\section{B. PROFESSIONAL DEVELOPMENT}

To allow an Agriculture teachers from SHS, NSHS, and EAHS to attend a conference that is directly related to the CTE model curriculum standards framework.

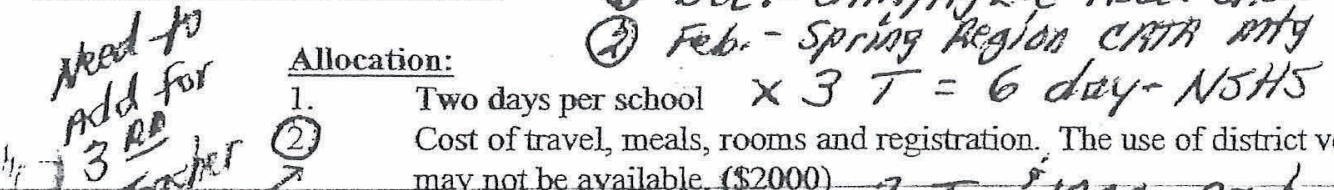

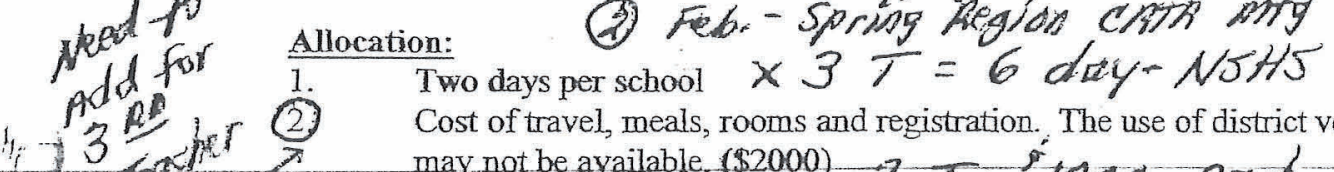

Q Dec. - CATH/Rg Ed 'Raed Shat' - 3 day

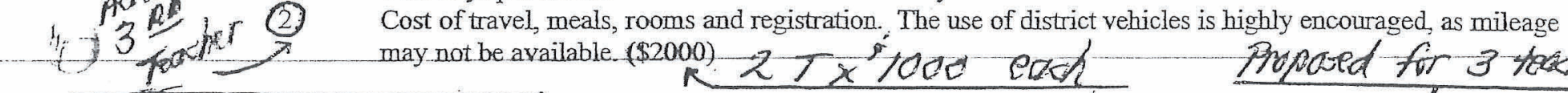

(1) Spring Rq. $\frac{\text { IFA Travel }}{\text { SHS - 2 days, } 2 \text { teachers }}$

FFP Mity

(2) Stafe Lar o d NSHS -2 days, 3 teachers $=$

C. INSTRUCTIONAL IMPRO VEMENT

(1) CAMA Fall arid spring, heg.

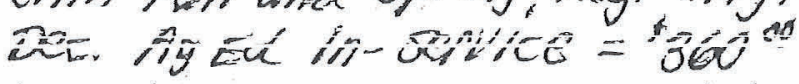

Bropored for 3 toweres

(2) state FFP Ldr. confer (ladging $/$ Reg $\left.^{\circ}\right)=660^{\circ}$

(3) state chits stiminer canf $=572300$

This is to select one or two program areas and purchase equipment and materials that will be used to improve or upgrade the

instructional materials to meet the aligned curricular standards and frameworks.

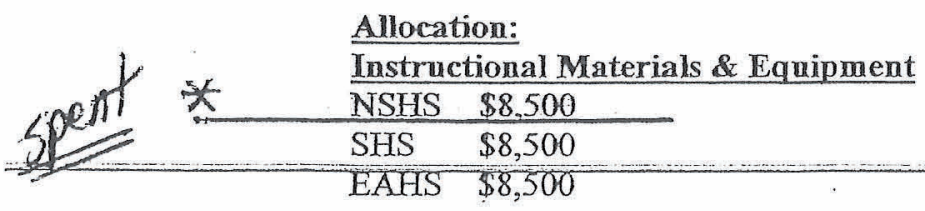

Please note: these allocations mary change due to total number of Career Tech Ed sections in the 2009-2010 master schedule.

You must have your principal's approval as well as our approval for use of these funds. Please include justification for all professional development and instructional improvement activities.

All purchase orders associated with these CTE allocations must be turned in by June 12, 2009 to Karla at the ROP Center.

$\mathrm{ED} / \mathrm{cg}$

')

Tim Vanoli, District Office

Augie Caresani, NSHS

Michael Romero, SHS

Darren Hershberger, EAHS 


\section{NSIIS Agriculture Department Stan I chiyana Tom Jones Stephanic Blodget}

\section{Memo}

$\begin{array}{ll}\text { To: } & \text { Mrs. Jean Ashen, Athletic Director } \\ \text { From: } & \text { Tom Jones, Ag/FFA Program } \\ \text { cC: } & \text { Mr. Uchiyama, Mrs. Blodgett } \\ \text { Date: } & \text { July 28, 2010 }\end{array}$

Re: 2009-2010 School Van Use Request

\section{How to Use This Memo Template}

With your approval, please schedule the Ag. Dept/FFA to use a school Van on the following dates and pickup/retum times. If available, we would like to use Van 151 or 103 for out-of-town trips. * indicates priority need for van use. Thank you and I await your timely response

\begin{tabular}{|c|c|c|c|}
\hline $\begin{array}{l}\text { Date \& time } \\
\text { Needed }\end{array}$ & Destination & Activity & $\begin{array}{l}\text { Date\& Time } \\
\text { Retumed }\end{array}$ \\
\hline $\begin{array}{l}\text { Aug } 4-6,2010 \\
(8 \mathrm{am})\end{array}$ & \multicolumn{2}{|c|}{ LakeNacimiento *FFA Leadership Camp } & $\begin{array}{c}\text { Aug. } 6,2010 \\
(6 \mathrm{pm})\end{array}$ \\
\hline $\begin{array}{l}\text { Aug } 21,2010 \\
(8 \mathrm{am})\end{array}$ & Monterey & $\begin{array}{l}\text { * Monierey County Fair } \\
\text { AS/AM Exhibit Entry }\end{array}$ & $\begin{array}{l}\text { Aug. } 21,2010 \\
{[7 \mathrm{pm}]}\end{array}$ \\
\hline $\begin{array}{c}\text { Aug } 30-\operatorname{Sep} 72010 \\
{[8 \mathrm{am}]}\end{array}$ & Monterey & $\begin{array}{l}\text { * Monterey County Fair } \\
\text { Student Trans/Super. }\end{array}$ & $\begin{array}{l}\operatorname{sep} 7,2010 \\
{[10 \mathrm{pm}]}\end{array}$ \\
\hline $\begin{array}{l}\operatorname{Sep} 11-12,2010 \\
{[8 \mathrm{am}]}\end{array}$ & Watsonville & $\begin{array}{l}\text { *Santa Cruz County Fair } \\
\text { ASIAM Exhibit Entry }\end{array}$ & $\begin{array}{l}\text { Sep } 11-12,2010 \\
\quad[8 \mathrm{pm}]\end{array}$ \\
\hline $\begin{array}{c}\operatorname{Sep} 17,2010 \\
{[3 \mathrm{pm}]}\end{array}$ & Watsonville & $\begin{array}{l}\text { * Santa Cruz County Fair-2 vans } \\
\text { Livestock Judge Contest }\end{array}$ & $\begin{array}{c}\text { Sep } 17,2010 \\
{[11 \mathrm{pm}]}\end{array}$ \\
\hline $\begin{array}{c}\text { Sep } 20,2010 \\
{[3 \mathrm{pm}]}\end{array}$ & Watsonville & $\begin{array}{l}\text { * Santa Cruz County Fair } \\
\text { Exhibit Removal }\end{array}$ & $\begin{array}{c}\operatorname{Sep} 20,2010 \\
{[9 \mathrm{pm}]}\end{array}$ \\
\hline $\begin{array}{l}\text { Oct } 3,2010 \\
{[9 \mathrm{am}]}\end{array}$ & $\begin{array}{l}\text { Hollister } \\
\text { [Bolado Park] }\end{array}$ & $\begin{array}{l}\text { San Benito County Fair } \\
\text { Livestock Judge Contest }\end{array}$ & $\begin{array}{l}\text { Oct } 3,2010 \\
\quad[6 \mathrm{pm}]\end{array}$ \\
\hline $\begin{array}{l}\text { Oct } 6,2010 \\
{[3 \mathrm{pm}]}\end{array}$ & Alvarez 2 Van & $\begin{array}{l}\text { S Section FFA } \\
\text { Meeting Ceremony Contest } \\
\text { Student Trans/Super. }\end{array}$ & $\begin{array}{l}\text { Oct } 6,2010 \\
{[10 \mathrm{pm}]}\end{array}$ \\
\hline
\end{tabular}

(See next page) 
opdete Nopo
Date \& Time
Destination
Activity
Date \& Time
Needed
Returned

\begin{tabular}{|c|c|c|c|}
\hline $\begin{array}{l}\text { Oct } 9-10,2010 \\
{[9 \mathrm{am}]}\end{array}$ & Hollister & $\begin{array}{l}\text { * Chapter FFA Officer } \\
\text { Leadership Conference }\end{array}$ & $\begin{array}{c}\text { Oct } 10,2010 \\
{[5 \mathrm{pm}]}\end{array}$ \\
\hline $\begin{array}{c}\text { Oct } 13,2010 \\
(3 \mathrm{pm}\}\end{array}$ & Paso Robles 2 Vans & $\begin{array}{l}\text { *Region FFA Freshman } \\
\text { Leadership Conference }\end{array}$ & $\begin{array}{l}\text { Oct } 13,2010 \\
{[6 \mathrm{pm}]}\end{array}$ \\
\hline $\begin{array}{l}\text { Nov } 4,2010 \\
\quad(3 \mathrm{pm})\end{array}$ & Gonzales & $\begin{array}{c}\text { *BIG and CO-OP Mkt } \\
\text { Contests }\end{array}$ & $\begin{array}{l}\text { Nov } 4,2010 \\
(11 \mathrm{pm})\end{array}$ \\
\hline $\begin{array}{c}\text { Dec } 8,2010 \\
(3 \mathrm{pm})\end{array}$ & King City & $\begin{array}{l}\text { * County FFA Public } \\
\text { Speaking Contest }\end{array}$ & $\begin{array}{l}\text { Dec 8, } 2010 \\
{[11 \mathrm{pm}]}\end{array}$ \\
\hline $\begin{array}{c}\operatorname{Jan} 21-22,2011 \\
(6 \mathrm{am})\end{array}$ & San Luis Obispo 2 Vans & $\begin{array}{l}\text { * State FFA Excellence } \\
\text { Leadership Conference }\end{array}$ & $\begin{array}{l}\operatorname{Jan} 22,2011 \\
(6 \mathrm{pm})\end{array}$ \\
\hline $\begin{array}{c}\text { Feb } 9,2011 \\
{[3 \mathrm{pm}]}\end{array}$ & Soledad & $\begin{array}{l}\text { County FFA Job } \\
\text { Interview Parli/Pro Contest }\end{array}$ & $\begin{array}{l}\text { Feb 10, } 2011 \\
{[9 \mathrm{pm}]}\end{array}$ \\
\hline $\begin{array}{c}\text { Feb } 10,2011 \\
{[7 \mathrm{am}]}\end{array}$ & $\begin{array}{l}\text { Salinas } \\
\text { (Growers Lane) }\end{array}$ & $\begin{array}{l}\text { *Moco AgEd Fam Day } \\
\text { Ag Ed Demonstrations }\end{array}$ & $\begin{array}{l}\text { Feb 10, } 2011 \\
{[6 \mathrm{pm}]}\end{array}$ \\
\hline $\begin{array}{r}\text { Feb } 9,2011 \\
{[3 \mathrm{pm}]}\end{array}$ & Tulare, CA 2 Vans & $\begin{array}{l}\text { * World Ag Expo Ag Equip. } \\
\text { Technology Field Trip }\end{array}$ & $\begin{array}{l}\text { Feb 10, } 2011 \\
{[11 \mathrm{pm}]}\end{array}$ \\
\hline $\begin{array}{c}\text { March 4, } 2011 \\
{[3011}\end{array}$ & Davis, CA & $\begin{array}{l}\text { *UC Davis Ag Judging } \\
\text { Siritis Cumputhitus }\end{array}$ & $\begin{array}{l}\text { March 5, } 2011 \\
\text { If }\end{array}$ \\
\hline $\begin{array}{l}\text { March 18, } 2011 \\
{[6 \mathrm{am}]}\end{array}$ & King City & $\begin{array}{l}\text { * Region FFA Meeting And } \\
\text { Leadership Contest Finals }\end{array}$ & $\begin{array}{c}\text { March } 18,2011 \\
{[8 \mathrm{pm}]}\end{array}$ \\
\hline $\begin{array}{l}\text { March } 25,2011 \\
{[3 \mathrm{pm}]}\end{array}$ & $\begin{array}{l}\text { Modesto, CA } \\
\text { (March 28) }\end{array}$ & $\begin{array}{l}\text { * Modesto JC Ag Judging } \\
\text { Skills Competition }\end{array}$ & $\begin{array}{c}\text { March 26, } 2011 \\
{[11 \mathrm{pm}]}\end{array}$ \\
\hline $\begin{array}{l}\text { March } 27,2011 \\
(8: 00 \mathrm{am})\end{array}$ & San Luis Obispo & $\begin{array}{l}\text { * Region FFA State Degree } \\
\text { \& Prof. Awards Ceremony }\end{array}$ & $\begin{array}{l}\text { March 27, } 2011 \\
(6 \mathrm{pm})\end{array}$ \\
\hline $\begin{array}{c}\text { April } 15,2011 \\
{[3 \mathrm{pm}]}\end{array}$ & Fresno & $\begin{array}{l}\text { * Fresno State College Ag } \\
\text { Judging Skills Contest }\end{array}$ & $\begin{array}{c}\text { April 16, } 2011 \\
\text { [Midnight] }\end{array}$ \\
\hline $\begin{array}{l}\text { April 16, } 2011 \\
{[3 \mathrm{pm}]}\end{array}$ & Fresno & $\begin{array}{l}\text { State FFA Leadership } \\
\text { Conference }\end{array}$ & $\begin{array}{l}\text { April 19, } 2011 \\
\text { [8 pm] }\end{array}$ \\
\hline $\begin{array}{l}\text { May } 6,2011 \\
{[3 \mathrm{pm}]}\end{array}$ & $\begin{array}{l}\text { San Luis } \\
\text { Obispo }\end{array}$ & $\begin{array}{l}\text { * State FFA Ag Judging } \\
\text { Skills Contest Finals }\end{array}$ & $\begin{array}{l}\text { May } 7,2011 \\
{[11 \mathrm{pm}]}\end{array}$ \\
\hline $\begin{array}{l}\text { May } 7-17,2011 \\
{[3 \mathrm{pm}]}\end{array}$ & King Clity & $\begin{array}{l}\text { * Salinas Valley Fair } \\
\text { Student Trans/Super. }\end{array}$ & $\begin{array}{l}\text { May 16, } 2011 \\
\quad[8 \mathrm{pm}]\end{array}$ \\
\hline $\begin{array}{l}\text { June } 1,2011 \\
\quad(3 \mathrm{pm})\end{array}$ & Gonzales & * Section FFA Elections & $\begin{array}{l}\text { June } 1,2011 \\
\quad(10 \mathrm{pm})\end{array}$ \\
\hline
\end{tabular}


AGRICULTURE DEPARTMENT COLLABORATION MEETING AGENDA/MINUTES

NORTH SALINAS HIGH SCHOOL (rev. 10/10)

MEETING DATE: $1 / 91 / / 8,201 /$

COPY TO: Dept. Files, Ag Staff, Principal

STAFF PRESENT: $\chi$ Mr. Jones, $\chi$ Mrs. Blodgett, $\chi$ Mr. Uchiyama, $\chi$ Mrs. Noroian MINUTES RECORDED BY: ___ Mr. Jones, __ Mrs. Blodgett, X Mr. Uchiyama, Mrs. Narolan

1. IMMEDIATE ATTENTION-ACTIVITIES, CONTESTS, TRIPS, PROGRAM MANAGEMENT ITEMS. (FYI, needs, supervision, travel, due dates, funding)

$(\mathbb{R}=$ responsible)

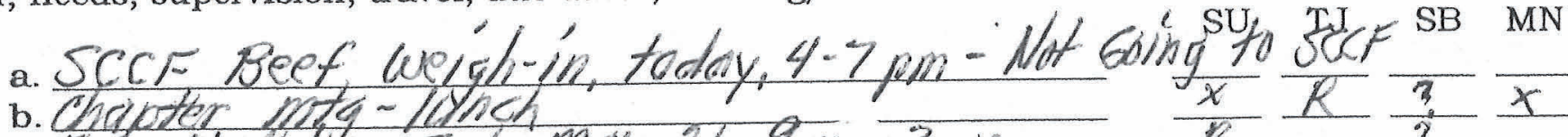

c. Faim workdy sat mey 21 , qam - $3 \mathrm{pm}$

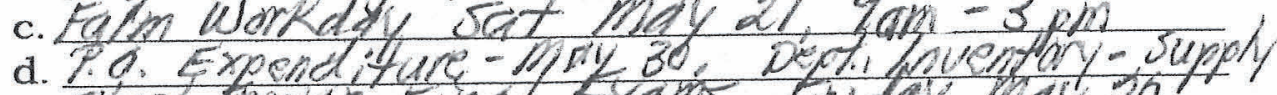

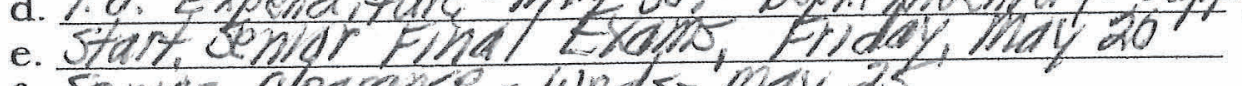

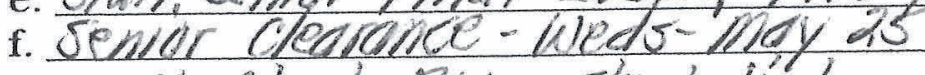
mce stock Buy-stant lift

2. MEETING SCHEDULE REVIEW---NOT PREVIOUSLY DISCUSSED.

(FYI, agenda plan, supervision, funding, travel, needs)

(admin. approval, teacher sub, other admin.)
a. $\mathrm{Ag}$ Booster Club Meeting.......Pfizer sfotibs I

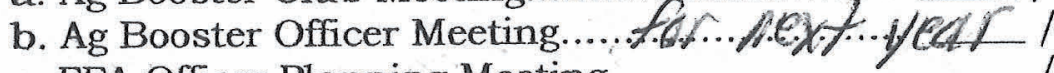
c. FFA Officer Planning Meeting..
d. Ag Advisory Committee Meeting.................. CATH
f. FFA Section / Region Contest.....................
g. FFA Section / Region Meeting....................
h. School Statt Meeting...................................
j.
k.
1. Next Department Collaboration Meeting.....hext fled

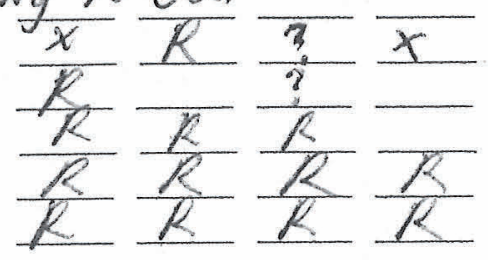

\section{DISCUSSION ITEMS--FY, NEEDS, PROBLEM SOLVING, ACTION PLAN, WHO DOES?.}

- DEPT. OPERATIONS, budget/purchasing, facilities, equipment, repairs, maintenance, travel.

-- CLASS supply, student problems, maintenance, repairs, AV, textbooks, storage.

-- AG. BOOSTERS, AG. Advisory, Community, CATA, Professional Development.

-- FFA OPERATIONS, Officer team, activities/contests, finances, awards/recognition.

-- SAEP OPERATIONS, School Farm, Project Supervision, County Fairs. 1) Mr. U Retirement date and future ofotis

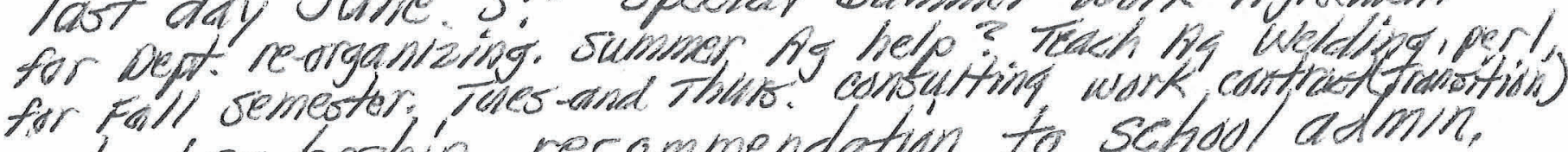
Dept. Leaderolife recommendatian to schoul denmen.

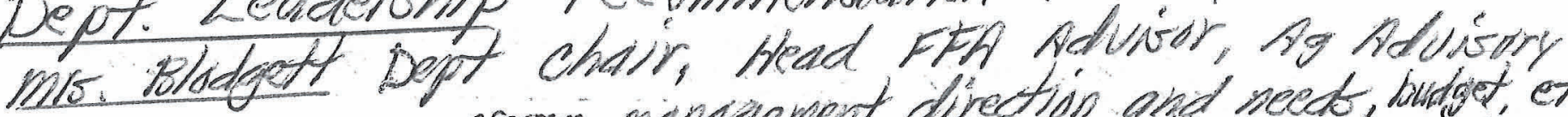
camm, over-see ag program minalgenent direction and secto buiget etc.

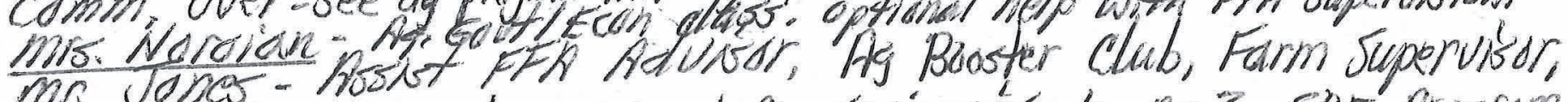

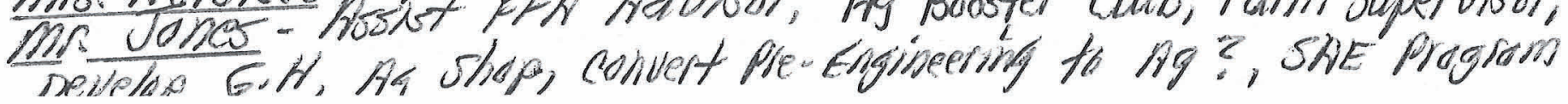


AGRICULTURE DEPARTMENT COLLABORATION MEETING AGENDA/MINUTES

NORTH SALINAS HIGH SCHOOL

(rev, 10/10)

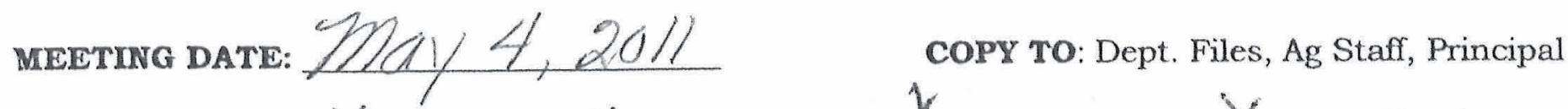

STAFF PRESENT: $\chi$ Mr. Jones, $\chi$ Mrs. Blodgett, $\chi$ Mr. Uchiyama, $X$ Mrs. Noroian

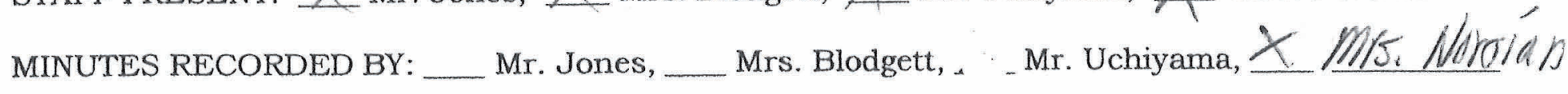

1. IMMEDLATE ATTENTION-ACTIVITIES, CONTESTS, TRIPS, PROGRAM MANAGEMENT ITEMS. (FYI, needs, supervision, travel, due dates, funding)

a. Project comp Award Banginet-tanífit, 6:15 pm b.3. SVE Progress Grade cheek - sid il c. Final SVE Cleanance-s/6/i

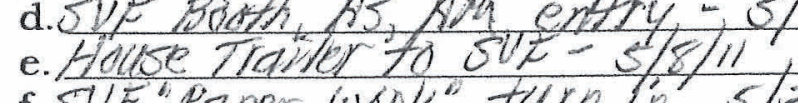

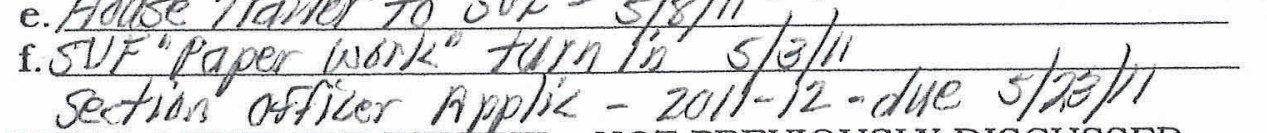

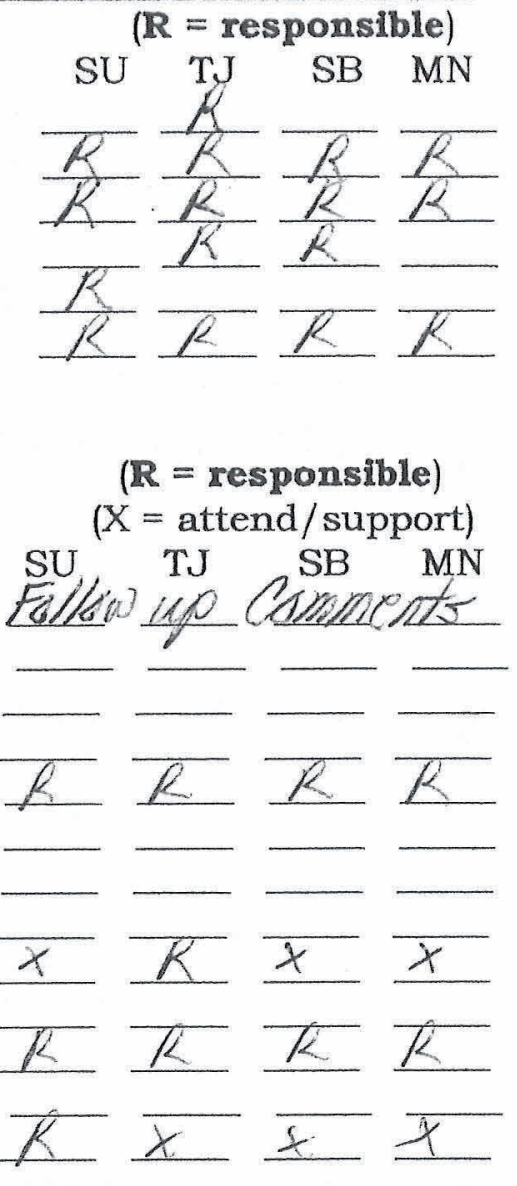
(FYI, agenda plan, supervision, funding, travel, needs) (admin. approval, teacher sub, other admin.)
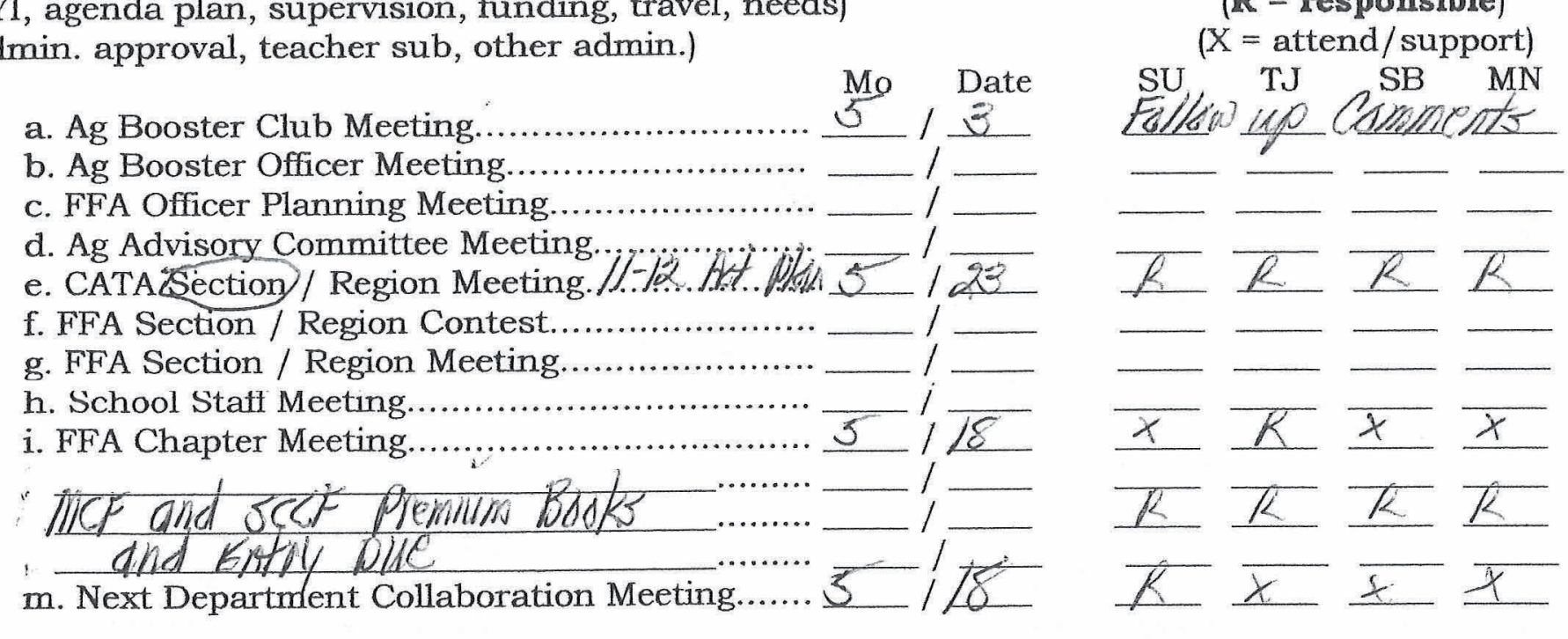

3. DISCUSSION ITEMS--FY, NEEDS, PROBLEM SOLVING, ACTION PLAN, WHO DOES?.

- DEPT. OPERATIONS, budget/ purchasing, facilities, equipment, repairs, maintenance, travel.

-- CLASS supply, student problems, maintenance, repairs, AV, textbooks, storage.

-- AG. BOOSTERS, AG. Advisory, Community, CATA, Professional Development.

- FFA OPERATIONS, Officer team, activities/contests, finances, awards/recognition.

-- SAEP OPERATIONS, School Farm, Project Supervision, County Fairs.

A. 2011-12 Chit g DULS - Farm available to paly prior to summer conference. Summer canto Reg and lodging

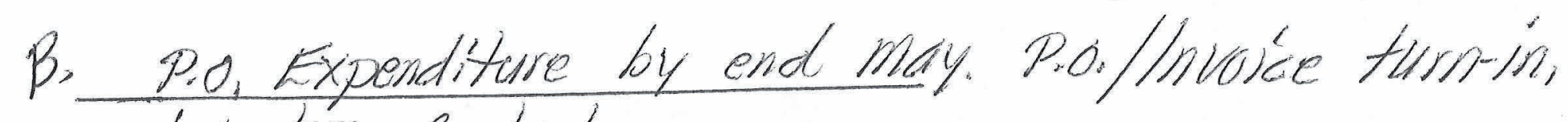
Inventory Control

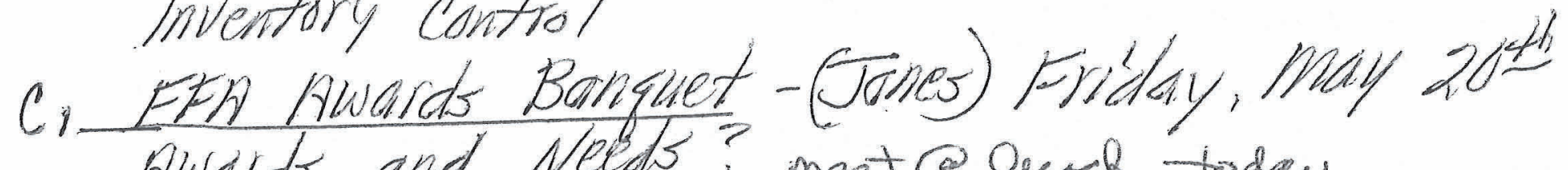
Awards and Needs? meet e curch today.

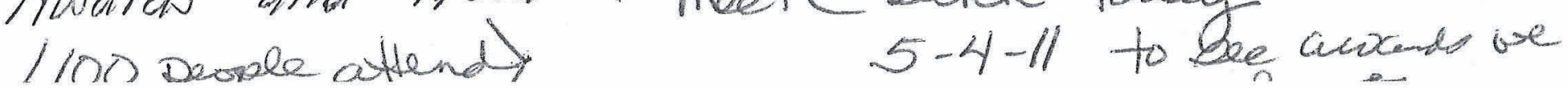


D Salinas Valley Fair

(1) Booth, AS, AM entry check in' Sort, styli ag truck + I van to take students

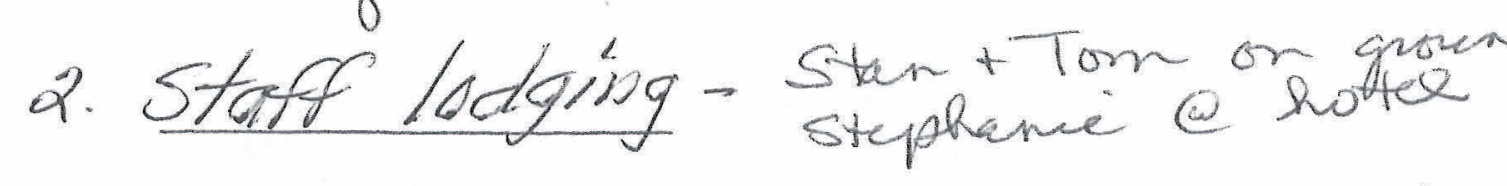

3. Stock, Equipment, Pen Display Status

stuff is out/heed to do decorations for pens

4. Bending

5. Teacher sub ( 12 days)

Stan 6 day p 3 days each
Tom

6. Teacher Ileal 4

7. Schorl Van Use

Utility Trailer

have one van for fair

8. Sat. Booster BBQ $\$ 2,500^{\circ 0}$ profit

q. Load out -sunday 5/15 everyone involved

10 Entry Out -Monday 5/he stan vale do

Emotes and SCCF steak buy. Summer Ag sign-up summer work? 
MEETING DATE: $7 \mathrm{pri} / 13,201 /$

COPY TO: Dept. Files, Ag Staff, Principal

STAFF PRESENT: $\Varangle$ Mr. Jones, $\chi$ Mrs. Blodgett, $\chi$ Mr. Uchiyama, $\Varangle$ Mrs. Noroian

MINUTES RECORDED BY: __ Mr. Jones, __ Mrs. Blodgett, $X$ Mr. Uchiyama,

1. IMMEDLATE ATTENTION-ACTIVITIES, CONTESTS, TRIPS, PROGRAM MANAGEMENT ITEMS. (FYI, needs, supervision, travel, due dates, funding)

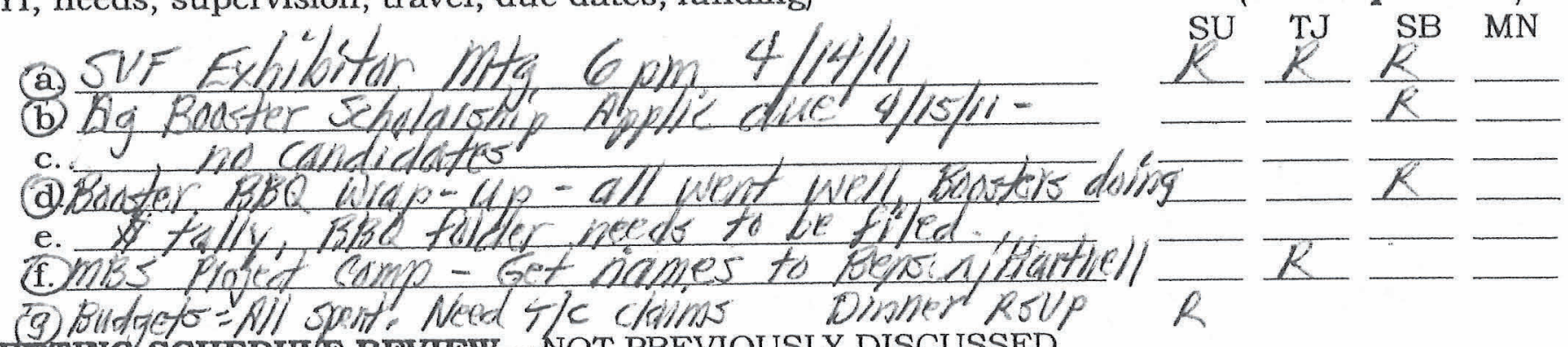

2. MEETIN S SCHEDULE REVIEW--NOT PREVIOUSLY DISCUSSED.

(FYI, agenda plan, supervision, funding, travel, needs)

(admin. approval, teacher sub, other admin.)

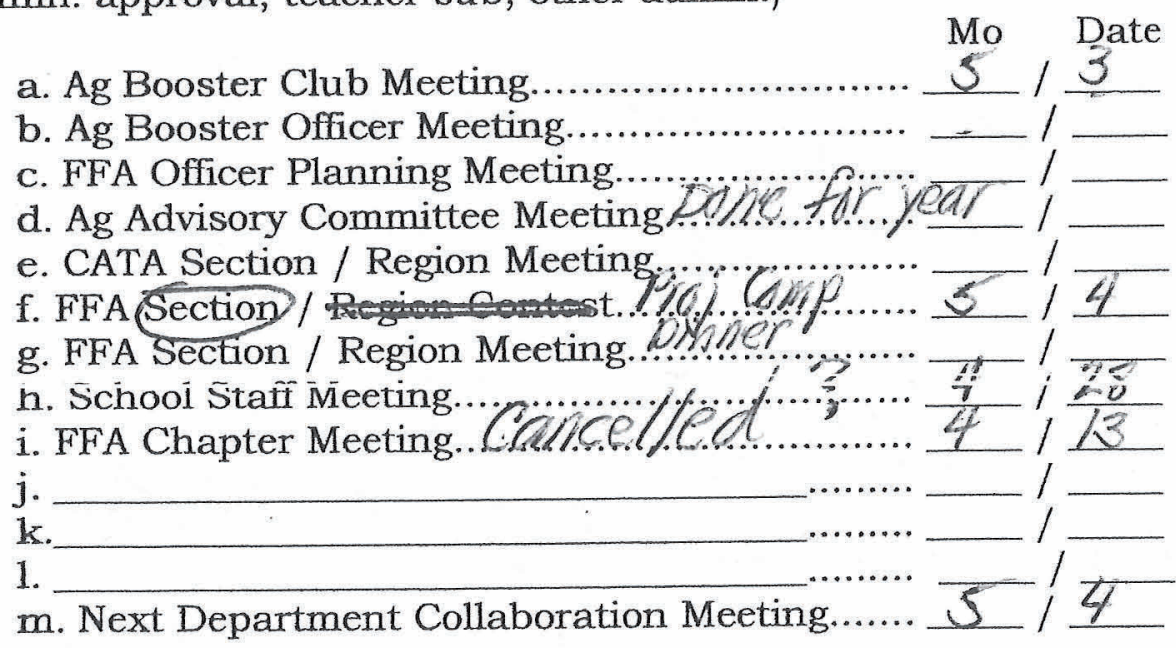

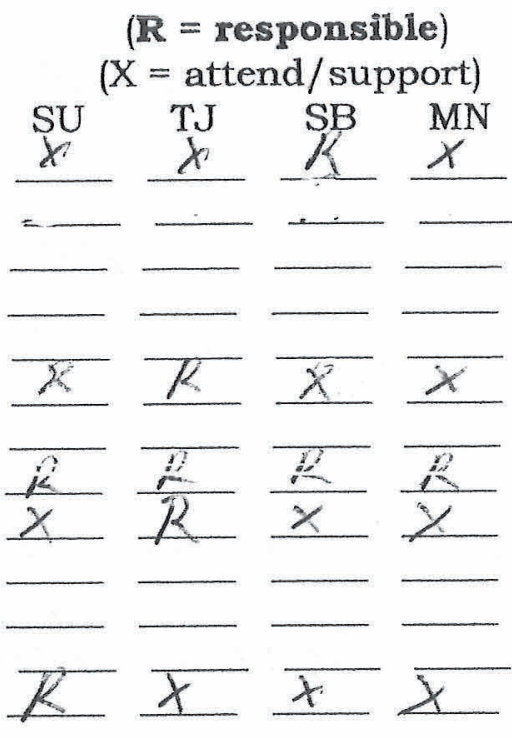

3. DISCUSSION ITEMS--FY, NEEDS, PROBLEM SOLVING, ACTION PLAN, WHO DOES?.

- DEPT. OPERATIONS, budget/purchasing, facilities, equipment, repairs, maintenance, travel.

- CLASS supply, student problems, maintenance, repairs, AV, textbooks, storage.

- AG. BOOSTERS, AG. Advisory, Community, CATA, Professional Development.

-- FFA OPERATIONS, Officer team, activities/contests, finances, awards/recognition.

-- SAEP OPERATIONS, School Farm, Project Supervision, County Fairs.

(A) SVF Ineligible student list - being made and list to principal, letters to parents. No grade checks or other papess returned. Talk to students

(B) State Ldir Conterence. intg. held, "paper work" out, transportation ok, Have 3 students still need to pay balance owed-Angela, Lausa, sarah, 
C) special VEM $\$$ proposals due to Karla by May, 20\% For 2011-12 finding. use proposal description form.

(D) VEA Gur. Dew. (3) Mtg, TUes, 4/26 Get own sub - Stor already processed = Jones?

(E) Start Equipment, Pen display, Feature Booths. stock prep, etc for 51/F w Workday during spring break?

(E) Farm maintenance Work to do? been 5 weeks since last workday

(6) $2011-12$ FEA OFficer Elections and parent -member award banquet stative J Junes (ii) Draft buyer letter, update resin bosk

(1) State CDE Final, May $7=B 16$ and Coups?

(J) Mci Beef weigh-in, May 3, 5-7 pm, MC

(1) other? 
AGRICULTURE DEPARTMENT COLLABORATION MEETING AGENDA/MINUTES

NORTH SALINAS HIGH SCHOOL

(rev. 10/10)

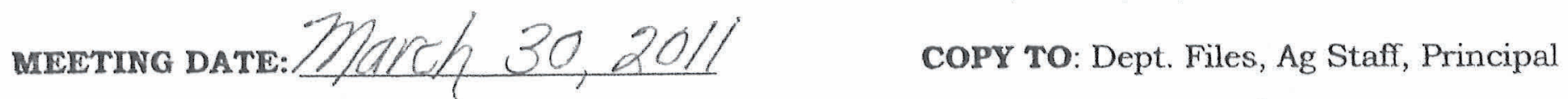

STAFF PRESENT: $X$ Mr. Jones, $X$ Mrs. Blodgett, $\underline{X}$ Mr. Uchiyama, $X$ Mrs. Noroian

MINUTES RECORDED BY: ___ Mr. Jones, ___ Mrs. Blodgett, $\chi$ Mr. Uchiyama,

1. IMMEDIATE ATTENTION -ACTIVITIES, CONTESTS, TRIPS, PROGRAM MANAGEMENT ITEMS.

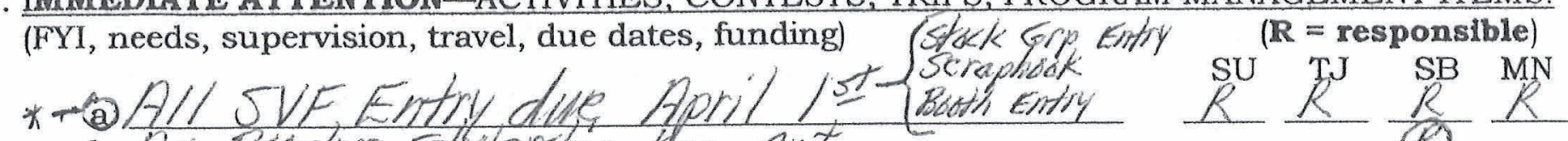

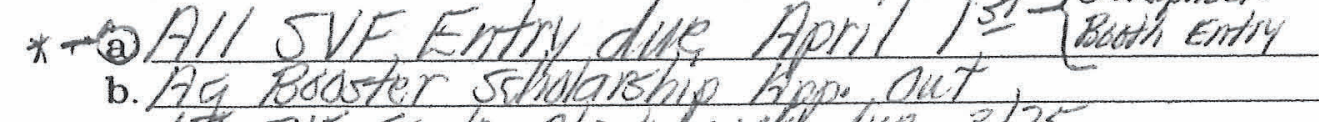

*-ai SVE Rabbit SAE WS Gillian rabbit sale us SVF Entry

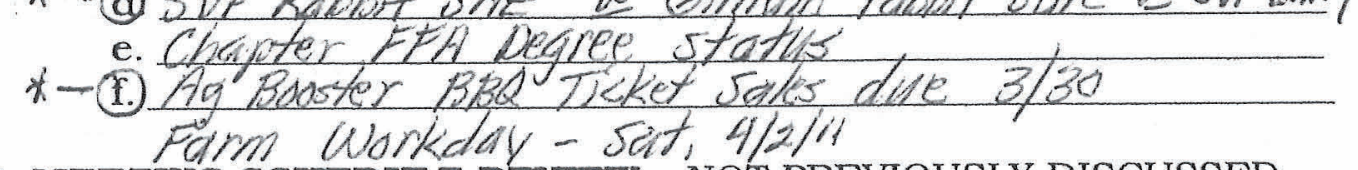

2. MEETING SCHEDULE REVIEW--NOT PREVIOUSLY DISCUSSED. (FYI, agenda plan, supervision, funding, travel, needs) (admin. approval, teacher sub, other admin.)

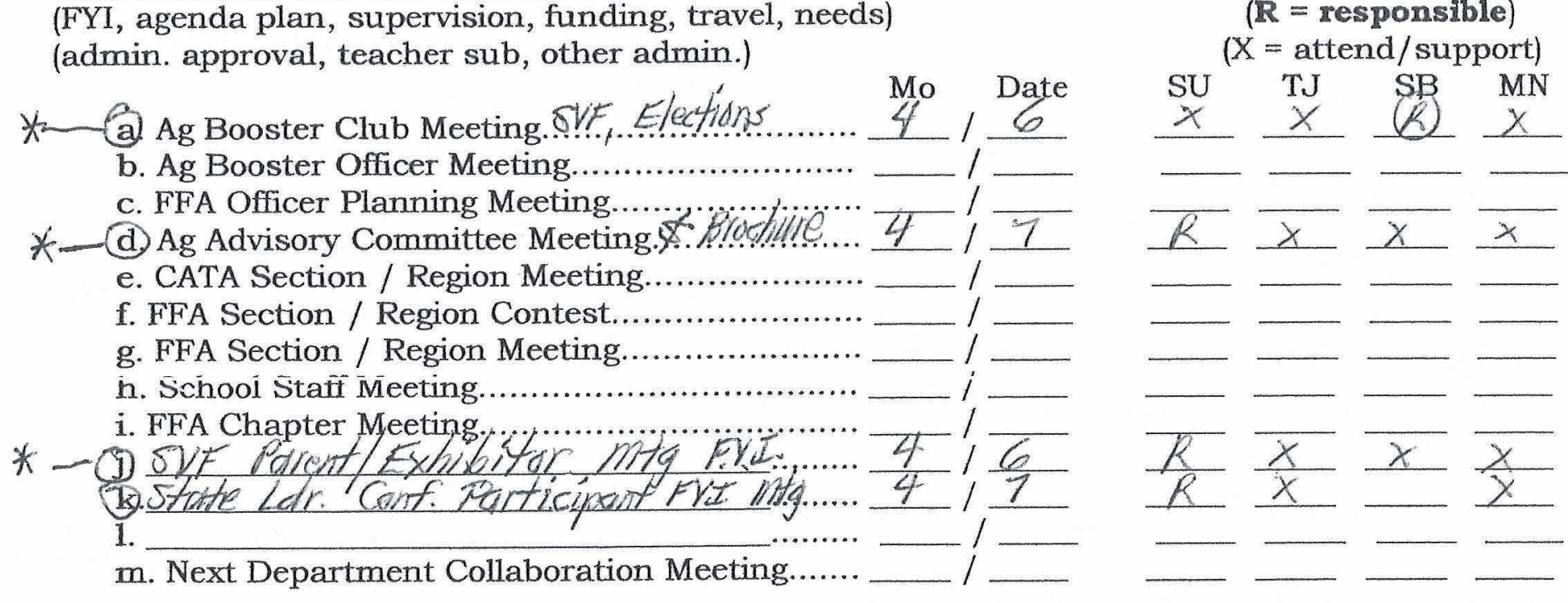

3. DISCUSSION ITEMS--FY, NEEDS, PROBLEM SOLVING, ACTION PLAN, WHO DOES?.

- DEPT. OPERATIONS, budget/purchasing, facilities, equipment, repairs, maintenance, travel.

- CLASS supply, student problems, maintenance, repairs, AV, textbooks, storage.

-- AG. BOOSTERS, AG. Advisory, Community, CATA, Professional Development.

- FFA OPERATIONS, Officer team, activities/contests, finances, awards/recognition.

-- SAEP OPERATIONS, School Farm, Project Supervision, County Fairs.

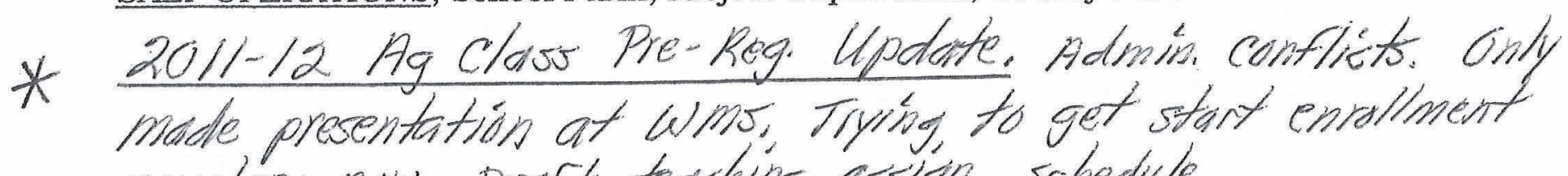
numbers now. Draft teaching assign. schedule

* sup leader pass fee- Io Pay. sending in this week.

Region s.t./Prof. Award Program. Thanks far going and supporting our students.

* All Po. spent by April 4 -hope process by 48) cut - 
State CDE Team entry by April 8 th

1. CoOp /marketing and 816 teams

2. Need 2 drivers - Ag Truck, sehod Van

* Spring Ag Industry Tour by Mg Bd u - 4/13/li?

Farm Lease with county up for renewal in 2012

1. Mn A to start contacts with getting approval processed.

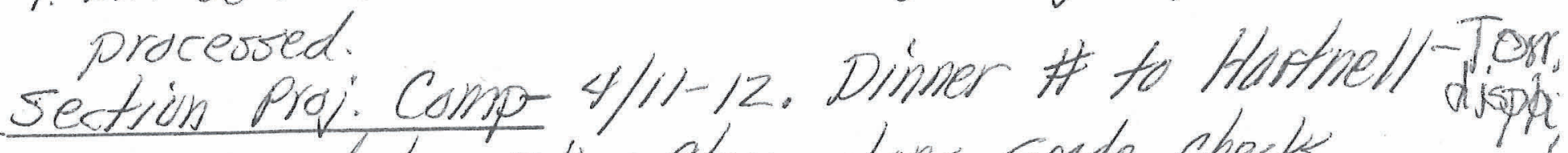

* SVF Due dates, Elíg. Clear plans, Grade checks, signed papers, etc.

1. List given to everyone. Teachers -please read.

2. Need all staff to emphasize compliance and help with task needs completion, etc.

* Ag Booster BBQ 4/a/il - Ready? Needs? Plan?

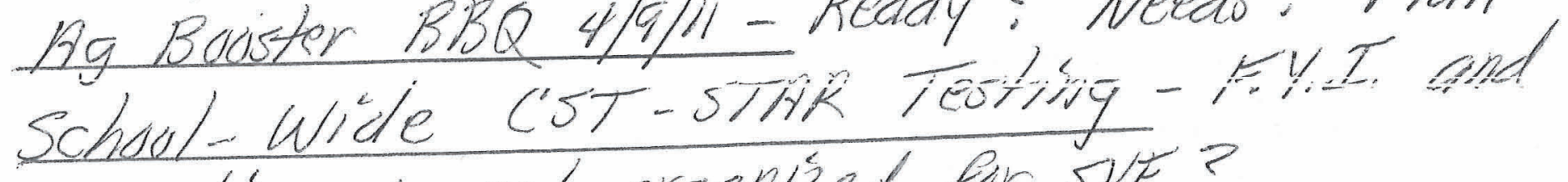
some time to get organized for SWF?

* CATA Summer Confer. Reg. and motel expense filling.

* Eth officer app. for 2011-12, Section Officer land, Banquet. When? Rm. Reservation done

1. Order banquet supply and awards.

3. Planning

2. Fix / proof read program history

mites?

* State Los. Confer - Eligibility and Pay clearance concerns. Into mints. 4/3/is

1. Mr. U, mr J. Mr. N to go

2. Need school van, ag truck. more? *

3. 5 ts h done. Reg and lintel paid. check on Motel keen 
AGRICULTURE DEPARTMENT COLLABORATION MEETING AGENDA/MINUTES

NORTH SALINAS HIGH SCHOOL (rev. 10,09)

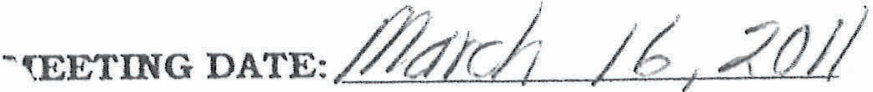

COPY TO: Dept. Files, Ag Staff, Principal

StafF Present: X. Mr. Jones, X. Mrs. Blodgett, X. Mr. Uchiyama, Y Mis. Nornald

MINUTES RECORDED BY:

Mr. Jones,

Mrs. Blodgett, X Mr. Uchiyama,

1. IMMEDIATE ATTENTION-ACTIVTTIES, CONTESTS, TRIPS, PROGRAM MANAGEMENT ITEMS.

(FY, needs, supervision, travel, due dates, funding)

$(\mathbb{R}=$ responsible $)$
a. Regin FFA MAt - All set

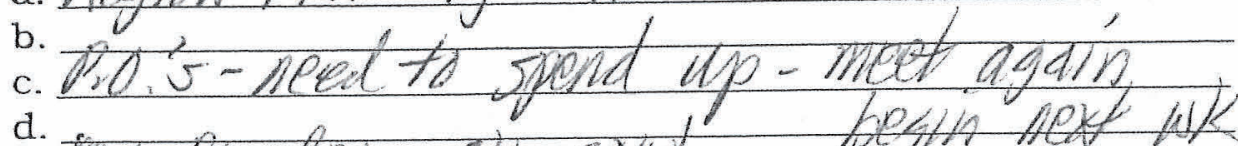
e. ThS pre-keg-OK Goud
f.

2. MEETING SCHEDULE REVIEW--NOT PREVIOUSLY DISCUSSED.

(FYI, agenda plan, supervision, funding, travel, needs)

(admin. approval, teacher sub, other admin.)
a. Ag Booster Club Meeting.
Mo Date
b. Ag Booster Officer Meeting.
c. FFA Officer Planning Meeting.
d. Ag Advisory Committee Meeting.
e. CATA Section / Region Meeting
f. FFA Section / Region Contest.
g. FFA Section / Region ivietiry.
h. School Staff Meeting.
i. FFA Chapter Meeting.
j. Dept.
chatr
1.
m. Next Department Collaboration Meeting.

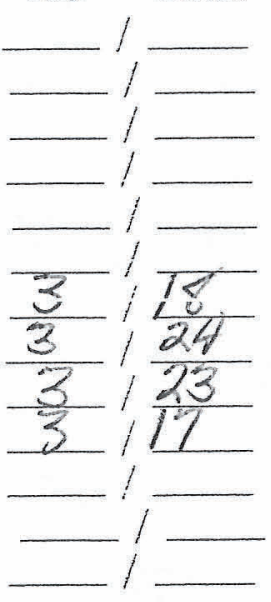 SU TJ $S B$

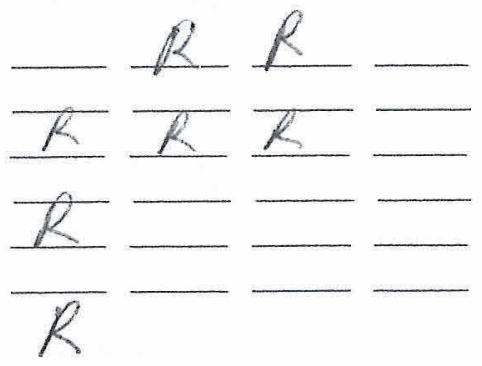

$(\mathbf{R}=$ responsible $)$

( $\mathrm{X}=$ attend $/$ support)

3. DISCUSSION ITEMS--FY, NEEDS, PROBLFM SOLVING, ACTION PLAN, WHO DOES?.

- DEPT. OPERATIONS, budget/purchasing, facilities, equipment, repairs, maintenance, travel.

-- CLASS supply, student problems, maintenance, repairs, AV, textbooks, storage.

- AG. BOOSTERS, AG. Advisory, Community, CATA, Professional Development.

-- FFA OPERATIONS, Officer team, activities/contests, finances, awards/recognition.

-- SAEP OPERATIONS, School Farm, Project Supervision, County Fairs.

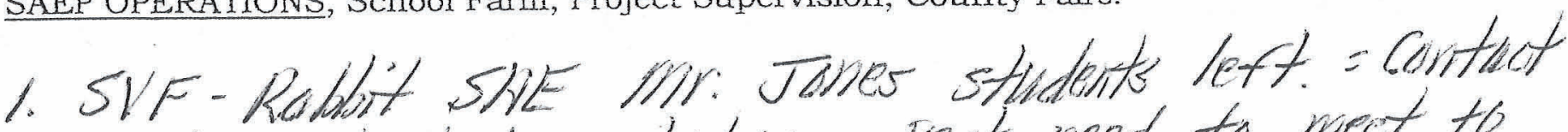

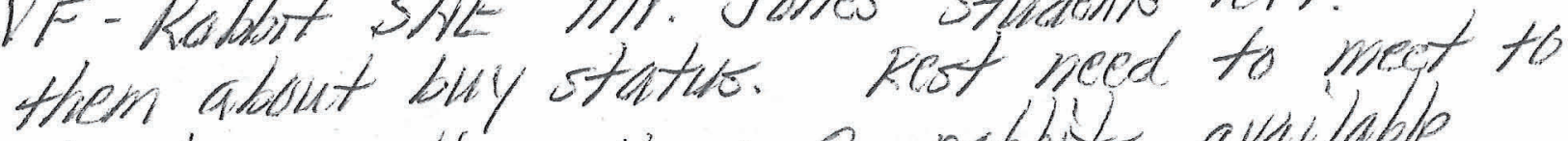

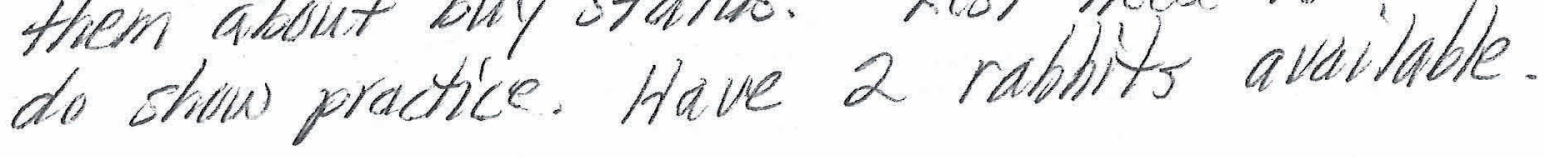

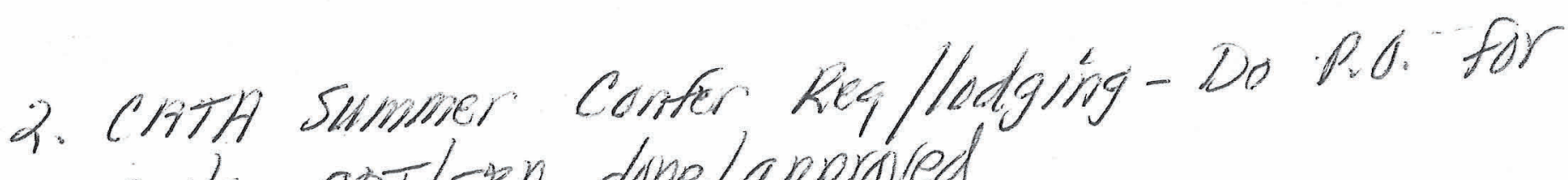
costs. oDT/sish done/approvel 
$\frac{\text { State ids confer mir. Jive call lintel confirm }}{\text { Get rest to poly balances owed }}$

Dept $=\$ 525$ e left to spend

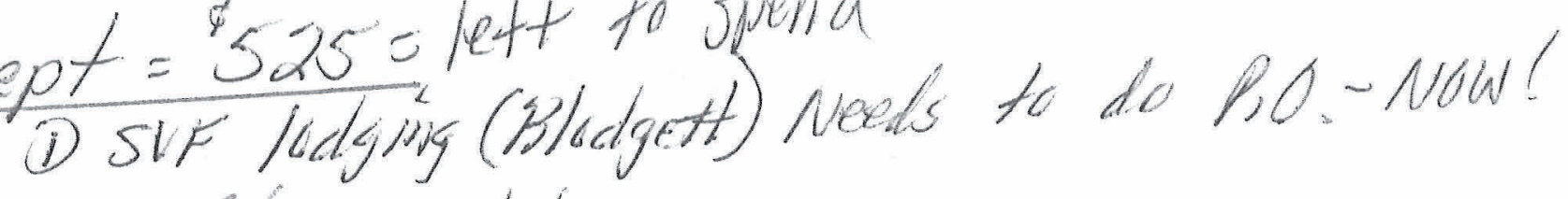

$\frac{A / G=18,874 \text { total }}{004500 \text { TO C }}$

(2) 1500 Repairs

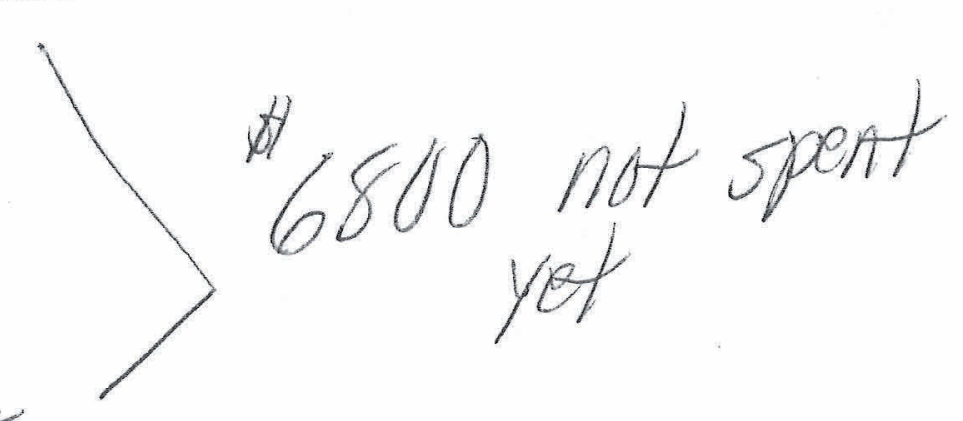

(3) 800 vehicle/ Bine

(4) 7960 list supply= left $J$ and B spend on shop and sciépce needs. check on open Do: needs

$$
\text { VEA = M rets: Supply= All spent }
$$

sulks

Traitel/Conf. - To Do

coth conf. Keg and lodging

Reg. S.F Degree Program-Rsilp to Gleg-now = 1"

A) OK - everyone has own tran. people

Reg. FFH into and Contorts imp ri' $18^{\text {th }}$

mir. Jones, mos. Bridgett with severs elect speech creed and Job hot kids dropped ait 

Dekalb nccomp Nurdrd-Cheyeme sievers
done and Sert

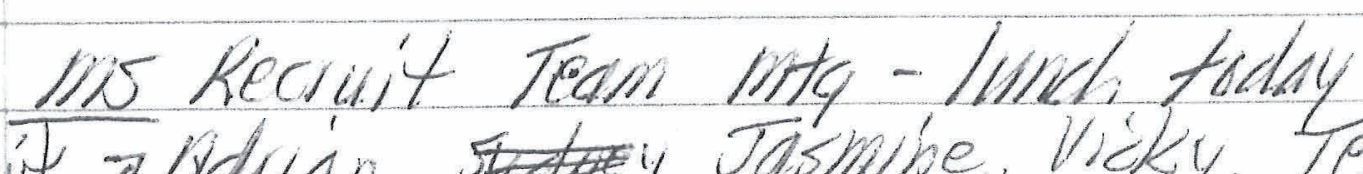

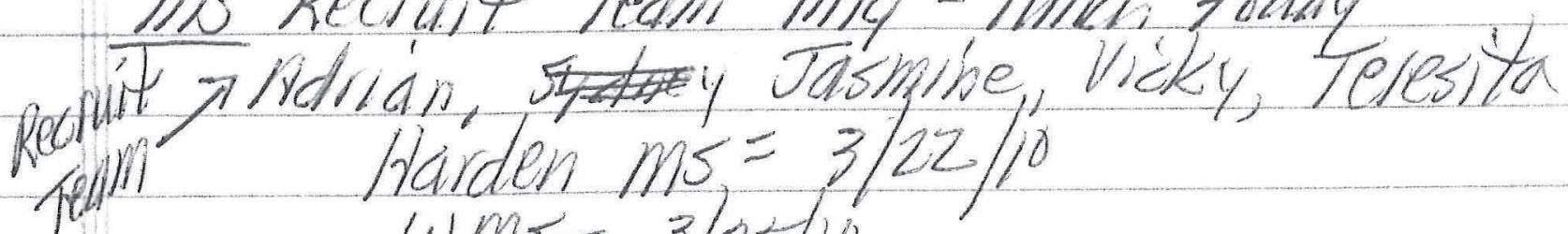

$$
\text { WMO }=3 / 25 / 10
$$

SHE - Steers - Weigh on Thus - 2/2tlli

shreep - Blidgett - Wosk with tuday, wom, shear.

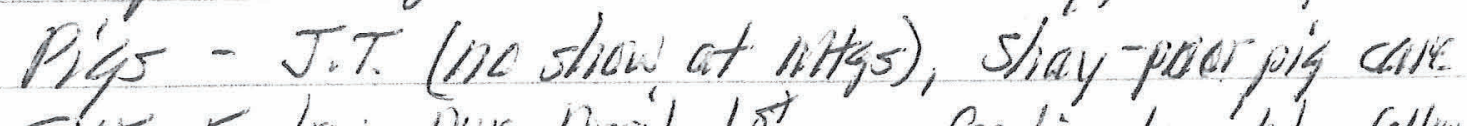

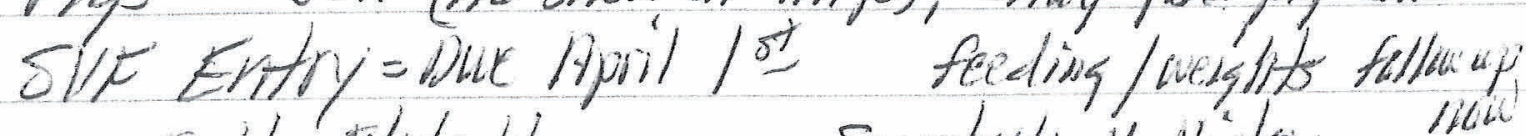
Eavth- Tildodgett seraplade-V, Vickey

Proj. Comp-Next time get connedurs involved - went of

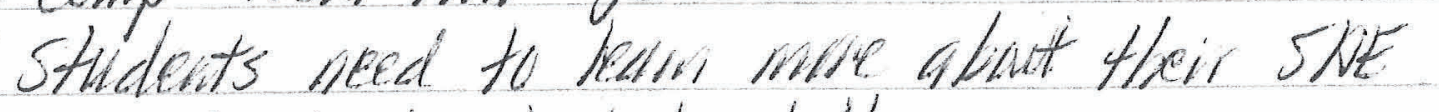
verd to teach students better. 


\section{NORTH SALINAS HIGH SCHOOL (rev. 10/10)}

VEETING DATE: $3 / 2 / 1 /$

COPY TO: Dept. Files, Ag Staff, Principal

STAFF PRESENT: $\chi \mathcal{X}$ Mr. Jones, $\chi$ Mrs. Blodgett, $\chi$ Mr. Uchiyama, $E X$ Mrs. Noroian

MINUTES RECORDED BY: ___ Mr. Jones, __ Mrs. Blodgett, $X$ Mr. Uchiyama,

1. IMMEDIATE ATTENTION-ACTIVITIES, CONTESTS, TRIPS, PROGRAM MANAGEMENT ITEMS. (FYI, needs, supervision, travel, due dates, funding)

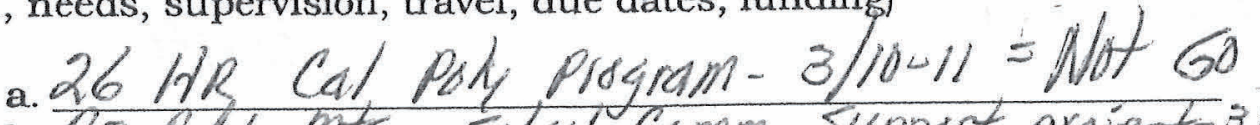

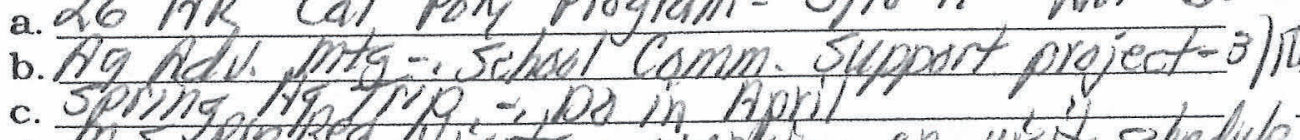

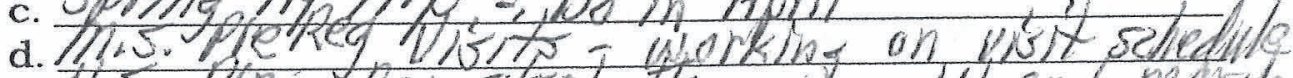

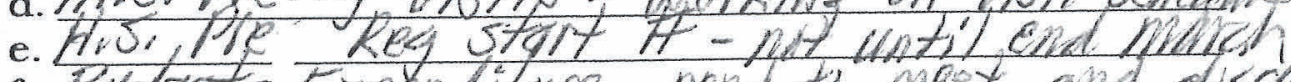
r. Budget - Expendifup - nepd ta pert and disciss neets.

( $\mathbb{R}=$ responsible)

2. MEETH G SCHEDULE REVIEW-NOT PREVIOUSLY DISCUSSED.

(FYI, agenda plan, supervision, funding, travel, needs)

(admin. approval, teacher sub, other admin.)

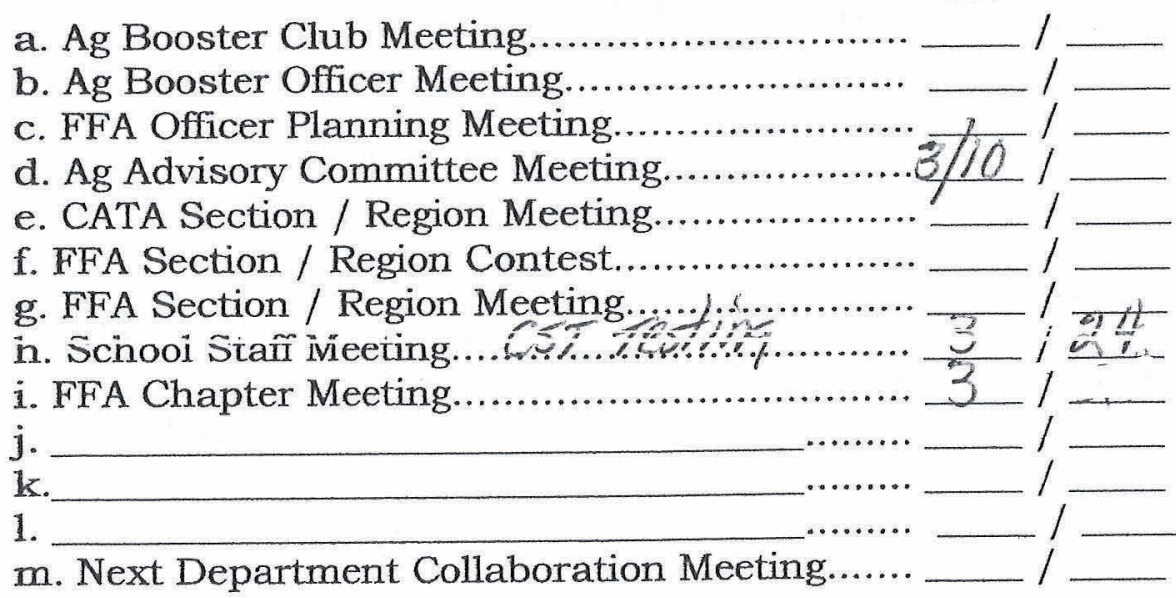

$(\mathbf{R}=$ responsible $)$ $(\mathrm{X}=$ attend/support $)$ SU TJ SB MN

\section{DISCUSSION ITEMS--FY, NEEDS, PROBLEM SOLVING, ACTION PLAN, WHO DOES?.}

- DEPT. OPERATIONS, budget/purchasing, facilities, equipment, repairs, maintenance, travel.

-- CLASS supply, student problems, maintenance, repairs, AV, textbooks, storage.

-- AG. BOOSTERS, AG. Advisory, Community, CATA, Professional Development.

- FFA OPERATIONS, Officer team, activities/contests, finances, awards/recognition.

-- SAEP OPERATIONS, School Farm, Project Supervision, County Fairs.

(1) Project Comp-Sat, March 12 - Jones/wordión cleanup day sat, march 5 -ofu habits in-? judges - Tmi, ploginet Wunch. Ag Bassiers provide sandulich, chips, encourage FY. a and Rules fentry form intg, weds, 3/8/l' 
(2) Ag Booster mtg

Ticket sates mm. B.

$\frac{1}{5}$ ticket $=1$ act credit

Publicity - Fin officers
(5) SVF Needs

* Phone \# list

f show contract Due $3 / 11$

+ stock Insurance bought=

setup, BBQ work day -mrs. B. to organize

Jackets - slow on orders. Need to promote other - pros. Comp. meal needs.

(3) Spring Reg mtg

Creed, Jab Interview, Reg. OFficer = work with them

Teachers mr. J, ms. B to go

(4)

\$16/Principal mtg - Commend/Recc. Discussion

Discussed not much positive progress made.

(5) CAHSEE Testing $3 / 8-9=F . Y . I$. 
AGRICULTURE DEPARTMENT COLLABORATION MEETING AGENDA/MINUTES

NORTH SALINAS HIGH SCHOOL

(rev. 10/10)

MEITING DATE: Weds, Fe l 16,2011

STAFF PRESENT: $\chi$ Mr. Jones, $\chi$ Mrs. Blodgett,
COPY TO: Dept. Files, Ag Staff, Principal Off CAmpus
Mr. Uchiyama, $\chi$ Mrs. Noroian MINUTES RECORDED BY: Mr. Jones, Mrs. Blodgett, Mr. Uchiyama, X MAS .N

1. IMMEDIATE ATTENTION -ACTIVITIES, CONTESTS, TRIPS, PROGRAM MANAGEMENT ITEMS. (FYI, needs, supervision, travel, due dates, funding)

a. Recommitment Dry

b. FFA WeeK /2/22-25

c. FFA Dieting $2 / 23$

d. Regional Mate ling $7 / 28$ CAA

f.

2. MEETING SCHEDULE REVIEW---NOT PREVIOUSLY DISCUSSED. (FYI, agenda plan, supervision, funding, travel, needs) (admin. approval, teacher sub, other admin.)

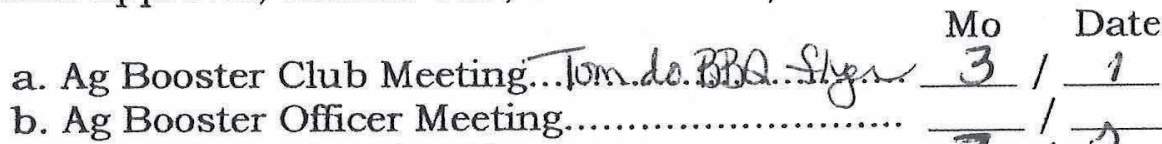

c. FFA Officer Planning Meeting.

d. Ag Advisory Committee Meeting.

e. CATA Section / Region Meeting

f. FFA Section / Region Contest.

g. FFA Section / Region Meeting................... $\frac{3}{3} / \frac{8}{2}$

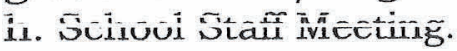

i. FFA Chapter Meeting j. Progress Comp-hochl $3 \ldots . . . .3, \frac{3}{3} / 2$

1.

m. Next Department Collaboration Meeting

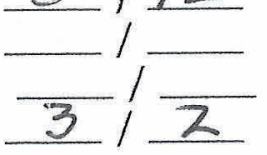

3. DISCUSSION ITEMS--FYI, NEEDS, PROBLEM SOLVING, ACTION PLAN, WHO DOES?.

- DEPT. OPERATIONS, budget/purchasing, facilities, equipment, repairs, maintenance, travel.

-- CLASS supply, student problems, maintenance, repairs, AV, textbooks, storage.

-- AG. BOOSTERS, AG. Advisory, Community, CATA, Professional Development.

-- FFA OPERATIONS, Officer team, activities/contests, finances, awards/recognition.

-- SAEP OPERATIONS, School Farm, Project Supervision, County Fairs.

* Expenditure of By chant funds. heed po's by?

need POs now for instructional supplies

due by march $15^{\text {th }}$

* Leading hip. State FF A conference Monty Stat os?

remind kids to pay their money. 
* State casa conf r pRey?

dort know status

$$
\text { * SVF Rubloits - ? }
$$
need to set up a meeting and
get rabbits

* School FARM Stephanie worked watepigkibs on Saturday get a lot done on Saturday - lots of mowing

Frosh kids must have constant supervision

Tom - has show practice every wad 4:30-6 pm

* FFA Week Activity Ph awning we due to recrutiman * Burg a rimes un on Friday (Jones)

* Pizza Feed e lure monday credit

Daily activity worth Activity credizes High School Reconktmext-2-17-1!

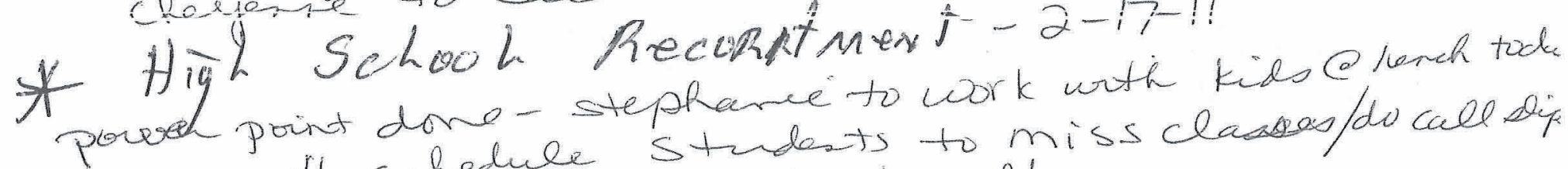
she will schedule students to miss classes/do call bi k 2 paras Angelawel be by herself

Recrutinant in Pm 730 last 20 minutes of each period 10-20 kids par prod point t chairs (Jones wei do)

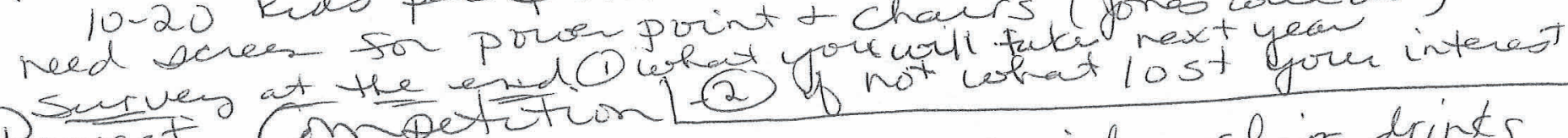

* Project Con petition will get sandwiches, chips, drinks for judges, FA members a parents present

3 judges (1) Gary Peterson

(2) Andy $2 \mathrm{kim}$ madatora

(3)? Caresani

tables +chairs $50 \mathrm{~m}$ will bring Margaret - project competition organization (Torr tu get last years info to tar) 


\section{AGRICULTURE DEPARTMENT COLLABORATION MEETING AGENDA/MINUTES}

\section{NORTH SALINAS HIGH SCHOOL (rev. 10/10)}

metring DATE: lleds, teb 2,201

COPY TO: Dept. Files, Ag Staff, Principal

STAFF PRESENT: ___ Mr. Jones,

Mrs. Blodgett,

Mr. Uchiyama,

Mrs. Noroian

MINUTES RECORDED BY: \ Mr. Jones, ___ Mrs. Blodgett, __ Mr. Uchiyama,

1. IMMEDIATE ATTENTION-ACTIVITIES, CONTESTS, TRIPS, PROGRAM MANAGEMENT ITEMS. (FYI, needs, supervision, travel, due dates, funding)

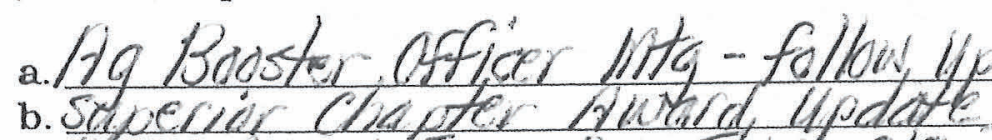

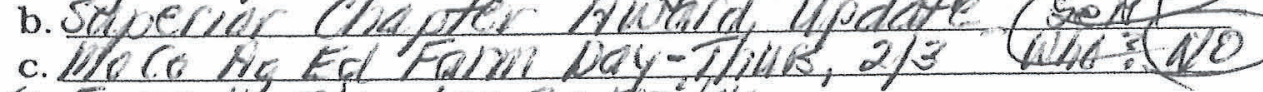

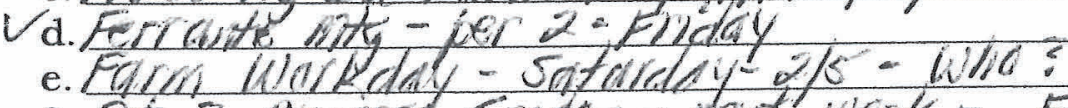

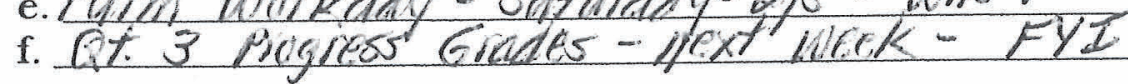

$(\mathbb{R}=$ responsible $)$

\section{MEETING SCHEDULE REVIEW--NOT PREVIOUSLY DISCUSSED.}

(FYI, agenda plan, supervision, funding, travel, needs)

(admin. approval, teacher sub, other admin.)

a. Ag Booster Club Meeting.

Mo Date

( $R=$ responsible)

b. Ag Booster Officer Meeting 1

c. FFA Officer Planning Meeting

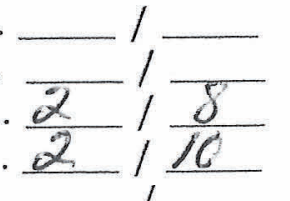

e. CATA Section / Region Meeting..................

g. FFA Section / Region Meeting....................

h. School staff Meeting.

i. FFA Chapter Meeting.

j.

k.

1.

m. Next Department Collaboration Meeting

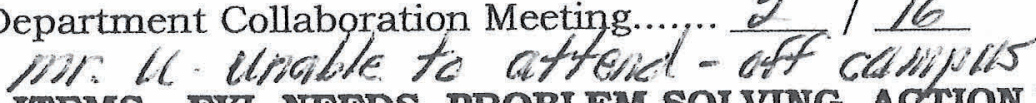

\section{DISCUSSION ITEMS--FY, NEEDS, PROBLEM SOLVING, ACTION PLAN, WHO DOES?.}

- DEPT. OPERATIONS, budget/purchasing, facilities, equipment, repairs, maintenance, travel.

-- CLASS supply, student problems, maintenance, repairs, AV, textbooks, storage.

-- AG. BOOSTERS, AG. Advisory, Community, CATA, Professional Development.

-- FFA OPERATIONS, Officer team, activities/contests, finances, awards/recognition.

-- SAEP OPERATIONS, School Farm, Project Supervision, County Fairs.

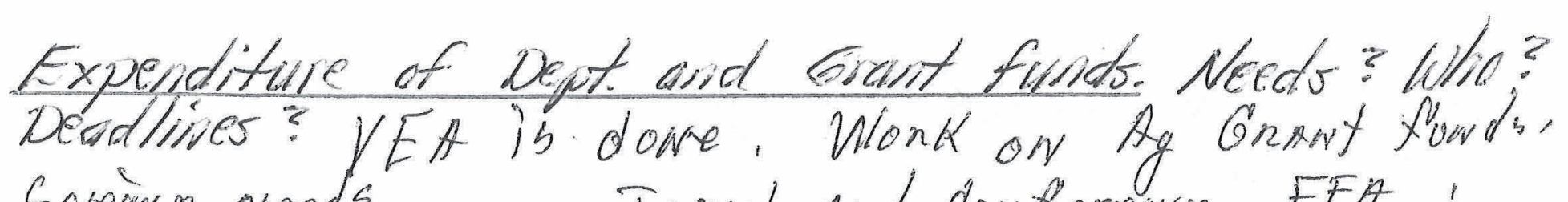
Screare needs shop wereds

oncenhouse, Finn

Jaskeh sod contenewe fon CITA CONA

FFA i

State fien lendostion corifer.

3 alutts 12 stodents

Money weeds Rogret in 
State CATA Canference-(June). Who" Reg finotel fee processing other liecels/ plans?

Rreg fon Boteh Pnocess popens

SWF Stock Needo? Last call-slieep, pigs. march $1{ }^{\text {th }}=60$ day ownership. ? Axy?

SUF Rabbits - 30 day, awnership = April $10^{\text {th }}$ same for Mreat Ribbiti. Whic? Neceds? Housing? Fair participation: Rabbit hist - Mest

Ag Advisory lintg- Jones - Community Suppont Group. and spring ragribusiness/lindustry Taur-witio? Aprit 14 th on $15 \times 2 \quad 2$ Tentchen sehool bos? students?

Scheol Farm-Pen repairs, fencing, wash rack, small basn roof, cleanup / trash hiauling. possithe cheanimy out thinigs me dowit obe

FFly week Activty Planning-Jones Actiritre: Furdromen

FYI- High Schenl and middle scheal 2011-12 Pre-Keg. coming up soon. ? ? set-up hecoinuert 


\section{AGRICULTURE DEPARTMENT COLLABORATION MEETING AGENDA/MINUTES NORTH SALINAS HIGH SCHOOL (rev. 10/10)}

MEETING DATE: Weds, J01/. 19 9011

COPY TO: Dept. Files, Ag Staff, Principal

STAFF PRESENT: X Mr. Jones, X Mrs. Blodgett, X Mr. Uchiyama, Mrs. Noroian MINUTES RECORDED BY: ___ Mr. Jones, ___ Mrs. Blodgett, \ Mr. Uchiyama,

1. IMMEDLATE ATTENTION-ACTIVITIES, CONTESTS, TRIPS, PROGRAM MANAGEMENT ITEMS. (FYI, needs, supervision, travel, due dates, funding)
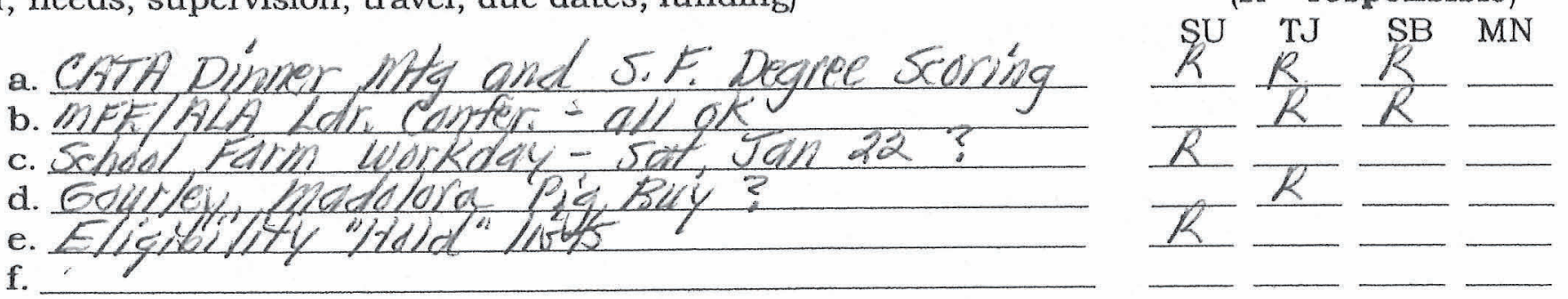

2. MEETING SCHEDULE REVIEW---NOT PREVIOUSLY DISCUSSED.

(FYI, agenda plan, supervision, funding, travel, needs)

(admin. approval, teacher sub, other admin.)
a. Ag Booster Club Meeting.
Mo Date
b. Ag Booster Officer Meeting $1, \frac{1}{12}$
c. FFA Officer Planning Meeting. $1 \frac{25}{10}$
d. Ag Advisory Committee Meeting
f. FFA Section / Region Contest. 1
g. FFA Section / Region Meeting....................
n. Sichooi staût Mieeting

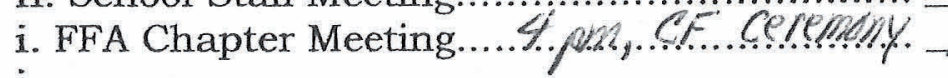
j.
k.
1.
m. Next Department Collaboration Meeting.

( $R=$ responsible)

3. DISCUSSION ITEMS--FY, NEEDS, PROBLEM SOLVING, ACTION PLAN, WHO DOES?.

- DEPT. OPERATIONS, budget/purchasing, facilities, equipment, repairs, maintenance, travel.

-- CLASS supply, student problems, maintenance, repairs, AV, textbooks, storage.

-- AG. BOOSTERS, AG. Advisory, Community, CATA, Professional Development.

-- FFA OPERATIONS, Officer team, activities/contests, finances, awards/recognition.

-- SAEP OPERATIONS, School Farm, Project Supervision, County Fairs.

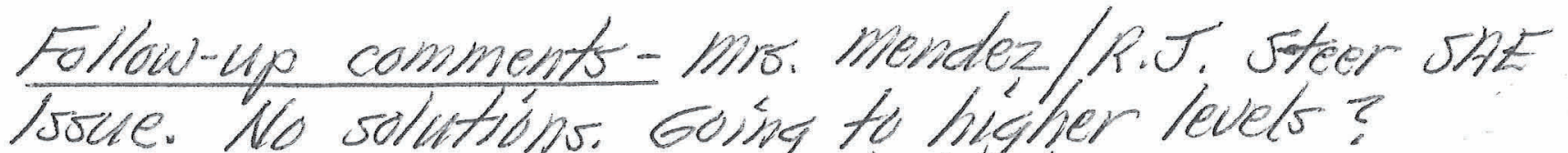
il need to setury winited. It

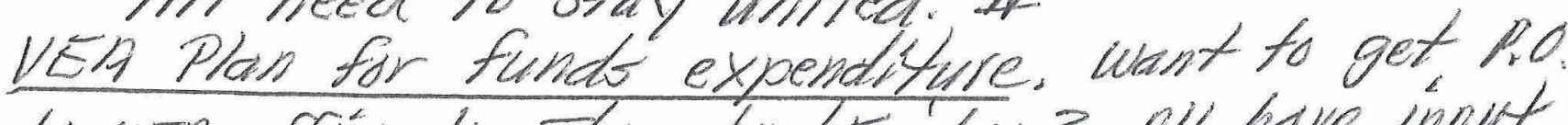

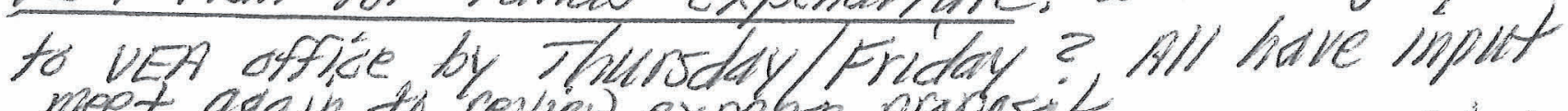
moet agan. to povien expopse

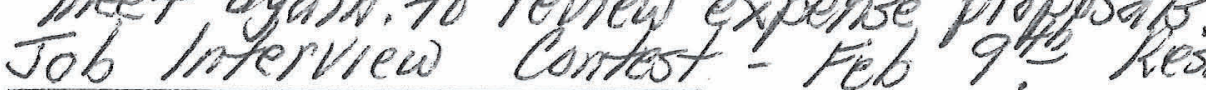
candiclates = talk with ndw. Give prep. materiab 
State FFA Lar. Confer. Reg and motel sign-ups. want to get sent to state by mid-Peb.

Need motel cost and get word out for sianups. Have 2 adult and 4 student rooms.

- upperclassmen, need be eligible

- Freshman jot yo. soph = Need to know who and deride Funds expenditure for Dept and Ag Grant funds. Need to meet after VEA funds spent open Pro, equipment science supply, Gout Econ? Get VEn finds spent first.

Curriculum Change Proposals = wove

mr. Blodgett work on Ag science 1-2 revision for "masters" project, Timeline? Dome by enot-of year (jib ind)

School Farm Maintenance and Repairs Work. General needs discussion. $\rightarrow$ continue discussions

FFA Manual Collection and boxed for use next year. Keep class oft for future -use. 
MeETING DATE: Hedo, Dec is, 2010

COPY TO: Dept. Files, Ag Staff, Principal

STAFF PRESENT: ___ Mr. Jones, Mrs. Blodgett, Mr. Uchiyama, Mrs. Noroian MINUTES RECORDED BY: ___ Mr. Jones, \ Mrs. Blodgett, __ Mr. Uchiyama,

1. IMMEDLATE ATTENTION-ACTIVITIES, CONTESTS, TRIPS, PROGRAM MANAGEMENT ITEMS. (FYI, needs, supervision, travel, due dates, funding)

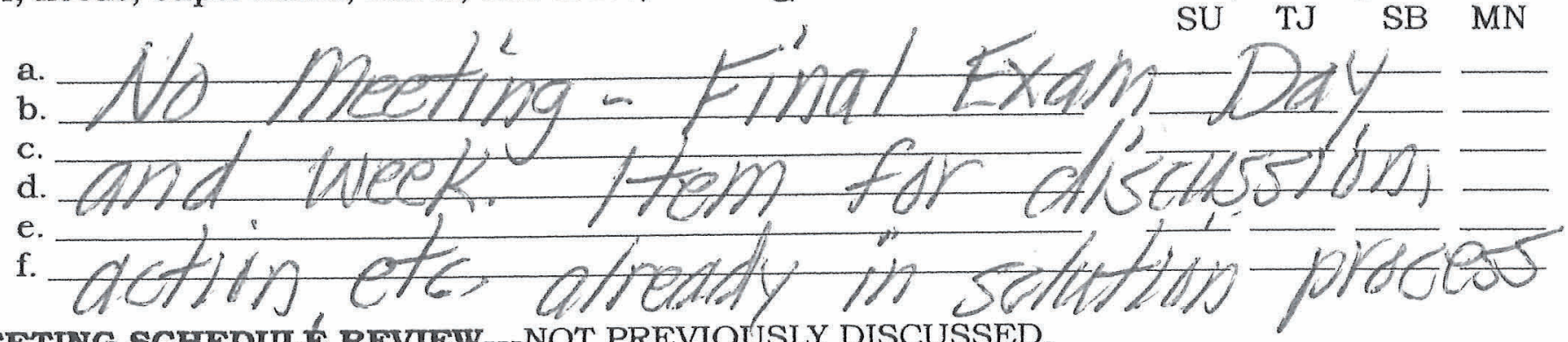

\section{MEETING SCHEDULE REVIEW---NOT PREVIOUSLY DISCUSSED.}

(FYI, agenda plan, supervision, funding, travel, needs) (admin. approval, teacher sub, other admin.)

a. Ag Booster Club Meeting.

b. Ag Booster Officer Meeting..

c. FFA Officer Planning Meeting....

d. Ag Advisory Committee Meeting.

e. CATA Section / Region Meeting.

f. FFA Section / Region Contest.....................

g. FFA Section / Region Meeting....................

h. Scchooi Staît Mieeting..

i. FFA Chapter Meeting.

j.

k.

1.

m. Next Department Collaboration Meeting

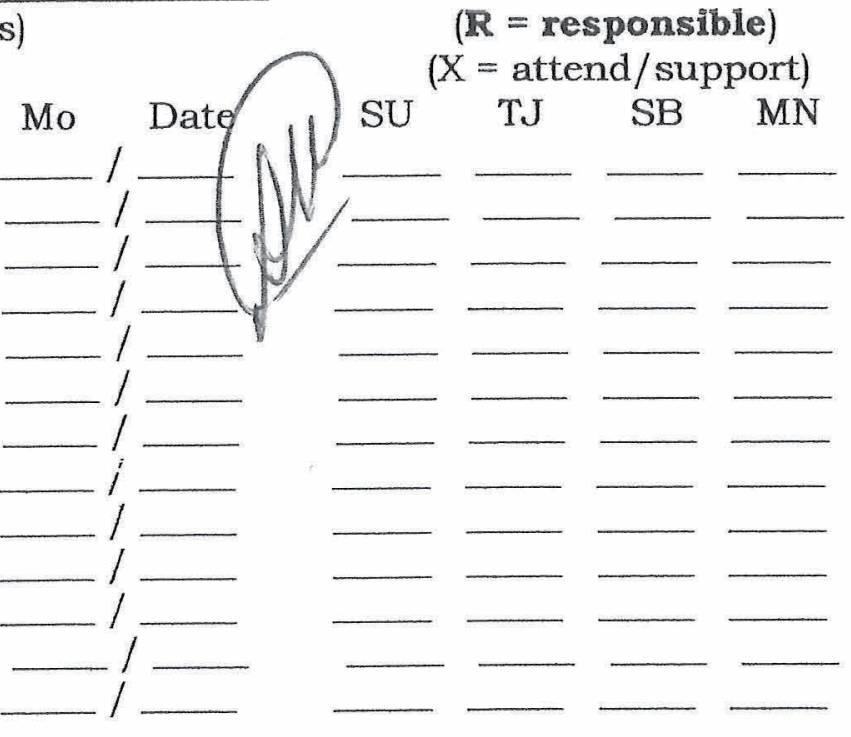

3. DISCUSSION ITEMS--FY, NEEDS, PROBLEM SOLVING, ACTION PLAN, WHO DOES?.

- DEPT. OPERATIONS, budget/purchasing, facilities, equipment, repairs, maintenance, travel.

-- CLASS supply, student problems, maintenance, repairs, AV, textbooks, storage.

-- AG. BOOSTERS, AG. Advisory, Community, CATA, Professional Development.

-- FFA OPERATIONS, Officer team, activities/contests, finances, awards/recognition.

-- SAEP OPERATIONS, School Farm, Project Supervision, County Fairs. 


\section{AGRICULTURE DEPARTMENT COLLABORATION MEETING AGENDA/MTNUTES} NORTH SALINAS HIGH SCHOOL (rev. 10/10)

- veting date: December $/, 2010$

COPY TO: Dept. Files, Ag Staff, Principal

STAFF PRESENT:

Mr. Jones,

Mrs. Blodgett,

Mr. Uchiyama,

Mrs. Noroian

MINUTES RECORDED BY: _ X Mr. Jones, ___ Mrs. Blodgett, __ Mr. Uchiyama,

1. IMMEDLATE ATTENTION-ACTIVITIES, CONTESTS, TRIPS, PROGRAM MANAGEMENT ITEMS. (FYI, needs, supervision, travel, due dates, funding)

a. State Ldr. Cont. Hatel Reorevation - Do Naw?

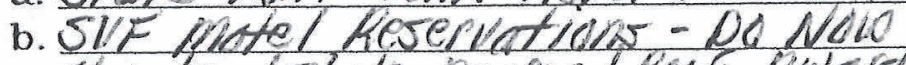

c. Chapter/state Degree/Profi hivard Completion

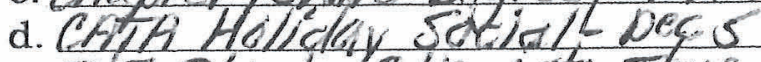

e. SIF Strek Q/A hat-THW; Dec 9 f. Ereenliend, Degree eepenany, DeC, lo

2. MEETING SCHEDULE REVIEW--NOT PREVIOUSLY DISCUSSED.

(FYI, agenda plan, supervision, funding, travel, needs)

(admin. approval, teacher sub, other admin.)

a. Ag Booster Club Meeting.

Moc, Date

b. Ag Booster Officer Meeting.

$\overline{\mathrm{DeC}} / \overline{7}$

c. FFA Officer Planning Meeting.

(1)

e. CATA Seetion / Region Meeting Kond

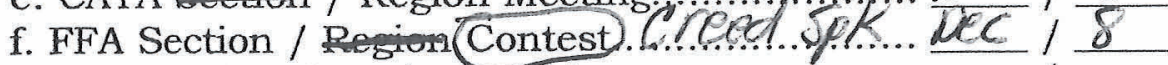

g. FFA Section / Region Meeting................... /

h. Śchooi Stañ İieeting.

i. FFA Chapter Meeting...

j. Dept. Clidir bieeting

Dec

$i$

Dee

1.

m. Next Department Collaboration Meeting.

Dec / $/ 5$

SU TJ SB MN

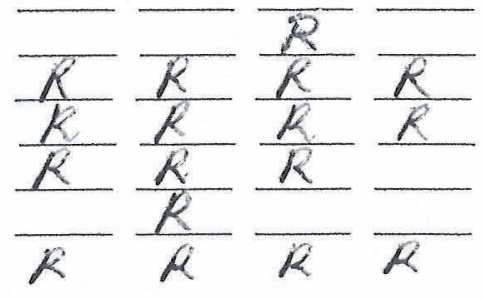

( $R=$ responsible)

( $\mathrm{X}=$ attend/support)

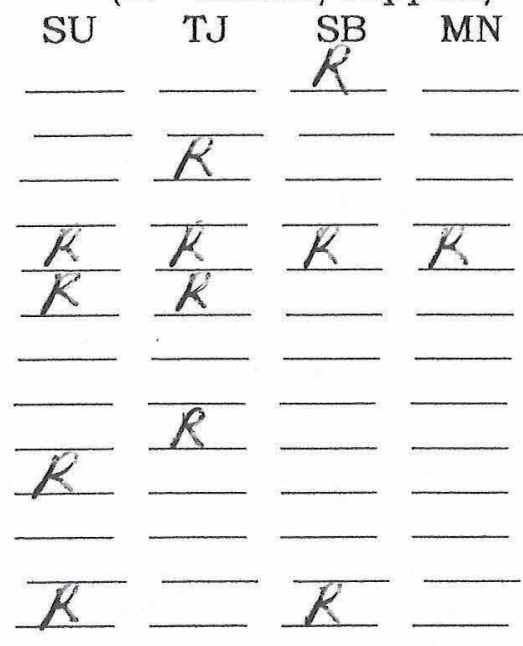

3. DISCUSSION ITEMS--FY, NEEDS, PROBLEM SOLVING, ACTION PLAN, WHO DOES?.

- DEPT. OPERATIONS, budget/purchasing, facilities, equipment, repairs, maintenance, travel.

-- CLASS supply, student problems, maintenance, repairs, AV, textbooks, storage.

-- AG. BOOSTERS, AG. Advisory, Community, CATA, Professional Development.

-- FFA OPERATIONS, Officer team, activities/contests, finances, awards/recognition.

-- SAEP OPERATIONS, School Farm, Project Supervision, County Fairs. A. Strite Ldr. Conference -2 male, / female teacher - (D) $/$ ib - 4/19

Number rooms - Rabsenve Bhock of RMS at Radisson 5 ams

B. Chapter Degree-Need to hidd/Stay in FFA office (J)

$$
\text { Doine by - 1/20 }
$$

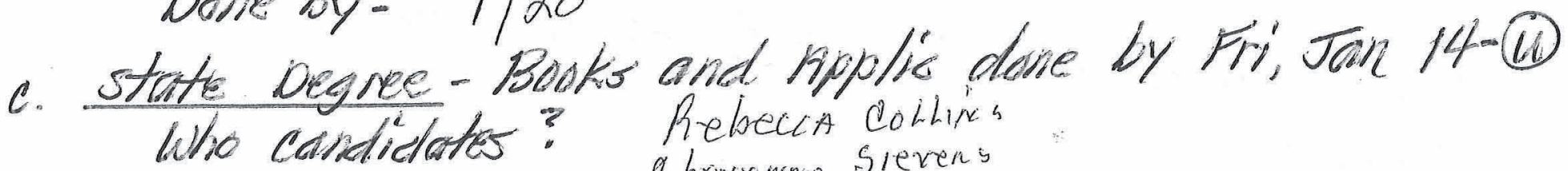

cheyerme sievens

,

Sydwey COAthitey 
D: SUF Stuck Q/A Parent inte-Thur, Dec. 9- (ii)

Program - Mir. U. At 6:30 pan C Rims in

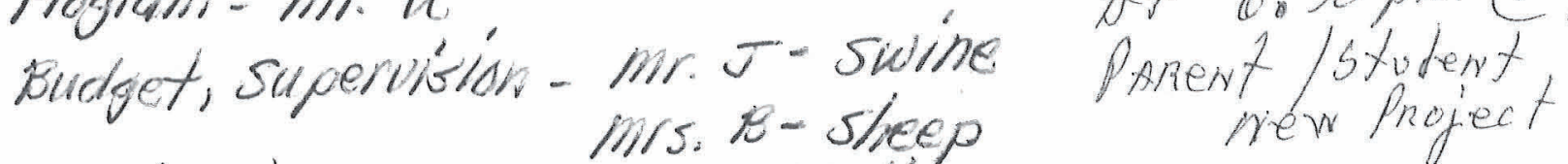

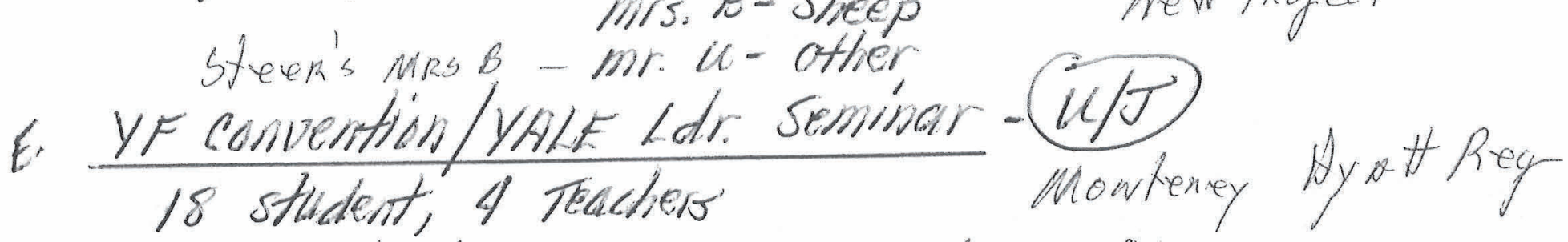
Transportation- 10;00 han Leave 8:30

F. Greenhand Degree Ceremony -(0) 12/10 Lookup Prim s 6. Ag Boosters, Dec 7. B

ming pot lack

'C' Wrap fundraising - weed parents ton monday 12-4p"

Jan. CATH Dinner - Phon in Precemben

"W. Calendar soles (II)

I. Salvation Army Kettle Drive -(3) Dec. 18 SBA?

5. Holiday canned Food Drive (UIN) - Ends on 12/8

K. Budget Expenditure - VEA, AI IG, Dept

1. Staff Duty and 5 year equip, facility,

m. Curriculum Development, Dist. Manual changes 


\section{AGRICULTURE DEPARTMENT COLLABORATION MEETING AGENDA/MINUTES}

\section{NORTH SALINAS HIGH SCHOOL (rev. 10/10)}

meeting date: Wed, Nov. 10,2010

COPY TO: Dept. Files, Ag Staff, Principal

STAFF PRESENT: $X$ Mr. Jones, $\chi$ Mrs. Blodgett, $\chi$ Mr. Uchiyama, $\chi$ Mrs. Noroian

MINUTES RECORDED BY: ___ Mr. Jones, __ Mrs. Blodgett, \ Mr. Uchiyama,

1. IMMEDIATE ATTENTION-ACTIVITIES, CONTESTS, TRIPS, PROGRAM MANAGEMENT ITEMS. (FYI, needs, supervision, travel, due dates, funding)

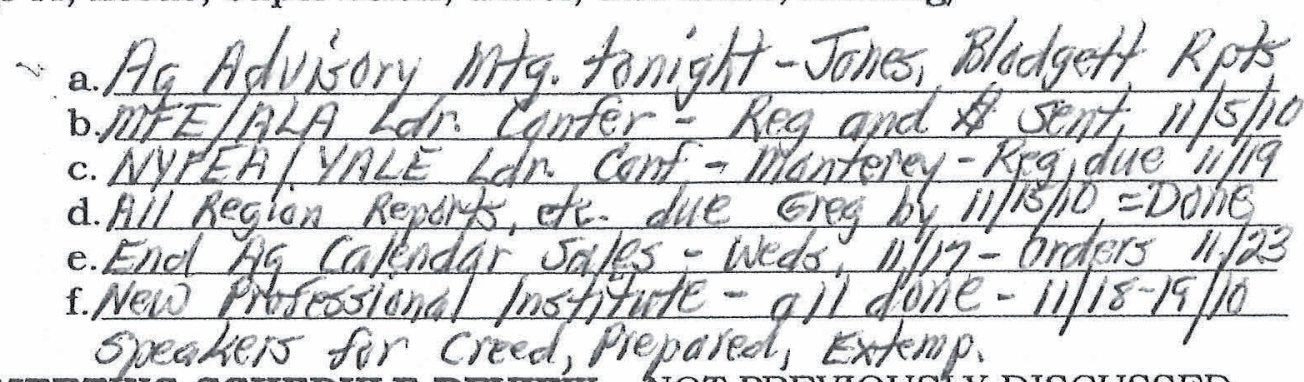

2. MEETING SCHEDULE REVIEW--NOT PREVIOUSLY DISCUSSED.

(FYI, agenda plan, supervision, funding, travel, needs)

(admin. approval, teacher sub, other admin.)

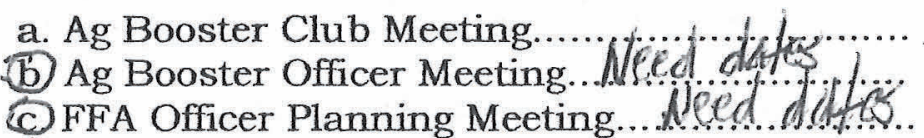
Mo Date
(d.)Ag Advisory Committee Meeting.
e. CATA Section / Region Meeting
f. FFA Section / Region Contest.
g. FFA Section / Region
h. School Staff Mieeting.

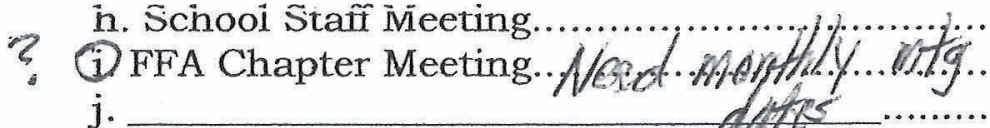
k.
1.
m. Next Department Collaboration Meeting.

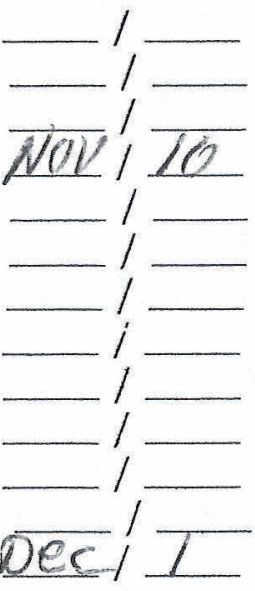

2

$?$
( $R=$ responsible)

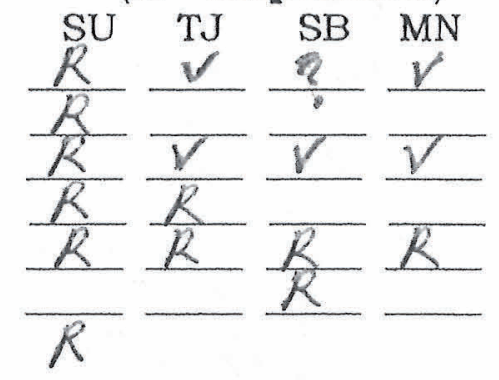

( $R=$ responsible)

$(\mathrm{X}=$ attend/support)

SU TJ SB MN

\section{DISCUSSION ITEMS--FY, NEEDS, PROBLEM SOLVING, ACTION PLAN, WHO DOES?.}

$V$ - DEPT. OPERATIONS(budget/purchasing) facilities, (equipment repairs, maintenance, travel.

$\checkmark$ - CLASS supply, student problems, maintenance, repairs, AV, textbooks, storage. $P . C$. Grales

-- AG. BOOSTERS, AG. Advisory, Community, CATA, Professional Development.

$\checkmark$-- FFA OPERATIONS, Officer team, activities/contests, finances, awards/recognition. fity fifey ?

$\checkmark-$ SAEP OPERATIONS, School Farm, Project Supervision, County Fairs. SAE tils out $11 / 9 / 10$

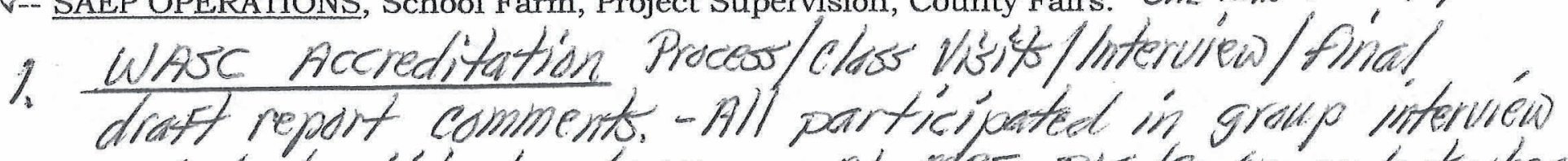

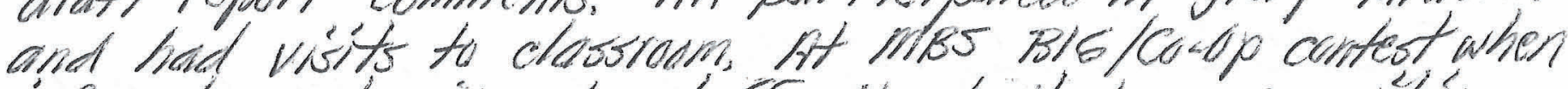

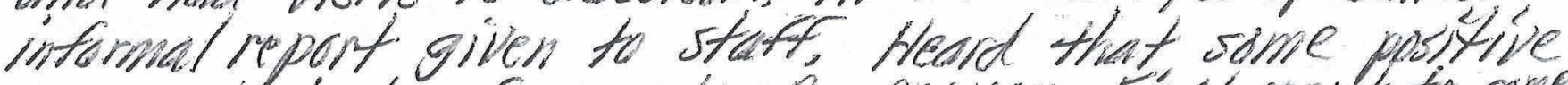

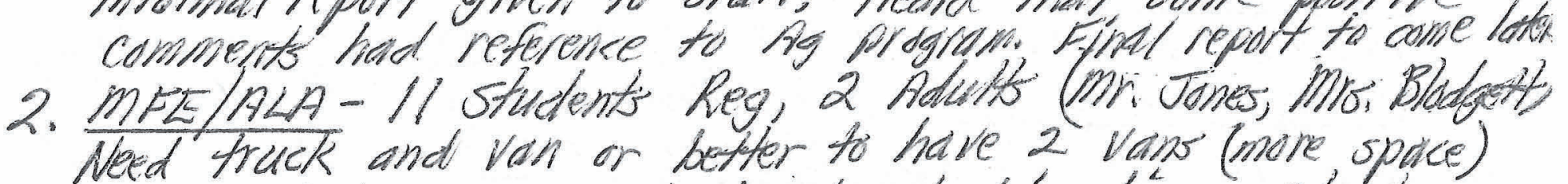
werd fruck and van or befter to have 2 vaw (mare, spoce)

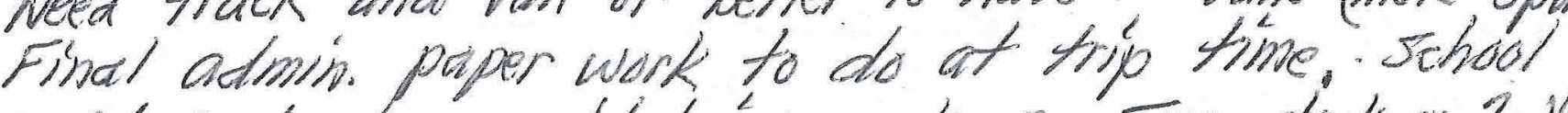

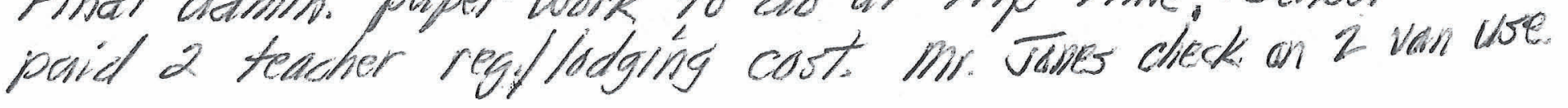


3. Ag Booster Mitty fillow-up Fry. I. / needs? - Blodgett Need ProA. Copy for all staff and Dept. records. Also. for, principal. Gaud to see printed agenda. Record of minutes and treasurer reports? Prep. for Jain coth dinner? minutes, Tres surer Rpt, Pion. need to be is Dept. files. 4. MB BS CoOp and BIG Contest Followup. All.

Hwards-Need get, of Team plaques and ribbons; BIG and Coop individual ribbons. Teams qualify for state fiscals contest in earl may, 2011.

4 a- COLC T.Y. letter follaw-up- Jones "Sore spot." R. Collins

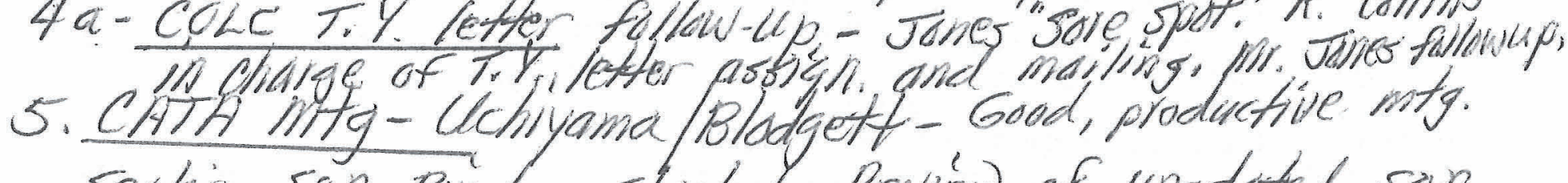
section sop Binder started. Review of updated sop at Jan. int g - send changes, te i to Maros Ruiz, shot

5. a -Officer Team Progress - mInty /purpose frequency- Jones 6. MBS-NYFEA/YALE - UChiyama

ODT filed. Good if as many teachers go as possible. " sol cost each participants. FEM pays for student.

1 truck t 2 vans $=16-18$ students. Reg due Nov. 19. Need all Names by Nov. 77 t - call in reg?

Ga- $\mathrm{Ag}$ calendar Sales - Not many order forms out. Need to encourage sales for wow. is turning. 7. AlG an-Site Revien-All. Process, preparations, documentation, draft rece/commend, final report. Thanks for everyone's help. Review seemed to go well. will return 2000 for full employment non-comply. Find report to come at later date, 8. GH, CF. S.F. Degrees-AII GH Ceremony- When? Planned for Dec, Chapter mtg. clearance for C.E and 5.5 Degree? Offerer = Co E Tigre? CF Degree done by? Not planned - need to do this?

9. Creed, Prepared, Extemp. speaking Contests -Dec. 8-K.C. Prepared maniseripots due weds Dea. I, RolF II/29/10 
AGRICULTURE DEPARTMENT COLLABORATION MEETING AGENDA/MINUTES

NORTH SALINAS HIGH SCHOOL

(rev. 10/09)

veEring DATE: Wed, Oct, 27,2010 COPY To: Dept. Files, Ag Staff, Principal StAFF PRESEnT: $x$ Mr. Jones, $x$ Mrs. Blodgett, $\underline{x}$ Mr. Uchiyama, $\underline{x}$ Mr. Mloroidn MINUTES RECORDED BY: Mr. Jones, Mrs. Blodgett, X Mr. Uchiyama, Moi Noroían

1. IMMEDIATE ATTENTION-ACTIVTTIES, CONTESTS, TRIPS, PROGRAM MANAGEMENT ITEMS. (FYI, needs, supervision, travel, due dates, funding) $(\mathbf{R}=$ responsible)

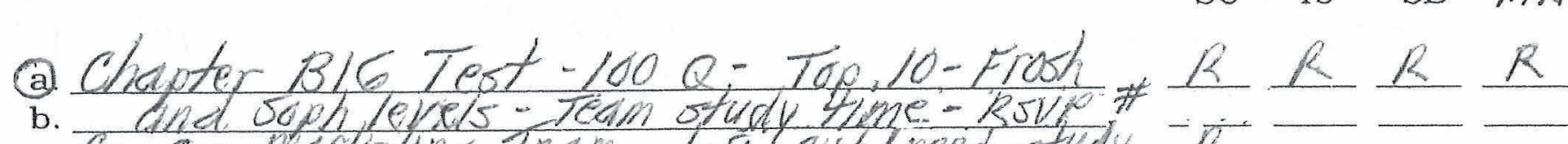

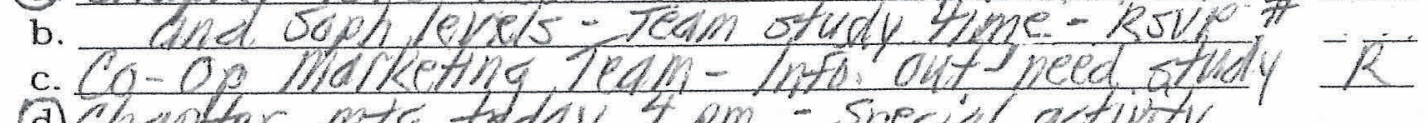

(d) chapter my today, 4 pm - special activity

(i) MET /hA Reg Pay due $10 / 29$ send by $10 / 5$ ?

$\bar{R} \div \bar{R}=$

2. MEETING SCHEDULE REVIEW--NOT PREVIOUSLY DISCUSSED. (FYI, agenda plan, supervision, funding, travel, needs) (admin. approval, teacher sub, other admin.)
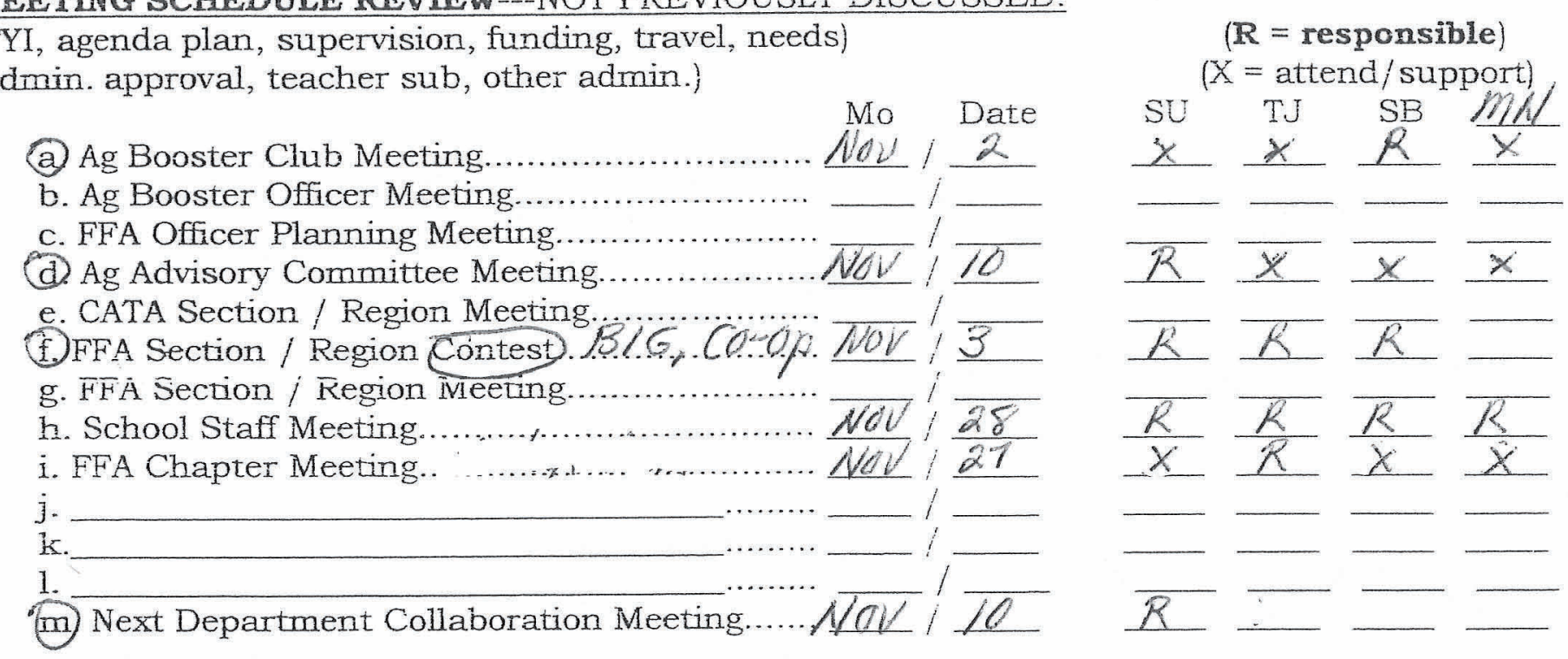

3. DISCUSSION ITEMS--FY, NEEDS, PRORLEM SOLVING, ACTION PLAN, WHO DOES?

- DEPT. OPERATIONS, budget/purchasing, facilities, equipment, repairs, maintenance, travel. -- CLASS supply, student problems, maintenance, repairs, AV, textbooks, storage.

-- AG. BOOSTERS, AG. Advisory, Community, CATA, Professional Development.

- FFA OPERATIONS, Officer team, activities/contests, finances, awards/recognition.

-- SAEP OPERATIONS, School Farm, Project Supervision, County Fairs.

AlG onsite Review Tues; Nov 2, 9 am

Review checklist criteria - all

Beard' intro and document needs list -all

Program Plan Info. Update - Jones

minty day needs. 
$\frac{\text { WA SC Visit Nav } 1-3}{\text { Work samples on View }}$

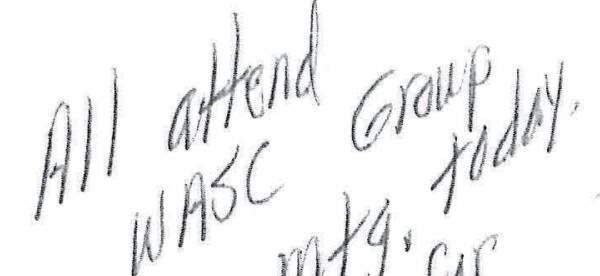

to

Review visit and meeting schedule pin for

Weds Nov 3-Report to school staffinil. int

Read Group report contents

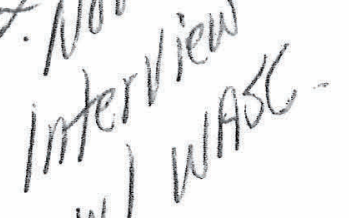

W

Ag Calendar Cookbook Gift wrap Salespush involvement.

05H vs Home Depot P.O. use and future - Dist to stop OSH use, Mid. stephanie to Home Depot. Charge list.

McF/sccF Sales check Rec. Book

clearance - Get done. Pickup As/ AM premium checks and projects. Give out/back.

Qt. Progress grades due 11/5/10 
AGRICULTURE DEPARTMENT COLLABORATION MEETING AGENDA/MINUTES

NORTH SALINAS HIGH SCHOOL (rev. 10/09)

Eeting Date: October 13,2010

COPY TO: Dept. Files, Ag Staff, Principal

staff PResent: $\chi$ mr. Jones, $X$ mrs. Blodgett, $X$ mr. Uchiyama, $X$ Mrs. Nordian MINUTES RECORDED BY: ___ Mr. Jones, __ Mrs. Blodgett, X Mr. Uchiyama,

1. IMMEDIATE ATTENTION-ACTIVITIES, CONTESTS, TRIPS, PROGRAM MANAGEMENT ITEMS. (FYI, needs, supervision, travel, due dates, funding)

$(\mathbf{R}=$ Iesponsible $)$

a. Ag Advisory Mtg-Oct 14 (Agenda revien)

c. Ereenhend confer. - oot 14

d.

e. R-2 Enrqliment and FFH Roster-oct is

f. Ag Studant Lenderobip Packet P. D. process.

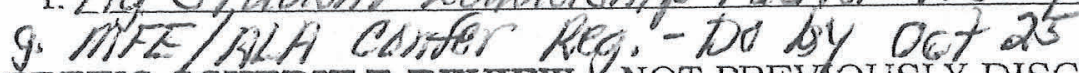

2. MEETWG SCHEDULE REVIEW-NOT PREVIOUSLY DISCUSSED.

(FYI, agenda plan, supervision, funding, travel, needs)

(admin. approval, teacher sub, other admin.)

Mo Date

SU TJ SB $m$ N

b. Ag Booster Officer Meeting.

c. FFA Officer Planning Meeting.

d. Ag Advisory Committee Meeting
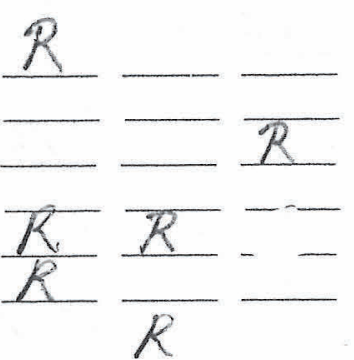

e. CATA Section / Region Meeting....................

f. FFA Section / Region Contest.....................

g. FHA Section $/$ Region ivieeting......................

h. School Staff Meeting.

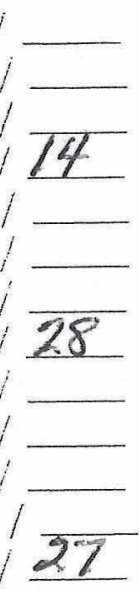

(i) FFA Chapter Meeting................................

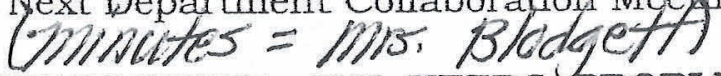

3. DISCUSSION ITEMS--FY, NEEDS, PROBLEM SOLVING, ACTION PLAN, WHO DOES?

- DEPT. OPERATIONS, budget/purchasing, facilities, equipment, repairs, maintenance, travel.

-- CLASS supply, student problems, maintenance, repairs, AV, textbooks, storage.

- AG. BOOSTERS, AG. Advisory, Community, CATA, Professional Development.

-- FFA OPERATIONS, Officer team, activities/contests, finances, awards/recognition.

-- SAEP OPERATIONS, School Farm, Project Supervision, County Fairs.

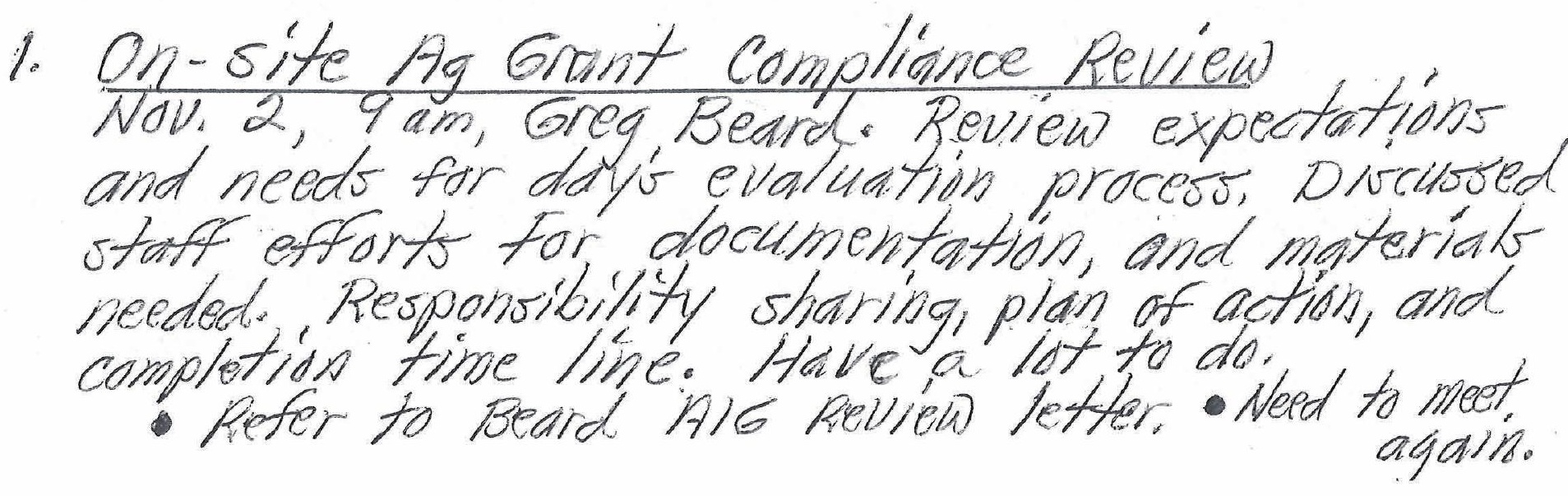


2. MBS B.1.6. and Coup Market Contest Status. SBA dine. Truck and 2 vans needed. Bl. G. study already started. wo chapter test on Tuesday oct. 26. mos. Blodgett make test score with scan tran sheet. Get co- op team

8. WASC Visit -Nov. 1-3. sign-up (J R-SK level) move Into to come later Get things cleared up. mir. Niwio working on school report.

4. R-2 Report FFA Roster Due Oct 15. AII done except for FEM roster ( 12 done). 340 members

5, Farm Work dove - Oct 16 and 30

Do on $16^{\text {th }}$ not on $30^{\text {th }}$ ( 1 gIG Keven reeds) stack per cleansing, fines being w sued. Barn graffiti t poistit-over by twist. main? Do wank order. Fy. I. fo mri Carcesany.

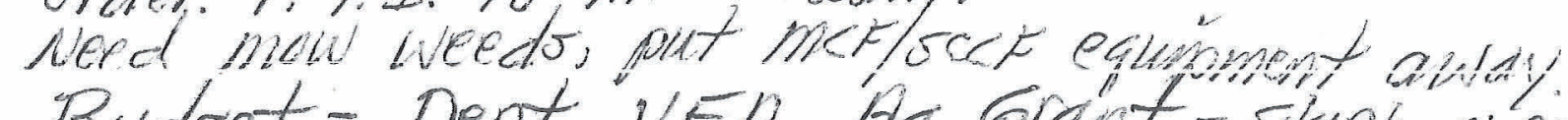

6. Budget -Dept, VEA, Ag Grant -start speeding 必

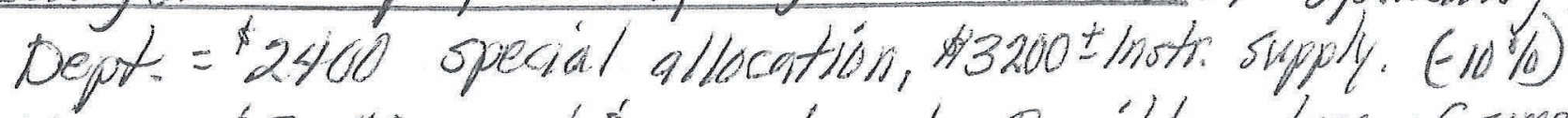

VEN $=8,500$ and 53000 travel. Passible return of sue H/G

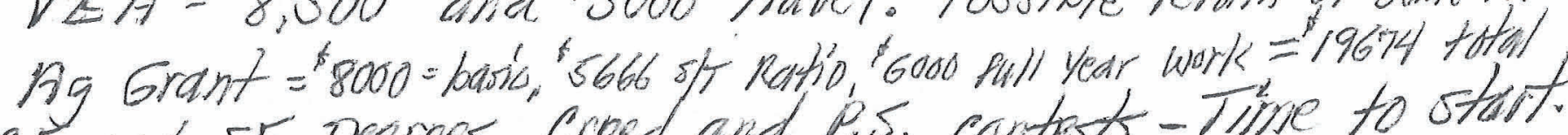

7. CF and SF Degrees, Creed and P.S. contests - Time to start.

8. Ag Curriculum -Earth, Chem, Gov/Econ, UC

approved. Book and curriculum, use.-

Need Copier for A.L.G. review and Dept.

Program Plan. Application to ag. standard o realistic? UC approval process for 2010\%1:

q. Staff Duty/Responsibility Assignment. Need to start review of job task assignments.

ito review of 5 year equip, facility, farm plans. 
AGRICULTURE DEPARTMENT COLLABORATION MEETING AGENDA/MINUTES

NORTH SALINAS HIGH SCHOOL

(rev. 10/09)

"mEeTING DATE: Sept. $79,90 / 0 \quad$ copy To: Dept. Files, Ag Staff, Principal

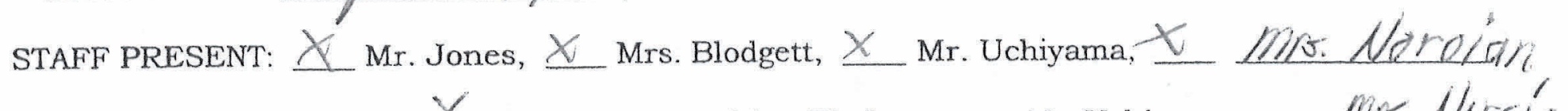

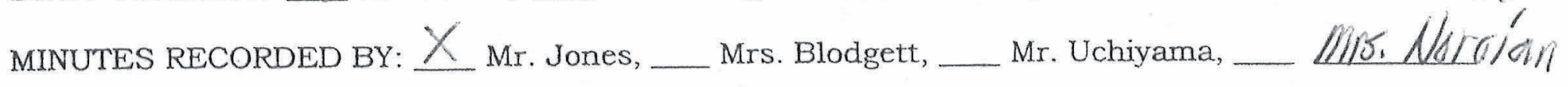

1. IMMEDIATE ATTENTION -ACTIVITIES, CONTESTS, TRIPS, PROGRAM MANAGEMENT ITEMS. (FYI, needs, supervision, travel, due dates, funding)

$(\mathbb{R}=$ responsible)
TU SB N MN

a. Sept. Clipper mt a and BBQ needs

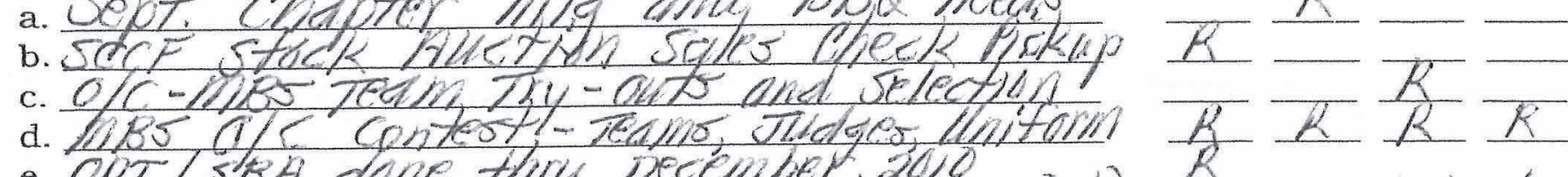

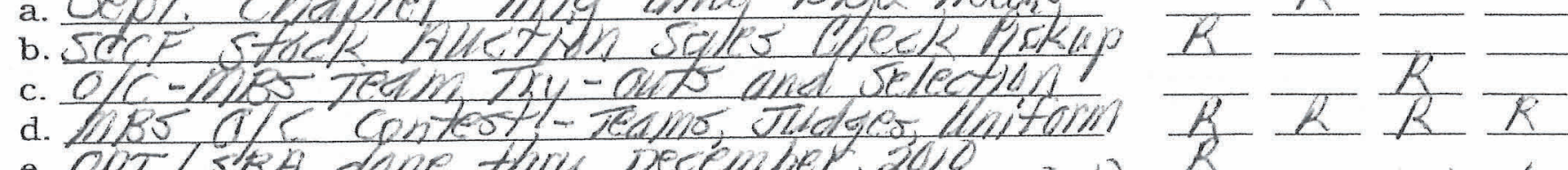

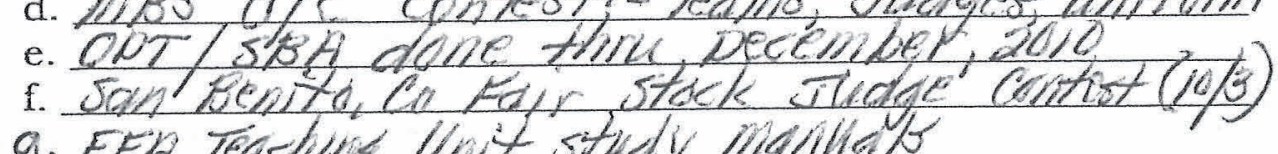

9. FFM TEaCh

2. MEETWG SCHEDULE REVIEW --NOT PREVIOUSLY (admin. approval, teacher sub, other admin.)
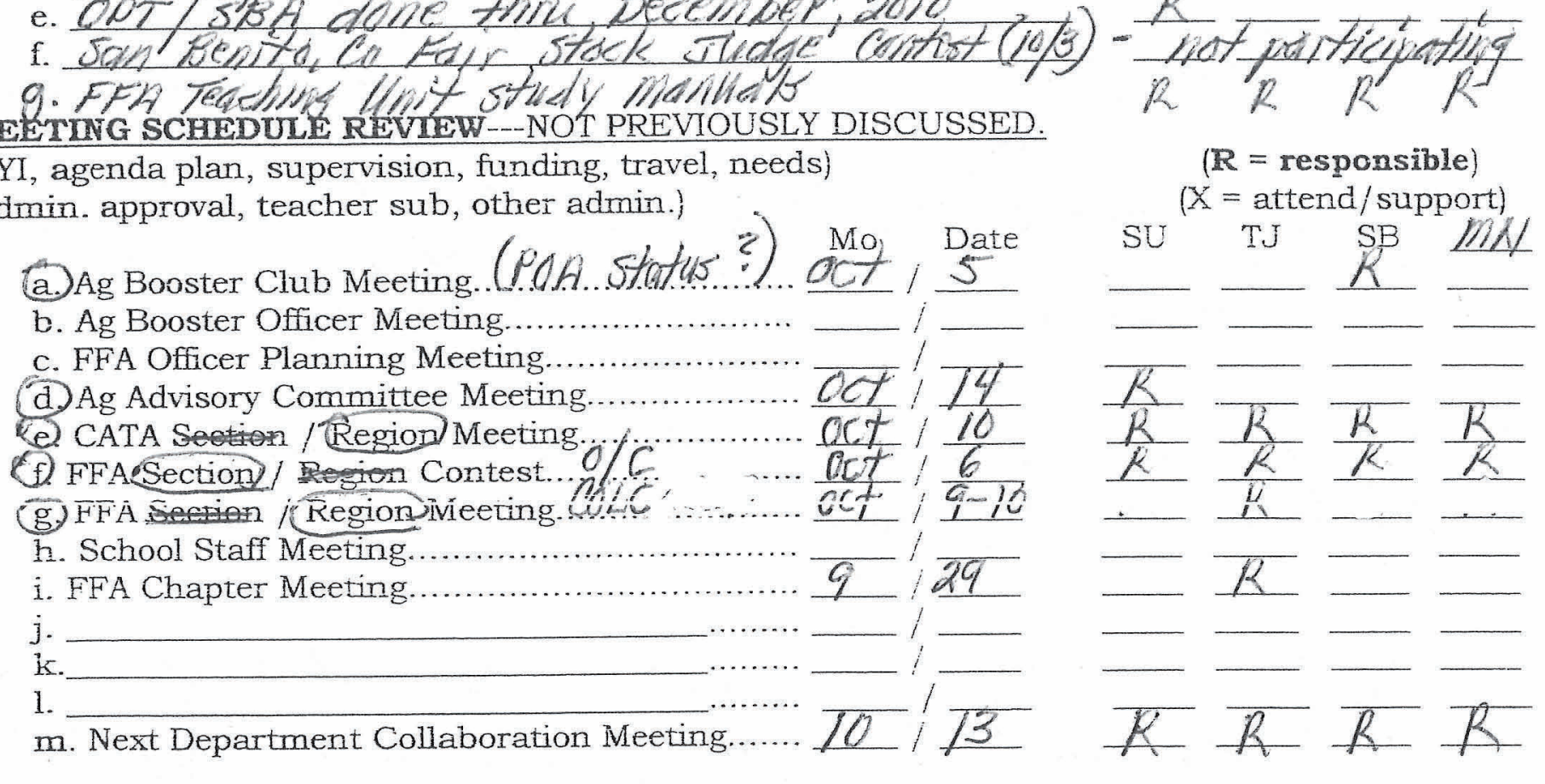

3. DISCUSSION ITEMS--FY, NEEDS, PROBLEM SOLVING, ACTION PLAN, WHO DOES?.

- DEPT. OPERATIONS, budget/purchasing, facilities, equipment, repairs, maintenance, travel. - CLASS supply, student problems, maintenance, repairs, AV, textbooks, storage.

-- AG. BOOSTERS, AG. Advisory, Community, CATA, Professional Development.

- FFA OPERATIONS, Officer team, activities/contests, finances, awards/recognition.

-- SAEP OPERATIONS, School Farm, Project Supervision, County Fairs.

I. FF i report to Solid Bod ad, $9 / 28 / 10$ - very impressive - FA presentation to school Bond, FAin and prognian Report

2. State FF H Roster. Filing and Po - Mr. Jones

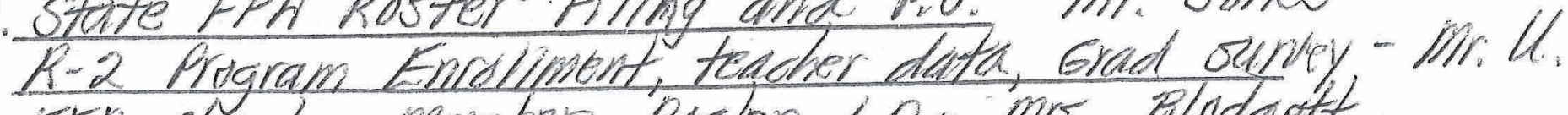

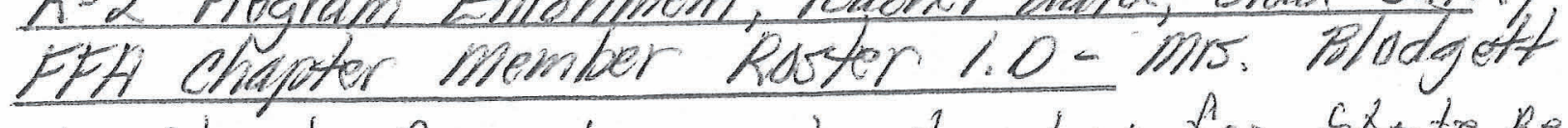
Ah sheet Doe to various teachers ton sxaterepont, 
3. MoCo and Somta Cruz Fair Wrap up of SMF Record books, she tists, etc. - Naed to plws studont to complete next 1-2 weeks. Equíment cleanup needs. All Fain wishp-up fon checks ASAP - SCCF'- Chent up

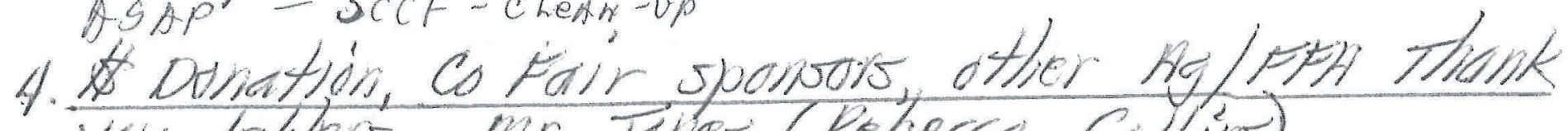
you, letteor - Mr joires. (Rebecca Collins) bend heber to Mrs. Woremsin, Mr. Cohbrean, 5. CF and State Degree Reguirement complotisn - start CF - Vicky, Silva St, Dopree - Chovene Seerens Aavgeha Wy quent Ris. Mevader hetobech collins Sy diney coskely

6. Use of Compiterized ReC. BK. - Year Tylem Gibson

6. Use of Compiterized ReC. BK. - Year Tylengibson year 2 hard or electronic copl/ cliolée? - All Discuss moviong to electnowic copy fon second yena stodents.

7. Experse Reimbusement, Non-class activity log for

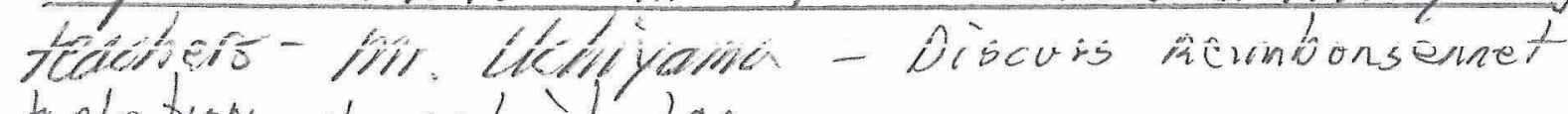
domphetion $f$ Actividy hog

8. Region FFA COLC and Chith witg, Oct 9-10 nereds. VA pay Reg \& for 4 Teachers. Hg Babtor paly FFH

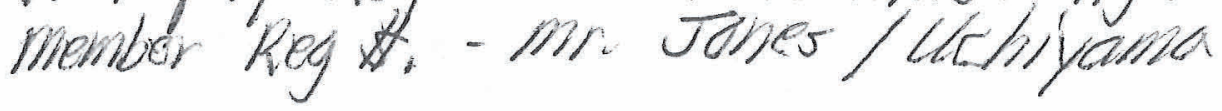

9. Greenhand Canference Thuro, Out 14-Mns. Bladgotf 19l) 7 spaces paid, 9/28/10.

10. AlG an-Jite Reviéa, Nov 2" update program plan, Revien criteria checklist, documentation for compliance, FF⿻ POA, o year plan, staff

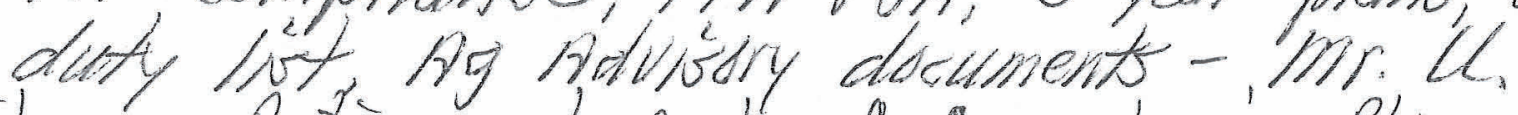
sphit up Duties and pants of Compnehensive Phaw. 
AGRICULTURE DEPARTMENT COLLABORATION MEETING AGENDA/MINUTES

NORTH SALINAS HIGH SCHOOL

(rev. 10/09)

"restring Date: Sept 15,2010 copy to: Dept. Files, Ag Staff, Principal

Staff Present: X Mr. Jones, X Mrs. Blodgett, $\Varangle$ Mr. Uchiyama, X MN. Noraian

MINUTES RECORDED BY: ___ Mr. Jones, X Mrs. Blodgett, __ Mr. Uchiyama,

1. IMMEDIATE ATTENTION -ACTIVITIES, CONTESTS, TRIPS, PROGRAM MANAGEMENT ITEMS.

(FY, needs, supervision, travel, due dates, funding)

$(\mathbb{R}=$ responsible $)$

a. Santa Cruz Fir stock Judqing/Auction Ring

(b) Back to school, Night $9 / 15 \%$ is

LU TU SB $\mathrm{hN}$

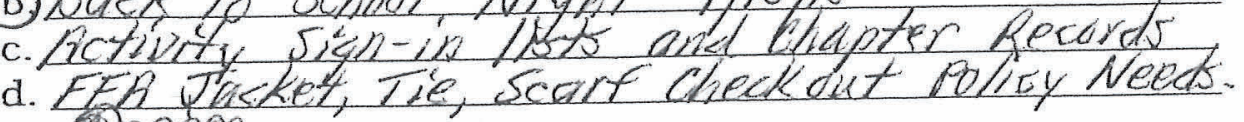

Angie

e. 000000

2. (FYI, agenda plan, supervision, funding, travel, needs) (admin. approval, teacher sub, other admin.)

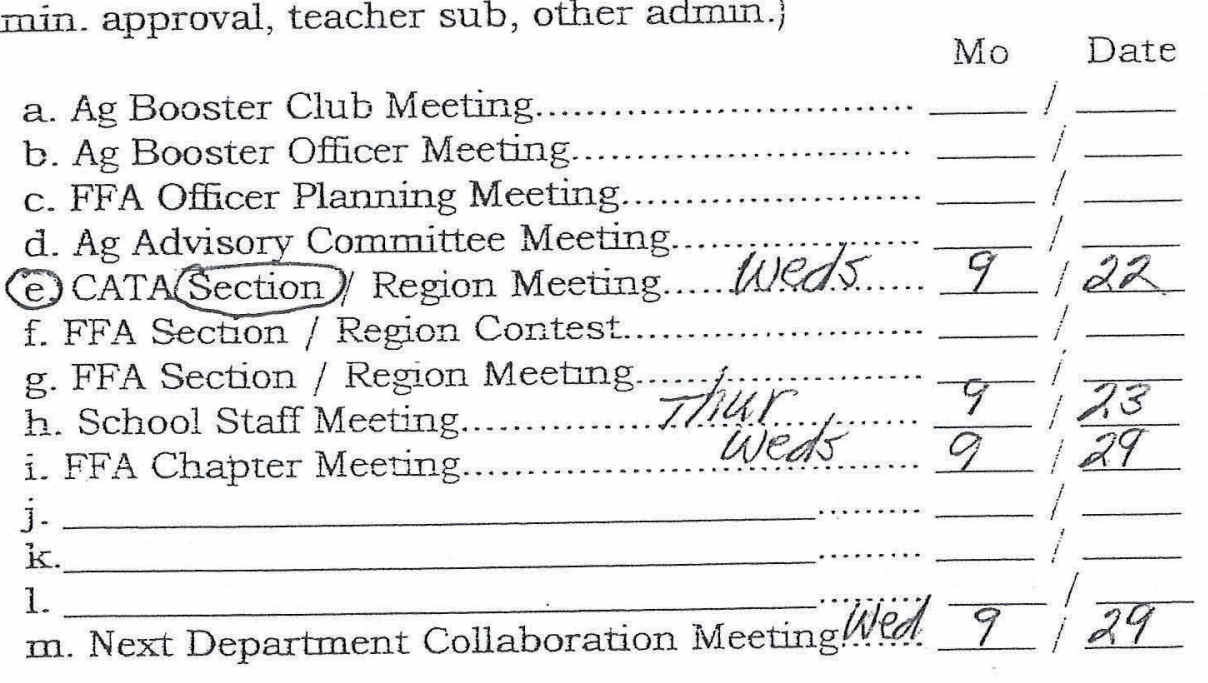

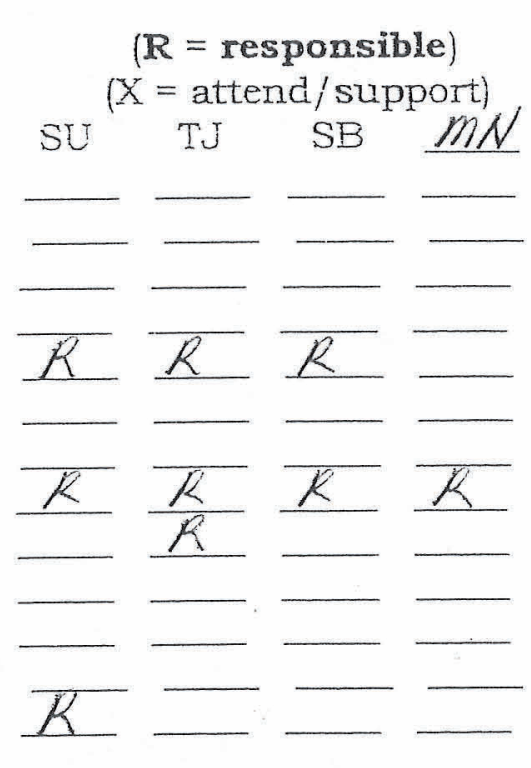

3. DISCUSSION ITEMS---FY, NEEDS, PROBLEM SOLVING, ACTION PLAN, WHO DOES?,

DEPT. OPERATIONS, budget/purchasing, facilities, equipment, repairs, maintenance, travel. - CLASS supply, student problems, maintenance, repairs, AV, textbooks, storage.

-- AG. BOOSTERS, AG. Advisory, Community, CATA, Professional Development.

- FFA OPERATIONS, Officer team, activities/contests, finances, awards/recognition.

-- SAEP OPERATIONS, School Farm, Project Supervision, County Fairs.

(1) Santa Cruz Co Fair -Supervision, shout status, weekend needs, load out, Entry Removal (Monday)

Livestock contest, Mutation' Ring (CC) setup. Feeder stat sale.

- Mr. la and Mrs. B going to auction

- ali to go Friday livestak judging, auction.

(2.) Poco Fair Exhibitor Clearance - Record Burns; pen Cleaning sales checks. (Thurs, q/16).

- Mrs Blodgeti to check pens. 
3. Veronica Corona pig removal form form.

- check pen did clean pen and add feed,

- out date is tuesday 9/91:

(4) Region COLC, at 9-10, Hollister-Supervision, Reg fees, Transportation, cosh mtg, Uniform Dress.

- Mr. Jones Mr. U going Saturday. All to go

- Booster to pay reg fee.

(3.) MB s open/closing minty ceremony Contest, oct 6, EAHS - Transportation, Teams, Judges, Uniform Dress

- Team practices. next week (hedresclay).

6. San Benito Co Fair Stack Judging Contest, Oct 3- ane vie going to go?

(7) Greensand Conference, Oct. 13-Registration, transportation, supervision, \# students," Teacher (Mrs. Bridgett)

- Mrs. B to dow load papen work

- L sub day for 2 teachers requested

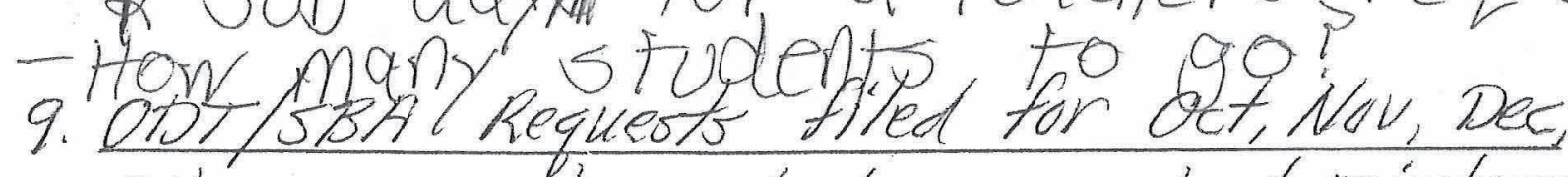

mIl on requests, sub day requests, to reimbursements

- Thanks to Mr. U.

(10) Oct 15 Dept/FFA Reports due to state

- R-2 Enrollment and Grad survey - Uchivama) All

- FEn state Roster - Jones

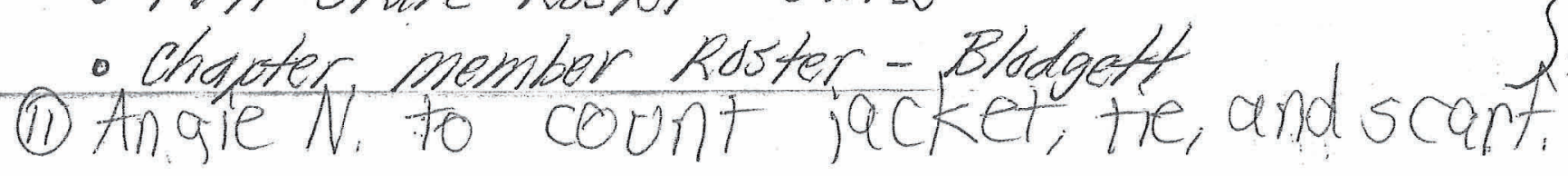


NORTH SALINAS HIGH SCHOOL (rev. 10/09)

ETING DATE: Sept. i, 2010

COPY TO: Dept. Files, Ag Staff, Principal

STAFF PRESENT: EX Mr. Jones, $x$ Mrs. Blodgett, $\chi$ Mr. Uchiyama, EX MF. Nordidn. (MoCo Fair)

MINUTES RECORDED BY:

Mr. Jones, Mrs. Blodgett, $\times$ Mr. Uchiyama,

MBs. Norgian

1. IMOMEDIATE ATTENTION-ACTIVITIES, CONTESTS, TRIPS, PROGRAM MANAGEMENT ITEMS.

(FYI, needs, supervision, travel, due dates, funding)

M. MCo Fair Events - sept $1-6$

b. As Bodster Budget P. A. - sept. 7

c. Ianfa Crun co Fair Evemts - sept. $11-20$

d. Farm workday - sept. II

e.

f.

2. MEETING SCHEDULE REVIEW---NOT PREVIOUSLY DISCUSSED.

(FYI, agenda plan, supervision, funding, travel, needs)

(admir. approval, teacher sub, other admin.)
a. Ag Booster Club Meeting.
$\frac{\text { Mo }}{9}, \frac{\text { Date }}{7}$
b. Ag Booster Officer Meeting.
c. FFA Officer Planning Meeting
d. Ag Advisory Committee Meeting.
e. CATASection/Region Meeting
f. FFA Section / Region Contest.
h. School Staff Meeting.
i. FFA Chapter Meeting.
j.
k.
1.
w. Next Department Collaboration Meeting.

3. DISCUSSION ITEMS--FY, NEEDS, PROBLEM SOLVING, ACTION PLAN, WHO DOES?

-- DEPT. OPERATIONS, budget/purchasing, facilities, equipment, repairs, maintenance, travel.

- CLASS supply, student problems, maintenance, repairs, AV, textbooks, storage.

-- AG. BOOSTERS, AG. Advisory, Community, CATA, Professional Development.

-- FFA OPERATIONS, Officer team, activities/contests, finances, awards/recognition.

-- SAEP OPERATIONS, School Farm, Project Supervision, County Fairs.

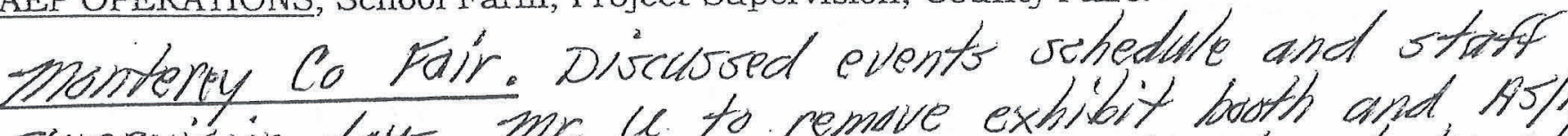

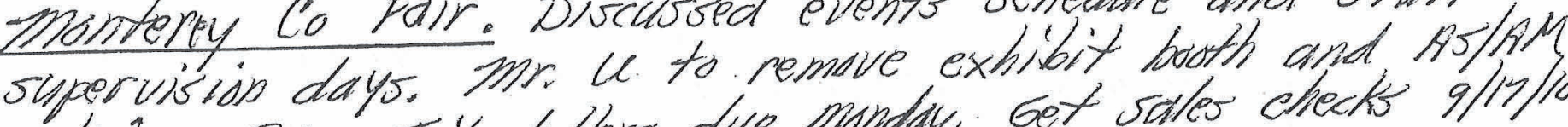
entives. Buyer T.y. lesters due monday. Get sales checks $9 / 13 / 10$. Santa Cruz Co Fair - Busth, As/an contry dive a/1ip/io stock weigh-in 8/13/10\%. stick confest and puction Ring set-up friday, 9/i?. Bowth, $195 /$ ism entry removalsupervivion delys. 
R-R FFA Raster Info. Collet all by $9 / 15$

Min. Jones to do chapter Raster filing.

Mr. U to do R-Z consillment and stiff Reporting

Mrs. B (Mg Boosters) do Fth chapter member roster list.

of e Minty Ceremony Contest - 2 Novice, 2 Bolvance, I officer Oct. 6, Alvarez H.5. Teams $=2 \mathrm{~N}, 2 \mathrm{~A}, 1$ officer Vans - Nh.

Teachers - all

start practice- up to teacher

Green hand Conference - weds, 10/13/10, Pass Robles

Vans - 1

Teachers -1

Roup pay by - 30 or

(InCH. I
How many - 7-12

on line Reg due-cherk aniline

Class hoad - See District Class Enrollment Ratio want exact class enrollment \#. will go to admin. to work on reduced class load. (1.H. Jab Classes 29 Gen. Academic 32 students)

solid Waste. material list to Jean when for school safety report. Do now corrosives, flammables use in dabs, etc. How dispose of? 
AGRICULTURE DEPARTMENT COLLABORATION MEETING AGENDA/MINUTES

NORTA SALINAS HIIGH SCHOOL (rev. 10/09)

- ne ting Date: Al lg $18,2010 \quad$ copy to: Dept. Files, Ag Staff, Principal StAfF PResent: $x$ Mr. Jones, $x$ Mrs. Blodgett, $x$ Mr. Uchiyama, Ex, Mrs. Neroian, MinUtes ReCORDED BY:__ Mr. Jones, __ Mrs. Blodgett, $X$ Mr. Uchiyama, _ Mo. Nordjan

1. IMMEDIATE ATTENTION-ACTIVITIES, CONTESTS, TRIPS, PROGRAM MANAGEMENT ITEMS. (FYI, needs, supervision, travel, due dates, funding)

$(\mathbf{R}=$ responsible)

ace Exhibitor mtg - Hunch

(b) MC E holp M check in and Booth setup

c. me stge k prep is tart now- shear, clipping

(d)

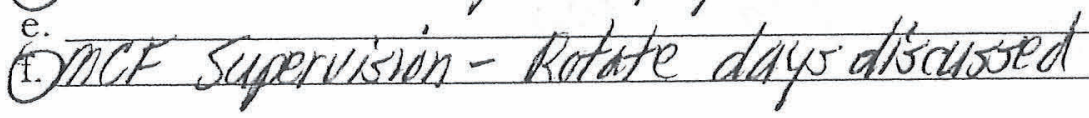

SO TU SB $M N$

$R$

$\frac{R}{R}=-$
$R=$
$R=$

2. MEETING SCHEDULE REVIEW---NOT PREVIOUSLY DISCUSSED. (FYI, agenda plan, supervision, funding, travel, needs) (admin. approval, teacher sub, other admin.)
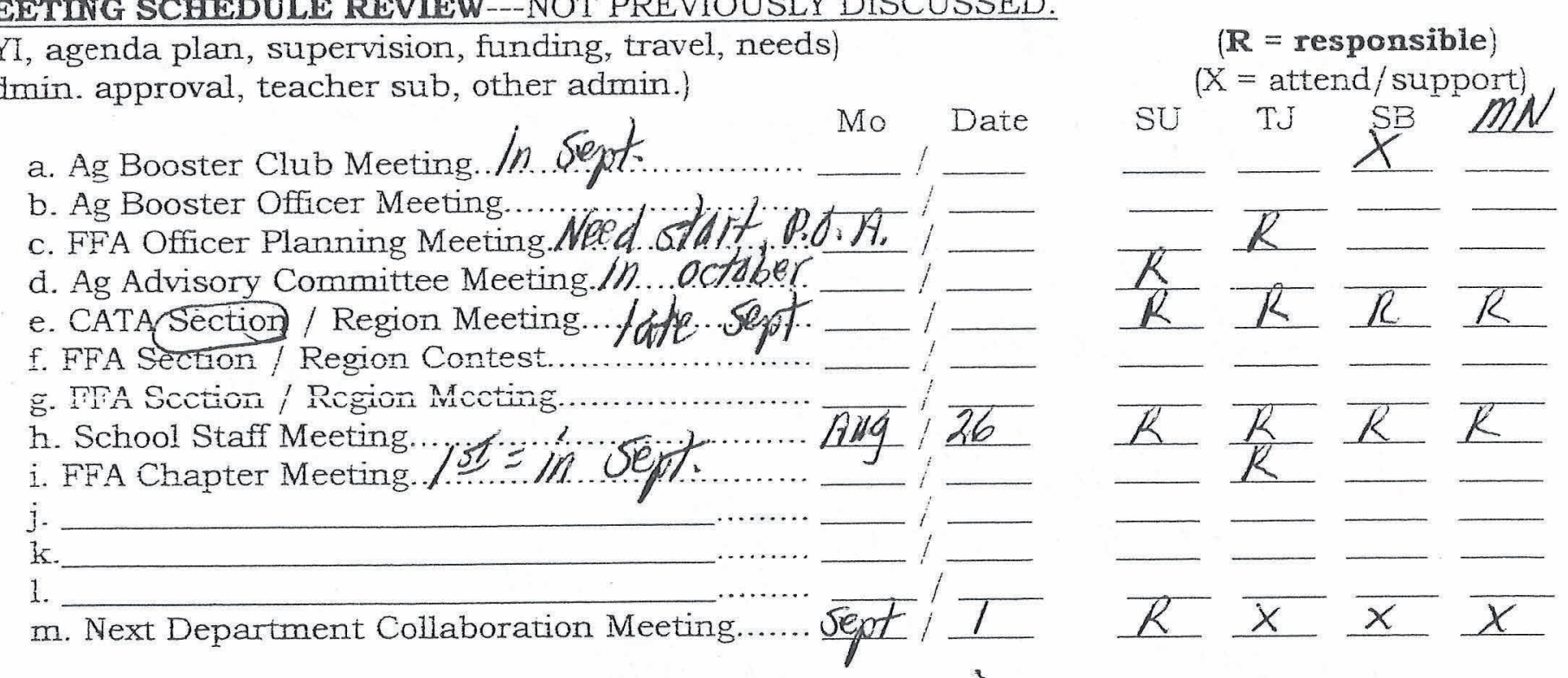

3. DISCUSSION ITEMS--FYI, NEEDS, PROBLEM SOLVING, AC̈TION PLAN, WHO DOES?.

- DEPT. OPERATIONS, budget/purchasing, facilities, equipment, repairs, maintenance, travel.

-- CLASS supply, student problems, maintenance, repairs, AV, textbooks, storage.

-- AG. BOOSTERS, AG. Advisory, Community, CATA, Professional Development.

-- FFA OPERATIONS, Officer team, activities/contests, finances, awards/recognition.

-- SAEP OPERATIONS, School Farm, Project Supervision, County Fairs.

mock sheep and swine, show practices needed.

mr. U work with Vicky on rabbit show practice DeF Draft buyer T.Y. letters due weds $8 / 25$ and show clearance. 8/25

Vicky-sccF lamb abbess on back -Vet remove $8 / 26$ mrs. Blodgett be there 
of c reams start? 8/18/10

(1) Dept. Mt ta Schedule - minute rotation meet on colas. days.

(2) mE J Sect L dr Pass at - pay to mr. U.

(3) officer/Lds Team -Class Nosing to stuff

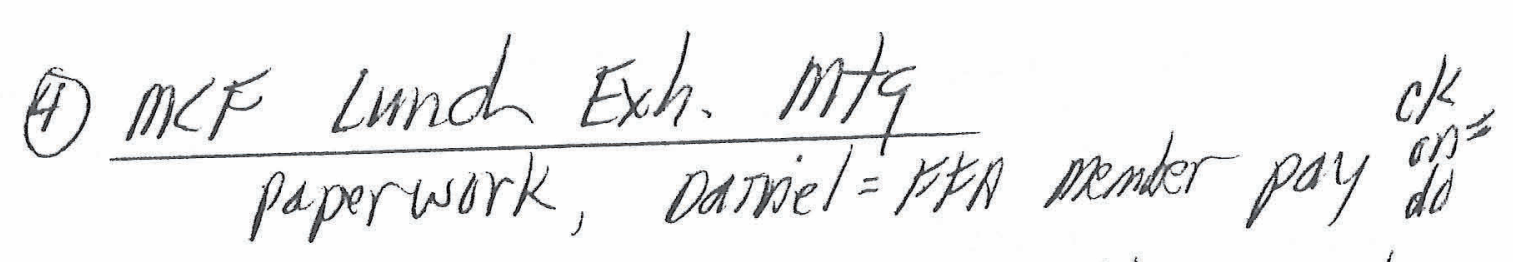

(5) she stack feeding, wt problems. Change feeds use fart fat, whole dits fo hold

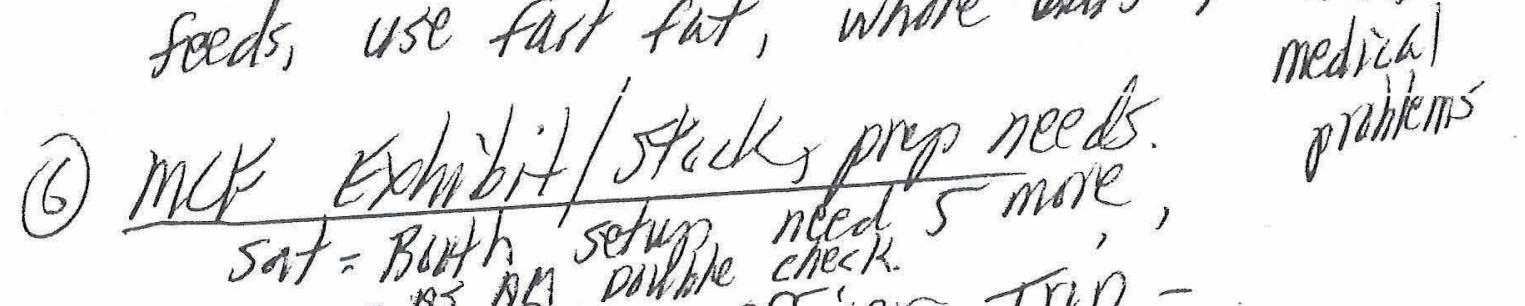

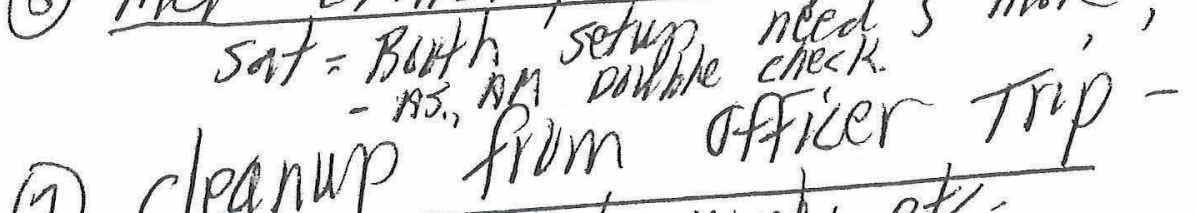
store meal supply, etc.

(8) Facility the memo- As hen

(9) Van Use schedute-Review sine approved

(10) Mr r- med hont-wirk help

(ii) Start prep data, for $R-2$ and 
2010-2015: SCHOOL FARM $1($ Old County Farm Site)

FIVE YEAR FACILITY IMPROVENENT and MAINTENANCE PLAN

TO BE REVIEWED EACH YEAR AND REVISED AS NECESSARY (rev. 10/10)

\section{ON-GOING MANTENANCE and IMPROVEMENT NEEDS.}

1. Weed control, trash removal, and road maintenance.

2. Repair/replace perimeter fencing, stock pen fencing, gates, and posts.

3. Barn roof, barn siding, stock pen roof, and rain gutter repairs.

4. Livestock pen doors, dog-proof fencing, and fence board repairs.

5. Water lines and outlets repair or replacement.

6. Fill dirt for stock pens, low ground areas, and entrance roadway.

7. Field spreading of livestock pen cleaning materials.

8. Purchase wire and wood livestock fencing materials, posts, gates, hardware, metal roof panels, etc.

9. Maintenance and repair of mowers and other power equipment.

10. Contract plant 14 acre perimeter field with oat hay or have County disc ground in fall and late spring.

11. Trim perimeter fence shrubs and trees.

12. District paid monthly maintenance service for portable rest room.

13. Maintenance of County installed water drainage systems along entrance roadway.

\section{TEAR SCHEDULE - SCHOOL FARM CONSTRUCTION, REPAIRS, and MAINTENANCE PLAN.}

DONE

2010-2011

\section{DESCRIPTION}

\section{Continue}

2. Install main water line shut-off valve between jail facility source and farm site.

3. Finish temporary east-west fencing along old north field fence line, with 3,12 ' access gates.

4. Replace water line to outer beef project barn.

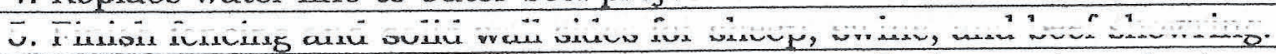

6. Finish perimeter security fencing and gate installation to sheep/swine pen area.

7. Install barn and outside security lighting system.

2011-2012

\begin{tabular}{|l|}
\hline \\
\hline \\
\hline \\
\hline \\
\hline \\
\hline \\
\hline
\end{tabular}

1. Replace beef pen roof support posts on west/jail side of barn.

2. Construct permanent east-west fence line to north field area, including 3, 12' access gates.

3. Replace lower level roof and sky light panels, and roof support posts for 3 beef pens on east side of main barn, install water lines and rain gutters.

4. Construct permanent north-south perimeter fence along west side of farm, next to roadway.

5. Start replacement/repair to perimeter fencing of north pasture area.

6. Finish permanent roof support structure and sheet metal covering to last 5 sheep/swine pens.

7. Renew farm lease with County. 5 year expiration, June, 2012.

2012-2013

\begin{tabular}{|l|l|}
\hline & 1. Construct rabbit/poultry production facility. \\
\hline 2. Construct 2 more isolation pens/area for sick livestock care.
\end{tabular}

3. Replace lower level roof and sky light panels for 3 beef pens on west/jail side of main barn, install water lines and rain gutters.

4. Build 6 covered $8^{\prime} \times 16^{\prime}$ sheep pens with water system.

5. Remodel swine farrowing barn and construct shade roof over connecting exercise pen.

6. Buy $8^{\prime} \times 40^{\prime}$ storage container to secure power equipment.

2013-2014

1. Construct/repair west side of outer beef project barn roof, roof support system, pen fencing and water line system

2. Construct permanent livestock loading pen and loading ramp next to wash rack area.

2014-2015

1. Construct/repair east side of outer beef project barn roof, roof support system, pen fencing, and water line system.

2. Construct beef wash area with fencing and cement pad. 
5 year school farm improve/maint. plan (page 2, 10/10)

\section{FUTURE CONSIDERATIONS}

1. Pour cement pads in back half of sheep and swine livestock pens.

2. Paint baras, fences, etc.

3. Install 1300 feet of ' ' permanent chain-link perimeter fencing.

4. Remove shrubs and old fence lines from pasture and outer areas.

5. Install main road entrance barrier/gate.

6. Construct road, farm ID, farm contributor/partnership sign.

7. Construct 4 holding pens in show ring.

8. Bleachers installed around show ring.

9. Develop vehicle parking area.

10. Repair 1.5 acre pasture perimeter fence, install cross fencing, and plant pasture grasses.

11. Plant assorted landscape trees, shrubs, and flowering plants around farm.

12. Make farm model and/or layout drawing.

13. Obtain loaner tractor with front-end loader, scraper, and disc/rototiller attachments.

PAST FARM IMPROVEMENTS AIN MAINTENANCE

(updated 10/10)

DONE

1996-1997

\begin{tabular}{|c|c|}
\hline $\mathrm{XX}$ & 1. Developed long-term farm-use agreement with County. \\
\hline $\mathrm{XX}$ & 2. Major scrap wood, trash, etc. cleanup and weeding of farm. \\
\hline XX & 3. Constructed practice show ring for sheep and swine. \\
\hline $\mathrm{XX}$ & 4. Repaired livestock wash rack. \\
\hline $\mathrm{XX}$ & 5. Repairs to perimeter fences. \\
\hline $\mathrm{XX}$ & 6. Constructed 10 sheep/swine, $8^{\prime \prime} \times 16^{n}$, pens with roofs. \\
\hline \multirow[t]{2}{*}{$\mathrm{XY}$} & 7 Pongired and evtended wrater line sustem to sheen and swine nens. \\
\hline & $1997-1998$ \\
\hline $\mathrm{XX}$ & 1. Developed long-term farm construction and improvement needs plan. \\
\hline $\mathrm{XX}$ & 2. Final school farm lease agreement with County approved---5 year lease, $\$ 1 /$ yr rent, take as is. \\
\hline $\mathrm{XX}$ & 3. Constructed/repaired 3 beef pens, $15^{\prime} \times 30^{\prime}$, on east side of main barn. \\
\hline $\mathrm{XX}$ & 4. Installed main water line to 3 new beef pens. \\
\hline $\mathrm{XX}$ & 5. Replaced crank and lift cable on portable beef scale. \\
\hline
\end{tabular}

\begin{tabular}{|c|c|}
\hline $\mathrm{XX}$ & 1. Major cleanup and weeding of farm. \\
\hline$X X$ & 2. Repairs to perimeter fences. \\
\hline $\mathrm{XX}$ & 3. Constructed 5 additional, $8^{\prime} \times 16^{\prime}$, sheep/swine pens with roofs. \\
\hline $\mathrm{XX}$ & 4. Extended water line system to 5 sheep/swine pens. \\
\hline $\mathrm{XX}$ & 5. Obtained fill dirt for stock pens and low ground areas. (County Road Dept.) \\
\hline $\mathrm{XX}$ & 6. Installed culvert drainage pipe at farm road entrance. (D. Wallace) \\
\hline XX & 7. First grading of road entrance. Lay 6 " gravel surface. (D. Wallace) \\
\hline $\mathrm{XX}$ & 8. Repaired/replaced lift weighing cables on pig/sheep scale. \\
\hline $\mathrm{XX}$ & 9. Constructed 2 sheep pens, $6^{\prime} \times 15^{\prime}$, on east side of barn. \\
\hline $\mathrm{XX}$ & 10. Constructed 5 small animal metal fenced wash rack pens. \\
\hline $\mathrm{XX}$ & 11. Dog proofing of 15 sheep/swine pens. \\
\hline & 1999-2001 \\
\hline $\mathrm{XX}$ & 1. Installed water lines and purchased 3 water tanks for beef pens on east side of main barn. \\
\hline $\mathrm{XX}$ & 2. School District installed portable rest room facility. Service contract paid by District. \\
\hline $\mathrm{XX}$ & 3. Removed 2 abandoned vehicles. (A \& S Metals) \\
\hline $\mathrm{XX}$ & 4. Second layer of surface material and grading of entrance road. (D. Wallace) \\
\hline $\mathrm{XX}$ & 5. Replaced main barn door tracking system and repairs to door. \\
\hline $\mathrm{XX}$ & 6. Dog proof and repair 3 beef pens, $15^{\prime} \times 30^{\prime}$, and 3 small animal pens of east side of barn. \\
\hline
\end{tabular}


5 year achool farm improve/maint. plan (page 3)

DONE

2001-2002

\begin{tabular}{|l|l|l|}
\hline XX & 1. Renewed 5 year farm lease with County---expires June 30, 2007.
\end{tabular} 2002-2003

\begin{tabular}{|l|l|l|l|l|}
\hline $\mathrm{XX}$ & 1 \\
\hline $\mathrm{XX}$ & 3 & \\
\hline
\end{tabular}

1. Repaired main entrance gate.

2. Gravel fill covering in front of barn door/parking area. (D. Wallace)

3. Perimeter fence line weed spraying. 2003-2004

\begin{tabular}{|l|l|}
\hline $\mathrm{XX}$ & 1. Major pruning of large shade tree. \\
\hline $\mathrm{XX}$
\end{tabular}

\begin{tabular}{l|l} 
XX & 2. Removed large wood and metal trash piles -8 each, 32 yard dumpster loads removed.
\end{tabular}

Service donated by Salinas BFI waste disposal service.

\begin{tabular}{l|l|l} 
XX & 3. Entrance road and front of barn surface fill and grading. (D. Wallace)
\end{tabular}

\begin{tabular}{l|l} 
XX & 4. Constructed shade support framework over wash rack pens.
\end{tabular} 2004-2005

\begin{tabular}{|l|l|}
\hline $\mathrm{XX}$ & 1. Repaired main barn roof panels and installed new ridge cap. (R. Wayman) \\
\hline $\mathrm{XX}$ & 2. Installed metal roofing over 12 sheep/swine pens. \\
\hline $\mathrm{XX}$ & 3. Increased size of show ring for sheep, swine and beef use. (J. Smith, A. Uribe)) \\
\hline $\mathrm{XX}$ & 4. Installed outer security fencing in front of 16 sheep/swine pens. (A. Uribe) \\
\hline
\end{tabular}
2005-2006

\begin{tabular}{|l|l|}
\hline $\mathrm{XX}$ & 1. Roof covering over 16 sheep and swine pens. \\
\hline $\mathrm{XX}$ & 2. Roof repair to outer beef project barn. \\
\hline $\mathrm{XX}$ & 3. Installed 2,12 foot access gates to small animal pen perimeter security fencing. (A. Uribe) \\
\hline
\end{tabular}
2006-2007

\begin{tabular}{|l|l|}
\hline$X X$ & 1. Installed rock border reinforcement along north side of County drainage pipe outlet. \\
\hline $\mathrm{Wy}$ & 2 . M \\
\hline $\mathrm{XX}$ & 3. County disk/mowing of 12 acre outer field area and County jail hillside. \\
\hline & 4. Started 5 year farm lease renewal process with County. \$5 lease fee paid to June, 2012. \\
\hline
\end{tabular}
2007-2008

\begin{tabular}{|l|l|}
\hline $\mathrm{XX}$ & 1. Roof repairs to 16 sheep/swine pens. \\
\hline $\mathrm{XX}$ & 2. Installed temporary stock fencing along west and north perimeter boundary of main farm area. \\
\hline $\mathrm{XX}$ & 3. Received sand-fill for sheep/swine pens. (Chapin Co.) \\
\hline $\mathrm{XX}$ & 4. Weed spraying of perimeter and inside fence lines. (A. Madolora) \\
\hline $\mathrm{XX}$ & 5. County disk/mowing of 12 acre outer field area and County jail hillside. \\
\hline $\mathrm{XX}$ & 6. Pumpkin planting and disking of pasture field area. (J. Ruiz) \\
\hline $\mathrm{XX}$ & 7. Verification of five year farm lease agreement renewal process with County. \\
\hline
\end{tabular}
2008-2009

\begin{tabular}{|l|l|}
\hline$X X$ & 1. Repairs to roof of outer beef barn. \\
\hline$X X$ & 2. Entrance road resurfacing (R. Mendez/Chapin Co.) \\
\hline$X X$ & 3. Rebuilding of roof structure for 5 swine/sheep pens. \\
\hline$X X$ & 4. Construction of 1 additional swine/sheep pen. \\
\hline$X X$ & 5. Installation of temporary livestock panel/T-post, west and south perimeter fences. \\
\hline$X X$ & 6. Construction of $2,6^{\prime} \times 16^{\prime}$ isolation pens for sick animals. \\
\hline$X X$ & 7. Underground water line repairs to 2 swine pens. \\
\hline
\end{tabular}
2009-2010

\begin{tabular}{|l|l|}
\hline $\mathrm{XX}$ & 1. Build roof structure and shade covering over stock wash rack pens. \\
\hline & \\
\hline & $2010-2011$ \\
\hline & \\
\hline & \\
\hline & \\
\hline
\end{tabular}




\title{
2010-2015: FIVE YEAR FACLITY IMPROVEMIENT ANd MANTTENANCE PLAN DEPARTMENT, CLASSROOM, SHOP, OUTDOOR ANTMAL LAB, GREENHOUSE, BMALL ANMMA BUILDING, and OUTDOOR STORAGE BUILDING
}

\author{
TO BE REVIEWED EACH YEAR AND REVISED AS NECESSARY (rev. 10/10)
}

\section{YUAR SCHEDULE - FACHITY OMPROVEMENT AND MANTTENANCE PLAN.}

\section{DONE 2010-2011}

DESCRIPTION

\begin{tabular}{|l|l|}
\hline & $\begin{array}{l}\text { 1. Small animal building: Install } 5 \text { security window screens, improve building ventilation. } \\
\text { 2. Small animal building: Install } 75 \text { foot fenced entry walkway from Kip Drive to building's } \\
\text { front entrance, including security lights. (student access during weekends, holidays, etc.) }\end{array}$ \\
\hline & $\begin{array}{l}\text { 3. Repair Ag. Mechanics shop dust collection system. } \\
\text { ag. building, classroom hallways, and outside buildings. }\end{array}$ \\
\hline $\begin{array}{l}\text { 5. Install decorative plants along Kip drive fence line. } \\
\text { 6. Replace Rm. 712 chalkboard with whiteboard surface. }\end{array}$ \\
\hline 7. Install base board system around livestock housing/show ring. \\
8. Greenhouse 2: Clean out floristry decorative supply.
\end{tabular}

8. Greenhouse 2: Clean out floristry decorative supply.

\section{1-2012}

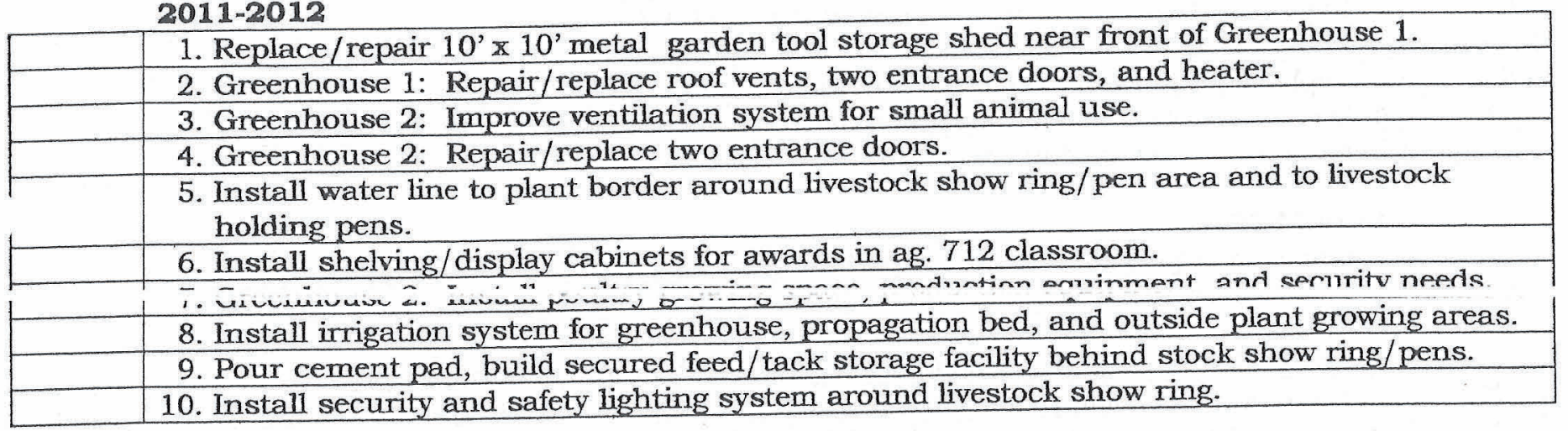

2012-2013

\begin{tabular}{|l|l|}
\hline & 1. Construct new steel storage rack and scrap metal storage bins in welding shop. \\
\hline 2. Rebuild welding shop supply store room cabinets, supply, and food dry goods storage shelves \\
\hline 3. Repaint shop power equipment \\
\hline 4. Repair/replacement of shop locker handles and doors. \\
\hline 5. Replace $10^{\prime} \times 10^{\prime}$ metal garden tool storage shed behind small animal building. \\
\hline
\end{tabular}

2013-2014

\begin{tabular}{|l|l|}
\hline & 1. Construct lath house and plant repotting work area. \\
\hline 2. Install wider door to outdoor storage building. \\
\hline 3. Rebuild oxy-acetylene welding work stations in welding shop. \\
\hline
\end{tabular}

2014-2015

\begin{tabular}{|l|l|}
\hline & 1. Rebuild arc welding work stations in welding shop. \\
\hline 2. Repaint welding and ag. mechanics classroom, shop, and teacher offices. \\
\hline
\end{tabular}


5 year facility improve/maint. plan (page 2, 10/10)

TUTURE CONSTOERATIONS

Annual neview of use/needs for outdoor lab facilities - growing aneas, water lines, lighting, landscaping, walkways, animal waste disposal, equipment storage, soil, bins, potting
area, security, etc.

2. Develop use/needs layout plan for ag. science lab addition to room 712 classroom.

3. Annual neview of use/needs for tack, feed supply, equipment storage, and security for livestock show ring/holding pen facility.

4. Install bleachers for out-door livestock show ring.

5. Build storage building for ag. truck, fair equipment, and stock trailer.

6. Install security fencing for out-door crop growing land lab.

7. Build plant re-potting area, soil storage bins, lath house plant growing facility.

8. Review layout plan for Ag. Mechanics Shop work stations, equipment placement, and storage
room use.

\section{PAST FACILITY IMPROVEMENT AND MATITENANCE WORK COMPLETED} (updated 10/10)

DONE

\begin{tabular}{|l|l|}
\hline $\mathrm{XX}$ & \\
\hline $\mathrm{XX}$ & \\
\hline $\mathrm{XX}$ & 3 \\
\hline $\mathrm{XX}$ & 4 \\
\hline $\mathrm{XX}$ & 5 \\
\hline
\end{tabular}

1996-1997

DESCRIPTION

1. Repaired fiberglass panels to Greenhouses 1 and 2.

2. Replaced roof on Greenhouse 2.

3. Replaced/repaired shop locker handles.

4. Installed second movie screen and more electrical outlets in classroom.

5. Installed heater unit in Greenhouse 1.

1998-1999

\begin{tabular}{|l|l|}
\hline $\mathrm{XX}$ & 1. Removed large pine tree in rear parking area. \\
\hline $\mathrm{YY}$ & 2 Doming \\
\hline
\end{tabular}

1999-2001 (includes school-wide reconstruction improvements)

\begin{tabular}{|l|l|}
\hline $\mathrm{XX}$ & 1. Increased electrical power breaker outlets to shop and classroom. \\
\hline $\mathrm{XX}$ & 2. Installed new classroom and shop heating/cooling system. \\
\hline $\mathrm{XX}$ & 3. Repainting of classroom, shop, and office rooms. \\
\hline $\mathrm{XX}$ & 4. Replaced key/lock and safety handles to all doors-one key fits all. \\
\hline $\mathrm{XX}$ & 5. Installed and connect independent ag. phoneline with District-wide phone system. \\
\hline $\mathrm{XX}$ & 6. Installed fire alarm and fire sprinkler systems in classroom and shop. \\
\hline $\mathrm{XX}$ & 7. Asphalt paving of outdoor surfaces not used for outdoor lab instruction. \\
\hline $\mathrm{XX}$ & 8. Removed old lath house structure next to Greenhouse 1. \\
\hline $\mathrm{XX}$ & $\begin{array}{c}\text { 9. Constructed } 45 \mathrm{x} 5 \text { foot livestock show ring with } 3 \text { holding pens and } 6 \text { foot high chain } \\
\text { link fence around show ring. }\end{array}$ \\
\hline $\mathrm{XX}$ & 10. Constructed $16 \mathrm{x} 24$ foot storage building for outdoor lab supply and equipment storage. \\
\hline $\mathrm{XX}$ & 11. Install drive-thru gate on Kip Drive side of ag. facility. \\
\hline
\end{tabular}

2001-2002

\begin{tabular}{|l|l|}
\hline $\mathrm{XX}$ & 1. Had made (Ag. Boosters) outdoor livestock facility sponsor sign. \\
\hline $\mathrm{XX}$ & 2.
\end{tabular}

2. Installed classroom and ag. office service lines for computer internet and E-Mail service.

2002-2003

\begin{tabular}{|l|l|}
\hline $\mathrm{XX}$ & 1. Replaced/repaired Greenhouse 1 growing benches and mist bed. \\
\hline $\mathrm{XX}$ & 2. Painted outdoor storage building. \\
\hline $\mathrm{XX}$ & 3. Developed $\mathrm{Ag} /$ school vehicle designated parking plan behind ag. shop. \\
\hline $\mathrm{XX}$ & 4. Installed outside water and gas valve outlet damage protection barriers. \\
\hline
\end{tabular} 
5 year facility improve/maint. plan (page 3)

DONE

\section{DESCRIPTION}

2003-2004

\begin{tabular}{|l|l|}
\hline$X X$ & 1. Repainted shop safety zone lines. \\
\hline
\end{tabular}

$\begin{array}{ll}\mathrm{XX} & \text { 2. Installed } 10 \text { inch radial arm saw. }\end{array}$

XX 3. Replaced shop wash basin, welding shop.

\begin{tabular}{l|l}
$\mathrm{XX}$ & 4. Installed 4 ceiling electrical drop outlet cords in shop.
\end{tabular}

2005-2006

\begin{tabular}{|l|l|l|l}
\hline$X X$ & 1. Built and installed $\mathrm{Ag} / \mathrm{FFA}$ display case in 700 hallway. (C. Riterbush) \\
\hline $\mathrm{XX}$
\end{tabular}

\begin{tabular}{l|l}
$\mathrm{XX}$ & 2. Installed canvas roof framing over livestock show ring/holding pns
\end{tabular}

2006-2007

\begin{tabular}{|l|l}
$\mathrm{XX}$ & 1. Installed canvas roofing over livestock show ring/pen area.
\end{tabular}

\begin{tabular}{l|l}
$X X$ & 2. Installed 6' wide classroom AV screen.
\end{tabular}

XX $\quad$ 3. Added Floristry Classroom building, surrounding growing grounds, and Greenhouse 2 to Department teaching facilities (convert to small animal production facility).

2007-2008

\begin{tabular}{|l|l|}
\hline $\mathrm{XX}$ & 1. Greenhouse 1: Replaced growing/propagation table. (S. Pfarr) \\
\hline $\mathrm{XX}$ & 2. Added Ag. Mechanics/Wood Shop, Rm. 730, to Department teaching facilities. \\
\hline $\mathrm{XX}$ & 2. Landscaping of front side of livestock show ring. (H. Jimenez) \\
\hline $\mathrm{XX}$ & 3. Greenhouse 1: Replaced plastic siding and roof. \\
\hline $\mathrm{XX}$ & 4. Greenhouse 1: Replaced electrical power panel and increased power breaker switches. \\
\hline $\mathrm{XX}$ & 5. Ag. Mechanics shop paint room exhaust/ventilation system repaired. \\
\hline
\end{tabular}

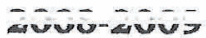

\begin{tabular}{|l|l|}
\hline $\mathrm{XX}$ & 1. Added Ag. Physical Science classroom, $\mathrm{Rm} .205$, to Department teaching facilities. \\
\hline $\mathrm{XX}$ & 2. Re-wired Ag. Mechanics Shop \\
\hline $\mathrm{XX}$ & 3. Install main water shut-off valve system to greenhouses and plant growing areas. \\
\hline $\mathrm{XX}$ & 4. Three vehicle parking spaces identified for Ag. Dept. use. \\
\hline
\end{tabular}

2009-2010

\begin{tabular}{|l|l|}
\hline $\mathrm{XX}$ & 1. Greenhouse 2: Clean out/remove floristry coolers and storage supplies. \\
\hline $\mathrm{XX}$ & 2. Small animal building: Build/install 40 rabbit cage system, production and show equip. \\
\hline $\mathrm{XX}$ & 3. Small animal building: Remove old floristry cooler. \\
\hline $\mathrm{XX}$ & 4. Cleanup area next to Greenhouse 1 and small animal building for plant growing use. \\
\hline $\mathrm{XX}$ & 5. Repaint ag. storage and small animal production buildings. \\
\hline $\mathrm{XX}$ & 6. Copper green treatment to mist bed. \\
\hline & \\
\hline & \\
\hline & \\
\hline
\end{tabular}




\section{0-2015: FIVE YEAR NEW EQUIPMENT PURCHASE, REPAIR, and REPLACEMENT PLAN DEPARTMENT, CLASSROOM, SHOP, OUTDOOR ANIMAL LAB, GREENHOUSE, and} SMALL ANIMAL BUILDING TO BE REVIEWED EACH YEAR AND REVISED AS NECESSARY (rev. 10/10)

\section{ON-GOING EQUIPMENT REPATRS, REPLACEMENT, and MANTENANCE NEEDS.}

1. Large shop power tool adjustments, wheel/blade replacement, lubrication, safety guard maintenance-table saw, radial arm saw, band saw, power hacksaw, bench grinders, drill press, sanders, buffer, air compressor, metal forge machines, planers, lathes, jointer, scroll/jig saws.

2. Sharpen/adjust, lubricate hydraulic metal shear blades, sheet metal shear blades, and sheet metal brake/bender.

3. Repair or replace welding torch bodies, regulators, hoses, cables and clamps, oxy-acetylene welding tips, cutting torch, welding leathers and gloves, welding masks.

4. Adjust, repair, clean MIG, plasma cutter, TIG, arc welding machines, and portable arc welder.

5. Repair or replace small metal and wood hand tools.

6. Power hand tool safety inspections, cord/plug replacements-drills, grinders, saws, sanders, routers, and jig saws.

7. Garden tool repair, replacement, sharpening---rakes, hoes, shovels, trowels, clippers, etc.

8. Large horticulture equipment adjustments, repairs, sharpening, lubrication---mowers, leaf blower, line trimmer, sprayer, shredder, cement mixer, rototiller, hedge trimmer, gas post hole digger.

9. Livestock grooming and cleaning equipment adjustments, lubrication, repairs, sharpening, replacement---small/large animal clippers and blades, fans, blowers, generator, trimming chutes, blocking tables, hoof grinder, wheel barrows.

10. Stock trailers, utility trailers, livestock scales, beef hoof trimming table, loading ramps, --repairs, adjustment, repainting, lubrication.

11. AV equipment cleaning, repair, bulb replacement, cord/plug replacement---TV, VCR, overhead projector, slide/filmstrip projectors, video camera, LCD projector, AV screens, llluminator projector, digital camera, portable PA system.

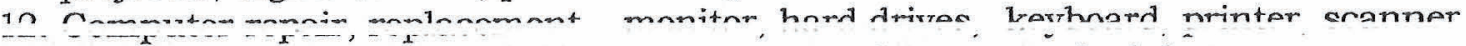

13. Agriculture truck service, maintenance, tire replacement schedule.

14. Photo copy machine maintenance and service contract.

5 YEAR SCHEDULE -- NEW EOUIPMENT PURCHASES, REPLACEMENT, And REPAIRS PLAN.

DONE

2010-2011

1. Purchase 1 teacher office computer and printer, Rm. 710 .

2. Construct livestock separation panels for ag. truck racks.

3. Service and Repair MIG welder \#1 and \#2.

4. Rebuild ag. truck bin for hauling welding scrap metal.

5. Rebuild 8 foot Department barbecue trailer.

6. Remove old welders and large power tools to District surplus equipment sales yard.

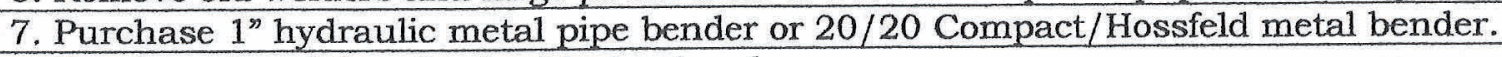

8. Purchase Panel Saw for Ag. Mechanics shop

\section{1-2012}

1. Purchase 3 compound and 3 dissecting microscopes.

2. Purchase cabinets to store 36 compound and 36 dissecting microscopes.

3. Purchase photo developer/printer for digital camera.

4. Purchase $2^{\text {nd }}$ TIG welder.

5. Purchase computerized metal cutting (Plasma Cam) machine.

6. Repair beef hoof trimming/tilt table.

7. Construct single hog transporting crate.

8. Repair portable air compressor unit.

9. Repair Troy-Built rototiller.

10. Purchase 2 classroom TV and VCR/DVD player, Rm. 712 and 730) 
5 year equip. plan (page $2,10 / 10$ )

DONE

2012-2013

1. Purchase 3 compound and 3 dissecting microscopes.

2. Replace floor of $16^{\prime}$ Hale stock trailer and convert into flatbed utility trailer.

3 . Replace 4 wood table vises in welding shop.

5. Repair 25 gallon herbicide sprayer and replace spray gun.

6. Construct livestock loading ramp for livestock trailer.

7. Replace floor and paint $3000 \mathrm{lb}$. livestock scale.

\section{3-2014}

1. Purchase $5^{\text {th }}$ MIG welder.

2. Purchase sheet metal roller for welding shop.

3. Construct sheep/swine squeeze chute.

\section{4-2015}

1. Purchase 2-horse trailer.

2. Purchase large, portable projector screen.

3. Purchase Wood Lathe

1. Purchase $18 \mathrm{hp}$ garden tractor.

\section{FUTURE CONSIDERATIONS}

PAST EQUIPMENT PURCHASES and REPATRS

(updated 10/10)

DONE

1987-1995

DESCRIPTION

\begin{tabular}{|l|l|l|}
$X X$ & 1. Purchased 4 customer owned Uxygen, $\# 2 D 1$, and 4 acerylene, $\# 4$ cymnaers. (Amerngas wela) \\
\hline
\end{tabular}

$\underline{\underline{X X}}$

$\underline{X X}$

2. Purchased 1 customer owned CO2, $50 \mathrm{lb}$, cylinder. (Amerigas Welding)

$\underline{X X}$

3. Purchased Sharp VL-L624 video camcorder, tripod, and accessories.

\begin{tabular}{l|l}
$\mathrm{XX}$ & 5. Purchased Panosonic VCR with remote. \\
\hline
\end{tabular} 1996-1997

\begin{tabular}{|l|l|}
\hline $\mathrm{XX}$ & 1. Repaired $8 \mathrm{Hp}$ Troy-Built rototiller. \\
\hline
\end{tabular}

XX 2. Purchased Contractor, $12^{n}$, portable miter power saw.

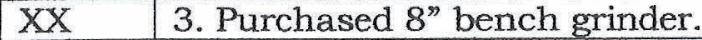

XX 4. Repaired 4 oxy. welding torch body tip couplings.

\begin{tabular}{l|l}
$\mathrm{XX}$ & 5. Built $3^{\text {rd }}$ steer trimming chute.
\end{tabular}

\begin{tabular}{l|l} 
XX & 6. Replaced ag. truck tool box. \\
\hline
\end{tabular}

XX 7 7. Repaired 4 oxy. welding regulators.

\begin{tabular}{|l|l|}
\hline$X X$ & 8. Purchased Hypotherm Max 20 Plasma Arc cutting system.
\end{tabular} 1997-1998

\begin{tabular}{|l|l|}
\hline $\mathrm{XX}$ & 1. Sharpened/adjusted metal shear blades. \\
\hline $\mathrm{XX}$ & 2. Repaired 6 oxy. welding torch body tip couplings. \\
\hline $\mathrm{XX}$ & 3. Purchased second computer and scanner. \\
\hline $\mathrm{XX}$ & 4. Repaired MIG welder $\# 1$. \\
\hline $\mathrm{XX}$ & 5. Cleaned/maintenance work on overhead projector. \\
\hline $\mathrm{XX}$ & 6. Repaired and cleaned 4 electric arc welders. \\
\hline $\mathrm{XX}$ & 7. Repaired 2 oxygen, 2 acetylene welding, and 1 CO2 regulator. \\
\hline $\mathrm{XX}$ & 8. Replaced oxy. welding hoses on 8 man welding unit. \\
\hline $\mathrm{XX}$ & 9. Purchased Stihl, FS-76, gas powered weed/brush trimmer. \\
\hline
\end{tabular}


5 year equip. plan (page 3)

DONE

1998-1999

\section{DESCRIPTION}

\begin{tabular}{|l|l|}
\hline $\mathrm{XX}$ & 1. Sharpened/adjusted metal shear blades. \\
\hline $\mathrm{XX}$ & 2. Repaired 4 oxy. welding torch body tip couplings. \\
\hline $\mathrm{XX}$ & 3. Cleaned/maintenance work on overhead projector. \\
\hline $\mathrm{XX}$ & 4. Repaired and cleaned 5 electric arc welders. \\
\hline $\mathrm{XX}$ & 5. Repaired 2 oxygen and 2 acetylene welding regulators. \\
\hline $\mathrm{XX}$ & 6. Repaired 2 Dukane filmstrip projectors. \\
\hline $\mathrm{XX}$ & 7. Purchased 8 garden hoes. \\
\hline $\mathrm{XX}$ & 8. Purchased HP Deskjet $895, \mathrm{CX} 1$, computer printer and speakers. \\
\hline $\mathrm{XX}$ & 9. Repaired Sharp video camera. \\
\hline $\mathrm{XX}$ & 10. Purchased Millermatic Vintage, $200 / 230, \mathrm{MIG}$ welder \# 1 with $\mathrm{CO}, 50 \mathrm{lb}$, cylinder. \\
\hline $\mathrm{XX}$ & 11. Purchased 1 small animal grooming clipper. \\
\hline
\end{tabular}
1999-2001

\begin{tabular}{|c|c|}
\hline $\mathrm{XX}$ & 1. Acquired 5 shop table vises from District surplus. \\
\hline $\mathrm{XX}$ & 2. Purchased 200 pound hanging sheep scale. \\
\hline $\mathrm{XX}$ & 3. Purchased beef clipper head for Sunbeam shears. \\
\hline $\mathrm{XX}$ & 4. Repaired plasma arc cutting unit and replaced filters. \\
\hline $\mathrm{XX}$ & 5. Purchased 2 Lister Stablemate sheep shears and 3 blade set for each. \\
\hline $\mathrm{XX}$ & 6. Replaced Delta, $10^{\prime \prime}$ radial arm saw. \\
\hline $\mathrm{XX}$ & 7. Purchased Miller, Mil 903367, Econo-TIG unit and argon cylinder. \\
\hline $\mathrm{XX}$ & 8. Purchased portable Milwaukee Sawzall Reciprocating saw. \\
\hline $\mathrm{XX}$ & 9. Purchased $5 \mathrm{Hp}$ Troy-Built push-type weed line trimmer. \\
\hline $\mathrm{XX}$ & 10. Purchased $6 \mathrm{Hp}$, Yazoo, 22 inch self-drive field mower. \\
\hline vo & 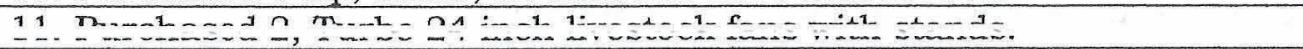 \\
\hline $\mathrm{XX}$ & 12. Purchased heavy duty 3 hole paper punch and paper cutter. \\
\hline $\mathrm{XX}$ & 13. Acquired sheet metal shear and pan/break from metal shop classroom. \\
\hline $\mathrm{XX}$ & 14. Acquired 2 four drawer file cabinets from school surplus. \\
\hline $\mathrm{XX}$ & 15. Received 36 new classroom desks from school. \\
\hline $\mathrm{XX}$ & 16. Purchased $3^{\text {rd }}$, Air Express II, livestock fitting blower. \\
\hline $\mathrm{XX}$ & 17. Acquired 7 inch bench grinder from metal shop classroom. \\
\hline $\mathrm{XX}$ & Constructed $2 \times 3$ foot portable barbecue pit for Department use. \\
\hline
\end{tabular}
2001-2002

\begin{tabular}{|l|l|}
\hline $\mathrm{XX}$ & 1. Purchased metal shear blade set. \\
\hline $\mathrm{XX}$ & 2. Purchased 1 small animal grooming clipper. \\
\hline $\mathrm{XX}$ & 3. Purchased 12 garden rakes and 4 shovels. \\
\hline $\mathrm{XX}$ & 4. Purchased Ford F-250, 3/4 ton, crew cab ag. truck, \#81. \\
\hline $\mathrm{XX}$ & 5. Purchased 7 compound lab. Microscopes. \\
\hline $\mathrm{XX}$ & 6. Purchased 6 dissecting microscopes. \\
\hline $\mathrm{XX}$ & 7.Purchased chicken hatching unit. \\
\hline $\mathrm{XX}$ & 8. Purchased North Star, $6-1 / 2 \mathrm{Hp}, 2500$ 1b. pressure washer. \\
\hline $\mathrm{XX}$ & 9. Purchased Stihl, \#025, 18 inch gas chain saw. \\
\hline $\mathrm{XX}$ & 10. Acquired 6 shop table vises from school surplus. \\
\hline $\mathrm{XX}$ & 11. Received 1 classroom, 1 teacher computer and printers from school. \\
\hline & 2002-2003 \\
\hline $\mathrm{XX}$ & 1. Purchased 7 compound lab microscopes and 6 dissecting microscopes. \\
\hline $\mathrm{XX}$ & 2. Repaired MIG welder \#1 wire feed unit. \\
\hline $\mathrm{XX}$ & 3. Sharpened sheet metal shear blades. \\
\hline $\mathrm{XX}$ & 4. Replaced hydraulic metal shear blades. \\
\hline $\mathrm{XX}$ & 5. Purchased used Sharp, sF 2025, photo copy machine. \\
\hline $\mathrm{XX}$ & 6. Acquired sheet metal roller unit from school surplus. \\
\hline
\end{tabular}


5 year equip. plan (page 4)

DONE

2003-2004

\section{DESCRIPTION}

\begin{tabular}{|l|l|}
\hline $\mathrm{XX}$ & 1, Purchased 6 compound microscopes and 8 dissecting microscopes. \\
\hline $\mathrm{XX}$ & 2. Purchased DR, 13 Hp self-driven brush mower for school farm. \\
\hline $\mathrm{XX}$ & 3. Purchased Honda, EM5000, Sxk, 5000 watt electricity generator. \\
\hline $\mathrm{XX}$ & 4. Purchased Sony Mavica, CD350, digital camera. \\
\hline $\mathrm{XX}$ & 5. Purchased Samsung mini DVD, SCD 107, video camcorder. \\
\hline $\mathrm{XX}$ & 6. Purchased Sharp, PG, C45x, LCD projector, speakers, and accessories. \\
\hline $\mathrm{XX}$ & 7. Purchased Govideo DVD recorder, VCR combo. \\
\hline $\mathrm{XX}$ & 8. Received donation of Hayes, 1974,2 horse trailer. \\
\hline $\mathrm{XX}$ & 9. Received 1 Sullivan beef trimming chute and $300+1 \mathrm{~b}$. swine/sheep scale donation. \\
\hline $\mathrm{XX}$ & 10. Purchased used swine farrowing crate/unit. \\
\hline
\end{tabular}
2004-2005

\begin{tabular}{|l|l|}
\hline $\mathrm{XX}$ & 1. Purchased Husqvarna, 21 inch lawn mower for school farm. \\
\hline $\mathrm{XX}$ & 2. Purchased DeWalt $3 / 8$ inch cordless drill. \\
\hline $\mathrm{XX}$ & 3. Purchased DeWalt $1 / 2$ inch electric drill. \\
\hline $\mathrm{XX}$ & 4. Repaired 2 steer trimming chutes. \\
\hline $\mathrm{XX}$ & 5. Built 2 $2^{\text {nd }}, 2^{\prime} \times 3^{\prime}$ portable Department barbecue pit. \\
\hline
\end{tabular}
2005-2006

\begin{tabular}{|c|c|}
\hline $\mathrm{XX}$ & rting poly \\
\hline $\mathrm{XX}$ & 2. Purchased two power groom Andis cordless clippers with extra battery and battery charger. \\
\hline $\mathrm{XX}$ & 3. Purchased 6 gallon Craftsman shop vacuum. \\
\hline $\mathrm{XX}$ & 4. Purchased Apollo 3400 open-head overhead classroom projector. \\
\hline $\mathrm{XX}$ & 5. Purchased 6 aluminum $33^{n} \times 48^{n}$ expandable trailer pen dividers with single lift gate. \\
\hline XX & 6. Purchased Echo EA55 gas post hole digger with $10^{\prime \prime}$ auger. \\
\hline $\mathrm{XX}$ & 7. Purchased Singaiwa 4 gallon back pack sprayer. \\
\hline $\mathrm{XX}$ & 8. Purchased pair of $6^{\prime}, 8^{\prime \prime}$ aluminum truck loading ramps. \\
\hline $\mathrm{XX}$ & 9. Purchased 3 compound micrascopes and 3 stereo (dissecting) microscopes. \\
\hline $\mathrm{XX}$ & 10. Purchased 2 clothes changing tents for County Fair use. (Cabela Shower Shelters) \\
\hline
\end{tabular}
2006-2007

\begin{tabular}{|c|c|}
\hline $\mathrm{XX}$ & 1. Purchased 1 cordless hog grooming clipper with extra battery and charger. \\
\hline $\mathrm{XX}$ & 2. Purchased 2, Sullivan, 8' long beef stall dividers for County Fair exhibits. \\
\hline $\mathrm{XX}$ & 3. Purchased lluma-vision overhead projector. \\
\hline $\mathrm{XX}$ & 4. Ag. Booster purchase of Parke, $2002,8^{\prime} \times 16^{\prime}$ equipment/utility trailer for SAE/Dept. use. \\
\hline $\mathrm{XX}$ & 5. Ag. Booster purchase of $10^{\prime} \times 10^{\prime}$ portable shade canopy for activity use. \\
\hline $\mathrm{XX}$ & 6. School purchase of $6^{\prime}$ wide classroom AV screen. \\
\hline $\mathrm{XX}$ & 7. Received TV, VCR, and cart. From school surplus. \\
\hline $\mathrm{XX}$ & 8. Purchased Miller Syncrowave 200 Runner TIG welder with 1 argon gas cylinder. \\
\hline $\mathrm{XX}$ & 9. Purchased Millermatic MIG welder, \#2, and 1 argon/CO2 cylinder. \\
\hline \multicolumn{2}{|r|}{$2007-2008$} \\
\hline $\mathrm{XX}$ & 1. Purchased 1 Hitachi CP-X300 LCD projector (District buy) \\
\hline $\mathrm{XX}$ & 2. Purchased $2,30^{n} \times 36^{n}$ poly hog sorting panels. \\
\hline $\mathrm{XX}$ & 3. Purchased $6,18^{\prime \prime}$ small livestock feeders. \\
\hline $\mathrm{XX}$ & 4. Purchased 1,96 " double hinged poly hog sorting panel. \\
\hline $\mathrm{XX}$ & 5. Purchased Dewalt, $12^{n}$ slide compound mitre saw, DW718. (ag. mech. Shop) \\
\hline $\mathrm{XX}$ & 6. Purchased Delta 17 laser drill press, 17-959L. (ag. mech. Shop) \\
\hline $\mathrm{XX}$ & 7. Purchased Powermatic, 8 Jointer, 1610077K. (ag. mech. Shop) \\
\hline $\mathrm{XX}$ & 8. Purchased Delta, 18" bandsaw, \#28-682. (ag. mech shop) \\
\hline $\mathrm{XX}$ & 9. Purchased Chicken Brooder, 3E 610 with Gro-off extension and wheeled base. \\
\hline $\mathrm{XX}$ & 10. Purchased 3 layer poultry growing cage unit, $\# 5$ PLC. \\
\hline $\mathrm{XX}$ & $\begin{array}{l}\text { 11. Purchased } 18,30^{n} \times 24^{n} \times 18^{n} \text { stacking rabbit cages with clean-out trays and wheels. } \\
\text { Assorted cage building tools and supplies purchased. }\end{array}$ \\
\hline
\end{tabular}


5 year equip. plan (page 5, 10/10)

\section{DONE}

\section{DESCRIPTION}

2007-2008

\begin{tabular}{|l|l|}
\hline $\mathrm{XX}$ & 12. Purchased $16,24^{\prime \prime} \times 24^{n} \times 14^{\prime \prime}$ stacking rabbit cages with clean-out trays and wheels. \\
\hline $\mathrm{XX}$ & 13. Purchased $18,36^{\prime \prime} \times 30^{n} \times 18^{n}$, stacking rabbit cages with clean-out rays and wheels. \\
\hline $\mathrm{XX}$ & 14. Purchased 36 assorted water bottles, feed bowls, hay racks for each cage. \\
\hline $\mathrm{XX}$ & 15. Purchased 2 rabbit scales (hanging and spring dial). \\
\hline $\mathrm{XX}$ & 16. Purchased 9 assorted rabbit nest box sizes. \\
\hline $\mathrm{XX}$ & 17. Purchased 2 Stone rabbit tattoo kits with extra letters and numbers. \\
\hline $\mathrm{XX}$ & 18. Purchased 1, 8 cage rabbit show table. \\
\hline $\mathrm{XX}$ & 19. Purchased 9 assorted size rabbit carrying/transport cages. \\
\hline
\end{tabular}
2008-2009

\begin{tabular}{|l|l|}
\hline $\mathrm{XX}$ & 1. Replaced 5 tires and rims for old Hale, 16' livestock trailer. (M. Johnson, 101 Trailers)) \\
\hline $\mathrm{XX}$ & 2. Purchased 2 Millermatic, 212, MIG welders, \#3 and \#4, with 2 argon/CO2 cylinders, 220. \\
\hline $\mathrm{XX}$ & 3. Purchased 1 Califone, PA10a, portable PA system. \\
\hline $\mathrm{XX}$ & 4. Purchased 1 Andis cordless hog grooming clipper with extra battery and charger. \\
\hline $\mathrm{XX}$ & 5. Purchased 1 Lister Stablemate sheep clippers with extra blades. \\
\hline $\mathrm{XX}$ & 6. Purchased 10 Jorgensen Wood Vises. \\
\hline $\mathrm{XX}$ & 7. Purchased Sharp AR-M277 Reconditioned Photo Copy Machine. \\
\hline $\mathrm{XX}$ & 8. Ag. Booster purchase of Digital Camera. \\
\hline $\mathrm{XX}$ & 9. Purchased PC-3 Nailer compressor combo kit (light wood project use). \\
\hline $\mathrm{XX}$ & 10. Purchased 2 Rigid 7-1/4 inch circular saws. \\
\hline $\mathrm{XX}$ & 11. Purchased 2 Rigid 3/8 inch corded drills. \\
\hline $\mathrm{XX}$ & 12. Donation (SV Ford-Skillett) of 3 Ink jet printers. \\
\hline $\mathrm{XX}$ & 13. Set-up 3 computer/printer system for FFA use (Tech. Core classroom). \\
\hline $\mathrm{XX}$ & 2009-2010 \\
\hline $\mathrm{XX}$ & 1. Purchase Jet Pro-14 inch Band Saw. \\
\hline $\mathrm{XX}$ & 3. Purchase 1 LCD projector. \\
\hline $\mathrm{XX}$ & 4. Purchase 2 teacher lap-top computers. \\
\hline $\mathrm{XX}$ & 5. Repair/replace Greenhouse \#1 heater. \\
\hline $\mathrm{XX}$ & 6. Purchase 22" Honda lawn mower for farm. \\
\hline $\mathrm{XX}$ & 7. Purchase second self-driven field mower for farm. \\
\hline $\mathrm{XX}$ & 8. Purchase portable air compressor unit for Ag. Truck. \\
\hline
\end{tabular}

2010-2011

\begin{tabular}{|l|l|}
\hline & \\
\hline & \\
\hline & \\
\hline & \\
\hline & $2011-2012$ \\
\hline & \\
\hline & \\
\hline & \\
\hline & \\
\hline
\end{tabular}


AGRICULTURE ADVISORY COMMITTEE

North Salinas High School (rev 10/10)

Thursday, Apri/7,2011

MEETING AGENDA and MONUTES

1. CALL To ORDER: 6:45 pm by Chairman Josh Ruiz'

2. INTRODUCTIONS (Guests and New Members) - Chairman

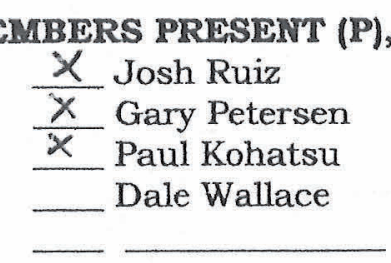

4. PREVIOUS MEETING MINUTES:

5. AG. MMPROVEMENT FUNDS BALANCE: $\$ 4209$

Q $\frac{5 x}{\frac{E x}{0 p}}$ is 4 Ag. Industry Rebec tea Collins Rick- IXmburti Angie Caresani embers.

$\frac{x}{x}$ Stephanie Blodgett Tom Jones

Stan Uchiyama

* Margaret Noroian

6. AG. DEPARTMENT UPDATE REPORT -- Stan Uchiyama, Tom Jones, Stephanie Blodgett, Margaret Noroian.

a. $2010-12$ Class Enrollment and staffing. Have 10 classes and

assignment for two tenches. Teacher 3 class assignment uncertain.

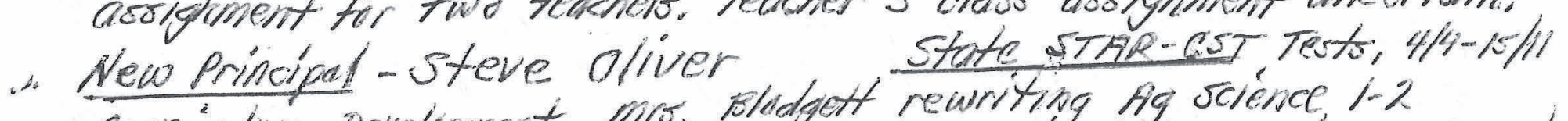

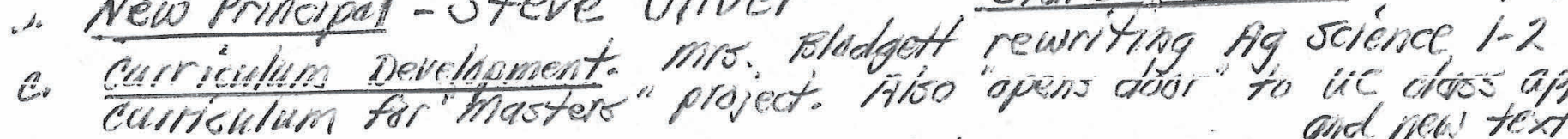

and neal texthok

d. Ag Booster, School Flarm/stiv- mo. Bladgett, and mi s Jones.

7. FF a chapter update RePort - Rebecca Collins

a. Past Events (achievements, value, etc.) summary:

Chapter Project Competition, Spring Region FFH meeting Region state Degree aid one Profieichey Awards (of 47 award areas $115 \mathrm{ks}$ sponsored 12 awards.)

b. Future Activities (information only):

Ag Booster/FFl BLQ, FFA officer Elections far $2011-12$

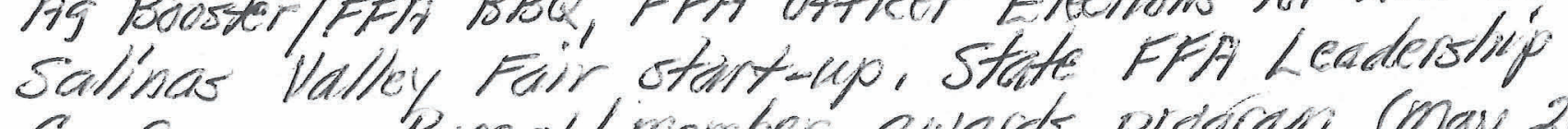

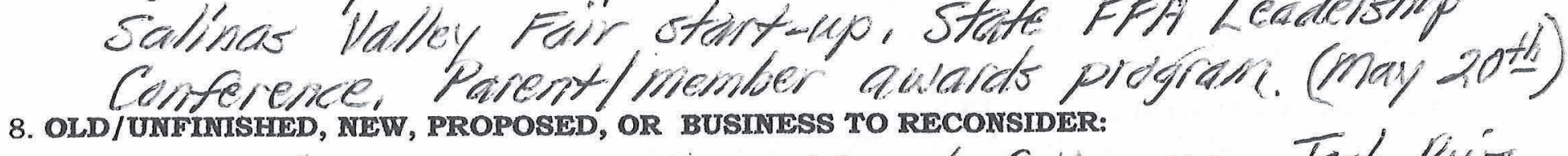

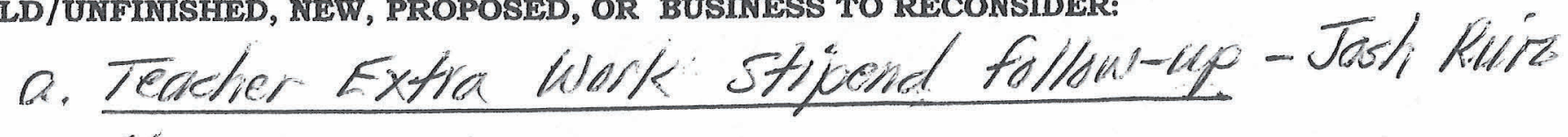
No progress. 
(continued - old/ unfinished, new, proposed, or business to reconsider)

Page 2

b. Salinas Valley Ag Industry Tour - Vashti, Maul, Dale Tour Chairmen- Josh, Paul, Date isp Wa te- We ats, Api Teaders-2, H student = 75-30, Tine 8:30 am- 2:30, Lunch- Eat on trip, Bus = Have, Teacher $56 \mathrm{~A}=$ App raved cast-schal pay sub is Grant pry hus -ok, commits dido approve " 00 to fund trip

* Feasible to still do? CSs Testing. partial stop schedule set-lup, student signal needed.

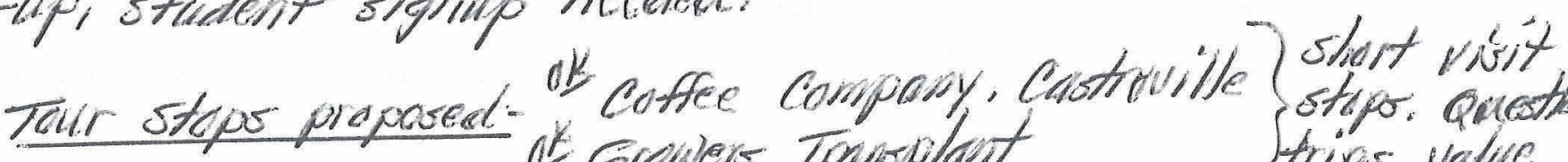
stipe. quotes fixes valine to herring.

(miss) (Petersen, madalara) to postpone trip to fall.

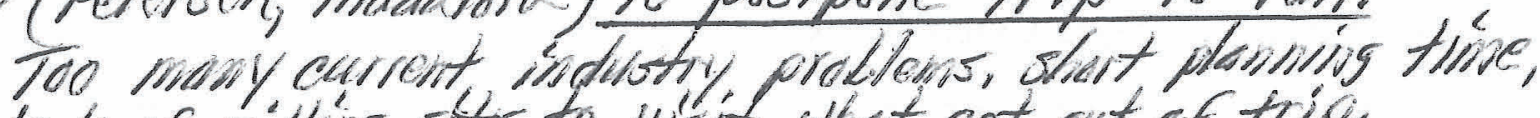
lack of willing st he to visit, whit get out of trip:

c. Ag Program direction / future? Need to review program goals, objectives, needs, future is ion, AIG commendation, and recommendations to give greater exposure of ag.

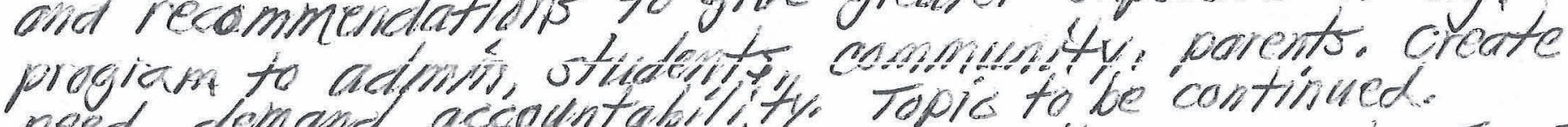

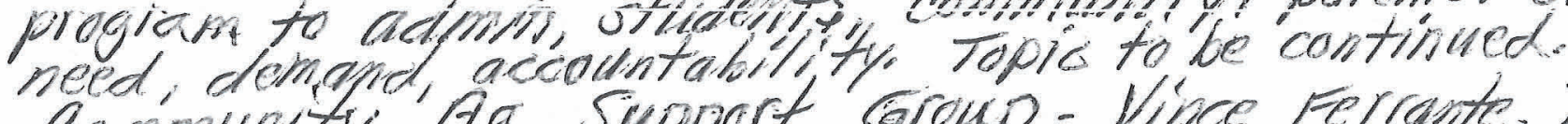

d. Community' Ag Support Group - Vince Ferrante, Tom Jones Brochure revision progress (also refer to llisflio start up proposal and draft initio letter).

Nothing done to reuse brochure.

to be continued.

9. NEXT MEETING AGENDA ITEMS:

a. To be determined

c. Ag Program Direction/Future-see it ten C, above

10. ANMOUNCEMENTS and REMINDERS:

a. Next meeting is: SGetfoller 28,201

b. Refreshments provided by: .

11. ADJoURNment: $8: / 5$ pm by chairman $105 / 2$

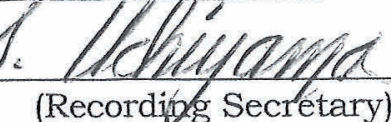




\section{AGRICULTURE ADVISORY COMMITTEE}

North Salinas High School (rev 10/10)

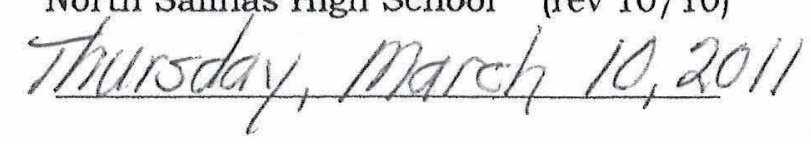

\section{MEETING AGENDA and MUNUTES}

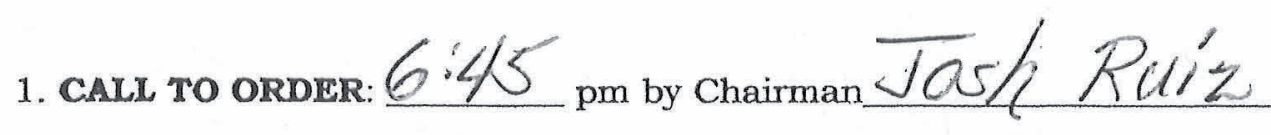

2. INTRODUCTIONS (Guests and New Members) - Chairman

3. MEMBERS PRESENT (P), ABSENT (no mark): Quorum is 4 Ag. Industry members.

$\not{X}$ Josh Ruiz
$\not$ Gary Petersen Paul Kohatsu

X Dale Wallace

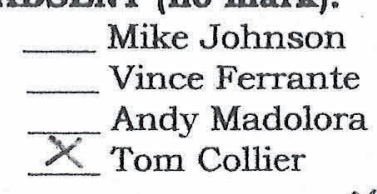

Mike Johnson

Andy Madolora

$x$ Tom Collier
$\frac{X}{X}$ Syble Beardslee

$\times$ Rebecca Collins Rick Winburn Augie Caresani $\frac{x}{x}$ Stephanie Blodgett

$\frac{X}{X}$ Tom Jones

Stan Uchiyama

Margaret Noroian

* mike Johnuan no langer able to attend.

4. PREVIOUS MEETING MINUTES: February 10,2011 mse Peteren Callier Thapdary meetings, meeting minutes as printed.

5. AG. IMPROVEMENT FUNDS BALANCE: $\$ 470913$

6. AG. DEPARTMENT UPDATE REPORT -- Stan Uchiyama, Tom Jones, Stephanie Blodgett, Margaret

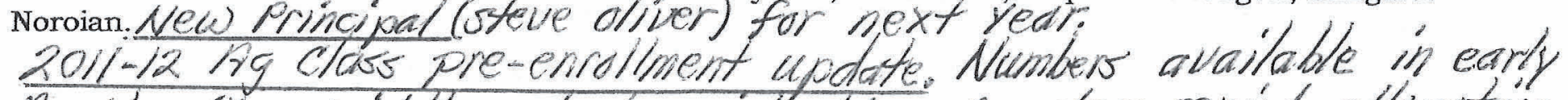
April, afier, middle sctidol registlatidn. Ag clas period allocatian will, be affected by pre-neg. numbers and reading class numbes inchease

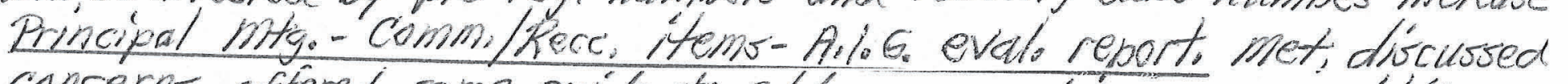

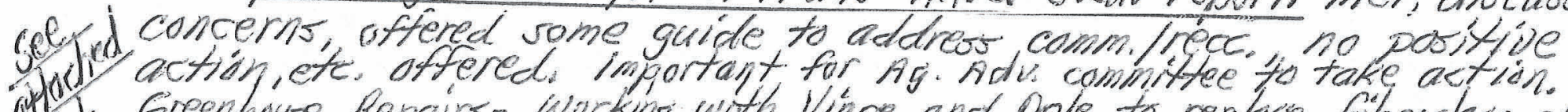

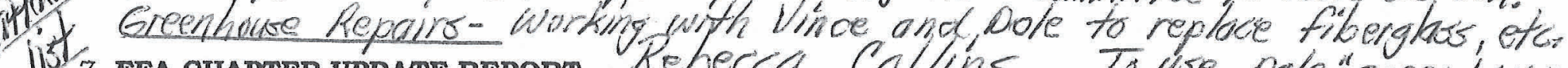

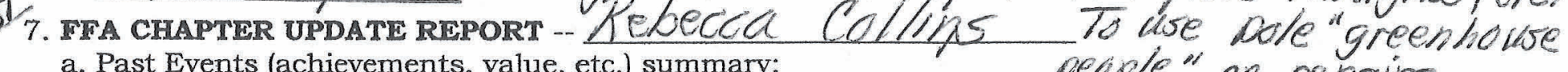
a. Past Events (achievements, value, etc.) summary:

FFA week Actiuities, - activity to promote ng lndustry, Ay program and for FFA conducted each day, with pizza. feed on last day df week. Discuserion and sllgrest to conduct ochool-uide FFA pramotion activitio.

b. Future Activities (information only):

chapter Praject Competitian, Spring Reginis EFA ntig. and cantest finat, Regian state Degree pulards; State FFA leciderotíp conference.

8. OLD/UNFINISHED, NEW, PROPOSED, OR BUSINESS TO RECONSIDER:

a. Follow-up teabler extra work stipend-J. Puiz frtion plan to revolve - who? linat? hiben? Nepds lefter

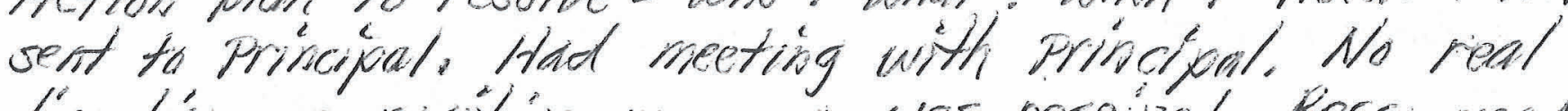

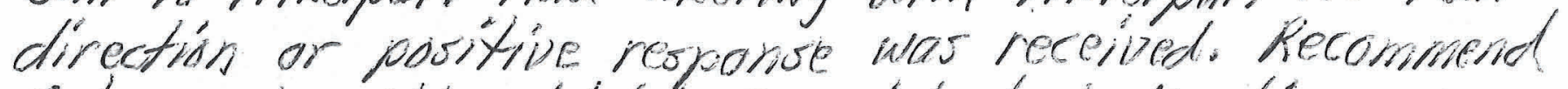

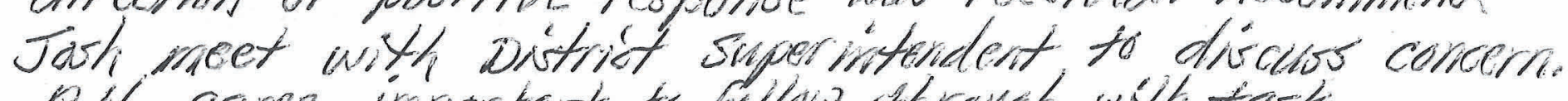

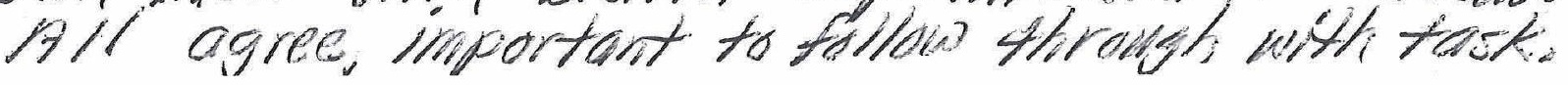


(continued - old/ unfinished, new, proposed, or business to reconsider)

Page 2

b. Salinas Valley Ag Industry Tour - Josh Ruiz.

Tour Chairmen -Josh and Paul Trip pate -April 13

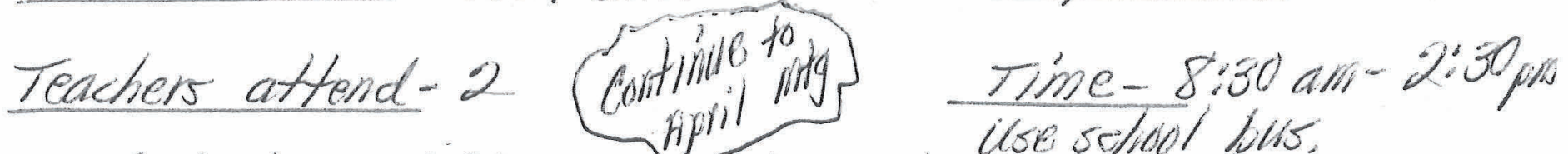

\# students -15-30 Zunch-Eat on trip. Bring own.

Cost -Approved at Feb. amity = 250 for teacher sub cost and

soar for shad bus use. (school or hic to pay burs cost).

Tour Visit schedule suggestions. 2-3 stops.

Try to keep trip stow wis him Castroville and salinas Valley,

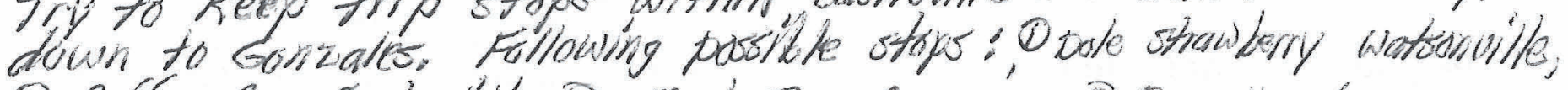

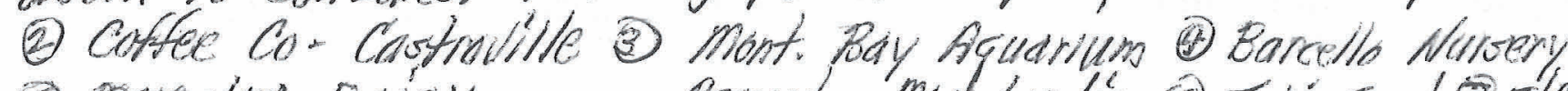

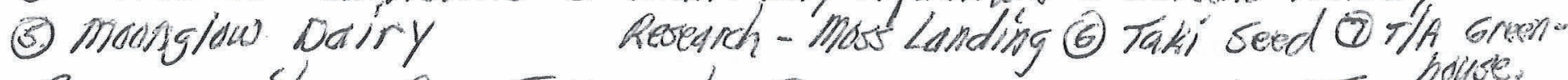

c. Community Ag support Program-Ferrarte, Jones. information brochure progress- Mir. Jones

Discussion. Need to define programs scope of fundraising and purpose of finds generated. keep perspective "dow nato

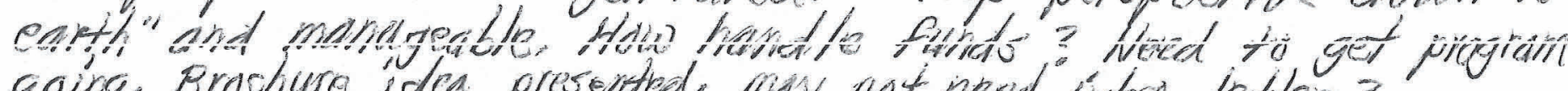

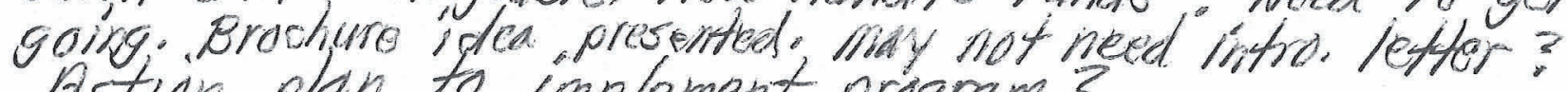
Action plan to implement program?

All liked draft brochure presented. Needs editing, and updated pictures. mir. Jones to work on improving brochure and present progress work at hor is meeting

d. Important need for committee to become more active, visible, aggressive to keep hglFth program viable9. NEXT MEETING AGENDA ITEMS:

a. SV $\mathrm{Ag}$ lndiestry Tour follow in o. future iv very uncertain? strate-wide concern.

b. Community Ag support Program

d. Future- Ag Program status, events, staffing, ether

10. ANMOUNCEMENTS and REMTNDERS:

a. Next meeting is: tacit' $201 /$ (last meeting for verdi)

b.

11. ADJOURNMENT: 8:45 pm by Chairman Jos R Ruiz

S. Uthisemente 


\section{COMMENDATIONS North Salinas High School

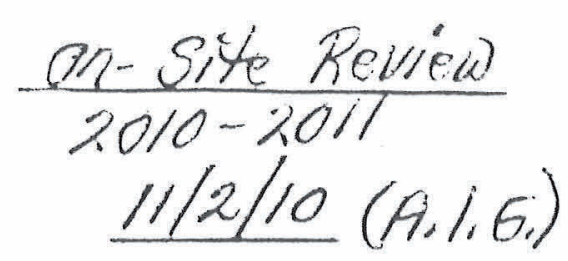

The Board of Education, District, and Site Administration for their support of the agriculture staff to implement and maintain the Agriculture Incentive Grant Program Quality Standards.

The Site Principal for recognizing the importance of a designated single Department structure for agriculture education.

The Agriculture Advisory Committee for their outstanding commitment and continuous support to provide for a quality agriculture education and FFA program for North Salinas High School students.

The Agriculture Department for their thorough preparation of documentation required to comply with the Quality Criteria Standards of the Agriculture Incentive Grant program.

The District for providing transportation and other unique program expenses associated with maintaining a quality agriculture education program.

The participation of the instructors in professional meetings and in-service activities sponsored jointly by the California Agriculture Teacher Association and the State Department of Education.

The instructors for the example set in neatness and organization for the agriculture facilities. Impressive!

\section{RECOMARTNA TIONS North Salinas High School}

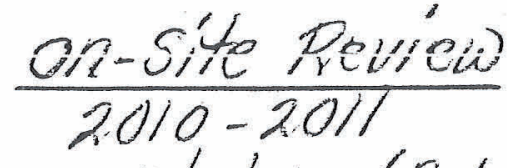

$11 / 2 / 10 \quad\left(1.1 . G_{0}\right)$

That the District provide full year employment for all agriculture teachers, specifically providing for AG/FFA leadership supervision school/ year stipends and Summer Agriculture program employment compensation.

That the Site Principal actively support the implementation of an Ornamental Horticulture teaching program to meet student non-livestock and plant science interests.

That the Administration and Agriculture Instructors develop a plan which would secure a classroom (205) and storage room to develop an Agriculture Science lab to enhance Ag. Science, Ag. Biology, Ag. Chemistry, and Ag. Earth Science instruction, leading to advanced technical studies and $\mathrm{UC}$ admission requirements.

That the school site and Administration actively support school farm building maintenance and electrical installation needs.

That the Horticulture Facilities be utilized.

That the Agriculture Department develop strategies to help retention within the program. 
AGRICULTURE ADVISORY COMMITTEE

North Salinas High School (rev 10/10)

Thursday, February 10, 2011

MEETING AGENDA and MINUTES

1. CALL To ORDER: 6:45 pm by Chairman Gary Petersen

2. INTRODUCTIONS (Guests and New Members) - Chairman

sydney Coakley-igg student guest

3. MEMBERS PRESENT (P), ABSENT (no mark): Quorum is $4 \mathrm{Ag}$. Industry members.

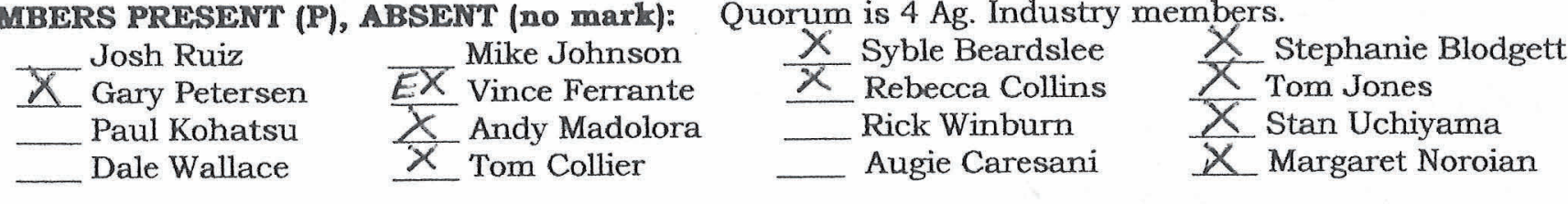

4. PREvious MeEting Minutes: Ms P (Bedrdslee, Madolora $)$ to approve the January 13,2011 meeting minutes as printed. 4011 - Added 1800 , Young Life

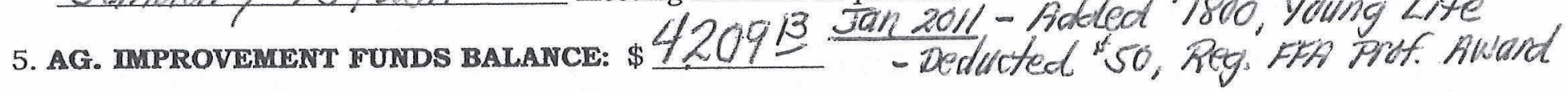

6. AG. DEPARTMENT UPDATE REPORT -- Stan Uchiyama, Tom Jones, Stephanie Blodgett, Margaret Noroian.

2011 Ag Class pre-registration process. Now in process of High school Reg. middle school. Wis ito in March. Hope prelim, \# early April. Final 2010 AlG. Compliance Report i Reviewed by committee. Noncompliance ( 2000 lows) with full year employ. criteria. Commendations and recommendations gisqussed. Future minty wi privoipal/staff to review. committee member update- Encourage prem les to find new member additions. suggest removal of members not attending meetings:

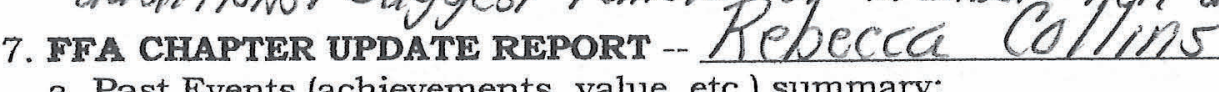
a. Past Events (achievements, value, etc.) summary:

state FFA Degree candidates, MFE/ALA header ship conference, Salinas Farm Day, FFA Tob Interview contest

b. Future Activities (information only):

FFA Week -Feb. 21-25. Daily activities planned chapter project competition (march id) -Judges needed. members interested = Petersen, madilara 8. OLD/ UNFINISHED, NEW, PROPOSED, OR BUSINESS TO RECONSIDER:

a. Follow-up-teacher extra work stipend - T. Ruiz Next step. Action Plan to resolve who? What? when? wash not in meeting attendance. sue is will a problem and noted as recommendation on Ale report. postponed to march meeting. 
(continued - old/ unfinished, new, proposed, or business to reconsider)

Page 2

b. Salinas Valley Ag Industry. Tour

Tour Leaders.

Teachers.

Date of trip.

Time-

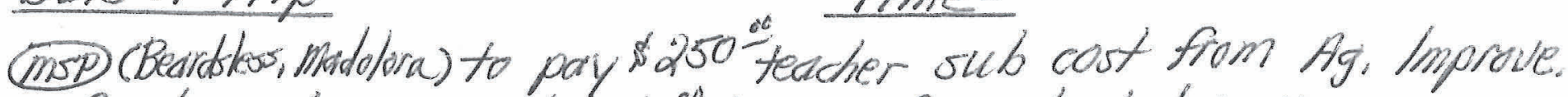
fund monies and so 500 to tory for school bus use. \#students-

school Bus Use-

Tour visit schedule

* Prefer sig. site visits in the salinas Valley

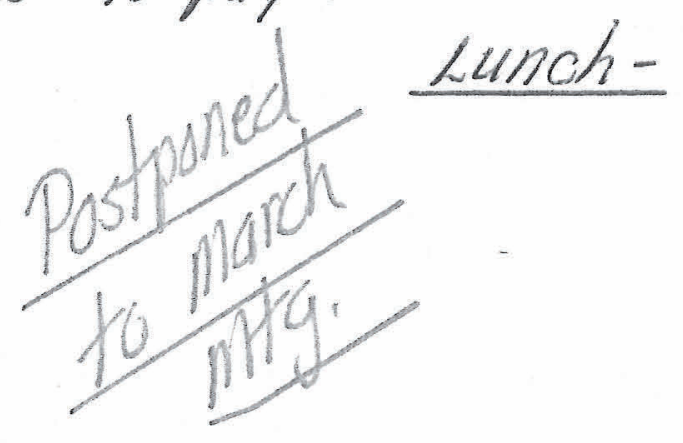

* mop (isadora, Collier.) That salinas valley Mg Tour have option of being conducted in fall months or sporing months diving odd years when si ha Tour is schedule, to be conducted.

C. Community Ag Support Program-Ferrante, Jones Status -Do we continue or drop this program idea? Agreed program is good concept and want to "work to Restart action plan - Who? What? When? Mr. Jones discussed past efforts and minimal progress done. Intro. Letter and needs list draft copies completed - both need content and format revision. Information brochure presented, reviewed, only. Report at march meeting. Start now/ future action plan?

a. Final Planning Needs - SV Ag Industry Tour

b. Community hi support Program

d.

10. ANNOUNCEMENTS and REMINDERS:

a. Next meeting is: Mrupday, larch 10, 2011

Refreshments provided by: Gary Petersen

b.

11. AdJOURNMENT: 8:30 pm by Chairman Gary Petersen,

1. Alflitínma 


\section{COMMENDATIONS \\ North Salinas High School}

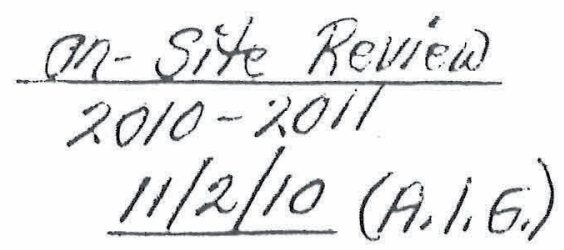

The Board of Education, District, and Site Administration for their support of the agriculture staff to implement and maintain the Agriculture Incentive Grant Program Quality Standards.

* * The Site Principal for recognizing the importance of a designated single Department structure for agriculture education. Very important to kecp thes stotis. low of The Agriculture Advisory Committee for their outstanding commitment and continuous support to provide for a quality agriculture education and FFA program for North Salinas High School students.

The Agriculture Department for their thorough preparation of documentation required to comply with the Quality Criteria Standards of the Agriculture Incentive Grant program.

* The District for providing transportation and other unique program expenses associated with maintaining a quality agriculture education program. $\angle 005$ hinld "shet dow" activities and opportunitier aweiloble to stiudests. The participation of the instructors in professional meetings and in-service activities sponsored jointly by the California Agriculture Teacher Association and the State Department of Education.

The instructors for the example set in neatness and organization for the agriculture facilities. Impressive!

\section{RECOMVINENDATIONS North Salinas High School}

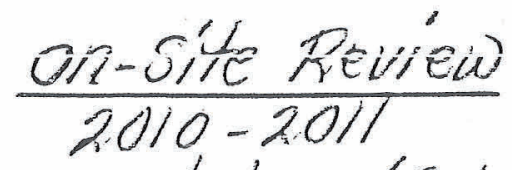

$11 / 2 / 10(1,1, G)$

* * That the District provide full year employment for all agriculture teachers, specifically providing for AG/FFA leadership supervision school/ year stipends and Summer Agriculture program employment compensation. Needs to le prioriy for copsinstiee action.

* That the Site Principal actively support the implementation of an Ornamental Horticulture teaching program to meet student non-livestock and plant science interests.

* * That the Administration and Agriculture Instructors develop a plan which would secure a classroom (205) and storage room to develop an Agriculture Science lab to enhance Ag. Science, Ag. Biology, Ag. Chemistry, and Ag. Earth Science instruction, leading to advanced technical studies and UC admission requirements. of oopot roienet.

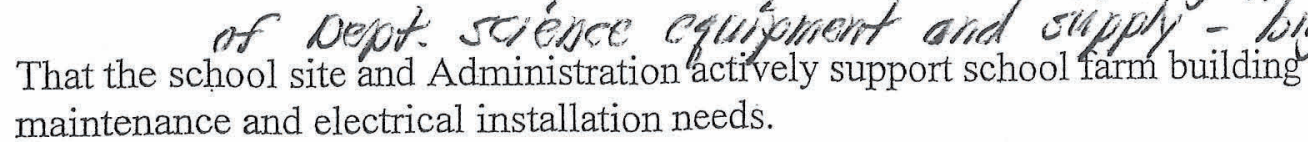

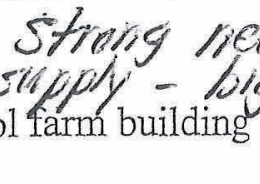 ort 


\section{AGRICULTURE ADVISORY COMMITTEE}

North Salinas High School (rev 10/10)

Therisday Jannary 13,2011

1. CALL to ORDER: 6: 40 pm by chairman_TOSh Ruic

2. INTRODUCTIONS (Guests and New Members) - Chairman

3. MEMBERS PRESENT (P), ABSENT (no mark): Quorum is $4 \mathrm{Ag}$. Industry members.

X Josh Ruiz Gary Petersen

X Paul Kohatsu Dale Wallace
Mike Johnson

Vince Ferrante

X Andy Madolora

Tom Collier

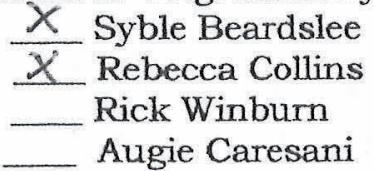

$X$ Stephanie Blodgett

$x$ Tom Jones

$X$ Stan Uchiyama

X Margaret Noroian

4. PREVIOUS MEETING MINUTES: Or.t if aud Noll 10

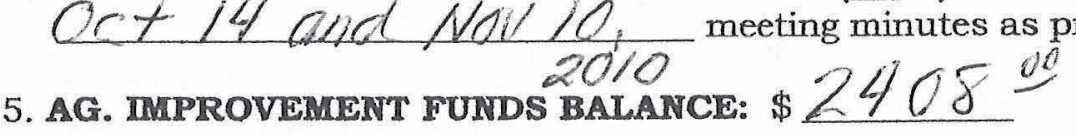

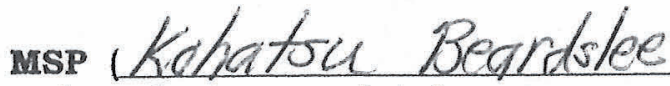
) to approve the meeting minutes as printed. Aide $\% 800$ from Voung life (T, Rü Pumpkin Grop). See dienda 8 -f

6. AG. DEPARTMENT UPDATE REPORT -- Stan Uchiyama, Tom Jonés, Stephanie Blodgett, Margaret Noroian.

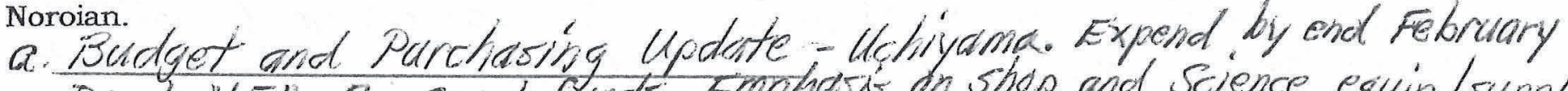

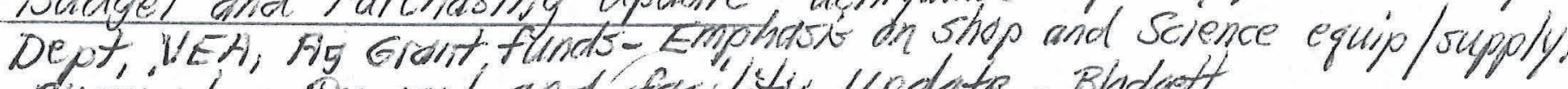

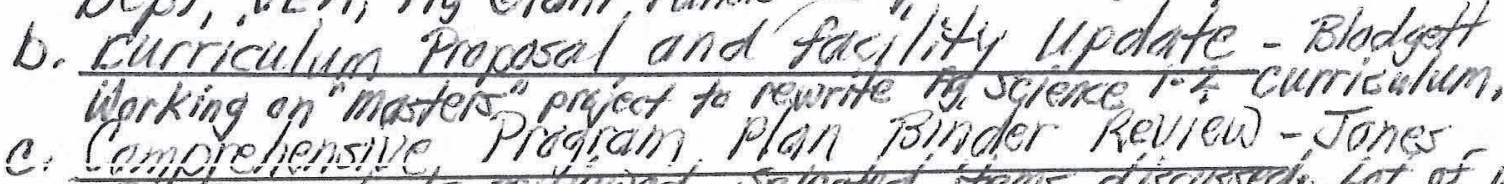

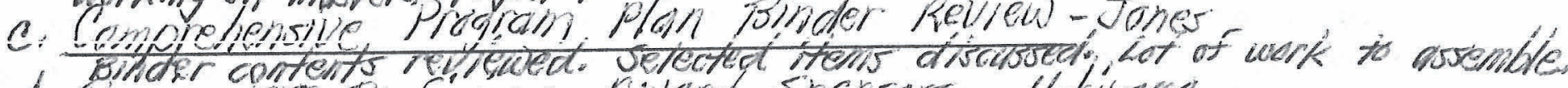

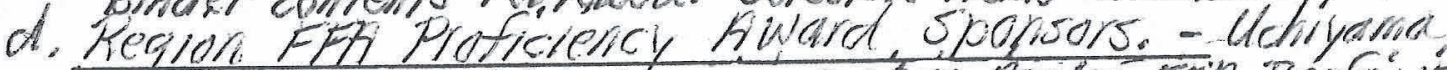

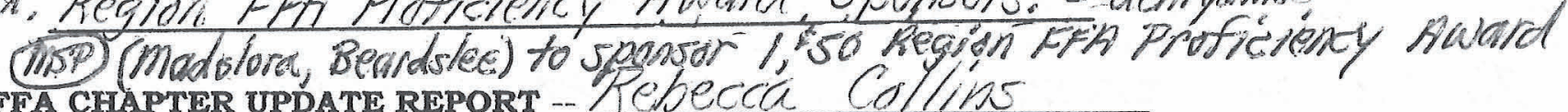

7. IFA CHAPTER UPDATE REPORT - $\mathrm{TCBPCCC}$
a. Past Events (achievements, value, etc.) summary

Ag Calendar Salles fulidraising, Creed and Publis spenting contests, Holiday canned Fiod Drive, SVIF stack linterest mita, National, Young Farmer converition, salinas-"Parade of Lights," Salvation Army "Kettle Drive."

b. Funure Activities (information only: State FFA, Degree candidates, MFE/MLA Ldr. Conference, Salinas Mo co Bg Ed Farm Day, Job lintervieal contest, spring livestack ShiE staitup.

8. OLD/UNIINISHED, NEW, PROPOSED, OR BUSINESS TO RECONSIDER:

a. Teacher Extra Work stipend Concern Status - T. Kuiz Visit made with Principal and letter of concem given in Yate october. Follow up contact made by J. Kuis, Discussionfaction. - No Pay $\rightarrow$ No wark $\rightarrow$ Direct affect on compliance, with inany AIG Criteringstandarift. Josh, to contact m. Curestani on action staths or who to vee to get action on concern. 


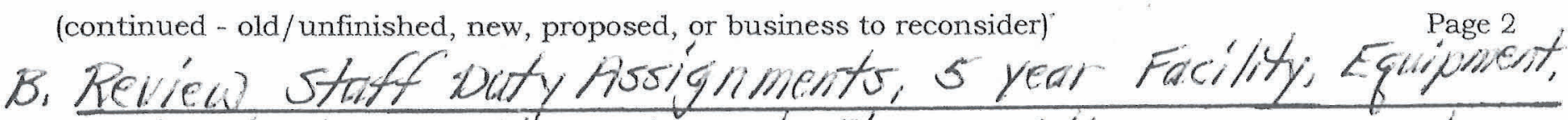

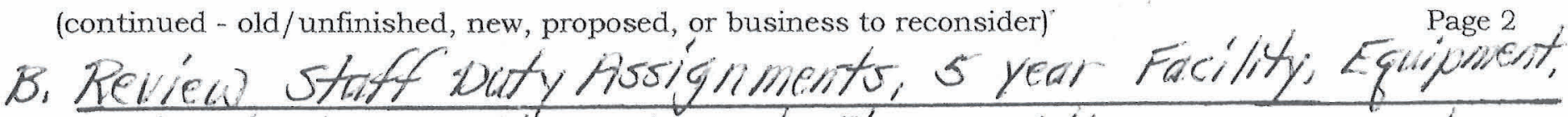

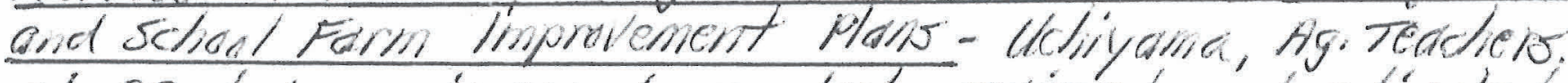

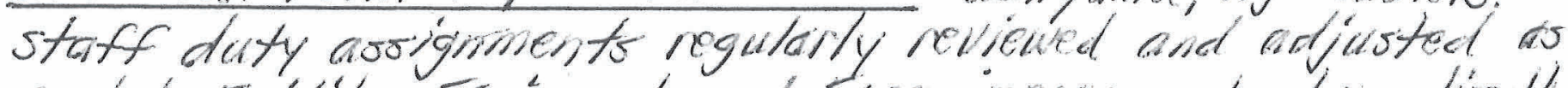
needed. Facility, Equipment, and Farm improvement plans directly

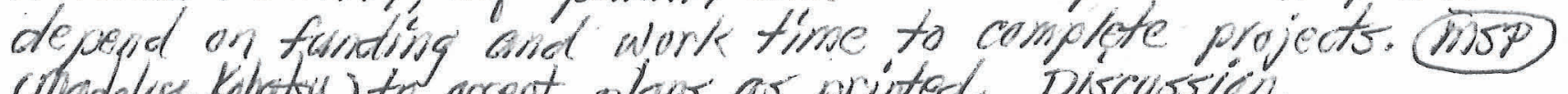

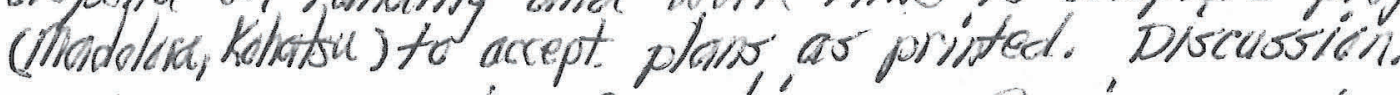

c. AlG. OnSite Compliance Review and final CDE evaluation report - Uchiyama (Continued from Nov aunty.) Review of Uchiyama's self evaluation commendationfreconmendation criteria and standards per nit check list and cot rep. discussions. Review? of final CDE onsite evaluations report. Refer to Nous, mtg.

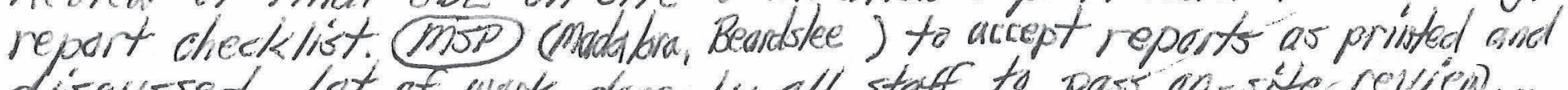

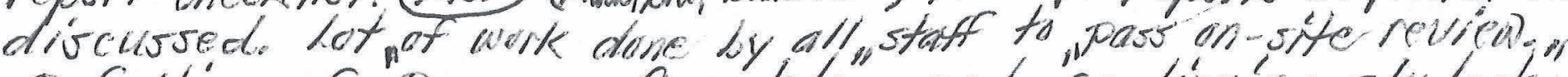
d. Definition of "Program Completer and "Continuing student" following definitions to be used in the management concerno/need of the averral ag. ed, program. Q" Program completer" - A student Who has completed 3 years of consecutive ag . oles enroll meet and identified by state EFH affiliation records. (2) "Continuing sturcteurA student who is in their second, third, or fourth year of consecutive ag.

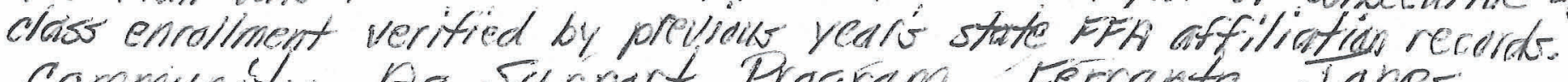

e. Community Ag Support Program-Ferrante, Sones

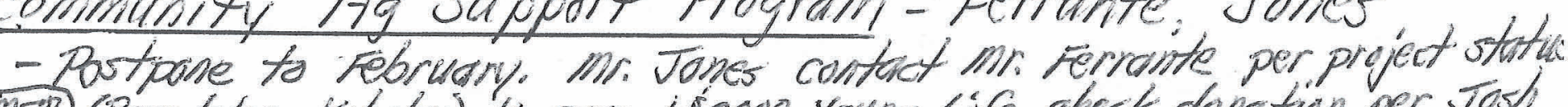

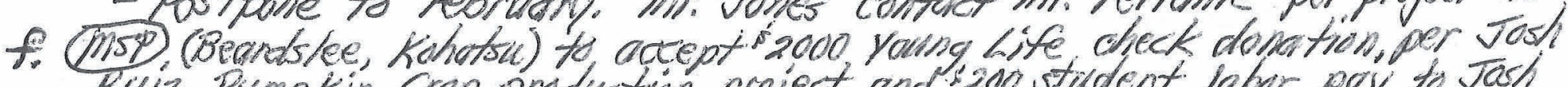
Ruiz Pumpkin crop production project, and "300 student labor pol to Nash

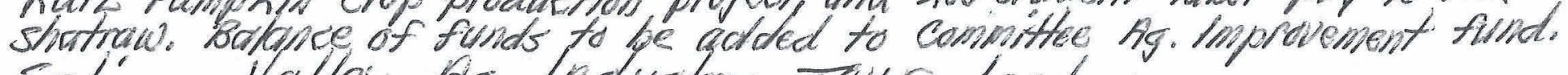
salinas Valley Mg Industry Tour. Leaders:

Date-Tues, March 15. Basic tour needs, site visits, ot e. discussed may 9. NEXT MEETING AGHIDA ITEMS:

a. Salinas Valley Rig industry, Tour

c. Community hg. support Program

d. have visitation problems with early date? Josh and Paul to check on some site operations. Continue setup 10. ANNOUNCEMENTS and REMIINDERS: 102011 details and discuss

a. Next meeting is: IMUs, FChradry 10.2011 at Febridaly meeting,

b.

11. ADJOURNMENT: $8: 30$ pm by chairman $100 / 2 \mathrm{R} 112$

(Recording /Secretary) 
AGRICULTURE ADVISORY COMMITTEE

North Salinas High School, (rev 10/10)

Weds, November 10,2010

No int g.

MEETING AGENDA and MINUTES

Quorum

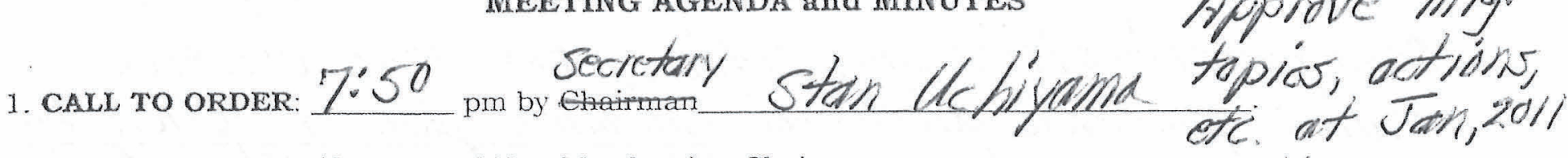

2. INTRODUCTIONS (Guests and New Members) -- Chairman

meeting

3. MEMBERS PRESENT

J__ Josh Ruiz

ABSENT (no mark):
Mike Johnson
Vince Ferrate
$\bar{X}$ Andy Madolora
Tom Collier

Quorum is $4 \mathrm{Ag}$. Industry members.

Stephanie Blodget

Dale Wallace

$\frac{X}{X}$ Syble Beardsley

Rick Windburn

Tom Jones

_. Angie Caresani

Margaret Noroian

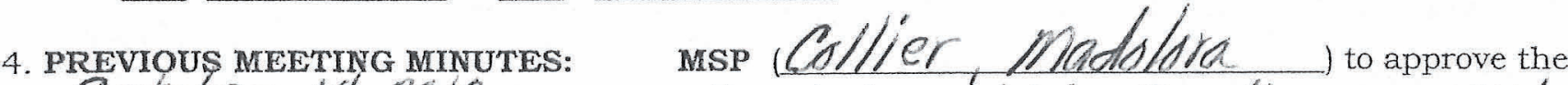
October $/ 4,2010$ meeting minutes as printed. Pending approval at

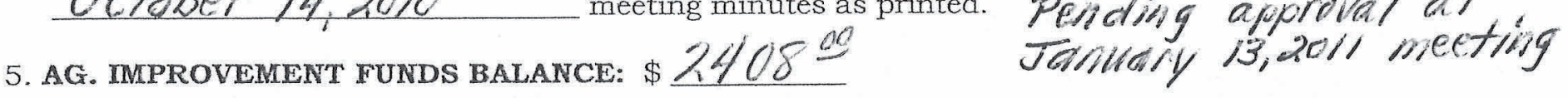

6. AG. DEPARTMENT UPDATE REPORT -- Stan Uchiyama, Tom Jones, Stephanie Blodgett, Margaret Noroian.

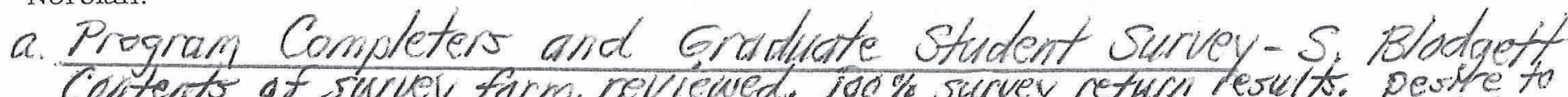

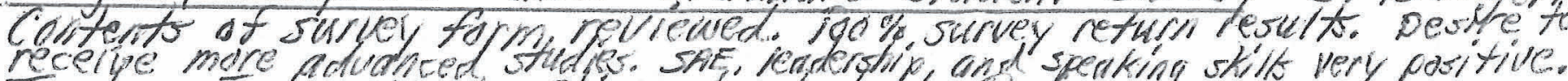

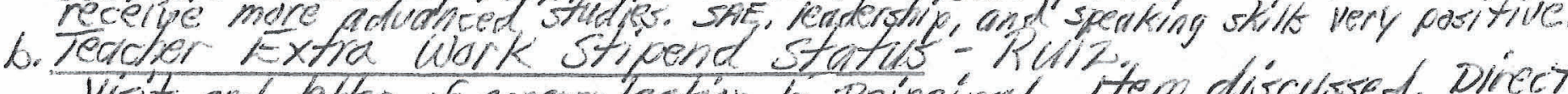

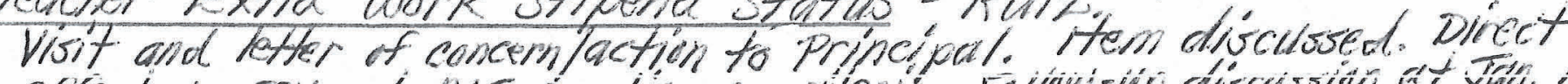

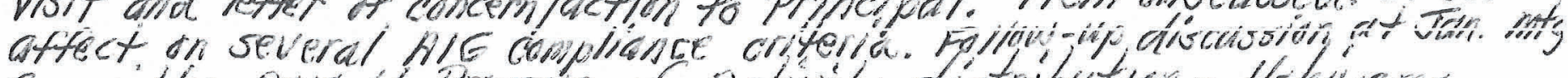

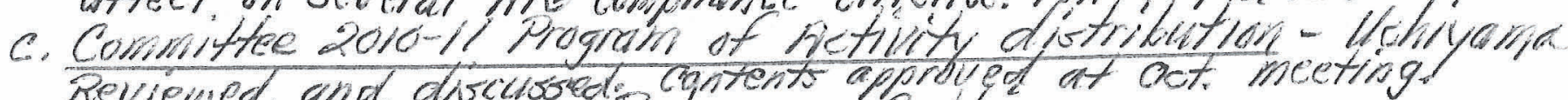
Reviewed aid divewsod pegntent appougd at

7. Past Events (achievements, value, etc.) summary:

MBS B.1.6. and Coop marketing contests Ag Calendar, Cookbook, Gift Wrap Fundraising Chapter Creed and Public speaking contest

b. Future Activities (information only): $M$ ts s creed and Public speaking Contests

8. OLD/UNFINISHED, NEW, PROPOSED, OR BUSINESS TO RECONSIDER:

a. Comprehensive Program Plan (Binder) Leview-Jones hot of document, information, and up dating, to program plan done by ag, staff: Copy filed with principal, Ag Dept Reg, super. Table of contents attached. Plan contents reviewed and of sicussed. Excellent reference to ag. program mangsement and operations needs-

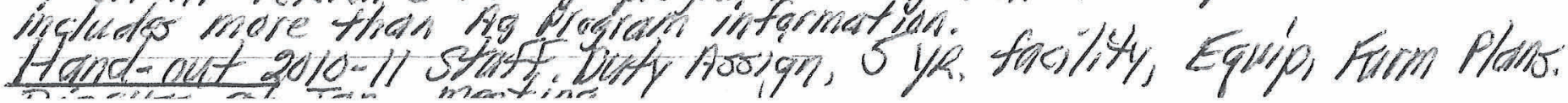


(continued - old/ unfinished, new, proposed, or business to reconsider)

Page 2

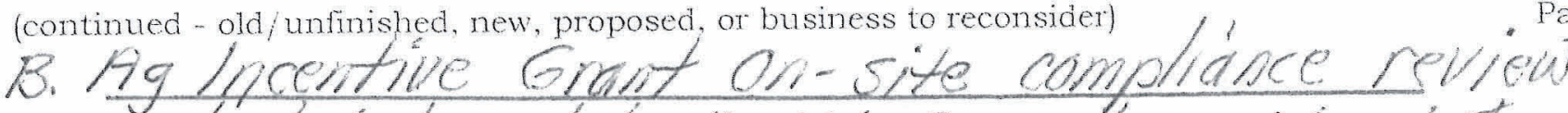

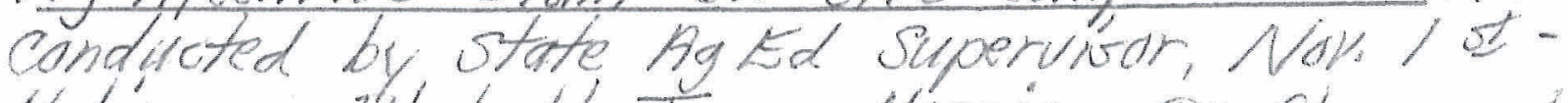
Uchingama, Bridgett, Jones, Noroian. Draft report, commendations/recommendations, and day's criteria review comments discussed. Guidance plan of action for future improvement and quality program growth Final report to come within next month. see separate draft evaluation checklist with comments. AIG compliance. checklist to be further discussed at January meeting. checklist good to use as "plan of action" source for, ag. program improvement and future program directions emphasis, on instruction.

C. Community Support Program - Ferrante, Tones

1. Dole Fresh Veg proposal fo upgrade greenhouse. Brief awareness of proposal letters received.

2. Intro solicitation lester draft to community - needs review and more direct thought it. mn sones to reviews by Jan mtg.

3. Draft list of projects and materials equipment needs. Best to have project, purpose, and, master in ts needed to complete project mi. Jones to review/rewritte by Jan. mtg.

D. Fundraising Proposal - Spring BRQ. Ruiz, Wallace

1 . Proposed date-

2. Draft Details

Post oping

$$
\text { In -change- }
$$

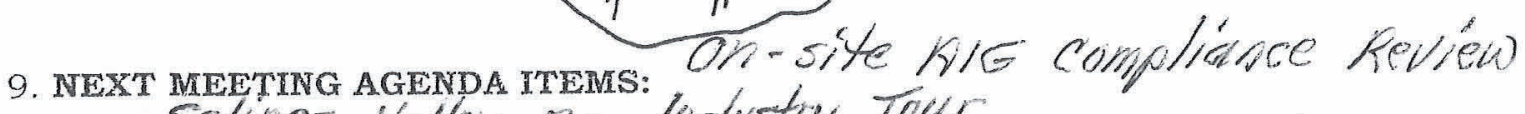
a. Salinas Valley $17 g$. Hedistry Tour

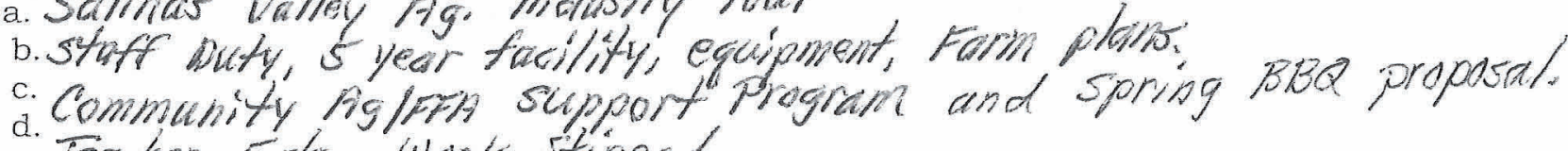
Tearier Extra work sifiend.

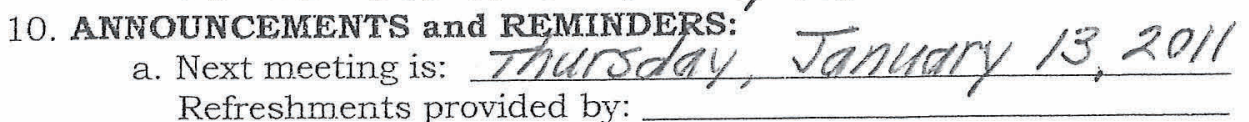

b.

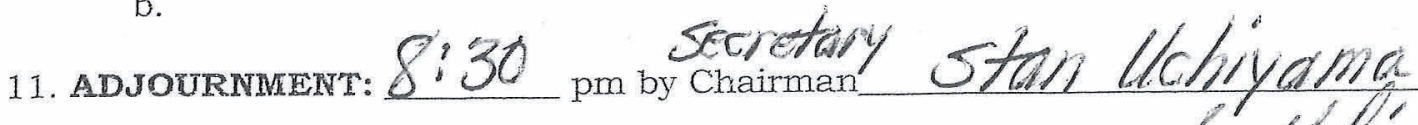

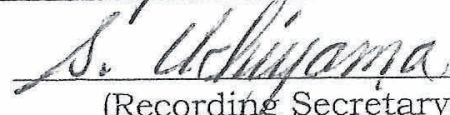

(Recording Secretary) 
AGRICULTURE ADVISORY COMMITTEE

North Salinas High School (rev 10/09)

Thursday, Oct. 14,2010

MEETING AGENDA and MINUTES

1. CALL To order. 6:50 pm by chairman Josh Ruiz

2. INTRODUCTIONS (Guests and New Members) - Chairman

Ag Booster Rep -sybil Beardsley

Ag student Rep-Rebecra Collins

3. MEMBERS PRESENT (P), ABSENT (no mark): Quorum is 4 Ag; Industry members.

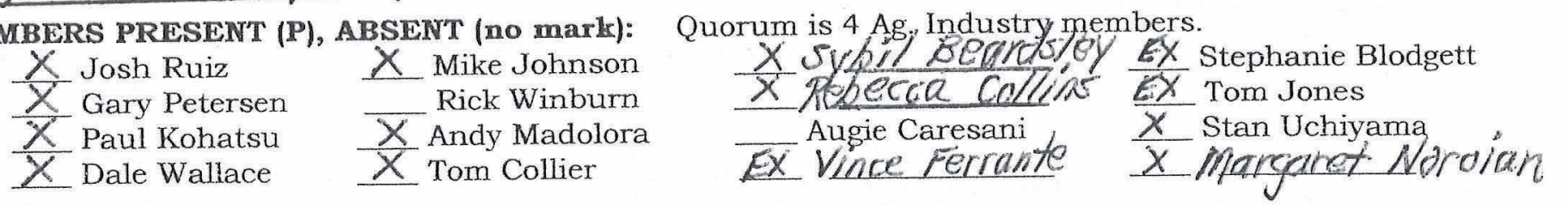

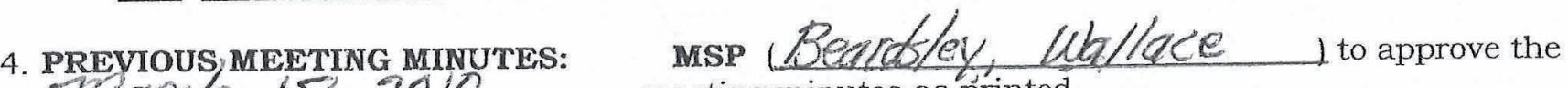
$210 / 2 / 8,2010$ meeting minutes as printed.

5. AG. IMPROVEMENT FUNDS BALANCE: $\$ 240890$

Margaret Noroían

6. AG. DEPARTMENT UPDATE REPORT -- Stan Uchiyama, Tom Jones, Stephanie Blodgett.

a. Staff Update and Introduction.

b. Ag class Enrollment -340 curduplicated students. Five shop type closures, Five Ag Earth science, 2 Ag chemistry, one Ag. Ecom/Govt, and one Intro, Rig sense classes.

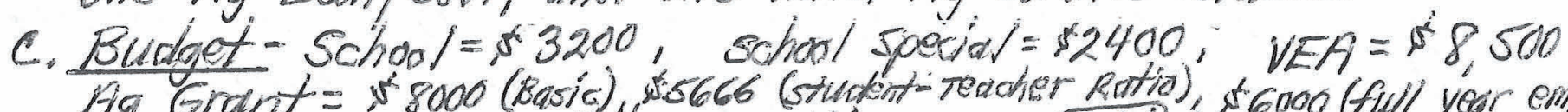

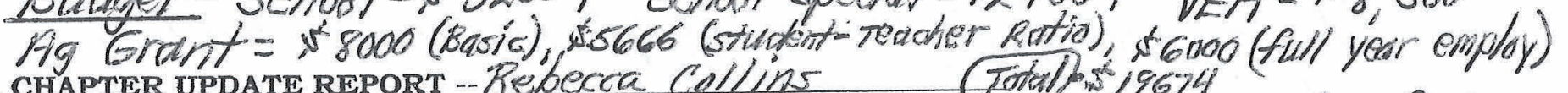
7. FF A CHAPTER UPDATE REPORT - Rebec ca Collins TOTHOW/9674

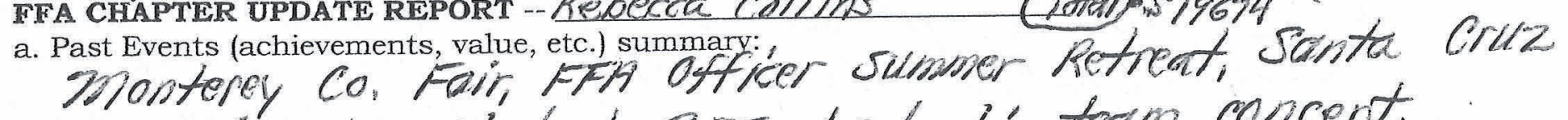
Co. Fair, New student BER, leadership team concept, chapter officer Leadership conference, of c contest.

b. Future Activities (information only): Green hand Conference, $B, 1, G$ and marketing contests.

8. OLD/UNFINISHED, NEW, PROPOSED, OR BUSINESS TO RECONSIDER:

a. Member Roster Up-dacte - address, phone \#, work place. mop (keresan, kohgtalu) to accept continued service for members presents fluent members to be corraded and status reported at November, meeting.

b. Ag/FFA/SWE stipend Misdate status. No stipend for two teacher extra big foin work task done.

Thoughts on continued problem. All members 
(continued - old/ unfinished, new, proposed, or business to reconsider)

Page 2 agree that out-of-class FEA, SAE, and support group torts and supervision activities, are important l integral to

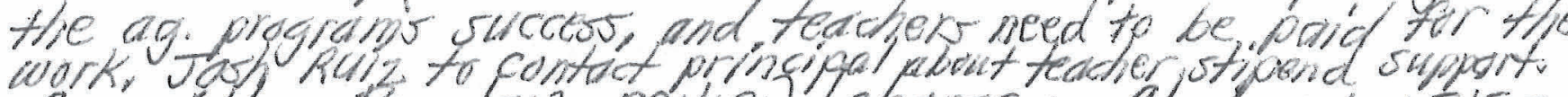

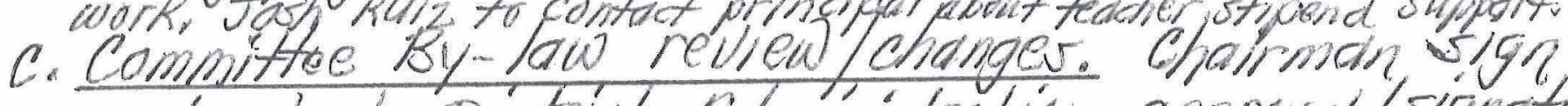
and start District Administration approval/signature process. ms p (Mirdadera, Collier) to approve Committee. constitution and Bylaw as reviewed/revised. Copy part of Program of hetwity packet. No changer from last year.

d. Program of Activity, review. Goal, meeting dates, year's activity guidelines. ms p (Johnson, anlace) to accept P. O. A. goals, and revisions, etc, as proposed. updated Poo. A. guidelines distributed at November meeting.

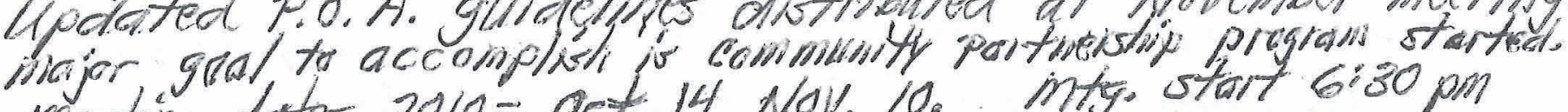

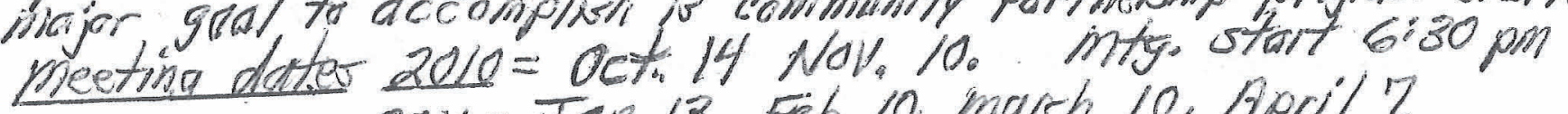
$2011=\operatorname{Jan} 13$, Feb.10, march 10, April T,

e. Ag Grant Compliance checklist 1-11 criteria review. State Dept of Ed, on-campicis reviews visit

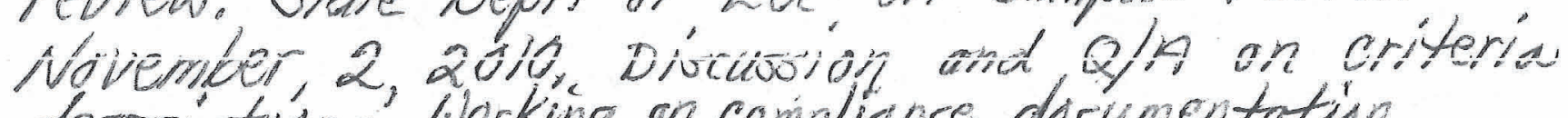
descriptions." Working on compliance documentation

1 Grant reduction possible on full-year employment and teacher -student ratio funding applied for up totroor?

(1). Ag Community Partnership Prog ran - Vince errant, Tom Jones

1. Update statue and future direction. Refer to march 18

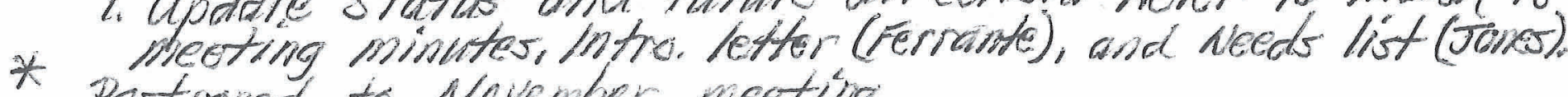

* Post opined to November meeting

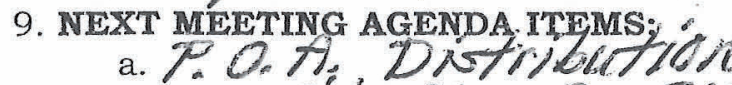

b. On-Site C DE $\Rightarrow g$ Grant compliance review.

c. Ag community Port nerohip Program

comprehensive Program plan -Selected content review.

10. ANNoUnCEMENTS and REMInDERS: $10,2810,6: 30 \mathrm{pm}$

Refreshments provided by: 21 . Nard al

11. ADJOURNMENT: 8.20 pm by chairman $\sqrt{2} / 2 / 1.4 \%$

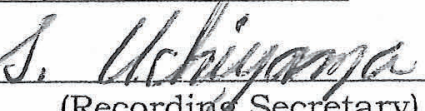

(Recording Secretary) 


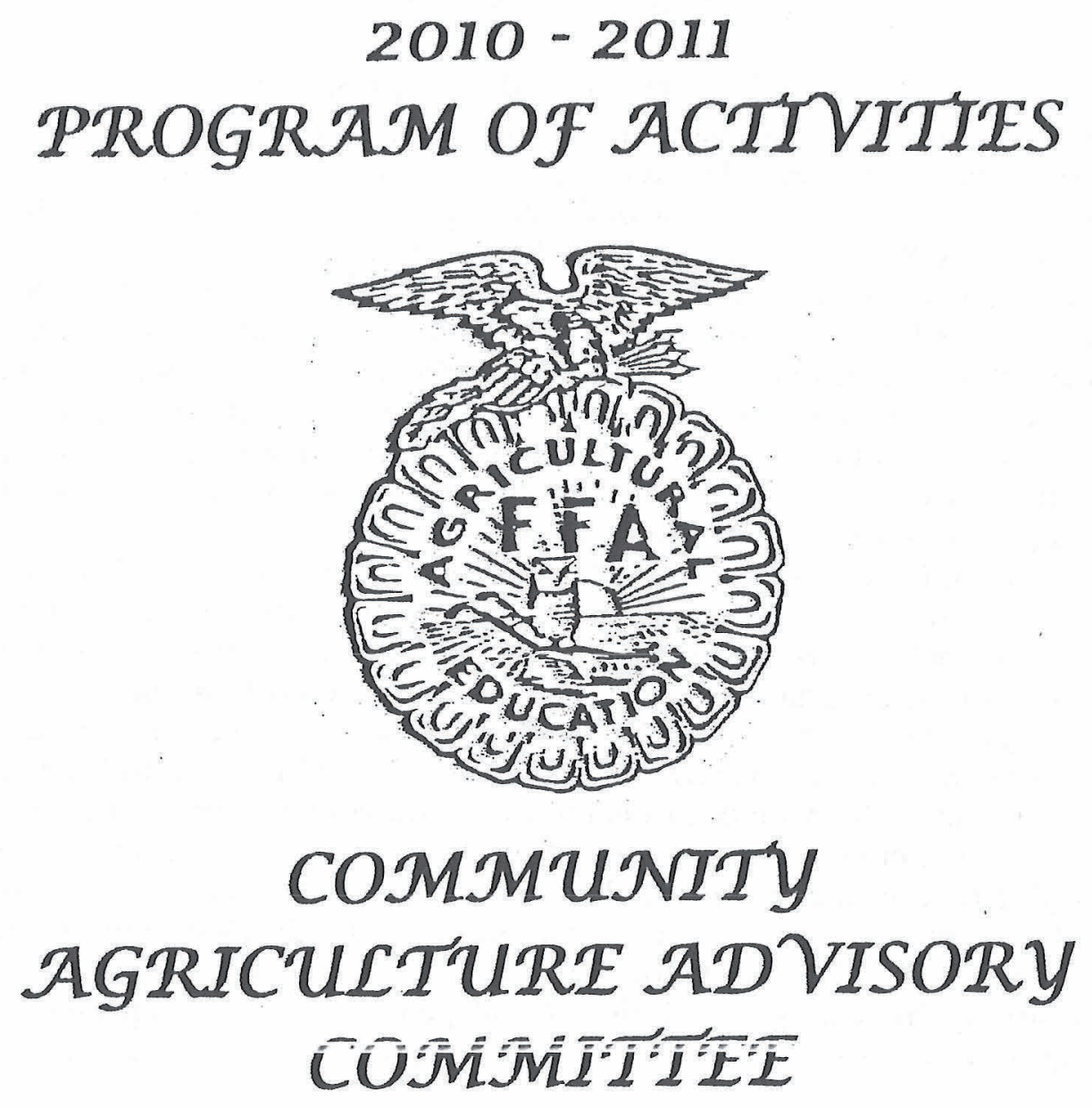

AGRISCIENCE AJD TECHINOLOGY $\mathcal{D E P} \mathcal{A R T M} \mathcal{T} \mathcal{N} \mathcal{T}$ NORTH SALINAS HIGH SCHOOL 


\title{
AGRICUCTURT ADVISORY COMMITITE
}

\author{
Organized April, 1973 \\ AgriScience and Technology Department \\ North Salinas High School \\ 55 Kip Drive Salinas, CA $93906 \quad$ (831) 796-7536
}

INT'RODUCTORY STATEMTNT

Advisory committees are established for using industry resource people to assist professional staffs at various levels of education. Agricultural education has a great need for such assistance, and when properly organized into a practical working group, benefits to local high school agriculture education programs are limitless.

Constant changes in agriculture and technology dictate the need for successful agriculturalist and other leaders in the industry to provide organized assistance to the high school agriculture education program. Agriculture today is a highly scientific, mechanized, and complicated business. New machines, technologies, materials, and methods are appearing constantly. As a result, employment skills and opportunities in agriculture change, develop, and expand rapidly. It is virtually impossible for an agriculture teacher to keep up to date with agricultural changes and carry a heavy work load at the same time.

Many areas in California are changing from rural to semi-urban and urban; yet, there is an increasing need for instruction in agriculture. The scope of high school agricultural teaching has widened and now includes preparation for careers and experiences in the occupational areas of agricultural mechanics, ag. sales and services, horticulture and landscaping, ag. business and international relations, crop science, rural recreation, pest management, animal science, forestry and natural resources management, environmental studies, education and research, as well as

preparation of skilled agricultural labor in each area. Instruction must now include the study of pollution, labor relations, environmental regulation, food safety, economics, biotechnology, and the resultant employment opportunities and requirements that exist in these new professional areas. The need for increased agricultural production and the development of new technologies demands better training in skills and techniques, and more individuals gainfully employed in specialized occupations, including completion of college educational programs. One of the

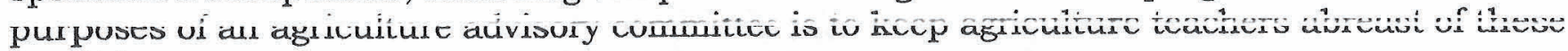
conditions.

California's high school agriculture education programs have a long history of utilizing advisory committees to assist in program improvement. The need and encouragement for local advisory committees in agriculture have been further implemented by the establishment of a state agriculture advisory committee. Additionally, a functional advisory committee is a quality indicator of a progressive agriculture education program. Industry advisory groups have no administrative or legislative authority and may not establish policy or take the place of the administrator or board of education. The function of high school agriculture advisory committee is to provide a two-way system of understanding and communication between the school and community, helping schools to be responsible to the changing needs of their communities.

\section{0-2011 AGRICUSTURT ADVISORY COMMITITE IEADERSHIT}

(committee member listing attached)

Chairman...................Mr. Josh Ruiz

Tanimura \& Antle

Vice Chairman............Mr. Gary Petersen

P \& P Sales

Secretary...............Mr. Stan Uchiyama, Teacher

Agriculture Program Manager 
2010-2011 JMETTING DATES

$\begin{array}{lll}\text { Thursday - October 14, 2010 } & 6: 30 \mathrm{pm} \\ \text { Wednesday - November 10,2010 } & 6: 30 \mathrm{pm} \\ \text { Thursday - January 13,2011 } & 6: 30 \mathrm{pm} \\ \text { Thursday - February 10,2011 } & 6: 30 \mathrm{pm} \\ \text { Thursday - March 10, 2011 } & 6: 30 \mathrm{pm} \\ \text { Thursday - April 7, 2011 } & 6: 30 \mathrm{pm} \\ & \text { (if needed ??) } & \end{array}$

\section{AGRICUCTURE ADVISORY COMMITTET GOALS FOR 2010-2011}

1. To add 2 industry committee members with emphasis on ornamental horticulture, ag. research, or ag. mechanics.

2. To sponsor Salinas Valley Ag. Industry tour in February or March.

3. To establish Ag. Community Partnership Program to support/sponsor NSHS agriculture education classroom, enterprise project program, and FFA leadership program needs.

\section{SCHOOL and DISTRICT ADMINISTRATION}

High School Administration

Principal

Mr. August Caresani $\quad 796-7510$

Admin. Intern/V.P

Mrs. Jean Ashen , 796-7514

Assist. Principal

Mr. John McKenzie $\quad$ 796-7516

Assist. Principal

Mr. Bernie Nuno

796-7512

Agriculture Department Staff

Mr. Stan Uchiyama

Dept. Chairman/Program Manager

Mr. Tom Jones

796-7500, ext. 3550

Mrs. Stephanie Blodgett

Mrs. Margaret Noroian
District Administration

Superintendent of Schools

Mr. James Earhart

796-7010

Assoc. Superintendent - Instruction

Mr. Tim Vanoli 796-7027

Assoc. Superintendent - Business

796-7013

Assoc. Superintendent - Human Resources

Mr. Alejandro Hogan 796-7037

Director, ROP/Career Education

Mr. Randy Bangs

$753-4173$

\section{0-2011 SCHOOL BOARD MEMBERS}

(April 16, 2009)

Phillip G. Tabera, President

Lila Cann

Cheryl Larison, Clerk
Kathryn Ramirez

Jim Reavis

Patty Saenz 
1. COMMITTEE CHAIRMAN: Josh Ruiz

Meeting agenda, oversee committee operations, project and/or committee assignments,

School Board reports, school Principal contacts, special program review meetings.

2. COMMITTEE VICE CHAIRMAN: Gary Petersen

Assume duties of Chairman during chairman's absence, coordination of committee assignments and projects with chairman, assist with School Board reports.

3. COMMITTEE SECRETARY - TREASURER (Parent Ag. Booster Club account): Stan Uchiyama Meeting notices, agenda and minutes printing, Ag Grant compliance reports, Ag Department update, needs and curriculum review, finance handling, maintain documents and records.

4. LIASON TO PRINCIPAL: Josh Ruiz.

Update report to Principal each semester and contacts as needed.

5. NEW MEMBERS: Dale Wallace and Rick Winburn

Member invitation letters and contacts for service.

6. CONSTITUTION and BY-LAWS: Stan Uchiyama

Annual review and approval by school administration.

7. AGRIBUSINESS TOUR or SPECIAL TRIPS: Gary Petersen and Paul Kohatsu.

Coordinate alternating tour of Salinas Valley Agribusiness tour (even number years) and World Ag Expo/Tulare Farm Equipment show trip (odd number years), including funding for transportation, entrance fees, teacher substitutes, other.

++ Members coordinate business contacts. schedules, transportation arrangements, funding resources, expense and other needs with trip Chairman.

8. VO-AG INCENTIVE GRANT, PROGRAM STANDARDS, COMPREHENSIVE PROGRAM PLAN, and PROGRAM CERTIFICATION REVIEWS: Stan Uchiyama and all committee members. Be familiar with ag. program quality standards and evaluation criteria. Be familiar with CTE Model Curriculum Standards for Agriculture, and program "Foundation" and "Pathway" standards. Conduct annual AIG compliance evaluation.

9. GRADUATE SURVEY: Stephanie Blodgett and Rebecca Collins

Conduct written survey, receive, evaluate, and report survey results.

10. FUNDING SUPPORT: Andy Madolora, Dale Wallace.

Fundraising activities for field trips, special equipment and supply purchases, other needs.

11. AG. BOOSTER SCHOLARSHIP PANEL: Tom Collier, Andy Madalora, Syble Beardslee Scholarship interview panel in late May.

12. CHAPTER SAE PROJECT COMPETITION: Josh Ruiz, Gary Petersen, Andy Madolora, Mike Johnson

Saturday judging competition in late March or early April.

13. AG. COMMUNITY PARTNERSHIP PROGRAM. Vince Ferrante and Tom Jones

14. MEETING REFRESHMENTS: Volunteer member and ag. staff rotation.

Provide light refreshment of choice. Coffee and assorted drinks provided at each meeting. 
OCTOBER, 2010

1. Program of Activity revisions, meeting dates, assignments, review monthly meeting topics.

2. Review Constitution and By-Laws, Chairman sign, school and district administration approval.

3. Review 2010-11 Ag Grant evaluation checklist, requirements, and compliance time line schedule.

4. Renew member service, new member additions, member roster update.

5. Review FFA/SAEP Grading Policy (integral components of classroom instruction).

6. Review Committee Ag Improvement Fund-status and guidelines. Fundraising project ??

7. Review school Ag. budget, Ag. Grant funding application and VEA funding allocations.

8. Update report on Department, staffing and classes, FFA, facility use and School Farm status.

9. Ag. Staff Duty and Responsibility assignment review.

NOVEMBER, 2010

1. Review AIG Quality Criteria \#1--Curriculum and Instruction. Foundation and Pathway standards

2. Review and validate Department and course descriptions, graduation credits, CSU/UC credits.

3. Review AIG Quality Criteria \#4-Qualified and Professional Personnel. Professional Development.

4. Review AIG Quality Criteria \#9-Program Accountability and Planning.

5. Send out June, 2010 graduate survey questionnaire. Review survey analysis form.

6. Approval of committee membership by School Administration and School Board.

7. Progress report to school Principal with completed POA.

8. Review October $15^{\text {th }}$ R-2 enrollment, FFA membership, and Graduate status reports.

NOVEMBER, 15, 2010. Due 2010-11 Ag Grant compliance evaluation and documentation: 5 year Facility and Equip. plans, Staff Duty Roster, FFA POA, Ag. Advisory member list and 5 meeting minutes. JANUARY, 2011

1. Review AIG Quality Criteria \#2-Leadership and Citizenship Development.

2. Review FFA Chapter Program of Activity and percent Chapter and member participation.

3. Review AIG Quality Criteria \#5-Facilities, Equipment, and Materials.

4. Review 5 year Department facility improvement and maintenance plan.

5. Review 5 year equipment purchase, repair, maintenance plan.

6. Review Livestock exhibitor County Fair eligibility policy/contract and school farm use agreement.

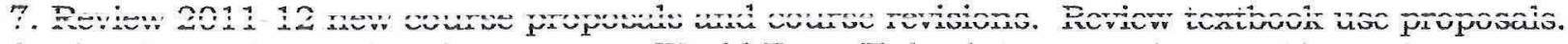

8. Plan SV Spring Agribusiness --or-- World Expo (Tulare) Farm Equipment Show trip.

9. Review 2009 Graduate Survey questionnaire and validate analysis.

10. Review O.H. 1 and Grow Orn. class curriculum and proficiency standards (odd number year).

11. Review Ag. Biology curriculum and proficiency standards (even number year).

FEBRUARY, 2011

1. Review AIG Quality Criteria \#8-Program Promotion.

2. Review AIG Quality Criteria \#10-Student-Teacher Class and SAE Ratio.

3. Review AIG Quality Criteria \#11-Full Year Employment.

4. Review AgSci 1-2 Core class curriculum and proficiency Standards (odd number year).

5. Review advanced AgSci classes: Animal/Plant Science, Livestock/Crops Production, Ag. Business Management-curriculum and proficiency standards (even number year).

6. Review 5 year School Farm facility improvement and needs plan.

7. Comprehensive Program Plan update review and validation.

8. Sponsor World Ag Expo (Tulare) Farm Equipment Show trip (even number years).

MARCH, 2011

1. Review AIG Quality Criteria \#6-Community, Business, Industry Involvement.

2. Review AIG Quality Criteria \#3-Practical Application of Agricultural Skills.

3. Review SAE Record Books, mock problem exercise, storage system, and SAEP update report.

4. Review AIG Quality Criteria \#7-Career Guidance. Post-secondary (Comm. College) articulation.

5. Review Student Data/4 Year Planning sheet and records storage system.

6. Review AgMech and Welding curriculum and proficiency standards (even number year).

7. Review Ag. Earth Sci., Chemistry, Govt/Econ curriculum 8sprof. standards (odd number year).

8. Status report of 2011-12 middle school recruitment and high school pre-registration.

APRIL, 2011

1. Conduct Spring Salinas area Agribusiness Tour (odd number year).

2. Update report to Principal, annual report to School Board.

3. Update report on year's Ag. Grant Expenditures and special project needs for 2011-12.

4. End-Of-Year barbecue/dinner meeting planning. 
2010-2011 AGRICULTURE ADVISORY COMMITTEE

(revised October, 2010)

NAME

Josh Ruiz, Chairman

Ag Production/Mgmt.

(November, 2003)

Gary Petersen, Vice Chair.

Ag Sales/Service

(November, 1991)

Paul Kohatsu

Ag Production

(March, 1992)

Dale Wallace

Ag Mechanics/Constr.

(November, 1999)

Mike Johnson

Livestock/Service

(October, 2007)

Rick Winburn

Ag Supply/Service

(November, 2003)

Andy Madolora

Ag Production/Mgmt

(October, 2005)

Tom Collier

Ag Sales/Service

(March, 2005)
BUSINESS

Tanimura \& Antle

1 Harris Road

Salinas 93901

Office: 455-4001

cell: 229-7259

P \& P Sales

449 Old Natividad Road

Salinas 93906

cell: $320-6138$

Church Bros, LLC/

True Leaf Farms

19065 Portola Drive

Salinas 93908

Office: $796-1000$

cell: (831) 809-4791

Don Chapin Company

440 Crazy Horse Cyn. Road

Salinas 93907

Office: $970-8163$

cell: $970-8163$

101 Livestock Market

4400 Highway 101

Aromas 95004

Office: 726-3303

Smurfit-Stone Containers

1078 Merrill Street

P.O. Box 81211

Salinas 93912

Office: $424-1831$

Tamagni Farms

P.O. Box 7398

Spreckels 93962

Office: $455-1233$

cell: $262-3473$

Collier Feed 86 Pet Supply

101 West Laurel Drive

Salinas 93906

Office: 443-6161
HOME

369 Barolo Circle

Greenfield 93927

674-1747

cell: 229-7259

449 Old Natividad Road

Salinas 93906

443-0566

24745 Foothill Drive

Salinas 93908

754-1945

cell: $809-4791$

535-3483

139 Rosarita Drive

Salinas 93906

757-4992

cell: 206-6006

4430 Highway 101

Aromas 95004

cell: $320-4237$

666 Middlefield Road

Salinas 93906

443-6130

cell: $970-1376$

708 Middlefield Road

Salinas 93906

443-1425

cell: $970-2099$

167 Chaucer Drive

Salinas 93901

422-6529

cell: $214-4452$ 
NAME

Vince Ferrante

Ag Production/Sales/Mgmt.

(January, 2009)

Syble Beardslee

Ag Booster Rep.

(October, 2010)

Graduate Student

Rebecca Collins

Ag Student Rep.

(October, 2010)

Mr. August Caresani

Principal, School Admin.

(November, 2003)

Stan Uchiyama, Secretary

Ag. Dept. Chairman

(February, 1973)

Tom Jones

Ay. TeacileI

(October, 2007)

Stephanie Blodgett

Ag. Teacher

(October, 2007)

Margaret Noroian

Ag. Teacher

(October, 2010)
BUSINESS

Dole Berry Company

Watsonville 95076

(831) 763-5237

Agriculture Department

North Salinas High School

North Salinas High School

55 Kip Drive

Salinas 93906

Office: $796-7510$

Agriculture Department North Salinas High School 796-7536, rooms $710-712$

Agriculture Department ivoriñ Saindas riigh Scinvoi 796-7500 ext. 3550 , rm. 730 or $796-7536$

Agriculture Department North Salinas High School 796-7500 ext. 3516, rm. 205 or $796-7536$

Agriculture Department North Salinas High School 796-7540, room
HOME

14680 Stonebridge Drive Morgan Hill 95037 (831) 233-9464

435 Old Natividad Road Salinas 93906 449-0658

cell: (831) 970-2715

19630 Agate Circle Salinas 93906 449-7175

P.O. Box 734

iviuss Liduciung 95039

cell: (559) 213-9715

1087 Harding St. Salinas 93906 cell: (530) 559-1135

274 Chaparral Street Salinas 93906 (831) 449-8536 cell: (831) 320-2234 


\section{AGRICUᄃTURE ADVISORY COMMITITE \\ "COMMUNITY PARTNERSHIP WITH EDUCATION" \\ AGRICULTURE DEPARTMENT \\ NORTH SALINAS HIGH SCHOOL \\ 55 KIP DRIVE $\quad$ SALINAS, CALIFORNIA 93906 \\ (831) 796-7536}

CONSTITUTION and BY-LAWS

FOUNDED APRIL, 1973

(Adopted, December, 1998)

(Revised, November, 2008)

\section{ARTICLE 1: THE COMMITTEE}

Section A: Statement: Programs of instruction in vocational agriculture should be advised by a committee representative of the agriculture interests of the community.

Section B: Rationale: Agricultural Education must be kept relevant to the changing nature of modern agriculture. The instructional program must be based on currently acceptable practices used in agriculture and its related industries in order for Vocational Agriculture students to succeed in the agriculture job market. This can happen more readily when teachers regularly consult with up-to-date successful agriculturists

\section{ARTICLE 2: PURPOSES AND LIMITATIONS}

Section A: The Agriculture Advisory Committee shall exist for the purpose of appraising the quality of the school's education in agriculture program and related instructional components, including its relationship within the school district's cduration in agrimblture framewrorle and gon!s.

Section B: The Agriculture Advisory Committee shall exist for as long as it shall be authorized by the Administration of the High School and the Board of Education, or when community input is necessary in appraising the quality indicators of instructional programs in vocational agriculture education.

Section C: The Agriculture Advisory Committee shall limit its activities to matters and issues which directly concern the instructional programs, policies, practices, progress-hindering problems, and general direction of the school's Department of Vocational Agriculture. All activities shall be ADVISORY ONLY.

Section D: The Agriculture Advisory Committee may direct its advice and/or recommendations toward the teachers of agriculture, the school administration, or the Board of Education, which-ever level that can provide satisfactory attention to such advice or recommendations.

\section{ARTICLE 3: FUNCTION AND DUTIES}

Section A: It shall be the primary duty of the Agriculture Advisory Committee to:

1. Study the needs of the agriculture community and vo-ag students which are related to the goals and objectives of the school's Department of Vocational Agriculture. 
2. Review and make recommendations regarding the goals, objectives, program components, and practices of the school's program of vocational education in agriculture; as study experience and community needs may warrant.

3. Aid and guide the Department of Vocational Agriculture in those activities which will lead to progress and modernization of the vocational education in agriculture program including, but not limited to, the following:

a. As needed, review and provide advice regarding courses and course content to be offered to high school and/or adult students.

b. Assist in developing student recruiting programs and/or offer counseling assistance as needed.

c. Provide advice on the instructional practices, facilities, and equipment available for program use; working towards improvement and modernization of instruction.

d. Assist in evaluating the total program or its components according to acceptable objectives and procedures, including special program standards or activities undertaken by the committee involving:

- Relevant instruction.

- Program completion standards review.

- Program management.

- Priorities review and development assistance.

- Follow-up surveys and placement of students.

- FFA component.

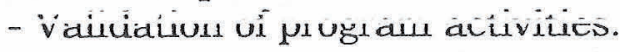

- Supervised Agricultural Experience Program component.

- Overall program planning.

Section B: As available, may serve as classroom resource persons to the instructional program.

Section C: Members shall annually review and set committee goals of service, review committee By-Laws, and Program of Activities guide.

Section D: If requested, assist in the selection process of new vo-ag staff personnel.

Section E: As needed, members shall serve as resource persons and spokesmen between the Department of Vocational Agriculture and the school administration, Board of Education, and community.

Section F: As needed, members may provide special assistance to work with groups participating in the maintenance and/or progress of the programs in vocational agriculture education.

Section G: As needed, members shall serve on ad-hoc committees formed by the general membership of this Agriculture Advisory Committee. 
Section H: As desired, committee members may undertake special projects or activities to assist AQ/FFA students in their FFA and SAEP efforts, including fundraising support.

\section{ARTICLE 4: MEMBERSHIP SELECTION AND UALIDATION}

Section A: Agriculture Advisory Committee membership shall be voluntary, non-paid service, and shall be limited only to the number and resources needed by the committee to accomplish its function and duties.

Section B: Members will be selected in such a way that they, at best, represent a cross-section of the farm and agribusiness community served by the local Department of Vocational Agriculture.

Section C: Qualifications of community members shall be determined by their successful employment or relationship within the local agriculture industry.

Section D: Members can be nominated for committee appointment by school board members, high school administration, teachers of vocational agriculture, or individual agriculture advisory comittee members.

Section E: As accepted, each Agriculture Advisory Comittee member shall be annually approved by the school principal and the Eoard of Education. Once approved by the Board of Education, addition of committee members shall only need to be approved ty the sthool poincipal

1. Initial membership roster shall be made complete by the Decemter meeting of each year=

Section $F$ : In case of yacancies, new members shall be appointed to fill those vacancies as described in the above selection and approual prosedures. Such new members shall serve only for the time remaining by the uacated position.

Section G: Upon initial contact, prospective committee members shall be notified of their nomination or appointment by the school principal, committee chairman, or their representative.

\section{ARTICLE 5: MEMBERSHIP COMPOSITION, LENGTH OF SERUICE AND VOTING}

Section A: Except for school employed personnel, each member appointed shail Eerue for a one (1) year term, corresponding with the academic year of the school.

Section B: By their consent, returning committee members are eligible for re-appointment to the committee at the first meeting of each school year.

Section C: The Agriculture Advisory Committee shall be composed of the following minimum number of representatives:

1. Six (b) community representatives from the local agriculture industry.

2. The school principal or one (1) of his administrativefunctioning representatives. 
3. At least one (1) junior or senior level vo-ag/FFA student.

4. If available, one (1) recently graduated vo-ao student who has completed a minimum of two years of enrollment and is currently employed in agriculture or is pursuing a postsecondary education in agriculture.

5. One (1) representative of the Parent Aoriculture Boosters club.

6. Each teacher of vo-ag employment at the local school.

Section D: Voting members shall include all committee members except teachers of vocational agriculture.

Gection E: Each voting committee member is entitled to one (1) vote and all prony wotes must be in writing and approved by the committee.

Section F: A quorum at meetings shall consist of four (4) members present, excluding va-ag teachers, school administration member, and high school student representative. For lack of a guorum, agenda items discussed, actions taken, etc. shall be classified as "unoficicial" or postponed until a later time.

\section{ARTICLE 6: MEETINGS}

Section A: At the first meeting of each academic year, usually in Detober, the Committee shall Establish its yearly meeting schedule: celecting a regular meeting day and week of the month. When a holiday falls upon a regular meeting date, such megting wil] , held on the following like-day or as decided by the comittee Chairman.

Section B: Comittes mettirge shall not continue for more than one hour and thirty minutes (90 minutes? after belrog called to order; unlese so extended by the committee members present.

Section C: Special meetings or sumer seruices may be called by the Chairman of the committee.

Section D: Because of the nature of member job comithents and/or out-oftown responsibilities, each comittee member is charged with the obligation of attending as many scheduled meetings as possible.

Section E: Each teacher of vocational agriculture should attend all metings. However, for multi-teacher Departments, at least one teacher shall attend every committee meeting.

Section F: An indiuidual may lose membership if he/she fails to attend three (3) successive meetings without a cause of absence directed to the committee chairman.

\section{ARTICLE 7: FINANCES}

Section A. The local school shall pay postage and duplicating costs for materials necessary in conducting the business of the comn t theg. 
Section B: Any special funds generated by the Agriculture Advisory Committee shall be deposited and accounted for through special agreement with the NSHS Parent AQ. Booster Club. Interest income earned from Committee funds shall be qiven to Parent $A g$. Booster Club as payment for handling services.

Section C: Expenditure of any special funds of the committee shall be for special needs, except those described in section $A$, and approved by the committee. Special needs shall be, but not limited to, class field trip costs, educational equipment and supplies, teacher substitute costs, student conference fees, committee member expenses for special meetings, and expenses for special committee programs

\section{ARTICLE 8: RECOMMENDATIONS AND ACTION}

Section A: Recommendations of importance to the progress and operations of the vocational agriculture program or its componerits shall be made in writing to the school principal for initial attention to or action on. A copy of written recomendations stall be given to the Vo-Ag Department. chairman for reference.

Section B: Actions on written recommendations made shall be in a timely manner and from the apgropriate level of school or district administration, whether it be informative, prohibitive; or positive in nature.

\section{ARTICLE 9: OFFICERS AND DLTIES}

Section A: The officers of the committee shall be chairman, ViceCharman: and secretary-Treasurer. These officers stiall be considered members of the Executive Cominittee.

Section B: The officers shall be annually elected or appointed by a majority yote of the comittee members present at the first meeting of the academic school year-

Section C: The Chairman shall be elected from the group of community members who have serued on the comittee for at least one

(1) year. The duties of the Committee Chairman Ehall be:

1. To preside at all committee metings and assist in developing meeting agendas.

2. To serve as Chairman of the Executive Comittee.

3. To appoint special adhoc committees which may include persons other than the committee members.

4. As necessary, to call special committee meetings.

5. To coordinate all committee activities and serve as a liason person or appoint a conmittee member to serve as a liason person between the committee and school administration, district administrators, Board of Education members, or agriculture industry members.

6. To make at least one (1) progress report to the school board each year. 
7. To meet with or select a committee representative to meet with the school principal, each semester, to discuss the prooress, needs, or concerns of the committee.

8. When applicable, to insure the selection of community conmittee member representatives to serve on established District-wide Agriculture Advisory, Adhoc, or Aoriculture Education steering committees, which-ever form shall be established.

Section $D:$ The duties of the Committee Vice-Chairman shall be:

1. To perform the duties of the Chairman during his/her absence, and to assist the chairman in coordinating committee activities, including assigned committee responsibilities.

2. When applicable, to be one of the committee representatives to serve on established District-wide Agriculture Advisory, Adhoc, or Agriculture Education steering committees, which-ever form is established.

Section E: The duties of the Committee Secretary-Treasurer shall be:

1. To prepare written notices of all reqular and special meatings and to mall them to all committee members and quests within one week prior to the scheduled meeting date.

2. To assist the chairman in meeting agenda preparations and to keep membere informed of committee meeting schedules and obligations.

3. To maintain a record of discussions, recomendations, actions taken, committee appointments, etc, and haue them auallable at each meeting.

4. To maintain a permanent record file of all comititeo activities, meeting minutes, attendance records, and other comittee materials.

5. To distribute minutes of committee meetings and copies of other comittee documents to members, principal, district administrators of vocational education, superintendent, and others who may be concerned with committee meetings and actions.

6. To deposit funds, monitor records, process expenditure approvals and make reqular financial reports to the committee on any special funds established by the agriculture aduisory committee,

Section F: The local high school facilities, equipment, and office staff shall be made auailable to assist committee officers in carrying out their appointed duties.

Section G: The duties of the Executive Committee are to direct special committee activities as directed by the chairman, committee, or as needed. 
Section G: The duties of the Executive Committee are to direct special committee activities directed by the Chairman or committee, including activities between meetings.

\section{ARTICLE 10: RECOGNITION OF SERVICE}

Section A: As allowed, recognition of service documents shall be awarded to committee members by the local high school or District Career Technical Education office.

Section B: A letter of appreciation, certificate of appreciation, or similar document of recognition shall be annually awarded to each member fully completing his/her term of committee membership service.

\section{ARTICLE 11: CHANGES IN CONSTITUTION AND/OR BY-LAWS}

Section A: Changes in the Constitution and/or By-laws can be proposed by any committee member and shall be reviewed by the Executive Committee.

Section B: Constitution and/or By-laws changes require a 30 day minimum review period before voting to adopt or reject the proposal by a majority vote of the committee members present when such vote is called for.

\section{ARTICLE 12: CONSTITUTION AND BY-LAWS APPROVAL}

Section A: The North Salinas High School Agriculture Advisory Committee shall annually review the committee Constitution and By-laws for up-dating.

Section B: The committee Constitution and By-laws shall be submitted every school year for approval by the school principal, vocational career technical education director, school superintendent, and school board chairman.

Section C: Signatures shall constitute approval of this Constitution and By-laws and shall officially recognize and support the activities of the NSHS Agriculture Advisory Committee, as outlined in Articles 2 and 3 of this document.

Section D: Copies of this document \$hall be poade available upon request.

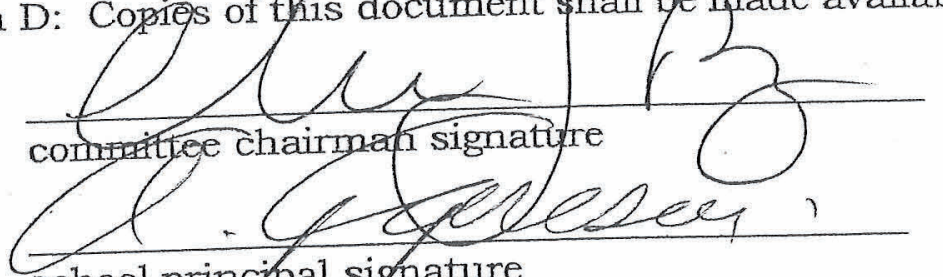

school principal signature
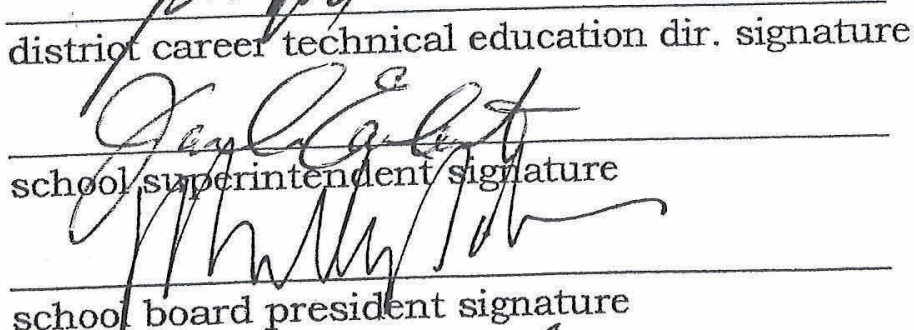

school board president signature

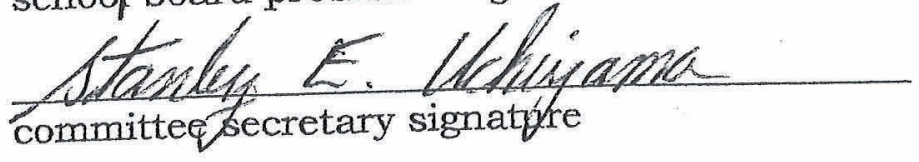

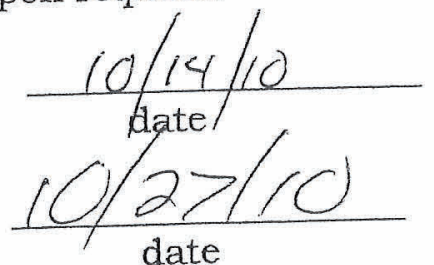
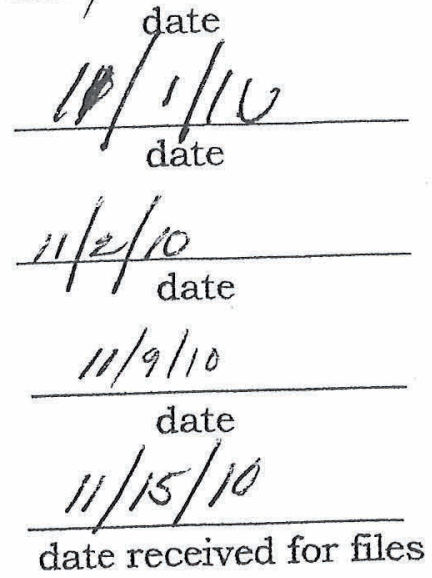


\title{
AGRICULTURE EDUCATION IMPROVEMENT FUND
}

\author{
PURPOSE AND USE GULDELINES \\ Community Agriculture Advisory Gorarflttee (rev 3/99) \\ Adopted: April 6, 1999. Mr. Paul Kohaj Chairman
}

PURPOSE. The primary purpose of the Agriculture Education Improvement Fund is to support the continuation and improvement of educational programs and services for Ag/FFA students at North Salinas High School. It is an additional funding resource and shall not supplant school funding obligations to the agriculture education program. To achieve its purpose, AgEd Improvement funds shall be used to support the following:

1. Agriculture Advisory Committee expenses necessary to complete special programs, meal functions, or other activities.

2. Field trip transportation, related expenses, and teacher substitute costs for educational activities directly involving students and unavailable through school funds.

3. School Farm repair/new construction materials purchase, special equipment needs or rentals, and Ag. Science/Shop class instructional material purchases, equipment repairs or new equipment purchases.

4. Augmented award monies for NSHS Champion market livestock. Medium/large Ag. Mechanics Best of Show constructed projects, first place Ag. Mechanics sweepstakes winner, and Best of Show Agriculture Science or Horticulture winners at local County Fairs.

5. Support of student fmancial hardship situations that hinder their involvement in agriculture education/FFA programs.

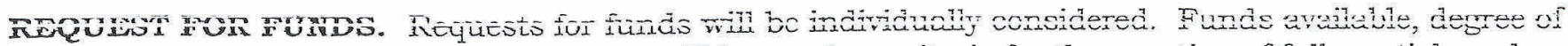
need, and existing financial commitments will be used as criteria for the granting of full, partial, or denying of request.

APPROVAL OF EXPENDITURES. Approval of expenditures shall be made by majority vote (quorum is 4) of industry committee members present at any regular monthly or special meeting of the Agriculture Advisory Committee. Approval shall be documented in meeting minutes. Approval of expenditures between meetings or summer months shall be made by the Committee Chairman, Vice Chairman, and any 2 industry committee members contacted. Such approval shall be reported at the next regular committee meeting and verified by meeting minutes.

ACCOUNTABIITY OF FUNDS. Per written agreement $(2 / 5 / 96)$, funds will be handled through the Parent Agriculture Booster Club's accounting system, including the depositing or disbursement of funds. Interest income earned shall be given to the Ag. Booster Club as payment for fund handling services. Verified by meeting minutes, account balance shall be reported at regular meetings of the Agriculture Advisory Committee.

FUNDING SOURCE. Funds raised and deposited shall be from non-School District sources. Money raised shall come from, but not limited to: direct mail solicitations, donations, special prize drawings, product sales, or other appropriate fundraising activities.

CHANGES TO USE GUIDELINES. As needed, amendments to, additions, and/or deletions of items to these guidelines shall be approved by a majority vote of industry committee members present at any regular business meeting. 


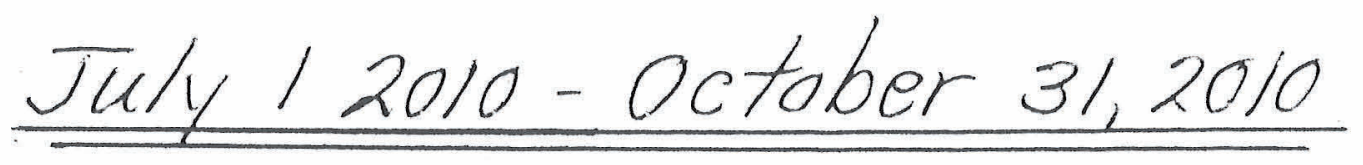

CALIIFORNIA DEPARTMENT OF EDUCATION

AGIRICULTURAL EDUCATION

INCENTIVE GRANT CHECKLIST

SCHOOL North Salinas High School

DATE

$11 / 2 / 10$

\begin{abstract}
AG DEPARTMENT CHAIR Stanley E. Uchiyama
QUALITY CRITERIA 1 - 9

Failure to meet any part of a Quality Criteria may result in the loss of $10 \%$ of the incentive funds up to a maximum of $25 \%$.

Loss of funds can be avoided with an approved variance request which may be granted for one year on any Quality Criteria 1-9.
\end{abstract}

\title{
QUALITY CRITERIA 10,11 or 12
}

Failure to meet either Quality Criteria 10, 11 or 12(when applied for) will result in the loss of the funds applied for in that criteria.

-epartment Head Signature

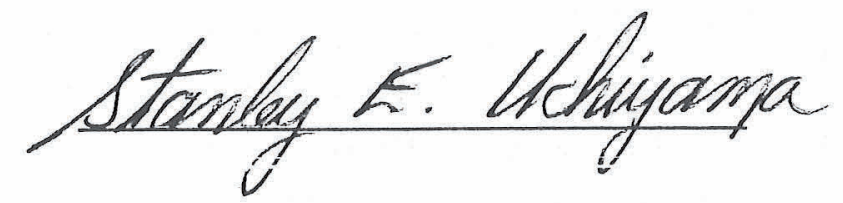

Advisory Committee Chairperson Signature

(for programs conducting Advisory Committee Reviews)

Regional Supervisor Signature

Advisory Committee Chair Contact information

\begin{tabular}{|c|c|}
\hline Name & Josh Ruiz \\
\hline Address & 369 Barolo Circle \\
\hline City & Greenfield, CA \\
\hline Phone & (831) 229-7259 \\
\hline
\end{tabular}

Zip 93927

Revised 1/10 


\section{INCENTIVE GRANT CHECKLIST}

\section{CURRICULUM \& INSTRUCTION}

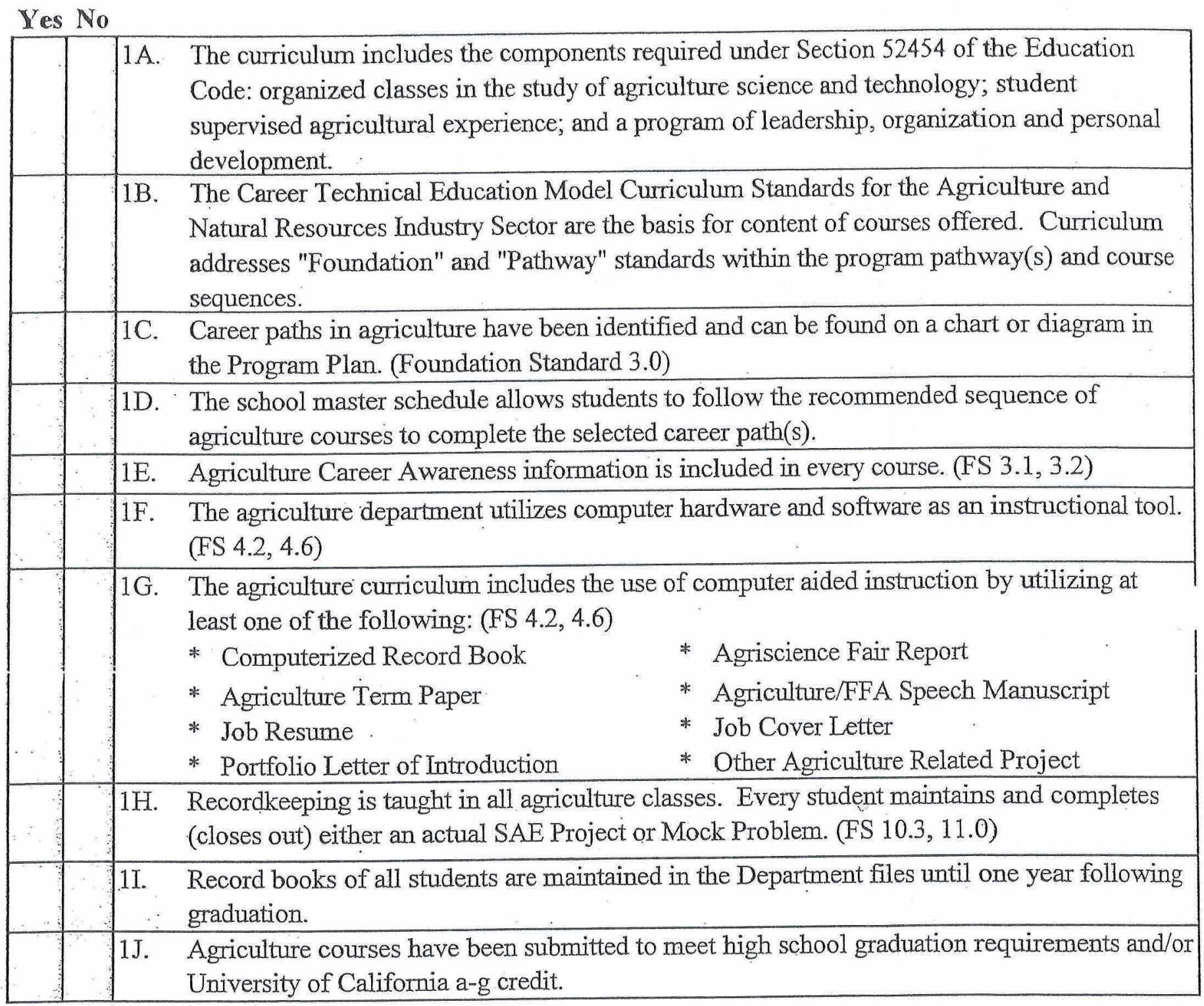

\section{LEADERSHIP \& CITIZENSHIP DEVELOPMENT}

\section{Yes No}

\begin{tabular}{|c|c|c|}
\hline & 2A. & An FFA Chapter has been chartered by the State Association or has been applied for. \\
\hline & 2B. & $\begin{array}{l}\text { A Chapter Program of Work is developed annually and a copy is furnished to the Regional } \\
\text { Supervisor by December 15th. }\end{array}$ \\
\hline & 2C. & Every student is given a grade based upon participation in leadership activities. \\
\hline & 2D. & All students enrolled in agriculture classes are affiliated with the State FFA Association. \\
\hline & 2E. & $\begin{array}{l}\text { Based on previous year's records, the department participated in a minimum of } 12 \text { activitie } \\
\text { as listed on the FFA Activities Check Sheet. (Attached) }\end{array}$ \\
\hline
\end{tabular}




\begin{tabular}{|c|c|}
\hline $2 \mathrm{~F}$. & $\begin{array}{l}\text { A minimum of } 80 \% \text { of the students participate in at least three leadership development } \\
\begin{array}{ll}\text { activities annually as verified by department records. Activities could include any three of } \\
\text { the following intra-curricular activities: (FS 7.0,9.1,9.2,9.3,9.6, 10.1) } \\
\text { * Local Best Informed Greenhand Contest } & \text { * Local Creed Speaking Contest } \\
\text { * Local Opening \& Closing Contest } & * \text { Local COOP Quiz Contest } \\
\text { * Local Program of Work Committee(s) } & * \text { Local Demonstration Fair } \\
\text { * Local Agriscience Fair Exhibition } & * \text { Local Public Speaking Contest } \\
\text { * Local Parliamentary Procedure Contest } & * \text { Chapter Meeting or Activity } \\
\text { * Any Section, Region, or State Activity } & * \text { Other Local Activities }\end{array}\end{array}$ \\
\hline
\end{tabular}

\section{PRACTICAL APPLICATION OF A GRICULTURAL SKILLS}

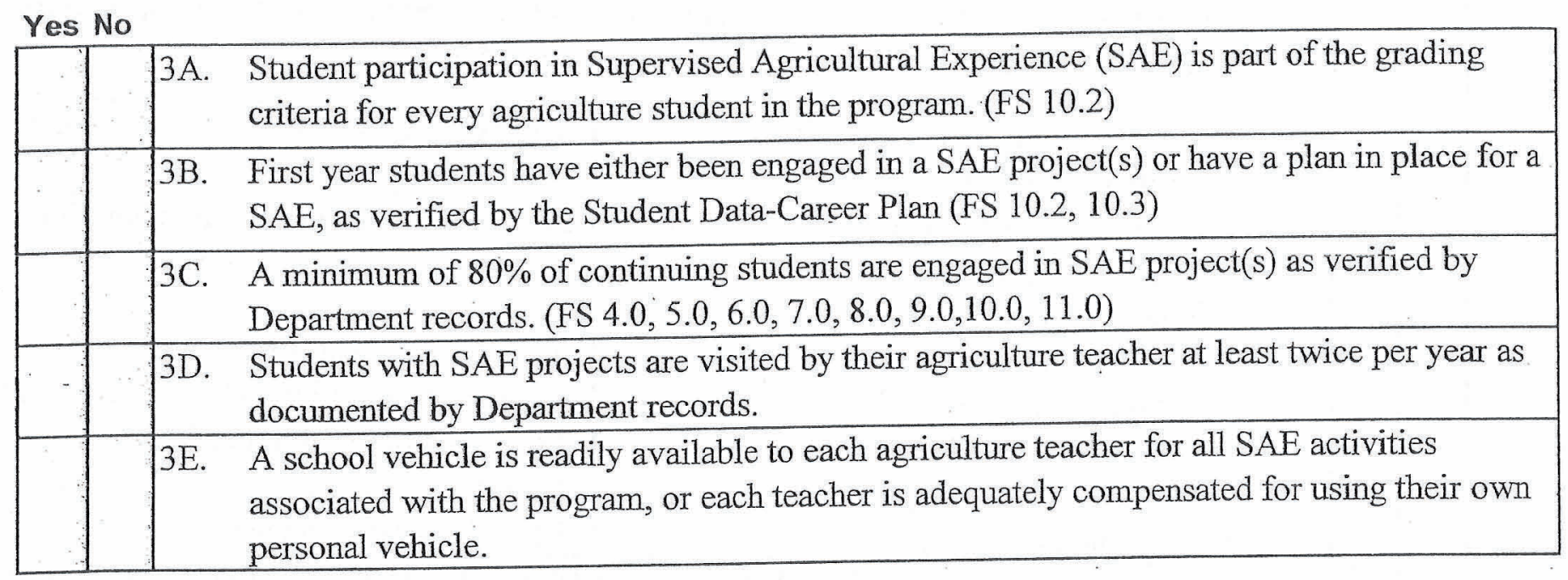

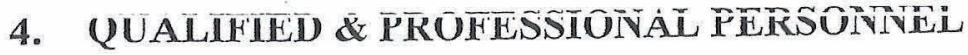

Yes No

\begin{tabular}{|c|c|c|}
\hline & 4A. & $\begin{array}{l}\text { Every agriculture teacher has the appropriate credential for teaching the subject(s) assigned. } \\
\text { Copy of authorizing credential(s) is in the Comprehensive Program Plan. }\end{array}$ \\
\hline & $4 B$. & $\begin{array}{l}\text { Based on the previous year's records, every agriculture teacher, teaching at least } 1 / 2 \text { time } \\
\text { agriculture, attends a minimum of four professional development activities: (Complete } \\
\text { attachment). }\end{array}$ \\
\hline & $4 \mathrm{C}$. & $\begin{array}{l}\text { The agriculture staff meets a minimum of twice a month. (This criteria does not apply to } \\
\text { single person departments - mark column N/A = Not Applicable) }\end{array}$ \\
\hline & 4D. & $\begin{array}{l}\text { A written record of minutes is kept of action taken during agriculture staff meetings } \\
\text { and is kept in Department files or the Comprehensive Program Plan. (This criteria does } \\
\text { not apply to single person departments - mark column N/A = Not Applicable) }\end{array}$ \\
\hline & & $\begin{array}{l}\text { Teachers are reimbursed for personal expenses they incur while participating in all approved } \\
\text { integral activities associated with FFA, SAE, and professional CATA in-service activities. }\end{array}$ \\
\hline
\end{tabular}

\section{FACILITIES, EQUIPMENT \& MATERIALS}

Yes No

\begin{tabular}{|r|ll|}
\hline & $\begin{array}{l}\text { 5A. } \\
\text { Modification of facilities and equipment has occurred when necessary, based on the } \\
\text { needs of students, including special populations. }\end{array}$ \\
\hline & $5 \mathrm{~B}$. & There is adequate storage space for materials, records, equipment and supplies. \\
\hline
\end{tabular}




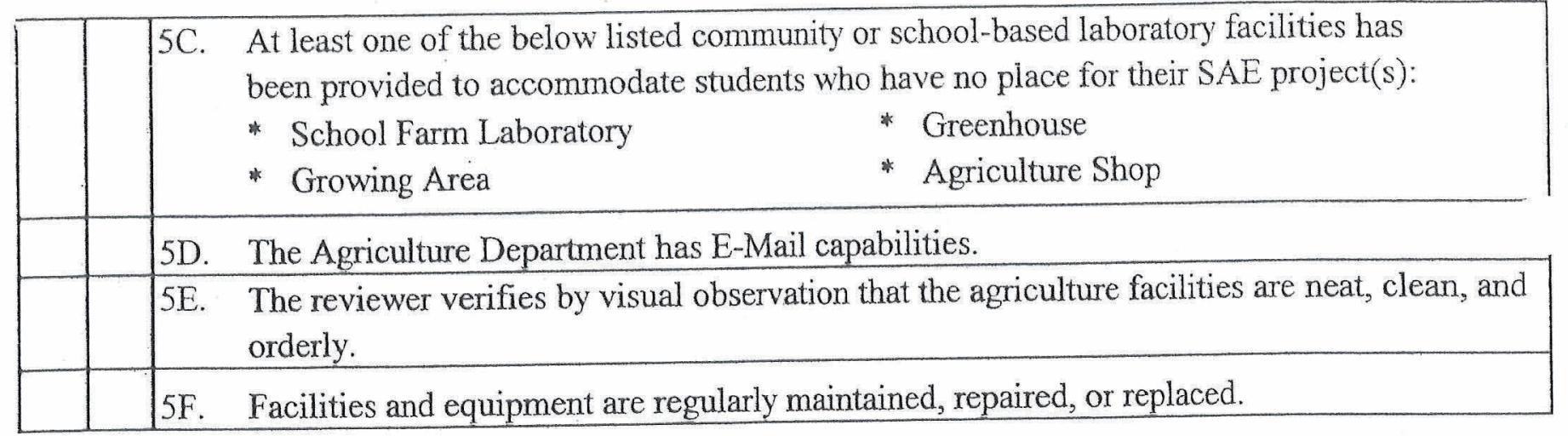

\section{COMMUNITY, BUSINESS AND INDUSTRY INVOLVEMENT}

\begin{tabular}{|c|c|c|}
\hline & $6 \mathrm{~A}$. & $\begin{array}{l}\text { The Advisory Committee is operational and reflects the committee membership as outlined } \\
\text { in the "Agricultural Education Advisory Committee Manual". }\end{array}$ \\
\hline & $6 \mathrm{~B}$. & $\begin{array}{l}\text { The Agricultural Advisory Committee meets at least twice each year. (Minutes are available } \\
\text { to verify meetings.) }\end{array}$ \\
\hline & $6 \mathrm{C}$. & $\begin{array}{ll}\text { The Agricultural Advisory Committee has assisted in the development or revision of the } \\
\text { following components of the Comprehensive Program Plan, as evidenced in the Ag. } \\
\begin{array}{ll}\text { Advisory Committee minutes } & * \text { Targeted Occupations } \\
\text { * Job Market Description } & * \text { Program Description - Courses, SAE, FFA } \\
* \text { Total Program Goals \& Objectives } & * \text { Program Completion Standards } \\
* \text { Course Subject Matter Outlines } & * \text { Current Year Budget } \\
* 5 \text { Year Facility \& Equipment Acquisition } & * \text { List of Active placement Sites } \\
\text { Graduate Follow Up } & \text { * }\end{array}\end{array}$ \\
\hline$x$ & $6 \mathrm{D}$. & $\begin{array}{l}\text { The contact information of the Advisory Committee Chair has been provided on the cover } 01 \\
\text { this checllist }\end{array}$ \\
\hline
\end{tabular}

\section{CAREER GUIDANCE}

Yes No

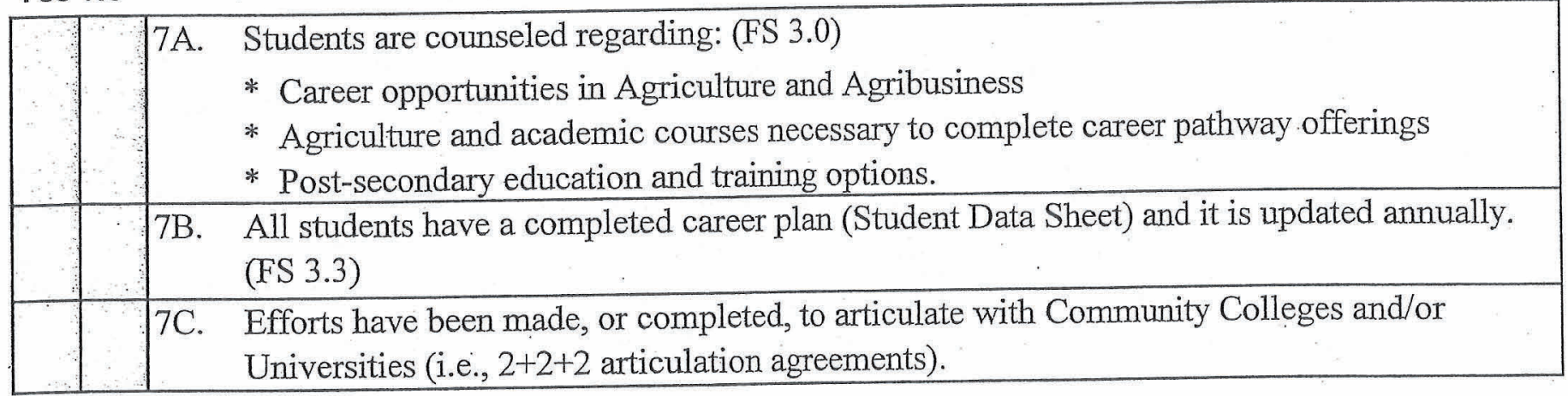

\section{PROGRAM PROMOTION}

Yes No

\begin{tabular}{|c|c|l|}
\hline & $8 \mathrm{~A}$. & $\begin{array}{l}\text { An Agricultural Education program recruitment brochure or similar document is used to } \\
\text { promote the program. }\end{array}$ \\
\hline & $8 \mathrm{~B}$. & $\begin{array}{l}\text { Students have alternative means of overcoming financial barriers to participate in program } \\
\text { activities. (Includes FFA, SAE, Leadership Activities.) }\end{array}$ \\
\hline & 8C. & The Agriculture Department conducts recruitment activities with local feeder schools. \\
\hline
\end{tabular}




\section{PROGRAM ACCOUNTABILITY \& PLANNING}

Yes No

\begin{tabular}{|c|c|c|}
\hline$\therefore$ & $9 \mathrm{~A}$. & $\begin{array}{l}\text { A Comprehensive Program Plan is on file with the Regional Supervisor and a copy is } \\
\text { retained in the local department files. }\end{array}$ \\
\hline & $9 \mathrm{~B}$. & $\begin{array}{l}\text { Updates of the Program Plan are sent to the Regional Supervisor by November 15th. These } \\
\text { updates include: (1) Five Year Equipment Acquisition Schedule; (2) Chart of Staff } \\
\text { Responsibilities; (3) FFA Program of Work; (4) Advisory Committee Roster; and (5) } \\
\text { Advisory Committee Minutes. }\end{array}$ \\
\hline & $9 \mathrm{C}$. & $\begin{array}{l}\text { A follow-up system is used which gathers the following information from program } \\
\text { * Status of employment or school enrolled within } \\
\text { * Opinion regarding the value and relevance of the agriculture program } \\
\text { * Suggestions for improving the agriculture program }\end{array}$ \\
\hline & 9D. & $\begin{array}{l}\text { The Graduate Follow Up data collected was entered with the On-line R2/FFA Roster Data } \\
\text { Entry by October 15th. }\end{array}$ \\
\hline & $9 \mathrm{E}$. & $\begin{array}{l}\text { The Agriculture Department analyzes their student retention numbers each year and } \\
\text { develops strategies to help increase retention within the program. }\end{array}$ \\
\hline & & $\begin{array}{l}\text { The R-2, AIG Expenditure Reports, and FFA Roster have been received by the Regional } \\
\text { Supervisor and/or State FFA Financial Coordinator on or before October } 15 \text { th. }\end{array}$ \\
\hline
\end{tabular}

QUALITY CRITERIA 10, 11 and 12 MUST BE SCORED DURING THE REVIEW PROCESS. HOWEVER, SCORES WILL ONLY COUNT IF THESE CRITERIA HAVE BEEN APPLIED FOR VIA THE AGRICULTURE INCENTIVE GRANT APPLICATION.

Yes No
\begin{tabular}{|c|c|c|c|}
\hline & $\begin{array}{l}\text { 10A. Shop and laboratory-based classes have no more than } 20 \text { students enrolled. Classroom-based } \\
\text { classes have no more than } 25 \text { students enrolled. }\end{array}$ \\
\hline & $\begin{array}{l}\text { 10B. The total number of students enrolled in agriculture classes does not exceed } 75 \text { students per } \\
\text { teacher. First year students enrolled in agriculture courses will be counted as .5 for purpose } \\
\text { of determining the total count only. (This does not pertain to class size.) }\end{array}$ \\
\hline
\end{tabular}

\section{FULU YEAR EMTPLOYMENT}

\section{Yes No}

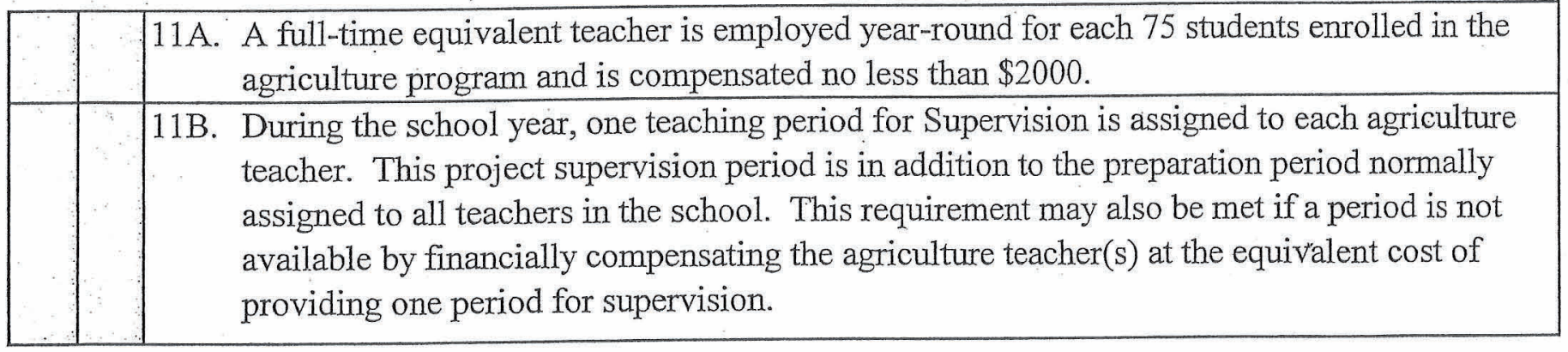

\section{PROGRAM ACHIEVEMENT}

Yes No

12A. The Agriculture Program meets the requirements of Program Achievement (attach checklist) 


\section{Budget}

At North Salinas High School our department's budget is lumped together as instructional supplies. In order to spend money we have to fill out a purchase order and get it approved by the district before the funds can be permitted. After funds are approved one of the school's secretaries will send off the order to the company we are ordering the supplies from. As we spend allocated money the district uses formula fund codes and subtracts the specific amount spent from that funding source. When the items ordered are received, we compare the items disclosed on the packing slip to the items in the actual order. When we have confirmed that the entire order has been received, the district then sends a check to the company to pay for the supplies. 


\section{$2010-2011$ \\ AGRICULTURE DEPARTMENT FINANCIAL SUPPORT RESOURCES \\ North Salinas High School (rev. 9/10) \\ (Source, Allocation, and Funded Areas)}

PROGRAM AREAS FUNDED: (allocations subject to change, pending program needs.)

a. Department: Operations, facilities, equipment, travel and conference.

b. Classroom Instruction: Ag. Science1-2, Ag. Earth Science, Ag. Chemistry, Ag. Govt/Econ, Ag. Welding, and Ag. Mechanics.

c. Integral Programs: Student Ag. Leadership, SAEP, School Farm, and Support Groups.

\section{INSTRUCTIONAT SUPPLY, EQUIPMENT, REPAMRS, ANA SERVICES.}

1. School Site Formula Funds. $\$ 5,600$

- Department and Class Supply, Equipment, and repairs........\$2,600

- Copy Machine Service Contract..........................................\$ 600

- 2009-10 Special School Carry-over Allocation ...................\$2,400

- $\$ 750$ per 3.2 FTE - spend on respective classroom supply.

2. VEA (Perkins) Funding $\$ 8.500$

- Department and Class Supply, Equipment, and repairs.

3. Vo-Ag Incentive Grant $\$ 15,174$

- Department and Class Supply

$\$ 8,674$

- New Equipment

$\$ 3,000$

- Equipment Repairs and Services

$\$ 2,500$

- Ag. Truck/Vehicle Operation.

$\$ 1,000$

\section{TEACHER TRAVEL AND CONFERENCE REGISTRATION/LODGING.}

1. VEA (Perkins)---3 Teachers ...(need request to add $4^{\text {th }}$ teacher)

$\$ 3,000$

- CATA RC fion Af́ctings and In Serrice--4 teachers

- State FFA Leadership Conference---3 teachers....................\$ 760

- CATA Summer Conference---3 teachers.........................\$2,490

(<\$750-AIG/NSHS? )

2. Vo-Ag Incentive Grant---4 teachers. $\$ 4,500$

- Department, CATA, FFA, SAEP, Professional Dev. $\$ 4,150$

- Spring CP Intern Teacher ??

3. School Site/Principal Formula Funds (pending approval)

Teacher Registration and Lodging Costs.

- Spring CP Intern Teacher ??.....................................\$ 400

- Master/Intern Teacher Conference (U) ...............................\$ 150

- Fourth Ag. Teacher................................................\$\$ 1,000

+ State FFA Ldr. Confer. Reg $\&$ Lodging.

+ SVF Lodging.

+ CATA Summer Confer. Reg \& Lodging.

NON-SALARY TOTAL .........\$38,324 
Page 2: 10-11 Ag. Dept. Financial Support

\section{SUBSTITUTE TEACHER SUPPORT}

1. VEA (Perkins)---3 teachers... (need request to add $4^{\text {th }}$ teacher)

$\$ 2,250$

18 days $\mathrm{x} \$ 125$ for Curriculum Development, Professional Improvement, and $\mathrm{Ag} / \mathrm{FFA}$ Leadership Events.

- 6 days District Ag. Curriculum Development.

- 3 days CATA December In-Service.

- 3 days Region FFA Meeting and Contest Finals.

- 3 days Spring Region CATA Meeting.

- 3 days State FFA Leadership Conference.

2. School Site---4 teachers $\$ 4,000$ 32 days x $\$ 125$ for Department, Curriculum Development, Professional Improvement, SAE, and Ag/FFA Leadership Events August through June.

\section{TEACHER CONTRACTED SERVICES}

1. Ag/FFA and SAEP Supervision Stipend-- 1 teacher. 3,067 - August,2010 thru June, 2011.

2. Summer Agriculture SAE/FFA Program Teacher Employment \$ 6,087 - 130 hours (July, Aug, June) x $\$ 37.53$ $\$ 4,878$

- Summer ROP Animal Care Class. $\$ 1,209$

3. Department Head Stipend. $\$ 1,916$ 
I - FINANCIAL SCHEDULE

Part A

\begin{tabular}{|c|c|c|c|c|c|}
\hline & & & A & B & C \\
\hline Line & $\begin{array}{l}\text { Acct. } \\
\text { No. }\end{array}$ & Classification & $\begin{array}{l}\text { Description of Item for } \\
\text { which Funds will be } \\
\text { Expended }\end{array}$ & $\begin{array}{l}\text { Incentive } \\
\text { Grant Funds }\end{array}$ & $\begin{array}{l}\text { Matching } \\
\text { Funds }\end{array}$ \\
\hline 1 & 4000 & Books \& Supplies & & $12,874.00$ & $18,000.00$ \\
\hline 2 & & . & Subtotal for 4000 & $\$ 12,874.00$ & $\$ 18,000.00$ \\
\hline 3 & \multirow[t]{6}{*}{5000} & \multirow{6}{*}{$\begin{array}{l}\text { Services and other Operating Expenses } \\
\text { such as: Personal Services of } \\
\text { Consultants, Staff Travel, and } \\
\text { Conference; Rentals, Leases, and } \\
\text { Repairs; Bus Transportation }\end{array}$} & 1 Travel \& Conference & $4,500.00$ & $4,500.00$ \\
\hline 4 & & & 2 Equip Serv/Repair & $1,500.00$ & $1,500.00$ \\
\hline 5 & & & 3 Vehicle/Bus Operation & 800.00 & 800.00 \\
\hline \multirow[t]{2}{*}{6} & & & 4 & & \\
\hline & & & 5 & & \\
\hline 7 & & & 6 & & \\
\hline 8 & & & Subtotal for 5000 & $\$ 6,800.00$ & $\$ 6,800.00$ \\
\hline 9 & \multirow[t]{5}{*}{6000} & \multirow{5}{*}{$\begin{array}{l}\text { Capital Outlay: Includes Sites and } \\
\text { Improvements of Sites; Buildings and } \\
\text { Improvement of Buildings; Equipment }\end{array}$} & 1 & & \\
\hline 10 & & & 2 & & \\
\hline \multirow[t]{2}{*}{11} & & & 3 & & \\
\hline & & & 4 & & \\
\hline 12 & & & 5 & 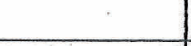 & \\
\hline $1-$ & $\because$ & $\therefore$ & Subtotal for 6000 & $\$ 0.00$ & $\$ 0.00$ \\
\hline 14 & & & $\begin{array}{c}\text { Total for } 4000-6000 \\
\text { Lines } 2,8,13\end{array}$ & $\$ 19,674.00$ & $\$ 24,800.00$ \\
\hline
\end{tabular}

TOTAL 2010-2011 Incentive Grant Allocation: $\$ 19,674.00$

Part B - Complete this portion if a waiver of the matching requirement was granted:

\begin{tabular}{|c|c|c|c|c|c|}
\hline & & & A & $B$ & $\mathrm{C}$ \\
\hline Line & $\begin{array}{l}\text { Acct } \\
\text { No. }\end{array}$ & Classification & $\begin{array}{c}\text { Description of ltem for } \\
\text { which Funds were } \\
\text { Expended }\end{array}$ & $\begin{array}{l}\text { Incentive } \\
\text { Grant Funds }\end{array}$ & $\begin{array}{c}\text { Amount of } \\
\text { Salary and } \\
\text { Benefits } \\
\end{array}$ \\
\hline 15 & 1000 & Salaries & $\begin{array}{l}\text { Teachers' Summer Service } \\
\text { Salaries }\end{array}$ & & $4,879.00$ \\
\hline 16 & 1000 & Salaries & $\begin{array}{l}\text { Teachers' Salaries for } \\
\text { Project Supervision Period }\end{array}$ & 8 & \\
\hline 17 & 3000 & Benefits & $\begin{array}{l}\text { Benefits for the Above } \\
\text { Items (1000) }\end{array}$ & & 661.00 \\
\hline 18 & 3 & & TOTAL & & $\$ 5,540.00$ \\
\hline
\end{tabular}




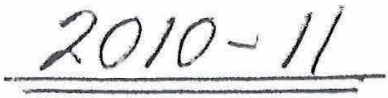

Mission Trails Regional Occupational Programs

867 East Laurell Drive

Salinas, CA 93905

TO: $\quad$ Agriculture and Natural Resources

FROM: $\quad$ Gina Muller

$$
\text { 1. mulec. }
$$

DATE: $\quad$ May 10,2010

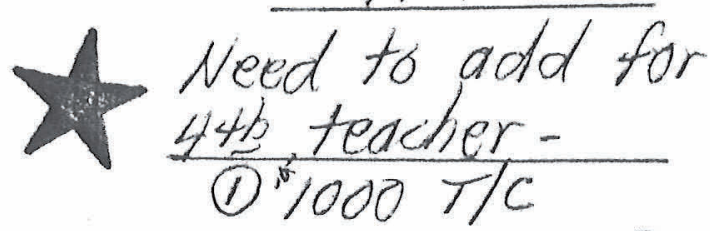

SUBJECT: $\quad$ Agriculture and Natural Resources VEA Allocations for 10-11

(2) 2 day curr. Deis sub. (3) 2 day FFA Travel. sub. As part of the district 5-year plan the following gives you the district allocation for the Agriculture and Natural Resources Department this school year. Please note that there are three district categories for this allocation.

(4) 2 day Prof, OQV, Sub.

\section{A. CURRICULUM DEVELOPMENT}

To align the Agriculture and Natural Resources Department with the state CTE model curriculum and framework. In addition CTE instructors will align their new curriculum with the embedded academic standards from Language Arts, Math, Science \& Social Studies.

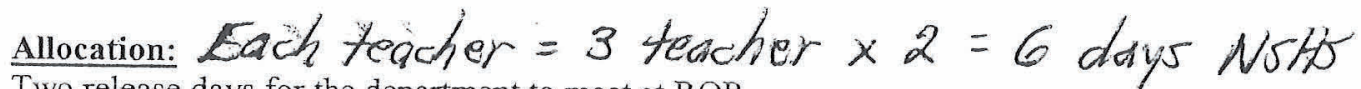
Two release days for the department to meet at ROP.

\section{B. PROFESSIONAL DEVELOPMENT}

$$
\text { * Ais pay teacher meal, other costs }
$$

To allow an Agriculture teachers from SHS, NSHS, and EAHS to attend a conference that is directly related to the CTE model

curriculum standards framework.

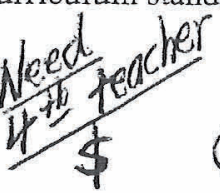

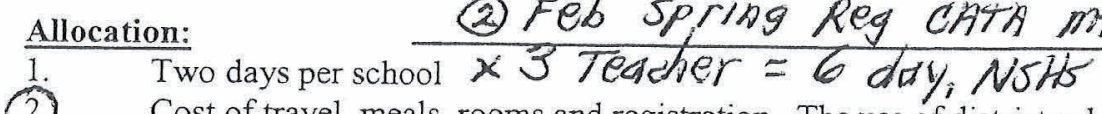

(1) DeC Ag Ed "Rond show" - 3 days

Cost of travel, meals, rooms and registration. The use of district vehicles is highiy encouraged, as mileage may not be available. (\$1000 per teacher) - Propased for 3 teaches, NJHe (50000)

(1) Spring Rer. FFA Travel \$ 7500

FFY MYT - KC SHS - 2 days, 1 teachers

2) state Lidr:

confs - Fresno

EAHS - 2 days, 3 teachers

NSHS - 2 days, 3 teachers

C. INSTRUCTIONAL IMPROVEMENT

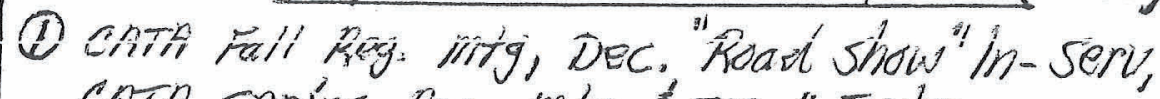
CATA spring Reg. Mntg. $\$ 500-4$ Teacher

(2) State FFh Ldr Conf. (Reg and lodging) Regiging:-400

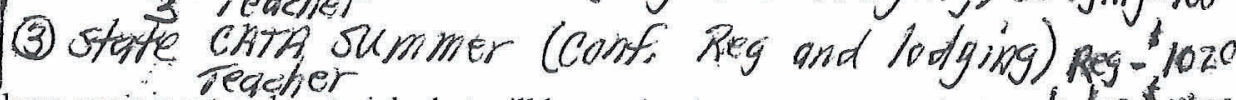

This is to select one or two program areas and purchase equipment and materials that will be used to improve or upgrade the /odge $-1 / 70$ instructional materials to meet the aligned curricular standards and frameworks.

$\frac{\text { * } \frac{\text { Allocation: }}{\text { Instructional Materials \& Equipment }}}{\frac{\text { NSHS } \$ 8,500}{\text { SHS } \$ 8,500}}$

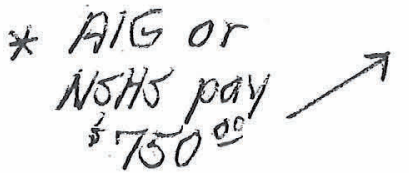

Please note: these allocations may change due to total number of Career Tech Ed sections in the 2010-2011 master schedule.

You must have your principal's approval as well as our approval for use of these funds. Please include justification for all professional development and instructional improvement activities.

All purchase orders associated with these CTE allocations must be turned in by June 03, 2010 to Karla at the ROP Center.

$\mathrm{SM} / \mathrm{km}$

$$
N_{0}=\text { changing mgmt system. weit until loste fill. }
$$

cc: Tim Vanoli, District Office

Augie Caresani, NSHS

Sascha Heckmann, SHS

Darin Hershberger, EAHS 
DATE: June 18, 2009

TO: Mr. A. Caresani, Principal, North Salinas High School

FROM: Stan Uchiyama, Vo-Ag Teacher

\section{SUBJECT: EXTRA WORK AGREEMENT PROPOSAL: 2009-2010 SUMMER AGRICULTURE PROGRAM VO-AG TEACHER EMILOYMENT}

NEED, IMPORTANCE, AND RATIONAL FOR SUMOMER VO-AG PROGRAMS. (Refer to SB-187 State Vo-Ag Advisory Committee Report adopted by State Board of Education; 2005-2006 Vo-Ag Incentive Grant Compliance Review Quality Standard Number 11, Full Year Employment and Standard Number 3, Practical Application of Occupational Skills.):

Effective instruction in Vocational Education in Agriculture extends far beyond the regular school day, school year, and school environment. The basic component of instruction occurs as group instruction in the classroom, shop, or field during regularly scheduled classes September through June. The unique character of California Vo-Ag education programs encourages continued instruction during the summer months, July through August.

The vocational agriculture teacher's supervision of student agricultural/occupationai experiences (SAE) is to (1) provide individual instruction as needed; (2) assist the studen in selecting and conducting occupational experiences which are relevant to his or her occupational objectives and which include opportunities to reinforce learning and apply skills and knowledge in class or shop; (3) counsel the student in employer-employee relationships; (4) assist in locating suitable empioyment for program students and graduates; (5) provide opportunities for student leadership development training and skills acquisition; and (6) provide supervisory functions and activities for student enterprise projects leading to their successful completion.

Since the instructional program is considered to be best accomplished on a year-round basis for. reasons stated previously, and since FFA is an integral part of the agriculture education instructional program, it follows that the FFA advisors(s) should be available to supervise and conduct those FFA leadership and enterprise project activities which are scheduled for the summer months and other times ouiside the regular class schedule. To meet the Summer Vo-Ag program objectives, needs of participating students and to maintain Ag. Program continuity, teacher compensation is necessary.

BACRGROUND: The 2007-08 Summer Vo-Ag program provided \$5314 (130 hours at \$37.53 per hour, inciuding teacher benefits) for 1 FTE agriculture teacher at North Salinas High School. Per - annual Ag. Incentive Grant specifications and compliance evaluations, \$4000 in additional state funds were received and used for non-salaried program expenditures during the 2008-09 school year.

For the 2009-10 year, our School will continue to have a summer ag. program that will involve 1 FTE ag. teacher. Our 2009-10 enrollment will, again, qualify our school to receive additional State Ag. Grant funding for non-salary Ag. Program use. Without 2009-10 District Summer Ag. Program salary support our school will not qualify to receive the full Ag. Grant funds available to us. Receiving the maximum amount of Ag. Grant funds is critical to the effective year-long operation of North High's active agriculture program. 
PROPOSAl: Two FTE North High ag. teachers be retained to be responsible for the conduct of student enterprise projects, school farm management supervision, FFA student leadership activities, applicable contact with incoming students and parents, Ag. Booster Club activities, summer program management needs, Ag. facilities and equipment maintenance, and supervision of student participation in the Monterey and Santa Cruz County Fairs. Documented teacher activity logs for summer employment have far exceeded the hourly allocations for any one past summer employment period. Minimum teacher duties are proposed and teachers will submit detailed activity description logs and time sheets to validate their performance of described duties. Although it is not equitable to surrounding School Districts providing summer $\mathrm{Vo}-\mathrm{Ag}$ employment for their teachers, the following 2009-10 Summer Vo-Ag Program employment plan is proposed:

1. Summer Ag. Program Support Expenses:

* Ag truck operational costs: Paid by our site budget allocations.

* Teacher expenses and private car mileage: Paid from our school's 2009-10 Ag. Grant or other available North High funding sources.

II. Ag. Teacher Summer Ag. Program Employment:

* Two FTE ag. teachers. 260 employment hours, $\$ 37.53$ hourly rate $=\$ 9,758$

(130 hours/FTE)

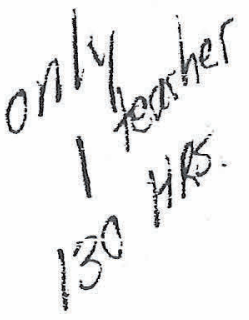

-- Teacher hour allocations are for July-August, 2009 and June, 2010. We will save an optional 8 hours for June, 2010 use.

- 70 students involved with 21 County Fair (MoCo and S.C.C.F) animal, 108 ag. mechanics, $47 \mathrm{ag}$. science, Chapler Feature Dooth, and livestock educational exhibits.

\section{MPLEMENTATION, COMPLIANCE CRITERIA, AND MONIMUM TEACHER DUTIES FOR SUMAMER VO-AG PROGRAM.}

1. Construct a calendar of activities for FFA leadership development and SAEP activities for July and August.

2. Descriptive activity logs will be kept by teachers to verify their performance of duties and shall be used to validate monthly salary allotments.

3. Teachers shall be responsible for the up-keep and maintenance of the agriculture truck, school farm facility, school site facilities, and equipment, as required by our school's ag. program needs.

4. Teachers will make reasonable project supervision contacts with involved students. Number of supervision visits/contacts made shall be according to student project type and/or needs.

5. Hourly salary time may include time spent for summer program/ag. department administration work, community relations, and other related activities of benefit to the supervised project, Vo-Ag and FFA summer program. Time spent shall be balanced with student supervision time. 
6. Teachers will conduct FFA and Ag. Program activities and supervise those events which are necessary to the proper functioning of the FFA Chapter and Vo-Ag programs during the summer months, including work with support groups, new activities, and other necessary duties related to successful summer program operations.

7. Out-of District travel (School Business Agreements) and reimbursement requests shall be approved by and/or filed with school administration.

8. Teachers make plans to contact or involve incoming freshman students and potential new students. Such plans shall be the design of each teacher, pending availability or reasonable implementation time.

RESPONSE: To allow for agriculture staff coordination of summer program duties, calendar schedule development and miscellaneous payroll filing deadlines, I await your timely response to my proposal. Thank you for your consideration.

Cc: Mr. T. Jones

Ms. S. Pfarr 


\section{Dept.

NORTH SALINAS HIGH SCHOOL AGRISCIENCE AND TECHNOLOGY DEPARTMENT

\section{MEMORANDUM}

TO: Jim Earhart, Tim Vanoli, R. Bangs, August Caresani, Cindy Fellows

FROM: Stan Uchiyama

SUBJ]ECT: 2010-20011 Vo-Ag Incentive Grant Application filing

DATE: June 16, 2010

Attached is a copy of our school's $\$ 19,674$ Vo-Ag Incentive Grant application for the 2010-2011 school year. Our Grant application will involve two and five-sixth teachers at North Salinas High School. In addition, I have attached the Grant's processing/approval timeline for your reference. I did take the liberty to identify that North High's application would be School Board approved by August 31, 2010.

It is understood that current State and District financial allocations may change in the next few months. As such, budget data on Part VI, Financial Schedule A will be revised to reflect those changes. Column C, matching funds of the Financial Schedule comes from 2009-2010 Ag. Progtam support funds to date and estimated 20010-2011 NSHS site, District, ROP allocations and Perkins (VEA) funding sources. Data was provided to me by NSHS, District, ROP/VEA, and transportation administrative personnel. As in the past, it is acceptable to use ROP-Flotistry funds to meet matching funds criteria for this grant. If needed, additional matching funds can be possibly added from $\mathrm{Ag}$ Booster and/or $\mathrm{Ag}$ Advisory Committee sources. All matching fund sources will be validated in the end-of-year's Report of Expenditures that must be filed by October 15, 2011.

I have applied for a compliance waiver to the Student-Teacher and Ptoject Supervision Ratio criteria \#10. If we are not granted the waiver, I will submit a revised Ag Grant budget reflecting this $\$ 5,666$ loss. As long as we do not supplant out District's, Perkins, and school's regular on-going expenditures for North High's Ag Program, I do not see any problems in submitting revised budgets for approval by our State $\mathrm{Ag}$ Ed Unit.

Thank you for your help in our Ag Incentive Grant application process and may our efforts achieve another successful application year. 


\section{Califomia Department of Education \\ AGRICULTURAL CAREER TECHNICAL EDUCATION INCENTIVE GRANT 2010-11 APPLICATION FOR FUNDING}

(Due Date: To be received in Regional Supervisor's Office by June 30, 2010)

April 2010

DATES OF PROJECT DURATION - JULY 1, 2010 TO JUNE 30, 2011

North Salinas High School

(School Site)
Salinas Union High School District

(District)

Certification: I hereby certify that all applicable state and federal rules and regulations will be observed; that to the best of my knowledge, the information contained in this application is correct and complete; and that the attached assurances are accepted as the basicconditions of the operations in this project/program for local participation and assistance.

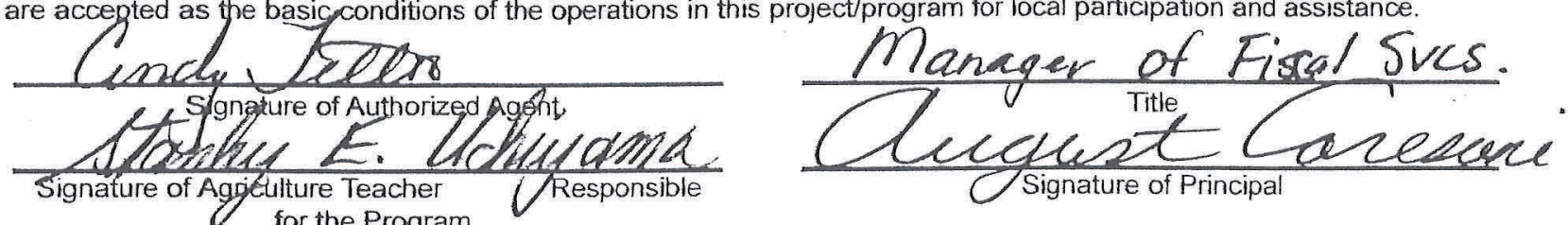

Contact Phone Number

(831) $796-7536$

Date of Approval of Local Agency Board:

Funds Requested - Part I

Part II

Part III

Part IV

Part V

$$
\text { 31-Aug-10 }
$$

Total

Number of Different Agriculture Teachers at Site:

\begin{tabular}{c}
\hline$\frac{31 \text {-Aug-10 }}{\$ \$ 5,000.00}$ \\
\hline$\$ 3,008.00$ \\
\hline$\$ 0.00$ \\
\hline$\$ 11,666.00$ \\
\hline 0.00
\end{tabular}

4

PART I - QUALITY CRITERIA 1-9 (REQUIRED) ALLOCATION

\begin{tabular}{l} 
Quality Criteria \\
\hline 1. Curriculum and Instruction \\
2. Leadership and Citizenship Development \\
3. Practical Application of Occupational Skills \\
4. Qualified and Competent Personnel \\
5. Facilities, Equipment and Materials \\
6. Community, Business and Industry Involvement \\
7. Career Guidance \\
8. Program Promotion \\
9. Program Accountability and Planning
\end{tabular}

Formal Variance Request must be included if requesting a variance. A variance is a proposed plan for bringing the program into compliance with required quality criteria. Variances should result in compliance prior to the following year's application. All variances must be approved with the application. Non-compliance with the terms of the approved variance will result in a loss of funds. 


\section{GENERAL ASSURANCES \\ (SDE 100A)}

The signature of the authorized agent on the cover page (Form 100) acknowledges that general assurance 1-13 on Form 100A will be observed.

1. Progress and services shall be in compliance with Title VI and Tivle VIl of the Civil Rights Acr of 1964, the California Fair Employment Practices Act, and Chapter 4 (commencing with Section 30) of Division 1 of Title 5, California Administrative Code. A statement of compliance with Tirle VI of the Civil Righrs Act of 1964 has been filed with the Superiateadent of Public lastmuction.

2. Program and services shall be in compliance with Title $\mathrm{XX}$ (nondiscrimination on the basis of sex) of the Education Amendments of 1972.

3. Programs and services shall be in compliance with the affumative action provisions of the Education Amendments of 1972 .

4. Programs and services shall be in compliance with the Age Discrimination Act of 1975.

5. Programs and services for handicapped persons shall be in compliance with the Education for All Handicapped Children Act of 1975, Section 613(a) and Section 504 of the Rehabilitation Act of 1973.

6. When federal funds are made available, they will be used so as to supplement, and to the extent practicable, increase the amount of state and local funds that would, in the absence of such federal funds, be made available for the uses specified in The State Plan, and in no case supplant such state or local fumds.

7. All state and federal statutes, regulations, program plans, and applications applicable to each program under which federal or state funds are made available through this application will be met by the applicant agency in its

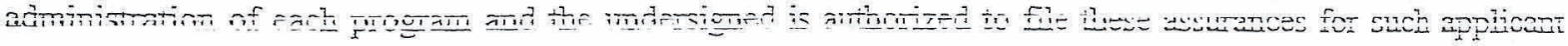
agency.

8. The local agency will use fiscal control and fund accounting procedures that will ensure proper disbursement of and accounting for, state and federal funds paid to that agency under each program.

9. The public agency shall make reports to the state agency or board and to the Secretary of Education as may reasonably be necessary to enable the state agency or board and the Secretary to perform their duties and will maintain such records and provide access to those records as the state agency or board or the Secretary deem necessary. Such records shall include, but not be limited to, records which fully disclose the amount and disposition by the recipient of those funds, the total cost of the activity for which the funds are used, the share of that cost provided from other sources, and such other records as will facilitate an effective andit. The recipient shall maintain such records for five years after the completion of the activities for which the funds are used.

10. Any application, evaluation, periodic program plan, or report relating to each program will be made readily available to parents and other members of the general public.

11. Auditable records of each participating school program will be maintained on file at the district office. (T5 3944; CFR 220.56)

12. The district board of trustees has adopted written procedures to ensure prompt response to complaints from parents, members of advisory committees, and members of other groups, within 30 days, and has disseminated these procedures to parent/community groups in the district. (T5 3951)

13. School districts participating in the incentive grant must certify that the funds will be used to supplement, not supplant the districts regular ongoing expenditures for the Agricultural Vocational Education program. 
1 - CONTINUED

Departmental Allocation: Meeting the criteria in PART I makes the program eligible for the following amounts based on the number of teachers in the program.

\begin{tabular}{lcc} 
Total Number of Teachers & Amount Eligible & Amount Requested \\
\hline One Teacher or Less & $\$ 4,000$ & \\
Two Teachers & $\$ 4.500$ & \\
Three Teachers or More & $\$ 5.000$ & $\$ 5.000 .00$ \\
\hline
\end{tabular}

PART II - PROGRAM ENROLLMENT ALLOCATION

Total Number of Students 2009-10 R2 Number Amount Requested

List Number from R2 Report (\$8/Member)

376

$\$ 3,008.00$

PART III - SAE AND RETENTION ALLOCATION

Number of State Degrees in 2009

Percent of Students (R2) Receiving

State Degree

SAE/Retention Standard Funds - If percentage of State

Degree recipients is $5 \%$ or greater, then you are eligible

FALSE

for $\$ 200$ per degree awarded. Maximum of $\$ 10,000$.

PART IV - QUALITY CRITERIA 10-11 (OPTIONAL) ALLOCATION

Schools which qualify for a Departmental Allocation may apply for additional amounts for each specific Quality Criteria (10 and 11) met.

* Amounts requested in Quality Criterion 10 will be the indicated amount for that criterion, multiplied by the fulltime equivalent (FTE). To count a preparation period, the teacher must be teaching Career Technical Education courses in Agriculture for $50 \%$ or more of their teaching periods.

* Amounts requested in Quality Criterion $11 \mathrm{~A}$ will be the indicated amount for each teacher who was compensated a minimum of $\$ 2,000$ for year-round employment.

* Amounts requested in Quality Criterion $11 \mathrm{~B}$ will he the indicated amount for each teacher who is provided a project supervision period. Project periods will be counted if the teacher has a preparation period as part of the regular teaching day.

Number of FTE Agriculture Teachers at Site:

2.833

List the Names of the Agriculture Teachers:

Stanley Uchiyama

$\frac{\text { Tom Jones }}{\text { (Alephanie Blodgett }}$

Criterion 10 - Student/Teacher Ratio

Criterion $11 \mathrm{~A}$ - Year-Round Employment

Criterion 11B - Project Supervision Period

TOTAL FUNDS REQUESTED PART IV

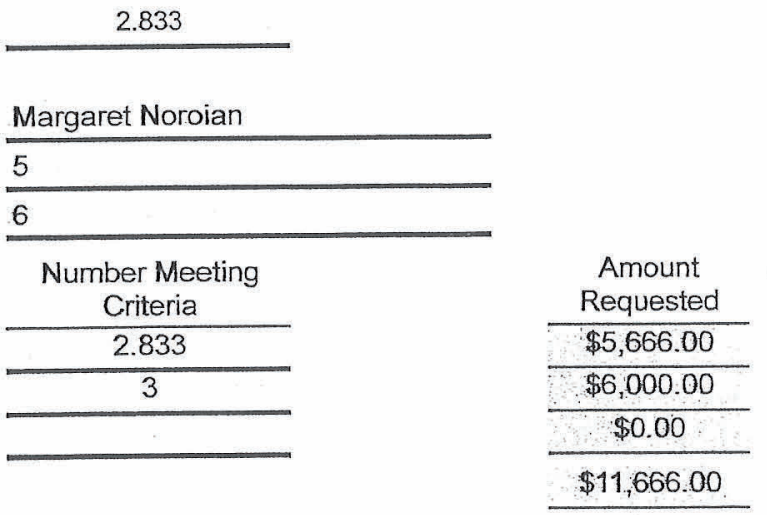

\section{PART V - QUALITY CRITERION 12 (OPTIONAL) ALLOCATION}

Quality Criterion 12 Form is attached and all criteria has been met. If the answer is yes, list $\$ 3,000$ (funds requesting) in space to the right. 
P. $\quad$ I-FINANCIAL SCHEDULE

Par A

\begin{tabular}{|c|c|c|c|c|c|}
\hline & & & A & $B$ & C \\
\hline Line & $\begin{array}{l}\text { Acct. } \\
\text { No. }\end{array}$ & Classification & $\begin{array}{l}\text { Description of ltem for } \\
\text { which Funds will be } \\
\text { Expended }\end{array}$ & $\begin{array}{l}\text { Incentive } \\
\text { Grant Funds }\end{array}$ & $\begin{array}{l}\text { Matching } \\
\text { Funds }\end{array}$ \\
\hline 1 & 4000 & Books \& Supplies & & $12,874.00$ & $18,000.00$ \\
\hline 2 & & & Subtotal for 4000 & $\$ 12,874.00$ & $\$ 18,000.00$ \\
\hline 3 & \multirow[t]{6}{*}{5000} & \multirow{6}{*}{$\begin{array}{l}\text { Services and other Operating Expenses } \\
\text { such as: Personal Services of } \\
\text { Consultants, Staff Travel, and } \\
\text { Conference; Rentals, Leases, and } \\
\text { Repairs; Bus Transportation }\end{array}$} & 1 Travel \& Conference & $4,500.00$ & $4,500.00$ \\
\hline 4 & & & 2 Equip Serv/Repair & $1,500.00$ & $1,500.00$ \\
\hline 5 & & & 3 Vehicle/Bus Operation & 800.00 & 800.00 \\
\hline \multirow[t]{2}{*}{6} & & & 4 & & \\
\hline & & & 5 & & \\
\hline 7 & & & 6 & & \\
\hline 8 & & & Subtotal for 5000 & $\$ 6,800.00$ & $\$ 6,800.00$ \\
\hline 9 & \multirow[t]{5}{*}{6000} & \multirow{5}{*}{$\begin{array}{l}\text { Capital Outlay: Includes Sites and } \\
\text { Improvements of Sites; Buildings and } \\
\text { Improvement of Buildings; Equipment }\end{array}$} & 1 & & \\
\hline 10 & & & 2 & & \\
\hline \multirow[t]{2}{*}{11} & & & 3 & & \\
\hline & & & 4 & & \\
\hline 12 & & & 5 & & \\
\hline - & $\therefore$ & & Subtotal for 6000 & $\$ 0.00$ & $\$ 0.00$ \\
\hline 14 & & & $\begin{array}{c}\text { Total for } 4000-6000 \\
\text { Lines } 2,8,13\end{array}$ & $\$ 19,674,00$ & $\$ 24,800.00$ \\
\hline
\end{tabular}

TOTAL 2010-2011 incentive Grant Allocation:

Part B - Complete this portion if a waiver of the matching requirement was granted:

\begin{tabular}{|r|r|l|l|l|r|r|}
\hline Line & $\begin{array}{l}\text { Acct } \\
\text { No. }\end{array}$ & \multicolumn{1}{|c|}{ Classification } & $\begin{array}{l}\text { A } \\
\text { Description of Item for } \\
\text { which Funds were } \\
\text { Expended }\end{array}$ & $\begin{array}{c}\text { Incentive } \\
\text { Grant Funds }\end{array}$ & $\begin{array}{c}\text { Amount of } \\
\text { Salary and } \\
\text { Benefits }\end{array}$ \\
\hline 15 & 1000 & Salaries & & $\begin{array}{l}\text { Teachers' Summer Service } \\
\text { Salaries }\end{array}$ & \\
\hline 16 & 1000 & Salaries & $\begin{array}{l}\text { Teachers' Salaries for } \\
\text { Project Supervision Period }\end{array}$ & & $4,879.00$ \\
\hline 17 & 3000 & Benefits & $\begin{array}{l}\text { Benefits for the Above } \\
\text { Items (1000) }\end{array}$ & & \\
\hline 18 & & & TOTAL & & 661.00 \\
\hline
\end{tabular}

TOTAL Amount of Waiver Requested: 


\section{NORTH SALINAS HIGH SCHOOL \\ AGRISCIENCE AND TECHNOLOGY DEPAR'TMENT}

\section{WATVER REQUEST: CRITERIA 10-STUDENT TEACHER RATIO}

Greg Beard, Regional Supervisor

Agriculture Education

State Department of Education

For the 2010-2011 school year, we are requesting a waiver consideration for the Student-Teacher tatio criteria \#10. We offer the following rationale.

BACKGROUND: Our cutrent District contract student-teacher ratio is 32 students in a classtoom and 29 students in a shop-type class. We have satisfactorily met the Incentive Grant Student-Teacher Ratio Criteria by our annual compliance review date and/or January of each school year. Our 2010-2011 school formula budget for agriculture will be $\$ 3,500$. These funds are used to cover all program expenses regardless of funding category. With additional financial support from Vo Ed-Perkins funds and the $\mathrm{Ag}$ Incentive Grant, we have managed to conduct an active and effective agricultural education/FFA program at out school.

REASONS FOR NON-COMPLIANCE AND CURRENT CRITERIA STATUS: With cooperative effort between our school administration and Agriculture Advisory Committee input, our progtam will employ four (4) agriculture teachers for this coming school year. In comparison to the Incentive Grant Student-Teacher ratio criteria (classtoom - 24 students; shop-type classes-20 students,) and based on June, 2010 pre-registration enrollment, we currently project an average agticulture science class size of 27 students and shop-type class of 28 students. It is expected that entollment for all classes will significantly change within the first 4-6 weeks of school. Based upon the teacher-SAEP supervision ratio of 75 students per FTE teacher, it is estimated that this number will be 78 for two and five/sixth teaching staff. Historically, both class entollment and supervision ratios decline with the first half of the Fall Semester term. Such a change

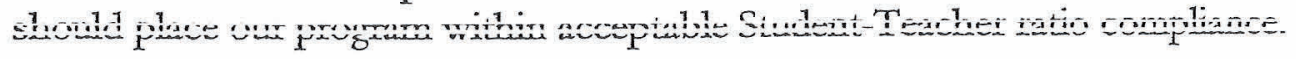

EFFORTS TO MEET CRITERIA AND PLANS FOR COMPLIANCE: Our school will be on a regular six (6) period school day for the 2010-2011 school year. It has been validated that about $15 \%$ of our all-year projected entollment numbers will be lost and/or "not seen" due to schedule changes or nonattendance/ Our thinking and class scheduling strategy is a "good faith" compliance, I will work with the $\mathrm{Ag}$ Advisory Committee and our School Principal to review class entollment balancing or limit strategies. Current enrollment data will be monitored in October 2010 and at the time of our 2010-2011 Incentive Grant compliance review in December 2010. At this same time, an updated enrollment analysis report will be filed with your office.

IMPACT OF VARIANCE APPROVAL AND REQUEST RESPONSE: With announced 2010-2011 plans for District and possible VEA funding teductions to North High's Ag.Ed. program, denial of our waiver request will cause a significant reduction in our total Incentive Grant funds for the 2010-2011 school year. This reduction will Create a hardship in our ability to provide the student services and instructional supplies needed to accommodate our current growth in student and program's needs. We are asking for a Criteria \#10 waiver to manage and adjust our program's growth trend without financial penalty for our efforts. To allow for the possible revision of our application, your timely response to our variance request is appreciated.

Sincerely and with good faith

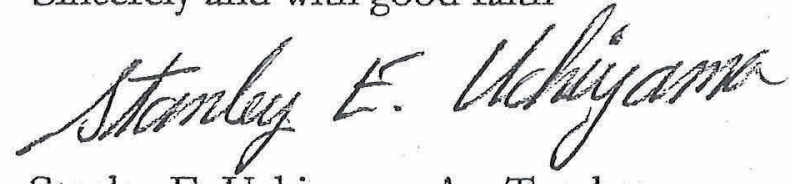

Stanley E. Uchiyama, Ag. Teacher and Ag. Program Manager

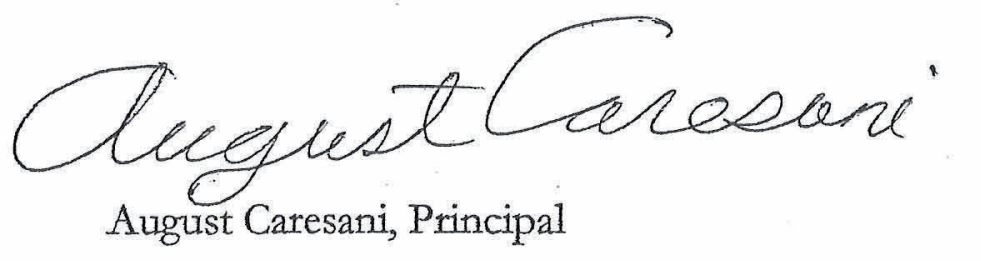

August Caresani, Principal 


\section{Timeline for \\ Agricultural Career Technical Education Incentive Grant \\ Fiscal Year (FY) 2010-11}

\begin{tabular}{|c|c|}
\hline Activity & Dates \\
\hline $\begin{array}{l}\text { Application form and announcement letters made } \\
\text { available to LEAs. }\end{array}$ & May 10,2010 \\
\hline $\begin{array}{l}\text { Agriculture and Home Economics Education Unit } \\
\text { staff available to assist LEAs. }\end{array}$ & May 10 - June 30,2010 \\
\hline Applications due to Regional Supervisors. & June 30,2010 \\
\hline $\begin{array}{l}\text { Regional Supervisors will review and summarize } \\
\text { applications. }\end{array}$ & June 30 - July 30,2010 \\
\hline $\begin{array}{l}\text { California Department of Education } \\
\text { apportionments established and LEAs notified. }\end{array}$ & September 15, 2010 \\
\hline $\begin{array}{l}\text { Program Enroliment Data Report }(\mathrm{R}-2) \text { due to } \\
\text { Regional Supervisors. }\end{array}$ & October 15,2010 \\
\hline $\begin{array}{l}\text { Report of Expenditures for previous year (2009- } \\
\text { 10) due to Regional Supervisors. }\end{array}$ & October 15,2010 \\
\hline $\begin{array}{l}\text { FFA Membership Rosters due to FFA Financial } \\
\text { Services Office. }\end{array}$ & October 15, 2010 \\
\hline On-site LEA reviews by Regional Supervisors. & October 15 - December 15,2010 \\
\hline $\begin{array}{l}\text { Final date for budget revisions to be submitted to } \\
\text { Regional Supervisors. }\end{array}$ & April 1, 2011 \\
\hline $\begin{array}{l}\text { Final } 25 \text { percent apportionment, based on any } \\
\text { adiustments, re!eased to districts and final report } \\
\text { form and notice of apportionment mailed to LEAs. }\end{array}$ & April 1, 2011 \\
\hline $\begin{array}{l}\text { Report of Expenditures for year }(2010-11) \text { due to } \\
\text { Regional Supervisors. }\end{array}$ & October 15, 2011 \\
\hline
\end{tabular}




\section{General Assurances}

Consolidated Application Part I and II general legal assurances for fiscal year 2009-10.

\section{ineral Assurances}

1. Programs and services are and will be in compliance with Title $\mathrm{VI}$ and Title VII of the Civil Rights Act of 1964; the California Fair Employment Practices Act, Government Code \$11135; and Chapter 4 (commencing with $\S 30$ ) of Division I of Title 5, California Code of Regulations (CCR).

2. Programs and services are and will be in compliance with Title IX (nondiscrimination on the basis of sex) of the Education Amendments of 1972. Each program or activity conducted by the LEA will be conducted in compliance with the provisions of Chapter 2, (commencing with \$200), Prohibition of Discrimination on the Basis of Sex, of Part 1 of Division 1 of Title I of the Education Code, as well as all other applicable provisions of state law prohibiting discrimination on the basis of sex.

3. Programs and services are and will be in compliance with the affirmative action provisions of the Education Amendments of 1972.

4. Programs and services are and will be in compliance with the Age Discrimination Act of 1975.

5. Programs and services for individuals with disabilities are in compliance with the disability laws. (PL 10517; 34 CFR 300, 303; and Section 504 of the Rehabilitation Act of 1973)

6. When federal funds are made available, they will be used to supplement the amount of state and local funds that would, in the absence of such federal funds, be made available for the uses specified in the state plan, and in no case supplant such state or local funds. (20 USC $\$ 6321$ (b)(1); PL 107-110 $\$ 1120 \mathrm{~A}(\mathrm{~b})(1))$

7. All state and federal statutes, regulations, program plans, and applications appropriate to each program under which federal or state funds are made available through this application will be met by the applicant agency in its administration of each program.

8. Schoolsite councils have developed and approved a Single Plan for Student Achievement for schools participating in programs funded through the consolidated application process, and any other school program they choose to include, and that school plans were developed with the review, certification, and advice of any applicable school advisory committees. (EC \$64001)

9. The local educational agency (LEA) will use fiscal control and fund accounting procedures that will ensure proper disbursement for state and federal funds paid to that agency under each program. (CCR T5, \$4202)

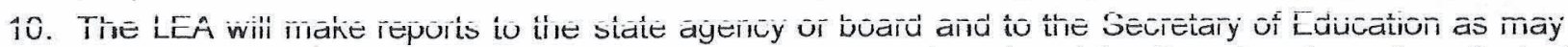
reasonably be necessary to enable the state agency or board and the Secretary to perform their duties and will maintain such records and provide access to those records as the state agency or board or the Secretary deems necessary. Such records will include, but will not be limited to, records which fully disclose the amount and disposition by the recipient of those funds, the total cost of the activity for which the funds are used, the share of that cost provided from other sources, and such other records as will facilitate an effective audit. The recipient shall maintain such records for three years after the completion of the activities for which the funds are used. (34 CFR 76.722, 76.730, 76.731, 76.734, 76.760; 34 CFR 80.42)

11. The local governing board has adopted written procedures to ensure prompt response to complaints within 60 days, and has disseminated these procedures to students, employees, parents or guardians, district/school advisory committees, and interested parties. (CCR T5, §4600 et seq.)

12. The LEA declares that it neither uses nor will use federal funds for lobbying activities and hereby complies with the certification requirements of 34 CFR Part 82. (34 CFR Part 82)

13. The LEA has complied with the certification requirements under 34 CFR Part 85 regarding debarment, suspension and other requirements for a drug-free workplace. (34 CFR Part 85)

14. The LEA provides reasonable opportunity for public comment on the application and considers such comment. (20 USC §7846(a)(7); 20 USC, §1118(b)(4); PL 107-110, §1118(b)(4)).

15. The LEA will provide the certification on constitutionally protected prayer that is required by PL 107-110, $\S 9524$ and 20 USC $\$ 7904$.

16. The LEA administers all funds and property related to programs funded through the Consolidated Application. (20 USC $\$ 6320(d)(1)$; PL 107-110, $\$ 1120(d)(1)$ )

17. The LEA will adopt and use proper methods of administering each program including enforcement of any obligations imposed by law on agencies responsible for carrying out programs and correction of deficiencies in program operations identified through audits, monitoring or evaluation. (20 USC $\$ 7846$ (a) (3)(B))

18. The LEA will participate in the Standardized Testing and Reporting program. (20 USC $\S 6316(a)(1)(A-D)$; 
PL 107-110, $\$ 1116(a)(1)(A-D) ; E C ~ \$ 60640$, et seq.)

19. The LEA assures that classroom teachers who are being assisted by instructional assistants retain their responsibility for the instruction and supervision of the students in their charge. (EC $\$ 45344(\mathrm{a})$ )

20. The LEA governing board has adopted a policy on parent involvement that is consistent with the purposes and goals of EC Section 11502. These include all of the following: (a) to engage parents positively in their children's education by helping parents to develop skills to use at home that support their children's academic efforts at school and their children's development as responsible future members of our society; (b) to inform parents that they can directly affect the success of their children's learning, by providing parents with techniques and strategies that they may utilize to improve their children's academic success and to assist their children in learning at home; (c) to build consistent and effective communication between the home and the school so that parents may know when and how to assist their children in support of classroom learning activities; (d) to train teachers and administrators to communicate effectively with parents; and (e) to integrate parent involvement programs, including compliance with this chapter, into the school's master plan for academic accountability. ( $E C$ $\S 11502,11504$ )

21. Results of an annual evaluation demonstrate that the LEA and each participating school are implementing Consolidated Programs that are not of low effectiveness, under criteria established by the local governing board. (CCR T5, §3942)

22. The program using consolidated programs funds does not isolate or segregate students on the basis of race, ethnicity, religion, sex, sexual orientation or socioeconomic status. (USC, Fourteenth Amendment; Calif. Constitution, art. 1, §7; Gov.C §§11135-11138; 42 USC §2000d; CCR T5, §3934)

23. Personnel, contracts, materials, supplies, and equipment purchased with Consolidated Program funds supplement the basic education program. (EC $\S \S 2002$, 52034(I), 52035(e)(1), 54101; CCR T5, §§3944, 3946)

24. At least 85 percent of the funds for School Improvement Programs, Title I, Title VI and Economic Impact Aid (State Compensatory Education and programs for English learners) are spent for direct services to students. One hundred percent of Miller-Unruh apportionments are spent for the salary of specialist reading teachers. (EC §63001; CCR T5, §3944(a)(b))

25. State and federal categorical funds will be allocated to continuation schools in the same manner as to comprehensive schools, to the maximum extent permitted by state and federal laws and regulations. (EC §48438)

26. Programs and services are and will be in compliance with Section 8355 of the California Government Code and the Drug-Free Workplace Act of 1988, and implemented at 34 Code of Federal Regulations (CFR) Part 84, Subpart F, for grantees, as defined at 34 CFR Part 84, Sections 84.105 and 84.110.

Back to Lega! Assurances

Questions: Anne Daniels | adaniels@cde.ca.gov | 916-319-0295

California Department of Education

1430 N Street

Last Reviewed: Wednesday, December 23, 2009

Sacramento, CA 95814 


\section{Drug-Free Workplace}

Certification regarding state and federal drug-free workplace requirements.

Alote: Any entity, whether an agency or an individual, must complete, sign, and return this certification with its ant application to the California Department of Education.

\section{Grantees Other Than Individuals}

As required by Section 8355 of the Califomia Government Code and the Drug-Free Workplace Act of 1988, and implemented at 34 Code of Federal Regulations (CFR) Part 84, Subpart F, for grantees, as defined at 34 CFR Part 84 , Sections 84.105 and 84.110

A. The applicant certifies that it will or will continue to provide a drug-free workplace by:

a. Publishing a statement notifying employees that the unlawful manufacture, distribution, dispensing, possession, or use of a controlled substance is prohibited in the grantee's workplace and specifying the actions that will be taken against employees for violation of such prohibition

b. Establishing an on-going drug-free awareness program to inform employees about:

1. The dangers of drug abuse in the workplace

2. The grantee's policy of maintaining a drug-free workplace

3. Any available drug counseling, rehabilitation, and employee assistance programs

4. The penalties that may be imposed upon employees for drug abuse violations occurring in the workplace

c. Making it a requirement that each employee to be engaged in the performance of the grant be given a copy of the statement required by paragraph (a)

d. Notifying the employee in the statement required by paragraph (a) that, as a condition of employment under the grant, the employee will:

1. Abide by the terms of the statement

2. Notify the employer in writing of his or her conviction for a violation of a criminal drug statute occurring in the workplace no later than five calendar days after such conviction

e. Notifying the agency, in writing, within 10 calendar days after receiving notice under subparagraph (d)(2) from an employee or otherwise receiving actual notice of such conviction. Employers of convicted empioyes must provide notice, including position titie, to every grent officer or other designee. Notice shall include the identification number(s) of each affected grant.

f. Taking one of the following actions, within 30 calendar days of receiving notice under subparagraph (d)(2), with respect to any employee who is so convicted:

1. Taking appropriate personnel action against such an employee, up to and including termination, consistent with the requirements of the Rehabilitation Act of 1973, as amended; or

2. Requiring such employee to participate satisfactorily in a drug abuse assistance or rehabilitation program approved for such purposes by a federal, state, or local health, law enforcement, or other appropriate agency

g. Making a good faith effort to continue to maintain a drug-free workplace through implementation of paragraphs (a), (b), (c), (d), (e), and (f).

B. The grantee may insert in the space provided below the site(s) for the performance of work done in connection with the specific grant:

Place of Performance (street address. city, county, state, zip code)

North Salinas High School (Salinas Union High School District) 55 Kip Drive

Salinas, CA 93906

reck [ ] if there are workplaces on file that are not identified here.

\section{Grantees Who Are Individuals}


As required by Section 8355 of the Califomia Govemment Code and the Drug-Free Workplace Act of 1988, and implemented at 34 CFR Part 84, Subpart F, for grantees, as defined at 34 CFR Part 84, Sections 84.105 and 84.110

A. As a condition of the grant, I certify that I will not engage in the unlawful manufacture, distribution, dispensing, possession, or use of a controlled substance in conducting any activity with the grant; and

B. If convicted of a criminal drug offense resulting from a violation occurring during the conduct of any grant activity, I will report the conviction to every grant officer or designee, in writing, within 10 calendar days of the conviction. Notice shall include the identification number(s) of each affected grant.

As the duly authorized representative of the applicant, I hereby certify that the applicant will comply with the above certifications.

Name of Applicant: Stan Uchiyama

Name of Program: Agricultural Education

Printed Name and Title of Authorized Representałive: August Caresani, Princjpa 1

Signature:

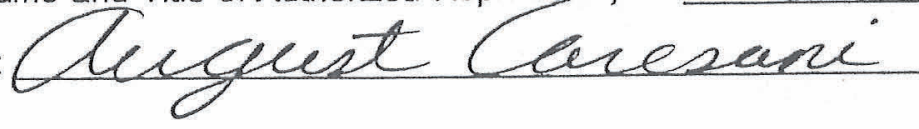
Date:

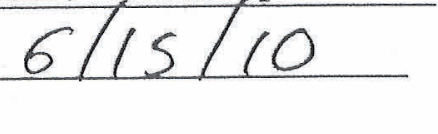

CDE-100DF (May-2007) - California Department of Education

Questions: Funding Master Plan I fmp@cde.ca.gov | 916-323-1544

California Department of Education

$1430 \mathrm{~N}$ Street

Sacramento, CA 95814
Last Reviewed: Wednesday, May 05, 2010 


\section{0-11 AGRICULTURE STAFF RESPONSIBILITIES DEPARTMENT PROGRAM MANAGEMENT AND OPERATIONS (rev. 10/10)}

AREAS: I. Department Operations-Program Management, Budget and Purchasing, Facilities and Equipment, Curriculum Development.

II. Support Groups and Professional Development-Ag. Booster Club, Ag. Advisory Committee, School and Faculty, Community and Service Organizations, Conferences and CATA.

III. FFA Operations/Activities, Leadership Development, CDE Teams and Events.

IV. SAEP Operations, Awards, Enterprise Supervision, County Fairs, School Farm.

* COMPLETE DUTIES PER DEPARTMENT, SCHOOL, AND DISTRICT POLICIES AND PROCEDURES.

** COMPLETE TRAVEL (SCHOOL BUSINESS AGREEMENTS) AND TRANSPORTATION, SUBSTITUTE TEACHER, FIELD TRIP/BUS, APPLICABLE PURCHASE ORDERS, AND STUDENT ABSENCE APPROVAL REQUESTS IN A TIMELY MANNER.

** COPY OF ALL REOUEST FORMS KEPT FOR DEPARTMENT REFERENCE RECORDS.

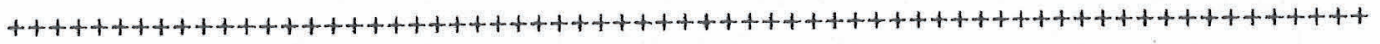

1. DEPARTMENT OPERATIONS.

page 1

Program Area and Responsibility

(XX--Direct Responsibility with other staff assistance)

Staff Member

\begin{tabular}{|c|c|c|c|c|}
\hline 1. PROGRAM MANAGEMENT & Uchiyama & Jones & $\begin{array}{l}\text { Blod- } \\
\text { gett }\end{array}$ & $\begin{array}{l}\text { Noro- } \\
\text { ian }\end{array}$ \\
\hline a. Program Manager/Department Chair/Head FFA Advisor & $\mathrm{XX}$ & & & \\
\hline b. Department Meeting Minutes --- Staff Rotation & $\mathrm{XX}$ & $\mathrm{XX}$ & $\mathrm{XX}$ & $\mathrm{XX}$ \\
\hline $\begin{array}{l}\text { c. Standard Operating Procedures, Policy Development, } \\
\text { Decision Making, and Implementation. }\end{array}$ & $\mathrm{XX}$ & $\mathrm{XX}$ & $\mathrm{XX}$ & $\mathrm{XX}$ \\
\hline 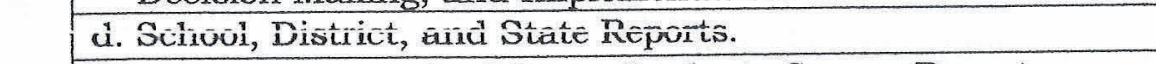 & $X Y$ & & & \\
\hline e. R-2 Enrollment, Staff Data, Graduate Survey Reports . & $\mathrm{XX}$ & & & \\
\hline f. State FFA Member Roster Filing and Payment. & & $\mathrm{XX}$ & & \\
\hline g. Ag. Incentive Grant Application Completion. & $\mathrm{XX}$ & & & \\
\hline h. Ag. Incentive Grant Criteria Items 1-11 Compliance. & $\mathrm{XX}$ & $\mathrm{XX}$ & $\mathrm{XX}$ & $\mathrm{XX}$ \\
\hline i. Ag. Incentive Grant Compliance/Financial Reports. & $\mathrm{XX}$ & & & \\
\hline j. Monthly Activity Calendar Development. & $\mathrm{XX}$ & $\mathrm{XX}$ & $\mathrm{XX}$ & $\mathrm{XX}$ \\
\hline k. Department, Student, SAEP Records Filing System. & $\mathrm{XX}$ & $\mathrm{XX}$ & $\mathrm{XX}$ & $\mathrm{XX}$ \\
\hline 1. Class Teaching Assignments and Class Scheduling. & $\mathrm{XX}$ & & & \\
\hline m. Program Promotion Activities and Awards. & $\mathrm{XX}$ & $\mathrm{XX}$ & $\mathrm{XX}$ & $\mathrm{XX}$ \\
\hline n. FFA/School Student Awards and Scholarships. & $\mathrm{XX}$ & $\mathrm{XX}$ & $\mathrm{XX}$ & $\mathrm{XX}$ \\
\hline o. Summer Agriculture Program Operations. & $\mathrm{XX}$ & & & \\
\hline $\begin{array}{l}\text { p. Year's Conference, Van Use, Sub-Teacher, and } \\
\text { Transportation Request to Principal, Athlet. Dir, VEA Dir. }\end{array}$ & $\mathrm{XX}$ & $\mathrm{XX}$ & & \\
\hline $\begin{array}{l}\text { q. School Business Agreement (travel, meals, confer, sub) } \\
\text { Requests to Principal and VEA/ROP Director. }\end{array}$ & $\mathrm{XX}$ & $\mathrm{XX}$ & $\mathrm{XX}$ & $\mathrm{XX}$ \\
\hline r. HS / Feeder School Recruit and Pre-Registration Activities. & $\mathrm{XX}$ & & $\mathrm{XX}$ & \\
\hline s. Co-Op School/Ag Intern Teacher Program. & $\mathrm{XX}$ & & & \\
\hline t. Teacher Non-Class Activity Records. & $\mathrm{XX}$ & $\mathrm{XX}$ & $\mathrm{XX}$ & $\mathrm{XX}$ \\
\hline u. Staff Duty/Responsibility Assignment Chart. & $\mathrm{XX}$ & $\mathrm{XX}$ & $\mathrm{XX}$ & $\mathrm{XX}$ \\
\hline v. Five Year Plans-Equipment, Facilities, School Farm. & $\mathrm{XX}$ & $\mathrm{XX}$ & $\mathrm{XX}$ & $\mathrm{XX}$ \\
\hline w. Comprehensive Program Plan Binder Update. & & $\mathrm{XX}$ & & \\
\hline x. Teacher Adjunct Duties and School Faculty Meetings. & $\mathrm{XX}$ & $\mathrm{XX}$ & $\mathrm{XX}$ & $\mathrm{XX}$ \\
\hline y. School Accreditation Process and Reports. & $\mathrm{XX}$ & & & \\
\hline z. Student Scholarship, Award, Job Recommendation Letters & $\mathrm{XX}$ & $\mathrm{XX}$ & $\mathrm{XX}$ & $\mathrm{XX}$ \\
\hline
\end{tabular}


Program Area and Responsibility

(XX--Direct Responsibility with other staff assistance)

Staff Member

\begin{tabular}{|c|c|c|c|c|}
\hline 2. BUDGET, SUPPLY, and PURCHASING & Uchiyama & Jones & $\begin{array}{l}\text { Blod- } \\
\text { gett }\end{array}$ & $\begin{array}{l}\text { Noro- } \\
\text { ian }\end{array}$ \\
\hline a. Budget, Purchasing Records and Reports. & $\mathrm{XX}$ & & & \\
\hline b. School Site Formula Funds Use. & $\mathrm{XX}$ & $\mathrm{XX}$ & $\mathrm{XX}$ & $\mathrm{XX}$ \\
\hline c. Ag. Incentive Grant Funds Use. & $\mathrm{XX}$ & $\mathrm{XX}$ & $\mathrm{XX}$ & $\mathrm{XX}$ \\
\hline d. VEA (Perkins) and/or Special VEA Funds Use. & $\mathrm{XX}$ & $\mathrm{XX}$ & $\mathrm{XX}$ & $\mathrm{XX}$ \\
\hline e. One-Time School, AIG, Other Special Funds Use. & $\mathrm{XX}$ & $\mathrm{XX}$ & $\mathrm{XX}$ & $\mathrm{XX}$ \\
\hline f. Ag. Science Class Instructional Supply Needs/Use & $\mathrm{XX}$ & & & \\
\hline g. Horticulture Class Instructional Supply Needs/Use & & $\mathrm{XX}$ & & \\
\hline h. Ag. Earth Sci. \& Ag. Chem. Class Ins. Sup. Need/Use & & & $\mathrm{XX}$ & \\
\hline i. Ag. Govt/Econ. Class Instructional Supply Needs/Use & & & & $\mathrm{XX}$ \\
\hline j. Ag. Welding Class Instructional Supply Need/Use & $\mathrm{XX}$ & & & \\
\hline k. Ag. Mechanics Class Instructional Supply Need/Use & $\mathrm{XX}$ & $\mathrm{XX}$ & & \\
\hline 1. ROP Animal Care Class Funds Use & & $\mathrm{XX}$ & & \\
\hline m. Dept/Class--Computer Equip. \& Supply Purchases & $\mathrm{XX}$ & $\mathrm{XX}$ & $\mathrm{XX}$ & $\mathrm{XX}$ \\
\hline n. Class Dept, FFA--AV Equip. and Supply Purchases. & $\mathrm{XX}$ & $\mathrm{XX}$ & $\mathrm{XX}$ & XX \\
\hline o. Purchase Order Completion. & $\mathrm{XX}$ & $\mathrm{XX}$ & XX & XX \\
\hline p. Open Purchase Order Account Monitoring. & $\mathrm{XX}$ & & & \\
\hline q. Teacher Expense Reimbursement Claim and Records. & $\mathrm{XX}$ & $\mathrm{XX}$ & $\mathrm{XX}$ & $\mathrm{XX}$ \\
\hline r. Shop Materials Special Expense Collection/Deposits. & $\mathrm{XX}$ & XX & & \\
\hline s. Resource Catalog File--Supply, Equip, AV, Curr, Text & $\mathrm{XX}$ & & & \\
\hline
\end{tabular}

\section{PACILITES and SCHOOL SITE EQUIPMENT}

\begin{tabular}{|c|c|c|c|c|}
\hline & & & & \\
\hline a. Master Plan - Facility Use and Development Planning & $\mathrm{XX}$ & $\overline{X X}$ & $\overline{X X}$ & $x x$ \\
\hline b. Ag. Office and Resource Room Maintenance. & $\mathrm{XX}$ & $\mathrm{XX}$ & $\mathrm{XX}$ & $\mathrm{XX}$ \\
\hline $\begin{array}{l}\text { c. Welding Shop ( } \mathrm{Rm} .710) \text { and Ag. Science Classrooms } \\
(\mathrm{Rm} .712) \text { Maintenance. }\end{array}$ & $\mathrm{XX}$ & & & \\
\hline d. Ag. Earth/Chem. Classroom (Rm. 205) Maintenance. & & & $\mathrm{XX}$ & \\
\hline e. Ag. Shop Classroom ( $\mathrm{Rm}$. 730) Maintenance. & & $\mathrm{XX}$ & & \\
\hline f. Ag. Grovt/Econ. Classroom (Rm. 845) Maintenance & & & & $\mathrm{XX}$ \\
\hline g. Hallway Display Cases and Bulletin Boards--Rotation & XX & $\mathrm{XX}$ & $\mathrm{XX}$ & \\
\hline h. Outside Storage Building Use and Maintenance. & $\mathrm{XX}$ & $\mathrm{XX}$ & $\mathrm{XX}$ & $\mathrm{XX}$ \\
\hline i. Land Space/Livestock Pens Use and Development. & $\mathrm{XX}$ & $\mathrm{XX}$ & $\mathrm{XX}$ & \\
\hline j. Greenhouse 1 -- Use, Development, Maintenance. & $\mathrm{XX}$ & $\mathrm{XX}$ & $\mathrm{XX}$ & \\
\hline k. Greenhouse 2 - Use, Development, Maintenance. & XX & & & \\
\hline 1. Horticulture Growing Supply (pots, soil, etc.) Storage. & & $\mathrm{XX}$ & & \\
\hline m. Small Animal Building Use and Maintenance. & XX & & & \\
\hline n. Agriculture Truck Use and Maintenance & $\mathrm{XX}$ & & & \\
\hline o. Dept. Copy Machine Use, Maint., Service Contract. & $\mathrm{XX}$ & & & \\
\hline $\begin{array}{l}\text { p. New Equipment, Equip. Replacement, and Repairs - } \\
\text { Shops, Sciences, O.H., General Needs. }\end{array}$ & $\mathrm{XX}$ & $\mathrm{XX}$ & $\mathrm{XX}$ & $\mathrm{XX}$ \\
\hline q. Audio Visual Equipment Use, Care, Maintenance. & $\mathrm{XX}$ & $\mathrm{XX}$ & $\mathrm{XX}$ & $\mathrm{XX}$ \\
\hline r. Equipment Inventory Records and I.D. Labeling. & $\mathrm{XX}$ & $\mathrm{XX}$ & $\mathrm{XX}$ & $\mathrm{XX}$ \\
\hline
\end{tabular}


1. DEPARTMENT OPERATIONS. (continued)

page 3

Program Area and Responsibility

(XX--Direct Responsibility with other staff assistance)

Staff Member

\begin{tabular}{|c|c|c|c|c|}
\hline 4. CURRICULUM DEVELOPMENT and & Uchiyama & Jones & $\begin{array}{l}\text { Blod- } \\
\text { gett }\end{array}$ & $\begin{array}{l}\text { Noro- } \\
\text { ian }\end{array}$ \\
\hline a. District Curriculum Manual Updating . & $\mathrm{XX}$ & & & \\
\hline b. New Class and Textbook Proposals. & $\mathrm{XX}$ & $\mathrm{XX}$ & $\mathrm{XX}$ & $\mathrm{XX}$ \\
\hline c. Class Textbook Inventory Responsibility/Control. & $\mathrm{XX}$ & $\mathrm{XX}$ & $\mathrm{XX}$ & $\mathrm{XX}$ \\
\hline d. Class Syllabus and Curriculum Development. & $\mathrm{XX}$ & $\mathrm{XX}$ & $\mathrm{XX}$ & $\mathrm{XX}$ \\
\hline e. School and College Curriculum Articulation Activities & $\mathrm{XX}$ & $\mathrm{XX}$ & $\mathrm{XX}$ & $\mathrm{XX}$ \\
\hline f. District-Wide Ag. Curriculum Development/Textbook. & $\mathrm{XX}$ & $\mathrm{XX}$ & $\mathrm{XX}$ & $\mathrm{XX}$ \\
\hline g. UC Approved Ag. Class Credit Monitoring. & $\mathrm{XX}$ & & $\mathrm{XX}$ & \\
\hline h. Curriculum Articulation With Other Subject Areas. & $\mathrm{XX}$ & $\mathrm{XX}$ & $\mathrm{XX}$ & $\mathrm{XX}$ \\
\hline
\end{tabular}

\section{SUPPORT GROUPS - AG. BOOSTERS, AG. ADVISORY, SCHOOL, COMMUNITY, CATA}
Program Area and Responsibility
(XX--Direct Responsibility with other staff assistance)
Staff Member

\begin{tabular}{|c|c|c|c|c|}
\hline 1. AG. BOOSTERS, AG. ADVISORY, CATA, SCHOOL & Uchiyama & Jones & $\begin{array}{l}\text { Blod- } \\
\text { gett }\end{array}$ & $\begin{array}{l}\text { Noro- } \\
\text { ian }\end{array}$ \\
\hline $\begin{array}{l}\text { a. Parent Agriculture Booster Club Advisor and POA } \\
\text { Development. }\end{array}$ & & & $\mathrm{XX}$ & \\
\hline $\begin{array}{l}\text { b. Agriculture Advisory Committee Advisor/Secretary } \\
\text { and POA Development. }\end{array}$ & $\mathrm{XX}$ & & & \\
\hline c. CATA Prof. Organization Member/Involvement. & $\mathrm{XX}$ & $\mathrm{XX}$ & $\mathrm{XX}$ & $\mathrm{XX}$ \\
\hline d. CATA Teacher Awards and Other Recognition. & $\mathrm{XX}$ & $\mathrm{XX}$ & $\mathrm{XX}$ & $\mathrm{XX}$ \\
\hline e. CATA Professional Development Growth Activities. & $\mathrm{XX}$ & $\mathrm{XX}$ & $\mathrm{XX}$ & $\mathrm{XX}$ \\
\hline f. Volunteer Service -- School Committees. & $\bar{x}$ & $\bar{x}$ & $\bar{x}$ & $x$ \\
\hline g. District VocEd Committees, School Board Programs & $\mathrm{XX}$ & $\mathrm{XX}$ & $\mathrm{XX}$ & $\mathrm{XX}$ \\
\hline h. Community Relations - Business, Ag, Civic Groups. & $\mathrm{XX}$ & $\mathrm{XX}$ & $\mathrm{XX}$ & $X X$ \\
\hline
\end{tabular}

III. FFA CHAPTER OPERATIONS/ACTIVITIES, LEADERSHIP DEVELOPMENT, CAREER (JUDGING TEAM) DEVELOPMENT EVENTS.

Program Area and Responsibility

(XX--Direct Responsibility with other staff assistance)

Staff Member

\begin{tabular}{|c|c|c|c|c|}
\hline 1. CHAPTER OPERATIONS & Uchiyama & Jones & $\begin{array}{l}\text { Blod- } \\
\text { gett }\end{array}$ & $\begin{array}{l}\text { Noro- } \\
\text { ian }\end{array}$ \\
\hline a. Chapter and FFA Officer Team Advisor. & & $\mathrm{XX}$ & & \\
\hline b. Program of Activity and Budget Development. & & $\mathrm{XX}$ & & \\
\hline $\begin{array}{l}\text { c. Chapter Activity, Applications, Leadership, and } \\
\text { History Record Files System. }\end{array}$ & $\mathrm{XX}$ & $\mathrm{XX}$ & $\mathrm{XX}$ & $\mathrm{XX}$ \\
\hline d. Chapter Constitution and By-Laws Review. & & $\mathrm{XX}$ & & \\
\hline e. Chapter Member Affiliation With State FFA Assoc. & & $\mathrm{XX}$ & & \\
\hline f. Chapter Member I.D. Roster Development. & & & $\mathrm{XX}$ & \\
\hline $\begin{array}{l}\text { g. FFA General (Act. 190), Foundation (Act. 195), } \\
\text { Financial Accounts and Records Monitoring. }\end{array}$ & $\mathrm{XX}$ & $\mathrm{XX}$ & $\mathrm{XX}$ & $\mathrm{XX}$ \\
\hline h. Activity Monies Collection/Deposits. & $\mathrm{XX}$ & $\mathrm{XX}$ & $\mathrm{XX}$ & $\mathrm{XX}$ \\
\hline i. Year's Member Activity Participation Records. & $\mathrm{XX}$ & & & \\
\hline j. FFA Chapter, Officer, Member, SAE Awards Supply. & & $\mathrm{XX}$ & & \\
\hline 1. Food Service Supply - Plates, Utensils, Cups, etc. & & $\mathrm{XX}$ & & $\mathrm{XX}$ \\
\hline
\end{tabular}


III. FFA CHAPTER OPERATIONS/ACTIVITIES, LEADERSHIP DEVELOPMENT, CAREER (JUDGING TEAM) DEVELOPMENT EVENTS. (continued)

Program Area and Responsibility

(XX--Direct Responsibility with other staff assistance)

Staff Member

\begin{tabular}{|c|c|c|c|c|}
\hline 1. CHAPTER OPERATIONS & Uchiyama & Jones & $\begin{array}{l}\text { Blod- } \\
\text { gett }\end{array}$ & $\begin{array}{l}\text { Noro- } \\
\text { ian }\end{array}$ \\
\hline $\begin{array}{l}\text { 1. FFA Equip., Paraphernalia, Leadership Supply, and } \\
\text { Uniform Clothing Inventory Control. }\end{array}$ & $\mathrm{XX}$ & $\mathrm{XX}$ & $x X$ & $\mathrm{XX}$ \\
\hline $\begin{array}{l}\text { m. Region, State, National Chapter Award Applications- } \\
\text { Superior Chapter, Student, Advisor, Admin., etc. }\end{array}$ & & $\mathrm{XX}$ & & \\
\hline n. Monthly Chapter Meeting Attendance. & $\mathrm{XX}$ & $\mathrm{XX}$ & $\mathrm{xX}$ & $\mathrm{XX}$ \\
\hline
\end{tabular}

\begin{tabular}{|c|c|c|c|c|}
\hline 2. PROMOTION, FFA DEGREES, FUNDRAISING ACTIVTTIES & Uchiyama & Jones & $\begin{array}{l}\text { Blod- } \\
\text { gett }\end{array}$ & $\begin{array}{l}\text { Noro- } \\
\text { ian }\end{array}$ \\
\hline a. Fall Round-Up New Member Barbecue. & & $\mathrm{XX}$ & & \\
\hline b. Santa Cruz Fair Stock Auction Ring Set-up & $\mathrm{XX}$ & & & \\
\hline c. Ag. Calendar/Cookbook/Gift Wrap Fundraising. & $\mathrm{XX}$ & & $\mathrm{XX}$ & \\
\hline d. Greenhand Degree Applications and Awards Program. & $\mathrm{XX}$ & $\mathrm{XX}$ & $\mathrm{XX}$ & $\mathrm{XX}$ \\
\hline e. Chapter FFA Degree Application and Awards Program & $\mathrm{XX}$ & $\mathrm{XX}$ & $\mathrm{XX}$ & $\mathrm{XX}$ \\
\hline f. State and American Degree Applications. & $\mathrm{XX}$ & $\mathrm{XX}$ & $\mathrm{XX}$ & $\mathrm{XX}$ \\
\hline g. Mo Co AgEd Farm Day Demo/Display/Guides & & & $\mathrm{XX}$ & \\
\hline H FFA Week Promotion, Activities, and Recreation. & & $\mathrm{XX}$ & & $\mathrm{XX}$ \\
\hline i. Beef Jerky Sales Fundraising. & & & $\mathrm{XX}$ & -1 \\
\hline j. Spring Ag. Booster Barbecue. & & & $\mathrm{XX}$ & \\
\hline $\begin{array}{l}\text { k. Year-End Parent-Member FFA Awards Banquet- } \\
\text { Awards Selection, Invite, Program Dinner, Set-up, } \\
\text { Clean-up }\end{array}$ & $\mathrm{XX}$ & $\mathrm{XX}$ & $\mathrm{XX}$ & $\mathrm{xx}$ \\
\hline
\end{tabular}

3. LEADERSHIIP DEVELOPMENT and CONFERENCES
\begin{tabular}{|l|c|c|c|c}
\hline a. Chapter, Section, Region, State Office Candidates. & Uchiyama & Jones & $\begin{array}{c}\text { Blod- } \\
\text { gett }\end{array}$ & $\begin{array}{c}\text { Noro- } \\
\text { ian }\end{array}$ \\
\hline b. Chapter Officer Training - Officer Summer Retreat & & $\mathrm{XX}$ & $\mathrm{XX}$ & $\mathrm{XX}$ \\
\hline c. Chapter and Section B.I.G. Contests. & $\mathrm{XX}$ & $\mathrm{XX}$ & $\mathrm{XX}$ & \\
\hline d. Chapter Officer Training - COLC. & & $\mathrm{XX}$ & & $\mathrm{XX}$ \\
\hline e. Greenhand Conference. & $\mathrm{XX}$ & $\mathrm{XX}$ & $\mathrm{XX}$ & \\
\hline f. Open/Closing Meeting Ceremony Contest Teams & $\mathrm{XX}$ \\
\hline g. Freshman/Sophomore FFA Creed Speaking Contest. & $\mathrm{XX}$ & $\mathrm{XX}$ & $\mathrm{XX}$ & $\mathrm{XX}$ \\
\hline h. Prepared/Extemporaneous Public Speaking Contest. & $\mathrm{XX}$ & $\mathrm{XX}$ & $\mathrm{X}$ & $\mathrm{XX}$ \\
\hline i. Excellence/Advanced Leadership Conference. & & $\mathrm{XX}$ & $\mathrm{XX}$ & \\
\hline j. Sac. Ldr. Exp. (SLE), State Band, Talent, Choir Applic. & $\mathrm{XX}$ & $\mathrm{XX}$ & $\mathrm{XX}$ & $\mathrm{XX}$ \\
\hline k. Parliamentary Procedure Contest - Team Coaching. & & & & \\
\hline 1. Region Spring FFA Meeting and Contest Finals. & $\mathrm{XX}$ & & $\mathrm{XX}$ & \\
\hline m. Ag. Co-Op Marketing Contest Team. & $\mathrm{XX}$ & & & \\
\hline n. State FFA Leadership Conference Housing Reserv. & & & $\mathrm{XX}$ & \\
\hline o. State FFA Leadership Conference Admin. 86 Super. & $\mathrm{XX}$ & $\mathrm{XX}$ & $\mathrm{XX}$ & \\
\hline p. National FFA Convention Trip. & $\mathrm{XX}$ & & & \\
\hline
\end{tabular}


III. FFA CHAPTER OPERATIONS/ACTIVITIES, LEADERSHIP DEVELOPMENT, CAREIER (JUDGING TEAM) DEVELOPMENT EVENTS. (continued)

\author{
Program Area and Responsibility \\ (XX--Direct Responsibility, X--Assistance) \\ Staff Member
}

4. CAREER DEVELOPMENT EVENTS and TEAM TRAMNING
\begin{tabular}{|l|c|c|c|c}
\hline a. MoCo Fair Livestock/Veg Crop Judging Contests. & $\mathrm{XX}$ & & $\begin{array}{c}\text { Blod- } \\
\text { gett }\end{array}$ & $\begin{array}{c}\text { Noro- } \\
\text { ian }\end{array}$ \\
\hline b. Santa Cruz Co. Fair Livestock Judging Contest. & & & $\mathrm{XX}$ & \\
\hline c. San Benito Co. Fair Livestock Judging Contest. & & $\mathrm{XX}$ & & \\
\hline d. Salinas Valley Fair Stock/Veg Crop Judge Contests. & $\mathrm{XX}$ & $\mathrm{XX}$ & $\mathrm{XX}$ & \\
\hline e. CDE Skills Team Training/Coaching: & & & & \\
\hline 1. Vegetable Crops Team & $? ?$ & & & \\
\hline 2. Livestock Judging Team & & & $?$ & \\
\hline 3. Ag. Mechanics Team & $? ?$ & $? ?$ & & \\
\hline 4. Ornamental Horticulture & & $? ?$ & & \\
\hline f. CDE Contest Participation: & & & & \\
\hline 1. UC Davis, Merced J.C, or Reedley J.C. Field Day & & & $\mathrm{XX}$ & \\
\hline 2. Modesto J.C. Field Day & & $\mathrm{XX}$ & & \\
\hline 3. Fresno State Field Day & $\mathrm{XX}$ & & & \\
\hline g. State FFA CDE Contest Finals Participation. & $\mathrm{XX}$ & $\mathrm{XX}$ & $\mathrm{XX}$ & \\
\hline
\end{tabular}

IV. SAEP (ENTERPRISE PROJECTS) - OPERATIONS, SUPERVISION, RECOGNITION, COUNTY FAIRS, SCHOOL FARM.

Program Area and Responsibility (XX-Direct Responsibility, X-Assistance)
Staff Member

\begin{tabular}{|c|c|c|c|c|}
\hline 1. OPERATIONS and MANAGEMENT OF SAE'S & Uchiyama & Jones & $\begin{array}{l}\text { Blod- } \\
\text { gett }\end{array}$ & $\begin{array}{l}\text { Noro- } \\
\text { ian }\end{array}$ \\
\hline a. Project Supervision Visitation Documentation. & $\overline{X X}$ & $\mathrm{XX}$ & $\mathrm{XX}$ & $\mathrm{xX}$ \\
\hline b. SAEP Billing, Money Collection, Deposits. & $\mathrm{XX}$ & $\mathrm{XX}$ & $\mathrm{XX}$ & \\
\hline c. Animal Medical, Grooming, and Feed Supply. & $\mathrm{XX}$ & & & \\
\hline $\begin{array}{l}\text { d. Livestock and Utility Trailers, Grooming Equipment, } \\
\text { Production/Show Equipment Use and Maintenance. }\end{array}$ & $\mathrm{XX}$ & $\mathrm{XX}$ & $\mathrm{XX}$ & \\
\hline e. SAEP Equipment Purchase, Repair, Maintenance. & $\mathrm{XX}$ & $\mathrm{XX}$ & $\mathrm{XX}$ & \\
\hline f. Record Book Start-up, Progress, Closing Evaluation. & $\mathrm{XX}$ & $\mathrm{XX}$ & $\mathrm{XX}$ & $\mathrm{XX}$ \\
\hline g. Livestock Exhibitor Eligibility Contracts. & $\mathrm{XX}$ & $\mathrm{XX}$ & $\mathrm{XX}$ & $\mathrm{XX}$ \\
\hline h. Livestock Insurance and Bank Loan Services. & $\mathrm{XX}$ & $\mathrm{XX}$ & $\mathrm{XX}$ & \\
\hline i. New Student Livestock Information Meeting. & $\mathrm{XX}$ & & & \\
\hline $\begin{array}{l}\text { j. SAEP (Act. 185) and School Farm (Act. 180) Financial } \\
\text { Accounts and Records Monitoring }\end{array}$ & $\mathrm{XX}$ & $\mathrm{XX}$ & $\mathrm{XX}$ & \\
\hline $\begin{array}{l}\text { 1k. SAEP O.H. (Act. 170) and Ag. Mech (Act. 175) Special } \\
\text { Projects Financial Accounts and Records Monitoring }\end{array}$ & $\mathrm{XX}$ & $\mathrm{XX}$ & $\mathrm{XX}$ & \\
\hline
\end{tabular}

2. SAE AWARDS and RECOGNTION
\begin{tabular}{|l|c|c|c|c}
\hline a. Greenhand, Chapter, State, and American Degrees. & $\mathrm{XX}$ & $\mathrm{XX}$ & $\mathrm{XX}$ & $\mathrm{XX}$ \\
\hline b. Proficiency Awards and Applications - All Levels. & $\mathrm{XX}$ & $\mathrm{XX}$ & $\mathrm{XX}$ & $\mathrm{XX}$ \\
\hline c. Chapter and Section Project Competition. & $\mathrm{XX}$ & & $\mathrm{XX}$ & \\
\hline d. Star Degrees - Ownership and Placement Enterprises & $\mathrm{XX}$ & $\mathrm{XX}$ & $\mathrm{XX}$ & $\mathrm{XX}$ \\
\hline
\end{tabular}


IV. SAEP (ENTERPRISE PROJECTS)-OPERATIONS, SUPERVISION, RICOGNITION, COUNTY FAIRS, SCHOOL FARM.

Page 6

Program Area and Responsibility

(XX-Direct Responsibility, X-Assistance)

Staff Member

\begin{tabular}{|c|c|c|c|c|}
\hline 3. SAE ENTERPRISE PROJECT SUPERVISION/ADVISORS & Uchiyama & Jones & $\begin{array}{c}\text { Blod- } \\
\text { gett }\end{array}$ & $\begin{array}{l}\text { Noro- } \\
\text { ian }\end{array}$ \\
\hline a. Ornamental Horticulture Production/Placement. & $\mathrm{XX}$ & $\mathrm{XX}$ & $\mathrm{XX}$ & $\mathrm{XX}$ \\
\hline b. Landscape Maintenance - Home/Placement. & $\mathrm{XX}$ & $\mathrm{XX}$ & $\mathrm{XX}$ & \\
\hline c. Vegetable and Crops Production - Home/Placement. & $\mathrm{XX}$ & & & \\
\hline d. Agriculture Mechanics - School/Placement. & $\mathrm{XX}$ & $\mathrm{XX}$ & & \\
\hline e. Beef and Dairy - Market, Breeding, Placement. & $\mathrm{XX}$ & & & \\
\hline f. Swine - Market, Breeding, Placement. & & $\mathrm{XX}$ & & \\
\hline g. Sheep and Goats - Market, Breeding, Placement. & & & $X X$ & \\
\hline h. Rabbit, Poultry, and Small Animal - Market, Breeding & $\mathrm{XX}$ & & & \\
\hline i. Pleasure Horses. & & & & \\
\hline j. Work Experience - Other Than Above. & $\mathrm{XX}$ & $\mathrm{XX}$ & $\mathrm{XX}$ & $\mathrm{XX}$ \\
\hline
\end{tabular}

4. COUNTY FAIR EXHIBIT ADVISOR SUPERVISION
\begin{tabular}{|l|c|c|c|c}
\hline a. Entry Form Completion - All Areas. & Uchiyama & Jones & $\begin{array}{c}\text { Blod- } \\
\text { gett }\end{array}$ & $\begin{array}{c}\text { Noro- } \\
\text { ian }\end{array}$ \\
\hline \begin{tabular}{l|c|} 
b. Ag. Science, Ag. Mechanics, O.H. Exhibits. \\
c. County Fair Ag. Feature Booth Exhibit.
\end{tabular} & $\mathrm{XX}$ & $\mathrm{XX}$ & $\mathrm{XX}$ & $\mathrm{XX}$ \\
\hline d. County Fair FFA Scrapbook Entry. & & $\mathrm{XX}$ & $\mathrm{XX}$ & $\mathrm{XX}$ \\
\hline e. MCF and SCC Fair Livestock Ed. Display Posters. & $\mathrm{XX}$ & & $\mathrm{XX}$ & \\
\hline $\begin{array}{l}\text { f. Fair Eligibility and Chaperone Contracts, Participation } \\
\text { and Housing Rules, Medical and Transport Waivers, } \\
\text { School Release Forms, Private Car Use Clearance. }\end{array}$ & $\mathrm{XX}$ & $\mathrm{XX}$ & $\mathrm{XX}$ & $\mathrm{XX}$ \\
\hline g. Salinas Valley Fair Supervision. & $\mathrm{XX}$ & $\mathrm{XX}$ & $\mathrm{XX}$ & \\
\hline h. Monterey County Fair Supervision. & $\mathrm{XX}$ & $\mathrm{XX}$ & $\mathrm{XX}$ & \\
\hline i. Santa Cruz County Fair Supervision. & $\mathrm{XX}$ & & & \\
\hline j. Junior Livestock Auction - Buyer Solicitation. & $\mathrm{XX}$ & $\mathrm{XX}$ & $\mathrm{XX}$ & \\
\hline
\end{tabular}

5. SCHOOL FARM OPERATION and MANAGEMENT
\begin{tabular}{|l|c|c|c|c}
\hline a. Farm Manager. & Uchiyama & Jones & $\begin{array}{c}\text { Blod- } \\
\text { gett }\end{array}$ & $\begin{array}{c}\text { Noro- } \\
\text { ian }\end{array}$ \\
\hline b. Farm Use Plan and Operation Policy Development. & $\mathrm{XX}$ & & & \\
\hline $\begin{array}{l}\text { c. Farm (Act. 180) Financial Accounting and Records } \\
\text { Monitoring. }\end{array}$ & $\mathrm{XX}$ & & & \\
\hline d. Farm Workday Schedule & $\mathrm{XX}$ & & & \\
\hline e. Facility Maintenance, Refuse Disposal, Weed Control & $\mathrm{XX}$ & & & \\
\hline f. Facility Repairs, Supplies, and Equipment Needs. & $\mathrm{XX}$ & $\mathrm{XX}$ & $\mathrm{XX}$ & $\mathrm{XX}$ \\
\hline g. New Construction Supplies, and Equipment Needs. & $\mathrm{XX}$ & $\mathrm{XX}$ & $\mathrm{XX}$ & \\
\hline h. Power Equipment Purchases, Repair, Maintenance. & $\mathrm{XX}$ & $\mathrm{XX}$ & $\mathrm{XX}$ & \\
\hline i. Farm/County Lease Terms Review and Renewal & $\mathrm{XX}$ & & & \\
\hline j. Student SAE Use Agreement. & $\mathrm{XX}$ & $\mathrm{XX}$ & $\mathrm{XX}$ & $\mathrm{XX}$ \\
\hline k. Equipment and Tool Maintenance and Repairs. & $\mathrm{XX}$ & $\mathrm{XX}$ & $\mathrm{XX}$ & $\mathrm{XX}$ \\
\hline
\end{tabular}




\section{Substitute Teacher Procedure}

Before getting a substitute at North Salinas High School we need an approved School Business Agreement by the principal as well as the district. When the school business agreement has been approved we go onto Aesop education online, which is an automated substitute placement and management system, and submit our absence. From there we write our lessons and go to the necessary event or conference. 


\section{AGRI CULTURE SCIEMEE $1-2$

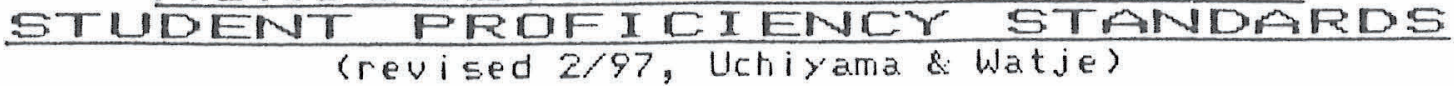

Upon completion of this course, all students will, at a minimum, have a satisfactory understanding of; be able to perform; or will have accomplished the following criteria identified as important proficiencies for Salinas Union High School District agriculture education students.

\section{$v_{\overline{7}}$ UNITED STATES AND INTERNATIONAL AGRICULTURE}

1. Be able to identify geographical locations (on map of U.S.) of production areas--corn, cotton, dairy, wheat, range and forage, citrus, vegetable, etc.

2. Understand the economic importance of the top 5 U.S. agricultural export and import commodities.

3. Have a knowledge of National Free Trade Agreement (NFTA) as it applies to agricuture.

4. Know what the USDA is and have a knowledge of what their function is.

\section{CALIFORNIA AGRI CULTURE}

1. Have a knowledge of the 7 California agricultural production regions and their main commodities produced.

$\bar{z}$. inderstand the economic importance of the Laífornia Agricuiture to the State's economy and economy of the United States.

3. Have a knowledge of the top 5 agricultural commodities produced in Cal ifornia.

4. Know 5 agricultural commodities that are 90-100\% produced in Cal ifornia.

5. Know what the CDFA is and have a knowlege of their services.

\section{MONTEREY COUNTY AGRI CULTURE}

1. Be able to describe the difference between North County and South County agriculture--commodities grown, growing season (climate) conditions, and water availability.

2. Kriow the top 10 agricultural commodities produced in Monterey county.

3. Have a krowledge of governmental agencies found in Monterey County and their function. Examples: Ag. Comissioner, Ag. Extension Service, Soil Conservation Society, etc. 
4. Have a knowledge of special agriculture interest groups and boards serving Monterey County. Example: Farm Bureau, Grower-Shipper. Vegetable Association, Young Farmers, Cattlemen's Associatin, Calif. Agricultural Production Association, Calif. Women in Agriculture, etc.

5. Understand the economic importance of Monterey County agriculture to local and state economies.

\section{ANIMAL SCIENCES}

1. Understand the importance of domestic livestock to food production and services to mankind.

2. Know the major external body parts of cattle, swine, sheep, horses, poúutry, rabbits, dairy goats, and dairy cattle.

3. Kriow the correct sex terms for cattle, swine, sheep, horses, poultry, rabbits, and goats.

4. Know the estrus and gestation cycle days for cattle, swine, sheep, horses, poultry, rabbits, and goats.

5. Be able to calculate the dressing percent for all meat animals."

6. Knowledge of and be able to calculate average daily gains, feed conversion ratios, and daily feeding percentages of all meat animals.

7. Identify three breeds of cattle, swine, sheep, horses, poultry, rabbits, goats, and dairy eattle.

8. Identify the wholesale cuts of cattle, swine, sheep, and poultry.

9. Know the difference between monogastric and ruminant digestive systems.

10. Understand the function of and be able to identify major body organs. Example: Heart, liver, lungs, small and large intestines, etc.

11. Understand the function of the respiratory and circulatory systems.

12. Identify the major parts of the male and female reproductive system.

13. Use a compound microscope to identify and label the parts a sperm cell.

14. Make written or oral report on a selected internal or external livestock parasite.

15. Know proper storage of, disposal of, and demonstrate safe handling of livestock medicines. Vaccines, dusts, oral bolus and powders, feed addi tives.

16. Know acceptable methods of restraining livestock and demonstrate proper techniques for intramuscular and subcutarieous injections, including proper injection locations.

17. Able to read and understand medicine label information and directions. 
18. Make written or oral report on a selected livestock disease.

19. Know proper methods of handling livestock for transport and safety in daily handling practices, including correct completion of CDFA cattle transportation slips.

20. Identify alfalfa hay, oat hay, grass hay, corn, oats, barley, wheat, milo, cottonseed, linseed, molasses, and fish meal feed ingredients.

21. Able to read and understand a feed label.

22. Know the difference between concentrate and roughage feeds, and purpose of levels of protein in feeds.

23. Understand acceptable feeding practices and daily nutritional requirements (feed and water needs) of livestock.

24. Define a balanced ration.

25. Know the acceptable market weights and grades for cattle, sheep, and swine.

26. List 10 acceptable management practices for the daily care of livestock.

27. Identify 10 commonly used pieces of equipment associated with veterinarian science, cattle, sheep, swine, and horse prduction, including describing their use.

28. Design a satisfactory housing and fencing facility for student selected livestock species.

29 Know normal body temperature range for selected livestock species, demonstrate how to take body temperature, and read a livestock

thermame ter.

30. Understand the major factors in selecting cattle, sheep, and swine for market arid breeding purposes.

31. Describe 1 commonly used methad of marketing Salinas Valley 1 ivestock.

\section{PLANT SCIENCE}

1. Understand the importance of oplants to food, shelter, and clothing production.

2. Identify the major plant parts and describe their function.

Example: roots, stems, leaves, bud, flower, and seed parts.

3. List 3 reasons why plants are important to mankind.

4. Understand the scientific classification system of plants.

5. Know the use classification of agricultural plants (vegetables, grains, fruits, nuts, citrus, vines, agronomic, forage, etc.) and i ist 3 examples of each.

6. Understand seed germination process and be able to calculate seed germination percent. 
7. List 3 differences between plant and animal cells.

8. Understand plant process of diffusion, osmosis, xylem and phloem tissue functions.

9. Identify 10 common edible portions of vegetable.

10. Make a collection of 10 vegetable crop seeds common to Salinas Valley.

11. Identify 5 weeds common to Salinas Valley.

12. Propagate plants from sexual and asexual methods.

13. Compléte a report on selected vegetable, ornamental, or other crop diseases.

14. Identify 5 harmful and beneficial insects common to agricultural plants.

15. Know the 4 layere of a soil profile and describe their characteristics.

16. Know the 3 primary elements (N-P-K) of a fertilizer mixture and the purpose of each element.

17. Know how to read a fertilizer label and calculate the actual fertilizer amount in the bag.

18. List 3 types of irrigation systems used in the Salinas Valley and describe how they are used.

19. Understand the importance of water quality and conservation to

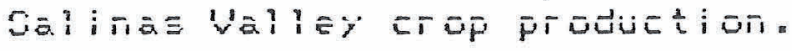

20. Understand the importance of safe handling, application, and disposal of agricultural chemicals.

21. Identify 10 large implements commonly used in Salinas Valley crop production.

22. Understand acceptable techniques used in harvesting, processing, storage, and transporting of major Salinas Valley crops.

23. Describe 1 commonly used method of marketing Salinas Valley crop products.

\section{LEADERSHIP}

1. Understand thé FFA organization structure, achievement opportunities, and FFA leadership programs by scoring $80 \%$ on local Best Informed Greenhand written test.

2. Complete minimum requirements for and receive the FFA Greenhand Degree.

3. Understand the basic principles of putlic speaking and give a 3 minute speech on an agricultural topic.

4. Demonstrate the ability to cooperatively work with other students by participating in 2 FFA Chapter activities or as a team member, in opening and Closing Ceremony and Parliamentary Procedure contest events. 
5. Understand 5 parliamentary procedure skills--main motion, amendment, refer to committee, previous question, postpone/table motion, and woting processes.

6. Attend and participate in 2 Chapter FFA meetings.

7. Construct a four year plan for personal leadership development.

\section{SUPERUISED OCCUPATIONAL EXPERIENCE}

1. Able to explain the difference between ownership and non-ownership enterprise programs.

2. Constritict a four year plan for SOE involvement, including plans for growth.

3. Complete application for Chapter or Section enterprise proficiency award.

\section{AGRICULTURE BUSINESS MANAGEMENT}

1 - List 3 reasons why record keeping is an important management practice.

2. Know the purpose of each section of the Calif. Vo-Ag Record Book and commonly used record keeping terms.

3. Keep an up-to-date Cal if. Vo-Ag Record Book on student selected enterprise by completing the following book sections:

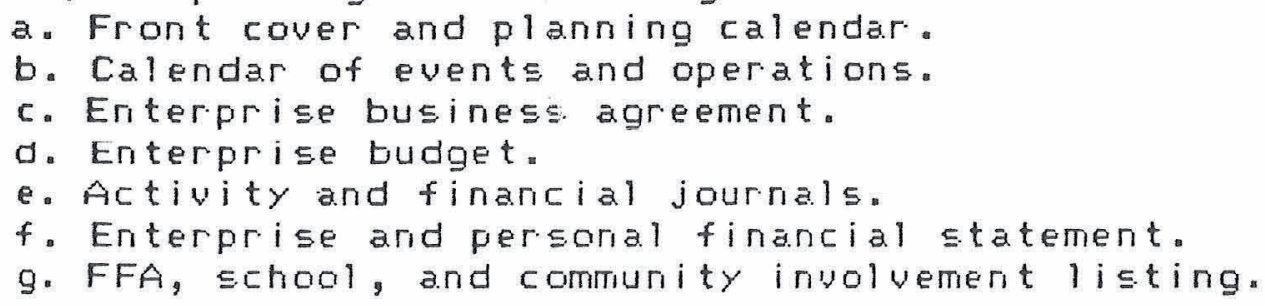

EMPLOYABILITY

1. Interpretate newspaper job listing descriptions.

2. Understand techniques for job search and types of jobs associated with levels of preparation and training.

3. Develop a personal resume using a computer.

\section{CAREERS}

1. Name the 7 ag; occupational areas and list 3 jobs related to each area.

2. Complete a job application in 20 minutes.

3. Complete student Program Planning and Career choice data sheet. 


\section{个 Hartnell College Secondary/Post Secondary Articulation Agreement}

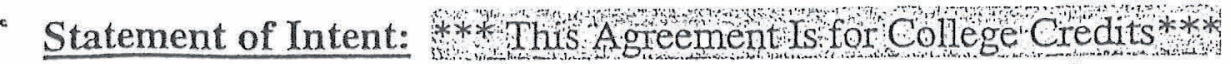

This agreement enables student to receive either a course waiver or college credit for completion of courses at * secondary level that are comparable to courses offered at Hartnell College. For secondary courses that

:fy a course waiver, completion of the course with a grade of " $C$ " or better will satisfy prerequisite requirements at Hartnell College for which the course is the equivalent to the prerequisite. For courses that are to receive Hartnell College credit, student must successfully complete a final examination, approve by Hartnell College faculty who normally teach the course in questions, or must successfully complete the Hartnell College Credit By Examination process. Unlike other nontraditional course at Hartnell College, which require 6 units of residency, students, upon successful completion of the process will receive college credit is based on the achievement of competencies through a courses, or sequence of courses, as defined in Attachment \#1, which specifies the condition of the articulation agreement.

Terms of the Agreement:

This agreement between Hartnell College \& Alvarez North Salinas, and Salinas High Schools.

Shall remain in force for a four-year period of time but shall be reviewed for possible revision each academic year. This review will include an examination of up-to-date course outlines and a discussion of current teaching methodologies. Either party of the agreement may terminate this agreement at the close of any school year by proper notice in writing to the Principal or Superintendent/President of the other party.

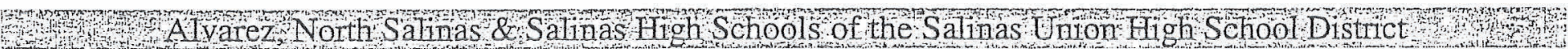
(SchooliDistrict)

agrees to certify, via the Course Waiver Form or the Credit by proficiency exam for those student who have successfully completed one year

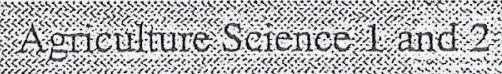

(Course (s) / Course Number(s))

lawluding the competencies listed on Attachment \#1 of this agreement. Upon receipt of either signed from the secondary educational institution name below. Hartnell College agrees to provide course waiver or college credit for Hartnell College courses(s)

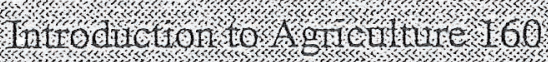

(Course(s)/ Course Number(s))

Articulation Contract Date:

Hartmell College

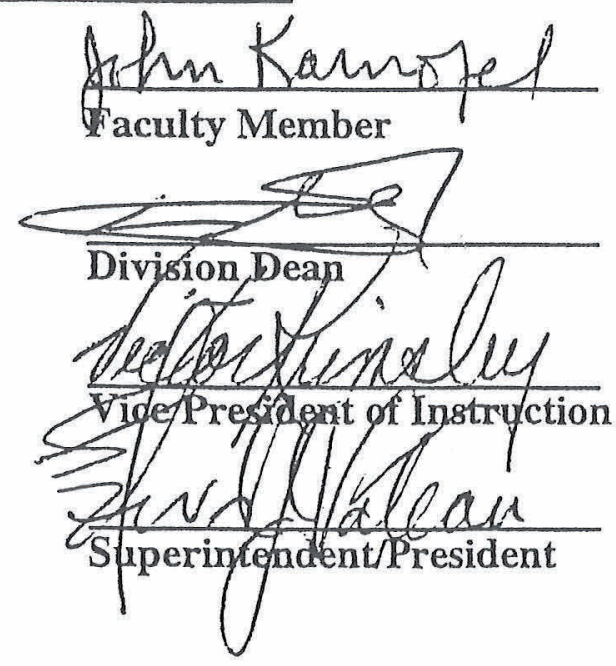

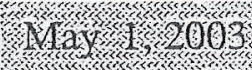

1.7. .2 .200 


\section{Ag. Scierice 1-2

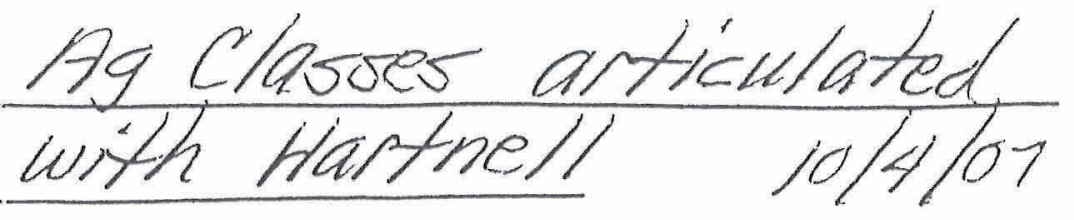 \\ Hig. Carstrution}

\begin{tabular}{|c|c|c|c|c|c|c|c|c|c|c|c|c|c|c|c|c|}
\hline 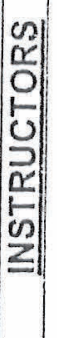 & 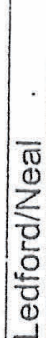 & 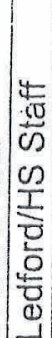 & $\left\{\begin{array}{l}\frac{4}{0} \\
\frac{0}{0} \\
0 \\
\frac{1}{5} \\
\frac{0}{0} \\
0 \\
\frac{0}{5} \\
0\end{array}\right.$ & $\mid \begin{array}{c}5 \\
\frac{5}{J} \\
0 \\
0 \\
0 \\
0 \\
0 \\
0 \\
\frac{0}{0} \\
0\end{array}$ & 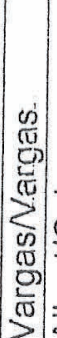 & 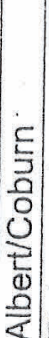 & $\begin{array}{l}\frac{2}{2} \\
\frac{0}{3} \\
\frac{d}{0} \\
\frac{1}{0}\end{array}$ & $\mid \frac{\frac{2}{0}}{\frac{0}{0}}$ & $\begin{array}{l}\frac{\vec{d}}{\bar{D}} \\
\frac{\pi}{2} \\
\frac{0}{2}\end{array}$ & $\frac{\frac{\partial}{\omega}}{\frac{0}{0}}$ & 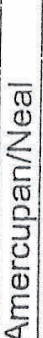 & 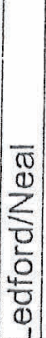 & $\frac{\frac{7}{\omega}}{\frac{\omega}{0}}$ & 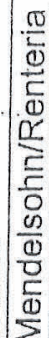 & 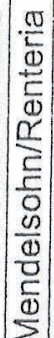 & 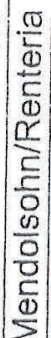 \\
\hline
\end{tabular}

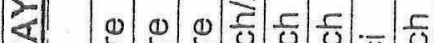

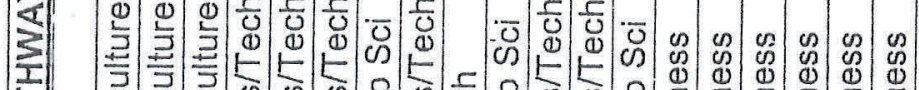

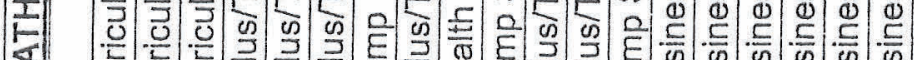

ㄴ. 可 它

क

$\mathscr{D} E$ E E E E E

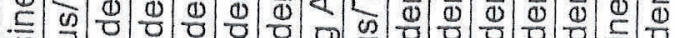

言学

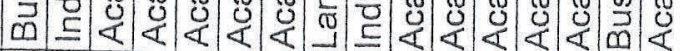

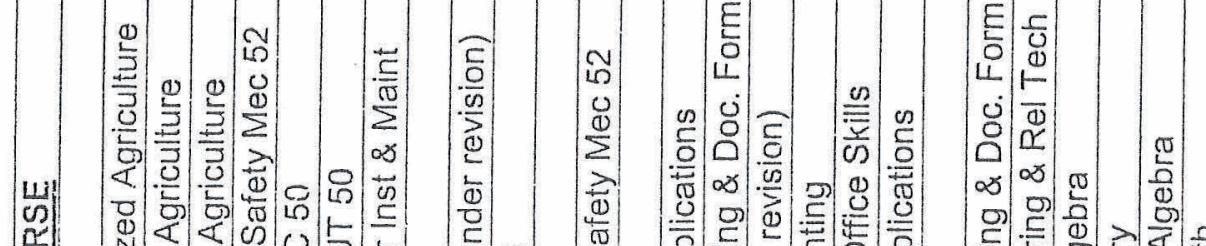

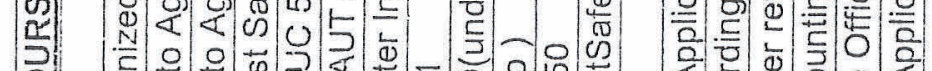

竞

돈은온 믇 등 늘

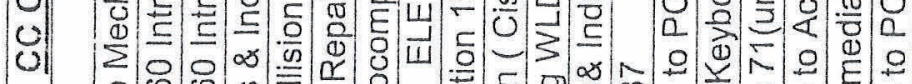

응

단:

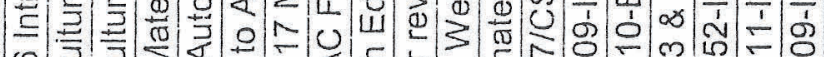

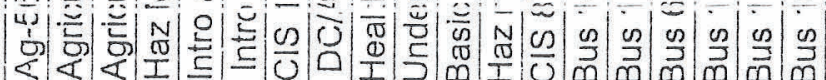

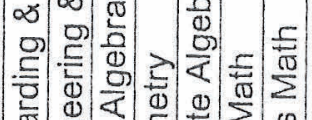

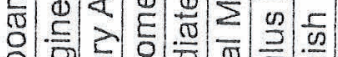

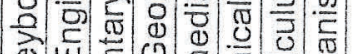

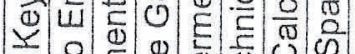

아를

D)

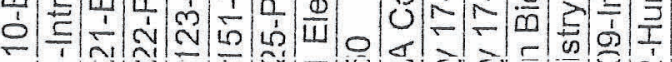

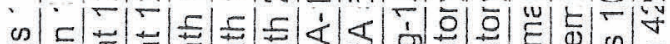

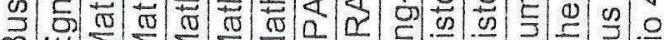

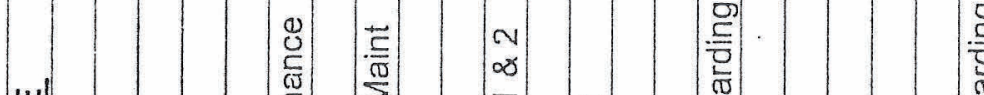

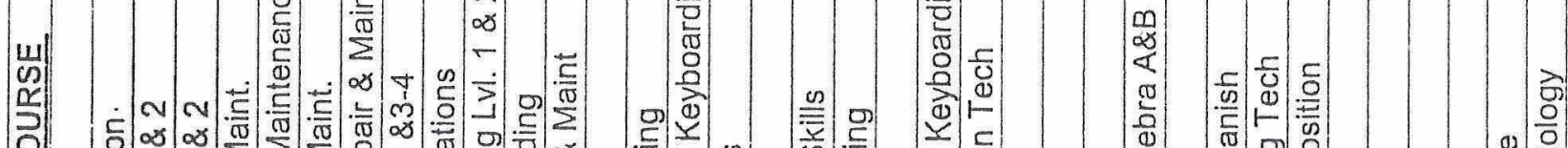

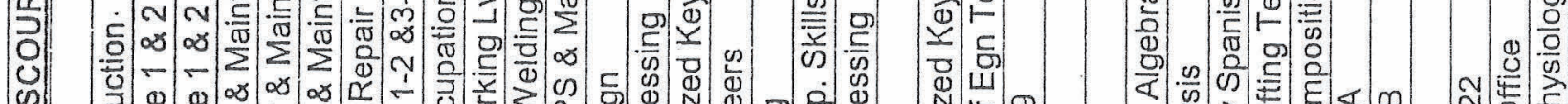 \\ (5) \\ क人

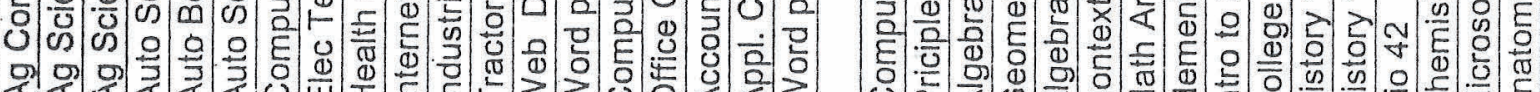

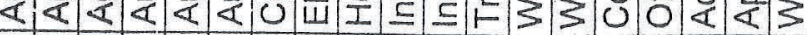 U人 ব)}

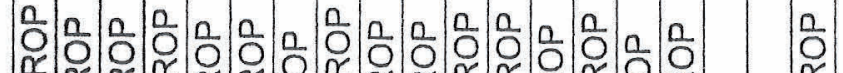

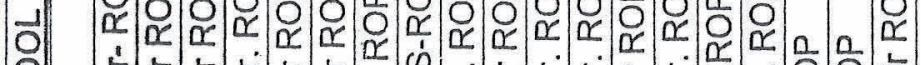

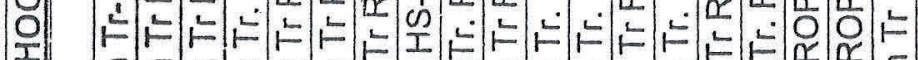
내

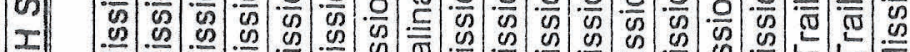

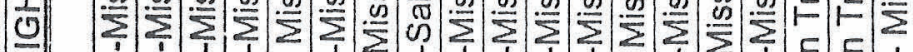
ด่

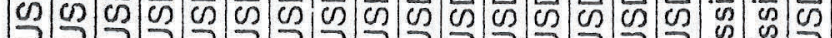

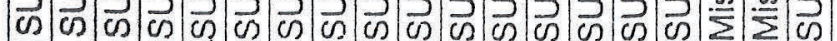

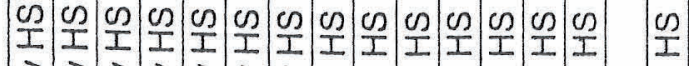

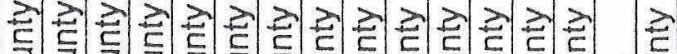

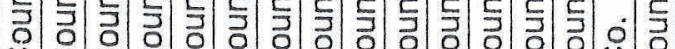

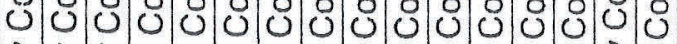

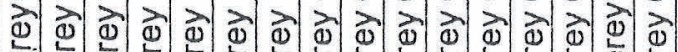

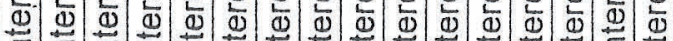

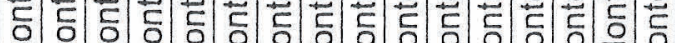
$\sum \sum \sum \sum \sum \sum \sum \sum \sum \sum \sum \sum \sum \sum \sum \sum$

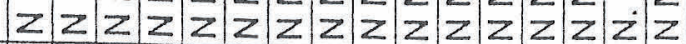

品 $======$

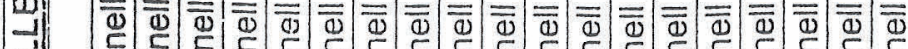

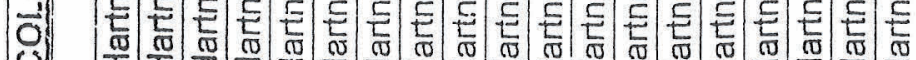
$\overline{\bar{\alpha}} \overline{\bar{\alpha}} \overline{\overline{0}} \overline{\overline{0}} \overline{\bar{\alpha}} \overline{\bar{\alpha}} \overline{\bar{\alpha}} \overline{\bar{\omega}} \overline{\overline{0}} \overline{\overline{0}} \overline{\bar{\alpha}} \overline{\bar{\alpha}} \overline{\bar{\omega}}$ 든 든든든든

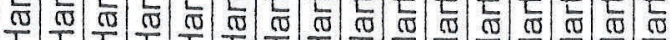

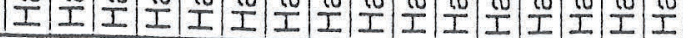


White-MCOE Copy

Canary-A/P File Copy

Pink-Ret. To Claimant With Check

1 nrod-Retain by Claimant
SALINAS UNION HIGH SCHOOL DISTRICT

\section{CLAIM FOR REIMBURSEMENT}

OF TRAVEL EXPENSES

claimant Stephanie BloqgeH Date August a009 Work Site or Address 1097 Handingsti Squinos. Cf q3906 Indicate where reimbursemeht check is to be returned or mailed.

\begin{tabular}{|c|c|c|c|c|c|c|c|}
\hline & & & & & & Misc & cellaneous \\
\hline Date & Location & Reason & Mileage & Lodging & Meals & Expense & Explanation \\
\hline $7 / 31 / 09$ & Salinas, CA & MoGoFair & & & & $5-$ & leader po \\
\hline $8 / 11 / 09$ & Monterey CA & H Moro Fain - & & & 12 & & lunch \\
\hline & & anima transpor & ation & & 20 & & dinner \\
\hline & & and weigh in & & & & & \\
\hline $8 / 12 / 09$ & Monterey CA & Moco Pain- & & & 12 & & lunch \\
\hline & & swine show doy & & & & & \\
\hline & & student supenh & 5100 & & & & \\
\hline $8 / 13 / 09$ & Monterey CA & MoCoFair- & & & 8 & & bneakfa \\
\hline & & sheep show day & & & 12 & & lunch \\
\hline & & student supen & sion & & & & \\
\hline$1 0 1 4 \longdiv { 4 0 9 }$ & Monteney CA & 110CoFair - & & & 12 & & lunch \\
\hline & & Ruiblit show ây & & & & & \\
\hline & & student supents & 5101 & & & & \\
\hline & & & & & & & \\
\hline & The conference b & orochure/fiyer with re & gistratio & breakdo & & & \\
\hline & must be attached & to claim. & & & & & \\
\hline & If registration fee & s include meals, cla & mant can & hot doub & claim. & & \\
\hline & & & & & & & \\
\hline Milea & tion Miles & X Rate & $\$$ & $\$$ & 76 & $\$ 5$ & \\
\hline
\end{tabular}

\begin{tabular}{|c|c|c|c|c|c|c|c|c|c|}
\hline Fund & Resource & PY & Goal & Function & Object & School & Local & Manager & Total Amount \\
\hline 0101 & 70100 & 0 & 11100 & 10000 & 520000 & 005 & 0000 & 025 & $\$ 81-$ \\
\hline & & & & & 520000 & & & & $\$$ \\
\hline & & & & & & & & & $\$$ \\
\hline & & & & & & & & & $\$$ \\
\hline
\end{tabular}

I hereby certify this claim is a true statement of actual and necessary expenses incurred in performance of District business.

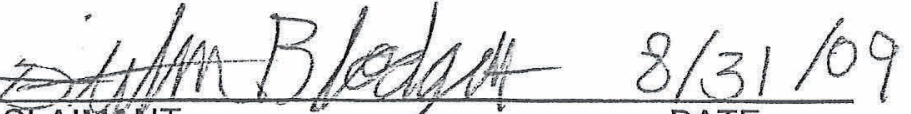

CLAIMANT

DATE
APPROVED BY

DATE

Please staple two copies of all backup to claim. See back of claim for description of required backup.

HSD $36(1 / 03)$ 
White-MCOE Copy

Canary-A/P File Copy

Pink-Ret. To Claimant With Check

is inrod-Retain by Claimant

\section{SALINAS UNION HIGH SCHOOL DISTRICT}

\section{CLAIM FOR REIMBURSEMENT \\ OF TRAVEL EXPENSES}

claimant Stephanie Blodgett Date Algust 2009-Septemie

indicate where reimbursement check is to be returned or mailed.

Miscellaneous

\begin{tabular}{|c|c|c|c|c|c|c|c|}
\hline Date & Location & Reason & Mileage & Lodging & Meals & Expense & Explanation \\
\hline $8 / 1569$ & Monteney, CA & Mo Co-stack & & & 8 & & bre \\
\hline & & auction day & & & 12 & & lunch \\
\hline & & Strudent sobem & $\operatorname{sic} n$ & & & & \\
\hline $8 / 1$ & Montens CA & MoCo-10adou & 1 & & 8 & & bmeqke \\
\hline & & and anand das & & & 12 & & lond \\
\hline & Montene, CA & Mo(o-still' & & & 12 & & lonch \\
\hline & & exhihit remo & (9) & & & & \\
\hline & & student trans & SOP. & & & & \\
\hline 81 & S9lings. CA & Santa Gure Ca & & & \& & 1.5 & $\operatorname{cases} \theta$ \\
\hline & & Fain-Leader Po & ss Fep & & & & tee \\
\hline, $18 / 09$ & Watsomille C & LSanta Cruz Co. & & & 20 & & dinnen \\
\hline & & Fain stock eg & ntest & & & & \\
\hline & & student transA & UP, & & & & \\
\hline $9 / 17 / 09$ & hatsonnille, C & Santu Cruz co & Fin & & 20 & & dinnen \\
\hline & \multirow{3}{*}{\multicolumn{5}{|c|}{$\begin{array}{l}\text { The conference brochufe/flyor with registration breakdown } \\
\text { must be attached to claim. } \\
\text { If registration fees include meals, claimant cannot double claim. }\end{array}$}} & & \\
\hline & & & & & & & \\
\hline & & & & & & & \\
\hline & & & & & & & \\
\hline & & X Rate_ & & $\$$ & 92 & $\$ 15$ & \\
\hline
\end{tabular}

\begin{tabular}{|c|c|c|c|c|c|c|c|c|c|}
\hline Fund & Resource & PY & Goal & Function & Object & School & Local & Manager & Total Amount \\
\hline 0101 & 70100 & 0 & 11100 & 10000 & 520000 & 005 & 0000 & 09 & $\$ 107$ \\
\hline & & & & & 520000 & & & & $\$ 1$ \\
\hline & & & & & & & & & $\$$ \\
\hline & & & & & & & & & $\$$ \\
\hline
\end{tabular}

I hereby certify this claim is a true statement of actual and necessary expenses incurred in performance of District business.

107

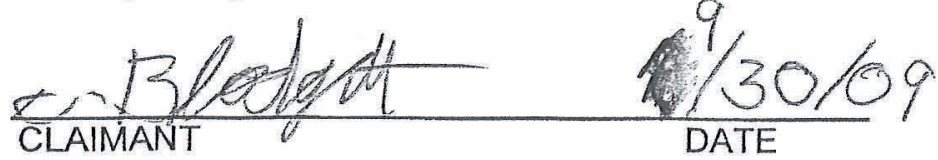

Please staple two copies of all backup to claim. See back of claim for description of required backup.

HSD $36(1 / 03)$ 
White-MCOE COpy

Canary-A/P File Copy

Pink-Ret. To Claimant With Check

I nrod-Retain by Claimant

\section{SALINAS UNION HIGH SCHOOL DISTRICT}

\section{CLAIM FOR REIMBURSEMENT \\ OF TRAVEL EXPENSES}

claimant Steplnanie Blodgett Date Octaber-Januany 2000

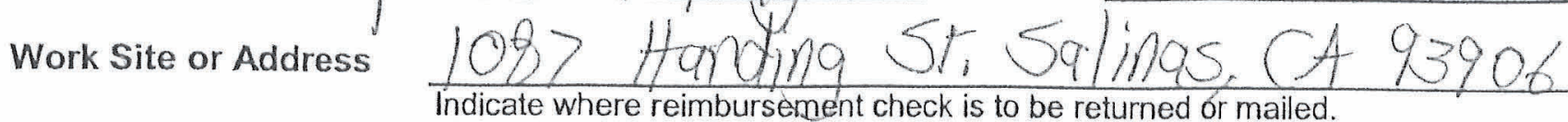

\begin{tabular}{|c|c|c|c|c|c|c|c|}
\hline \multirow{3}{*}{\begin{tabular}{|c|} 
Date \\
$9 / 19 / 69$ \\
\end{tabular}} & \multirow{2}{*}{ Location } & \multirow[b]{2}{*}{ Reason } & \multirow[b]{2}{*}{ Mileage } & \multirow[b]{2}{*}{ Lodging } & \multirow[b]{2}{*}{ Meals } & \multicolumn{2}{|c|}{ Miscellaneous } \\
\hline & & & & & & Expense & Explanation \\
\hline & atsonvilleca & Santa Cne Co & & & 8 & & breakta \\
\hline & & Fair Auction & $y$ & & 12 & & lunch \\
\hline & & student transal & Sp & & & & \\
\hline $10 / 11 / 09$ & Hollisten, CA & Fall, CATA mta & $T$ & & 8 & & breakfas \\
\hline & & and FFA COU & C & & 12 & & lunch \\
\hline $12 / 9 / 09$ & King City, CA & FFA Cneed and & & & 20 & & dinnen \\
\hline & & pahlic speaking & & & & & \\
\hline & & contests, student & $2000 / A$ & ans & & & \\
\hline $14 / 09$ & Paso Rahles, a & Regionol AaEd & & & 8 & & breakta \\
\hline & & CAYA In-Sen & ce & & & & \\
\hline & & Mta- Cal Poly $\$$ & 20 & & & & \\
\hline $1 / 16 / 10$ & Los Banos, G & SHFLireśluck & $-5 q t$ & & 8 & & breakta \\
\hline & Turlock CA & turlock+Men & ced & & 20 & & dimpep \\
\hline & Merred, CA & stodent trans & 500 & & & & \\
\hline & The conference br & rochure/flyer with reg & istration & breakdo & & & \\
\hline & must be attached & to claim. & & & & & \\
\hline & If registration fees & include meals, clain & nant can & lot doubl & claim. & & \\
\hline & & $s=1$ & & & & & \\
\hline & & $\mathrm{x}$ & & $\$$ & $\$ 96$ & $\$$ & \\
\hline
\end{tabular}

\begin{tabular}{|c|c|c|c|c|c|c|c|c|c|}
\hline \multirow{5}{*}{$\begin{array}{l}\text { Fund } \\
0 \mid 01\end{array}$} & Resource & PY & Goal & Function & Object & School & Local & Manager & Total Amount \\
\hline & 70100 & 0 & 11100 & 10000 & 520000 & 005 & 0000 & 0.5 & $\$ 96$ \\
\hline & & & & & 520000 & & & & $\$$ \\
\hline & & & & & & & & & $\$$ \\
\hline & & & & & & & & & $\$$ \\
\hline
\end{tabular}

I hereby certify this claim is a true statement of actual and necessary expenses incurred in performance of District business.

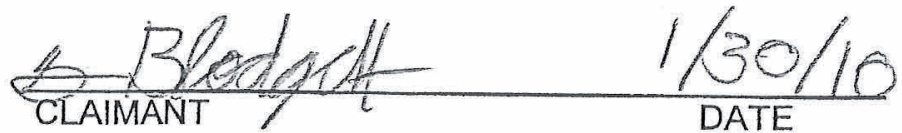

Please staple two copies of all backup to claim. See back of claim for description of required backup. 
White-MCOE Copy

Canary-A/P File Copy

Pink-Ret. To Claimant With Check

r mrod-Retain by Claimant
SALINAS UNION HIGH SCHOOL DISTRICT

\section{CLAIM FOR REIMBURSEMENT \\ OF TRAVEL EXPENSES}

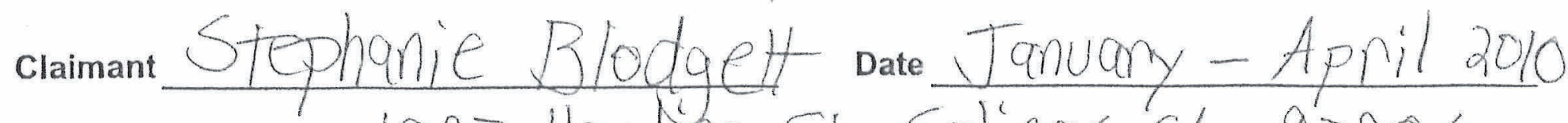

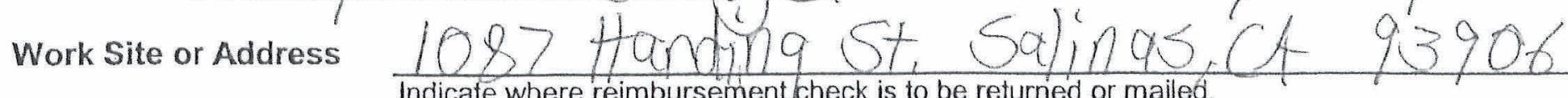
Indicate where reimbursement check is to be returned or mailed.

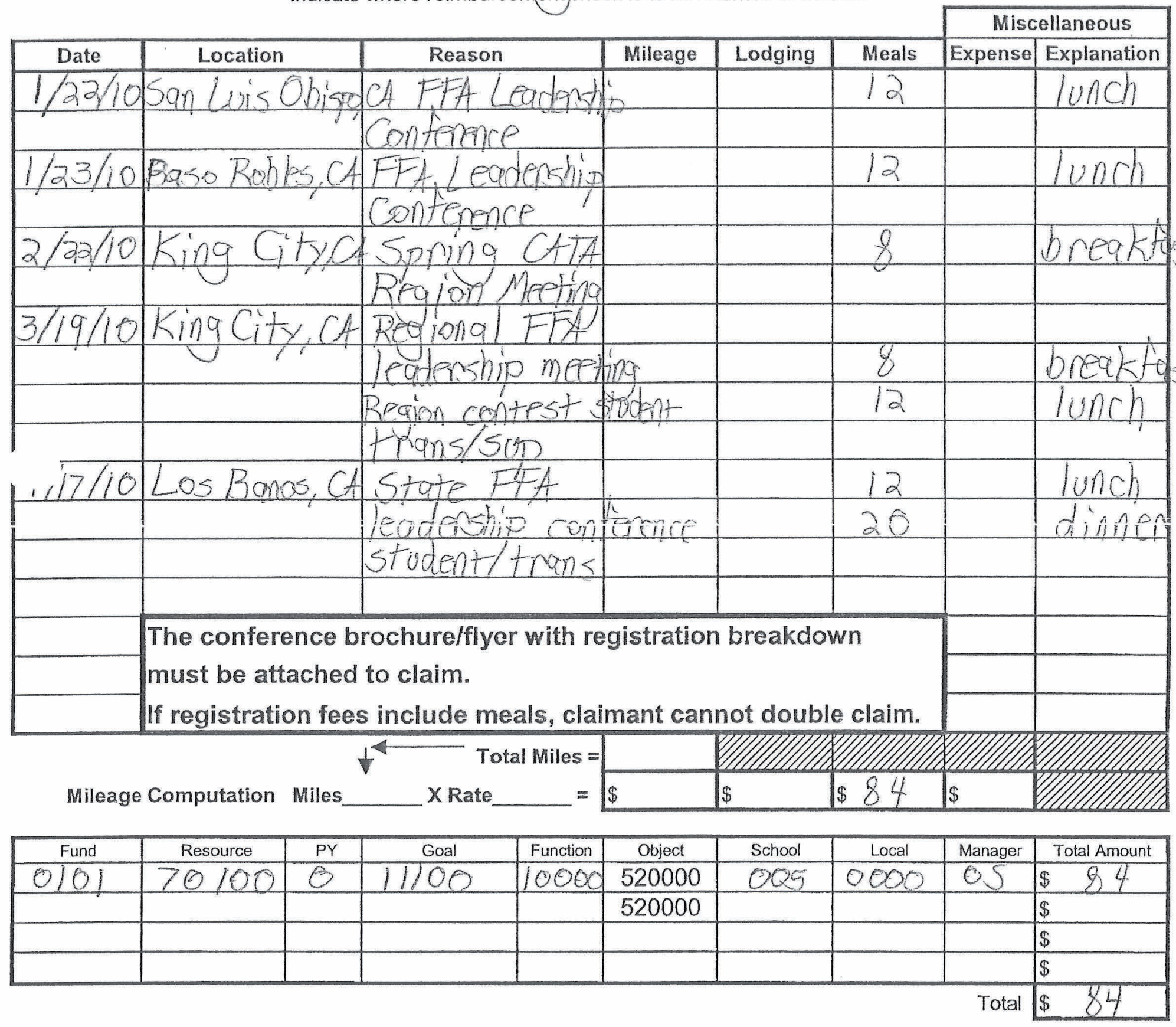

I hereby certify this claim is a true statement of actual and necessary expenses incurred in performance of District business.

$\therefore 2$ Retent $4 / 30 / 10$

CLAIMANT DATE
APPROVED BY

Please staple two copies of all backup to claim. See back of claim for description of required backup.

HSD $36(1 / 03)$ 
White-MCOE Copy

Canary-A/P File Copy

Pink-Ret. To Claimant With Check

Ir anrod-Retain by Claimant
SALINAS UNION HIGH SCHOOL DISTRICT

\section{CLAIM FOR REIMBURSEMENT \\ OF TRAVEL EXPENSES}

claimant Stephanie Blogloft Date Manch-April aolo

Work Site or Address

1087 Handina St, Salinas, CA 93906

Indicate where reimbursement check is to be returned or mailed.

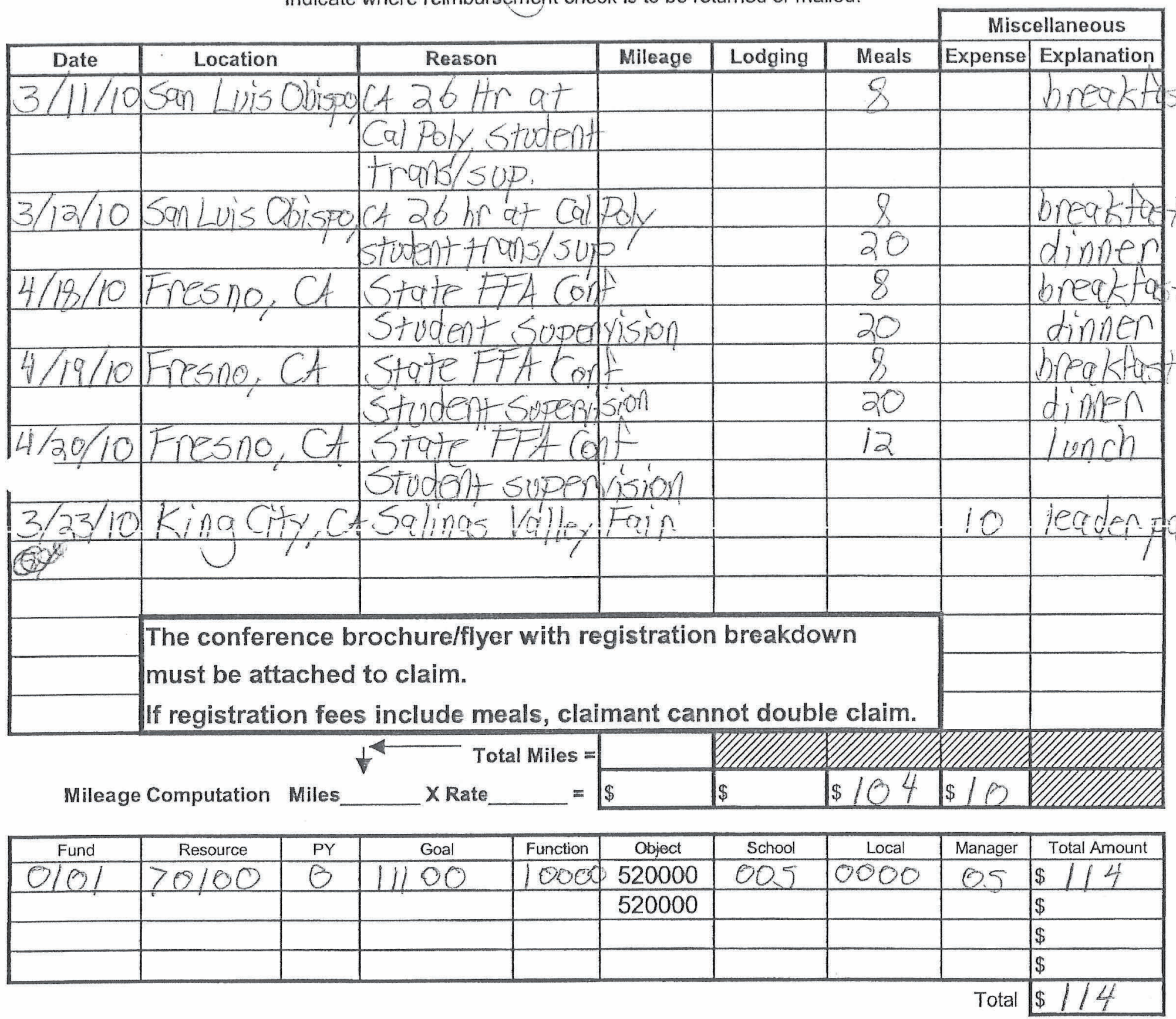

I hereby certify this claim is a true statement of actual and necessary expenses incurred in performance of District business.

- Plateil 4/39/10

CLAIMANT

DATE

APPROVED BY

DATE

Please staple two copies of all backup to claim. See back of claim for description of required backup.

HSD $36(1 / 03)$ 
White-MCOE Copy

Canary-A/P File Copy

Pink-Ret. To Claimant With Check

r mrod-Retain by Claimant

\section{SALINAS UNION HIGH SCHOOL DISTRICT}

\section{CLAIM FOR REIMBURSEMENT \\ OF TRAVEL EXPENSES}

claimant Stephanie Blodgett Date May 2010

Work Site or Address 1097 Hading St, Salinas. A 9390

Indicate where reimbursement check is to be returned or mailed.

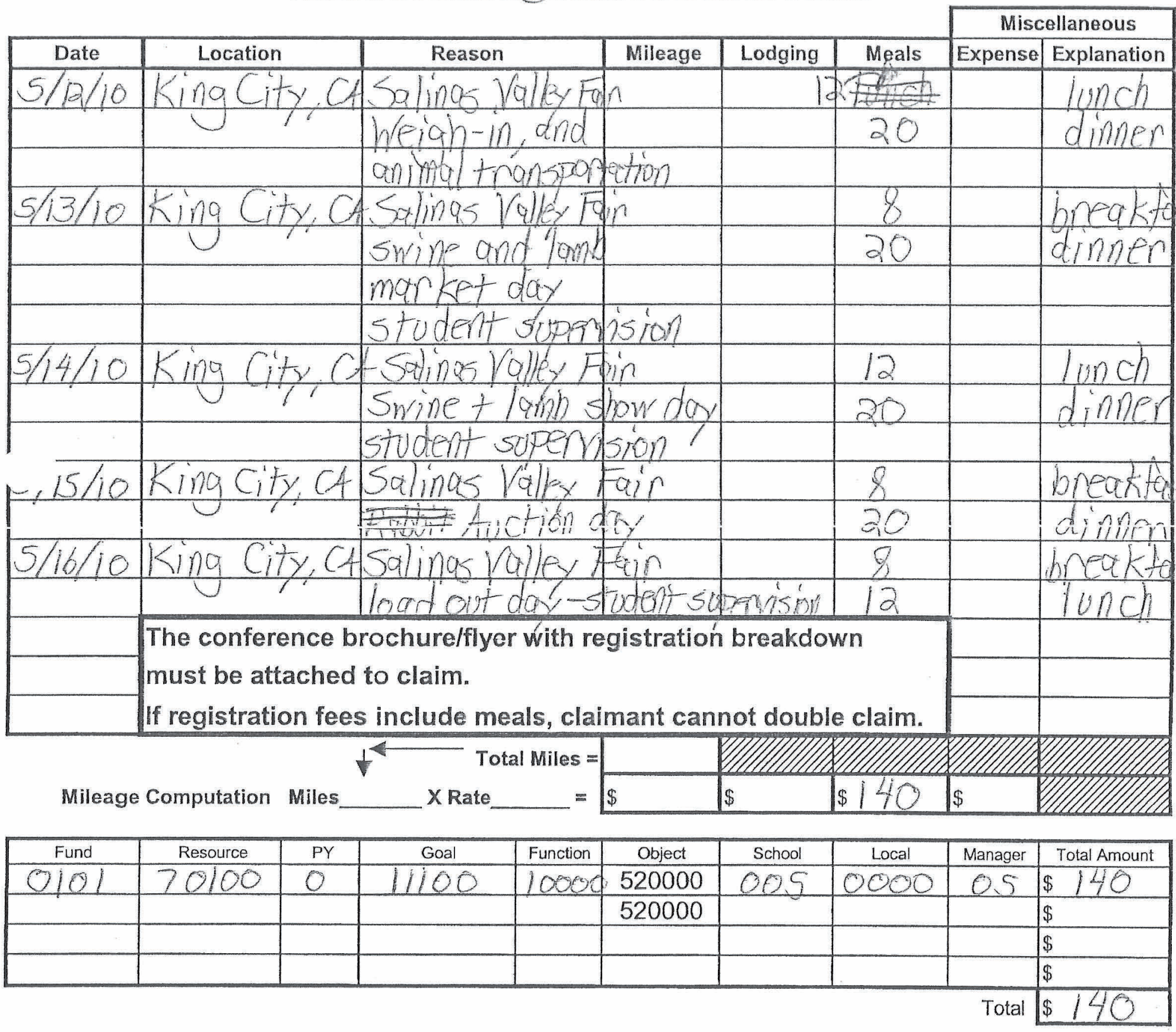

I hereby certify this claim is a true statement of actual and necessary expenses incurred in performance of District business.

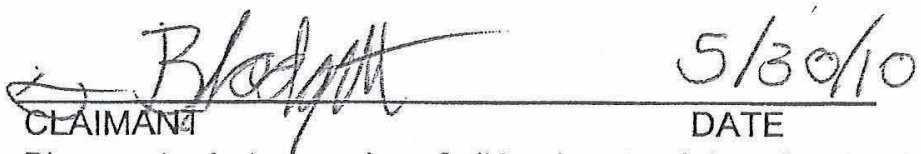

Please staple two copies of all backup to claim. See back of claim for description of required backup. 


\section{ALINAS HIGH SCHOOL \\ Salinas Union High School District \\ 55 Kip Drive, Salinas, CA 93906 \\ Phone (831) 796-7500 Fax (831) 796-7505}

August Caresani, Principal

Jill Camron, Assistant Principal

Bernardo Nuño, Assistant Principal

Mr. Greg Beard, Region Supervisor

Agriculture Education

State Department of Education

Agriculture Education and Comm. Department

Cal Poly State University

San Luis Obispo, California 93407

Dear Mr. Beard:

Our school's 2010-11 Agricultural Education Incentive Grant included $\$ 5,666$ approved for Criteria \#10, Student-Teacher ratio. Our October 15, 2010 R-2 enrollment report validated a 340 unduplicated student count and the following class enrollment data for 2.83 FTE teachers.

Ag. Earth Science $=30$ students

Ag. Earth Science $=24$ students

Ag. Earth Science $=26$ students

Ag. Chemistry $=26$ students

Ag. Chemistry $=21$ students
Ag. Mechanics 1-4 = 21 students

Ag. Earth Science $=24$ students

Ag. Earth Science $=26$ students

Ag. Econ/Govt. $=20$ students

October 27, 2010

In a letter filed with our original 2010-11 Incentive Grant Application, I offered rationale and requested consideration for a Student-Teacher ratio and non-financial penalty compliance waiver/variance. I also stated that a follow-up enrollment report would be filed by the November 15, 2010 due date of our program's final Incentive Grant compliance review report. In keeping with my commitment, below is an updated class enrollment analysis report.

For Criteria \#10, STUDENT-TEACHER RATIO, per the 2010-11 Agriculture Program Quality Criteria checklist, I submit the following CLASS ENROLLMENT DATA OF UNDUPLICATED students for your evaluation.

** STUDENT-TEACHER RATIO STANDARD: Shop and Laboratory classes have not more than 20 students. All other classes have no more than 25.

Total Unduplicated 2010-11 Class Enrollment --.-328 Students, October 26, 2010

Ag. Earth Science $=29$ students

Ag. Earth Science $=23$ students

Ag. Earth Science $=26$ students

Ag. Chemistry $=25$ students

Ag. Chemistry $=21$ students
Ag. Mechanics 1-4 $=20$ students

Ag. Earth Science $=24$ students

Ag. Earth Science $=25$ students

Ag. Econ/Govt $=18$ students
Ag. welding 1-4 $=21$. students Ag. Mechanics $1-4=25$ students Ag. Mechanics 1-4 $=25$ students Ag. Mechanics 1-4 $=24$ students

Ag. Science 1-2 $=22$ students

++ Teacher-Student class period average ratio: Ag. Science 1:23 and Shop classes $=1: 23$

For comparison, our District's contracted teacher-student class load ratio is:

Fall Semester

Classroom $=32$ student

Shop/Lab = 30 students Shop $/$ Lab $=26$ students
Spring Semester

Classroom $=29$ students

Shop $/$ Lab $=26$ students

mnoroian(a)salinas.k12.ca.us, jashen@,salinas.k12.ca.us 


\section{North Salinas High School}

\section{AGRICULTURAL SCIENCE CLASS AND LABORATORY ACTIUITY AWARENESS LETTER (reU. 2/04)}

Dear. Parent/Guardian of:

$$
\text { (student name--neatly print full riame? }
$$

Over the caurse of the year, agriscience students will have the cpportunity to experience many different animal and plant production aspects of vocatianal science that they may never otheruise see or participate in. These include, but are not limited to docking/castrating of livestock, handling livestack medicines, administering vaccinations, hoof trimming, treating animal wounds and suturing, performing autopsies notching and ear. tagging, harvesting/processing of iarge and small mest animals, and expasure to certain easily purchased plant pesticides fertilizers and plant grawthriesl th products. While we teel these are great practical application experiences for students, we realize not everyone feels the same, so the following applies:

- No one has to participate.

- Na student is forced to watch, touch, etc. They have the opticin of staying in an appointed location. Their grades will not be affected.

- Studente are asked to infarm the ir parents both before and after. the activity, using proper terminolggy they have learned.

- Ariy animals harvested/frocesed are dorie so for a purpase. They are raised as market animals, and reed to be processed for a buyer ar consumer.

- Students will not handle or be furposely exposed to restricted use chemical $\equiv$.

- Equipment and material handling safety practiceswill te taught to all students and they will be required ta follow the respective safe use and handling procedures.

If you have ary questions or concerns, please do not hesitate to call the North Sal inas High Sichool Agrieulture Department at (331) $796-7536$ or gur School Principel at (831) 7\%6-7510. Thank you for your understanding and sogperation.

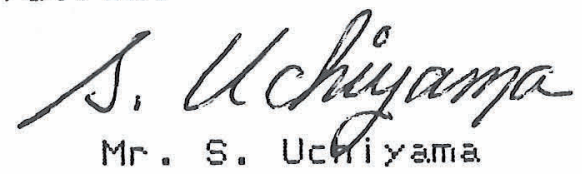

Agr i Science Teacher.
Sincerely,

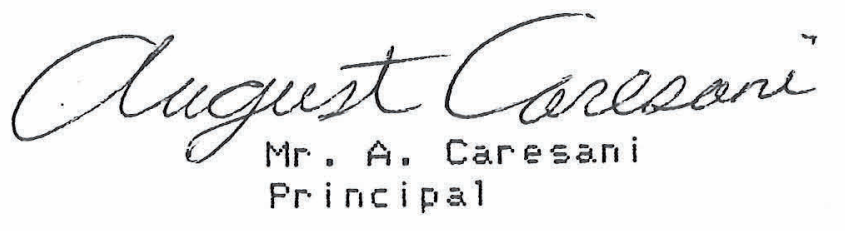

I have read the above, and underetand what my child may be exposed to.

(parent/guardian signature)

$$
\text { spros }
$$

(printed parent/guardian names

(date)

Parent/guardian comments: 